Computer Science \& Information Technology 19 

David C. Wyld

Natarajan Meghanathan

Dhinaharan Nagamalai

\section{Computer Science \& Information Technology}

Fourth International conference on Computer Science \& Information Technology (CCSIT)

Sydney, Australia, February 21 22, 2014 


\section{Volume Editors}

David C. Wyld,

Southeastern Louisiana University, USA

E-mail: David.Wyld@ selu.edu

Natarajan Meghanathan,

Jackson State University, USA

E-mail: nmeghanathan@jsums.edu

Dhinaharan Nagamalai

KTO Karatay University, Turkey

E-mail: dhinthia@yahoo.com

ISSN : $2231-5403$

ISBN : 978-1-921987-27-4

DOI : 10.5121/csit.2014.4201 - 10.5121/csit.2014.4242

This work is subject to copyright. All rights are reserved, whether whole or part of the material is concerned, specifically the rights of translation, reprinting, re-use of illustrations, recitation, broadcasting, reproduction on microfilms or in any other way, and storage in data banks. Duplication of this publication or parts thereof is permitted only under the provisions of the International Copyright Law and permission for use must always be obtained from Academy \& Industry Research Collaboration Center. Violations are liable to prosecution under the International Copyright Law.

Typesetting: Camera-ready by author, data conversion by NnN Net Solutions Private Ltd., Chennai, India 


\section{Preface}

Fourth International conference on Computer Science \& Information Technology (CCSIT-2014) was held in Sydney, Australia, during January 21 22, 2014. The Second International Conference on Signal, Image Processing \& Pattern Recognition (SIPP 2014), Second International Conference on Artificial Intelligence, Soft Computing (AISC-2014), The Third International conference on Parallel, Distributed Computing technologies \& Applications (PDCTA-2014), Third International conference on Natural language Processing(NLP-2014) were collocated with the CCSIT-2014. The conferences attracted many local and international delegates, presenting a balanced mixture of intellect from the East and from the West.

The goal of this conference series is to bring together researchers and practitioners from academia and industry to focus on understanding computer science and information technology and to establish new collaborations in these areas. Authors are invited to contribute to the conference by submitting articles that illustrate research results, projects, survey work and industrial experiences describing significant advances in all areas of computer science and information technology.

The CCSIT 2014, SIPP 2014, AISC 2014, PDCTA 2014, NLP 2014 Committees rigorously invited submissions for many months from researchers, scientists, engineers, students and practitioners related to the relevant themes and tracks of the workshop. This effort guaranteed submissions from an unparalleled number of internationally recognized top-level researchers. All the submissions underwent a strenuous peer review process which comprised expert reviewers. These reviewers were selected from a talented pool of Technical Committee members and external reviewers on the basis of their expertise. The papers were then reviewed based on their contributions, technical content, originality and clarity. The entire process, which includes the submission, review and acceptance processes, was done electronically. All these efforts undertaken by the Organizing and Technical Committees led to an exciting, rich and a high quality technical conference program, which featured high-impact presentations for all attendees to enjoy, appreciate and expand their expertise in the latest developments in computer network and communications research.

In closing, CCSIT 2014, SIPP 2014, AISC 2014, PDCTA 2014, NLP 2014 brought together researchers, scientists, engineers, students and practitioners to exchange and share their experiences, new ideas and research results in all aspects of the main workshop themes and tracks, and to discuss the practical challenges encountered and the solutions adopted. The book is organized as a collection of papers from the CCSIT 2014, SIPP 2014, AISC 2014, PDCTA 2014, NLP 2014.

We would like to thank the General and Program Chairs, organization staff, the members of the Technical Program Committees and external reviewers for their excellent and tireless work. We sincerely wish that all attendees benefited scientifically from the conference and wish them every success in their research. It is the humble wish of the conference organizers that the professional dialogue among the researchers, scientists, engineers, students and educators continues beyond the event and that the friendships and collaborations forged will linger and prosper for many years to come.

David C. Wyld

Natarajan Meghanathan Dhinaharan Nagamalai 



\section{Organization}

\section{General Chairs}

David C. Wyld

Natarajan Meghanathan

\section{Steering Committee}

Abdul Kadhir Ozcan

Brajesh Kumar Kaushik

Dhinaharan Nagamalai

Eric Renault

John Karamitsos

Khoa N. Le

\section{Program Committee Members}

Southeastern Louisiana University, USA Jackson State University, USA

The American University, Cyprus Indian Institute of Technology - Roorkee, India Wireilla Net Solutions PTY Ltd, Australia Institut Telecom-Telecom SudParis, France University of the Aegean, Samos, Greece University of Western Sydney, Australia

\author{
A Vadivel \\ A.G.Ananth \\ A.Kannan \\ Abdellatif BERKAT \\ Achhman Das Dhomeja \\ Ajay K Sharma \\ Alejandro Regalado Mendez \\ Alvin Lim \\ Amandeep Singh Thethi \\ Asghar gholamian \\ Ashok kumar Sharma \\ Ayad salhieh \\ Azween Bin Abdullah \\ Balaji Raj N \\ Binod Kumar Pattanayak \\ Buket Barkana \\ Carlos E. Otero \\ Ch.V.Rama Rao \\ Choudhari \\ D.Minnie \\ Deepak Laxmi Narasimha \\ Denivaldo LOPES \\ Dinesh Chandrajain \\ Ferdin Joe J \\ G.M. Nasira
}

National Institute of Technology Trichy, India

R.V. College of Engineering-Bangalore, India

K.L.N. College of Engineering, India

Abou-Bekr Belkadd University (Tlemcen), Algeria

University of Sindh, Pakistan

Dr B R Ambedkar NIT, India

Universidad del Mar. Mexico

Auburn University, USA

Guru Nanak Dev University Amritsar, India

Babol University of Technology, Iran

YMCA Institute of Engineering, India

Australian College at Kuwait, Kuwait

Universiti Teknologi Petronas, Malaysia

JJ College of Engineering and Technology, India

Siksha O Anusandhan University, India

University of Bridgeport, USA

The University of Virginia's College at Wise, USA

Gudlavalleru Engineering College, India

Bhagwati Chaturvedi College of Engineering, India

Madras Christian College, India

University of Malaya, Malaysia

Federal University of Maranhao - UFMA, Brazil

University of RGPV, India

Prathyusha Institute of Tech. \& Management, India

Sasurie College of Engineering, India 
Hao Shi

Hao-En Chueh

Henrique J. A. Holanda

Indrajit Bhattacharya

Jalel Akaichi

Jestin Joy

Jyoti Singhai

Jyotirmay Gadewadikar

K. Chitra

kalikiri nagi reddy

Khoa N. Le

Krishna Prasad E S N Ponnekanti (KP)

Krishnaveni

L.Jaba Sheela

lakshmi Rajamani

Lylia Abrouk

M. Dinakaran

M. P. Singh

M.Hemalatha

M.P Singh

M.Pravin Kumar

Madhan KS

Michel Owayjan

Mohammed Ali Hussain

Mohd. Ehmer Khan

Monika Verma

Narottam C. Kaushal

Nitiket N Mhala

Nour Eldin Elmadany

P.Ashok Babu

P.Shanmugavadivu

P.Thiyagarajan

Patrick Seeling

Pravin P. Karde

Premanand K.Kadbe

R. Murali

R.Baskaran

Rahul Vishwakarma

Raman Maini

Richard Millham

Roberts Masillamani

S.Sapna

S.Senthilkumar

Salman Abdul Moiz

Sandhya Tarar

Sanjay K, Dwivedi

Sanjay Singh
Victoria University, Australia

Yuanpei University, Taiwan, R.O.C.

UERN - Universidade do Estado do Rio Grande do

Norte

Kalyani Govt. Engg. College, India

University of Tunis, Tunisia

Federal Institute of Science and Technology, India

Electronics and Communication Deptt-MANIT, India

Alcorn State University, USA

Govt Arts College for Women, India

NBKR Institute of Science \& Technology, India

University of Western Sydney, Australia

Aditya Engineering College-Kakinada, India

Avinashilingam University for Women, India

Anna University, India

Osmania University, India

University of Burgundy, France

VIT University - Vellore, India

National Institute of Technology Patna, India

Karpagam University, India

National Instituite of Technology, India

K.S.R College of Engineering, India

Infosys Technologies Limited, India.

AUST, Lebanon

Sri Sai Madhavi Institute of Science \& Tech., India

Al Musanna College of Technology, Sultanate of Oman

Punjab Technical University, India

NIT Hamirpur, India

B.D.College of Engineering - Sewagram, India

Arab Acadmy for Science and Technology, Egypt

D.M.S.S.V.H. College of Engineering, India

Gandhigram Rural Institute - Deemed University, India

Pondicherry University, India

University of Wisconsin, USA

HVPM's College of Engg. \& Tech. - Amravati, India

Vidya Pratishthan's College of Engineering, India

Dr. Ambedkar Institute of Technology, Bangalore

Anna University - Chennai, India

Tata Consultancy Services,ACM, India

Punjabi University, India

University of Bahamas, Bahamas

Hindustan University, India

K.S.R College of Engineering, India

NIT - Tiruchirappalli, India

Centre for Development of Advanced Computing, India

Gautam Buddha University, India

Ambedkar Central University Lucknow, India

Manipal University, India 
Sanjoy Das

Sherif S. Rashad

Shin-ichi Kuribayashi

Shrirang.Ambaji.Kulkarni

Sundarapandian V

T Venkat Narayana Rao

Tien D. Nguyen

Tuli Bakshi

Utpal Biswas

V.Radha

Vijayanandh. R

Wichian Sittiprapaporn

wided oueslati

Zuhal Tanrikulu
Jawaharlal Nehru University, India

Morehead State University, USA

Seikei University, Japan

National Institute of Engineering, India

Vel Tech Dr. RR \& Dr. SR Technical University, India

Hyderabad ITM , India

Coventry University, UK

Calcutta Institute of Technology(WBUT), India

University of Kalyani, India

Avinashilingam University, India

Bharathiar Univ, India

Mahasarakham University, Thailand

l'institut superieur de gestion de tunis, Tunisia

Bogazici University, Turkey

\section{Technically Sponsored by}

Computer Science \& Information Technology Community (CSITC)

Software Engineering \& Security Community (SESC)
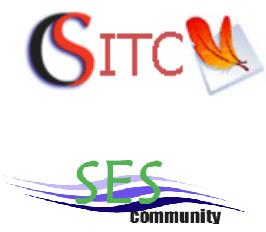

Digital Signal \& Image Processing Community (DSIPC)

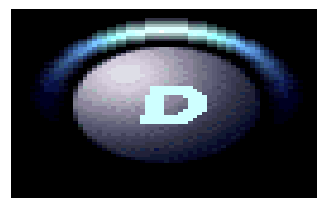

\section{Organized By}

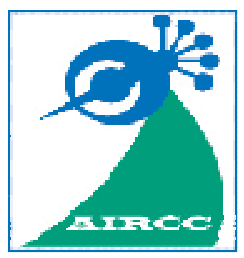

ACADEMY \& INDUSTRY RESEARCH COLLABORATION CENTER (AIRCC) 


\section{TABLE OF CONTENTS}

\section{Computer Science \& Information Technology}

ECSM: Energy Efficient Clustering Scheme for Mobile M2M Communication

Networks.

Mohammed Saeed Al-kahtani

Mining Developer Communication Data Streams.

Andy M. Connor, Jacqui Finlay and Russel Pears

A Distributed Agent Media Access Framework

Craig M. Gelowitz, Luigi Benedicenti and Raman Paranjape

Multilevel Techniques for the Clustering Problem.

Noureddine Bouhmala

Multi-Dimensional Customization Modelling Based on Metagraph for SAAS

Multi-Tenant Applications.

Ashraf A. Shahin

Effect of Colours in Manual Data Typing. Melih Kirlidog

Backtracking Based Integer Factorisation, Primality Testing and Square Root

Calculation

Mohammed Golam Kaosar

Human Interaction in the Regulatory of Telecommunications Infrastructure

Deployment in South Africa

Sharol Sibongile Mkhomazi and Tiko Iyamu

Parameter Space and Comparative Analyses of Energy Aware Sensor

Communication Protocols

Ittipong Khemapech

A Method to Identify Potential Ambiguous Malay Words Through Ambiguity

Attributes Mapping: An Exploratory Study.

Hazlina Haron and Abdul Azim Abd. Ghani 
Towards A Semantic for UML Activity Diagram Based on Institution Theory

for it's Transformation to Event-B Model.

Aymen Achouri

An Explicit Trust Model Towards Better System Security.

Orhio Mark Creado, Bala Srinivasan, Phu Dung Le and Jefferson Tan

Energy Efficient Load Balanced Routing Protocol for Wireless Sensor

Networks.

Alghanmi Ali Omar and ChongGun Kim

User Preferred Color Combination Design Using Interactive Genetic

Algorithm.

Tad Gonsalves and Ayumi Kawai

Process-Driven Software Development Methodology for Enterprise

Information System.

Kwan Hee Han and Yongsun Choi

Development and Evaluation of a Web Based Question Answering System for

Arabic Language.

Heba Kurdi, Sara Alkhaider and Nada Alfaifi

Signal, Image Processing \& Pattern Recognition

Coherence Enhancement Diffusion Using Robust Orientation Estimation.

Mohammad A. U. Khan, Tariq M. Khan, Wadee Al-Halabi, Hiba Shahid

and Yinan Kong

A Fast PU Mode Decision Algorithm for H.264/AVC to HEVC Transcoding 215 Jiunn-Tsair Fang, Zong-Yi Chen, Tsai-Ling Liao and Pao-Chi Chang

Off-Line System for the Recognition of Handwritten Arabic Character 227 Ahmed Sahloul and Cheng Suen.

A Novel Global Threshold-Based Active Contour Model.

Nuseiba M. Altarawneh, SuhuaiLuo, Brian Regan, ChangmingSun

Automatic Estimation of Live Coffee Leaf Infection Based on Image

Processing Techniques.

Eric Hitimana and Oubong Gwun

DAC for High Speed and Low Power Applications Using Abacus 267 Shankarayya G. Kambalimath 
Visual Saliency Model Using Sift and Comparison of Learning Approaches 275 Hamdi Yalin Yaliç

Violent Scenes Detection Using Mid-Level Violence Clustering. Shinichi Goto and Terumasa Aoki

Hybrid Technique Based on N-GRAM and Neural Networks for Classification of Mammographic Images.

Pradnya Kulkarni, Andrew Stranieri, Siddhivinayak Kulkarni, Julien Ugon and Manish Mittal

Natural Language Processing Through Different Classes of Machine

Learning. Harsh Jain and Keshav Mathur

3D Modelling, Simulation and Prediction of Facial Wrinkles Sokyna Al-Qatawneh, Ali Mehdi and Thamer Al Rawashdeh

\section{Artificial Intelligence, Soft Computing}

Neural Network Approach to Railway Stand Lateral SKEW Control.

Peter Mark Beněs, Matouš Cejnek, Jan Kalivoda and Ivo Bukovsky

Another Adaptive Approach to Novelty Detection in Time Series. Matouš Cejnek, Peter Mark Beněs and Ivo Bukovsky

Resource Allocation Using Metaheuristic Search. Andy M. Connor and Amit Shah

Are Evolutionary Algorithms Required to Solve Sudoku Problems?. 365 Sean McGerty and Frank Moisiadis

Hybrid Ant Colony Optimization for Real-World Delivery Problems Based on Real Time and Predicted Traffic in Wide Area Road Network. 379 Junichi Ochiai and Hitoshi Kanoh

The Research of Induced Current in Coils When the Process of Motion of Magnetically Levitated Planar Actuators Rougang Zhou, Yunfei Zhou, Guangdou Liu and Xiao Tu

Bayesian Methods for Assessing Water Quality. Khalil Shihab and Nida Al-Chalabi 
A Recommender System Sensitive to Intransitive Choice and Preference

Reversals.

Amir Konigsberg and Ron Asherov

Parallel Guided Local Search and Some Preliminary Experimental Results

for Continuous Optimization.

Nasser Tairan, Muhammad Asif Jan and Rashida Adeeb Khanum

Parallel, Distributed Computing technologies \& Applications

Design and Implementation of a Cache Hierarchy-Aware Task Scheduling for Parallel Loops on Multicore Architectures.

Nader Khammassi and Jean-Christophe Le Lann

Real Time Face Detection on GPU Using OPENCL

Narmada Naik and Rathna.G.N

Positive Impression of Low-Ranking Microrn as in Human Cancer

Classification.

Feifei Li, Yongjun Piao, Meijing Li, Minghao Piao and Keun Ho Ryu

Natural language Processing

Latent Semantic Word Sense Disambiguation Using Global

Co-Occurrence Information.

Minoru Sasaki

Modeling of Speech Synthesis of Standard Arabic Using an Expert

System.

Tebbi Hanane and Azzoune Hamid

A Model of Correlated Ageing Pattern for Age

Ranking.

Onifade O.F.W and Akinyemi J.D, University of Ibadan, Nigeria 


\title{
ECSM: ENERGY EFFICIENT CLUSTERING SCHEME FOR MOBILE M2M COMMUNICATION NETWORKS
}

\author{
Mohammed Saeed Al-kahtani \\ Computer Engineering Dept., Salman bin Abdulaziz University, Saudi Arabia \\ alkahtaniesau.edu.sa
}

\begin{abstract}
Scheduling the active and idle period of machine type communication devices (MTC) such as RFID tags, sensors and smart meters are significantly important to achieve energy efficiency in the emerging machine to machine (M2M) communication networks, which comprises thousands of resource constrained MTC devices (i.e., low data rate, energy and bandwidth). However, only a few studies exist in the literature on node scheduling schemes of M2M communication networks. Most of these schemes consider only the energy efficiency of MTC devices and do not support mobility. Thus, we introduce an energy efficient, node scheduling scheme for mobile M2M (ECSM) communication networks. The ECSM scheduling scheme selects a minimum number of active MTC devices in each cluster and increases the probability of network coverage. Simulation results show that the ECSM scheduling scheme outperforms the existing cluster-based and well-known mobility centric LEACH-M and LEACH-ME schemes in terms of network energy consumption and lifetime.
\end{abstract}

\section{KEYWORDS}

Machine to Machine (M2M) communications, MTC devices, Node Scheduling, Network Coverage, Mobility.

\section{INTRODUCTION}

Machine to machine (M2M) communication networks are becoming popular in real-time monitoring, surveillance, and security applications since they are connected to a large number of machine type communication (MTC) devices. These devices have low energy, bandwidth and memory. They are integrated with sensors or RFID tags that result in multi-coverage or redundant data transmissions. Thus, these devices dissipate energy very fast and also create channel congestion and longer data transmission delay. Scheduling the active and sleep cycles of MTC devices is one of the most effective solutions to reduce energy consumption. This is because scheduling allows a small number of devices, which cover the network area to be in active mode and the rest of the MTC devices in inactive mode. Fig. 1 illustrates such an M2M communication framework that requires scheduling the sleep and wake-up time of MTC devices. However, only a

David C. Wyld et al. (Eds) : CCSIT, SIPP, AISC, PDCTA, NLP - 2014

pp. 01-11, 2014. (C) CS \& IT-CSCP 2014

DOI : $10.5121 /$ csit.2014.4201 
few studies exist in literature on coverage-aware node scheduling schemes, especially for sensorbased M2M communication networks. Most of these approaches do not support mobility. For instance, LEACH-M [4] and LEACH-ME [6, 7], CBR [3] are well known mobility-centric clustering protocols. However, these approaches either allow data redundancy or do not provide complete network coverage.

Thus, we introduce ECSM - an energy efficient cluster-based, node scheduling scheme for mobile M2M communication networks. This approach is scalable and application independent. The proposed ECSM approach uses a primary cluster head $(\mathrm{PCH})[1,2]$ and a number of secondary cluster heads (SCHs) in each cluster of the network. The PCH is responsible for selecting SCHs and active MTC devices, transmitting control messages to the member devices of the network, collecting and aggregating data from the active member devices and transmitting to the MTC gateway. The SCHs are scheduled to wake-up a predefined time interval to check the energy status of the PCH. If the PCH fails due to energy shortage an $\mathrm{SCH}$ with the most residual energy becomes the PCH. The ECSM scheme achieves energy efficiency by reducing the number of active MTC devices as compared to existing LEACH-M and LEACH-ME protocols.

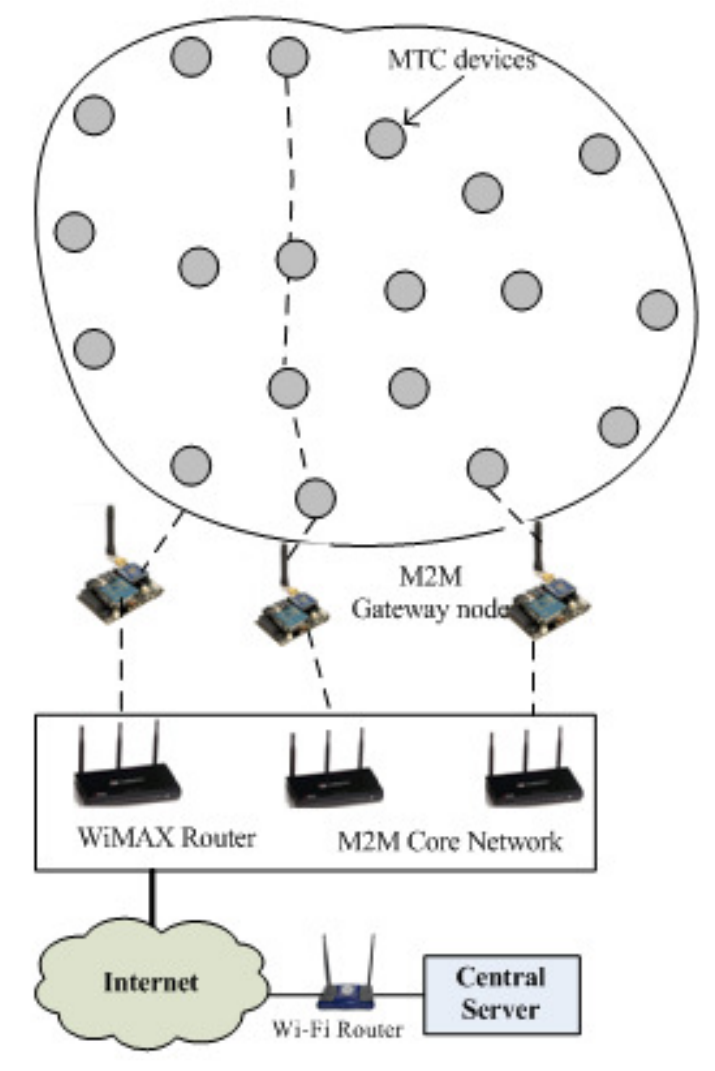

Figure 1. Sleep wake-up scheduling in M2M communication network

The remainder of this paper is organized as follows. Section 2 presents literature review on existing node scheduling schemes. Section 3 presents the working principle of ECSM node scheduling scheme along with some assumptions. Section 4 evaluates the performance of the ECSM scheduling scheme. Section 5 concludes the paper with some future research possibilities. 


\section{RELATED WORKS}

Only a few studies exist in literature on the node scheduling schemes of machine to machine (M2M) communication networks. The work done by Elkheir et al. [5] proposes a co-operative sleep wakeup scheduling mechanism for M2M communications where a number of relay nodes are selected as active to cooperate source nodes for transmitting data to the destination based on their optimal residual energy. The rest of the nodes are kept in sleep mode for a fixed time interval. However, determining the optimal sleep time is a great challenge in M2M communication network because it comprises thousands of heterogeneous devices. Another work done in [11] proposes integrated hybrid MAC and network topology control scheme (i.e., cross layer concept of MAC and network layer with multi-channel TDMA scheme) for M2M communication networks. However, this approach does not consider the sleep scheduling of MTC devices.

We also present a number of sleep scheduling mechanisms of sensors since sensors are the main constituents of M2M communication networks. For instance, in intra-cluster node scheduling algorithm [9], the sink node selects a few active nodes in a cluster to provide full network coverage. All other nodes remain in sleep mode to reduce network energy consumption. A random scheduling scheme is introduced in [10], where nodes randomly join in a disjoint set of nodes. The set of nodes works in a round robin fashion. This scheme does not require any priori localization of the sensor nodes. However, an uneven random distribution of nodes in the node set affects the performance in terms of network lifetime. Thus, the work done by Lim and Bleakley propose a multiple subset (MULS) of active node scheduling scheme [12] to eliminate the problems of random scheduling scheme. This approach uses and gathers exploratory data to find relationship among data sensing at different nodes based on which clusters are formed with nodes having stronger data relationship. A number of subsets of nodes are selected as active in each cluster to work in round robin fashion. However, MULS is not effective for sensing the unwanted events that rarely occur in a cluster and cannot be easily predicted. MULS might also fail to ensure the full network connectivity. The work done by Hwang et al. [8] introduces a network coverage-aware cluster-based node scheduling approach. This approach works by dividing the network into clusters and group the member nodes into sponsor sets based on the nodes residual energy and neighborhood information. This approach allows only one sponsor set of a cluster to be active at each round and all other sponsor sets into sleep mode. However, most of these approached do not support mobility. LEACH-M [4], LEACH-ME [6, 7], CBR [3], CBR-MWSN [3] are well-known mobility centric clustering protocols that can be used in M2M communication networks. These protocols also incorporate node scheduling using time division multiple access (TDMA) schemes which are not energy efficient.

\section{ECSM NODE SCHEDULING SCHEME}

We assume that MTC devices are homogeneous in terms of their initial energy and know their locations. The M2M area network is square shaped since network of any shape can be circumscribed into a square. Moreover, MTC devices are homogeneous in terms of mobility. M2M consists of a large number of MTC devices. Thus, it is expected that if an MTC device moves out of a cluster another device enters the cluster with a high probability. The ECSM scheme works into the following phases. 


\subsection{Network Setup phase}

The MTC gateway or BS divides the network into a number of square-sized clusters since network area of any shape can be circumscribed into a square. The maximum distance between any two points in a cluster is less than or equal to the communication range of a wireless MTC device (e.g., sensor). The MTC gateway assigns ID to each cluster and determines its area through local mapping. MTC devices transmit their coordinates to the MTC BS through multihop communications. The BS assigns an ID to each node based on the cluster they belong to. For example, $n_{i j}$ is the ID of $j$-th node in cluster $i$.

Cluster Head Selection - each cluster will have a primary cluster head (PCH) and a number of secondary cluster head (SCHs). The PCH is responsible for coordinating member MTC devices, collecting data from MTC devices nodes and sending the aggregate data to the MTC gateway. The SCHs are used as alternatives to the PCHs since they take over the responsibilities of PCHs whenever the PCH fails.

Initially, the MTC gateway randomly selects an MTC device in each cluster as a PCH and informs all member MTC devices the ID of PCH $[1,2]$. Fig. 4 illustrates that the MTC device with ID 6 is randomly selected as a PCH. Then, the PCH selects a number of devices as SCHs, which are within the sensing range of the $\mathrm{PCH}$. The SCHs are kept in sleep mode and scheduled to wake-up at a pre-defined timeslot to check the energy status of the $\mathrm{PCH}$. The SCH transmits a "Hello" message to the $\mathrm{PCH}$ whenever it wakes-up. If the residual energy of the PCH goes beyond a threshold $\left(E_{t h}\right)$ value the PCH replies with "ACK-LOW" message. Once the SCH replies with a message that it has taken over the responsibility of $\mathrm{PCH}$ the $\mathrm{PCH}$ goes to the sleep mode. The new PCH informs all member MTC devices. If the residual energy of the $\mathrm{PCH}$ is much more than the $E_{t h}$, the $\mathrm{PCH}$ replies $\mathrm{SCH}$ with an "ACK-OK" message. Similarly, the alternatives (or neighboring) MTC devices of the active MTC devices of a cluster wake up at the predefined timeslot and transmit "Hello" messages to the active devices. The active MTC devices reply with either "ACK-LOW" or "ACK-OK" messages based on their energy status, as we already discussed.

The threshold energy, $E_{t h}$ of an MTC device is dynamically adjusted to balance the network energy consumptions. Initially, a certain percentage (e.g., 40\%) of the node remaining energy is set to $E_{t h}$. If the remaining energy of the $\mathrm{PCH}$ goes below $E_{t h}$ and an $\mathrm{SCH}$ is found with higher than the $E_{t h}$ the $\mathrm{SCH}$ becomes $\mathrm{PCH}$. If no $\mathrm{SCH}$ is found, $E_{t h}$ is set to the residual energy of a node, which has the lowest energy among all MTC devices including PCH and SCHs. However, $E_{t h}$ cannot be reduced after a certain energy level, $E_{\text {fail }}$ (e.g., $5 \%$ ), which we call the failed energy level. A node is considered as dead/failed if its residual energy goes below $E_{\text {fail }}$.

Active MTC Device Selection - the PCH selects a number of MTC devices in each cluster, which provide network coverage and remain in active state. The work done in [15] is used to select the active MTC devices. The rest of the MTC devices of the cluster remain in inactive sleep state. However, at least one alternative device is selected for each active MTC device, $a_{1}$. Alternative devices are also scheduled to wake up at predefined timeslots to check the energy status of $a_{1}$. If the residual energy of $a_{1}$ goes below $E_{t h}, a_{1}$ goes into the sleep state and the 
alternative device for $a_{1}$ works as an active MTC node. The selection of active MTC devices is reinitiated if no alternative device exists due to nodes failure or mobility. Figs. $2-5$ demonstrate the active node selection process of the ECSM scheduling scheme.

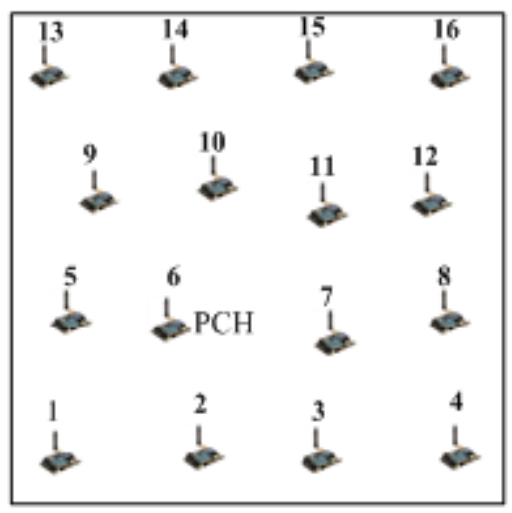

Figure 2. Primary Cluster Head (PCH) selection

Fig. 2 shows that the MTC device 6 of the cluster is randomly selected as a PCH by the MTC gateway. Fig. 3 shows the two furthest points $A$, and $B$ of the cluster, which has distance equal to the communication range, $R \mathrm{c}$. Hence, if the $\mathrm{PCH}$ is located at the point $A$, the MTC device at the point $B$ can still communicate with the $\mathrm{PCH}$. We also assume that the cluster is divided into a number of small squares, where $l$ is the length of a side of the square (Fig. 5) and has the following relationship with $R \mathrm{~s}$.

$$
R_{s}{ }^{2}=(2 l)^{2}+(2 l)^{2} \Rightarrow l=\frac{R_{s}}{2 \sqrt{2}}
$$

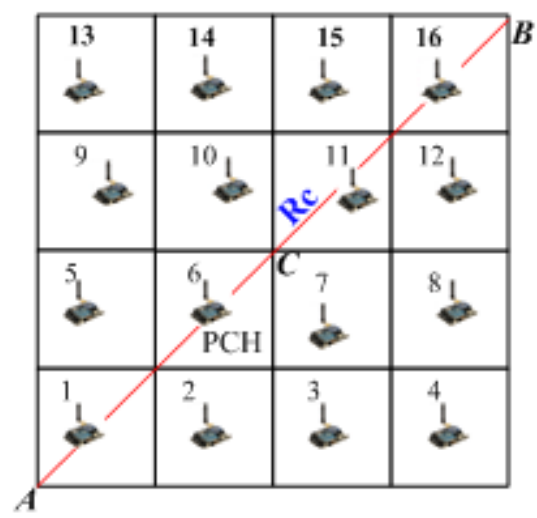

Figure 3. Divide a cluster into a number of small squares 


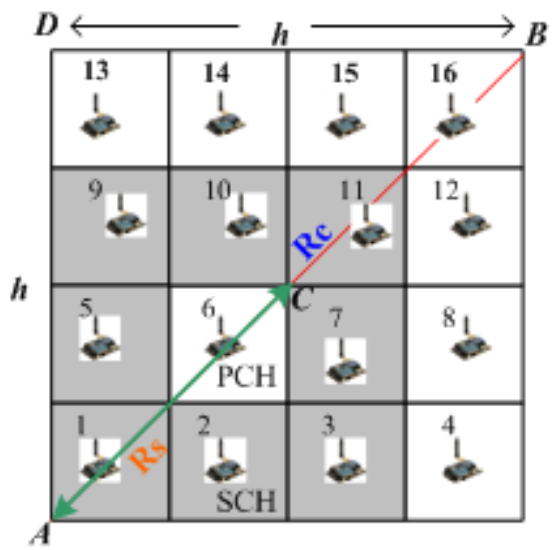

Figure 4. Secondary Cluster Head (SCH) selection

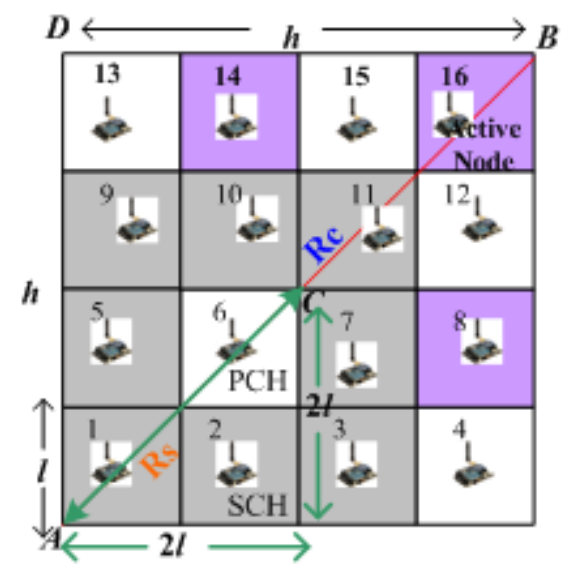

Figure 5. Active nodes selection for cluster and network coverage

This will ease the selection process of active MTC devices because an MTC sensing device that is located in a square can cover (sense) the area of all neighboring squares. Thus, once a device in a square is selected as active, nodes in all neighboring can be kept in sleep mode (inactive). From Fig. 5 we also find that

$$
R_{c}^{2}=h^{2}+h^{2} \Rightarrow h=\frac{R c}{\sqrt{2}}
$$

Thus, the area, $A_{c}$ of a cluster (square) is approximated as

$$
A_{c}=h \times h=\frac{R_{c}}{\sqrt{2}} \times \frac{R_{c}}{\sqrt{2}}=\frac{R c^{2}}{2}
$$

Fig. 4 illustrates that the SCHs (i.e., the devices that reside in ash colored squares having ID 1, 2, $3,5,7,9,10,11)$ are within the sensing range, $R$ s (i.e., distance, AC) of the PCH or device 6.

Once SCHs are selected the PCH notifies the IDs of SCHs to all member MTC devices. All member devices can also calculate the number of devices in their neighboring squares, which is used as a node degree. The device with the highest node degree is selected as an active member device. Other active members are selected from the rest of members, which are not the neighbor 
of an active member and has the highest node degree among the rest of member nodes. Fig. 5 illustrates this active member selection process. The nodes that reside in the violet colored squares, 8, 14, 16 are active MTC devices. Other member nodes 4, 12, 15, 13 remain in sleep mode and are scheduled by the PCH to wake-up at the predefined time period to check the energy status of neighboring active MTC devices.

The active MTC sensing devices at the border area of each cluster also provide network cover over some area of neighboring clusters since the neighboring squares of the borderline MTC devices are located in the neighboring cluster. For instance, the neighboring squares of the MTC device 14 are located in its neighboring cluster. Thus, the MTC device 14 covers the area of those squares in neighboring cluster. Let us assume that $P$ is an active MTC device that resides in the neighboring square of node 14 in the adjacent cluster. Neither $P$ nor node 14 covers the area of all neighboring squares of each other. Thus, the ECSM scheduling algorithm cannot completely eliminate redundant data sensing. However, by reducing the number of active MTC devices the ECSM scheme achieves energy efficiency.

\subsection{Mobility Management}

The MTC devices can be mobile since they are attached to objects such as human body, car, animal that make them mobile. This section presents how mobility of MTC devices is incorporated in the ECSM scheduling, especially when PCHs, SCHs, active and alternative MTC devices move.

Mobility of PCHs - whenever a PCH moves inside or outside of the cluster, an SCH that wakesup at the next timeslot becomes PCH only if its residual energy is more than $E_{t h}$ and notifies the member MTC devices of the cluster. If the PCH (that moved) still resides inside the cluster it becomes either an $\mathrm{SCH}$ if it is within the sensing range of the new $\mathrm{PCH}$ or a regular cluster member. If the $\mathrm{PCH}$ moves outside of the cluster it joins a new cluster, $C_{\mathrm{i}}$ by sending a "JOINREQUEST" message to the PCH of $C_{\mathrm{i}}$.

Mobility of SCHs - each PCH has a number of SCHs. Thus, if an SCH moves into another location of the cluster, which is still within the sensing range of the $\mathrm{PCH}$ the role of SCH does not change. Only the PCH updates the location information of the SCH. If the SCH moves inside the cluster but outside the sensing range of the $\mathrm{PCH}$, the $\mathrm{PCH}$ deletes the $\mathrm{SCH}$ from its neighbor list and also the scheduled timeslot of the SCH. However, the PCH still has a number of SCHs. The $\mathrm{SCH}$ (that moved) becomes a regular cluster member, neighbor of an active MTC device and remains in sleep mode.

Mobility of active and alternative MTC devices - if an active MTC device $x$ moves from its current location to another location of the cluster, the alternative device $y$ that wakes-up first becomes active and notifies its PCH. Then, $x$ is kept in sleep mode if the new location of $x$ is already covered (most likely) by turning its radio-off. Otherwise, $x$ is again selected as an active device. If $x$ moves out of the cluster the alternative node $y$ that wakes up first becomes active. If the selection of $y$ as an active node still leaves some area of the network uncovered, another sleeping MTC device $z$ that covers those areas becomes active whenever $z$ wakes up. 


\section{Performance Evaluation}

This section presents the energy model, simulation model and results.

\subsection{Energy Model}

The proposed ECSM scheduling scheme follows the energy model used in $[3,6,10]$. In this model, the energy consumption for transmitting a data packet of size $k$ bits over a distance $d$ is represented by

$$
E_{T X}(k, d)=k \times \varepsilon_{\text {elec }}+k \times \varepsilon_{f_{S}} \times d^{\alpha}
$$

where $\varepsilon_{\text {elec }}$ and $\varepsilon_{f_{s}}$ represent energy consumption of the transmitter/receiver circuitry for each bit data, and RF amplifiers for propagation loss, respectively. The constant $\alpha$ is used to represent propagation loss such as $\alpha=2$ for the straight line of sight or free space data propagation.

\subsection{Simulation Setup and Results}

A simulation model is designed to measure the performance of the proposed ECSM scheduling scheme in terms of network energy consumptions, network lifetime, and number of data transmissions. The network energy consumption is defined as the energy consumption of MTC devices for transmitting, receiving, and aggregating data for a certain number of rounds where a round comprises a network setup phase and a number of steady phases. Network lifetime is defined as the remaining energy of the network after a certain number of rounds. We compare the proposed ECSM scheduling scheme with existing mobility centric LEACH-M and LEACH-ME schemes.

Table 1. Simulation parameters and their values

\begin{tabular}{|c|c|}
\hline Parameter & Value \\
\hline Network area & $100 \mathrm{~m} \times 100 \mathrm{~m}$ \\
\hline Number of nodes & Maximum 200 \\
\hline Number of clusters & $4-8$ \\
\hline Coordinate of base station & $(100 \times 105)$ \\
\hline Transmission energy consumptions & 50 nJoule/bit \\
\hline Energy consumption in free space & $0.01 \mathrm{nJoule} / \mathrm{bit} / \mathrm{m}^{2}$ \\
\hline Energy consumptions in idle state & $0.00185 \mathrm{nJoule} / \mathrm{sec}$ \\
\hline Initial energy of each node & 3 Joule (2 AA batteries of 1.5 volt each) \\
\hline Data transmission rate & $250 \mathrm{Kbps}$ \\
\hline Velocity & $2-6$ meters/second \\
\hline
\end{tabular}

A network of size $100 \mathrm{~m} \times 100 \mathrm{~m}$ is used in the simulation model where MTC devices or sensors are randomly deployed. Table I presents simulation parameters and their respective values. The simulation is run for a fixed number of clusters, and nodes by varying the number of rounds. We set the number of clusters, and nodes to 4 and 100, respectively, and place the MTC gateway outside all clusters at the coordinate $(55,105)$. Node moves at the speed of $2-6$ meters/second. Figs. $6-8$ illustrate the performance of the proposed ECSM node scheduling scheme and compare it with the existing LEACH-M and LEACH-ME scheduling schemes in terms of network energy consumption and network lifetime. 


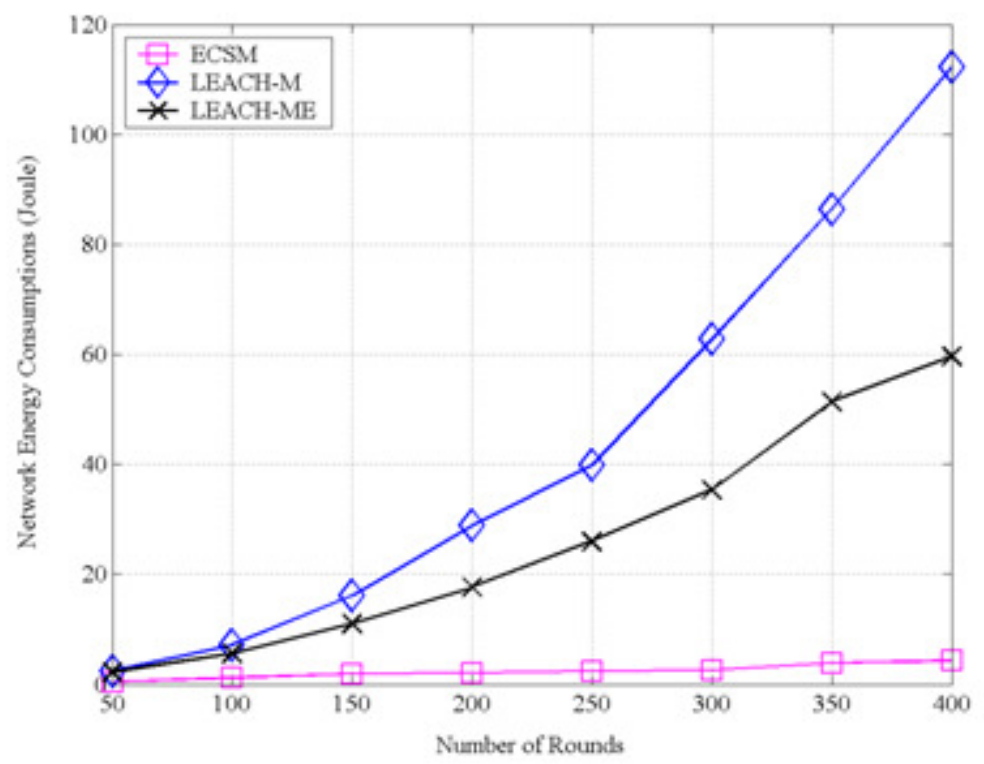

Figure 6. Network energy consumptions over a number of rounds

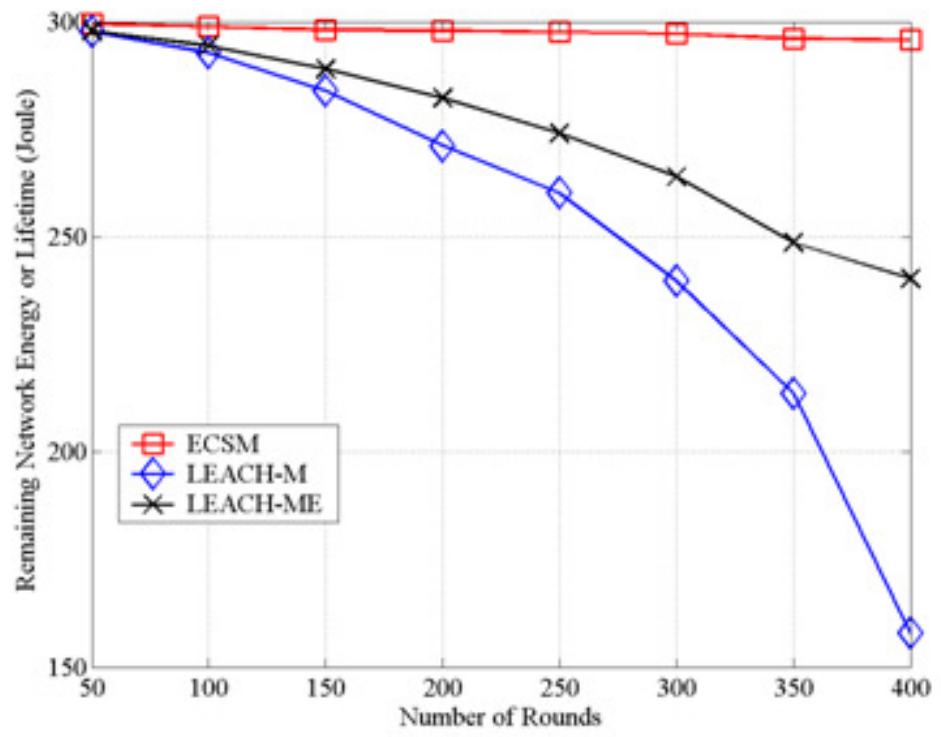

Figure 7. Network lifetime in terms of remaining network energy over a number of rounds 


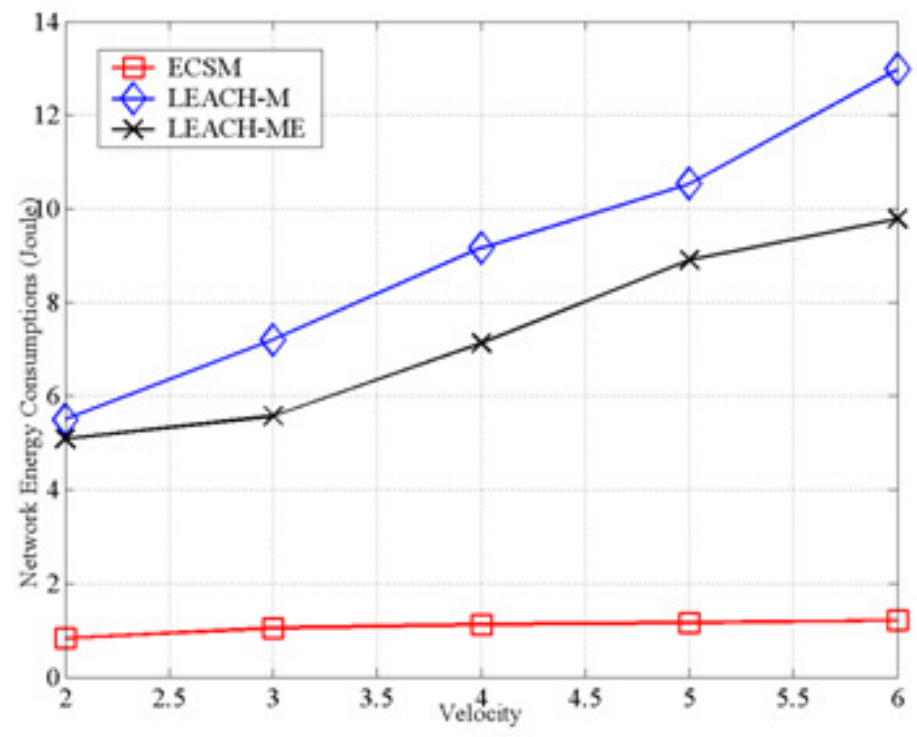

Figure 8. Network energy consumptions varying the velocity.

Fig. 6 demonstrates that the ECSM scheduling scheme consumes much less energy as compared to the existing LEACH-M and LEACH-ME protocols because the number of active member devices are more than that in ECSM scheme. Moreover, LEACH-M and LEACH-ME protocols require a large number of message transmissions in network setup phase and a large number of packets are lost if the $\mathrm{CH}$ keeps moving before selecting a new $\mathrm{CH}$ for the next round. Fig. 7 shows that the lifetime of ECSM scheduling scheme is much more than those of LEACH-M and LEACH-ME protocols. Fig. 8 demonstrates that network energy consumption of ECSM, LEACH-M and LEACH-ME increase a little bit for increasing the velocity of MTC devices since the MTC devices travel a long distance at higher velocity and are expected to reside far away from the $\mathrm{CH}$ that consumes more energy. However, there is also the possibility that MTC devices come closer to the $\mathrm{CH}$ from a distant place.

\section{CONCLUSION}

This paper introduces Energy efficient, Cluster-based Scheduling scheme for Mobile M2M communication networks (ECSM). The ECSM scheme selects a number of active machine type communication (MTC) devices that provides network coverage and alternative devices for each active device to support fault tolerance. Moreover, a number of secondary cluster heads (SCHs) are also selected for each primary $\mathrm{CH}(\mathrm{PCH})$. Performance analysis and simulation results show that the ECSM scheduling scheme has longer network lifetime as compared to existing LEACH$\mathrm{M}$ and LEACH-ME protocols. Moreover, the ECSM scheme supports mobility of sensor nodes. In future, we plan to compare the performance of ECSM scheduling scheme in terms of end-toend delay and packet loss ratio and also with more existing scheduling schemes. 


\section{REFERENCES}

[1] Al-kahtani M.S., Mouftah H.T., "A stable clustering formation infrastructure protocol in mobile ad hoc networks" IEEE International Conference on Wireless and Mobile Computing, Networking and Communications (WiMob'05), pp. 406-413, Aug 22-24, 2005, Canada.

[2] Al-kahtani M.S., Mouftah H.T., "Enhancements for clustering stability in mobile ad hoc networks", Q2SWinet'05 - Proceedings of the First ACM Workshop on Q2S and Security for Wireless and Mobile Networks, pp. 112-121, October 13, 2005, Montreal, Canada.

[3] S. A. B. Awwad, C. K. Ng, N. K. Noordin and M. F. A. Rasid, "Cluster based routing protocol for mobile nodes in wireless sensor network," in Collaborative Technologies and Systems, 2009. CTS '09. International Symposium on, 2009, pp. 233-241.

[4] Do-Seong Kim, Yeong-Jee Chung, "Self-Organization Routing Protocol Supporting Mobile Nodes for Wireless Sensor Network", the First International Multi-Symposiums on Computer and Computational Sciences (IMSCCS'06), 2006

[5] Elkheir G.A., Lioumpas A.S., Alexiou A., "Energy efficient cooperative scheduling based on sleepwake mechanisms," Wireless Communications and Networking Conference Workshops (WCNCW), 2012 IEEE , vol., no., pp.238,242, 1-1 April 2012.

[6] Gu-ping Zheng and Yu Zhou, "An Energy-aware Cluster Protocol for Wireless Sensor Networks," Innovative Computing, Information and Control, 2007. ICICIC '07. Second International Conference on, pp. 473-473, 2007.

[7] B. Huang, F. Hao, H. Zhu, Y. Tanabe and T. Baba, "Low-Energy Static Clustering Scheme for Wireless Sensor Network," Wireless Communications, Networking and Mobile Computing, 2006. WiCOM 2006. International Conference on, pp. 1-4.

[8] Hwang S.F., Su Y.Y., Lin Y.Y., Dow C.-R., "A Cluster-Based Coverage-Preserved Node Scheduling Scheme in Wireless Sensor Networks," Mobile and Ubiquitous Systems: Networking \& Services, 2006 Third Annual International Conference on, pp.1-7, July 2006.

[9] Jian-bo X., Li P., "A New Intra-cluster Node Scheduling Algorithm of Clustering Data Collection Protocol in WSNs," Electronic Computer Technology, International Conference on, pp.582-586, 2022 Feb. 2009.

[10] Jiang J., Fang L., Wen J., Wu G., Zhang H., "Random Scheduling for Wireless Sensor Networks", Parallel and Distributed Processing with Apps, IEEE International Symposium on, pp.324-332, 10-12 Aug. 2009.

[11] Kim E.-J., Heo S.-P., Chong H.-J, Jung H.-W., "Integrated hybrid MAC and topology control scheme for M2M area networks," Network Operations and Management Symposium (APNOMS), 2012 14th Asia-Pacific , vol., no., pp.1,5, 25-27 Sept. 2012

[12] Lim J.C., Bleakley C.J., "Extending the lifetime of sensor networks using prediction and scheduling", Intelligent Sensors, Sensor Networks and Information Processing, ISSNIP 2008. International Conference on, pp.563-568, 15-18 Dec, 2008.

[13] Karim L., Nasser N. and Salti T.E., "Routing on Mini-Gabriel Graphs in Wireless Sensor Networks", the IEEE International Conference on Wireless and Mobile Computing, Networking and Communications (WiMob), pp. 105-110, China, October 2011.

\section{AUTHOR}

Dr. Mohammed Al-Kahtani received M.Sc. and Ph.D. in Electrical Engineering from Colorado State University in 2001 and University of Ottawa in 2005, respectively. He is currently an Assistant Professor at the College of Computer Science and Engineering, as well as being the Dean and Founder of the IT and Distance Learning Deanship, Salman bin Abdulaziz University, Saudi Arabia.

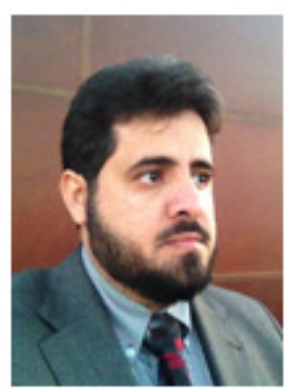


INTENTIONAL BLANK 


\title{
MINING DEVELOPER COMMUNICATION DATA STREAMS
}

\author{
Dr Andy M. Connor ${ }^{1}$, Dr Jacqui Finlay ${ }^{2}$ and Dr Russel Pears ${ }^{2}$ \\ ${ }^{1}$ CoLab, Auckland University of Technology, Private Bag 92006, \\ Wellesley Street, Auckland, NZ \\ andrew. connoreaut.ac.nz \\ ${ }^{2}$ School of Computing \& Mathematical Sciences, Auckland University of \\ Technology, Private Bag 92006, Wellesley Street, Auckland, NZ
}

\begin{abstract}
This paper explores the concepts of modelling a software development project as a process that results in the creation of a continuous stream of data. In terms of the Jazz repository used in this research, one aspect of that stream of data would be developer communication. Such data can be used to create an evolving social network characterized by a range of metrics. This paper presents the application of data stream mining techniques to identify the most useful metrics for predicting build outcomes. Results are presented from applying the Hoeffding Tree classification method used in conjunction with the Adaptive Sliding Window (ADWIN) method for detecting concept drift. The results indicate that only a small number of the available metrics considered have any significance for predicting the outcome of a build.
\end{abstract}

\section{KEYWORDS}

Data Mining, Data Stream Mining, Hoeffding Tree, Adaptive Sliding Window, Jazz

\section{INTRODUCTION}

Software development projects involve the use of a wide range of tools to produce a software artefact, and as a result the history of any given software development may be distributed across a number of such tools. Recent research in this area [1] has described the different types of artefacts that can be used to reconstruct the history of a software project. These include the source code itself, source code management systems, issue tracking systems, messages between developers and users, meta-data about the projects and usage data.

IBM's Rational Team Concert is a fully integrated software team collaboration and development tool that automatically captures software development processes and artefacts [2]. The tool has been produced as part of the Jazz project at IBM and the development repository has been released for research purposes. The Jazz repository contains real-time evidence that provides researchers the potential to gain insights into team collaboration and development activities within software engineering projects [3]. With Jazz it is possible to extract the interactions 
between contributors in a development project and examine the artefacts produced. Such interactions are captured from user comments on work items, which is the primary communication channel used within the Jazz project. As a result Jazz provides the capability to extract social network data and link such data to the software project outcomes.

This paper describes an attempt to fully extract the richness available in the IBM Jazz data set by representing the emergence of develop communication as a data stream as a means of predicting software build outcomes. Traditional data mining methods and software measurement studies are tailored to static data environments. These methods are typically not suitable for streaming data which is a feature of many real-world applications. Software project data is produced continuously and is accumulated over long periods of time for large systems. The dynamic nature of software and the resulting changes in software development strategies over time causes changes in the patterns that govern software project outcomes. This phenomenon has been recognized in many other domains and is referred to as data evolution, dynamic streaming or concept drifting. However there has been little research to date that investigates concept drifting in software development data. Changes in a data stream can evolve slowly or quickly and the rates of change can be queried within stream-based tools. This paper describes an initial attempt to fully extract the richness available in the Jazz data set by constructing predictive models to classify a given build as being either successful or not, using developer communication metrics as the predictors for a build outcome.

\section{BACKGROUND \& RELATED WORK}

The mining of software repositories involves the extraction of both basic and value-added information from existing software repositories [4]. The repositories are generally mined to extract facts by different stakeholders for different purposes. Data mining is becoming increasingly deployed in software engineering environments $[3,5,6]$ and the applications of mining software repositories include areas as diverse as the development of fault prediction models [7], impact analysis [8], effort prediction [9, 10], similarity analysis [11] and the prediction of architectural change [12] to name but a few.

According to Herzig \& Zeller [2], Jazz offers huge opportunities for software repository mining but such usage also comes with a number of challenges. One of the main advantages of Jazz is the provision of a detailed dataset in which all of the software artefacts are linked to each other in single repository. This simplifies the process of linking artefacts that exist in different repositories. To date, much of the work that utilizes Jazz as a repository has focused on the convenience provided by this linking of artefacts, such as bug reports to specification items, along with the team communication history. Researchers in this area have focused on areas such as whether there is an association between team communication and build failure [13] or software quality in general [14]. Other work has focused on whether it is possible to identify relationships among requirements, people and software defects [15].

Previous research has investigated the prediction of build outcomes for the Jazz repository by developing decision models based on the extraction of software metrics from the source code included in the repository [16] including the modeling of the available data as a data stream in order to apply data stream mining techniques [17] to facilitate the mining of project data on the fly to provide rapid just-in-time information to guide software decisions. The research presented in this paper extends the work of applying data stream mining techniques by considering the role 
of developer communication metrics in the prediction of build outcomes. The data used in this work is consistent with previous work [17] in that the developer communication metrics are extracted from the same builds as were used for extracting software metrics which allows direct comparison of the predictive power of the two approaches.

\section{THE JAZZ REPOSITORY}

What makes the Jazz repository unique is that there is full traceability between a wide range of software artefacts. The Jazz team itself is globally distributed and therefore the existing repository data provides the opportunity to data mine developer communication, bug databases and version control systems at the same time. Within the repository each software build may have a number of work items associated to it. These work items represent various types of units of work and can represent defects, enhancements and general development tasks. Work items provide traceable evidence for coordination between people as they can also be commented. In addition to this they are one of the main channels of communication and collaboration used by contributors of the Jazz project.

That being said there are, of course, other channels of communications which are not captured by work items, these include email, on-line chats and face-to-face meetings. Even though these elements are not captured, exploration of communication on work items offers a non-intrusive means to explore much of the collaboration that has occurred during the Jazz project. The Jazz team itself is fairly large, with 66 team areas for approximately 160 contributors that are globally distributed over various sites across the United States of America, Canada and Europe.

To explore the communication between contributors involved in builds, social network metrics are derived from the communication networks that are present within work items. Each work items is able to be commented on and this is the main task-related communication channel for the Jazz project. This enables contributors to coordinate with each other during the implementation of a work item. There are many elements, in regards to contributors, of a work item to consider. This makes the process of constructing a social network a little more challenging. In doing so some basic assumptions about the data has been made. Work items can have various contributors assigned to various roles, for example there are creators, modifiers, owners, resolvers, approvers, commenters and subscribers. For the purposes of this research a social network is constructed similar to the work presented by Wolf et al. [13].

For each social network constructed, nodes represent contributors involved with a build. A series of directed edges/links represent the communication flow from one contributor to another. A build can have any number of work items associated with it. Therefore the social networks generated at the work item level are required to be propagated to the build level for analysis of its impact on build success. To do this if a contributor appears within multiple work items that are associated with a single build, only one node is created to represent that contributor (there are no duplicate nodes). However additional edges are added to reflect entirely new instances communication that takes place between contributors. All edges within a network are treated as unique (there are no duplicate edges). This is because it would threaten the validity of metrics such as density. For example if a as a network that is fully connected has a density of 1 . If there are edges which represent each individual flow of communication the density metric would no longer be valid (potentially being greater than 1), which would make comparisons between networks metrics challenging. 
For this research, roles which are used to construct the network nodes include committers of change sets, creators, commenters and subscribers. Committers of change sets for a build are presented as node, as they have a direct influence on the result of the build. Creators of a work item are communicating the work item itself with other members of the team. Commenters are contributors that are discussing issues about a work item. Subscribers are people who may be interested on the status of a work item as it has impact on their own work/other modules. In order to generate the edges between nodes, rules have been implemented to establish connections between people.

From these elements constructing the social networks for each build, the metrics are calculated are:

- Social Network Centrality Metrics:

- Group In-Degree Centrality, Group Out Degree Centrality, Group InOut-Degree Centrality, Highest In-Degree Centrality, Highest Out-Degree Centrality

- Node Group Betweenness Centrality and Edge Group Betweenness Centrality

- Group Markov Centrality

- Structural Hole Metrics:

- Effective Size and Efficiency

- Basic Network Metrics:

- Density, Sum of vertices and sum of edges

- Additional Basic Count Metrics:

- Number of work items the communication metrics were extracted from

- Number of change sets associated with those work items

The process of generating the underlying data consisted of first selecting the appropriate builds for analysis. As the intention was to allow comparison with previous work [17], only builds that had associated source code were selected. In total this resulted in 199 builds of which 127 builds were successful and 72 failed. The builds were comprised of 15 nightly builds (incorporating changes from the local site), 34 integration builds (integrating components from remote sites), 143 continuous builds (regular user builds) and 7 connector Jazz builds. Builds were ordered chronologically to simulate the emergence of a stream of data over time and then work items extracted for each build to allow the social network for the build to be constructed.

\section{DATA STREAM MINING}

Most software repositories are structured that over time the underlying database grows and evolves resulting in large volumes of data. The data from this perspective arrives in the form of streams. Data streams are generated continuously and are often time-based. In large and complex systems the data, arriving in a stream form, takes its toll on resources, particular the need for large capacity data storage. In some cases it is impossible to store the entire steam, which is the case for the Jazz repository. This is because streams themselves can be overwhelming. Often in these cases the data is processed once and then is disposed of. 
The implications of data stream mining in the context of real-time software artefacts is yet to be fully explored. Currently there is little research that has explored whether or not stream mining methods can be used in Software Engineering in general, let alone for predicting software build outcomes. In large development teams software builds are performed in a local and general sense. In a local sense developers perform personal builds of their code. In a general sense the entire system is built (continuous and integration builds). These builds occur regularly within the software development lifecycle. As there can be a large amount of source code from build to build, the data and information associated with a build is usually discarded due to system size constraints. More specifically, in the IBM Jazz repository, whiles there are thousands of builds performed by developers; only the latest few hundred builds can be retrieved from the repository. Data stream mining offers a potential solution to provide developers real-time insights into fault detection, based from source code and communication metrics. In doing so it enables developers to mitigate risks of potential failure during system development and maintenance and track evolutions within source code over time.

This work revolves around the use of a data stream mining techniques for the analysis of developer communication metrics derived from the IBM Jazz repository. For this purpose the Massive Online Analysis (MOA) software environment was used [29]. Data streams provide unique opportunities as software development dynamics can be examined and captured through the incremental construction of models over time that predict project outcome. Two outcomes are possible: success, or failure. A successful outcome signals that each of the constituent work items in a project has been built as per the specification and that the items have been integrated with each other without generating any errors. On the other hand, the failure outcome is caused by one or more work items malfunctioning and/or errors being generated in the integration process.

Instances within the stream are sorted via the starting date/time property of a software build (oldest to newest) to simulate software project build processes. Using the Hoeffding tree [18], a model is built using the first 20 instances for training. Prediction is performed on each of the remaining 179 instances which are used to incrementally update the model built. Furthermore, as the outcomes are known in advance, model accuracy can be evaluated on an ongoing basis. This was possible as we used a static dataset which contained pre-assigned class labels to simulate a data stream environment. The methods used to mine the simulated data stream are described in the following section.

\subsection{Hoeffding Tree}

Decision trees were selected as the machine learning outcome for this research. This choice was influenced by the fact that the decision tree has proven to be amongst the most accurate of machine learning algorithms while providing models with a high degree of interpretability. However, the basic version of the decision tree algorithm in its basic form cannot be deployed in a data stream environment as it is incapable of incrementally updating its model, a key requirement in a data stream environment. As such, an incremental version, called the Hoeffding tree was deployed.

The Hoeffding tree is a commonly used incremental decision tree learner designed to operate in a data stream environment [19]. Unlike in a static data environment decision tree learners in a data stream environment are faced with the difficult choice of deciding whether a given decision node in the tree should be split or not. In making this decision, the information gain measure that drives 
this decision needs to take into account not just the instances accumulated in the node so far, but must also make an assessment of information gain that would result from future, unseen instances that could navigate down to the node in question from the root node. This issue is resolved through the use of the Hoeffding bound [20].

The Hoeffding bound is expressed as:

$\in=\sqrt{\frac{R^{2} \ln \left(\frac{1}{\delta}\right)}{2 n}}$

Where $\mathrm{R}$ is a random variable of interest, $\mathrm{n}$ is the observations and $\mathrm{d}$ is a confidence parameter. Essentially, the Hoeffding bound states that with confidence $1-\delta$, the population mean of $R$ is at least $\bar{r}-\in$, where $\bar{r}$ is the sample mean computed from $n$ observations.

In the context of data mining, the variable $\mathrm{R}$ is the information gain measure which ranges from 0 to 1 in value. One key advantage of the bound is that it holds true irrespective of the underlying data distribution, thus making it more applicable than bounds that assume a certain distribution, for example the Gaussian distribution.

The Hoeffding tree implementation available from MOA was used and coupled with a concept drift mechanism called the Adaptive Sliding Window (ADWIN) which was also available from the MOA environment. Most machine learner algorithms in a data stream environment use fixed size sliding window widths to incrementally maintain models. Sliding windows offer the advantage of purging old data while retaining a manageable amount of recent data to update models. However, while the concept of windows is attractive in the sense that memory requirements are kept within manageable bounds, fixed sized window are often sub-optimal from the viewpoint of model accuracy. This is due to the fact that concept change or drift may not align with window boundaries. When changes occur within a window, all instance before the change point should be purged leaving the rest of the instances intact for updating the model built so far. The ADWIN approach has many merits in terms of detecting concept drifts in dynamic data streams.

\subsection{Concept Drift Detection}

ADWIN works on the principle of statistical hypothesis testing. It maintains a window consisting of all instances that have arrived since the last detected change point. In its most basic form, the arrival of each new instance causes ADWIN to split the current window into two sub-windows, left and right. The sample means of the data in the two sub-windows are compared under a null hypothesis $\mathrm{H} 0$ that the means across the sub-windows are not significantly different from each other. If HO is rejected, concept drift is taken to have occurred and ADWIN shrinks the window to only include instances in the right sub-window, thus removing instances in the left window representing the "old" concept. Simultaneously, the Hoeffding tree is updated to remove subtree(s) representing the old or outdated concept. 


\section{EXPERIMENTAL RESULTS}

The experimental approach used in this work involves simulating a data stream by stepping through historical data in a sequential manner. The aim is to track key performance aspects of the predictive model as a function of time as well as also quantifying the level of drift in the features used by the model that determine build outcomes over the progression of the data stream over time. This experimentation revealed that the model was robust to concept drift as the overall classification accuracy recorded a steady increase over time.

Due to the limited size of the data set the default value of grace period for the Hoeffding tree is lowered from the default 200 instances. At the beginning of this set of experiments various grace periods were trailed to see whether or not the beginning set of training instances had an effect on the final classification accuracy. The results indicated that if the grace period is set too high it will result in a loss of final accuracy, as the initial model built is over fitted to the data. In terms of results for sections 4.10.1 and 4.10.2 a grace period of 20 was found to generate the highest level of accuracy for the 199 instances. The split confidence is 0.05 and the tie threshold option is set to 0.1 .

\subsection{Hoeffding Tree Classification}

The graph presented in Figure 1 presents the trend of overall classification accuracy for builds over time using the Hoeffding Tree method for the developer communication metrics. It is observed that after approximately 100 builds the prediction accuracy begins to stabilize and improve. This is to be expected because at the start of the training process insufficient instances exist, resulting in model under-fitting. The final overall prediction accuracy is approximately $63 \%$ which is only a nominal improvement from the earlier prediction accuracies which start around $52 \%$. The overall trend shows that, as more instances are trained, the classification accuracy steadily improves but does not appears to have the same predictive power as source code metrics for which the same datastream mining approach produces models that have an overall accuracy of approximately 72\% [17]. Figure 2 and Figure 3 show the sensitivity measures for actual successful and failed builds.

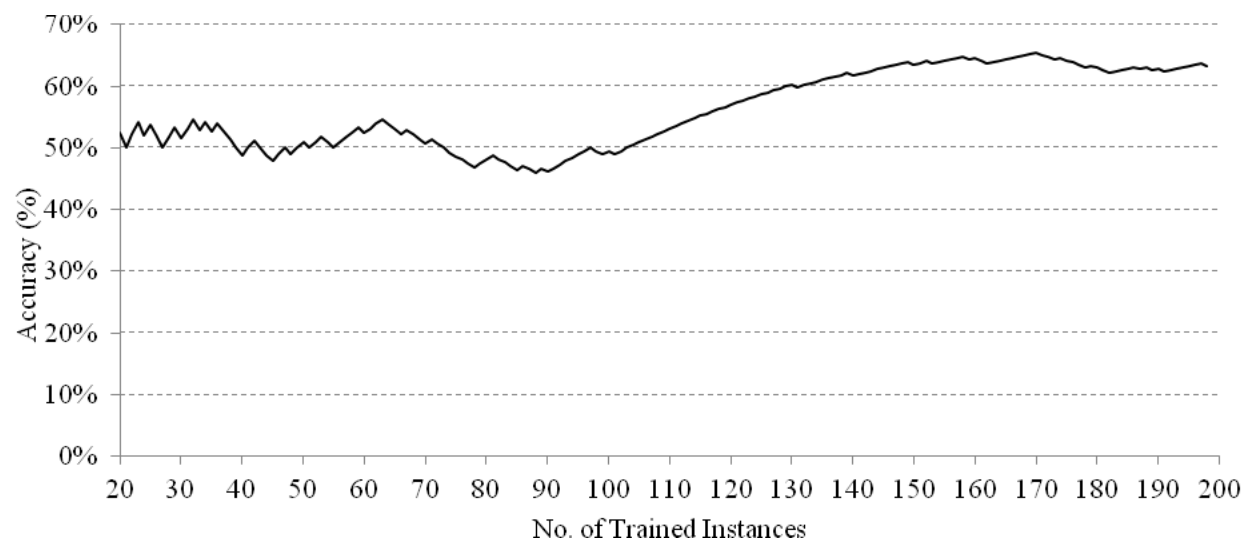

Figure 1. Overall prediction accuracy 


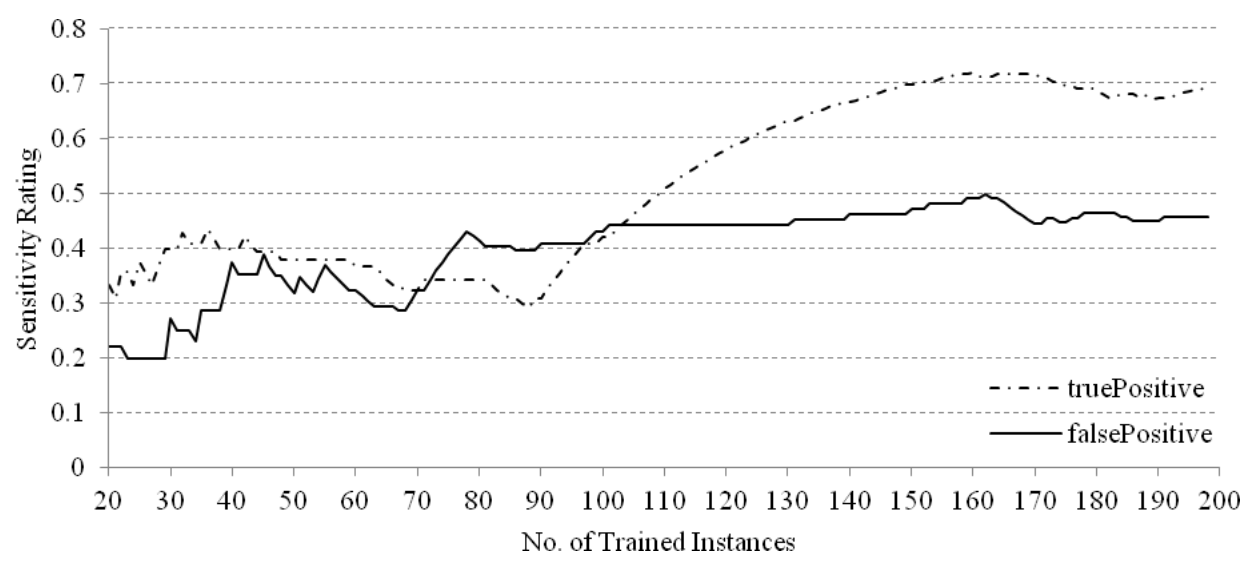

Figure 2. Sensitivity measures for successful builds

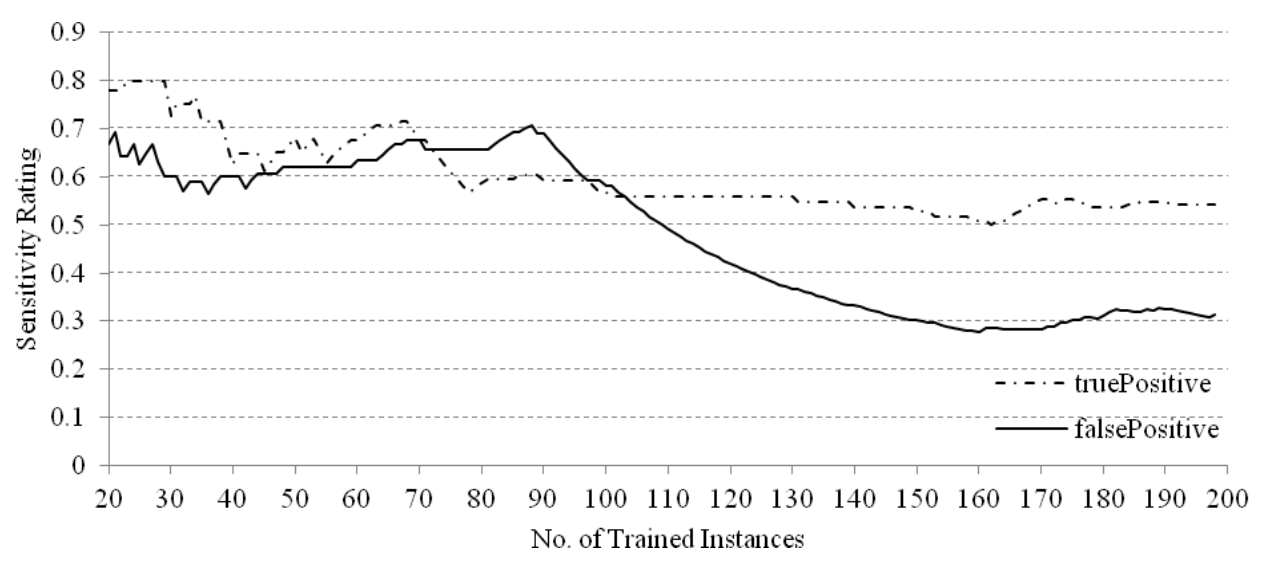

Figure 3. Sensitivity measures for failed builds

The results shown in Figure 2 and Figure 3 demonstrate that the evolution of the decision tree model is a complex scenario. Over the duration of the simulated data stream, the proportion of successful builds that are correctly classified by the decision tree steadily increases to around $70 \%$., Over the same simulated period the proportion of failed builds that are correctly classified drops from a very high initial value to around 54\%. Clearly the initial value is a result of the lack of data causing model under-fitting, but the outcomes of the end of simulating the data stream support observations made in previous work $[16,17]$ that it is significantly more challenging to identify a failed build than it is a successful one. In this case it appears that the developer communication metrics are equally as effective in this regard when compared to source code metrics [17]. From the false positive data it is clear that there is a significant problem in failed builds being misclassified as successful builds.

To fully understand the application of the Hoeffding tree approach it is important to analyze the emergence of the decision tree model, not just the final model itself. By examining Figure 1 it would seem reasonable to conclude that the minimum number of instances required to develop a classification tree that is reasonably stable would be around 100 instances. It is at this point that 
the prediction accuracy starts to stabilize and show a trend to improving asymptotically. However, an examination of the Hoeffding tree outcomes at this point shows that no actual decision tree has been generated by the model at this point in time. In fact, the Hoeffding tree approach has not identified a single feature that has sufficient predictive power to use effectively. The approach is therefore attempting to classify a new build in the data stream against the majority taken over all instances and all attribute values. So, for example, if there were $60 \%$ successful builds, then all builds would be labeled success. This is an exceptionally degenerate case where severe model under-fitting is occurring due to lack of training examples resulting in no clear predictors. The Hoeffding tree approach identifies an actual decision tree only after 160 builds. This first decision tree identifies only a single attribute against which to classify a given build and the resulting decision tree is shown in Figure 4.

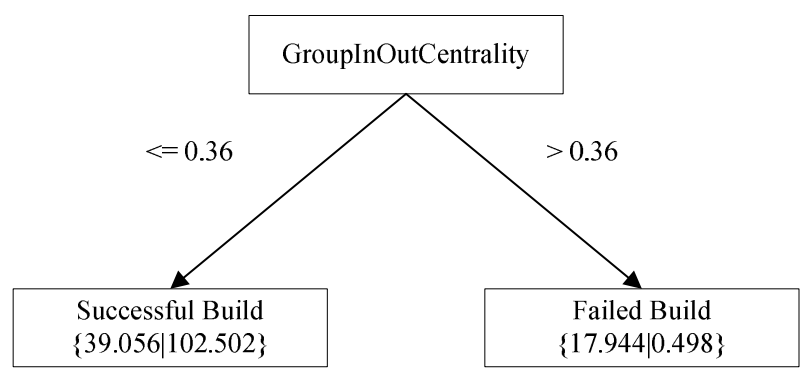

Figure 4. Decision tree at 160 builds

This decision tree indicates that the metric GroupInOutCentrality has emerged as the only significant predictor of success and failure. This outcome differs somewhat from previous work investigating the use of developer communication in predicting build outcomes for the Jazz repository [13] which indicated that there were no individual metrics that were statistical significant in terms of predicting outcomes. The leaves of the tree show the predicted outcome and the numeric values represent the votes used in the majority vote classifier. The value on left represents the weighted votes for failed builds and the value on the right represented the weighted votes for successful builds (i.e. failed builds I successful builds). Again, this indicates that there are a large number of failed builds being mis-classified as successful builds. So whilst it may appear that the outcomes differ from previous studies [13] there is not sufficient evidence to suggest that GroupInOutCentrality is a strong indicator of outcomes, particularly for the builds that would be of most interest, i.e. failed builds.

One of the advantages of the use of the ADWIN approach for concept drift detection and the result in terms of the Hoeffding tree approach, that results in only detecting and responding to changes that are statistically significant. After the initial model emerges at 160 builds there are no significant changes for a further 10 builds. At 180 builds such a change is detected but rather than see a different model appear it is expected that the model will evolve. The resulting model is shown in Figure 5. 


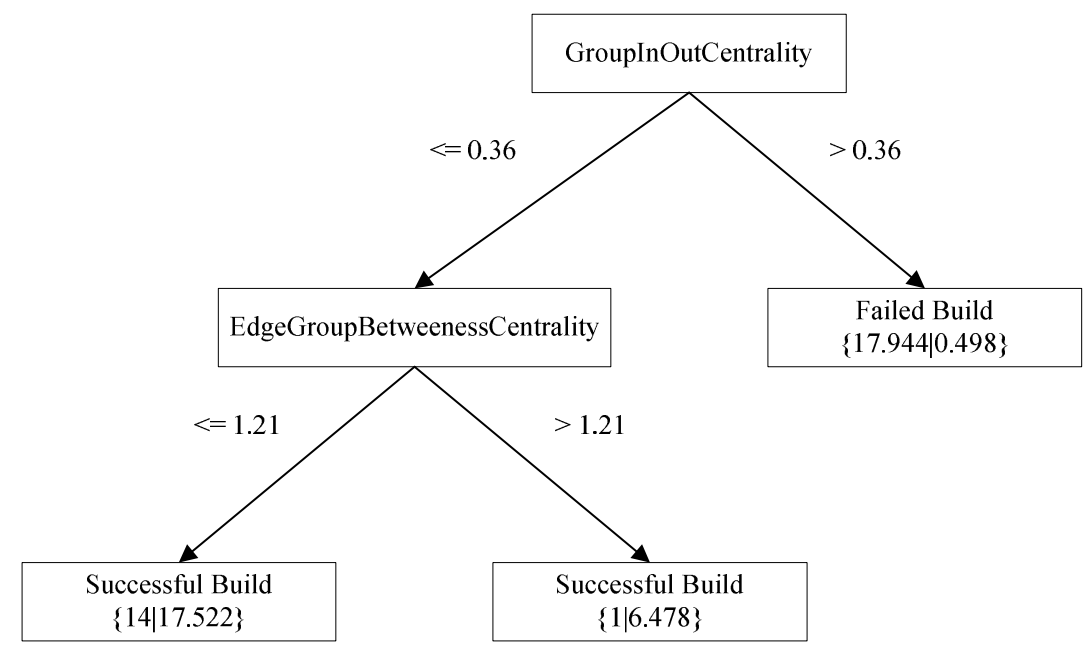

Figure 5. Decision tree at 180 builds

The addition of EdgeGroupBetweennessCentrality as a new metric has an interesting effect in that it refines the classification of successful builds only and does not improve the classification of failed builds at all. Of the 39 builds used in this incremental change, 15 of them are failed builds that are mis-classified as successful builds by the addition of the new metric. In percentage terms, the false positive rate for successful builds in these leaves of the tree is $38 \%$ which compares to a false positive rate for successful builds in Figure 4 of 27\%. Therefore it is arguable that the model that has evolved is in fact a worse prediction model than its predecessor because whilst it has improved classification for successful builds this has been at the cost of a reduced ability to classify failed builds.

However, such variations are very small to the point of being insignificant and the reduction in quality of the model may be reversed in the long term as more data is captured.

\subsection{Comparison with k-NN Clustering}

A full validation of the Hoeffding tree approach by comparing to other methods has not yet been completed, however an initial comparison to the k-nearest neighbor (k-NN) algorithm has been undertaken. In this case, the initial 20 builds are excluded from the analysis and the k-NN algorithm is trained on the remaining 179 builds, of which 116 were successful builds and 63 were failed. The final prediction accuracies of the Hoeffding tree and the k-NN when applied to communication network metrics are shown in Table 1 , where $\mathrm{k}=5$. The Hoeffding tree has performed better in terms of overall correctly classified instances for both successful and failed build outcomes when compared to the k-NN method. 
Table 1. Comparison of Hoeffding Tree \& k-NN (179 instances).

\begin{tabular}{|l|l|c|c|}
\cline { 3 - 4 } \multicolumn{2}{c|}{} & $\begin{array}{c}\text { Hoeffding } \\
\text { Tree }\end{array}$ & $\mathrm{k}-\mathrm{NN}$ \\
\hline Successful Builds & Correctly Classified Instances & 83 & 80 \\
\cline { 2 - 4 } & Incorrectly Classified Instances & 33 & 36 \\
\hline \multirow{2}{*}{ Failed Builds } & Correctly Classified Instances & 32 & 23 \\
\cline { 2 - 4 } & Incorrectly Classified Instances & 31 & 40 \\
\hline Overall Accuracy of Prediction & $64.24 \%$ & $57.54 \%$ \\
\hline
\end{tabular}

\section{LIMITATIONS \& FUTURE WORK}

Most of the limitations in the current study are products of the relatively small sample size of build data from the Jazz project. With only 199 builds available it is difficult to truly identify significant metrics and evaluate the efficacy of the data stream mining approach. It has been observed that predicting failure is more challenging than predicting success and that not predicting failure doesn't mean that success has been predicted. This is due to the fact that the build successes and failures overlap in feature space and "failure" signatures have a greater degree of fragmentation than their "success" counterparts. This overlap is a strong symptom of the fact that some vital predictors of software build failure have not been captured in the Jazz repository. This is consistent with previous that utilized source code metrics for build outcome prediction. It is an open question as to whether any such predictors can indeed be quantified in a form suitable for use in a machine learning predictive context, however future work will investigate the combination of software metrics with developer communication metrics.

\section{CONCLUSIONS}

This paper presents the outcomes of an attempt to predict build success and/or failure for a software product by modeling the emergence of developer communication as a datastream and applying datastream mining techniques. Overall prediction accuracies of $63 \%$ have been achieved through the use of the Hoeffding Tree algorithm. This is a lower prediction accuracy than is obtained when source code metrics are mined as a datastream [17].

This research has presented a potential solution for encoding developer communication metrics as data streams. In the case of Jazz the data streams would be provided when a software build was executed, though this study simulated such a datastream from historical data. The real-time streams can be run against the model which has been generated from software build histories. From the real-time based predictions developers may delay a build to proactively make changes on a failed build prediction. One of the advantages of building predictive models using data stream mining methods is that they do not have large permanent storage requirements.

The results have shown that data stream mining techniques holds much potential as the Jazz environment, as the platform can continue to store the latest builds without losing relevant information for a prediction model that has been built over an extended series of (older) software builds. Future work will investigate the combination of developer communication metrics with source code metrics [17] into a unified predictive model. 


\section{ACKNOWLEDGEMENTS}

Our thanks go to IBM for providing access to the Jazz repository and the BuildIT consortium that has funded this research.

\section{REFERENCES}

[1] D. Rodriguez, I. Herraiz, and R. Harrison, "On software engineering repositories and their open problems," in 2012 First International Workshop on Realizing Artificial Intelligence Synergies in Software Engineering (RAISE), 2012, pp. 52-56.

[2] K. Herzig and A. Zeller, "Mining the Jazz Repository: Challenges and Opportunities," Mining Software Repositories MSR '09. 6th IEEE International Working Conference, pp. 159-162, 2009.

[3] T. Nguyen, A. Schröter, and D. Damian, "Mining Jazz: An Experience Report," Infrastructure for Research in Collaborative Software, pp. 1 - 4, 2009.

[4] I. Keivanloo, C. Forbes, A. Hmood, M. Erfani, C. Neal, G. Peristerakis, and J. Rilling, "A Linked Data platform for mining software repositories," in Mining Software Repositories (MSR), 2012 9th IEEE Working Conference on, 2012, pp. 32-35.

[3] T. Xie, S. Thummalapenta, D. Lo, and C. Liu, "Data mining for software engineering," Computer, vol. 42, pp. 55-62, 2009.

[5] M. Halkidi, D. Spinellis, G. Tsatsaronis, and M. Vazirgiannis, "Data mining in software engineering," Intelligent Data Analysis, vol. 15, pp. 413-441, 2011.

[6] T. Xie, J. Pei, and A. E. Hassan, "Mining Software Engineering Data," in Software Engineering Companion, 2007. ICSE 2007 Companion. 29th International Conference on, 2007, pp. 172-173.

[7] O. Vandecruys, D. Martens, B. Baesens, C. Mues, M. De Backer, and R. Haesen, "Mining software repositories for comprehensible software fault prediction models," Journal of Systems and Software, vol. 81, pp. 823-839, 2008.

[8] G. Canfora and L. Cerulo, "Impact analysis by mining software and change request repositories," in Software Metrics, 2005. 11th IEEE International Symposium, 2005, pp. 9 pp.-29.

[9] R. Moser, W. Pedrycz, A. Sillitti, and G. Succi, "A Model to Identify Refactoring Effort during Maintenance by Mining Source Code Repositories," in Product-Focused Software Process Improvement. vol. 5089, A. Jedlitschka and O. Salo, Eds., ed: Springer Berlin Heidelberg, 2008, pp. 360-370.

[10] C. Weiss, R. Premraj, T. Zimmermann, and A. Zeller, "How Long Will It Take to Fix This Bug?," presented at the Proceedings of the Fourth International Workshop on Mining Software Repositories, 2007.

[11] T. Sager, A. Bernstein, M. Pinzger, and C. Kiefer, "Detecting similar Java classes using tree algorithms," presented at the Proceedings of the 2006 international workshop on Mining software repositories, Shanghai, China, 2006.

[12] J. Ratzinger, T. Sigmund, P. Vorburger, and H. Gall, "Mining Software Evolution to Predict Refactoring," in Empirical Software Engineering and Measurement, 2007. ESEM 2007. First International Symposium on, 2007, pp. 354-363.

[13] T. Wolf, A. Schroeter, D. Damian, and T. Nguyen, "Predicting build failures using social network analysis on developer communication," Proceedings of the IEEE International Conference on Software Engineering (ICSE), Vancouver, 2009.

[14] M. Di Penta, "Mining developers' communication to assess software quality: Promises, challenges, perils," in Emerging Trends in Software Metrics (WETSoM), 2012 3rd International Workshop on, 2012, pp. 1-1.

[15] H. Park, and Baek, S., "An empirical validation of a neural network model for software effort estimation," Expert Systems with Applications, vol. 35, pp. 929-937, 2008.

[16] J. Finlay, A. M. Connor, and R. Pears, "Mining Software Metrics from Jazz," in Software Engineering Research, Management and Applications (SERA), 2011 9th International Conference on, 2011, pp. $39-45$. 
[17] J. Finlay, R. Pears, R. \& A.M. Connor "Data stream mining for predicting software build outcomes using source code metrics", Information \& Software Technology, 2014, 56( 2), 183-198

[18] A. Bifet, G. Holmes, B. Pfahringer, J. Read, P. Kranen, H. Kremer, T. Jansen, and T. Seidl, "MOA: A Real-Time Analytics Open Source Framework," in Machine Learning and Knowledge Discovery in Databases. vol. 6913, D. Gunopulos, T. Hofmann, D. Malerba, and M. Vazirgiannis, Eds., ed: Springer Berlin / Heidelberg, 2011, pp. 617-620.

[19] B. Pfahringer, G. Holmes, and R. Kirkby, "Handling Numeric Attributes in Hoeffding Trees," in Advances in Knowledge Discovery and Data Mining. vol. 5012, T. Washio, E. Suzuki, K. Ting, and A. Inokuchi, Eds., ed: Springer Berlin Heidelberg, 2008, pp. 296-307.

[20] W. Hoeffding, "Probability Inequalities for Sums of Bounded Random Variables," Journal of the American Statistical Association, vol. 58, pp. 13-30, 1963/03/01 1963.

\section{AUTHORS}

Andy Connor is a Senior Lecturer in CoLab and has previously worked in the School of Computing \& Mathematical Sciences at AUT. Prior to this he worked as a Senior Consultant for the INBIS Group on a wide range of systems engineering projects. He has also worked as a software development engineer and held postdoctoral research positions at Engineering Design Centres at the University of Cambridge and the University of Bath

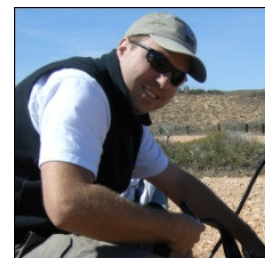

Jacqui Finlay completed her PhD in Computer Science at Auckland University of Technology, investigating the use of data mining in the use of software engineering management. Previously she was employed as Senior Systems Architect at GBR Research ltd.

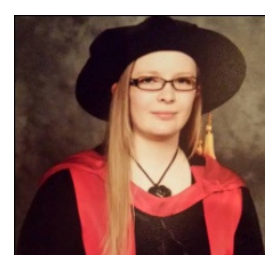

Russel Pears is a Senior Lecturer in the School of Computing \& Mathematical Sciences at AUT, where he teaches Data Mining and Research methods. He has a strong interest in Machine Learning and is currently involved in several projects in this area, including classification methods for imbalanced data sets, dynamic credit scoring methods, contextual search techniques and mining high speed data streams. 
Computer Science \& Information Technology (CS \& IT)

INTENTIONAL BLANK 


\title{
A DISTRIBUTED AGENT MEDIA ACCESS FRAMEWORK
}

\author{
Craig M. Gelowitz ${ }^{1}$, Luigi Benedicenti ${ }^{2}$ and Raman Paranjape ${ }^{3}$ \\ ${ }^{1}$ Software Systems Engineering, University of Regina, \\ Regina, Saskatchewan, Canada \\ craig.gelowitz@uregina.ca \\ ${ }^{2}$ Software Systems Engineering, University of Regina, Regina, \\ Saskatchewan, Canada \\ luigi.benedicenti@uregina.ca \\ ${ }^{3}$ Electronic Systems Engineering, University of Regina, Regina, \\ Saskatchewan, Canada \\ raman.paranjapeduregina.ca
}

\begin{abstract}
This paper presents a distributed software mobile agent framework for media access. The access and delivery of media is enhanced by an agent architecture framework that utilizesmobile agent characteristics to provide access and delivery of media. Migrating agents to the media source and destination devices enables the framework to discover the properties of devices and media within networks dynamically. The mobile agents in this framework make decisions and work together to enable access and delivery of media through the transcoding of media based on the properties of the data path and device constraints.
\end{abstract}

\section{KEYWORDS}

Distributed Media Access, Mobile Software Agents, Real-time Transcoding \& Media

Framework

\section{INTRODUCTION}

Mobile agent software technology provides the methodology used to create the framework. The following subsections introduce mobile agents and the distributed framework. In section two, the framework approach is presented and identifies some common issues. In section three, the design of the framework is presented. In section four, the issues identified in section two are discussed with respect to the framework. In section five, the video transcoding methodology results are discussed and section six concludes the paper with potential future work.

\subsection{Mobile Agents}

The mobile agent paradigm is a branch of traditional computing areas such as distributed computing and artificial intelligence. The differentiators mobile agents provide over the David C. Wyld et al. (Eds) : CCSIT, SIPP, AISC, PDCTA, NLP - 2014 pp. 27-36, 2014. (C) CS \& IT-CSCP 2014

DOI : $10.5121 /$ csit.2014.4203 
traditional computing areas are the properties of mobility, reactivity, persistence, social ability and autonomy. Mobile agents are able to react to their environment, communicate, negotiate and migrate to network resources $[1,2]$.

Mobile agents are created to be goal oriented. When a mobile agent requires a resource to complete a task, it will move to that resource and execute until the resource is no longer required. Mobile agents will migrate to resources when required to reach an execution goal $[3,4]$. As a result, the agent model is a useful paradigm to create distributed applications that have the ability to migrate and provide a media access service.

An agent's communicative ability increases the potential advantages of the mobile agent paradigm. This ability enables the development of multi-agent systems. Multi-agent systems differ somewhat from single agent systems. A multi-agent system must include provisions to handle interaction and the current context of other agents in the system. In a multi-agent system, agents or resources can be added or removed from a system as services are initiated or removed. Mobile agents are able to adjust to changing conditions such as network nodes, devices, media services or other agents which may be available or unavailable dynamically. Multi-agent systems can be created that use agent communication to collaborate and work together to provide services, reach a desired outcome or achieve a common execution goal $[5,6]$.

For these reasons mobile agents are useful for deploying a media access service. Mobile agents exhibit characteristics such as flexibility, reliability and scalability. They are also able to provide different types of distributed applications and services to users with desired preferences and outcomes $[7,8]$.

\subsection{The Distributed Agent Framework}

Mobile agents exhibit characteristics such as autonomy, mobility, flexibility and the ability to collaborate and communicate [9]. The distributed agent framework inherits these characteristics from the mobile agent paradigm. These characteristics provide useful mechanisms to provide media access.

The framework provides users with access to media services through the use of agent mobility and communication. Multiple agents work together to move through local and global networks and create message paths for the transfer and consumption of media through networks. Users are given the ability to access media across networks and between devices. Agents within this framework also provide other functions for media access such as the determination of media availability, transcoding of content, media serving and media caching.

A user is able to transfer and consume media at selected devices within the framework. Agents within the framework collaborate to determine the current properties of media resources and the current network conditions in order to execute the appropriate steps to provide content to the user.

\section{APPROACH}

The approach taken by the developed prototype for media access utilizes the framework to create a communication path for the real-time transfer of media data. The following section provides a short overview of the framework. 


\subsection{Distributed Agent Framework Approach}

The framework provides a mobile agent enabled mechanism to allow users to share content between devices. The following diagram illustrates an overview of the agent system and its migration and data path which provides content between example private networks.

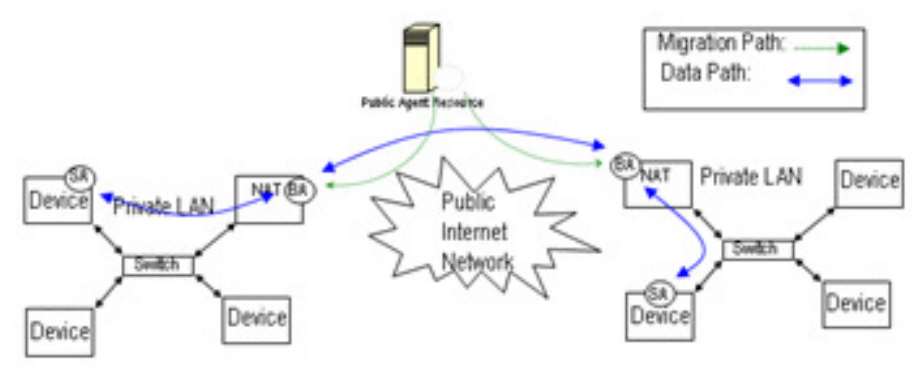

Figure 1. Agent Migration Overview

The interface to the system is provided by a web-server through a web browser interface. The public agent resource is where mobile agents are launched when a media service is initiated by a user. The web-server initiates mobile agent actions for migration and dynamic set-up of the data path between devices.

The framework enables access to content by transferring media data through agent message relaying. The interface enables users to select a source gateway and a destination gateway followed by a source device and a destination device. After the selections are made, mobile agents move out from the public resource to the selected source and destination. The media currently available on the source device is presented to the user through the interface. After a media selection is made, the media is transferred across the networks by relaying messages through the bridge agents from the source device to the destination device. Depending on the type of media available, the bandwidth available between networks and the nature of any timesensitive selected media such as video, the agents will perform actions to compensate for these conditions to provide real-time consumption at the destination.

\subsection{Media Framework Issues}

The framework provides a mechanism for content access between devices. The framework faces some challenges which are presented here:

\subsubsection{Network Connection}

In order to provide user access to media across networks, agents must traverse the network and create a data path for media information. In the case of local private networks, the internal devices are behind a NAT (network address translation) router. This makes it difficult for data paths to be established when they are initiated from the public network. Some peer-to-peer applications use NAT traversal protocols such as TURN [10] and STUN [11]. Agents can provide an alternate mechanism to create a data path. Multiple agents can use message forwarding to relay messages and create a data path for media transfer or application communication $[12,13]$. 


\subsubsection{Bandwidth Constraints}

The available bandwidth between networks can be highly variable. Video and other time sensitive data such as audio require a certain level of bandwidth to be available for real-time consumption of content. A system that provides real-time media services to a community of users needs to account for available bandwidth between devices.

\subsubsection{Media Availability}

Mobile agents can aide in file and media access $[14,15]$. An agent's ability to migrate enables them to move directly to a resource to inspect content. Upon arrival at the media resource an agent can determine the availability and context of the media. Agents can use this information to make decisions with respect to delivering the content to a destination.

\subsubsection{Scalability and Context}

Mobile agents require a platform to migrate and execute. This implies that available devices must have an agent execution platform. The availability of these platforms can be dynamic in nature. Devices and network nodes may be available or unavailable at any given time. In the case of a large community of devices and networks, a framework must be able to handle the dynamic nature of available devices in a scalable manner.

\section{DESIGN AND IMPLEMENTATION}

The following sections provide further explanation of the framework with respect to media access and its delivery. The current prototype implements the architecture illustrated below:

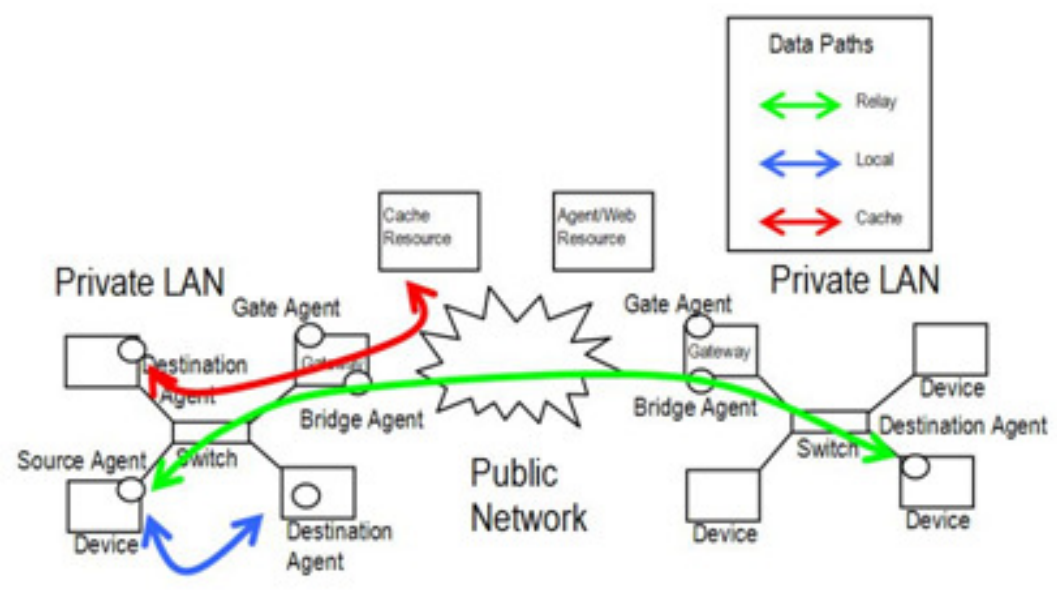

Figure 2. Agent Communication Paths

The figure shows two private networks where agents have moved out to their respective locations to provide a media service. The numbers of private networks that are part of the system are not restricted as a community of users might span multiple networks. The public resource provides the user interface to the system and the launching point for agents to migrate out to devices. 


\subsection{Device Selection}

After the respective source and destination networks have been chosen, a list of devices currently available within the selected networks are made available to the user.

A message is sent to each selected gateway requesting the information about the devices connected to their respective internal networks. The gate agents respond to the public resource with their respective list of devices connected to their internal private networks. The device list for each gateway is created dynamically using a broadcast mechanism which allows the gate agent to keep an updated list of respective devices available on the internal network. Any device in the local network which is executing the mobile agent environment will make it itself known to the gate agent and dynamically be added to the list of devices. In this way, the user is presented with a current list of source and destination devices.

After the source and destination devices are selected, bridge agents and source/destination agents are created at the public repository and move to their respective locations as seen in figure 2 .

\subsection{Media Selection}

When the source agent arrives at the source device, the agent inspects the device for available media. The list of available media is sent back to the user interface for selection. Once a selection is made, the framework determines how to transfer the media.

Within the developed prototype, there are currently four fundamental alternatives for media transfer/consumption. The prototype determines which alternative to use based on the state of the system. The four alternatives are as follows:

\subsubsection{Agent Message Passing}

Message passing is the default mechanism for media transfer. Media data is passed using agent messaging by relaying messages through the bridge agents and to the final destination as seen in figure 2. The source agent uses the bandwidth determined between the devices through-out the data path to adjust media for real-time transfer. As an example and in the specific case of video data, the source agent will begin transcoding video to a resolution and bit rate achievable by the available bandwidth to transfer.

\subsubsection{Local Data Stream}

This approach includes an alternate mechanism for media transfer which is performed only in the local area network case. When the identical source and destination network are chosen, the agents will move out and setup in the same fashion as they do when they set up across separate networks. The difference in this case is that the source and destination agents are able to communicate directly without relaying messages using the bridge agents. As such, it becomes no longer necessary to use message passing to transfer media data. The source agent (where the requested file originates) transforms itself into a streaming media service. It opens an IP port for streaming available content and proceeds to wait for a connection from the destination agent. The destination agent connects to the port directly with the appropriate streaming directives. The media is now streamed directly between the agents on the local area network and displayed in its original form. Once the process begins there is very little overhead with respect to bandwidth or computational resources other than what is normally required to consume the content. The gateway and its resources are not utilized because no gateway resource is required after the initial setup has taken place. 


\section{DISCUSSION}

The following section discusses the challenges identified in section two with respect to the framework.

\subsection{Network Connection}

The agents within the framework traverse the public to private network using a series of consecutive hops. Source and destination agents move first to the respective network gateways by travelling to their public IP addresses first from the public resource. The internal IP addresses of the devices are now available for migration once they have made the first hop to the gateway. The internal IP address of a source or destination device is known through information gathered from the gate agents.

Agent to agent data paths are handled by a relay mechanism using the bridge agents on the gateways. The source and destination agents communicate by relaying messages through the bridge agents. Other data paths such as access to the cache or the public resource is handled in the normal fashion whereby the initiation of the path can originate from within the private network because an agent is executing within the network. The cache and public resource exist at publicly addressable IPs and can be accessed from any device that has connection to the public network.

\subsection{Bandwidth Constraints}

The framework handles bandwidth constraints by making decisions based on the content required and the conditions of the system. It will attempt to provide content in the most appropriate way to allow users within the community the highest quality experience it can deliver. The methods presented included various ways that media can be accessed and transferred within the framework to adjust for bandwidth constraints.

\subsection{Media Availability}

The framework determines media availability by sending an agent directly to the source of the media. In this way, inspection of the file system locally provides a list of media currently available at the device. This also allows the system to determine the context and properties of the media at the source. As such, an agent can make decisions locally about how to handle a media sharing request using the information gathered.

\subsection{Scalability and Context}

The prototype provides an automatic discovery mechanism. By default, the agent platform accepts broadcast query packets and responds to those queries with its IP address, device name and the names of agents currently executing on its platform. This allows the gate agent to query its local subnet for currently available devices. The lists of devices within the larger system of networks are distributed and not centrally located. A query from the system to a gate agent will dynamically identify the current available platforms/devices for the selected network. This distribution of the current system context also provides the prototype the ability to easily determine the current state of the system on a request by request basis. The framework does not centrally maintain the state of the system and as such the community of networks and users can potentially grow in a scalable manner. The mechanism mimics the well-known DNS mechanism and provides the framework a dynamic way of determining currently available devices and content in a distributed and on-demand manner. 


\section{RESULTS}

The results discussed in this section refer to video media because video media requires the framework to adjust for bandwidth and device constraints. To determine a transcoding methodology for the distributed agent framework the following tests were performed.

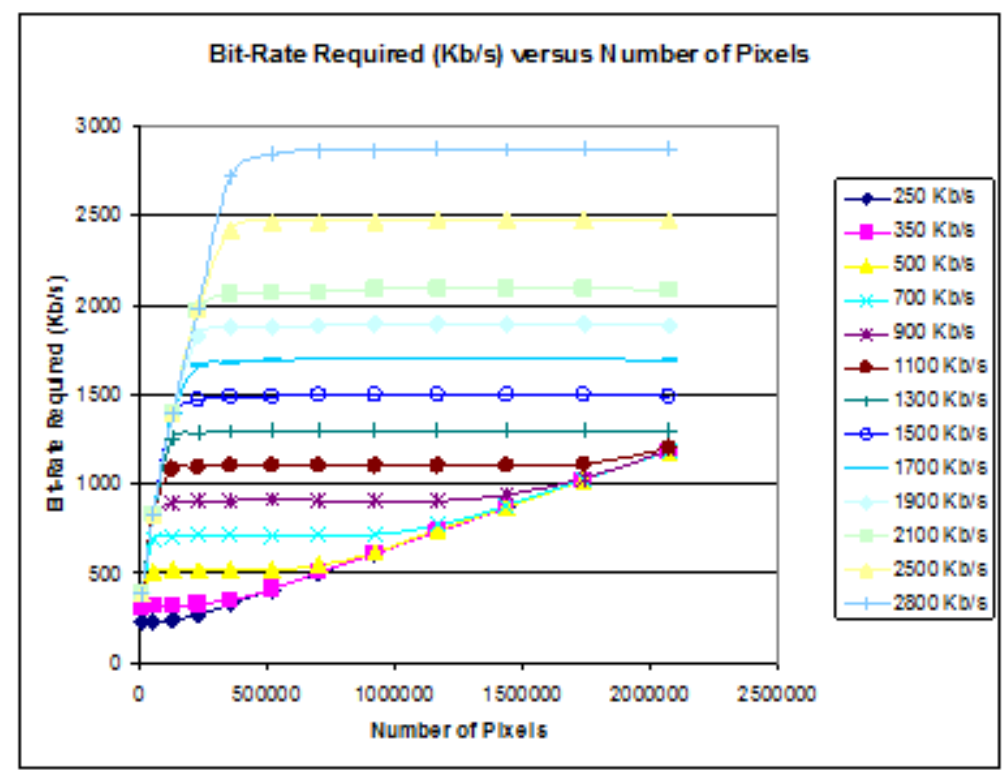

Figure 3. Multiple Transcodes of One Video Media File

Figure 3 graphs the resulting bit-rate of numerous encoding trials for the same video media. Each line represents a specific bit-rate parameter where the resolution parameter was varied and corresponds to the number of pixels identified in the graph. The Y-axis is the resulting average bit-rate of the media and the $\mathrm{X}$-axis is the number of pixels of the resolution selected for the transcode.

The region to the left of the horizontal region is a saturation region because the number of bits required to represent the video information reaches a saturation point. The saturation area indicates that these smaller resolutions do not require the addition of more bits to encode the information and is reflected by a resulting lower average bit-rate than the parameter selected for encoding. The horizontal region shows the convergence of the transcode toward the bit-rate parameter selected for the encoding process. The diagonal region to the right of the horizontal region indicates a shortage of bits in the encoding parameter to provide higher resolutions at the selected bit-rate parameter.

With respect to the framework, it is the source agent's responsibility to select the transcoding parameters and account for bandwidth constraints. The resolution parameter selected for a given encode can greatly affect the quality of the resulting viewing experience at the destination.

It is desirable for the agents to set the encoding parameters to the maximum resolution of a given destination device, but as shown in Figure 3, if the resolution of the destination device is to the right of the horizontal region for a given bit-rate parameter, the resulting bit-rate will be higher 
than the parameter selected. This translates into a failed real-time viewing experience because the available bandwidth is lower than the resulting transcoded bit-rate. The media transfer will not be able to keep up with the encoded bit-rate. The bit-rate parameter selection is further complicated by the fact that different video media do not exhibit consistent encoding characteristics.

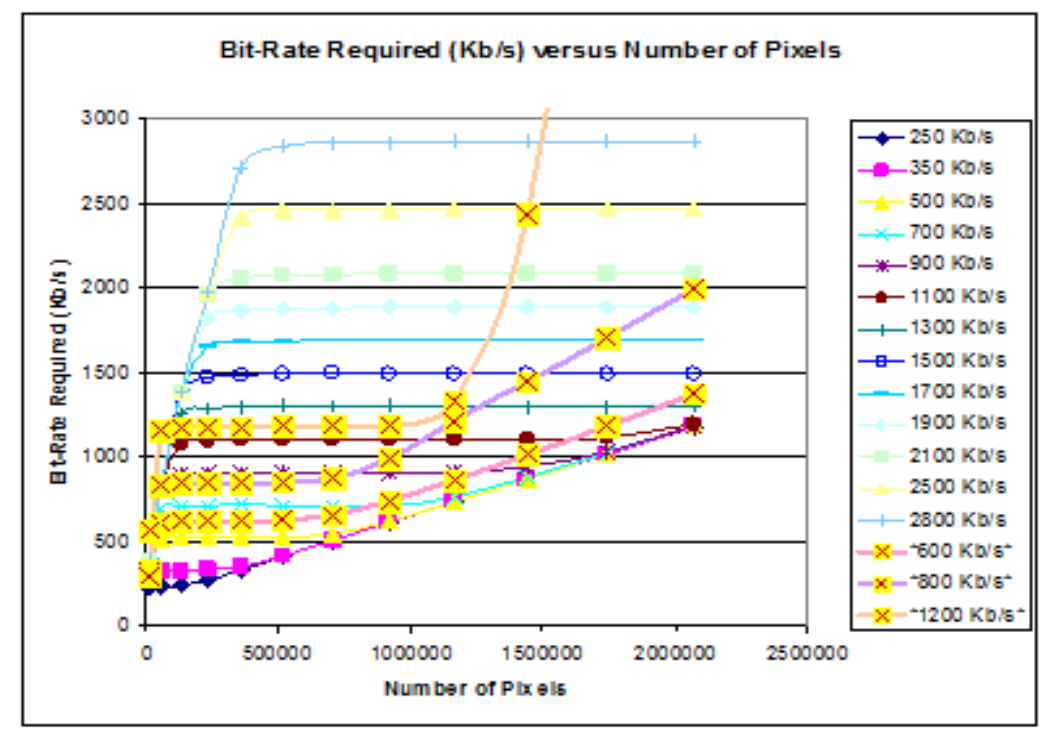

Figure 4. Multiple Transcodes of Various Video Media Files

Figure 4 shows the same graph as Figure 3 with overlays of different video transcoding lines as indicated by the lines and boxes with the X's. Different video media data exhibit similar characteristics for media encoding with respect to the three regions, but the size of the horizontal region varies. This depends primarily on the amount of changes and detail contained in the original video images per unit time.

The agents in the system are not able to predict the point at which the graph of a given media selection will go from the approximate zero slope of the horizontal region to a positive slope of the corresponding diagonal region. Despite the variable characteristics for encoding different media, the decision regarding the encoding of a video file is much less complex because of the known properties of the original media file and the known constraints of the situational context that is provided through agent execution at local devices.

The source agent is aware of the properties of the original media file and the resolution of the destination device. The two most important factors with respect to the parameters selected for real-time transfer are known imposed constraints. These factors are the bandwidth availability constraints and the resulting resolution of the transcode. The following is a simple decision table based on these two important considerations: 
Table 1. Agent Decision Table

\begin{tabular}{|c|c|l|}
\hline BW available $>$ = BW Orig. & Res. Dest. $>$ = Res. Orig. & Action \\
\hline Yes & Yes & $\begin{array}{l}\text { Adaptation: } \\
\text { Not Required }\end{array}$ \\
\hline Yes & No & $\begin{array}{l}\text { Adaption: } \\
\text { Resolution decrease }\end{array}$ \\
\hline No & Yes & $\begin{array}{l}\text { Adaptation: } \\
\text { Bit-rate decrease }\end{array}$ \\
\hline No & No & $\begin{array}{l}\text { Adaptation: } \\
\text { Bit-rate decrease } \\
\text { Resolution decrease }\end{array}$ \\
\hline
\end{tabular}

For the first two cases, the bandwidth available is greater than the bandwidth required for transferring the media data. As such, the source agent does not have to make any bit-rate changes to the media. In the first case, increasing the resolution of the original file does not provide any new information. If the destination device has a higher resolution than the original media it makes little sense to increase the resolution from the original resolution. The media should simply be displayed at full screen on the destination device. In the second case, decreasing of resolution will likely result in a decreased bit-rate for the resulting encode and so the bandwidth constraint can be ignored and an equal bit-rate can be used.

It is in the last two cases where the real-time adaptation decisions need to be made with respect to the parameters selected for a resulting transcode. It is the parameters adjusted for bandwidth constraints that pose the greatest difficulty in determining the optimal parameters for transcoding. However, since the source agent inspects the properties of the original media before transcoding begins, it can determine the average number of bits-per-pixel used by the original media file given its current resolution and codec.

It is these properties that provide bounding constraints for agent decision making which significantly reduces the complexity of the decision. The upper bound of the bit-rate parameter is determined through bandwidth estimation and therefore the only decision left to be made is the resulting resolution. The evaluation of the bits-per-pixel of the original file also provides a bounding constraint for the source agent. A decrease in the resulting average bits-per-pixel may force the transcode to the right in the standard encoding graph and into the diagonal region.

This would increase the resulting bit-rate and not meet the real-time constraints of the bandwidth available as discussed previously. The resulting resolution is calculated by determining the maximum resolution that can be achieved which results in the number of bits-per-pixel being greater than or equal to the observed number of bits-per-pixel found in the original file. It is generally understood that lower bits-per-pixel values are a result of similar quality higher resolution encodes. The lower bound constraint of the bits-per-pixel of the original media ensures the resolution cannot be towards the right-side of the graph at a lower resolution.

\section{CONCLUSiON}

This paper presents a distributed framework for media access. Mobile agents use their unique properties to provide delivery methods and access to desired content on distributed devices. 
The framework provides access to distributed content through a web-browser interface that is used to send media from one device to another. These devices can be local devices or devices across private networks.

The prototype functions as discussed but there still exists several opportunities for further research and development. The framework could be more standards compliant by utilizing well established protocols like UPnP (universal plug-n-play) to provide integration into other IP service systems. The framework provides a discovery mechanism for media available but does not include the ability for an automated search of media. The user is expected to know what device the desired media is on. Other opportunities include further optimization of the current system and further authentication through user location technologies.

\section{REFERENCES}

[1] Bradshaw, J (editor). "Software Agents," AAAI Press /MIT Press, Cambridge, Massachusetts, 1997.

[2] Wooldridge, M. and Jennings, N. R., "Intelligent agents: Theory and practice," Knowl. Eng., Rev. 10, 2, 115-152, 1995.

[3] Harrison, C. G., Chess, D. M. and Kershenbaum, A., "Mobile Agents: Are They a Good Idea?" IBM Research Report, 1995.

[4] Gray, R. S., Cybenko, G., Kotz, D. and Rus, D., "Mobile Agents: Motivations and State of the Art," Handbook of Agent Technology, J. Bradshaw, ed., 2001.

[5] Tozicka, J., Jakob, M.and Pechoucek, M., "Market-Inspired Approach to Collaborative Learning," Cooperative Information Agent X, 2006.

[6] Dutta, P. S., Jennings, N. R. and Moreau, L., "Cooperative information sharing to improve distributed learning in multi-agent systems," Journal of AI Research 24 407-463, 2005.

[7] Günter, M., Braun, T., "Internet Service Delivery Control with Mobile Agents," Extended Abstract for the 2nd International Symposium on Agent Systems and Applications / Mobile Agents, September 2000, Zürich, Switzerland.

[8] Kotz, D. and Gray, R. S., "Mobile agents and the future of the Internet," SIGOPS Oper. Syst. Rev. 33(3):7-13, 1999.

[9] Franklin S., Graesser A., "Is it an agent, or just a program? A taxonomy for autonomous agents", Third International Workshop on Agent Theories, Architectures, and Languages, Springer-Verlag, 1996

[10] Rosenberg, J., Huitema, C. and Mahy R., "Traversal Using Relay NAT (TURN)", Internet Draft, draft-rosenberg-midcom-turn-08, Sept. 2005.

[11] Rosenberg, J., Weinberger, J., Huitema, C. and Mahy R., "STUN - Simple Traversal of User Datagram Protocol (UDP) Through Network Address Translators (NATs)," RFC 3489, March 2003.

[12] Satoh, I., "Building reusable mobile agents for network management," Systems, Man and Cybernetics, Part C, IEEE Transactions on Volume 33, Issue 3, Page(s):350 - 357, 2003.

[13] Chen, R.Y. and Yeager, B., "Java mobile agents on project JXTA peer-to-peer platform ," Proceedings of the 36th Annual Hawaii International Conference 2003 Page(s):10 pp., System Sciences, 2003.

[14] Gendelman, E., Bic, L., Dillencourt, M.B., "Fast File Access for Fast Agents," Int'l Conf. on Mobile Agents, Atlanta, GA, Dec. 2001.

[15] Villate, Y., Illarramendi, A. and Pitoura, E., "Data Lockers: Mobile-Agent Based Middleware for the Security and Availability of Roaming Users," Data In CoopIS, 2000. 


\title{
MULTILEVEL TECHNIQUES FOR THE CLUSTERING PROBLEM
}

\author{
Noureddine Bouhmala \\ Department of Maritime Technology and Innovation, \\ Vestfold University College , Norway \\ noureddine.bouhmala@hive.no
}

\begin{abstract}
Data Mining is concerned with the discovery of interesting patterns and knowledge in data repositories. Cluster Analysis which belongs to the core methods of data mining is the process of discovering homogeneous groups called clusters. Given a data-set and some measure of similarity between data objects, the goal in most clustering algorithms is maximizing both the homogeneity within each cluster and the heterogeneity between different clusters. In this work, two multilevel algorithms for the clustering problem are introduced. The multilevel paradigm suggests looking at the clustering problem as a hierarchical optimization process going through different levels evolving from a coarse grain to fine grain strategy. The clustering problem is solved by first reducing the problem level by level to a coarser problem where an initial clustering is computed. The clustering of the coarser problem is mapped back level-bylevel to obtain a better clustering of the original problem by refining the intermediate different clustering obtained at various levels. A benchmark using a number of data sets collected from a variety of domains is used to compare the effectiveness of the hierarchical approach against its single-level counterpart.
\end{abstract}

\section{KEYWORDS}

Clustering Problem, Genetic Algorithm, Multilevel Paradigm, K-Means.

\section{INTRODUCTION}

The amount of data kept in computers is growing at a phenomenal rate. However, extracting useful information has proven extremely a challenging task. Often, traditional data analysis tools and techniques simply are not adequate to support these increases demands for information. Data mining steps in to solve these needs using a combination of data analysis methods with sophisticated algorithms to automatically analyse and extract knowledge from data. Cluster Analysis which belongs to the core methods of data mining is the process of discovering homogeneous groups called clusters. Given a data-set and some measure of similarity between data objects, the goal in most clustering algorithms is maximizing both the homogeneity within each cluster and the heterogeneity between different clusters. In other words, objects that belongs to the same cluster should share many features, but are very dissimilar to objects not belonging to that cluster [1] . The clustering problem is NP-Complete [2] and it is considered one of the most

David C. Wyld et al. (Eds) : CCSIT, SIPP, AISC, PDCTA, NLP - 2014

pp. 37-51, 2014. (C) CS \& IT-CSCP 2014

DOI : $10.5121 /$ csit.2014.4204 
and challenging problems due to its unsupervised nature. It is important to make a distinction between supervised classification and unsupervised clustering.

In supervised classification, the analyst has available sufficient knowledge to generate representative parameters for each class of interest. This phase is referred to as training. Once trained, a chosen classifier is then used to attach labels to all objects according to the trained parameters. In the case of clustering analysis, a clustering algorithm is used to build a knowledge structure by using some measure of cluster quality to group objects in classes. The primary goal is to discover concepts structure in data objects. The paper is organized as follows: Section 2 presents a short survey of techniques for the clustering problem. Section 3 explains the clustering problem while Section 4 describes the genetic algorithm and the K-Means algorithm. Section 5 introduces the multilevel paradigm, while section 6 presents the experimental results. Finally, Section 7 presents a summary and possible future work.

\section{A SHORT SURVEY OF Algorithm}

Cluster analysis has been a hot topic of research due to its applicability in many disciplines including market segmentation [3], image processing [4], web mining [5], and bio-informatics [6] to name just a few. This has stimulated the search for efficient clustering approximation algorithms which can be broadly be divided into three main types: hierarchical, partitional, and local search methods. Hierarchical clustering algorithms [7] construct a hierarchy of clusters using either agglomerative or divisive style. The agglomerative style starts with each data object in its own cluster, and at each step, the closest pair of clusters are merged using a metric of cluster proximity. Different agglomerative algorithms differ in how the clusters are merged at each level. With divisive clustering, all data objects are initially placed in one cluster and clusters are repeatedly split in two until all data objects are in their own cluster. On the other hand, Nonhierarchical or partitional clustering [8] are based on iterative relocation of data objects between clusters. The set of data objects is divided into non-overlapping clusters such that each data object lies in exactly one cluster. The quality of the solution is measured by a clustering criterion. At each iteration, the algorithm improves the value of the criterion function until convergence is reached. The algorithms belonging to this class generate solutions from scratch by adding to an initially empty partial solution components, until a solution is complete. They are regarded as the fastest approximate methods, yet they often return solutions of inferior quality. Finally, local search methods constitute an alternative to the traditional partitional techniques. These techniques offer the advantage of being flexible. They can be applied to any problem (discrete or continuous) whenever there is a possibility for encoding a candidate solution to the problem, and a mean of computing the quality of any candidate solution through the so-called cost function. They have the advantage that they could escape more efficiently from local minima. They start from some initial solution and iteratively try to replace the current solution by a better one in the light of the cost function in an appropriately defined neighbourhood of the current solution. Their performances depend highly on finding a tactical interplay between diversification and intensification. The former refers to the ability to explore many different regions of the search space, whereas the latter refers to the ability to obtain high quality solutions within those regions. Examples include genetic algorithms [9] [10], Tabu Search [11], Grasp [12]. 


\section{The Clustering Problem}

The clustering Problem can be defined as follows: Given a finite set of $\mathrm{N}$ data objects where each object is a finite set of attributes or feature from which it can be identified. A relation defining the constraints on the resulting clusters. The relation to be respected by all the formed clusters is that no pairs of clusters should have a data object in common. A solution to the clustering problem requires the partitioning of the $\mathrm{N}$ data objects into a set of $\mathrm{K}$ clusters such that objects in the same cluster are more similar to each other than to those in other clusters. Searching all possible clustering alternatives would not be possible. Because of this reason, there is a considerable interest in the design of heuristics to solve the clustering problems using a cost function that quantifies the goodness of the clusters on the basis of the similarity or dissimilarity measures of the data objects. A commonly used cost function is the sum of squared distances of the data objects to their cluster representatives. Euclidean distance is the most widely used distance function in the clustering context.

\section{AlgorithMS}

\subsection{Genetic Algorithms}

Genetic Algorithms [13] are stochastic methods for global search and optimization and belong to the group of Evolutionary Algorithms. They simultaneously examines and manipulates a set of possible solution. Given a specific problem to solve, the input to GAs is an initial population of solutions called individuals or chromosomes. A gene is part of a chromosome, which is the smallest unit of genetic information. Every gene is able to assume different values called allele. All genes of an organism form a genome which affects the appearance of an organism called phenotype. The chromosomes are encoded using a chosen representation and each can be thought of as a point in the search space of candidate solutions. Each individual is assigned a score (fitness) value that allows assessing its quality. The members of the initial population may be randomly generated or by using sophisticated mechanisms by means of which an initial population of high quality chromosomes is produced. The reproduction operator selects (randomly or based on the individual's fitness) chromosomes from the population to be parents and enters them in a mating pool. Parent individuals are drawn from the mating pool and combined so that information is exchanged and passed to off-springs depending on the probability of the cross-over operator. The new population is then subjected to mutation and enters into an intermediate population. The mutation operator acts as an element of diversity into the population and is generally applied with a low probability to avoid disrupting cross-over results. Finally, a selection scheme is used to update the population giving rise to a new generation. The individuals from the set of solutions which is called population will evolve from generation to generation by repeated applications of an evaluation procedure that is based on genetic operators. Over many generations, the population becomes increasingly uniform until it ultimately converges to optimal or near-optimal solutions. Below are the various steps used in the proposed genetic algorithm.

\subsubsection{Fitness function}

The notion of fitness is fundamental to the application of genetic algorithms. It is a numerical value that expresses the performance of an individual (solution) so that different individuals can 
be compared. The fitness function used by the genetic algorithm is simply the Euclidean distance.

\subsubsection{Representation}

A representation is a mapping from the state space of possible solutions to a state of encoded solutions within a particular data structure. The encoding scheme used in this work is based on integer encoding. An individual or chromosome is represented using a vector of $\mathrm{n}$ positions, where $\mathrm{n}$ is the set of data objects. Each position corresponds to a particular data object, i.e, he ith position (gene) represents the ith data object. Each gene has a value over the set $\{1,2, \ldots . \mathrm{k}\}$. These values define the set of cluster labels.

\subsubsection{Initial population}

The initial population consists of individuals generated randomly in which each gene's allele is assigned randomly a label from the set of cluster labels.

\subsubsection{Cross-over}

The task of the cross-over operator is to reach regions of the search space with higher average quality. New solutions are created by combining pairs of individuals in the population and then applying a crossover operator to each chosen pair. The individuals are visited in random order. An unmatched individual i_l is matched randomly with an unmatched individual i_m. Thereafter, the two-point crossover operator is applied using a cross-over probability to each matched pair of individuals. The two-point crossover selects two randomly points within a chromosome and then interchanges the two parent chromosomes between these points to generate two new offspring. Recombination can be defined as a process in which a set of configurations (solutions referred as parents) undergoes a transformation to create a set of configurations (referred as off-springs). The creation of these descendants involves the location and combinations of features extracted from the parents. The reason behind choosing the two point crossover are the results presented in Icite $\{$ crossover\} where the difference between the different crossovers are not significant when the problem to be solved is hard. In addition, the work conducted in [14] shows that the twopoint crossover is more effective when the problem at hand is difficult to solve.

\subsubsection{Mutation}

The purpose of mutation which is the secondary search operator used in this work, is to generate modified individuals by introducing new features in the population. By mutation, the alleles of the produced child individuals have a chance to be modified, which enables further exploration of the search space. The mutation operator takes a single parameter $\mathrm{p} \_\mathrm{m}$, which specifies the probability of performing a possible mutation. Let $\mathrm{I}\left\{\mathrm{c} \_1, \mathrm{c} \_2, \ldots, \mathrm{c} \_\mathrm{k}\right\}$ be an individual where each of whose gene $c_{-} i$ is a cluster label. In our mutation operator, each gene $c_{-} i$ is mutated through flipping this gene's allele from the current cluster label $\mathrm{c}_{-} \mathrm{i}$ to a new randomly chosen cluster label if the probability test is passed. The mutation probability ensures that, theoretically, every region of the search space is explored. The mutation operator prevents the searching process from being trapped into local optima while adding to the diversity of the population and thereby increasing the likelihood that the algorithm will generate individuals with better fitness values. 


\subsubsection{Selection}

The selection operator acts on individuals in the current population. During this phase, the search for the global solution gets a clearer direction, whereby the optimization process is gradually focused on the relevant areas of the search space. Based on each individual fitness, it determines the next population. In the roulette method, the selection is stochastic and biased towards the best individuals. The first step is to calculate the cumulative fitness of the whole population through the sum of the fitness of all individuals. After that, the probability of selection is calculated for each individual.

\subsection{K-Means Algorithm}

The K-means [15] is a simple and well known algorithm used for solving the clustering problem. The goal of the algorithm is to find the best partitioning of $\mathrm{N}$ objects into $\mathrm{K}$ clusters, so that the total distance between the cluster's members and its corresponding centroid, representative of the cluster is minimized. The algorithm uses an iterative refinement strategy using the following steps:

1) This step determines the starting cluster's centroids. A very common used strategy is to assign random $\mathrm{k}$ different objects as being the centroids.

2) Assign each object to the cluster that has the closest centroid. In order to find the cluster with the most similar centroid, the algorithm must calculate the distance between all the objects and each centroid.

3) Recalculate the values of the centroids. The values of the centroid are updated by taking as the average of the values of the object's attributes that are part of the cluster.

4) Repeat steps 2 and 3 iteratively until objects can no longer change clusters.

\section{The Multilevel Paradigm}

The multilevel paradigm [16] is a simple technique which at its core involves recursive coarsening to produce smaller and smaller problems that are easier to solve than the original one. The multilevel paradigm consists of four phases: coarsening, initial solution, projection and refinement. The coarsening phase aims at merging the variables associated with the problem to form clusters. The clusters are used in a recursive manner to construct a hierarchy of problems each representing the original problem but with fewer degrees of freedom. This phase is repeated until the size of the smallest problem falls below a specified reduction threshold. Then, a solution for the problem at the coarsest level is generated, and then successively projected back onto each of the intermediate levels in reverse order. The solution at each child level is improved before moving to the parent level. A common feature that characterizes multilevel algorithms, is that any solution in any of the coarsened problems is a legitimate solution to the original problem. The multilevel paradigm comprises the following steps:

\subsection{Reduction Phase:}

The first component in the multilevel framework is the so-called coarsening or reduction phase. Let P_0 (the subscript represents the level of problem scale) be the set of data objects to be clustered. The next coarser level $P_{-} \_1$ is constructed from $P \_0$ using two different algorithms. The first algorithm is a random coarsening scheme (RC) The data objects are visited in a random order. If a data object $\mathrm{O} \_\mathrm{i}$ has not been matched yet, then a randomly unmatched data object $\mathrm{O}_{-} \mathrm{j}$ 
is selected, and a new data objects $\mathrm{O} \_\mathrm{k}$ (a cluster) consisting of the two data objects $\mathrm{O} \_\mathrm{i}$ and $\mathrm{O} \_\mathrm{j}$ is created. The set of attributes of the new data object $\mathrm{O} \_\mathrm{k}$ is calculated by taking the average of each attribute from $\mathrm{O}_{-} \mathrm{i}$ and its corresponding one from $\mathrm{O}_{-} \mathrm{j}$. Unmerged data objects are simply copied to the next level. The second coarsening algorithm distance coarsening (MC) exploits a measure of the connection strength between the data object which relies on the notion of distance. The data objects are visited in a random order. However, instead of merging a data object O_i with a random object $\mathrm{O} \_\mathrm{j}$, the data object $\mathrm{O} \_\mathrm{i}$ is merged with $\mathrm{O} \_\mathrm{m}$ such that Euclidean distance function is minimized. The new formed data objects are used to define a new and smaller problem and recursively iterate the reduction process until the size of the problem reaches some desired threshold .

\subsection{Initial Clustering}

The reduction phase ceases when the problem size shrinks to a desired threshold. Initialization is then trivial and consists of generating an initial clustering ( $\mathrm{S} \_\mathrm{m}$ ) for the problem using a random procedure. The clusters of every individual in the population are assigned a random label from the set of cluster labels.

\subsection{Projection Phase}

The projection phase refers to the inverse process followed during the reduction phase. Having improved the quality of the clustering on level $\mathrm{S}_{-}\{\mathrm{m}+1\}$, this clustering must be extended on is parent level $S_{-} m$. The extension algorithm is simple; if a data object $\mathrm{O} \__{-} \mathrm{i}$ in $\mathrm{S}_{-}\{\mathrm{m}+1\}$ is assigned the cluster label c_l, then the merged pair of data objects that it represents, O_l, O_m in S_m are also assigned the cluster label c_1.

\subsection{Improvement or Refinement Phase}

The idea behind the improvement phase is to use the projected clustering at level $S_{-}\{m+1\}$ as the initial clustering for the level S_m for further refinement using GA or K-Means described in the previous section. Even though the clustering at the level $S_{-}\{m+1\}$ is at a local minimum, the projected clustering may not be at a local optimum with respect to S_m. The projected clustering is already a good solution and contains individuals with low function value, GA and K-means will converge quicker to a better clustering. As soon as the population tends to loose its diversity, premature convergence occurs and all individuals in the population tend to be identical with almost the same fitness value. During each level, the genetic algorithm is assumed to reach convergence when no further improvement of the best solution has not been made during five consecutive generations.

\section{EXPERIMENTAL RESULTS}

\subsection{Benchmark Instances and Parameter Settings}

The performance of the multilevel paradigm is compared against its single variant using a set of instances taken from real industrial problems. This set is taken from the Machine Learning Repository website (http://archive.ics.uci.edu/ml/datasets). Due to the randomization nature of the algorithms, each problem instance was run 100 times. The tests were carried out on a DELL machine with $800 \mathrm{MHz}$ CPU and $2 \mathrm{~GB}$ of memory. The code was written in $\mathrm{C}$ and compiled with 
the GNU C compiler version 4.6. The following parameters have been fixed experimentally and are listed below:

-Crossover probability $=0.85$

-Mutation probability $=0.01$

-Population size $=50$

-Stopping criteria for the reduction phase: The reduction process stops as soon as the size of the coarsest problem reaches $10 \%$ the size of the original problem.

-Convergence during the refinement phase: If there is no observable improvement of the cost Euclidean distance cost function during 5 consecutive generations (GA) or iterations (for KMeans), both algorithms are assumed to have reached convergence and the improvement phase is moved to a higher level

\subsection{Analysis of Results}

The plot in Figures [1]-[8] show the evolution of the cost function versus the quality of the clustering. The plots suggest that cluster problem solving with GA happens in two phases. In the first phase, the cost function decreases rapidly and then flattens off as we reach the plateau region, marking the start of the second phase. The plateau region spans a region in the search space where the best value of the cost function remains unchanged. The plateau region may be of a short length depending on whether the algorithm possesses some sort of mechanisms capable of escaping from it, otherwise the algorithm stagnates and a premature convergence of the algorithm is detected. A closer look at Figures 1-2 show that the quality of the clustering reaches its highest value at $0.88 \%$ and continues to get marginally worse $(0.87 \%)$ while the value of cost function is slightly decreasing. The plots depicted in Figures 3-4 show that the quality of the clustering drops from $0.80 \%$ to $0.79 \%$ before GA enters a premature convergence state. On the other hand, the curve of the cost function continues to decrease showing some improvement. An improvement of $37 \%$ in the cost function led to no improvement in the quality of the clustering. The same phenomenon is detected with K-Means algorithm. The plots at Figures 5-6 reveal that the quality of the clustering is at its maximum value $(0.91 \%)$ and suddenly get worse by almost $6 \%$ while the cost function is showing an improvement by a factor of 7\%. Finally the plots at Figures 7-8 confirm that the quality of the clustering is getting worse after reaching a peak at 0.91 while the cost function is indicating the opposite and attaining slightly low values. These observations demonstrate that the cost function scores do not capture the quality of the clustering making it an unsuitable metric to apply for maximizing both the homogeneity within each cluster and the heterogeneity between different clusters. Figures 9-11 show the impact of the two coarsening schemes on the final cost function score. In most cases, the curve of MC remains lower compared to $\mathrm{RC}$ during the different levels and may reach the same level as $\mathrm{RC}$ or maintains its superiority until GA converges. The main conclusion that may be drawn from these plots is that $\mathrm{MC}$ is at least as good as RC or better as it provides a lower cost function value. The experimental results demonstrated the K-Means combined with MC delivers better clustering than K-Means with RC in 4 out of the 8 cases (up to 11\%), similar results in one case and does worse in two cases ( up to $31 \%$ ) while requiring between $15 \backslash \%$ and $55 \%$ more time. When GA is considered, MC outperforms RC in 3 cases (up to $24 \%$ ), while it performs $2 \%$ worse in only one case. The time of GA combined with MC ranges from $2 \%$ to $19 \%$ of the time of GA combined with RC. 
Comparing the two multilevel algorithms using MC as the chosen coarsening scheme, MLVLGA produces better quality in 3 out of 8 cases and the difference in quality ranges from $2 \%$ to $24 \%$. For the remaining 3 cases where MLVL-K-Means does better, the improvement is only marginally better (between $0.9 \%$ and $2 \%$ ). Looking at the time spent MLVL-K-Means, in all the cases requires the least amount of time (up to $99 \%$ faster).With regard to the multilevel paradigm, it is somewhat unsatisfactory that its ability to enhance the convergence behavior of the two algorithms is not conclusive. However, This does not seem to be in line with with the general success established in other combinatorial optimization problems such as the graph partitioning problem [16] and the satisfiability problem [17]. The reason behind this sort of convergence behaviour observed in the multilevel paradigm is not obvious but we can speculate. As pointed earlier, the multilevel paradigm requires that any solution in any of the coarsened problems should induce a legitimate solution on the original problem. Thus at any stage after initialisation the current solution could simply be extended through all the problem levels to achieve a solution of the original problem. This requirement is violated in our case. The attributes of each object formed during each child level are calculated by taking the average of the attributes of two different objects from the parent level. The consequence of this procedure is that the optimization is carried out on different levels each having its own space. The clustering obtained at the coarse space and the original space do not have have the same cost with respect to the objective function.

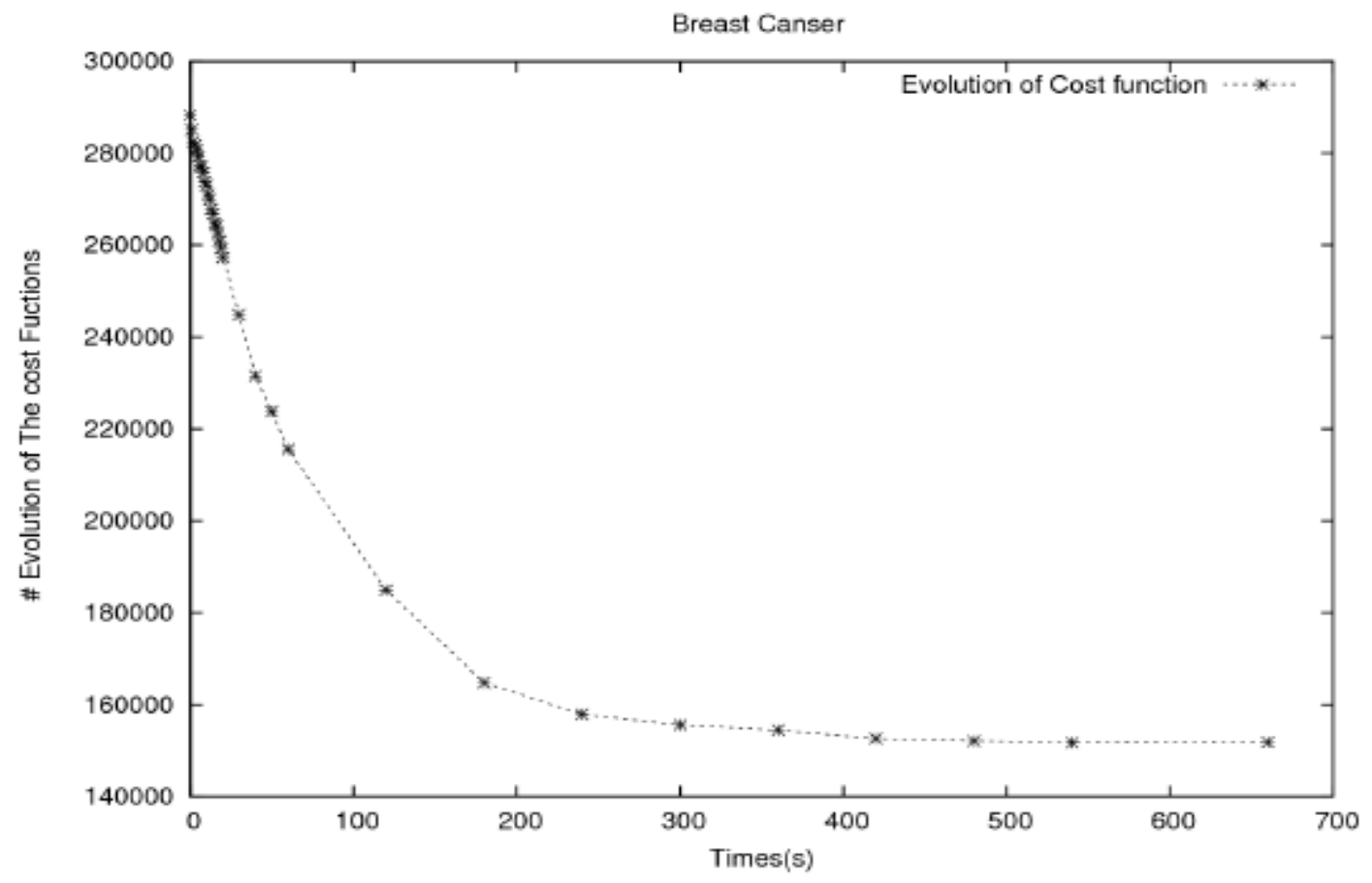

Figure 1. Average development for 100 runs. Evolution of the Euclidean cost function for BreastCanser 


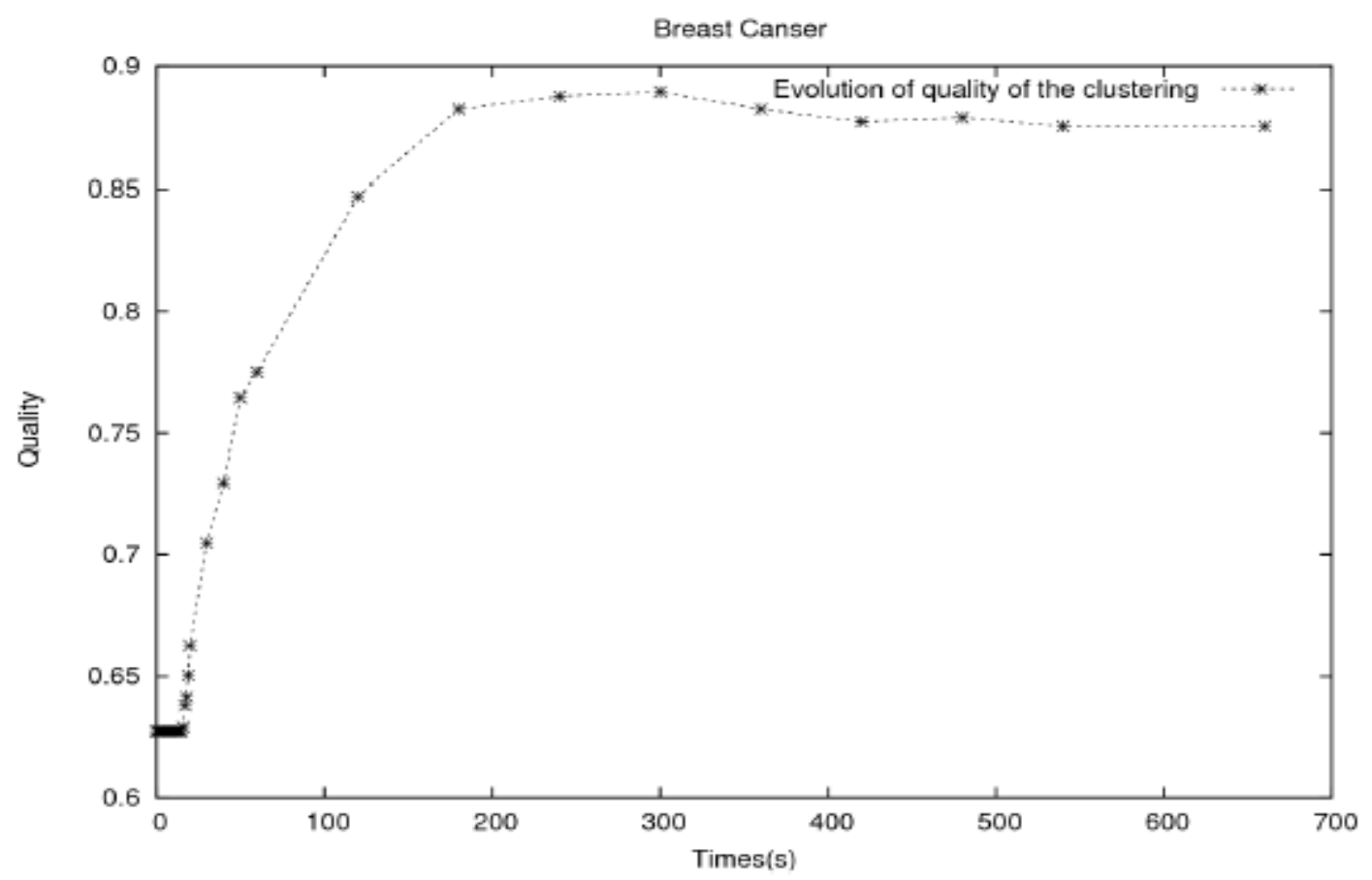

Figure 2. Average Development for 100 Runs Evolution of the Quality of the Clustering for BreastCancer

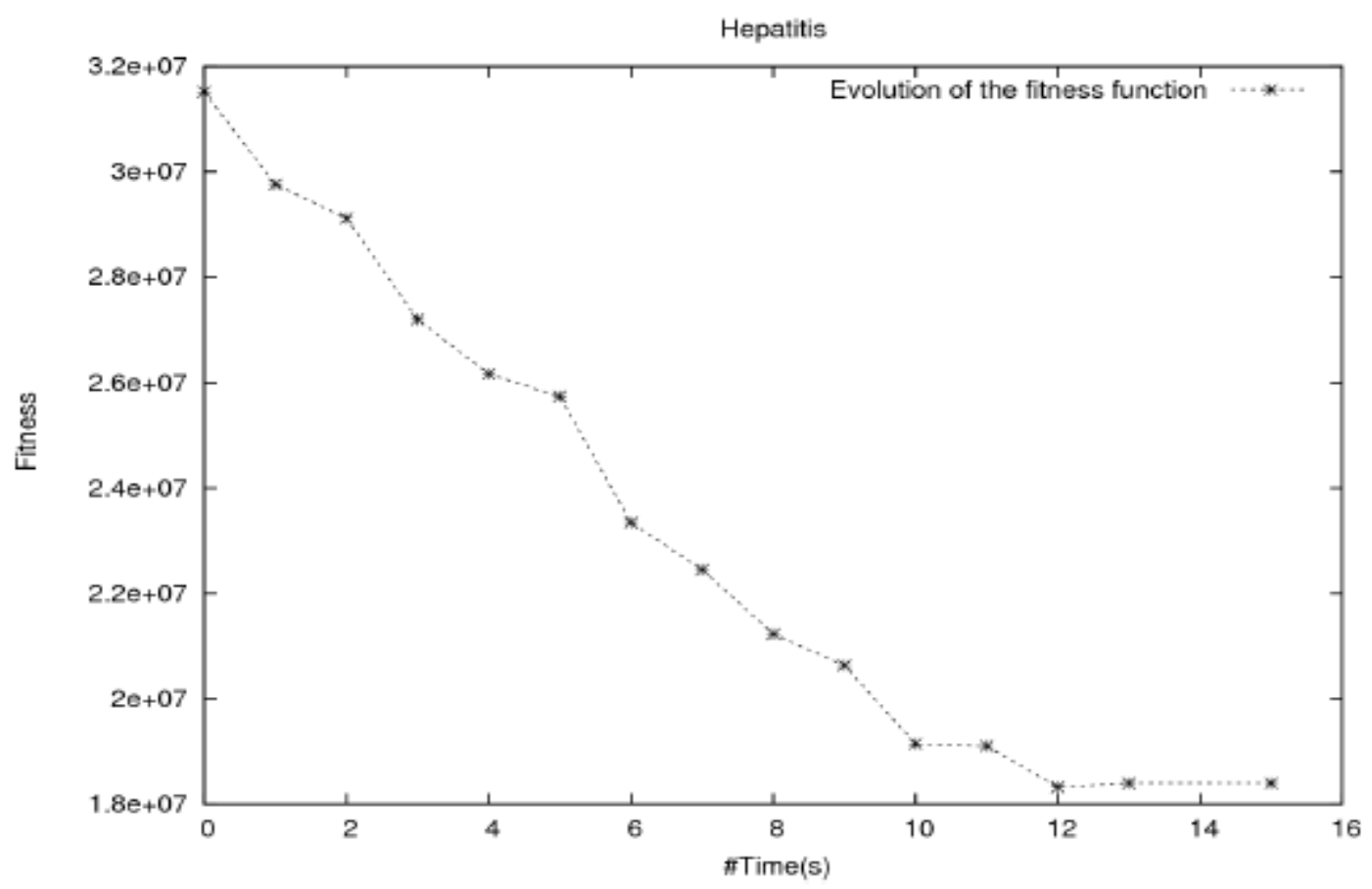

Figure 3. Average Development for 100 Runs. Evolution of Euclidean Cost Function for Hepatitis 


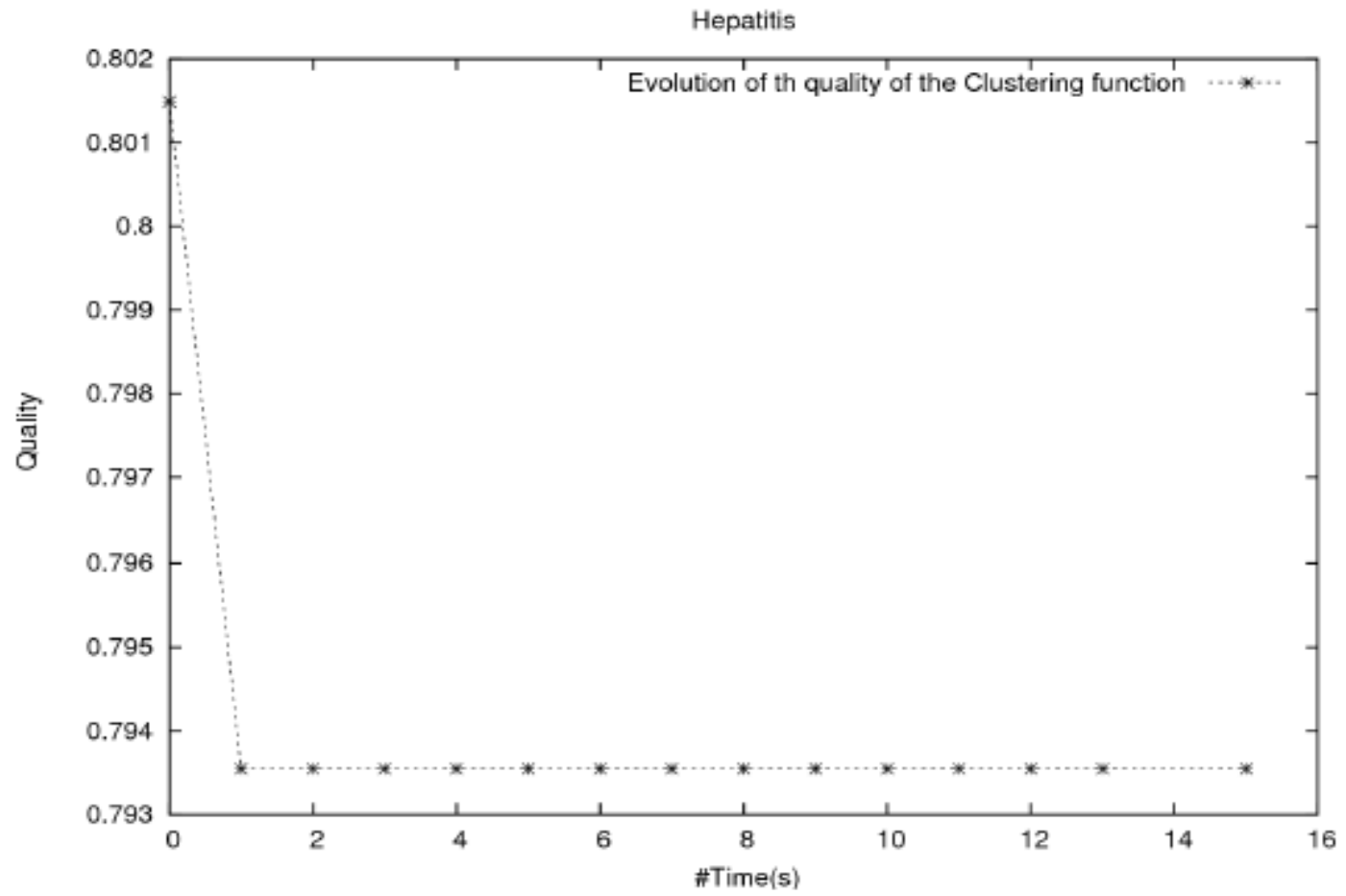

Figure 4. Average Development for 100 Runs. Evolution of the Quality of the Clutering for Hepatitis

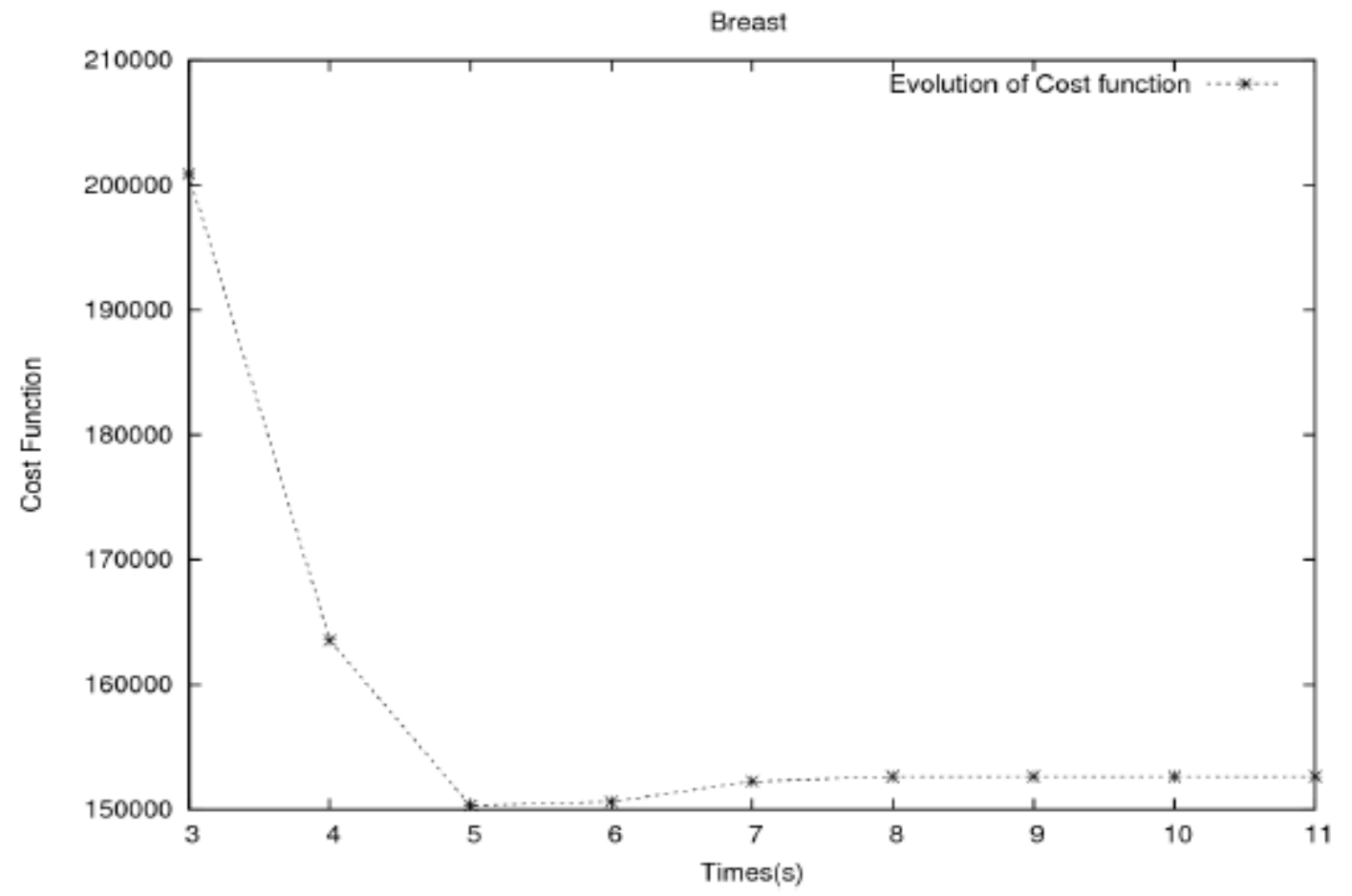

Figure 5. Average Development for 100 Runs. Evolution of Euclidean Cost Function for Breast. 


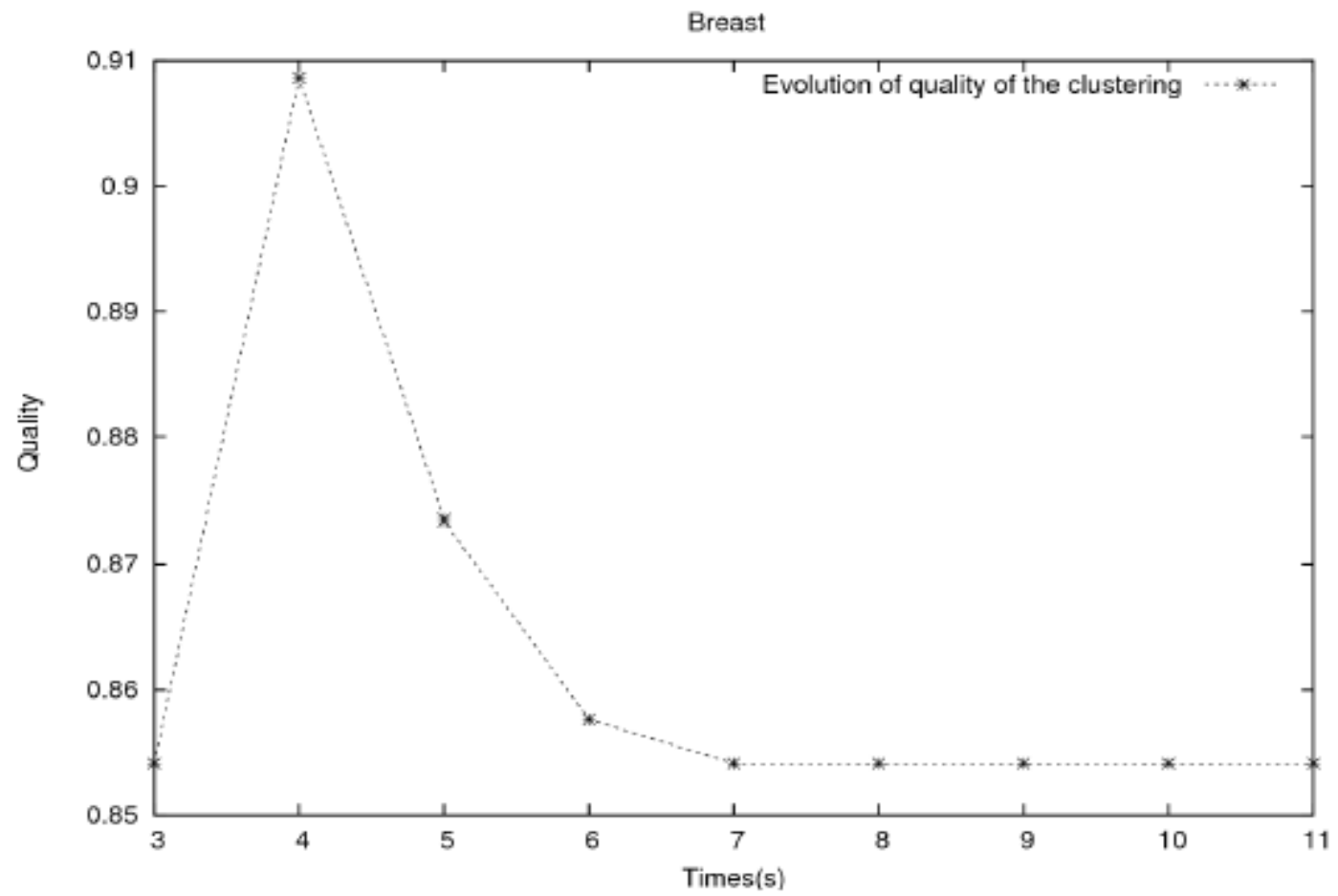

Figure 6. Average Development for 100 Runs. Evolution of the Quality of the Clustering for Breast.

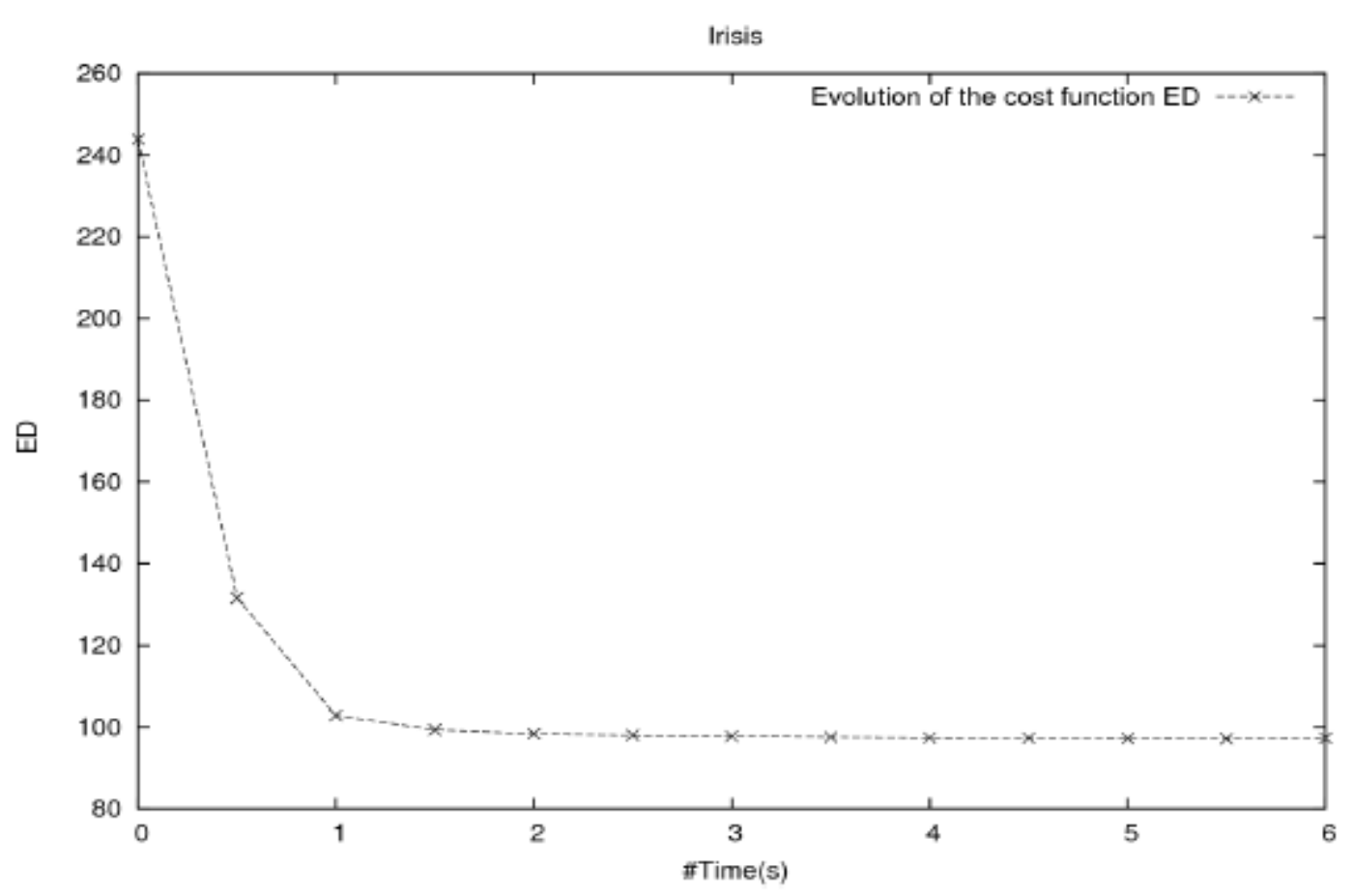

Figure 7. Average Development for 100 Runs. Evolution of Euclidean Cost Function for IRISIS 


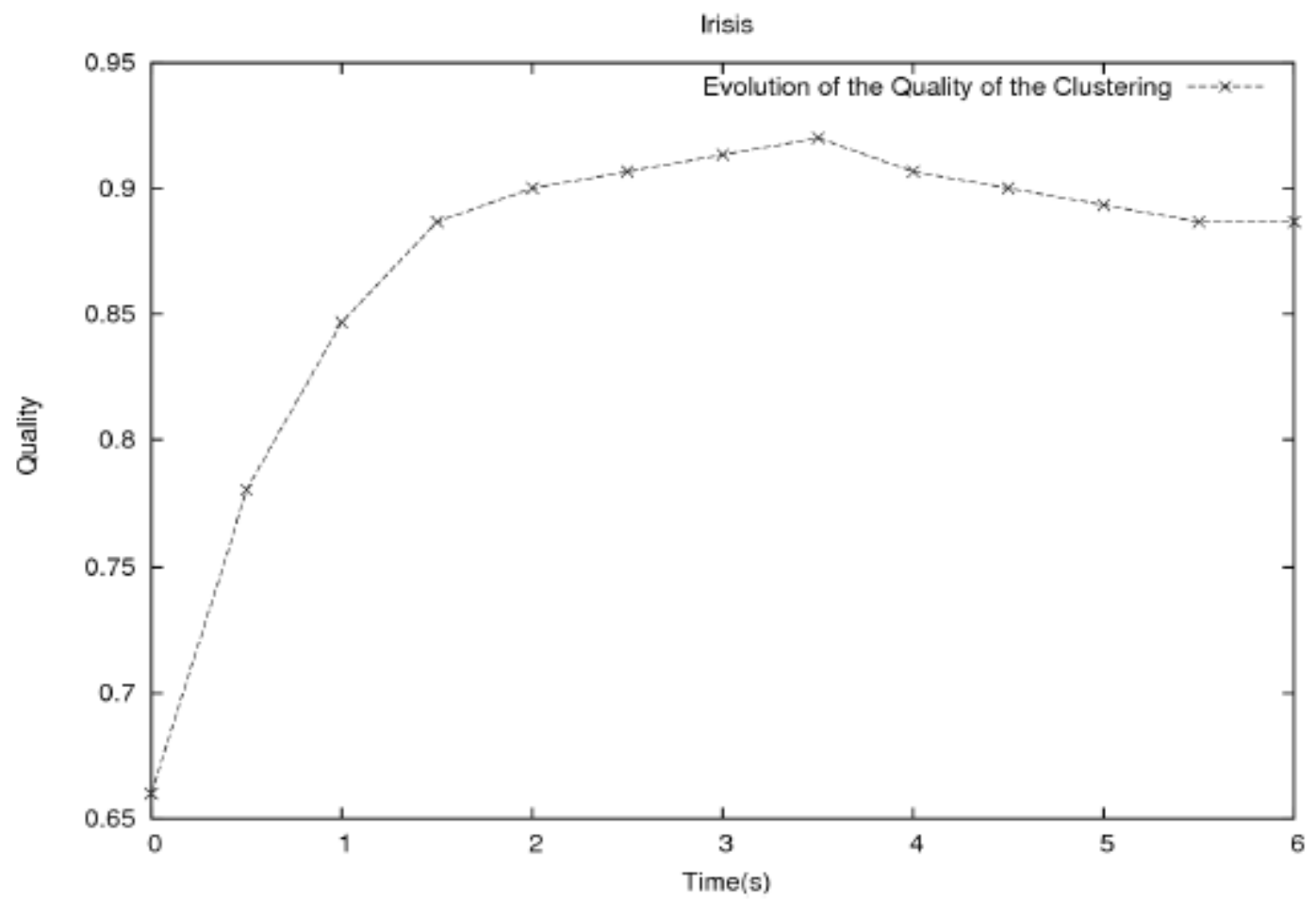

Figure 8. Average Development for 100 Runs. Evolution of the Quality of the Clustering for IRISIS.

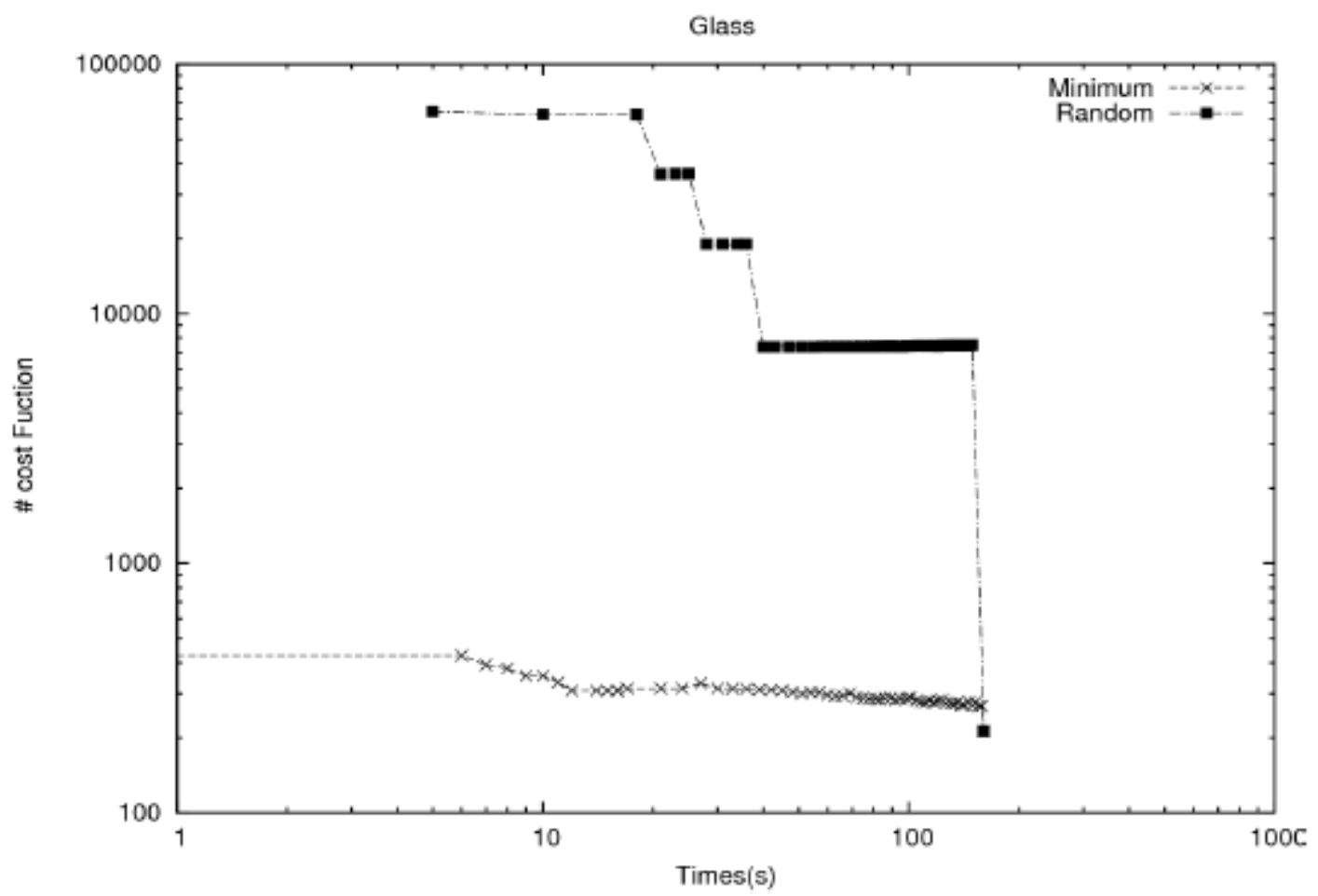

Figure 9. Comparison of Coarsening Schemes for Glass Figure 


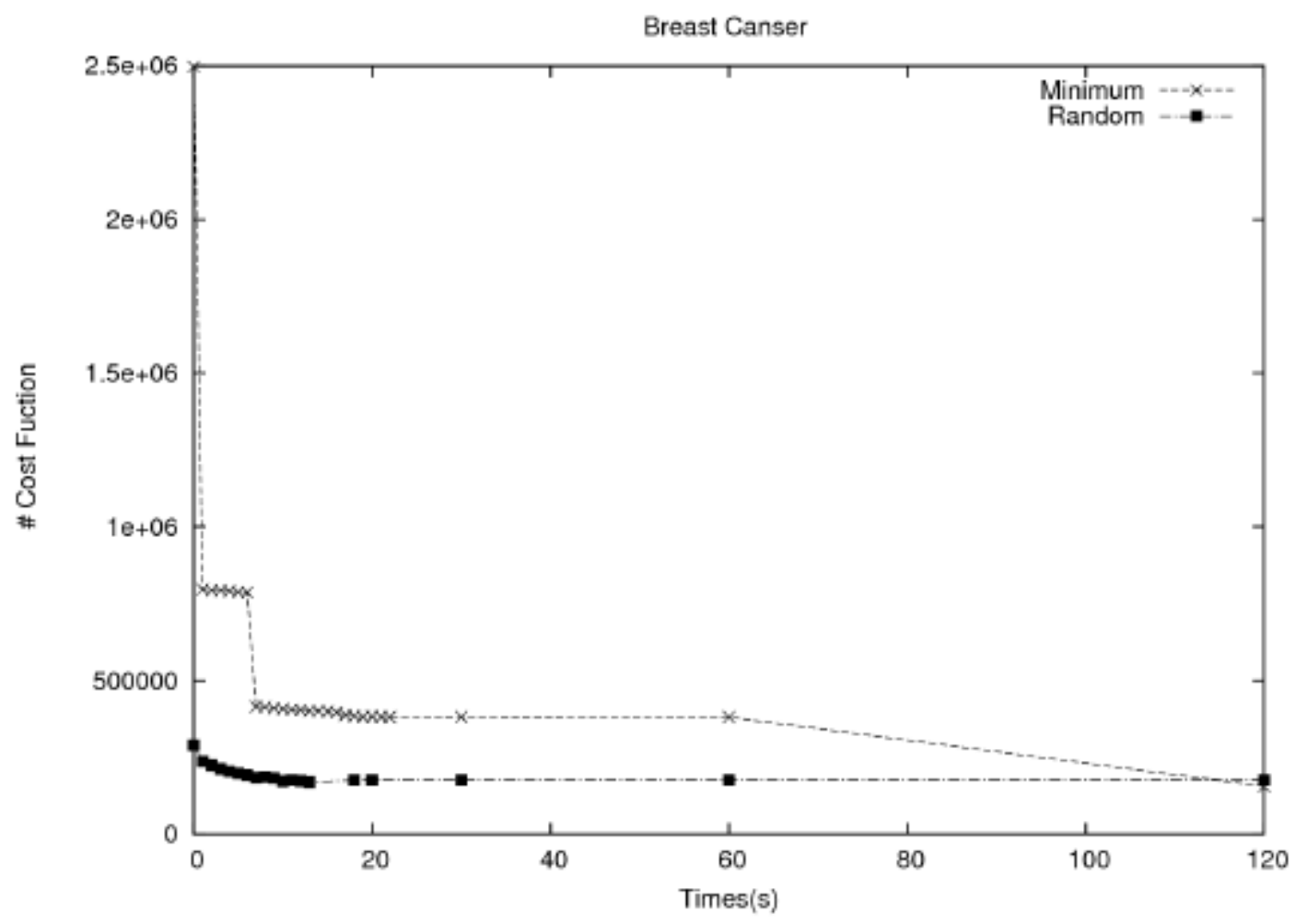

Figure 10. Comparison of Coarsening Schemes for Breastcancer

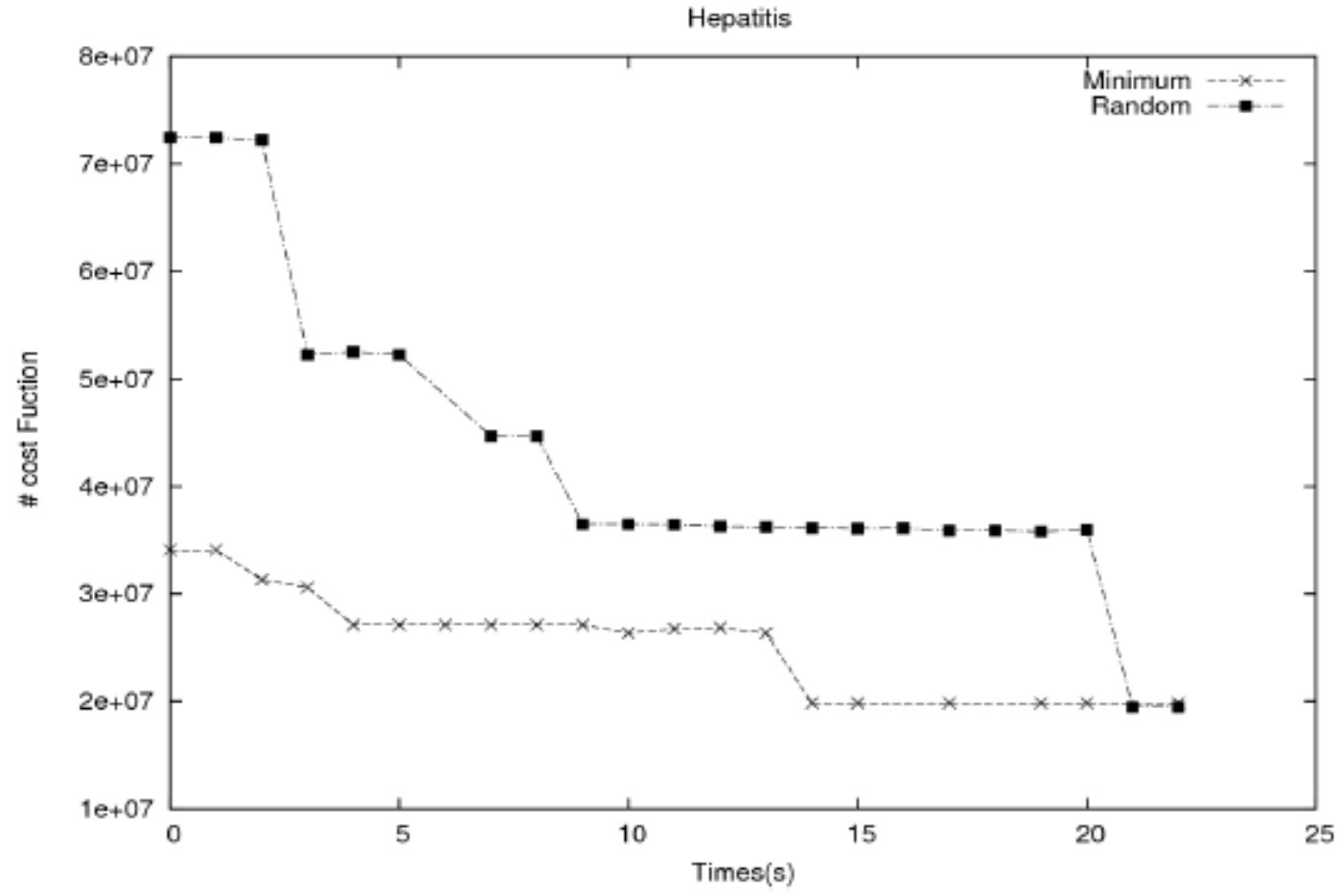

Figure 11. Comparison of Coarsening Schemes for Hepatitis 


\section{CONCLUSIONS}

This paper introduces a multilevel scheme combined with the popular K-Means and genetic algorithm for the clustering problem. The first conclusion drawn from the results at least for the instances tested in this work generally indicate that the Euclidean Distance cost function widely used in literature does not capture the quality of the clustering making it an unsuitable metric to apply for maximizing both the homogeneity within each cluster and the heterogeneity between different clusters. The coarsening methods used during the coarsening phase have a great impact on the quality of the clustering. The quality of the clustering provided by $\mathrm{MC}$ is at least as good or better compared to RC regardless of which algorithm is used during the refinement phase. To summarise then, the multilevel paradigm can improve the asymptotic convergence of the original algorithms. An obvious subject for further work would be the use of different cost functions and better coarsening schemes so that the algorithms used during the refinement phase work on identical search spaces. A better coarsening strategy would be to let the merged objects during each level be used to create coarser problems so that each entity of a coarse problem $\mathrm{P} \_\mathrm{k}$ is composed of $2^{\wedge} \mathrm{k}$ objects. The adopted strategy will provide K-Means and GA to work on identical search spaces during the refinement phase.

\section{REFERENCES}

[1] B.S Everitt, S. Landau, M. Leese (2001) Cluster Analysis, Arnold Publishers.

[2] M.R. Garey, D.S. Jhonson, H.S. Witsenhausen (1982). "The complexity of the generalized LlyodMax problem". IEEE Trans Info Theory 28 (2), pp255-256.

[3] J.P. Bigus ( 1996) Data Mining with Neural Networks, McGraw-Hill.

[4] A.K. Jain, R.C. Dubes. (1988) Algorithms for Clustering Data. Prentice Hall.

[5] G. Mecca, S. Raunich, A. Pappalardo. ( 2007) "A New Algorithm for Clustering Search Results. Data and Knowledge Engineering", Vol. 62, pp504-522.

[6] P.M. BertoneGerstein (2001) " Integrative Data Mining: The New Direction in BioinformaticsMachine Learning for Analzing Genome-Wide Expression Profiles" , IEEE Engineering in Medicine and Biology, Vol. 20, pp33-40.

[7] Y. Zhao and G. Karypis ( 2002) "Evaluation of hierarchical clustering algorithms for document datasets ", In Proc. of Int'l.Conf. on Information and Knowledge Management, pp515-524.2002.

[8] S. Zhong,J.Ghosh (2003) "A comparative study of generative models for document clustering", In SIAM Int. Conf. Data Mining Workshop on Clustering High Dimensional Data and Its Applications, San Francisco, CA.

[9] D.P.F. Alckmin, F.M.Varejao (2012) " Hybrid Genetic Algorithm Applied to the Clustering problem", Revista Investigacion Operational, Vol.33, NO. 2, pp141-151.

[10] B. Juans, S.U. Guan (2012) "Genetic Algorithm Based Split-Fusion Clustering", International Journal of Machine Learning and Computing, Vol. 2, No. 6.

[11] K. Adnan, A. Salwani, N.M.Z. Ahmad. (2011) "A Modified Tabu Search Approach for The Clustering Problem. Journal of Applied Sciences ", Vol. 11 Issue 19.

[12] D.O.V. Matos, J.E.C. Arroyo, A.G. dos Santos, L.B. Goncalves (2012) " A GRASP based algorithm for efficient cluster formation in wireless sensor networks. Wireless and Mobile Computing, Networking and Communications (WiMob) ", 2012 IEEE 8th International Conference on , vol., no., pp187-194.

[13] D.E. Goldberg ( 1989) Genetic Algorithms in Search, Optimization, and Machine Learning , Addison-Wesley, New York.

[14] W. Spears ( 1995) "Adapting Crossover in Evolutionary Algorithms " Proc of the Fourth Annual Conference on Evolutionary Programming, MIT Press, pp367-384. 
[15] J.B. MacQueen ( 1967 ) "Some methods for classification and analysis of multi- variate observation" , In: In Le Cam, L.M and Neyman, J., editor, 5 Berkeley Symposium on Mathematical Statistics and Probability. Univ. of California Press

[16] C. Walshaw ( 2008) "Multilevel Refinement for Combinatorial Optimization: BoostingMetaheuristic Performance", in C. Blum et al., pp261-289, Springer, Berlin.

[17] N. Bouhmala. (2012) "A Multilevel Memetic Algorithm for Large Sat-Encoded Problems ", Evolutionary Computation, Vol.20 (4), pp641-664.

\section{AUTHOR}

Master Thesis from University of Bergen, Norway, $\mathrm{PhD}$ Thesis in Computer Science from the University of Neuchatel in Switzerland. His research interests include MetaHeuristics, Parallel Computing, Data Minning.

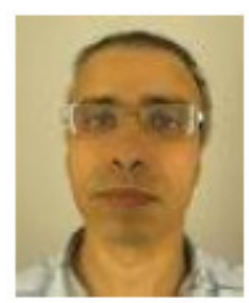


INTENTIONAL BLANK 


\title{
Multi-Dimensional Customization MODELLING BASED ON METAGRAPH FOR SAaS Multi-Tenant Applications
}

\author{
Ashraf A. Shahin ${ }^{1,2}$ \\ ${ }^{1}$ College of Computer and Information Sciences, \\ Al Imam Mohammad Ibn Saud Islamic University (IMSIU) \\ Riyadh, Kingdom of Saudi Arabia \\ ashraf_shahen@ccis.imamu.edu.sa \\ ${ }^{2}$ Department of Computer and Information Sciences, \\ Institute of Statistical Studies \& Research, Cairo University, Egypt
}

\begin{abstract}
Software as a Service (SaaS) is a new software delivery model in which pre-built applications are delivered to customers as a service. SaaS providers aim to attract a large number of tenants (users) with minimal system modifications to meet economics of scale. To achieve this aim, SaaS applications have to be customizable to meet requirements of each tenant. However, due to the rapid growing of the SaaS, SaaS applications could have thousands of tenants with a huge number of ways to customize applications. Modularizing such customizations still is a highly complex task. Additionally, due to the big variation of requirements for tenants, no single customization model is appropriate for all tenants. In this paper, we propose a multidimensional customization model based on metagraph. The proposed mode addresses the modelling variability among tenants, describes customizations and their relationships, and guarantees the correctness of SaaS customizations made by tenants.
\end{abstract}

\section{KEYWORDS}

Cloud Computing, Software as a Service (SaaS), Multi-tenancy, Customization Modelling, Metagraph

\section{INTRODUCTION}

Software as a Service (SaaS) is a cloud computing service model in which pre-built applications are delivered to customers as a service [1]. The main goal of the SaaS providers is attracting a significant number of tenants for their SaaS applications. However, the functionality and quality that individual customers require from a software application can differ [2]. This forces the SaaS providers to deploy multiple software applications customized for each set of users, which results in increasing cost of infrastructure and making it difficult to maintain and update. To overcome with this problem, the SaaS providers deploy software applications allow tenant-specific configuration and customization. For customization, some elements of an application need to be customized, including graphic user interfaces (GUI), workflow, service, and data models [3]. Several researches have attempted to support configuration and customization of these elements $[1,4,5,6,7]$.

David C. Wyld et al. (Eds) : CCSIT, SIPP, AISC, PDCTA, NLP - 2014

pp. 53-63, 2014. (C) CS \& IT-CSCP 2014

DOI : $10.5121 /$ csit.2014.4205 
One of the most prominent approaches for achieving the tenant-specific configuration and customization is providing an application template with unspecified parts that can be customized by each tenant $[7,8]$. These unspecified parts are called customization points of an application [3]. For each customization point, a set of components are provided to achieve variations in tenants' requirements. A tenant customizes each customization point by selecting components from the corresponding set or by defining new components.

However, modularizing such customizations still is a highly complex task. The complexity, in general, comes from the following sources. First, the SaaS applications could have hundreds of customization points with thousands of components for achieving variations in tenants' requirements. Second, the relationships and dependences of components are more complex. It is possible for some components to require other components or for some components to conflict with others (figure 1 shows the relationships of components from different customization points). Third, due to the big variation of requirements for the tenants, no single customizations model is appropriate for all tenants. To address this variation, the developers need to provide software applications with different modularizations.

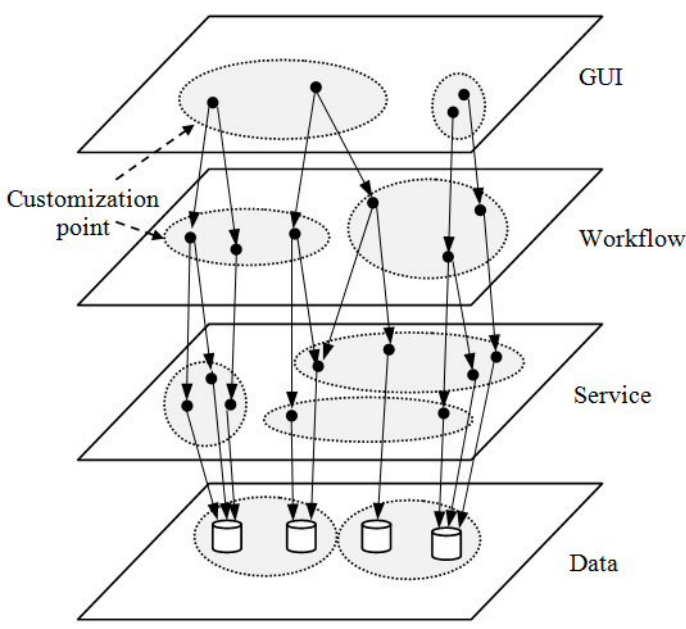

Figure 1. Relationships of components from different customization points

Multi-dimensional separation of concerns (MDSOC) is separation of concerns that allows developers to partition overlapping or interacting concerns in new and existing software products along multiple dimensions of decomposition and composition. MDSOC allows developers to identify new concerns, and new dimensions of concern, incrementally and simultaneously, at any time [9]. Another important property for MDSOC is on-demand remodularization, which is the ability to change the way in which a system is modularized without having to refactoring, reengineering, or other invasive change [10,11]. A detailed description of MDSOC approach is available in [9].

In this paper, we apply MDSOC concepts to address the modelling variability among tenants. Applying MDSOC allows developers to provide independent dimension for each type of concerns and allows tenants to customize concerns that crosscut several customization points independently. For example, tenants that are concerned with security can customize the security concerns in security dimension only. This results in distinct customization models, which are easier to handle than one large model. On the other hand, we use metagraph to describe dependences between components of each concern. Metagraph is a graphical structure in which edges represent directed relationships between sets of elements [12]. Using metagraph tools, we can combine the separated models to validate the customization points' values made by tenants. 
This paper is organized as the following. Section 2 discusses the related work in customization modelling. Section 3 describes our multi-dimensional customization model. Section 4 evaluates the proposed model. Section 5 concludes this paper.

\section{RELATED WORK}

Several researchers have tried to modularize customizations in SaaS applications [13, 14, 15]. In [13], the authors propose a method for modelling customization process based on Temporal Logic of Actions, and propose a verification algorithm to check tenant's customization and to guarantee that each step in customization will not cause unpredictable influence on system and follow the related rules defined by SaaS provider. In [3], the authors propose a customization model based on metagraph to describe relationships between customization points and propose an algorithm to calculate related sets when one customization point is changed. In [14], the authors propose a multi-granularity customization relationship model that uses directed graph to describe relations in the customization process. The authors introduce a verification algorithm to guarantee the correctness of SaaS customization products that are obtained by the proposed model. In [15], the authors model the customization process using orthogonal variability model and propose a guided semi-automated customization based on mining existing tenants' customization.

In [16], the authors propose an approach for model-level customization management, which raises the customization management to the level of business services. They specify variation points using so-called specialization patterns, which have been originally developed to support task-driven specialization of application frameworks. In [17], the authors propose multi-level customization model for SaaS applications. To reduce duplication of customization, the proposed model supports customization sharing among different virtualized applications in a tenant area. The proposed model reduces repeated customization operations by allowing lower-level applications to inherit high-level applications' customizations. However, most of current research work has not addressed the modelling variability among tenants.

\section{Multi-Dimensional CuSTOMization MOdelling BASED ON METAGRAPH}

In this section, we begin by giving an overview of the basic features of metagraphs from [12]. This is followed by a description of the proposed model. Finally, we will propose two algorithms to validate customizations made by tenants.

\subsection{Metagraphs}

Metagraphs are graphical structures in which edges represent directed relationships between sets of elements. They extend both directed graphs (by allowing multiple elements in vertices) and hypergraphs (by including directionality in edges).

Definition 1 (metagraph): Given a finite generating set $X=\{x i, i=1 \ldots I\}$, a metagraph is an ordered pair $S=\langle X, E\rangle$, in which $E$ is set of edges $E=\left\{e_{k}, k=1 \ldots K\right\}$. Each edge is an ordered pair $e_{k}=\left\langle V_{k}, W_{k}\right\rangle$, in which $V_{k} \subseteq X$ is the invertex of the edge $e_{k}$ and $W_{k} \subseteq X$ is the outvertex. The coinput of any $x \in V_{k}$ is $V_{k} \backslash\{X\}$ and the cooutput of any $x \in W_{k}$ is $W_{k} \backslash\{X\}$. Also $V_{k} U W_{k} \neq$ $\Phi$ for all $k$.

For example, the metagraph in Figure 2 can be represented as follows:

$\mathrm{S}=\langle\mathrm{X}, \mathrm{E}\rangle$, where 
$\mathrm{X}=\left\{\mathrm{x}_{1}, \mathrm{x}_{2}, \mathrm{x}_{3}, \mathrm{x}_{4}, \mathrm{x}_{5}, \mathrm{x}_{6}\right\}$ is the generating set, and

$\mathrm{E}=\left\{\mathrm{e}_{1}, \mathrm{e}_{2}, \mathrm{e}_{3}\right\}$ is the set of edges where each edge can be specified as pair ( for example $e_{1}=$ $\left.\left\langle\left\{x_{1}, x_{2}\right\},\left\{x_{3}, x_{4}\right\}\right\rangle\right)$

Invertex $\left(\left\langle\left\{x_{1}, x_{2}\right\},\left\{x_{3}, x_{4}\right\}\right\rangle\right)=\left\{x_{1}, x_{2}\right\}$,

Outvertex $\left(\left\langle\left\{x_{1}, x_{2}\right\},\left\{x_{3}, x_{4}\right\}\right\rangle\right)=\left\{x_{3}, x_{4}\right\}$,

Coinput $\left(x_{1},\left\langle\left\{x_{1}, x_{2}\right\},\left\{x_{3}, x_{4}\right\}\right\rangle\right)=\left\{x_{2}\right\}$,

Cooutput $\left(x_{3},\left\langle\left\{x_{1}, x_{2}\right\},\left\{x_{3}, x_{4}\right\}\right\rangle\right)=\left\{x_{4}\right\}$,

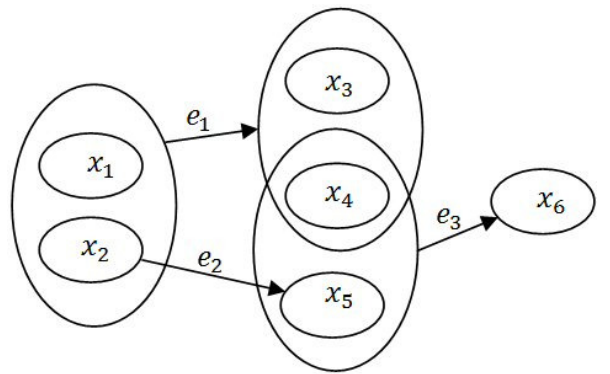

Figure 2. Metagraph example

Definition 2 (simple path): Given a metagraph $\mathrm{S}=\langle\mathrm{X}, \mathrm{E}\rangle$, a simple path from a source $x \in X$ to a target $x^{\prime} \in X$ is a sequence of edges $h\left(x, x^{\prime}\right)=\left\langle e_{l}^{\prime}, l=1, \ldots, L\right\rangle$ such that $x \in V_{1}^{\prime}, x^{\prime} \in W_{L}^{\prime}$, and $W_{i}^{\prime} \cap V_{i+1}^{\prime} \neq \Phi, \forall i=1, \ldots, L-1$. The coinput of $\mathrm{x}$ is $\left(\bigcup_{l=1}^{L} V_{l}^{\prime} \backslash \bigcup_{l=1}^{L} W_{l}^{\prime}\right) \backslash\{x\}$ and the cooutput of $\mathrm{x}^{\prime}$ is $\bigcup_{l=1}^{L} W_{l}^{\prime} \backslash\left\{x^{\prime}\right\}$.

The length of a simple path is the number of edges in the path; thus, the length of $h\left(x, x^{\prime}\right)=$ $\left\langle e_{l}^{\prime}, l=1, \ldots, L\right\rangle$ is L. For example, in the metagraph of Figure 2, $\left\langle\mathrm{e}_{1}, \mathrm{e}_{3}\right\rangle$ is a simple path from $\mathrm{x}_{2}$ to $\mathrm{x}_{6}$ with length 2 . The coinput of $\mathrm{x}_{2}$ is $\left\{x_{1}, x_{5}\right\}$, and the cooutput of $\mathrm{x}_{6}$ is $\left\{x_{3}, x_{4}\right\}$.

Definition 3 (metapath): Given a metagraph $S=\langle X, E\rangle$, a metapath $M(B, C)$ from a source $B \subseteq X$ to a target $C \subseteq X$ is a set of edges $E^{\prime} \subseteq \mathrm{E}$ such that $(1) \mathrm{e}^{\prime} \in \mathrm{E}^{\prime}$ is on a simple path from some element in $\mathrm{B}$ to some element in $\mathrm{C},(2)\left[\mathrm{U}_{\mathrm{e}^{\prime}} \mathrm{V}_{\mathrm{e}^{\prime}} \backslash \mathrm{U}_{\mathrm{e}^{\prime}} \mathrm{W}_{\mathrm{e}^{\prime}}\right] \subseteq \mathrm{B}$, and (3) $\mathrm{C} \subseteq \mathrm{U}_{\mathrm{e}^{\prime}} \mathrm{W}_{\mathrm{e}^{\prime}}$. For example, in Figure 2, one metapath from $x_{1}$ to $x_{6}$ is $M\left(\left\{x_{1}, x_{2}\right\},\left\{x_{6}\right\}\right)=\left\{e_{1}, e_{2}, e_{3}\right\}$.

Definition 4 (adjacency matrix): the adjacency matrix $A$ of a metagraph is a square matrix with one row and one column for each element in the generating set $X$. The $i j$ th element of $A$, denoted $\mathrm{a}_{\mathrm{ij}}$, is a set of triples, one for each edge $e$ connecting $\mathrm{x}_{\mathrm{i}}$ to $\mathrm{x}_{\mathrm{j}}$. Each triple is of the form $\left\langle C I_{e}, C O_{e}, e\right\rangle$ in which $C I_{e}$ is the coinput of $\mathrm{x}_{\mathrm{i}}$ in $e$ and $C O_{e}$ is the cooutput of $\mathrm{x}_{\mathrm{j}}$ in $e$. For example, the adjacency matrix for the metagraph in Figure 2 is shown in Figure 3.

\begin{tabular}{c|c|c|c|c|c|c|} 
& $x_{1}$ & $x_{2}$ & $x_{3}$ & $x_{4}$ & $x_{5}$ & $x_{6}$ \\
\hline$x_{1}$ & $\phi$ & $\phi$ & $\left\{\left\langle\left\{x_{2}\right\},\left\{x_{4}\right\}, e_{1}\right\rangle\right\}$ & $\left\{\left\langle\left\{x_{2}\right\},\left\{x_{3}\right\}, e_{1}\right\rangle\right\}$ & $\phi$ & $\phi$ \\
\hline$x_{2}$ & $\phi$ & $\phi$ & $\left\{\left\langle\left\{x_{1}\right\},\left\{x_{4}\right\}, e_{1}\right\rangle\right\}$ & $\left\{\left\langle\left\{x_{1}\right\},\left\{x_{3}\right\}, e_{1}\right\rangle\right\}$ & $\left\{\left\langle\phi, \phi, e_{2}\right\rangle\right\}$ & $\phi$ \\
\hline$x_{3}$ & $\phi$ & $\phi$ & $\phi$ & $\phi$ & $\phi$ & $\phi$ \\
\hline$x_{4}$ & $\phi$ & $\phi$ & $\phi$ & $\phi$ & $\phi$ & $\left\{\left\langle\left\{x_{5}\right\}, \phi, e_{3}\right\rangle\right\}$ \\
\hline$x_{5}$ & $\phi$ & $\phi$ & $\phi$ & $\phi$ & $\phi$ & $\left\{\left\langle\left\{x_{4}\right\}, \phi, e_{3}\right\rangle\right\}$ \\
\hline$x_{6}$ & $\phi$ & $\phi$ & $\phi$ & $\phi$ & $\phi$ & $\phi$ \\
\hline
\end{tabular}

Figure 3 . The adjacency matrix of the metagraph in figure 2 
Definition 5 (closure matrix): the closure of adjacency matrix A, denoted $\mathrm{A}^{*}$, represents all simple paths of any length in the metagraph. The ijth element of $A^{*}$, denoted $a_{i j}^{*}$, is a set of triples, one for each simple path $\mathrm{h}\left(\mathrm{x}_{\mathrm{i}}, \mathrm{x}_{\mathrm{j}}\right)$ of any length connecting $\mathrm{x}_{\mathrm{i}}$ to $\mathrm{x}_{\mathrm{j}}$. The closure matrix of Figure 3 appears in Figure 4.

Definition 6: Given a generating set $X$ and two metagraphs $S_{1}=\left\langle X, E_{1}\right\rangle$ and $S_{2}=\left\langle X, E_{2}\right\rangle$ with adjacency matrices $A_{1}$ and $A_{2}$ respectively, then the sum of the two adjacency matrices is the adjacency matrix of the metagraph $\mathrm{S}_{3}=\left\langle\mathrm{X}, \mathrm{E}_{1} \cup \mathrm{E}_{2}\right\rangle$ with components $\left(A_{1}+A_{2}\right)_{i j}=a_{i j}^{1} \cup a_{i j}^{2}$.

\begin{tabular}{c|c|c|c|c|c|c|} 
& $x_{1}$ & $x_{2}$ & $x_{3}$ & $x_{4}$ & $x_{5}$ & $x_{6}$ \\
\hline$x_{1}$ & $\phi$ & $\phi$ & $\left\{\left\{\left\{x_{2}\right\},\left\{x_{4}\right\}, e_{1}\right\rangle\right\}$ & $\left\{\left\langle\left\{x_{2}\right\},\left\{x_{3}\right\}, e_{1}\right\rangle\right\}$ & $\phi$ & $\left\{\left\langle\left\{x_{2}, x_{5}\right\},\left\{x_{3}, x_{4}\right\},\left\{e_{1}, e_{3}\right\rangle\right)\right\}$ \\
\hline$x_{2}$ & $\phi$ & $\phi$ & $\left\{\left\{\left\{x_{1}\right\},\left\{x_{4}\right\}, e_{1}\right\rangle\right\}$ & $\left\{\left\{\left\{x_{1}\right\},\left\{x_{3}\right\}, e_{1}\right\rangle\right\}$ & $\left\{\left\langle\phi, \phi, e_{2}\right\rangle\right\}$ & $\left\{\left\{\left\{x_{1}, x_{5}\right\},\left\{x_{3}, x_{4}\right\},\left\{e_{1}, e_{3}\right\rangle\right\rangle,\left\{\left\{x_{4}\right\},\left\{x_{5}\right\},\left\{e_{2}, e_{3}\right\rangle\right\}\right\}$ \\
\hline$x_{3}$ & $\phi$ & $\phi$ & $\phi$ & $\phi$ & $\phi$ & $\phi$ \\
\hline$x_{4}$ & $\phi$ & $\phi$ & $\phi$ & $\phi$ & $\phi$ & $\left\{\left\langle\left\{x_{5}\right\}, \phi, e_{3}\right\rangle\right\}$ \\
\hline$x_{5}$ & $\phi$ & $\phi$ & $\phi$ & $\phi$ & $\phi$ & $\left\{\left\langle\left\{x_{4}\right\}, \phi, e_{3}\right\rangle\right\}$ \\
\hline$x_{6}$ & $\phi$ & $\phi$ & $\phi$ & $\phi$ & $\phi$ & $\phi$ \\
\hline
\end{tabular}

Figure 4. The closure of the adjacency matrix in Figure 3

Definition 7: A metagraph $\mathrm{S}_{1}=\left\langle\mathrm{X}_{1}, \mathrm{E}_{1}\right\rangle$ is said to be a sub-metagraph (SMG) of another metagraph $\mathrm{S}_{2}=\left\langle\mathrm{X}_{2}, \mathrm{E}_{2}\right\rangle$ if $\mathrm{X}_{1} \subseteq \mathrm{X}_{2}$ and $\mathrm{E}_{1} \subseteq \mathrm{E}_{2}$. A metagraph $\mathrm{S}_{1}$ is an input independent $S M G$ of a metagraph $S_{2}$ if every element of $S_{1}$ that is not a pure input is determined only by edges within $\mathrm{S}_{1}$. A metagraph $\mathrm{S}_{1}$ is an output independent $S M G$ of a metagraph $\mathrm{S}_{2}$ if every element of $S_{1}$ that is not a pure output is used only by edges within $S_{1}$. A metagraph $S_{1}$ is an independent $S M G$ of a metagraph $\mathrm{S}_{2}$ if it is both input independent and output independent.

\subsection{The proposed model}

Consider $C P=\left\{c p_{i}, i=1, \ldots, I\right\}$ is the set of all customization points in SaaS application. For each customization point $c p_{\mathrm{n}} \in C P$, there is a set of components $C_{n}=\left\{x_{k}, k=1, \ldots, K\right\}$ provided by the SaaS application developer. Tenant customizes customization point $c p_{n}$ by selecting a subset of components $T C_{n} \subseteq C_{n}$.

Definition 8 (concern): Each concern that is important for tenants is represented by a metagraph $C N_{i}=\left\langle X_{c n_{i}}, E_{c n_{i}}\right\rangle$, where $X_{c n_{i}}$ is a set of all components that address this concern from all customization points' component sets (as shown in figure 5), and $E_{c n_{i}}$ is the relationships of these components. 


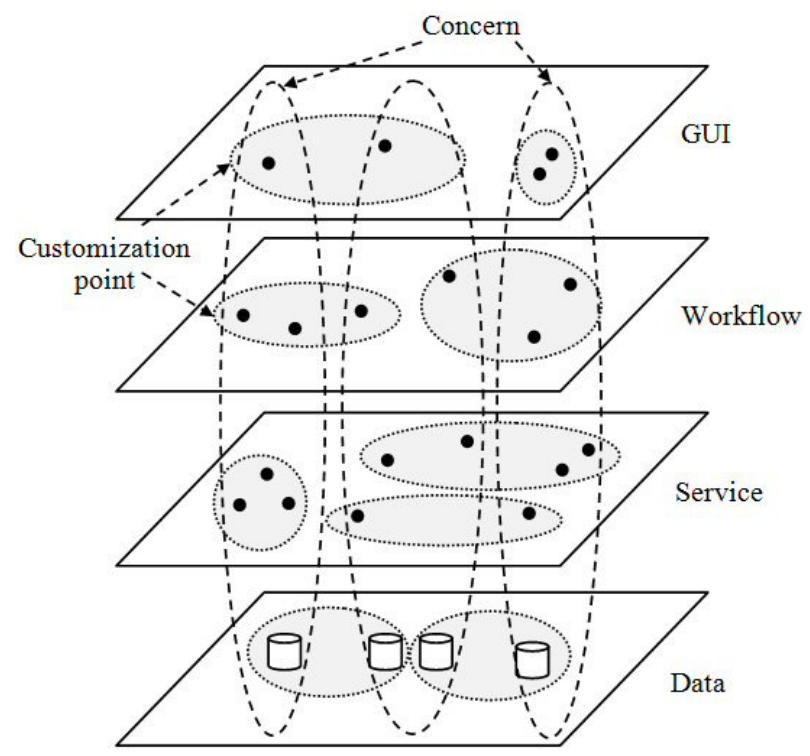

Figure 5. Concerns crosscut several customization points

In this paper, we concerned with "required by" relationship. Sometimes component requires more than one component. To express this state we add "and" vertex to the metagraph. For example, figure 6 shows that components $\mathrm{x}_{1}$ and $\mathrm{x}_{2}$ are required by component $\mathrm{x}_{4}$ and components $\mathrm{x}_{2}$ and $\mathrm{x}_{3}$ are required by component $\mathrm{x}_{5}$.

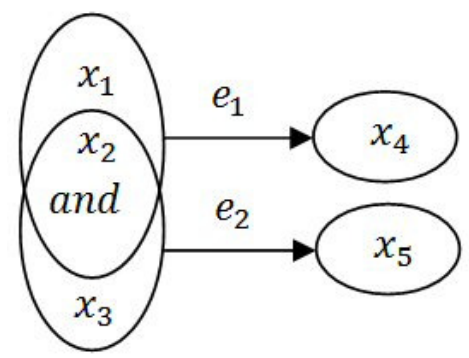

Figure 6. Metagraph example

Concerns are considered to overlap if their component sets are not disjoint. If there is a component address more than one concern, the required components can be different depending on the role played by this component in each concern.

Tenant customizes concern by selecting some components from its component set. The customized concern can be represented as a sub-metagraph $T C N_{i} \subseteq C N_{i}$. To guide tenants during customization process, closure matrix of each concern is generated to return all possible customization paths of this concern. Tenants review these paths and make their decisions.

Definition 9 (dimension of concerns): Dimension of concerns is a type of concerns that is important for tenants (e.g., performance, security) such that each dimension of concerns $D_{d}=$ $\left\{c n_{i}, i=1, \ldots, I\right\}$ contains a set of concerns whose component sets are disjoint and each concern is an independent sub-metagraph. Dimension can be represented as a Metagraph $D_{d}=\left\langle X, E_{d}\right\rangle$, where $X$ is the set of all components in SaaS application, and $E_{d}=E_{c n_{1}} \cup E_{c n_{2}} \cup \ldots \cup E_{c n_{n}}, E_{c n_{m}}$ is a set of edges for one of the concerns belong to this dimension. The adjacency matrix of each dimension is the sum of all adjacency matrixes of its concerns. 
Each dimension, has one concern called the None concern. All components in SaaS application that do not address any concern in this dimension address the None concern. This means that each component in the SaaS application will be located at exactly one concern in each dimension.

Definition 10 (SaaS application): A SaaS application is a set of dimensions $D=\left\{d_{j}, j=1, \ldots, J\right\}$, such that every concern is in exactly one dimension in $D$. A SaaS application can be represented as a metagraph $S=\langle X, E\rangle, X$ is the set of all components in SaaS application, and $E=E_{1} \cup E_{2} \cup$ $\ldots \cup E_{n}, E_{m}$ is the set of edges for one of the dimensions in this application.

Definition 11 (customization operations): is what a tenant does to customize the SaaS application to meet his requirements. In this paper, we define two types of customization operations: add component, and delete component. Tenant customizes SaaS application by a sequence of these operations such that each operation moves SaaS application from a valid state to another valid state.

Definition 12 (customization model): customization for each tenant are represented as a set $T D=$ $\left\{t d_{j}, j=1, \ldots, J\right\}$, where $t d_{j}$ is the set of customized concerns in dimension $d_{j}$. each tenant's customization is a sub-metagraph from SaaS application metagraph.

Tenants customize SaaS application by selecting dimensions that are important for them (e.g., performance, security), and select concerns from each dimension (e.g., authentication, authorization, and encryption in security dimension). Finally, tenants can customize each concern by specifying components from its component set.

\subsection{Customization validation}

Tenant customizes the SaaS application using a sequence of customization operations. To validate this sequence, we validate each operation based on the customizations that are constructed by the previous operations. If tenant requests to add new component to a specific concern in his customization, algorithm 1 shows how to validate and perform this operation. Algorithm 1 adds new components only if their requirements already exist in the tenant's customization. Algorithm 2 deletes components from the tenant's customization only if these components are not required by any component in the tenant's customization. Algorithm 2 deletes all edges that contain the deleted component in its outvertex without any cooutput. To minimize run-time and memory usage for the proposed algorithms, the only required rows or columns of adjacency matrixes are constructed.

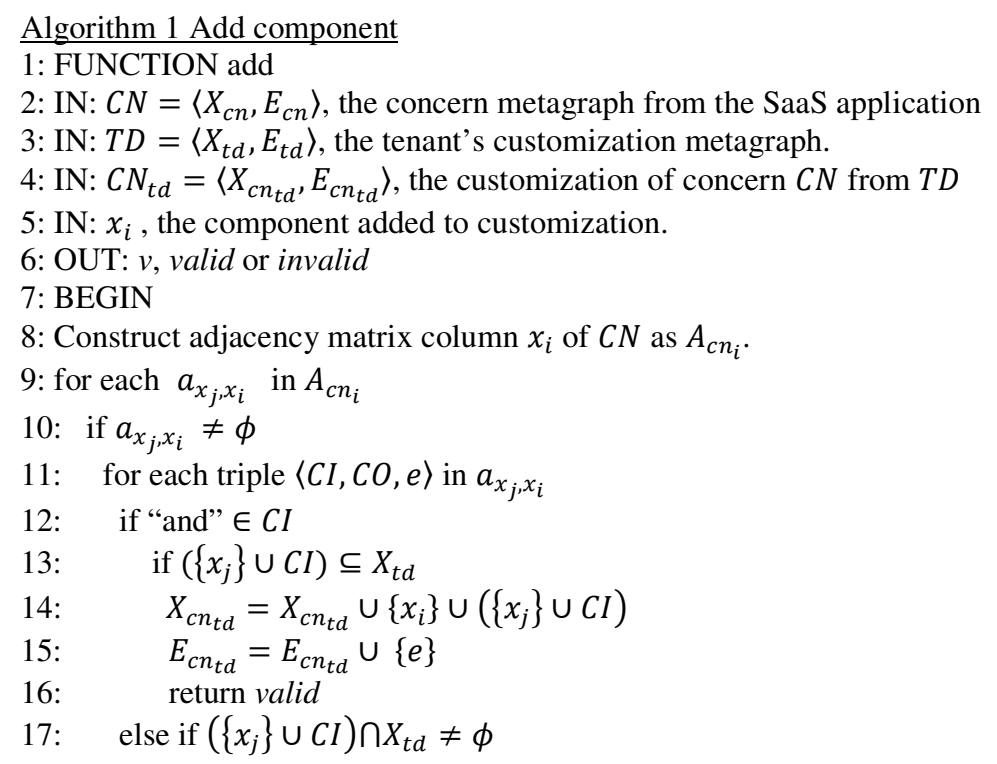



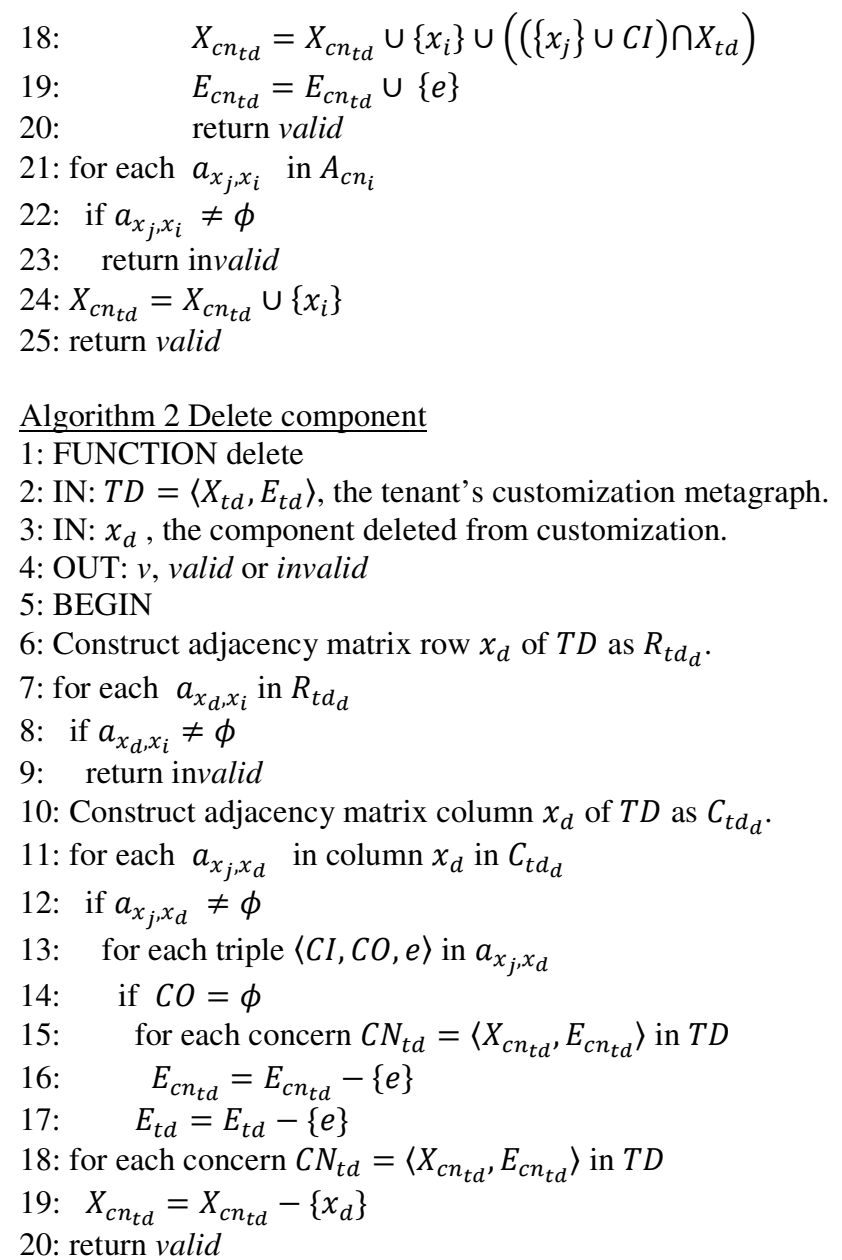

\section{Evaluation}

There are a number of benefits for the proposed model. Some of these benefits have been explored below:

- Customization complexity: by applying MDSOC, the proposed model allows tenant to customize one concern at a time instead of customization point. Concerns cut across multiple customization point to collect components that are related to a specific area of interest. In other words, tenant customizes customization points incrementally. Which enables tenant easily customizes the customization points and easily understands their dependencies. On the other side, provider follows the same steps in developing. Instead of developing all components of a specific customization point, provider develops components of a specific concern.

- SaaS upgradeability: in some SaaS application, tenants may be encountering some issues when SaaS providers upgrade their applications. The proposed model allows providers to add new concerns and new dimensions at any time without having to reengineering existing ones. Providers can upgrade each concern independently because each concern is modeled as independent sub-metagraph and is unaffected by changes that may occur within its associated dimension. Using metagraph tools, providers can expect the effects of their upgrades on the tenants' customizations within each concern. 
- Duplication of customization: the SaaS providers need to reduce duplication of customization to exploit the economies of scale [2]. Most of duplications of customization come from allowing tenants to define new components. This makes the SaaS providers face a broad spectrum of tenants' customizations and makes it difficult to drive commonalities amongst the customizations across tenants. By allowing providers to model a large number of components and enabling tenants to handle these components easily, providers can provide a wide range of components to reduce duplication of customization.

- Customization correctness: the proposed algorithms and metagraph tools had been used to ensure the correctness of customizations made by tenants.

However, with the proposed model, providers can face some difficulties in specifying disjoint concerns and their components in each dimension. We believe that, graph decomposition tools can help providers in specifying these concerns.

To evaluate the run-time performance of the proposed model and algorithms for differing application sizes, we had generated customization models with different sizes and random dependences. Figures 7 shows the average response time for each model size.
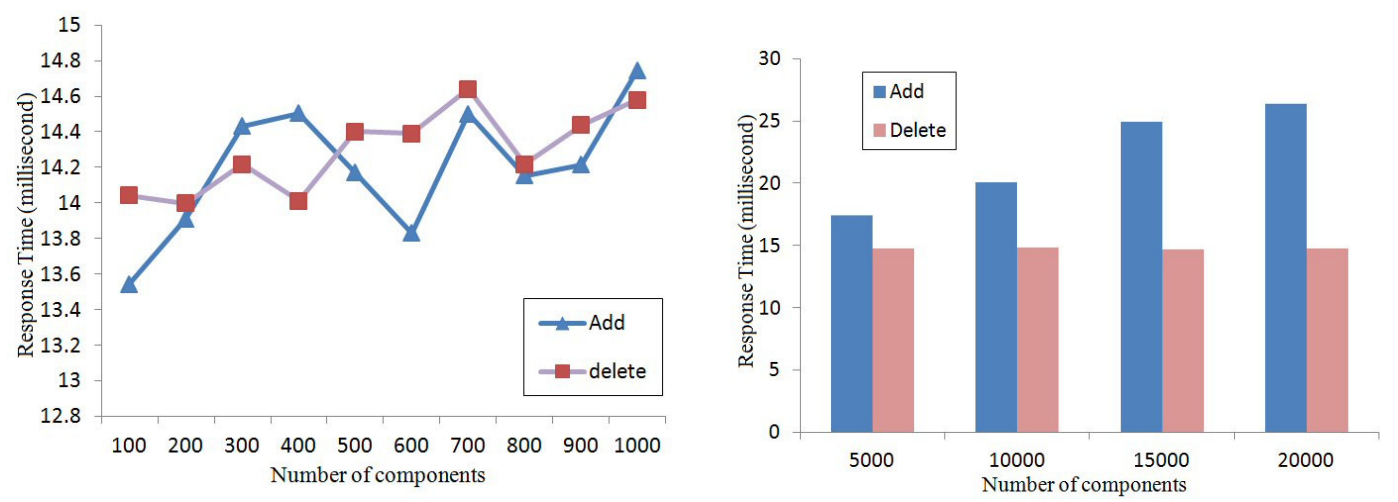

Figure 7. Response time for different application sizes

To evaluate the run-time performance of the proposed model and algorithms for varying numbers of users, we had generated model with size 500 components and had performed add and delete operations on the generated model with a large number of concurrent requests from users. The results are described in Figure 8.
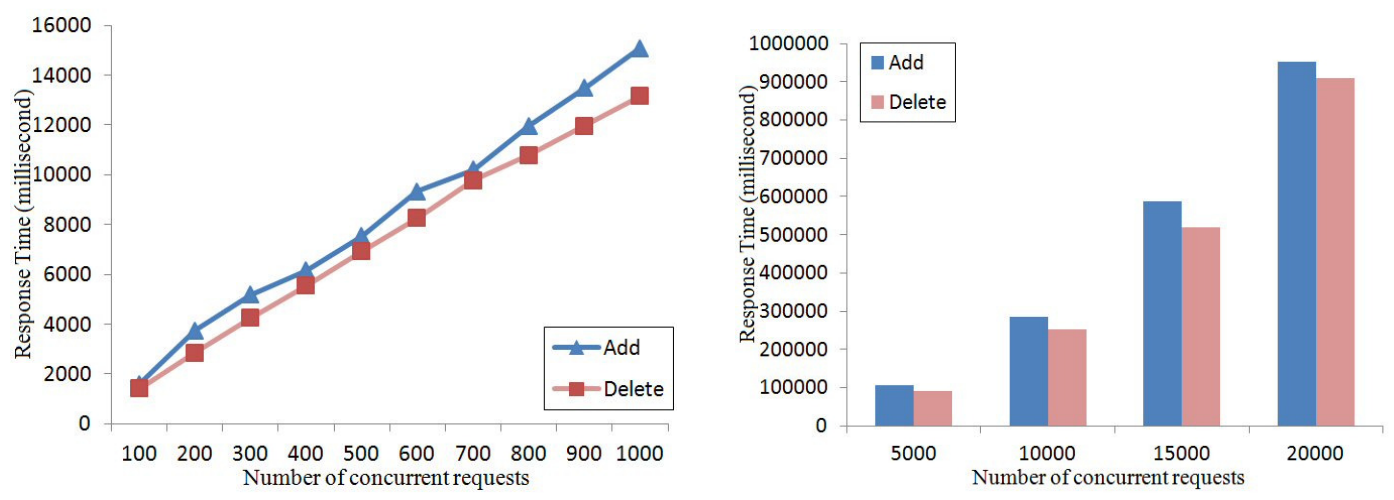

Figure 8. Response time for different numbers of concurrent requests 


\section{CONCLUSION}

In this paper, we presented a multi-dimensional customization model. The proposed model applied the MDSOC concepts to address the modelling variability among tenants and described components' dependences using metagraph.

The main benefits of our model are: (i) simplifying customization process by allowing tenants to incrementally customize customization points; (ii) increasing SaaS upgradeability by allowing providers to add new concerns and new dimensions at any time without having to reengineering existing ones; and (iii) reducing duplication of customization by minimizing the needs for define new components. Additionally, we proposed algorithms that can judge whether tenant's customization action has satisfied all the dependency, and prevent the invalid action.

However, specifying disjoint concerns and their components in each dimension (which is beyond the scope of this paper) still is a challenge for the proposed model. We can use graphdecomposition tools to specify these concerns.

Finally, we can conclude that, applying MDSOC concepts to customization models addresses the modelling variability among tenants. Our future work will be to improve the proposed model to guide tenants during the customization process based on mining existing tenants' customizations.

\section{REFERENCE}

[1] W. Lee and M. Choi, "A multi-tenant web application framework for SaaS," 2012 IEEE Fifth International Conference on Cloud Computing, vol. 0, pp. 970-971, 2012.

[2] R. Mietzner, A. Metzger, F. Leymann, and K. Pohl, "Variability modeling to support customization and deployment of multi-tenant-aware software as a service applications," in ICSE Workshop on Principles of Engineering Service Oriented Systems, PESOS 2009, 2009, pp. 18-25.

[3] C. Lizhen, W. Haiyang, J. Lin, and H. Pu, "Customization modeling based on metagraph for multitenant applications," in 2010 5th International Conference on Pervasive Computing and Applications (ICPCA), 2010, pp. 255-260.

[4] P. Aghera, S. Chaudhary, and V. Kumar, "An approach to build multi-tenant SaaS application with monitoring and SLA," in 2012 International Conference on Communication Systems and Network Technologies (CSNT), 2012, pp. 658-661.

[5] M. Pathirage, S. Perera, I. Kumara, and S. Weerawarana, "A multi-tenant architecture for business process executions," in 2011 IEEE International Conference on Web Services (ICWS), 2011, pp. 121128.

[6] J. Lee, S. Kang, and S. J. Hur, "Web-based development framework for customizing java-based business logic of SaaS application," in 14th International Conference on Advanced Communication Technology (ICACT), 2012, pp. 1310-1313.

[7] H. Moens, E. Truyen, S. Walraven, W. Joosen, B. Dhoedt, and F. De Turck, "Developing and managing customizable software as a service using feature model conversion," in Network Operations and Management Symposium (NOMS), 2012 IEEE, 2012, pp. 1295-1302.

[8] W. Sun, X. Zhang, C. J. Guo, P. Sun, and H. Su, "Software as a service: Configuration and customization perspectives," in Congress on Services Part II, 2008. SERVICES-2. IEEE, 2008, pp. $18-25$.

[9] B. Pekilis, "Multi-dimensional separation of concerns and IBM Hyper/j," University of Waterloo, Canada, Tech. Rep., January 2002.

[10] P. Tarr, H. Ossher, W. Harrison, and S. Sutton, "N degrees of separation: multi-dimensional separation of concerns," in Proceedings of the 1999 International Conference on Software Engineering, 1999, pp. 107-119.

[11] H. Ossher and P. Tarr, "Multi-dimensional separation of concerns and the hyperspace approach," in Software Architectures and Component Technology, ser. The Springer International Series in Engineering and Computer Science, Ed. Springer US, 2002, vol. 648, pp. 293-323.

[12] A. Basu and R. W. Blanning, Metagraphs and Their Applications. Springer US, 2007, vol. 15, pp. 1525 . 
[13] S. Luan, Y. Shi, and H. Wang, "A mechanism of modeling and verification for SaaS customization based on TLA," in Web Information Systems and Mining, ser. Lecture Notes in Computer Science, Springer Berlin Heidelberg, 2009, vol. 5854, pp. 337-344.

[14] H. Li, Y. Shi, and Q. Li, "A multi-granularity customization relationship model for SaaS," in International Conference on Web Information Systems and Mining, WISM 2009, 2009 , pp. 611-615.

[15] W.-T. Tsai and X. Sun, "SaaS multi-tenant application customization," in 2013 IEEE 7th International Symposium on Service Oriented System Engineering (SOSE), 2013, pp. 1-12.

[16] H. Hadaytullah, K. Koskimies, and T. Systa, "Using model customization for variability management in service compositions," in IEEE International Conference on Web Services, ICWS 2009, 2009, pp. 687-694.

[17] L. Kong, Q. Li, and X. Zheng, "A novel model supporting customization sharing in SaaS applications," in 2010 International Conference on Multimedia Information Networking and Security (MINES), 2010, pp. 225-229. 


\title{
EFFECT OF COLOURS IN MANUAL DATA TYPING
}

\author{
Melih Kirlidog \\ Department of Computer Engineering, Marmara University, Istanbul, Turkey \\ School of IT, North-West University Vaal Campus, South Africa \\ melihk76@gmail.com
}

\begin{abstract}
Although there is a large body of literature on research into colour in human-computer interaction, the overwhelming majority of the literature emphasises the cognition by computer users. However, colour is also important in this interaction when users manually type data into a computer. This paper investigates the effect of colour combinations on manual data typing. To this end, three experiments were conducted where the subjects were requested to read several texts with different colour combinations and re-type them in the same screen. Typing accuracy and speed is measured as the dependent variable across different colour combinations. Three experiments were conducted as such. In the first experiment, display and input windows were close to each other and in the second one they were located in the opposite ends of the screen. The third experiment was a subset of the first one with reversed foreground and background colours. It was found that different colour combinations had varying effects on data typing performance and proximity of the display and input windows was not a significant factor for typing accuracy in a 17-inch screen. The effect of reversing the foreground and background colours was inconclusive with the colour combinations used.
\end{abstract}

\section{KEYWORDS}

Human-computer interaction (HCI), Colour Combinations, Data Typing, Manual Data Entry

\section{INTRODUCTION}

Human-computer interaction (HCI) can have two directions of information flow, namely from human to computer and vice versa. The flow in the human-to-computer direction can be carried out by several methods using different types of hardware, such as direct manipulation through point-and-click with a mouse, [25] keystrokes on a keyboard and the use of a joystick. The most common type of interaction in the computer-to-human direction is via the computer screen, and the only method employed on the screen is the use of shapes and figures with different colours.

Although there is a large body of literature on the use of colour in computer-to-human interaction using a video display terminal (VDT), this is not true for human-to-computer interaction. In other words, the effect of colour and colour combinations on manual data entry on a chromatic VDT is a neglected area in HCI research. The present article investigates the effect of colour combinations on manual data entry performance. To this end, a series of experiments was David C. Wyld et al. (Eds) : CCSIT, SIPP, AISC, PDCTA, NLP - 2014 pp. 65-76, 2014. (C) CS \& IT-CSCP 2014

DOI : $10.5121 /$ csit.2014.4206 
conducted in which the subjects were requested to read various texts on the screen with different colour combinations and re-type them on the same screen.

Since it is not possible to distinguish the cognitive and psycho-motor functions in the readingand-typing cycle, the "manual data entry performance" in this research involves not only the data typing activity, but also the activity of reading from screen. The colour pairs in the display and input windows are the same in a trial. Thus, a combination of these two activities is measured for different colour pairs where the performance is measured by the accuracy and speed of the data typed. In other words, in effect, what is being measured is not only the isolated data entry activity, but also the cognition performance as an input mechanism to data entry.

\section{COMMON TASKS AND DIRECTIONALITY IN HCI}

An overwhelming majority of HCI research is based on human cognition, and the subjects in this line of research usually act as passive observers who are requested either to find irregularities in a given text or perform a reading activity against time. However, manual data typing into the computer is a more important and common task for many computer users, particularly for manual transaction entry clerks. Unlike the screen reading and observation activity, where most colourbased HCI research has been done, manual data typing is a more complicated activity, because the former is only a subset of the latter. In the manual data typing activity computer users not only have to read from either a computer screen or some other medium such as paper, but they also have to perform the psycho-motor action of typing on a keyboard, an activity which also involves reading the screen and observation per se. In other words, computer-to-human interaction is only a cognitive action whereas human-to-computer interaction is a two-phase action in which the first phase mainly involves cognition and the second phase involves both cognition and psycho-motor activity. The directionality is also different in these two kinds of actions. The former is a unidirectional cognitive activity where the information flows from the screen to the user and the latter is clearly a bidirectional activity.

Unlike the older command line interfaces where the user usually manipulated the computer through a series of typed commands, the modern graphical user interface (GUI) environment offers many convenient methods, including direct manipulation. Currently, direct manipulation of the computer through point-and-click with a mouse is the most common method of interacting with computers. However, although less frequent, manual data entry on a keyboard is also used. This method can still be used for various purposes such as short-cutting the navigation through GUI by experienced users, or for performing manual transactions on forms on the screen. Although in the latter case pointing and clicking can also be used to navigate to a particular field on the screen, the bulk of the action is manual data typing into the relevant fields.

\section{COLOUR COMBINATIONS IN HCI}

Almost all computer screens were monochromatic until a few decades ago. This meant that computer users and purchasing officers in companies had to decide on a fixed foreground and background colour combination of the computer monitor at purchase. The 1990s witnessed two important developments that increased the importance of colour in human-computer interaction. Firstly, chromatic screens became widespread and replaced the monochromatic screens, and secondly, the World Wide Web, which is a highly "colourful" medium, was introduced. The web 
and the related software development tools offered great flexibility to designers not only in the selection of colour, but also in other interface features such as layout, design and aesthetics. Currently almost all computer displays are chromatic and all software development platforms enable developers to use colour effectively. Although chromatic monitors are commonly used both in web-based and non-web-based applications, most of the research on colour-related human-computer interaction has been done on web-based applications, [7], [5], [18] possibly due to the prevalence of the web.

Current research on colour combinations on VDTs can be categorised in three groups [7]. In the first group attempts are made to measure readability through visual search tasks where subjects have to identify misspelled words or to find special words in a paragraph of nonsensical anagrams [16], [17], [12]. In the second group readability is measured in terms of the time required to read a specific text [23], [27]. In the third group visual performance is measured as the percentage of correctly recognised characters or words in a text [24], [10].

Although there is some conflicting evidence in the literature it is generally recommended that extremes of colour combinations such as red-green and red-blue should not be used [21] and rather those colours be used conservatively with a maximum of four colours in a single display [25].

Several authors warn about visual discomfort and poor visual performance resulting from the inappropriate use of colours [13], [22]. Some authors have stressed the importance of colour combinations in reading performance [17], [22], [26] and aesthetics [5], [17], [11].

Shieh and Lin [24] found that colour combination significantly affected not only visual performance but also subjective preference. They also found that a blue foreground and a yellow background resulted in both the best performance and the greatest preference rating, whereas purple on red had the worst rating. It is argued that red and blue are on the opposite ends of the spectrum and their combination on the display unit will strain the muscles surrounding the eye [25], [13]. This strain will result in difficulty in reading. The same is true for yellow on purple and magenta on green.

As stated above, there is a substantial body of research on the colour combinations in the humancomputer interface but there is not much research on the effects of inverted colour combinations on common interface tasks such as reading. In one of the few studies on inverted colour combinations, Nielsen [14] argued that the contrast is the same in an inverted colour scheme, yet "it throws people off a little and slows their reading slightly." A number of studies have found that dark text on light background (positive polarity) has better readability than its inverse combination (negative polarity) [6], [19], [1] while some others have found no significant benefit of positive polarity [17], [12], [5].

Some aspects of retention involving colours have been dealt with in HCI research. Noiwan and Norcio [15] investigated the retention of colours in screen banner graphics in a cross-cultural research. Hall and Hanna [5] found that different combinations of web page text/background colours do not have a statistically significant effect on retention. The cycle of reading a text and re-typing it on the keyboard involves the retention of information in memory. In this case, the information is a sentence or a part of a sentence, and it is investigated whether proximity on the screen affects memory retention. 
It must also be stated that some findings of the HCI research on colours are contradictory. As opposed to the research that found colour combinations having significant effect on text search, perceived aesthetics, and reading, some authors found insignificant effects on these tasks. For example, Pace [16] found no significant difference in text search performance among 24 colour combinations and Radl [19] found that, regardless of the specific colour combination, higher levels of contrast appeared to lead to better readability.

\section{RESEARCH DESIGN}

Data manipulation in this research was accomplished by special-purpose software. The software opened two windows on the screen. The first window displayed an English text in a particular combination of foreground and background colours and the subjects were requested to enter the same text manually in the second window which had the same colour combination (Figure 1).

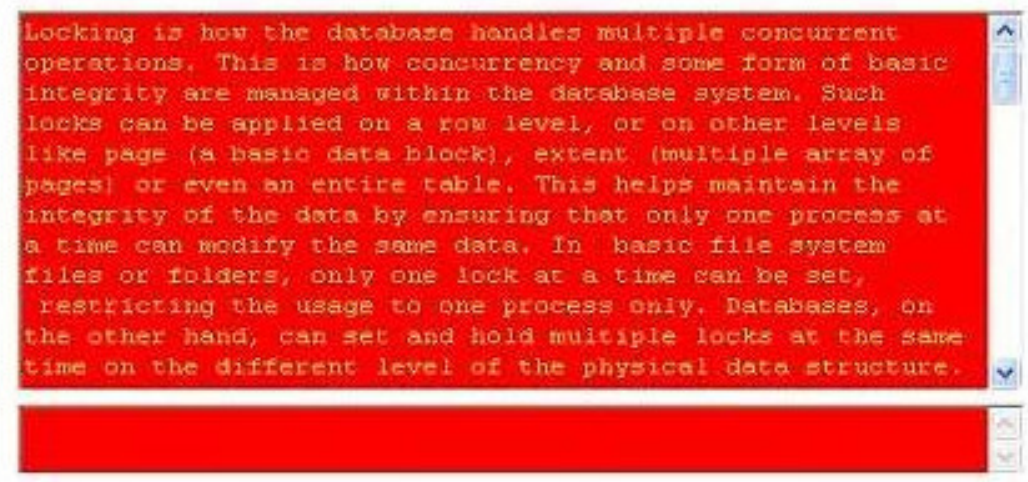

Seconds Remaining: $\quad 64$

Figure 1. Display and entry windows in experiment 1 , trial 1

The displayed texts were stored in the database and the software enabled the database to capture the texts typed by the subjects. The research is a within-subjects design which consisted of 19 trials in three experiments. Each trial had the same text for all subjects. The trials had different colour combinations and different texts of about 400 words each. Hence, a total of 19 texts were involved in the research. In each trial the subjects had 120 seconds for reading the texts in the display window and simultaneously typing them in the entry window. A counter displayed the remaining time during the trial and the software closed both windows automatically after the time had expired.

The performance measured in each trial was the number of correctly typed words in 120 seconds, during which the subjects could type only a certain portion of the given text. In order to familiarise the subjects with the study they were requested to perform an extra trial before starting the actual experiments. The reason for using different texts in the trials was to avoid possible retention of the texts in the subjects' memories across the trials. The texts were selected from an 
introductory computer engineering textbook that was used in an undergraduate course. Since all the subjects had completed that course, they were familiar with the material in the book.

The following rules were applied for calculating the number of correctly typed words:

- Mistyped words were not counted.

- Partially typed words due to time expiry at the end of each trial were not counted.

- In the case of a missing space between two words one of them was not counted, e.g. "dependingon" was counted as one word.

- Lack of a space after a comma or full stop was not counted as an error.

- Missing commas or full stops were not counted as errors.

- Mistyping of lower case and upper case words was not counted as an error.

- A space between words exceeding one character was not counted as an error.

- The words had to be exactly the same as those in the text. For example, typing "subschema" instead of the "sub-schema" in the text was counted as an error.

- Erroneously repeated words were counted as an error.

- A penalty of minus one word was applied in the case of missing a word or block of words.

In the first experiment which contained 13 levels of an independent variable (i.e. colour combination) it was aimed to gauge the typing performance where the two windows were located quite close to each other. In this experiment, the input window was located just under the display window of the text. The distance between the two windows was $0.2 \mathrm{~cm}$ (Fig. 1). The colour combinations used in this experiment are shown in Table 1 with the RGB colour codes.

In order to capture the subjective evaluations of aesthetics and readability, a new window appeared on the screen for data capturing after each trial in the first experiment. The subjects entered two evaluations in this phase through radio buttons in 5-point Likert scale: "I do not like this colour combination" and "It is difficult to read and enter data with this colour combination" (1: completely agree; 5 : completely disagree).

The second experiment aimed to investigate the effect of difference in proximity on typing performance - the two windows were at a distance from each other, i.e. at opposite ends of the screen. The display window was in the upper left corner of the screen and the input window was in the bottom right. The closest distance between the two was $11.5 \mathrm{~cm}$ in this experiment. Three randomly chosen colour pairs used in the first experiment were used in this experiment. Table 5 shows these pairs and descriptive statistics in this experiment.

In the third experiment the aim was to investigate typing performance when the foreground and background colours were reversed. To this end, the reverse of the randomly chosen three colour pairs from the first experiment were used and the screen layout was similar to that of the first experiment, i.e. the windows were $0.2 \mathrm{~cm}$ apart. The colour combinations used in this experiment are shown in Table 7 along with descriptive statistics. 


\subsection{The subjects}

Four female and 26 male computer engineering undergraduate students participated in this research. The average age of the subjects was 23.7, with a standard deviation of 1.53. All the subjects had normal or corrected visual acuity and none of them reported any colour vision deficiencies. Four of the subjects were left-handed. Due to their field of study, the subjects were heavily involved with computers. The starting age at which they became involved ranged from eight to eighteen years, and the mean value for total years of involvement was 9.4 years with a standard deviation of 2.7. Since their medium of instruction is English, the subjects were fluent in both reading and writing in English.

Since the research was mainly based on the performance of fast and accurate data entry, the subjects had to be stimulated for these tasks for the experiment. To this end, better performers were promised additional credits for a course they were attending as well as ten gift books. The experiments were conducted in a computer laboratory. Since the laboratory contained only 20 computers, the experiments were organised in two sessions with two groups of subjects. The subjects were randomly assigned to the groups. The first group comprised 14 students, all of whom were given some credits for participating in the experiment. However, only half of the highest performing subjects (i.e. seven students) were given additional credits. In the same way, only the ten highest performing of the 16 subjects in the other group got the books promised.

\subsection{Software and hardware used}

The VDTs used in the experiments were 17-inch Hewlett-Packard model L1710s which use thin film transistor liquid crystal display (TFT-LCD) technology. The experiments were performed at values of $60 \mathrm{~Hz}$ frequency and 1280 x 1024 pixels resolution. The computers used Microsoft Vista as the operating system and the software was developed using the Microsoft.Net platform. SQL Server 2008 was used for storing the data. The subjects were instructed to use MS Internet Explorer browser instead of Mozilla Firefox which is commonly used in the Department. The reason for this was somewhat paradoxical: Firefox defaults to a higher level of functionality in the context of this experiment by underlining mis-spelled English words. This would have allowed subjects to correct inaccurate entries, which was regarded as "noise" that distorts the real outcome of data entry accuracy.

\section{RESULTS AND DISCUSSION}

\subsection{First experiment (display and input windows in close proximity)}

In this experiment display and input windows are close to each other. The colour pairs used and the mean of correct words for each trial are shown in Table 1 along with other descriptive data such as subjective evaluations of aesthetics and readability. 
Table 1. Descriptive statistics and Tukey HSD test for the first experiment

\begin{tabular}{|c|c|c|c|c|c|c|c|c|c|c|c|}
\hline \multirow{2}{*}{$\begin{array}{l}\text { Tri } \\
\text { al }\end{array}$} & \multirow[b]{2}{*}{$\begin{array}{l}\text { Foreground } \\
\text { (RGB code) }\end{array}$} & \multirow[b]{2}{*}{$\begin{array}{l}\text { Background } \\
\text { (RGB code) }\end{array}$} & \multirow[b]{2}{*}{$\mathbf{N}$} & \multirow[b]{2}{*}{$\begin{array}{l}\text { "Dist } \\
\text { ance" }\end{array}$} & \multicolumn{3}{|c|}{ \# Correct words } & \multicolumn{2}{|c|}{ Aesthetics } & \multicolumn{2}{|c|}{ Readability } \\
\hline & & & & & Mean & St. dev. & $\begin{array}{c}\text { Tukey } \\
\text { group (*) }\end{array}$ & Mean & St. dev. & Mean & $\begin{array}{l}\text { St. } \\
\text { dev. }\end{array}$ \\
\hline 17 & green ( $\# 00$ ffo 0$)$ & orange (\#ffa 500) & 30 & 2 & 58.6 & 15.1 & $\mathrm{C}$ & 3.37 & 0.76 & 3.53 & 0.82 \\
\hline 12 & green $(\# 00 \mathrm{ff} 00)$ & yellow (\#ffffo0) & 30 & 1 & 51.9 & 15.1 & $\mathrm{~B}, \mathrm{C}$ & 3.47 & 0.86 & 3.40 & 0.93 \\
\hline 13 & orange $(\# \mathrm{ffa} 500)$ & blue $(\# 0000 \mathrm{ff})$ & 30 & 3 & 51.9 & 16.2 & $\mathrm{~B}, \mathrm{C}$ & 2.93 & 0.94 & 2.97 & 0.89 \\
\hline 1 & yellow ( $\#$ ffffoo) & red $(\#$ ffo 000$)$ & 30 & 2 & 51.5 & 16.3 & $\mathrm{~B}, \mathrm{C}$ & 2.53 & 1.07 & 2.67 & 0.96 \\
\hline 8 & blue $(\# 0000 \mathrm{ff})$ & yellow (\#ffffo0) & 30 & 2 & 50.6 & 15.7 & $\mathrm{~B}, \mathrm{C}$ & 3.47 & 0.90 & 3.73 & 0.83 \\
\hline 10 & orange ( $\#$ ffa 500$)$ & yellow (\#ffffo0) & 30 & 1 & 49.8 & 15.4 & $\mathrm{~A}, \mathrm{~B}, \mathrm{C}$ & 2.33 & 0.66 & 2.43 & 0.73 \\
\hline 15 & green $(\# 00 \mathrm{ffo} 0)$ & blue $(=0000 \mathrm{ff})$ & 30 & 1 & 49.0 & 14.4 & $A, B, C$ & 1.40 & 0.50 & 1.40 & 0.50 \\
\hline 6 & purple $(\# 800080)$ & $\operatorname{red}(\#$ ffo 000$)$ & 30 & 5 & 48.4 & 14.7 & $A, B, C$ & 1.63 & 0.61 & 1.73 & 0.83 \\
\hline 3 & blue $(\# 0000 \mathrm{ff})$ & $\operatorname{red}(\# \mathrm{ffo} 000)$ & 30 & 4 & 45.3 & 14.5 & A, B & 2.30 & 1.09 & 2.47 & 1.11 \\
\hline 5 & orange $(\# \mathrm{ffa} 500)$ & $\operatorname{red}(\# \mathrm{ffo} 000)$ & 30 & 1 & 43.6 & 15.3 & A, B & 2.00 & 0.83 & 1.97 & 0.72 \\
\hline 7 & green ( $\# 00$ ffo 0$)$ & $\mathrm{red}(\# \mathrm{ff0} 000)$ & 30 & 3 & 42.9 & 11.4 & A, B & 2.17 & 1.12 & 2.30 & 1.12 \\
\hline 16 & purple $(\# 800080)$ & orange (\#ffa 500) & 30 & 4 & 41.6 & 10.0 & A, B & 1.33 & 0.71 & 1.13 & 0.35 \\
\hline 14 & purple $(\# 800080)$ & blue ( $\# 0000 \mathrm{ff})$ & 30 & 1 & 38.2 & 11.9 & $\mathrm{~A}$ & 2.73 & 0.94 & 2.57 & 0.97 \\
\hline
\end{tabular}

(*) Combinations with the same letter are not significantly different among themselves

The table is sorted in descending order of the mean of the number of correct words column. The subjects scored highest in the green-on-orange trial and lowest in the purple-on-blue.

In order to investigate the effect of colour combinations on the speed and accuracy of data entry, an ANOVA test (analysis of variance) was performed where colour combinations were the factors, i.e. independent variables, and correct words constituted the dependent variable. The group sizes were equal, which satisfied the requirement of the ANOVA test. The Shapiro-Wilk test for normality was conducted on the data and it revealed that the distribution was normal only for experiments 12,13 and 17 at $\alpha=0.05$. The data in the other experiments did not have a normal distribution. Nevertheless, the ANOVA test was conducted since the robustness of the test affected by normality is a matter of degree [20], [8]. Prior to the ANOVA test, Levene's test was performed to determine the homogeneity of variances. The test revealed that variances for the groups were not significantly different (Levene statistics $0.782 ; \mathrm{p}=0.669$ ), and thus the assumption for ANOVA was satisfied. The ANOVA test result was $F(12,377)=4.280, p=$ 0.000; which meant that the null hypothesis could be rejected and that there were statistically significant performance differences for manual data entry for different colour combinations. Since the group variances and sample sizes were homogeneous, a Tukey HSD post-hoc test was done to determine the statistically significant different groups among the colour combinations.

The argument about the difficulty of human processing for colour pairs that are distant from each other [25], [13] was also tested with the data of the first experiment. To this end, each colour used in the experiment was given a number that corresponds to its wavelength. Table 2 shows the approximate wavelengths of each colour used in this study and the corresponding number used for an approximate calculation within colour pairs. The "distances" within colour pairs in the trials were calculated as the absolute value of the differences of these numbers and they are shown in Table 1. 
Table 2. Approximate colour wavelengths in the spectrum and the number used for calculating the "distance" within colour pairs

\begin{tabular}{|l|c|c|}
\hline Colour & Wavelength $(\mathbf{n m})$ & Number in the scale \\
\hline Infrared & $>1000$ & \\
\hline Red & 670 & 6 \\
\hline Orange & 610 & 5 \\
\hline Yellow & 575 & 4 \\
\hline Green & 530 & 3 \\
\hline Blue & 470 & 2 \\
\hline Purple & 430 & 1 \\
\hline Ultraviolet & $<300$ & \\
\hline
\end{tabular}

Table 3 shows the Pearson's correlations between the distances and other parameters. Although there was no significant correlation between wavelength distances in colour pairs and correct words, the correlation between the distances and perceived aesthetics was significant. This was also true for distances and readability. Thus, this result partially supports the argument that distant colours are not suitable for human processing. Table 3 also shows that although the correlation between the subjective evaluations of aesthetics and readability is quite high, it is low between correct words and perceived aesthetics. The same is true for correct words and readability.

Table 3. Pearson's correlations for dependent variables in the first experiment $(n=390)$

\begin{tabular}{|l|c|c|c|c|}
\hline & $\begin{array}{c}\text { Mean } \\
\text { (\# correct } \\
\text { words) }\end{array}$ & $\begin{array}{c}\text { Perceived } \\
\text { aesthetics }\end{array}$ & Readability & $\begin{array}{c}\text { Distance in } \\
\text { spectrum }\end{array}$ \\
\hline Mean (\# correct words) & & $0.112^{*}$ & $0.205^{* *}$ & -0.040 \\
\hline Perceived aesthetics & & & $0.813^{* *}$ & $-0.231^{* *}$ \\
\hline Readability & & & & $-0.207^{* *}$ \\
\hline Distance in spectrum & & & & \\
\hline
\end{tabular}

$* \mathrm{p}<0.05$

$* * \mathrm{p}<0.01$

Although a MANOVA test for investigating the relationships between colour combinations and the dependent variables of correct words, perceived aesthetics and readability would be desirable, such a test would be meaningful only if the correlations were moderate [9]. Hence, instead of MANOVA, two separate ANOVA tests were performed where colour combinations were the factors in both tests and perceived aesthetics and readability were the dependent variables.

Table 4. Test for homogeneity of variances for aesthetics and readability in the first experiment

\begin{tabular}{|c|c|c|c|c|}
\hline & Levene's test & df1 & df2 & Significance \\
\hline Aesthetics & 3.007 & 12 & 377 & 0.000 \\
\hline Readability & 5.394 & 12 & 377 & 0.000 \\
\hline
\end{tabular}

As shown in Table 4, Levene's test indicated that the variances were not homogenous both for perceived aesthetics and readability. Therefore Welch and Brown-Forsythe tests were performed for determining whether there were statistically significant differences in perceived aesthetics and readability across different colour combinations. The significance of Welch and Brown-Forsythe tests both for perceived aesthetics and readability was equal to 0.000 , which meant that the null 
hypothesis could be rejected for both. Hence, the differences were significant. For perceived aesthetics $\mathrm{F}(12,377)=21.85, \mathrm{p}<0.05$, and for readability $\mathrm{F}(12,377)=26.27, \mathrm{p}<0.05$.

\subsection{Second experiment (display and input windows are distant from each other)}

Some of the colour pairs that were used in the first experiment were also used in the second experiment. As stated, the aim in this experiment was to gauge the extent to which the proximity of the two windows affected the speed and accuracy of data entry. To this end, results of the second experiment were compared with the first experiment results for the same data sets. The colour pairs used in this experiment are shown in Table 5 along with the descriptive statistics. The pairs are sorted according to the mean of the correct words.

Table 5. Descriptive statistics for the second experiment (windows distant from each other)

\begin{tabular}{|c|c|c|c|c|c|c|}
\hline Trial & Foreground & Background & $\begin{array}{c}\text { Corresponding trial in } \\
\text { experiment 1 }\end{array}$ & $\mathbf{N}$ & $\begin{array}{c}\text { Mean } \\
\text { (\# correct words) }\end{array}$ & St. dev. \\
\hline 2 & yellow & red & 1 & 30 & 50.0 & 12.8 \\
\hline 4 & blue & red & 3 & 30 & 46.1 & 10.6 \\
\hline 9 & blue & yellow & 8 & 30 & 44.0 & 12.0 \\
\hline
\end{tabular}

Since there were only three sets of data for which we wished to gauge the difference, three t-tests were performed instead of ANOVA. The results of these tests are shown in Table 6.

Table 6. t-test for varying proximity $(\mathrm{p}<0.05)$

\begin{tabular}{|l|c|c|c|c|c|}
\hline & \multicolumn{2}{|c|}{ Levene's test } & \multicolumn{4}{c|}{ t-test } \\
\hline Trial pair (foreground, background) & F & Sig. & df & t & Sig. (2-tailed) \\
\hline 1-2 (yellow, red) & 0.358 & 0.552 & 58 & 0.389 & 0.699 \\
\hline 3-4 (blue, red) & 1.379 & 0.245 & 58 & -0.234 & 0.816 \\
\hline 8-9 (blue, yellow) & 0.680 & 0.413 & 58 & 1.831 & 0.072 \\
\hline
\end{tabular}

Higher significance values of Levene's test indicated that the variability of each group was approximately equal, satisfying the requirement for t-tests. This was true for all three experiments.

The t-tests indicated that, contrary to what one would expect, there were no statistically significant differences of typing speed and accuracy arising from the difference in proximity on the screen.

\subsection{Third experiment (foreground and background colours are reversed)}

In the third experiment the aim was to gauge the difference by reversing the foreground and background colours. For this experiment some of the colour pairs in the first experiment were also used. Descriptive data which are sorted according to the mean of the correct words in this experiment are shown in Table 7: 
Table 7. Descriptive statistics for the third experiment (reversed foreground and background colours)

\begin{tabular}{|c|c|c|c|c|c|c|}
\hline Trial & Foreground & Background & $\begin{array}{c}\text { Corresponding trial in } \\
\text { experiment 1 }\end{array}$ & $\mathbf{N}$ & $\begin{array}{c}\text { Mean } \\
\text { (\# correct words) }\end{array}$ & $\begin{array}{c}\text { St. } \\
\text { dev } \\
\text {. }\end{array}$ \\
\hline 20 & red & yellow & 1 & 30 & 53.1 & 15.0 \\
\hline 18 & red & blue & 3 & 30 & 50.1 & 17.7 \\
\hline 19 & yellow & blue & 8 & 30 & 42.5 & 11.1 \\
\hline
\end{tabular}

Three t-tests for three pairs of colour combinations were performed for this experiment. The tests were performed for pairs in the first experiment and the third one. The results are shown in Table 8:

Table 8. t-test for reversed foreground and background colours $(\mathrm{p}<0.05)$

\begin{tabular}{|l|c|c|c|c|c|}
\hline & \multicolumn{2}{|c|}{ Levene's test } & \multicolumn{3}{c|}{ t-test } \\
\hline Trial pair (foreground, background) & F & Sig. & df & T & Sig. (2-tailed) \\
\hline 18-3 (red, blue) - (blue, red) & 1.085 & 0.302 & 58 & 1.141 & 0.259 \\
\hline $19-8$ (yellow, blue) - (blue, yellow) & 2.053 & 0.157 & 58 & -2.320 & 0.024 \\
\hline 20-1 (red, yellow) - (yellow, red) & 0.001 & 0.972 & 58 & 0.404 & 0.688 \\
\hline
\end{tabular}

Levene's tests indicated that variances were homogeneous for all tests. The t-tests revealed that there was no statistically significant difference for the blue-red and yellow-red combinations, but the difference for the blue-yellow combination was significant.

\section{CONCLUSION, LIMITATIONS AND FUTURE WORK}

The investigation in this research was based on three HCI concepts, namely colour combinations, visual performance and manual data entry. Manual data entry performance is measured in terms of correct words entered in read-and-type cycles. Subjective considerations of colour combinations on perceived aesthetics and ease of readability and data entry were also evaluated.

It was found that different colour combinations had varying effects on the performance of manual data entry and that these effects were statistically significant. This is in conformity the majority of earlier research. There were also significant differences on subjective evaluations for aesthetics and readability of colour combinations.

Human memory is heavily involved in the read-and-type cycle. The cycle involves mainly three steps, namely visual cognition, temporary storage of data in human memory, and the typing process. When all other independent variables are kept constant, difference in length between the display and input windows can be expected to lead to decrease in performance, because larger distance can lead to increase in the cycle time which in turn can slow down the process. Nevertheless, it was found that the effect of proximity on a 17-inch screen in a read-and-type cycle was insignificant when the display and the typing windows had the same colour combination.

The effect on performance of reversing the foreground and background colours was somewhat inconclusive. The two trials in this experiment showed insignificant differences, but the difference in one trial was significant. This can be due to the fact that there is indeed a pattern that 
the read-and-type process is affected by reversing some colour pairs and not affected by reversing some others. This can be the topic of further research that involves more colour combinations.

There are some limitations on these findings which mainly stem from the implementation of the RGB colour system in VDTs. While different intensities of the primary colours red, green and blue produce different colours, those colours are also represented in the HSV (hue, saturation, value) colour model where the hue is the dominant wavelength or colour and the value is luminance or brightness. The saturation is the colour intensity spanning from "colourless" or grey to the maximum "colourfulness." These parameters are hardware-dependent and different hardware may produce slightly different colours for the same HSV and RGB codes. Fukuzumi et al. [3] argue that the hue spans various levels of saturation and occupies a large area in a chromaticity coordinate diagram. Hence, a particular colour such as green can take various forms. It is even more difficult to determine the appearance of a particular colour on VDTs due to the hardware difference where even different models of the same manufacturer may produce slightly different results [4]. Additionally, factors such as display resolution in different multimedia devices may lead to slight differences in colour perception [5]. However, these limitations are not applicable for the second and third experiments in this research.

This research is introductory in this specific area of HCI, and further research needs to be done to address its limitations. Such research should also contain other colour combinations that were not used in this research. This has important implications for practice, because as stated before, manual data typing in a "colourful" environment is a quite common computer task in today's world.

\section{REFERENCES}

[1] Bauer, D. \& Cavonius, C.R. (1980). Improving the legibility of visual display units through contrast reversal. In: E. Grandjean \& E. Vigliani (Eds.) Ergonomic aspects of visual display terminals (pp. 137-142) London: Taylor \& Francis.

[2] Radl, G.W. (1980). Experimental investigations for optimal presentation-mode and colours of symbols on the CRT-screen. In E. Grandjean \& E. Vigliani (Eds.), Ergonomic aspects of visual display terminals (pp. 127-136) London: Taylor \& Francis.

[3] Fukuzumi, S., Yamazaki, T., Kamijo, K., \& Hayashi, Y. (1998). Physiological and psychological evaluation for visual display colour readability: A visual evoked potential study and a subjective evaluation study. Ergonomics, 41(1), 89-108.

[4] Gnambs, T., Appel, M., \& Batinic, B. (2010). Color red in web-based knowledge testing. Computers in Human Behavior, 26(6), 1625-1631.

[5] Hall, R. \& Hanna, P. (2004). The impact of web page text-background colour combinations on readability, retention, aesthetics and behavioural intention. Behaviour \& Information Technology, 23(3), 183-195.

[6] Hill, A.L. \& Scharff, L.V. (1997). Readability of screen displays with various foreground/background color combinations, font styles, and font types. In Proceedings of the 11th National Conference on Undergraduate Research (pp. 742 - 746) Austin, TX.

[7] Humar, I., Gradisar, M., \& Turk, T. (2008). The impact of color combinations on the legibility of a web page text presented on CRT displays. International Journal of Industrial Ergonomics, 38(11-12), 885-899.

[8] Kong, S.C. \& Kwok, L.F. (2005). A cognitive tool for teaching the addition/subtraction of common fractions: a model of affordances. Computers \& Education, 45(2), 245 - 265.

[9] Leech, N.L., Barrett, K.C., \& Morgan, G.A. (2005). SPSS for intermediate statistics, 2nd ed. Mahwah, NJ: Lawrence Erlbaum Associates, Inc. 
[10] Lin, C.C. \& Huang, K.C. (2006). Effects of ambient illumination and screen luminance combination on character identification performance of desktop TFT-LCD monitors. International Journal of Industrial Ergonomics, 36(3), 211-218.

[11] Lindgaard, G. (2007). Aesthetics, visual appeal, usability and user satisfaction: what do the user's eyes tell the user's brain? Australian Journal of Emerging Technologies and Society, 5(1), 1-14.

[12] Ling, J. \& van Schaik, P. (2002). The effect of text and background colour on visual search of web pages. Displays, 23(5), 223-230.

[13] Matthews, M.L. (1987). The influence of colour on CRT reading performance and subjective comfort under operational conditions. Applied Ergonomics, 18(4), 323-328.

[14] Nielsen, J. (2000). Designing web usability: the practice of simplicity. Indianapolis, IN: New Riders Publishing.

[15] Noiwan, J. \& Norcio, A.F. (2006). Cultural differences on attention and perceived usability: investigating color combinations of animated graphics. International Journal of Human-Computer Studies, 64(2), 103-122.

[16] Pace, B.J. (1984). Color combinations and contrast reversals on visual display units. In Proceedings of the Human Factors Society 28th Annual Meeting (pp. 326-330) Santa Monica, CA.

[17] Pastoor, S. (1990). Legibility and subjective preference for color combinations in text. Human Factors, 32(2), 157-171.

[18] Pearson, R. \& van Schaik, P. (2003). The effect of spatial layout and link colour in web pages on performance in a visual search task and an interactive search task. International Journal of HumanComputer Studies, 59(3), 327-353.

[19] Radl, G.W. (1980). Experimental investigations for optimal presentation-mode and colours of symbols on the CRT-screen. In E. Grandjean \& E. Vigliani (Eds.), Ergonomic aspects of visual display terminals (pp. 127-136) London: Taylor \& Francis.

[20] Rutherford, A. (2001). Introducing ANOVA and ANCOVA: a GLM approach. London: Sage Publications.

[21] Sanders, M.S. \& McCormick, E.J. (1993). Human factors in engineering and design. Singapore: McGraw-Hill.

[22] Shieh, K.K. \& Chen, M.T. (1997). Effects of screen color combination, work-break schedule, and workplace on VDT viewing distance. International Journal of Industrial Ergonomics, 20(1), 11-18.

[23] Shieh, K.K., Chen, M.T., \& Chuang, J.H. (1997). Effects of color combination and typography on identification of characters briefly presented on VDTs. International Journal of Human Computer Interaction, 9(2), 169-181.

[24] Shieh, K.K. \& Lin, C.C. (2000). Effects of screen type, ambient illumination, and colour combination on VDT visual performance and subjective preference. International Journal of Industrial Ergonomics, 26(5), 527-536.

[25] Shneiderman, B. \& Plaisant, C. (2005). Designing the user interface, 4th ed. Boston, MA: PearsonAddison Wesley.

[26] Wang, A.H. \& Chen, C.H. (2003). Effects of screen type, Chinese typography, text/background color combination, speed, and jump length for VDT leading display on users' reading performance. International Journal of Industrial Ergonomics, 31(4), 249-261.

[27] Wu, J.H. \& Yuan, Y.F. (2003). Improving searching and reading performance: the effect of highlighting and text color coding. Information \& Management, 40(7), 617-637.

\section{AUTHOR}

Melih Kirlidog has BSc degree from Middle East Technical University, Turkey and MBA (in MIS) and PhD degrees from University of Wollongong, Australia. He has worked in the ICT industry in Turkey and Australia for over twenty years. Currently he is working as a faculty member in Marmara University, Turkey.

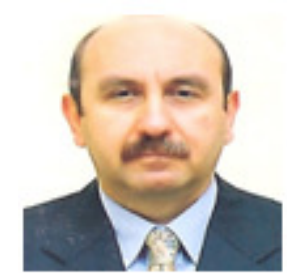




\title{
BACKTRACKING BASED INTEGER FACTORISATION, PRIMALITY TESTING AND SQUARE ROOT CALCULATION
}

\author{
Mohammed Golam Kaosar \\ School of Computing and Mathematics \\ Charles Sturt University Australia \\ mkaosarecsu.edu. au
}

\begin{abstract}
Breaking a big integer into two factors is a famous problem in the field of Mathematics and Cryptography for years. Many crypto-systems use such a big number as their key or part of a key with the assumption - it is too big that the fastest factorisation algorithms running on the fastest computers would take impractically long period of time to factorise. Hence, many efforts have been provided to break those crypto-systems by finding two factors of an integer for decades. In this paper, a new factorisation technique is proposed which is based on the concept of backtracking. Binary bit by bit operations are performed to find two factors of a given integer. This proposed solution can be applied in computing square root, primality test, finding prime factors of integer numbers etc. If the proposed solution is proven to be efficient enough, it may break the security of many crypto-systems. Implementation and performance comparison of the technique is kept for future research.
\end{abstract}

\section{KEYWORDS}

Information Security, Crypto-system, Factorization, Primality test, Backtracking.

\section{INTRODUCTION}

Integer factorisation is known as the decomposition of a composite integer number into small divisors. As for example, 91 is a composite integer which is a composition of 7 and 13, i.e. $91=7$ $\mathrm{x} 13$. Both 7 and 13 are known as the factors of 91 . If the factor is a prime number, then it is known as prime factor. In the above example, both of them are prime factors. As the size of the number increases, it becomes very difficult to find its factors. Sometimes, a sophisticated algorithm running in the fastest computer may take hundreds of years to find a factor of a large number. As a matter of fact, many cryptographic algorithms, such as RSA [11], use a big number (1024 or 2048 bits) in generating keys with the assumption that, fastest technique with the help of many computers would not be able to factorise that number within a practically feasible time.

This paper proposes a new technique to decompose a composite integer into two factors using backtracking technique. Repetitive application of the proposed technique will find all possible

David C. Wyld et al. (Eds) : CCSIT, SIPP, AISC, PDCTA, NLP - 2014

pp. 77-83, 2014. (C) CS \& IT-CSCP 2014

DOI : $10.5121 /$ csit.2014.4207 
factors of a given integer number. The proposed solution also can be used in testing the primality and finding the square root of an integer (if there exist any).

The impact of the proposed solution can be tremendous, depending upon its performance. If it can factorise a big number (as big as the number used in some crypto-systems) within a practically feasible amount of time, it would make a big change in the field of Cryptography. Further impact, implementation and performance analyses are kept for future research.

The rest of the paper is organised as follows: Section 2 presents existing solutions for factorising integer numbers in brief. Section 3 discusses the proposed solution with an example and Section 4 concludes the paper with some future research directions.

\section{EXISTING SOLUTIONS}

There have been many efforts proposed to factorize an integer number. The use of prime factors in crypto-systems increased much research interest to finding a practical solution to factorise a big integer. Integer factorization intrinsically leads towards the solution of primality testing and finding square root of an integer number. Some of the existing solutions are as follows:

- Trial Division: Trial division algorithm finds whether a given integer $\mathrm{N}$ is divisible by any positive number less than $\mathrm{N}$. This is a simple and brute force approach which is very time consuming to find a solution.

- Wheel Factorisation: This is a graphical method of factorising an integer. In this method, natural numbers are marked around the wheel to form spokes of primes and their multiples.

- Rho Methods: Rho ( $\rho$ ) method generates $\rho_{1}, \rho_{2}, \rho_{3} \ldots$ where $\rho_{i+1}=\rho_{i}^{2}+10, \rho_{1}=1$ and parameter 10 is chosen by users. This method finds factors of $\mathrm{N}$ by computing $\operatorname{gcd}(\mathrm{N}$, $\left.\left(\rho_{2^{-}} \rho_{1}\right)\left(\rho_{4}-\rho_{2}\right) \ldots\left(\rho_{2 n}-\rho_{n}\right)\right)$. Parameter $n$ is also chosen by user. This method initially was proposed by Pollard [8]. Some variations, improvements and optimisations of Rho methods are proposed in - [9], [2], [1] etc.

- Fermat's and Euler's Factorisation: Fermat's Factorisation technique [7] represents odd integer $\mathrm{N}$ as $\mathrm{a}^{2}-\mathrm{b}^{2}$ where both $\mathrm{a}$ and $\mathrm{b}$ are positive integer. Thus, $\mathrm{N}$ is equal to $\mathrm{a}^{2}-\mathrm{b}^{2}=$ $(a+b)(a-b)$ and factors are $(a+b)$ and $(a-b)$. In Euler's Factorisation technique [6], $N$ is represented as $a^{2}+b^{2}$ and $c^{2}+d^{2}$, where $a, b, c$ and $d$ are positive integers.

- Other Methods: Various other methods of factorisation algorithms are: group factorisation technique of Pollard's $\rho-1$ [8], William's $\rho+1$ factorisation [12], Lenstra's elliptic curve factorization [5]; Dixon's factorization [3]; Quadratic sieve factorization [10] etc.

\section{PROPOSED SOLUTION}

This section presents the proposed solution to factorize an integer with a simple example. The solution is also extended for primality testing and square root calculation. 


\subsection{Factorising an Integer}

Let us consider, a composite $m$ bit integer number $R$, binary representation of which is $r_{m}, r_{m-1}, \ldots r_{2}, r_{1}$. Also assume the binary representation of $R$ 's two factors $A$ and $B$ are $a_{\ell}, a_{\ell-1}, \ldots a_{2}, a_{1}$ and $b_{\ell}, b_{\ell-1}, \ldots b_{2}, b_{1}$ respectively, where both of them are $\ell$ bit long. Therefore, $R=A \times B$. Their standard multiplication (also known as grade-school multiplication) is demonstrated in Fig.1. The method of multiplication to be correct, $n_{i}$ has to be equal to $r_{i}$ for all $i=1$ to $\mathrm{m}$ where $m=2 \ell-1$.

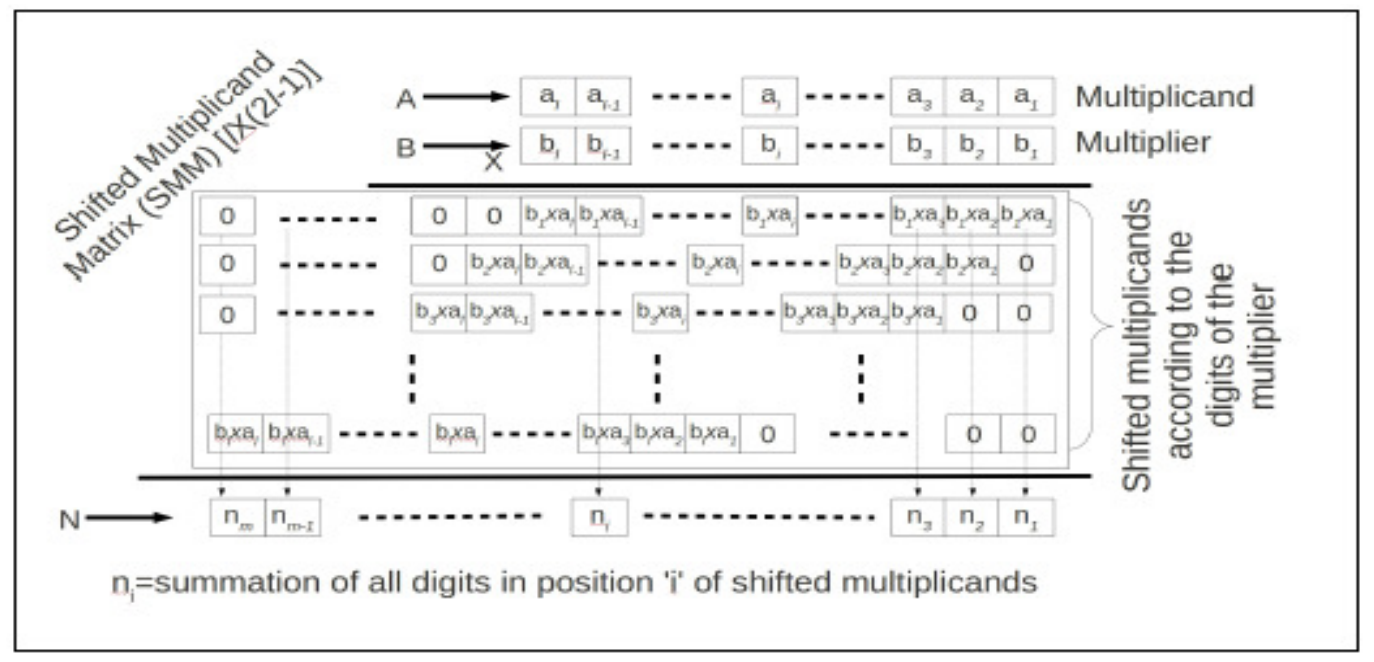

Figure 1: Binary standard multiplication of A and B to produce $\mathrm{N}$

Shifted Multiplicands Matrix (SMM), as shown in Figure 2, has the dimension of $\ell \times(2 \ell-1)$ with entry as follows:

$$
\begin{aligned}
& S M M[i][j]=0 \quad \text { for }(j<i) \text { and }(j \geqslant(\ell+i)) \\
& \text { or }
\end{aligned}
$$

$$
S M M[i][j]=b_{i} \times a_{j-(i-1)}
$$

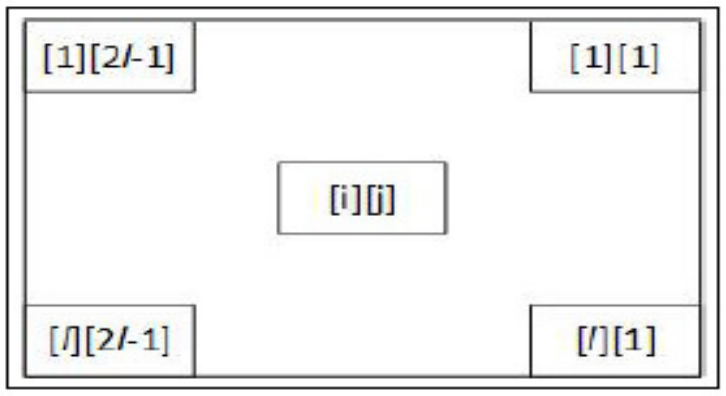

Figure 2: SMM block diagram 
Each bit in $\mathrm{N}$ is a binary summation of bits of SMM's corresponding positions, as displayed in Equation 2. Where, $S_{k}$ and $C_{k}$ are the sum and carry bit of SMM entries at column $k$.

$$
\begin{aligned}
S_{k} & =C_{k-1}+\sum_{j=1}^{\ell} S M M[j][k] \\
C_{k} & =S_{k} / 2 \\
n_{k} & =S_{k} \% 2
\end{aligned}
$$

Further detail of binary multiplication method can be found in [4].

Now an algorithm is to be developed which would choose $a_{i}$ and $b_{i}$ in such a way that, $n_{k}$ calculated in Equation 2 becomes equal to $r_{k}$, for all $k \leq(2 l-1)$. Following example explains how a number is factorised using the proposed technique.

\subsubsection{An Example}

Say an integer $\mathrm{R}=12$ and hence, $\mathrm{m}=4$. We have to find two factors, $\mathrm{A}$ and $\mathrm{B}$, or R. Binary representation of R, A, B and S M M, as discussed in Section 3.1, are now showed in step 1 of Fig. 3, where fields of A and B are empty and entries of S M M are set to 0 initially.

We need to consider every bit of $\mathrm{R}$ from right to left. In Step 2 we try to choose values for $\mathrm{a} 1, \mathrm{~b} 1$ and S M M [1] [1] such that $n 1$ calculated using Equation 2 becomes equal to $r 1$. Therefore, S M M [1] [1] must be 0 . Possible values of $\{\mathrm{a} 1, \mathrm{~b} 1\}$ could be $\{0,0\}$ or $\{1,0\}$ or $\{0,1\}$, but cannot be $\{1,1\}$. Let us say we choose $\{0,0\}$. Now immediately we can set $\mathrm{S} \mathrm{M} \mathrm{M}$ [2] [2] = $S \mathrm{M} \mathrm{M}[3][3]=0$, since $\mathrm{a}_{1}$ would make these values to 0 regardless of the value of $b_{i}, i=1$ to $l$. Similarly S M M [1] [2] = S M M [1] [3] = 0, since b1 would make these values to 0 regardless of the value of $a_{i}, i=1$ to $\ell$.

Now, we move to the next bit of R in step 3. Values of SMM in column 2 are already consistent. We have the option to choose any value for $\left\{a_{2}, b_{2}\right\}$. Say us choose $\{1,1\}$. Therefore, $S M M$ [2][3] can be set to 1 . Similarly we consider for $r_{3}$ in Step 4. Only one position of S M M in this column is left which has to be 0 to be consistent with $r_{3}$. Choose any value for $\{a 3, b 3\}$. Let us say we choose $\{0,0\}$. Hence S M M [2][4] and S M M [2][5] have to be 0 . But in the next column (column 4), all values become 0 . Therefore, a conflict arises, since the summation could not produce a value equal to $\mathrm{r} 4$. Hence, a backtracking will be necessary for $\mathrm{r} 3$. Now, let us try with the values of $\{a 3, b 3\}$ an $\{1,1\}$ in Step 5. This time it creates conflict too. In next step, let us try with $\{a 3, b 3\}-\{1,0\}$ in step 6 . Now the values of A, B and S M M becomes consistent with that of $\mathrm{R}$. Therefore, two factors of $\mathrm{R}$ would be $\mathrm{A}=6$ and $\mathrm{B}=2$. 


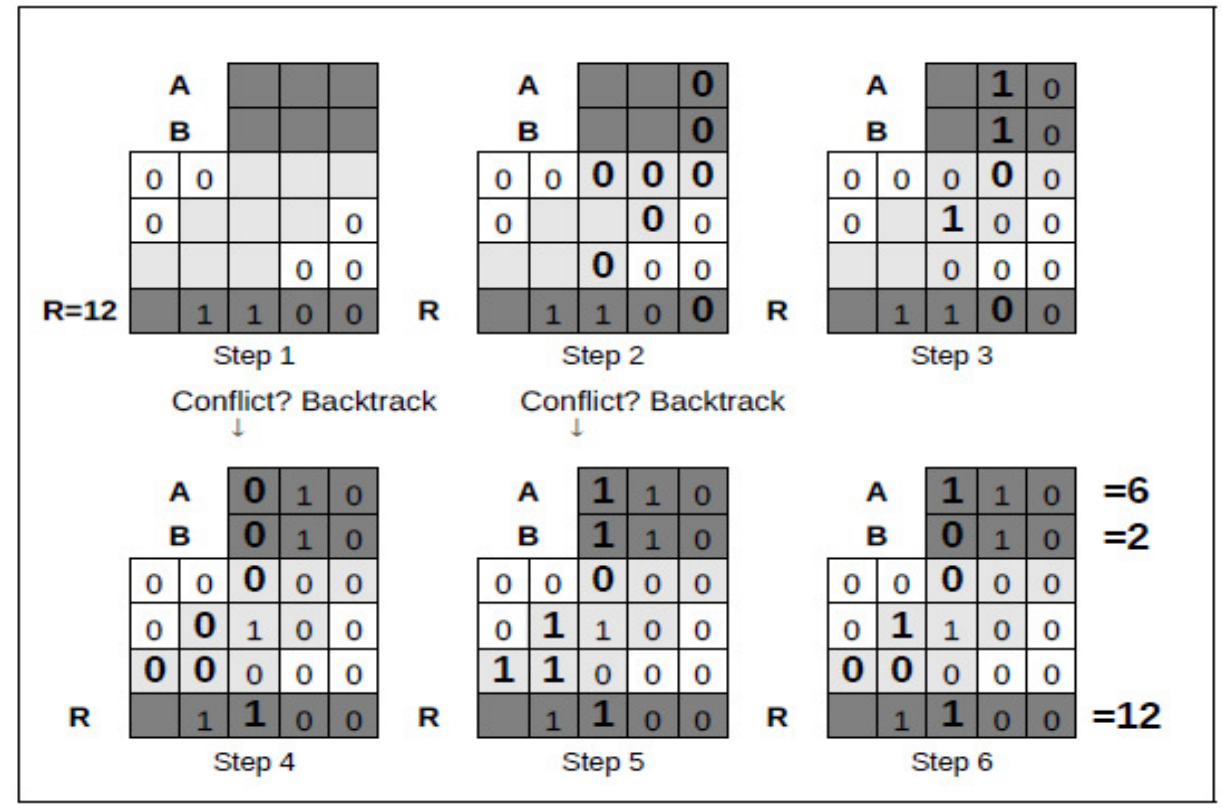

Figure 3: Steps of factorisation for the example

\subsubsection{The Algorithm}

The proposed solution appears in the following algorithm in a concised manner:

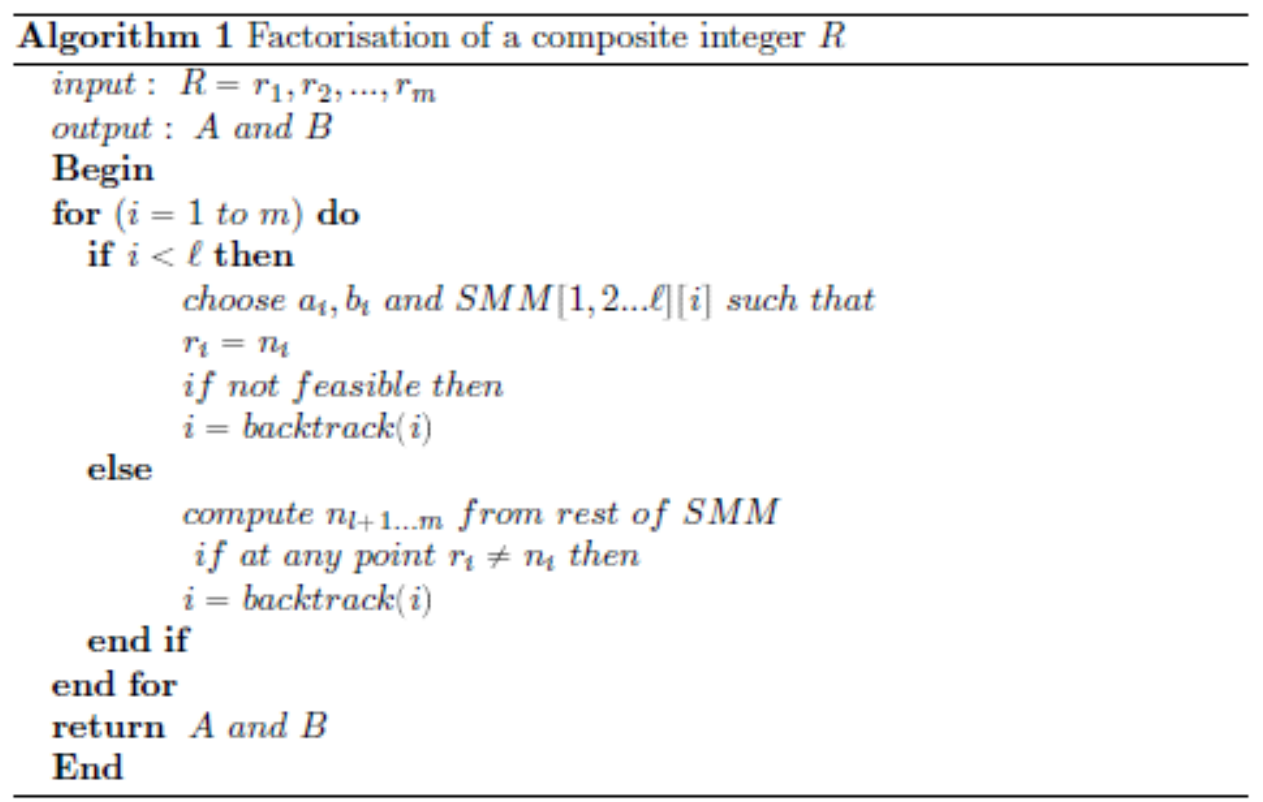




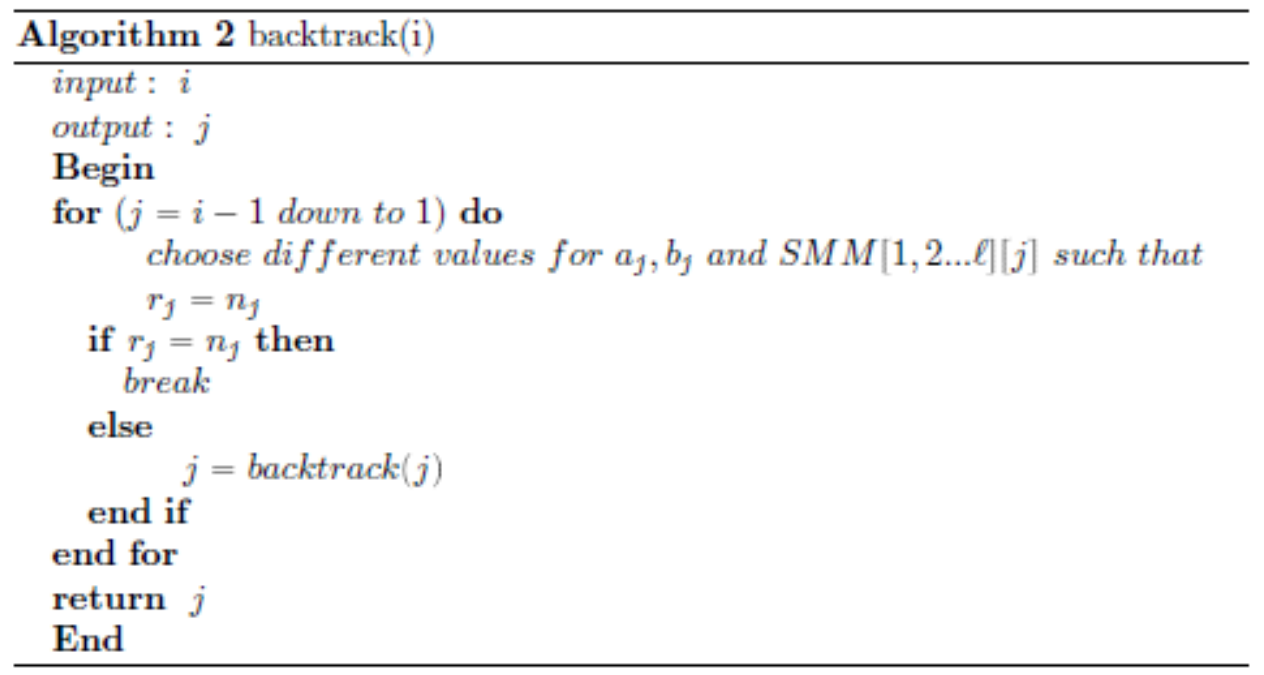

\subsection{Primality Test and Square Root}

The proposed factorisation technique can easily be extended for the following solutions:

\section{Square Root}

If we set or require, $\mathrm{A}$ and $\mathrm{B}$ must be equal, then we get the square root of $\mathrm{R}$ (if it is a perfect square) in A and B. In this case, the computation even would be quicker, since more values of SMM would be pre-set.

Primality Test

If the proposed algorithm finds no solution after finishing all steps then, $(\mathrm{R})$ is a prime number.

\section{CONCLUSION AND FUTURE WORK}

In spite of the existence of many factorisation techniques, cryptographic algorithms are still in work with the assumption that, factorising a very large number would take too long time to be practical for the crypto-system to be insecure. Therefore, a new solution in the factorisation family should draw much attention to the crypto-community. In this paper, only the algorithm is discussed in brief without considering its implementation and performance evaluation. If the proposed solution performs such that, it becomes capable to factorise those big numbers, then it may break the security of some crypto-systems, such as RSA.

In future, the algorithm can be implemented to measure its performance. It is also important to see whether this solution can factorise very big numbers used in some crypto-systems. If fails, still its performance can be compared with other existing factorisation solutions. A hybrid approach also can be thought to engage this solution with others. The algorithm itself can be improved by introducing some pruning and optimisation techniques too. 


\section{REFERENCES}

[1] Richard P. Brent. An improved monte carlo factorization algorithm. BIT Numerical Mathematics, 20:176-184, 1980. 10.1007/BF01933190.

[2] Richard P. Brent. Factorization of the tenth fermat number. MATH.COMP, 68:429-451, 1999.

[3] J. D. Dixon. Asymptotically fast factorization of integers. Math. Comp.,36 (153):255260, 1981.

[4] Harris and David. Digital Design and Computer Architecture : From Gates to Processors. Elsevier, Burlington 2007, 2007.

[5] A.K. Lenstra. Fast and rigorous factorization under the generalized riemann hypothesis. Indagationes Mathematicae (Proceedings), 91(4):443-454, 1988.

[6] James Mckee. Turning euler's factoring method into a factoring algorithm. Bulletin of the London Mathematical Society, 28, 1996.

[7] James McKee. Speeding fermat's factoring method. Math. Comput.,68(228):1729-1737, October 1999.

[8] J. M. Pollard. Theorems on factorization and primality testing. Mathematical Proceedings of the Cambridge Philosophical Society, 76:521-528,1974.

[9] J. M. Pollard. A monte carlo method for factorization. BIT Numerical Mathematics, 15:331-334, 1975. 10.1007/BF01933667.

[10] Carl Pomerance. The quadratic sieve factoring algorithm. In Thomas Beth, Norbert Cot, and Ingemar Ingemarsson, editors, Advances in Cryptology, volume 209 of Lecture Notes in Computer Science, pages 169-182. Springer Berlin / Heidelberg, 1985. 10.1007/3-540-39757-417.

[11] R. L. Rivest, A. Shamir, and L. Adleman. A method for obtaining digital signatures and public-key cryptosystems. Commun. ACM, 21(2):120-126, 1978.

[12] H. C. Williams. A p + 1 method of factoring. Math. Comput., 39:225-234,1982.

\section{AUTHOR}

Dr. Mohammed Kaosar is one of the faculty members in the School of Computing and Mathematics, Faculty of Business, Charles Sturt University, Australia. He has wide experience of teaching various ICT courses in several universities in Australia, KSA and Bangladesh. Previously, he used to work as a post-doc research fellow after his completion of PhD from the School of Engineering and Science, Victoria University Melbourne, Australia. Prior to that, Dr. Kaosar finished his MS in Computer Engineering and BSc in Computer Science and Engineering in the year of 2006 and 2001 from KSA and Bangladesh respectively. He also has experience of working in many academic, research and commercial projects. He has published good number of research papers in high quality journals and conferences including, IEEE Transactions on Knowledge and Data

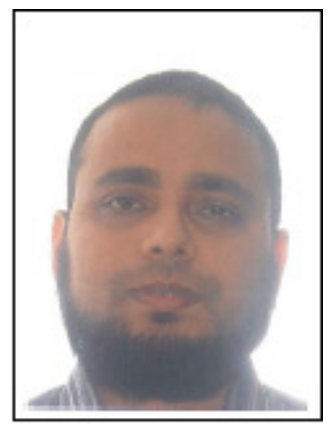
Engineering (TKDE), Data \& Knowledge Engineering (DKE), Computer Communications, IEEE International Conference on Data Engineering (ICDE- 2012) etc. He is an active member of various professional associations including IEEE, EAI. 


\title{
HUMAN INTERACTION IN THE REGULATORY OF TELECOMMUNICATIONS INFRASTRUCTURE DEPLOYMENT IN SOUTH AFRICA
}

\author{
Sharol Sibongile Mkhomazi ${ }^{1}$ and Tiko Iyamu ${ }^{2}$ \\ ${ }^{1}$ Department of Office Management and Technology, Tshwane University of \\ Technology, Pretoria, South Africa \\ mkhomazissetut.ac.za \\ ${ }^{2}$ Polytechnic of Namibia, Department of Business Computing, Windhoek, \\ Namibia \\ connectvilla@gmail.com
}

\begin{abstract}
Telecommunications is increasingly vital to the society at large, and has become essential to business, academic, as well as social activities. Due to the necessity to have access to telecommunications, the deployment requires regulations and policy. Otherwise, the deployment of the infrastructures would contribute to environment, and human complexities rather than ease of use.

However, the formulation of telecommunication infrastructure deployment regulation and policy involve agents such as people and processes. The roles of the agents are critical, and are not as easy as it meant to belief. This could be attributed to different factors, as they produce and reproduce themselves overtime.

This paper presents the result of a study which focused on the roles of agents in the formulation of telecommunication infrastructures deployment regulation and policy. In the study, the interactions that take place amongst human and non-human agents were investigated. The study employed the duality of structure, of Structuration theory as lens to understand the effectiveness of interactions in the formulation of regulations, and how policy is used to facilitate the deployment of telecommunications infrastructure in the South African environment.
\end{abstract}

\section{KEYWORDS}

Regulatory Authority, Telecommunications, Infrastructure sharing, Structuration theory, Human Interaction

\section{INTRODUCTION}

Infrastructure sharing is a concept that advocates on negotiated terms the sharing of network resources within geographical locations by two or more telecommunications network service providers [1]. Globally, telecommunications infrastructures (broadband) are deployed in urban and rural areas and these infrastructures can be shared. Sharing network infrastructure is relevant for both fixed and mobile telecommunications operators in helping to undertake the expansion of telecommunications networks in both urban and rural communities. Sharing telecommunications David C. Wyld et al. (Eds) : CCSIT, SIPP, AISC, PDCTA, NLP - 2014 pp. 85-97, 2014. @ CS \& IT-CSCP 2014

DOI : $10.5121 / \mathrm{csit} .2014 .4208$ 
infrastructure limits duplication and, enhances investment, product innovation and improved customer services [2], and reduces the infrastructure deployment costs for network service providers. It is further supported by [1] that building shared networks will lower the operators' capital investment and increase infrastructure roll-out speed. They further assert that operational costs can be saved, which is the key driver for sharing existing mature networks. However, the amount that an operator can save depends upon the depth of sharing arrangements [2].

The deployment and performance of shared telecommunications infrastructure (such as broadband) is significantly influenced by different national regulatory institutions, political processes and regulations [3]. These network infrastructures need to be managed and maintained with sound regulatory systems. In one of its strategic documents of 2009, [4] stated that the rapid rate, at which broadband technologies are deployed, requires regulations and policies for its guidance. [4] articulation and proposal for regulations and policies are mainly to avoid irregularities in the deployment, as well as to improve the technologies' efficiency and effectiveness.

Regulation plays an important role in the telecommunication industry. Regulatory structures represent key factors for innovative processes in the infrastructure sectors as they guide the direction of development and deployment of technology infrastructure [5]. These include price regulation, rules on network accessibility and environmental regulations. Therefore its sustainability relies on the legislation and regulatory structures of the country [6]. With distributed infrastructure and innovative regulations, telecommunication infrastructures such as broadband can provide high-end services to the business sector, as well a range of low-cost, highquality services to all [7].

This article presents the use of duality of structure from the perspective of Structuration theory to understand the effectiveness of regulatory in facilitating the deployment of shared telecommunications infrastructure. The focus is to understand how non-technical factors enable and constrain the development and implementation of telecommunications infrastructure sharing regulations.

\section{RESEARCH APPROACH}

To understand the roles and impact of regulatory and policy on telecommunication infrastructure deployments, a real-world situation was solicited through the case study and qualitative methods. [8] described the case study as method for eliciting natural setting. Qualitative research is a good inquiry process of understanding a social context [9]. In this vain, [10] described the method as a process which allows experience or perceptions to be shared. Based on the objectives of the research which was to understanding the roles and impact of regulation and policy on deployment of telecommunication infrastructures, probing of response was essential. The qualitative method allows for follow-up such was "why", "how", and "what" [11].

Capricon Regulatory Authority (CRA) was selected for the study. CRA is the main regulatory body in the South Africa. The organisation was instituted under the South African act of 1994. A total of four employees were interviewed within the organisation. The interviewees included two senior managers and two junior staff members. This was to draw balance in the data gathered.

The interviews approach was used in the data collection [12]. [13] described the interviews approach as a data collection method that produces first-on-hand accounts of experience, opinion, and perception from the respondents. Data was analysed using Structuration theory's duality of structure as a lens: to understand how and why interactions amongst actors were carried out in the manner that they did, in attempt to deploy telecommunication infrastructures; and to understand the impact of the roles of the agents in the deployment of telecommunication infrastructures. 


\section{STRUCTURATION THEORY}

Structuration theory (ST) is a theory which constitutes agents and structure within a social phenomenon. The social structure is drawn upon by agents, to consciously or unconsciously produce and reproduce their actions [14]. [15] argued that ST allows us to examine how people (agents) enact structures which shape their emergent and situated use of technology as they interact with it in their ongoing practices. As shown in Figure 1, the duality of structure draws and associate different factors together during agents' reproductive actions. Thus, it becomes difficult to analyse these events separately.

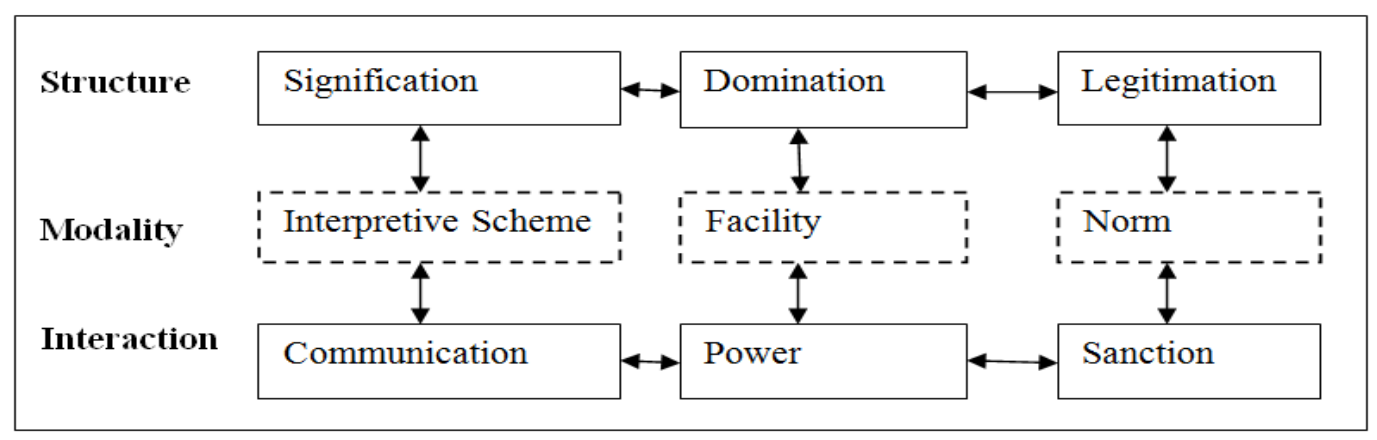

Figure 1: Duality of structure [14]

The modalities (interpretive schemes, facility and norms) link human actions (communication, power and sanction) with the structurational components (signification, domination and legitimation) [16]. The human actors' communication involves the use of interpretive schemes which are stocks of knowledge that human actors draw upon in order to make sense of their own and other's actions. These human actors referred by [17] as agents, thereby produce and reproduce structures of meanings which are termed structures of signification [18].

The human agents utilise power in interaction by drawing on facilities such as the ability to allocate material and human resources; in so doing, they create, reinforce or change structures of domination. Structuration theory's view of power is positive as it involves the exploitation of resources that allows things to get done [19]. [20] further point out that resources are the means through which intentions are realised, goals are accomplished and power is exercised.

The human agents sanction their actions by drawing on norms or standards of morality as deemed acceptable in the organisation. Thus maintaining or modifying structures of legitimation [16]. Norms or standards influence actions which may lead to changes on how rules and resources influence interactions and, the reinforcement of the norms upon which these interactions are based [21].

\section{TELECOMMUNICATION INFRASTRUCTURE DEPLOYMENT REGULATION}

The deployment of telecommunications' infrastructures includes technologies that enable high speed transfer of multi-media and high bandwidth information [22]. The deployment of telecommunications' infrastructures is socio-technical in nature [5]. This is primarily because of the technical and non-technical such as people and process components that are in involved in the deployment. Many countries, including South Africa ensure that legal requirements are met as part of the processes for telecommunication's regulatory matter [7].

Regulation is critical role on how and where telecommunications infrastructure are deployed and shared. Apart from the geographical location, regulatory structures have a major impact not only 
on the functioning and performance of national telecommunications but also on the comparative global performance of telecommunications [23]. [24] argued that the role of regulations is important in investment decision making of telecommunication companies as it helps to determine or shape the direction of their return on investment (ROI).

In South Africa regulatory and policy activities in telecommunication markets are strictly regulated by CRA. It is the sole telecommunications regulator in the country maintaining a competitive and socially responsive communications industry. South Africa derives its legislative mandate from the country's Telecommunications Act of 1996, Competition Act of 1998, the Broadcasting Act of 1999, the CRA Act of 2000 and the Electronic Communications Act of 2005 (ECA) [7]. The CRA develops regulations and policies, issues licenses to telecommunications companies, and also manages the frequency spectrum [25].

Telecommunication infrastructures are increasingly unconditional for information societies across the world. Telecommunication infrastructure facilitate, support and enable transparent system, wider dissemination of information, as well as guarantees freedom of speech for technology users [26]. Therefore the effectiveness of regulatory policies is critical in facilitating infrastructure deployment and sharing arrangements among the telecommunication companies, and the communities. The expansion of telecommunication infrastructure through sharing of infrastructure is a strategic process that necessitates co-operation among competitors, and it is subject to explicit involvement by telecommunication regulatory authorities to enforce implementation [22].

\section{STRUCTURATION VieW OF TELECOMMUNiCATION REgUlatory DEVELOPMENT}

The formulation of regulations for telecommunications infrastructure was carried out within rules and regulations of the country. The regulations facilitate the telecommunications operations in terms of infrastructure deployment. The resources required in the formulation and implementation of telecommunications regulations included technology and people. There were also processes involved.

The organisation, CRA employed both internal and external rules and regulations when formulating the governance and guidance for telecommunication's activities. The internal rules and regulations (standards and procedures) were based on the organisation's objectives and strategy. The external rules and regulations were mainly from the stakeholders including the National Government and Municipal authorities of the country.

The regulations and policies were considered critical as determine geographical location for the deployment of telecommunication infrastructures. This made the formulators of the regulations and policies to be powerful. However, the skill required to carry out such tasks were scarce. One of the employees' views on the importance of skilled resources was stated as follows: "The organisation employed people in all available positions. The challenge is whether they can do what is required of them is another matter".

There is mutual dependency among the regulators, the processes, rules and resources in the efficiency of developing and implementing telecommunications regulation throughout the country. The Table 1 below summarises the dependency. 


\subsection{DIMENSIONS OF THE DUALITY OF STRUCTURE}

Table 1: Development and implementation of telecommunication regulation

\begin{tabular}{|c|c|c|}
\hline Signification & Domination & Legitimation \\
\hline $\begin{array}{l}\text { Regulation and guidance of the } \\
\text { deployment of } \\
\text { telecommunications } \\
\text { infrastructure is of importance to } \\
\text { the government and the general } \\
\text { community of the country. }\end{array}$ & $\begin{array}{l}\text { The Ministry (Department) of } \\
\text { Communication mandates the } \\
\text { relevant Government agency to } \\
\text { formulate regulation on the } \\
\text { deployment of telecommunications } \\
\text { infrastructure in the country. }\end{array}$ & $\begin{array}{l}\text { The organisation (CRA) } \\
\text { carries out its responsibilities } \\
\text { and accountability within } \\
\text { rules and regulations as set } \\
\text { out by the government of the } \\
\text { country. }\end{array}$ \\
\hline Interpretive scheme & $\begin{array}{l}\text { Facility } \\
\end{array}$ & Norms \\
\hline $\begin{array}{l}\text { The government through CRA } \\
\text { regulates the activities regarding } \\
\text { locations where } \\
\text { telecommunications } \\
\text { infrastructures are deployed in } \\
\text { the country. The CRA assesses } \\
\text { the impact of } \\
\text { telecommunications } \\
\text { infrastructure on the } \\
\text { communities. }\end{array}$ & $\begin{array}{l}\text { The organisation (CRA) relies on the } \\
\text { requirements (Government } \\
\text { directive), people and processes to } \\
\text { carry out its mandates in the } \\
\text { formulation and management of the } \\
\text { regulations. }\end{array}$ & $\begin{array}{l}\text { The CRA have rules and } \\
\text { regulations through which } \\
\text { they developed, implemented, } \\
\text { and facilitated the } \\
\text { deployment of } \\
\text { telecommunications } \\
\text { infrastructure in the } \\
\text { communities and the country } \\
\text { at large. }\end{array}$ \\
\hline Communication & Power & Sanctions \\
\hline $\begin{array}{l}\text { The regulations as formulated by } \\
\text { the organisation are shared with } \\
\text { all the stakeholders, following } \\
\text { structures and channels as } \\
\text { defined by the Constitution and } \\
\text { bylaws of the country. }\end{array}$ & $\begin{array}{l}\text { The organisation is solely } \\
\text { responsible for the regulation of the } \\
\text { deployment of telecommunications } \\
\text { infrastructure in the country. This } \\
\text { authority is bestowed on the } \\
\text { organisation by the Constitution of } \\
\text { the country. The executive } \\
\text { committee is accountable for the } \\
\text { activities of the organisation. While } \\
\text { the Minister of communication } \\
\text { oversees the activities of the } \\
\text { organisation on behalf of the } \\
\text { Government. }\end{array}$ & $\begin{array}{l}\text { The regulations which are } \\
\text { formulated by the } \\
\text { organisation require approval } \\
\text { of the internal process } \\
\text { (executive committee) and } \\
\text { the Minister of } \\
\text { Communications. }\end{array}$ \\
\hline
\end{tabular}

\section{DUALITY OF STRUCTURE: SIGNIFICATION AND COMMUNICATION}

The Capricon regulatory authority (CRA) is accountable to the country's Minister of Communications. This was in order to ensure that there were sufficient regulations and policies to the telecommunications industry, and to protect citizens against negative effect of telecommunications infrastructure deployment. According to one of the employees, "the core business of the authority (CRA) is to monitor the activities of the telecommunication industry". The organisation's focus was considered essential in that it assist in creating an enabling environment for developing country as South Africa.

One of the criticalities for the regulations and policies was to guide against telecommunication service providers deploying infrastructures in locations as they so wish. This made the regulations and policies, as well as the organisation significant to the communities and government. For example, in a recent report by Pretoria News, edition of 14 August 2011, there was a protest by residents of Constantia, Cape Town over the deployment of Vodacom (one of South African service providers) towers in their community. The community was of the view that telecommunications towers will compromise their health. 
The regulations and policies were in a sense also considered to be of important to the service providers as it provide an umpiring status amongst them. This controlled competitiveness in the deployment of their infrastructures, particularly in areas considered to be strategic.

However, there seemed to be a gap in CRA's infrastructure deployment regulations. The implications of gap in the regulations and policies resulted in inappropriate deployment of telecommunications infrastructure in the different locations across the country. One of the employees of CRA briefly explained that "the incumbents are using the limitation of regulations to their defence for not deploying telecommunications infrastructure appropriately".

There are also external rules and processes such as municipal bylaws that were regarded as critical to the deployment of telecommunications infrastructure. However the challenge is that these rules are not formulated in conjunction with the organisation (CRA), and has a major impact on how telecommunications infrastructure could be deployed in the country. This could be attributed to lack of information sharing or different interpretations of shared information. This was associated to the ways and manners in which the information is shared. One of the managers, explained that "There are different municipal bylaws guiding the deployment of infrastructure, and that the inconsistencies in municipalities' bylaws created a complicated process for operators deploying telecommunications infrastructure in different locations".

Within the organisation (CRA), the information regarding the Minister's policy directive was shared with stakeholders who were involved in the formulation of regulations and policies. According to one of the employees, "the Minister of Communications" policy directives are communicated to the organisation's (CRA) Council for regulatory development. The Council shares this information with different stakeholders which are involved in the formulation of the regulations and policies". Communication was considered to be an integral part of regulatory development and implementation plans. The stakeholders who were involved in formulating telecommunications regulations and policies were expected at all times, to understand the importance of regulating the telecommunications industry. The structures and channels that were required were also understood by the stakeholders. However, the structures amongst other factors gave some individuals and groups certain power, and source of domination.

\section{DUALITY OF STRUCTURE: DOMINATION AND POWER}

As already established, the formulation of regulations and policies for the deployment of telecommunications infrastructures in the country was the responsibility of the CRA by virtue of the mandate bestowed upon them by the Ministry of Communications, as allowed by the constitution of the country. The Council of the CRA was therefore solely responsible and accountable for the regulations and policies guiding telecommunication infrastructure in the country.

Telecommunication infrastructure such as base stations and site towers were some of the facilities considered in regulatory development. To facilitate the deployment of telecommunications infrastructure in different locations, sharing of infrastructure was considered for rationale such as cost, and to reduce competitiveness. The CRA therefore formulated regulations to facilitate and manage the deployment of shared telecommunications infrastructures. The telecommunications operators were expected by law, to adhere to telecommunications regulations in terms of how and where infrastructures are deployed in the geographical locations across the country. This was intended to protect the sanity of the environment, as well as protect the interest of the communities.

The organisation had policy that guided how telecommunications infrastructure could be shared among the telecommunications companies. The policy was named or tagged "Facility Leasing". The Facility Leasing regulations was formulated to help facilitate efficient and appropriate infrastructure deployment. One of the employees explained that: "the facility leasing regulation 
defines the essential facilities that network operators (telecommunications companies) could use or apply in the deployment of their telecommunications infrastructure”. The Facility Leasing regulation was also intended to provide guidelines, and enable sharing of infrastructure among telecommunications companies in the country. The Facility Leasing served as a source of power to the CRA to manage and control the telecommunication companies. On another hand, it deprived one company from dominating others, particularly when it comes to size and financial muscles.

However, there seem to be some challenges in the finalisation of the Facility Leasing regulations. As a result, the telecommunications companies have not been able to apply the regulation in some areas such as sharing of the spectrum technology. The challenges include technical know-how to properly define and articulate technologies terms of reference for the telecommunications companies. One of the employees expressed himself as follows: "there are many challenges with the Facility Leasing regulation, as a result, it is not executable. This is because it was not properly developed". The challenge was attributed to lack of availability of sufficient resources such as people.

The role of people was considered to be vital to the development and implementation of telecommunications regulations and policies. However the organisation (CRA) lacked the necessary skills which were required doe the formulation of its regulations and policies. This had impact of on the relationship between the telecommunication companies and the CRA on one hand, on another hand, amongst the telecommunication companies in the country. This was primarily because the power to lead which were the regulations was weak due to lack of skilled resources.

The lack of available skilled personnel was attributed to insufficient fund. Two of the interviewees explained that "the organisation do not receive enough funds which would enable them to recruit qualified skilled personnel. This therefore impacted the quality of regulations and policies that we formulate". Also, according to another interviewee, "the Minister of Communication was responsible for the appointment of Council members and they are responsible to communicate their activities within the organisation to the Minister, in return". The Council was the highest decision making body of the organisation. This amplified the power of the Minister over CRA.

Unfortunately the organisation depended on the Government for funding in order to carry out their mandates. According to one of the interviewees, "we are dependent on the Minister of Communications for financial support in order to carry out our day-to-day responsibilities". The implication of such dependent led to control and political manipulation of the organisation's activities. According to one of the interviewees, "the Minister of Communication has the final word on our regulatory and policy matters". Through this type of funding model, the government asserted its power and dominance over CRA and the telecommunications companies in the country. At the time of this study, this was the norm and was legitimised and accepted by the stakeholders such as the telecommunications companies, the communities and CRA.

\section{DUALITY OF STRUCTURE: LEGITIMATION AND SANCTIONS}

Capricon Regulatory Authority (CRA) was legally authorised by the government of South Africa to regulate the activities of telecommunication industry, particularly to those that have impact on the communities. As already established above, CRA provided governance, and were the custodian of all regulatory development and implementation in the telecommunication industry. Some of the participants pointed out as follows: "the Minister of Communications' policy dictated their objectives and directions". In this case, the organisation needed to get approval for all its activities including recruitment of personnel. For example, as pointed out by one of the interviewees, "the organisation needs to get approval from the Minister to appoint international 
consultants and if the Minister does not approve of what the organisation requests, he pulls tight on the pay strings".

The legitimation and approval of CRA activities were driven through three-way dimensional approach: the CRA, Department of Communications, and the Minister of Communications. This had impact on the efficiency of regulatory and policy by CRA. One of the managers tried to explain the process and rational for the approach as follows: "the Department of Communication was the bridge between the CRA and the Minister. That the communication between CRA and the Minister has to go through the Department of Communication, this was based on the 1994 on which the CRA was established".

The formulation and implementation of regulations and policies were guided by external and internal rules and interests. The rules were followed during formulation of telecommunications regulations. The internal rules included the processes which were set by the organisation for its day-to-day business operations. External rules were set or directed by stakeholders such as the Ministry of Communications and municipalities bylaws.

However, there was a mutual dependency between the municipalities' bylaws and the regulations that were implemented by the organisation. The bylaws were considered as a tool that was used to ease some challenges in the deployment of telecommunications infrastructures in different locations. The bylaws were fundamental in that each of the geographical location across the country had its unique requirements. One of the interviewees pointed out: "It was a very complicated process for the telecommunications operators to deploy infrastructure in different locations, and that the challenge was due to lack of coordination in addressing the different bylaws set by municipalities". Despite the challenges, the CRA, government and the communities accepted the development and implementation of the regulations that facilitated the telecommunications' activities in the deployment of infrastructures in the country.

\section{FACTORS INFLUENCING TELECOMMUNICATION REGULATIONS AND POLICIES}

From the analysis presented above, some factors were found to influence the formulation of regulations and policing which guided the telecommunications infrastructure deployment. The factors, include government, organisational politics, regulatory accessibility, communication and organisational structure and technical know-how, as depicted in Figure 2, and discussed below.

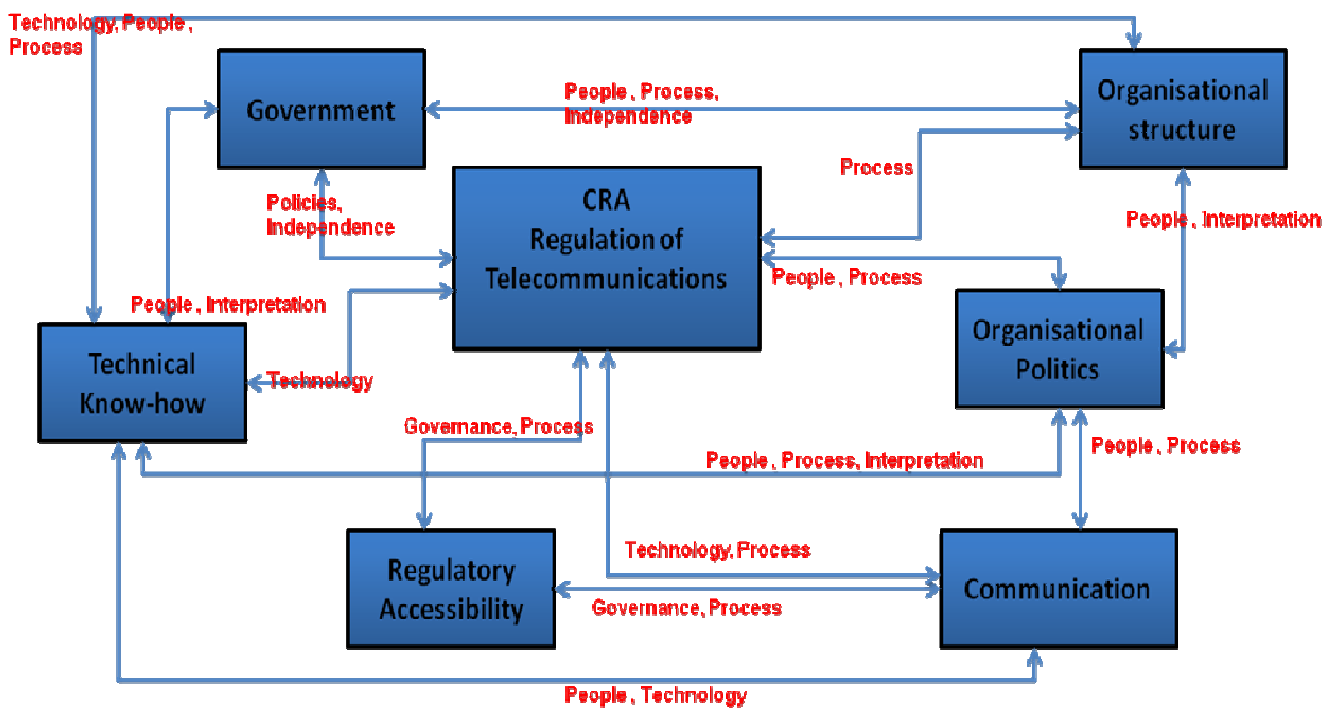

Figure 2: Components of telecommunication regulatory 


\section{GOVERNMENT}

As revealed in the analysis, government has a significant role in the formulation or regulations and policies by CRA, which were intended to guide the deployment of telecommunications infrastructure in the country. This was to ensure sustainability, control of competitiveness in the industry, as well as long-term innovation through sharing capability.

The interest and role of the government was defined around power to control the activities of the telecommunication through the establishment of CRA. By so doing, the government created obligatory passage point for CRA, meaning the organisation could not act based on its own assertion. This had negative impact on the operations of the organisation. For example, their privilege to access funds for its operations was not based on their scope of their scope of activities, but on the discretion of the Minister of Communication, which sometimes created uncertainty.

However, the there were some positive implication in government interference to CRA activities. It gave the organisation the political strength and muscle to manage and get the telecommunication to adhere to its regulations and policies. This implication of government interference was also positive from the perspectives of the community, as witnessed in the case of protest in the Cape Town area.

The CRA clearly depended on the government for funding and thus the autonomy of the organisation was undermined. This made the relationship between CRA and the government to be one sided, and was based on power for control. This had impact on the deployment of telecommunications infrastructures, and how they could be shared. The government interference was a manifestation of politics which emanated from interactions amongst the agents of the stakeholders.

\section{Organisational Politics}

Organisational politics existed in the course of formulation of regulations and policies for deployment of telecommunications infrastructures. The politics emanated from stakeholders' intent to control how the regulations and policies are developed. This could also be attributed to how information was communicated and interpreted, which were based on interests.

The organisational politics as experienced by CRA was influenced by many different factors, such as power to control and signification of presence, from both internal and external sources. The government and CRA wanted, at the same time, maintain their presence in how telecommunication companies deploy infrastructures within the communities. The power to control, which bestowed on the government, was aided by the fear that the telecommunication companies could embark on aggravated competition for space and location at the expense of the communities.

Politics is never stable and permanent. It is often driven by interest, as the government interest as in this case. This has serious implication on the existence, as well as the type of service delivered by the CRA in South Africa. As the government's interest shift focuses, the CRA changes direction, causing instability for the telecommunication companies. This in-turn impacts the types and quality of service that the communities get from the telecommunication companies overtime and space of occurrence.

The manifestation of the organisational politics can be further described as destructive. For example, due to government interference, formulation of regulation which is intended to guide sharing of infrastructures such spectrum technology has not been finalised as at the time of the study. This has implications on the telecommunications operator's decision making in deploying telecommunications infrastructure in certain areas of the country. For example, rural communities 
in some areas such as Malolisa in the Province of Limpopo suffer the consequence of the politics. Sharing of spectrum was intended to facilitate faster deployment of telecommunications infrastructure for shared network coverage.

Also, the manifestation of politics ignited the decision to sometime withheld fund, which deprived the CRA from recruiting qualified personnel, which sometimes derailed activities, as revealed in the analysis. Organisational politics and organisational structure influence, and depend on each other to exist and make a difference. As such, it is difficult, or lack of sustenance to address one without the other.

\section{ORGANISATIONAL STRUCTURE}

The organisational structure of CRA was relied upon in the distribution and allocation of tasks when it came to formulation of regulations and policies for the deployment of telecommunication infrastructures. Based on its role, the organisational defined how interactions were carried, and shaped the relationship amongst employees in the organisation, on one hand. On another hand, the structure of the organisation shaped the both interaction and relationship between CRA and the government, as well as the telecommunication companies.

The structure of the organisation dictated how roles and responsibilities were allocated amongst employees. The allocation of tasks had impact on how employees related with one another, and their subsequent interaction in carrying out their ultimate tasks, which was the formulation of regulations and policies. Somehow, the structure of the organisation was not clear and transparent, at least to external stakeholders.

The organisational structure of CRA was interpreted by some stakeholders as complicated, and as well undermined by some influencing factors or agent such as the government. This was attributed to ineffectiveness in CRA performance of its activities. This was because the Minister of Communication played a dominant role in the organisation's recruitment process. The Minister appointment of CRA's Council member made it difficult for the organisation to make decisions that were contrary to Government's interest. This can have an influence on the organisation's ability to regulate the telecommunications industry free of Government interference.

The structure within which the CRA operated extended beyond its boundaries. As part of the structure, there was a bridge created between CRA and the Minister of Communications. The bridge was occupied by the Department of Communications. This had a negative impact on how the organisation carried out its activities, as a result of bureaucracy, and multi interpretation of any and all events.

The Department of Communications made use of the structure to exert own dominance and power within legitimated frame. For example, the Department of Communications sometimes decided on which information was to be brought to the attention of the Minister of Communications and which information require their authorisation and approval. Also, the organisational structure influenced and shaped technical know-how in the formulation of regulations and policies in the deployment of telecommunications' infrastructures in the country.

\section{TECHNICAL KNOW-HOW}

To implement sufficient and efficient regulations it was crucial to have skilled and competent employees within the organisational structure. Lack of funding and the way the organisation was structured had impact on the recruitment of the required skills. The organisation lacked sufficient skilled people to carry out its strategic objective. Although the people formulate regulation, they cannot implement it. There was a need for skilled people with the appropriate technical know- 
how as it is a lack of such knowledge that causes problems with regulations such as spectrum sharing.

It was clear that there was a challenge regarding un-executable regulations for facilitating the telecommunications infrastructure deployment in different locations. This created challenges for licensed telecommunications operators to carry out their operations in deploying shared telecommunications infrastructure throughout the country.

Government's intervention and the dependence on government for funding have led to CRA not being able to employ the appropriate skilled persons to fulfil the role of regulating and implementing the regulations required to ensure the effective distribution and allocation of spectrum and infrastructure sharing.

\section{COMmunication}

The effect of the organisational politics shaped and influenced how information was communicated, and interpreted by employees as well as the stakeholders. The organisation relied on communication to carry out its mandates. Unfortunately, it was not only used for that purposed, but also for personal interest.

Some employees including stakeholders shared and interpreted information in accordance to their personal interests. In the same vain, others understood their roles and responsibilities based on their interest. Unfortunately, the information and their interpretations were followed in executing their daily activities.

Another critical aspect was that the communication amongst employees within the organisation took a different shape from the communication which happened externally, between the CRA, and the government, as well as the telecommunications companies. Due to factors such as organisational politics, and organisational structure, communication channels were not effective, messages did not reach audiences accurately.

This has an impact on the deployment of shared telecommunications infrastructure in the country. Proper communication channels will also improve productivity and, enhance those policies that have been developed through correct communication channels. However, in spite of the challenge of communication channels, the organisation and Government accepted the regulatory development and implementation facilitating the telecommunications environment.

\section{REGULATORY ACCESSIBILITIES}

The communication channels had an impact on how information was shared in the organisation. Based on our empirical evidences, it is fair to say that information sharing, and access to processes and procedures were limited in CRA. This had impact on the end-product, regulations and policies procedures.

Even though the organisation published its regulations on the website, the processes that were followed as well as the determining factors, drivers and requirements remained a secret. The secrecy could be attributed to many factors, such as the roles of government, the interaction amongst stakeholders, and independence of the organisation, which were questionable acts.

In summary, the interest of the government and the communities was driven by the significance which was associated to the services of the telecommunications, and how the infrastructures were deployed. The significance as interpreted by the CRA influenced how information was shared, and how communication was carried out. The government exerted its dominance, using the 
recourse as source of power over the CRA. At the end, the processes and procedures including the outcome (regulations and policies) were accepted as norm.

Interpretation, a further step in sense making of the findings was carried out. This was to gain a deeper understanding of why those factors as found in the analysis existed in the way that they did.

\section{CONCLUSIONS}

The study has empirically proven and revealed that the role played by non-technical factors such as people, processes and politics are critical to the development of telecommunication regulations and policies. The factors have a major impact on the effectiveness and efficiency in regulatory development and implementation. The study would therefore be important to telecommunications managers, the regulatory authority, government, as well the communities at large to gain better understanding of the impact and implications of the actions of non-technical factors.

The use of duality of structure from the perspective of Structuration theory (ST) was useful in understanding how events and activities were produced and reproduced overtime and space in the formulation of regulations policies for the deployment of telecommunication infrastructures in the country. Within the frame of the duality of structure, it was possible to follow the interactions which took place amongst the agents, and understand how significant was associated to facilities, and how events transformed themselves and become norm. This would be difficult or impossible to achieve without ST.

\section{REFERENCES}

[1] Berkers, F., Hendrix, G., Chatzicharistou, I., De Haas, T. \& Hamera, D. (2010) To Share Or Not To Share? Business aspects of network sharing for Mobile Network Operators. ICIN, BMMP Workshop, Berlin.

[2] Chanab, L., El-Darwich, B., Hasbani, G. \& Mourad, M. (2007) Telecom Infrastructure sharing: Regulatory Enablers and Economic Benefits [Online]. Available from: http://www.boozallen.com/media/file/Telecom_In, 20 October 2010.

[3] Naidoo, R., Kaplan, D. \& Fransman, M. (2005) The South African Telecoms Innovations Systems and teh Diffusion of Broadband [Online]. Available from: http://radian.co.za/docs/BROADBAND_paper_final.pdf. [Accessed: June 2010].

[4] INTEL, (2009) Realising the benefits of Broadband: White Paper. [Online]. Available from: http://www.intel.com/content/dam/www/publications. [Accessed: 14 March 2010].

[5] Markard, J. (2009) Characteristics of infrastructure sectors and implications for innovation processes. Discussion paper for the workshop on environmental innovation in infrastructure sectors. Karisruhe, 29 October 2009.

[6] Roman, E. S. (2009) Bringing broadband access to rural areas: A step-by-step approach for regulators, policy makers and universal access program administrators. Paper presented at the Nineth Global Symposium for Regulators, Beirut, Lebanon.

[7] Gillwald, A. (2001) Case Study: Broadband the case of South Africa. Paper presented at the ITU regulatory implications of broadband workshop, Geneva, 2 - 4 May 2001. Available: http://www.itu.int/osg/spu/ni/broadband/workshop/southafricafinal.pdf. [Accessed: 20 May 2010].

[8] Yin, R.K. (2009) Case study research: Design and methods, California: Sage Publications.

[9] Cresswell, J. W. (2003) Research designs: Qualitative, quantitative and mixed methods approaches, Thousand Oaks: Sage Publications.

[10] De Vos, A. S., Strydom, H., Fouche, C. B. \& Delport, C. S. L. (2002) Research at Grass Roots: For the social sciences and human service professions, Pretoria: Van Schaik.

[11] Saunders, M., Lewis, P. \& Thornhill, A. (2007) Research Methods for Business Students, 4th ed. Harlow, UK: Prentice Hall.

[12] Babbie, E. (2005). The basic of social research, Canada: Thomson.

[13] Polkinghorne, D. E. (2005) "Language and meaning: Data collection in Qualitative research", Counselling Psychology Journal, Vol. 52, No. 2, pp137-145. 
[14] Giddens, A. (1984) The Constitution of society: Outline of the theory of Structuration, Berkely, CA: University of California Press.

[15] Orlikowski, W. J. (2000) "Using technology and constituting structures: a practice lens for studying technology in organisations", Organisational Science, Vol.11. No. 4, pp 404-428.

[16] Walsham, G. (1993) Interpreting Information Systems in Organisations, Chichester: John Wiley \& Sons.

[17] Giddens, A. (1979) Central problems in social theory, Macmillan: Basingstoke, UK.

[18] Akgün, A. E., Byrne, J. \& Keskin, H. (2006)"Organizational intelligence: a Structuration view", Journal of Organizational Change Management, Vol. 2, No. 3, pp272 - 289.

[19] Chiasson, M. C. \& Saunders, C. (2005) "Reconciling diverse approaches to opportunity research using structuration theory", Journal of Business Venturing, 20:747-767.

[20] Orlikowski, W. J. \& Robey, D. (1991) "Information Technology and the Structuring of Organisation. Information Systems Research, Vol. 2, No. 2, pp143-169.

[21] Iyamu, T. \& Roode, D. (2010) "The use of Structuration Theory and Actor Network Theory for Analysis: Case Study of a Financial Solution in South Africa", International Journal of ActorNetwork Theory and Technological Innovation, Vol. 2, No. 1, pp1-26.

[22] Papacharissi, Z. \& Zaks, A. (2006) "Is Broadband the future? An analysis of broadband technology potential and diffusion", Telecommunications Policy, Vol. 30, (2006), pp64-75.

[23] Fransman, M. (2006) Global broadband battles: Why the US and Europe lag while Asia leads. Standford California: Standford University Press.

[24] Sutherland, D., Aranjo, S., Egert, B. \& Kozluk, T. (2009) Infrastructure Investment: Links to growth and the role of public Policies. OECD Economic department Working Papers, No 686:OECD Publishing.

[25] Tweheyo, A. (2009) Spectrum Management Policy framework for mobile communication: A case study of Uganda [Online]. Available from: http://dspace.mak.ac.ug/bitstream/123456789/600/3/twehenyo=asaph-cit-pgd-report.pdf. [Accessed: 16 August 2010].

[26] Chinn, M. D. \& Fairlie, R. W. (2004) The Determinants of the Global Digital Divide: A crosscountry analysis of computer and Internet penetration. Institute University of Wisconsin, Madison, WI.

\section{AUTHORS}

Dr. Sharol Sibongile Mkhomazi received her Master's degree in Business Information Systems from Tshwane University of Technology, Pretoria, South Africa in 2009 and her PHD in Computer Science and Data Processing in 2013 from Tshwane University of Technology. She is currently an HOD in the department of Office Management and Technology, Faculty of Management Sciences at Tshwane University of Technology. Her main research activities involve Telecommunication Infrastructure management, Knowledge Management and Research methodology.

Professor Tiko Iyamu holds a $\mathrm{PhD}$ in Information Systems. Currently, he is a Professor of Informatics, Chair of Health Informatics at the Namibia University of Science and Technology, Windhoek, Namibia. He also serves as a Professor Extraordinaire at the Department of Computer Science, University of the Western Cape, South Africa.

Before taken fulltime appointment in academic in 2009, Tiko held several positions in both Public and Private Institutions in South Africa. He was System Analyst and

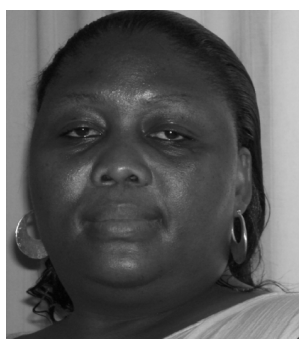
Technologist at both Nedcor Investment Bank and Metropolitan Life, respectively. He became the Chief Architect at the City of Cape Town in 1999. Thereafter, he joined Old Mutual as IT Architect, from 2001 to 2008. Iyamu's last corporate experience was at a Telecommunica tion c ompany (MWeb), as Head of Architecture \& Governance.

Iyamu's interests and focus areas include Mobile Computing, Enterprise Architecture, Information Technology Strategy, Actor Network Theory and Structuration Theory. He has published widely in book, book chapters, journals and conference proceedings. Tiko is an Associate Editor of the International Journal of Actor-Network Theory and Technological Innovation (IJANTTI). 
INTENTIONAL BLANK 


\title{
Parameter Space ANd Comparative ANALYSES OF ENERGY AWARE SENSOR COMMUNiCATION PRotocols
}

\author{
Ittipong Khemapech ${ }^{1}$ \\ ${ }^{1}$ School of Science and Technology, University of the Thai Chamber of \\ Commerce, Bangkok, Thailand \\ ittipong_utcc@utcc.ac.th
}

\begin{abstract}
Energy conservation is one of the important issues in communication protocol development for Wireless Sensor Networks (WSNs). WSNs are a shared medium system, consequently a Medium Access Control (MAC) protocol is required to resolve contention. The feature of the MAC together with the application behavior determines the communication states which have different power requirements. The power level used for a transmission, will affect both the effective range of the transmission and the energy used. The Power \& Reliability Aware Protocol (PoRAP) has been developed to provide efficient communication by means of energy conservation without sacrificing reliability. It is compared to Carrier Sense Multiple Access (CSMA), Sensor-MAC (S-MAC) and Berkeley-MAC (B-MAC) in terms of energy consumption. The analysis begins with a parameter space study to discover which attributes affect the energy conservation performance. Parameter settings in the Great Duck Island are used for comparative study. According to the results, PoRAP consumes the least amount of communication energy and it is applicable when the percentage of slot usage is high.
\end{abstract}

\section{KEYWORDS}

Energy Aware, Sensor, Communication Protocols, Comparative Analysis

\section{INTRODUCTION}

Wireless sensor networks (WSNs) are an important area of research. Data is transmitted to a destination without the need for communication cables. There are power and resource constraints upon WSNs, in addition WSNs are a shared medium system. The features of the Medium Access Control (MAC) protocol together with the application behaviour shape the communication states of the node. As each of these states have different power requirements the MAC protocol impacts upon the operation and power consumption efficiency.

The Power \& Reliability Aware Protocol (PoRAP) has been developed [1]. Its main objectives are to provide efficient data communication by means of energy conservation without sacrificing required reliability. This has been achieved by using direct communication, adaptive power adaptation and intelligent scheduling. There are some scenarios where direct communication is applicable and a significant amount of communication energy can be saved.

In this paper a comparative evaluation of PoRAP is presented. Energy consumption of PoRAP is compared with three protocols which adopt a multiple hop and random access approach. As such they may be deployed in a wider range of settings than PoRAP. This comparison focuses on

David C. Wyld et al. (Eds) : CCSIT, SIPP, AISC, PDCTA, NLP - 2014

pp. 99-114, 2014. (C) CS \& IT-CSCP 2014

DOI : $10.5121 /$ csit.2014.4209 
environments where it is appropriate to use PoRAP. The three protocols are Carrier Sense Multiple Access (CSMA) [2], Sensor Medium Access Control (S-MAC) [3] and Berkeley MAC (B-MAC) [4]. Both B-MAC and S-MAC are specifically developed for low duty cycle applications and CSMA is the default MAC protocol in TinyOS which is the chosen operating system for PoRAP development [5].

The remaining parts of this paper are organised as follows: Section 2 describes the methodology used in the comparative analysis. The Great Duck Island (GDI) [6], a production habitat monitoring system, and the calculation of energy consumption are summarized. Section 3 investigates how each parameter affects the energy consumption. The details of a comparative study are provided in Section 4. The GDI scenario is used and the methodology in [4] is adopted. Finally, the conclusion is stated in Section 5.

\section{Methodology}

This paper focuses on analysis of communication energy of the protocols. This section describes the Great Duck Island (GDI), a production habitat monitoring wireless sensor network (WSN) that is used in the analysis. Further, the calculation of energy consumption is outlined.

\subsection{Great Duck Island (GDI)}

The GDI is an important production WSN application. It is a habitat monitoring system [6]. Tiered architecture was used; the first tier consisted of sensor patches and gateways. There were up to 100 sensors in each patch. The gateway acted as a relay node. Data communication within each patch was either single or multi-hop depending on the distances between sensors and their gateway. The second tier provided connectivity between multiple patches.

The motes remained at their original positions throughout a 9-month operation. The selected sampling period in GDI was 5 minutes or 300s [6]. The data payload was 36 bytes and the required duty cycle was approximately $1.7 \%$ [4]. Several parameter settings in GDI and those given in [4] are used in this comparative study. Energy consumption required by each protocol is calculated and compared to the GDI scenario.

\subsection{Calculation of Energy Consumption}

In order to determine the effects of parameter space, the methodology given in [4] is adopted. The chosen metric is average energy usage per second. It is defined as a ratio of total energy consumed by a source to the total number of transmitted data bits in 1 second. The total energy consumption is the summation of energy used for control packet reception, data packet transmission, listening and sleeping. The data payload is used for calculating the total number of sent bits.

According to the methodology, communication delays required for a specific size of data payload is calculated with respect to a 1 -second interval by taking the sampling period into consideration. The sampling period is defined as an interval in seconds between two data collections and transmissions. The inverse of the sampling period indicates the amount of data being collected and transmitted within a second or the data reporting rate. For example, a 10 second sampling period means that a source sends one piece of 36 byte data every 10 seconds. It can also be stated that, on average, the source sends 3.6 bytes of data every second.

There are four communication modes including sending, receiving, listening and sleeping except in CSMA where the nodes do not sleep. An interval for each communication mode is computed based upon the 1-second interval. The required energy is the product of the communication 
interval and the relevant power. As the communication protocol in [4] was developed for multihop sensor networks, each node located within the communication range of the sending node has to receive the transmitted packets and the number of neighbors is included in the calculation of reception energy. The duration computations are detailed as follows:

\subsubsection{Transmission}

The duration required for data transmission per second is equal to the product of the data reporting rate, which is dependent upon application's requirement in packets per second, the number of bytes being transmitted and the duration required for transmitting 1 byte of data.

\subsubsection{Reception}

The receiving duration is equal to the products of the attributes used in the transmission and the number of neighbors. Hence, each source has to receive all of the incoming messages from its neighbors.

\subsubsection{Listening}

The listening period is equal to the product of total durations required for wakeup and carrier sensing in CSMA, B-MAC and also synchronization between nodes in S-MAC. The wakeup interval is obtained from [7] where the measurements were conducted directly from Tmote Sky motes. In total $4.18 \mathrm{~ms}$ was required for starting the radio voltage regulator, starting the radio oscillator, preparing the packet, loading the radio FIFO and setting the radio to transmission mode.

\subsubsection{Sleeping}

The sleeping period is the average time per second after subtracting transmission, reception and listening.

After obtaining all communication durations, they will be multiplied by the power in milli-watts $(\mathrm{mW})$ to get energy consumption. According to [8], the required power for data transmission depends upon the power settings. For example, the power of 25.50 and $52.20 \mathrm{~mW}$ are used at the minimum and maximum settings. The required power for receiving, listening and sleeping is respectively $59.10,1.10$ and $0.06 \mathrm{~mW}$. The selected data payload size is set to 36 bytes. The number of neighbours ranges from 1 to 100 nodes. The data sampling periods of 10, 50, 100 and 300 s are used in the parameter space study.

\section{Analysis of Parameter Space of Protocols}

The main objective of parameter space analysis is to investigate how each parameter affects the energy consumption. Several attributes required for the calculation are provided in the previous section. As each protocol has its own set of different message exchanges, the details of communication delay calculations are described separately.

\subsection{Berkeley-MAC (B-MAC)}

The driving force of B-MAC development is to support low duty cycle communication in environmental monitoring systems such as Great Duck Island (GDI) [6]. The sensors in GDI operated at approximately $1.7 \%$ duty cycle. After physical data collection and transmission, the sensors were in sleep mode for 5 minutes. 
Three main parameters affecting the energy consumption include the check interval, the data sampling period and the number of neighbours. The check interval indicates the duration of the source listening to the incoming signal. An inverse of the check interval is therefore the channel sampling frequency. B-MAC provides reliable data reception by preamble transmission. The preamble length must be at least the check interval. The size of preamble must be included in the total number of sent and received bytes.

The data sampling period illustrates the duration between two consecutive data collections and transmissions and its inverse indicates the data reporting rate in packets per second. The number of neighbours is important to the calculation of reception duration as all nodes located within the communication range of the sending node have to receive the message.

\subsubsection{Parameter Settings}

The settings in [4] apply in this analysis such as data payload size and the check intervals of 10 , 20, 50, 100 and 200ms. An average energy consumption of up to 100 neighbours is computed.

\subsubsection{Results}

Figure 1 shows the effects of check interval and sampling period on the average energy usage in B-MAC.

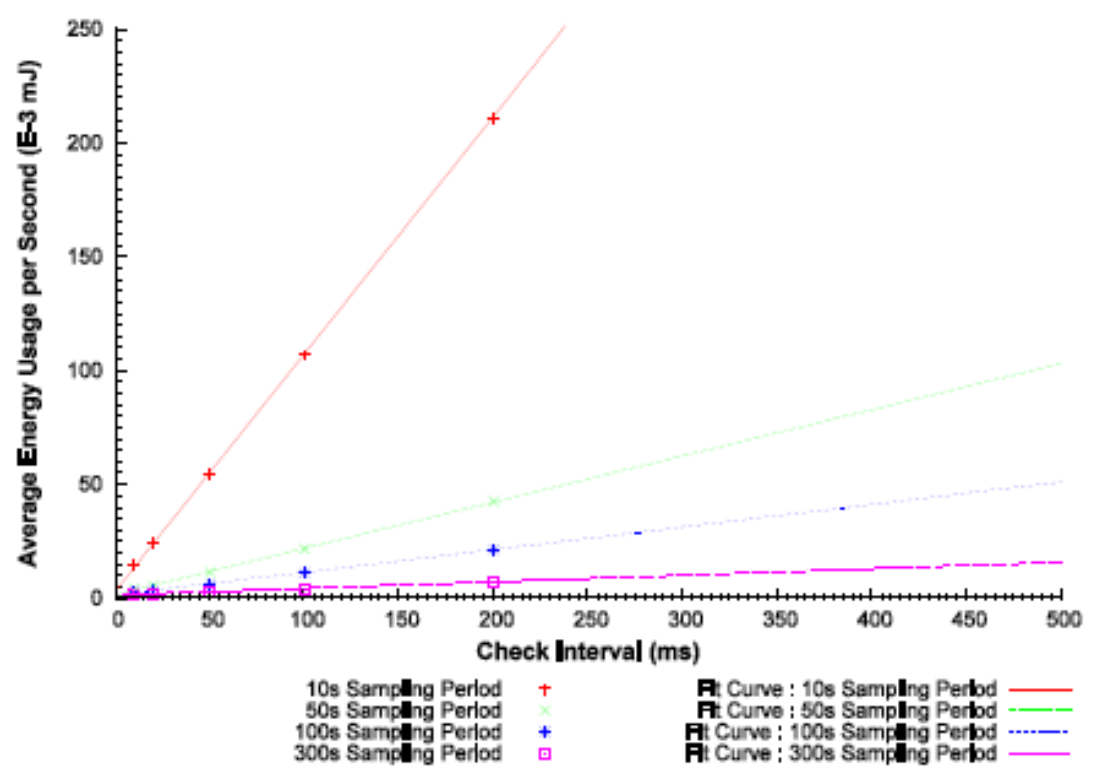

(a) At various check intervals 


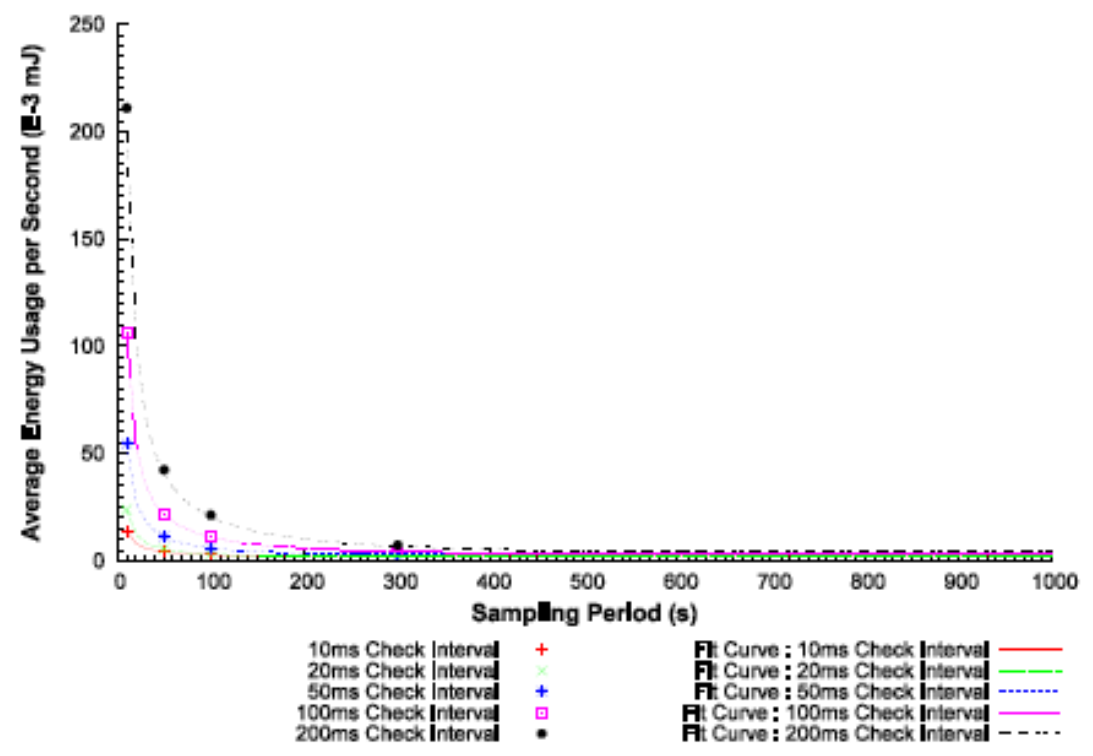

(b) At various sampling periods

Figure 1. Average energy usage in B-MAC

Linear relationships are observed in Figure 1(a) whilst inverse relationships are used in the plots in Figure 1(b). The R-square values for both figures are over 0.99 or more than $99 \%$ of data can fit. A smaller amount of energy will be used if a shorter check interval is used. However, the shortest duration is $4.18 \mathrm{~ms}$ as it is required for starting and initialising the hardware components [7].

More energy is required for a longer check interval if a sampling period is fixed. However, less energy is consumed if the sensors sample the data less frequently. A significant reduction in energy is observed when the sampling period is decreased from 10 to $100 \mathrm{~s}$. The degree of reduction is lower when a shorter check interval is used. For example, at a 200ms check interval, almost 10 times the energy is consumed if a sampling period of 100s is used instead of 10s whilst approximately 3 times the energy is saved at a $10 \mathrm{~ms}$ check interval.

The data sampling rate and check interval affects the transmission and reception energy. The number of neighbours is included in order to compute the reception duration and corresponding energy. Preamble transmission and reception can be considered as an overhead in B-MAC and increases with the number of neighbours. The source transmits its data to all of its neighbours located within the communication range. Each neighbour has to listen and receive their data. Routing is also conducted in order to forward the data to its destination. The cost in terms of energy consumption is added to both sender and receiver to provide a reliable data reception at the receiver. The receiver's wakeup schedule also affects the energy consumption.

\subsection{Sensor-MAC (S-MAC)}

Like B-MAC, S-MAC is also a contention-based protocol specifically developed for multi-hop wireless sensor networks. Additional frames are required for synchronization and hidden node problem avoidance. A source exchanges its schedule by sending a SYNC frame to the neighbours. Traditional RTS/CTS handshake is adopted in order to avoid collisions caused by transmissions from nodes which are not located within each other's ranges. The ACK frame is 
also used for data reception acknowledgement. Transmissions and receptions of additional control frames affect the energy consumption.

The effects of the three main parameters; active duration, data reporting rate and number of neighbours on energy consumption per bit of data are studied. According to [3], the default active period is set to $115 \mathrm{~ms}$. Carrier sensing, frame transmissions and receptions occur within this period. Energy consumption is equal to the product of communication delay and power. The data reporting rate is required to compute the number of packets per second. The data reporting rate is the inverse of the data sampling rate per second.

\subsubsection{Parameter Settings}

Several settings in [3] and [9] apply in this analysis. The lengths of SYNC (Synchronization), RTS (Request-To-Send), CTS (Clear-To-Send) and ACK (Acknowledgement) are set to 8, 20, 14 and 14 bytes, respectively. The active intervals of 115, 250, 500, 750 and 1,000ms are used.

\subsubsection{Results}

Figure 2 shows the effects of active interval and sampling period on the average energy usage in S-MAC.

At a specific sampling period, the average energy usage per second linearly increases with the active interval. However, the default duration is $115 \mathrm{~ms}$ [3]. A lower active interval cannot accommodate the transmission and reception delays when there are many neighbours.

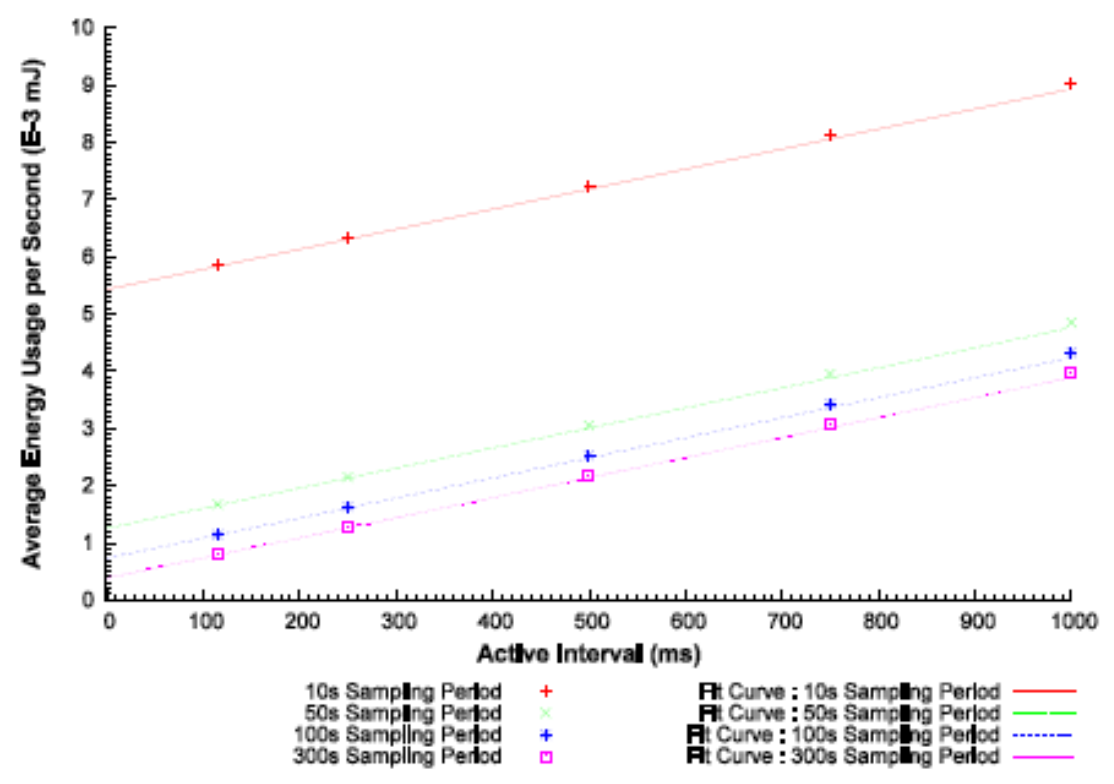

(a) At various active intervals 


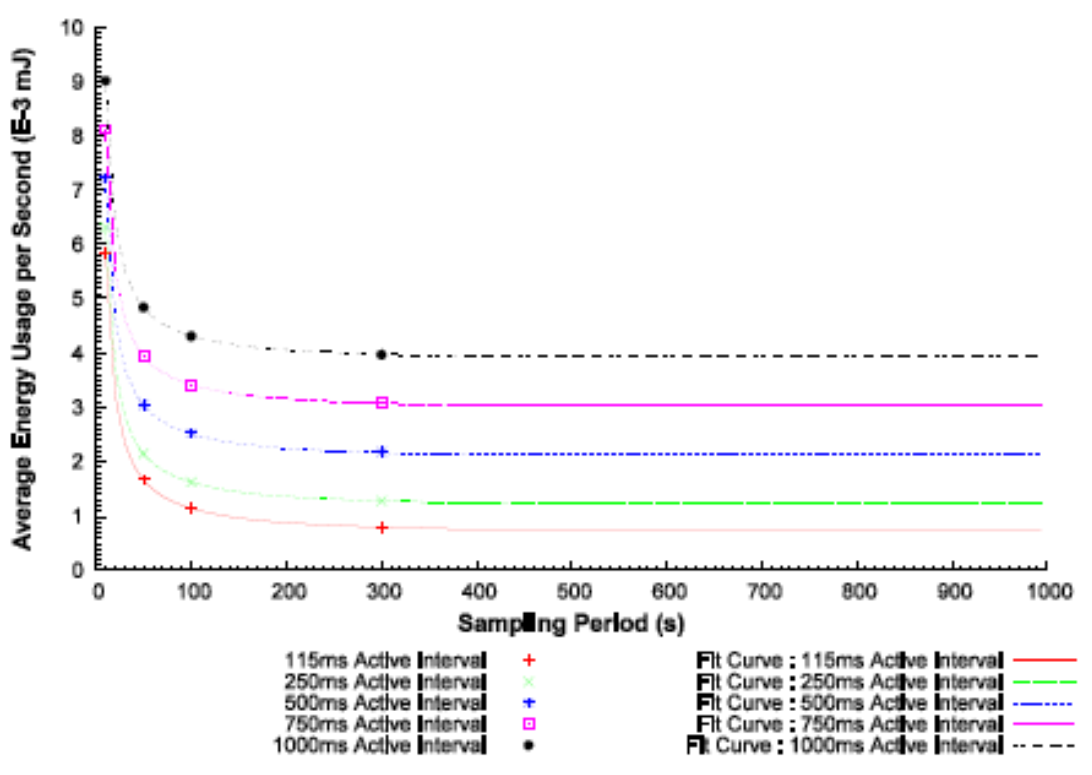

(b) At various sampling periods

Figure 2. Average energy usage in S-MAC

A considerable reduction in energy usage is observed at longer sampling periods. Up to 10 times the energy can be saved if the sensors sample every 300s instead of 10s. Figure 2(b) demonstrates an inverse technique used for fitting the plots of the relationship between the sampling period and energy usage. A significant reduction in energy consumption will be obtained if the sensors sample every 100s or longer. The main reason is that the sensor can be in sleep mode longer. The $\mathrm{R}$-square values are over 0.99 .

Several control frames including SYNC, RTS, CTS and ACK frames can be considered as overheads in S-MAC. The number of control transmissions and receptions increases with the number of neighbours. The sending source exchanges its scheduling information with its neighbors. An RTS is sent if the source has data to send. The DATA frame is not delivered unless the source receives the CTS. The receiver sends the ACK frame after the DATA is received. The minimum active duration is $115 \mathrm{~ms}$ as specified in [3]. In order to yield a low duty cycle, the data sample period should be high. For example, the sources sample data every $11.5 \mathrm{~s}$ to achieve a $1 \%$ duty cycle. For a higher number of neighbours, the source which samples data more frequently requires more energy than the one with a longer sampling period.

\subsection{Carrier Sense Multiple Access (CSMA)}

Carrier Sense Multiple Access with Collision Avoidance (CSMA/CA) is the default MAC protocol in TinyOS. Prior to transmission, the source senses the medium in order to detect whether there are ongoing activities. As the sources listen all the time, the listening energy accounts for a large proportion of the total communication energy. The effects of the data sampling period and the number of neighbors on energy consumption per bit of data are studied.

\subsubsection{Parameter Settings}

The selected data payload size is 36 bytes and four application data sampling periods are used as in the B-MAC and S-MAC analyses. 


\subsubsection{Results}

Figure 3 shows the effects of sampling period on the average energy usage in CSMA. Like BMAC and S-MAC, less frequent data sampling results in less energy usage. This is because there are fewer data communications within a specific interval. Approximately 4 milli-joules $(\mathrm{mJ})$ is required if the sensors sample every $100 \mathrm{~ms}$ or longer.

Idle listening is an important overhead as the sources constantly listen to the signal. The sampling period and the number of neighbours affect the energy consumption. A high amount of energy is required when the network includes many sources and they sample the medium more often.

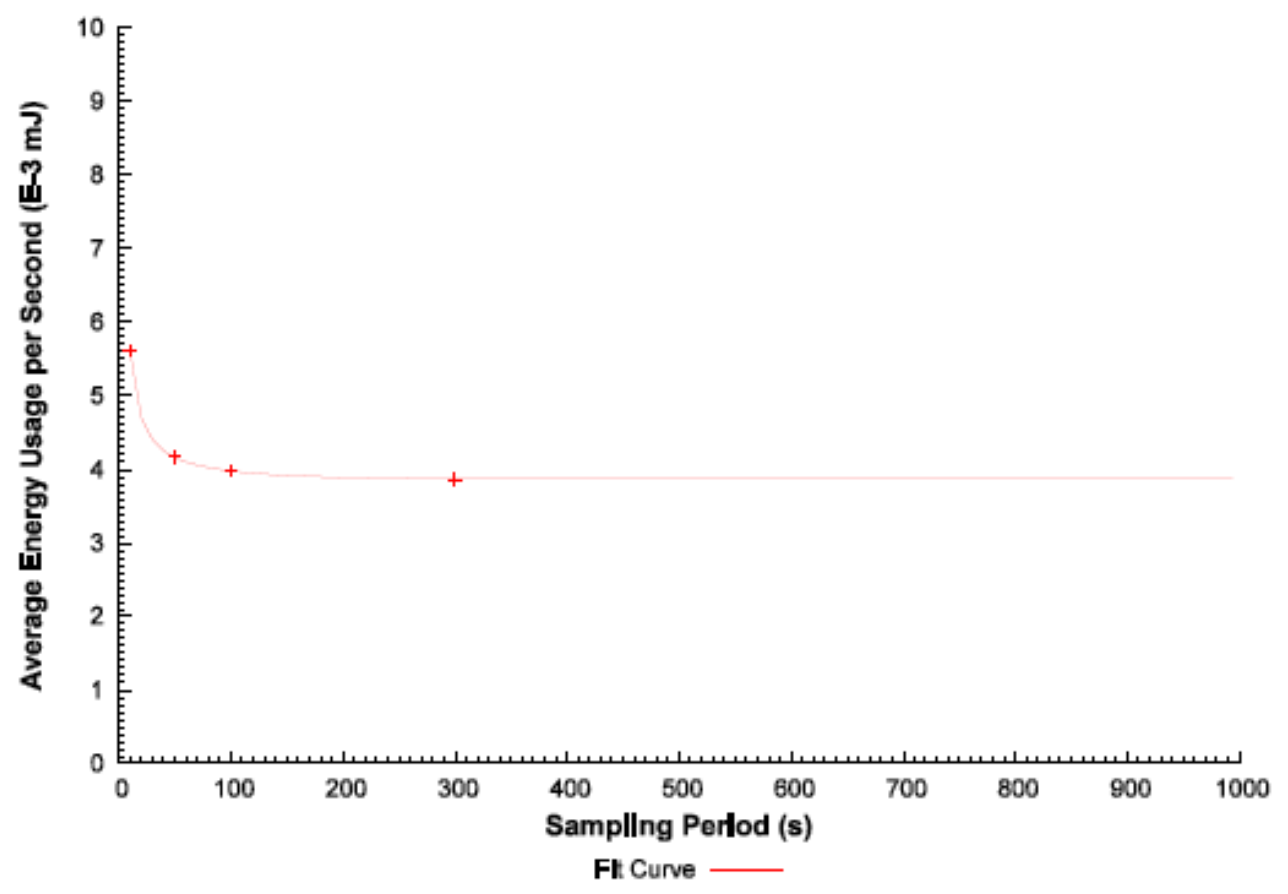

Figure 3. Average energy usage in CSMA

\subsection{Power \& Reliability Aware Protocol (PoRAP)}

There are two main parameters in PoRAP. The control packet size is directly dependent upon the number of sources. A byte of payload is required in the control packet for notifying the transmission power adaptation to every four sources because two bits are required for signalling power adaptation to each source. The duration of the control packet reception and the corresponding energy requirement for the four sources is the same. The effects of sampling periods and number of sources on the energy consumption are considered in this section.

\subsubsection{Parameter Settings}

The selected number of sources, data payload size and application data sampling periods are the same as in the previous analyses. As PoRAP is specifically designed for direct communication, the maximum power is always used in the analysis.

\subsubsection{Results}

Figure 4 shows the effects of sampling period on the average energy usage in PoRAP. A similar observation is obtained in Figure 4 compared to Figure 1 to Figure 3. A longer sampling period 
results in a lower average energy usage per second. The reduction in energy in PoRAP is the least compared to B-MAC, S-MAC and CSMA. This is because the packet is delivered directly to the base station and no data forwarding is required. The overhead is only related to the number of sources. However, PoRAP cannot accommodate a high frequency of transmission especially when there are many sources as it is a schedule-based protocol. It has to wait for all sources to complete their transmissions to start a new communication cycle.

Control packet reception can be considered as an important overhead in PoRAP. The size of control packet is related to the number of sources. According to the parameter settings, a source consumes approximately 0.2 to 0.3 milli-joules $(\mathrm{mJ})$ in transmitting one bit of data. Like other protocols, a higher amount of energy is required when there are more sources in the network and the sampling period is lower.

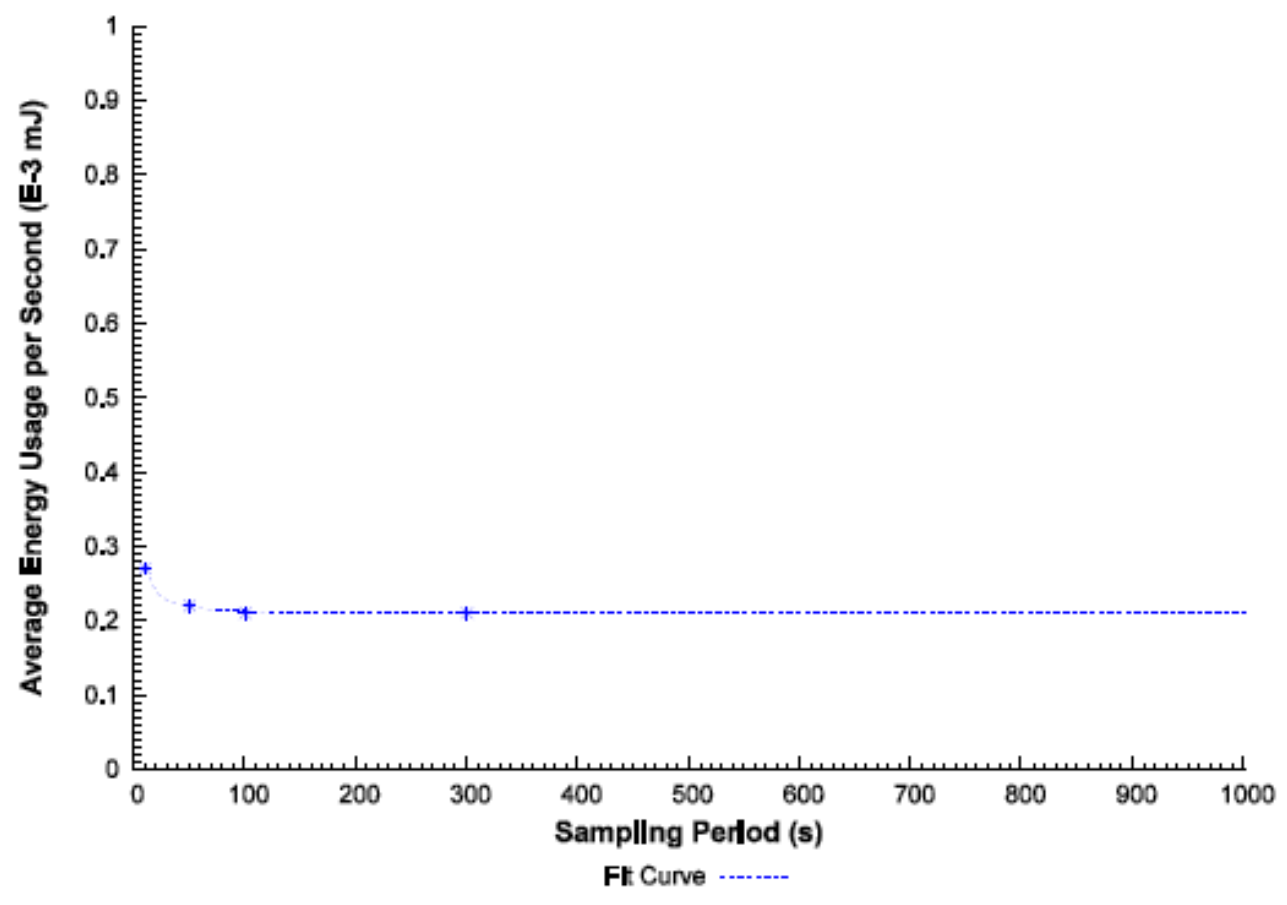

Figure 4. Average energy usage in PoRAP

\section{Comparative Study}

This section aims to compare the protocols' performances in terms of energy consumption based on the Great Duck Island (GDI) project [6]. In GDI, the sources sent their data every 5 minutes, that is the sampling period was 300s. A data payload size of 36 bytes was used [4]. The selected numbers of sources are 1, 10,50 and 100. The chosen check interval for B-MAC is $10 \mathrm{~ms}$ as it was used in [4]. The selected active interval for S-MAC is $115 \mathrm{~ms}$ as it is the default value according to [3]. Figure 5 shows the comparison in energy consumption. The sampling periods of $10,50,100$ and 300 s are used to indicate the differences in the results. 


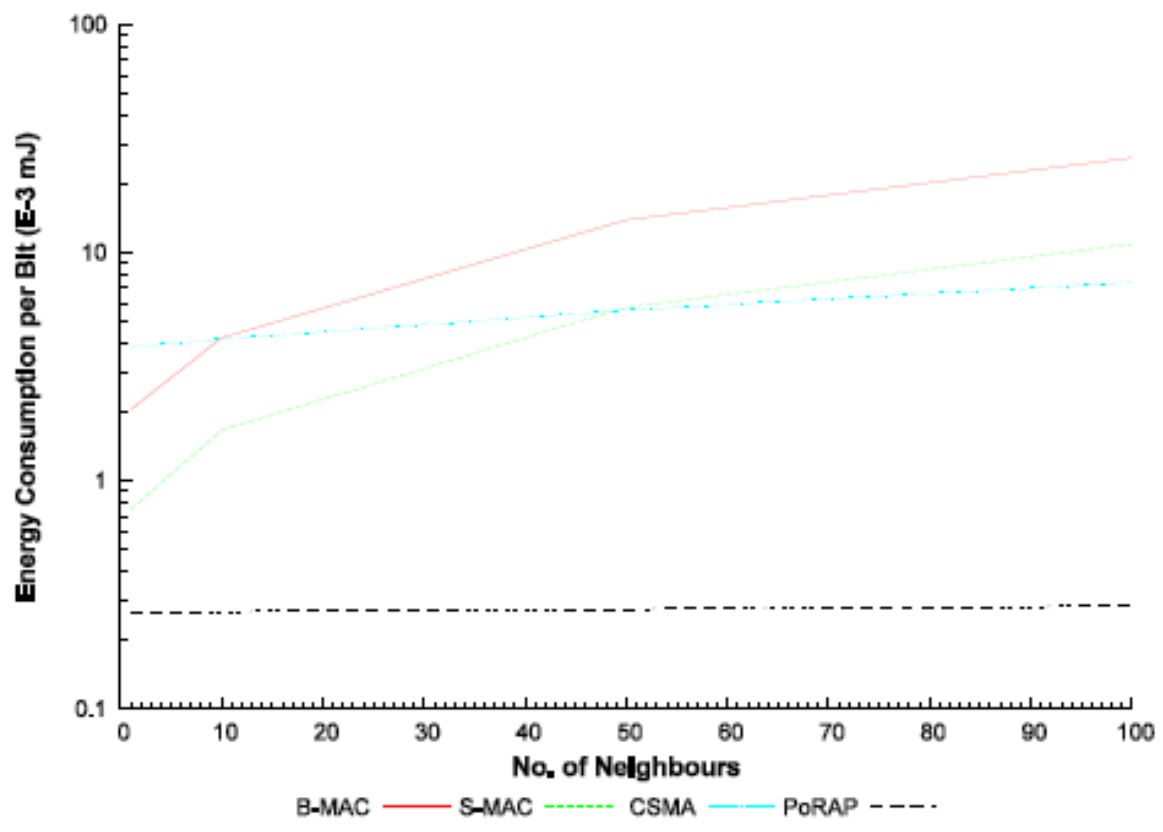

(a) 10s sampling period

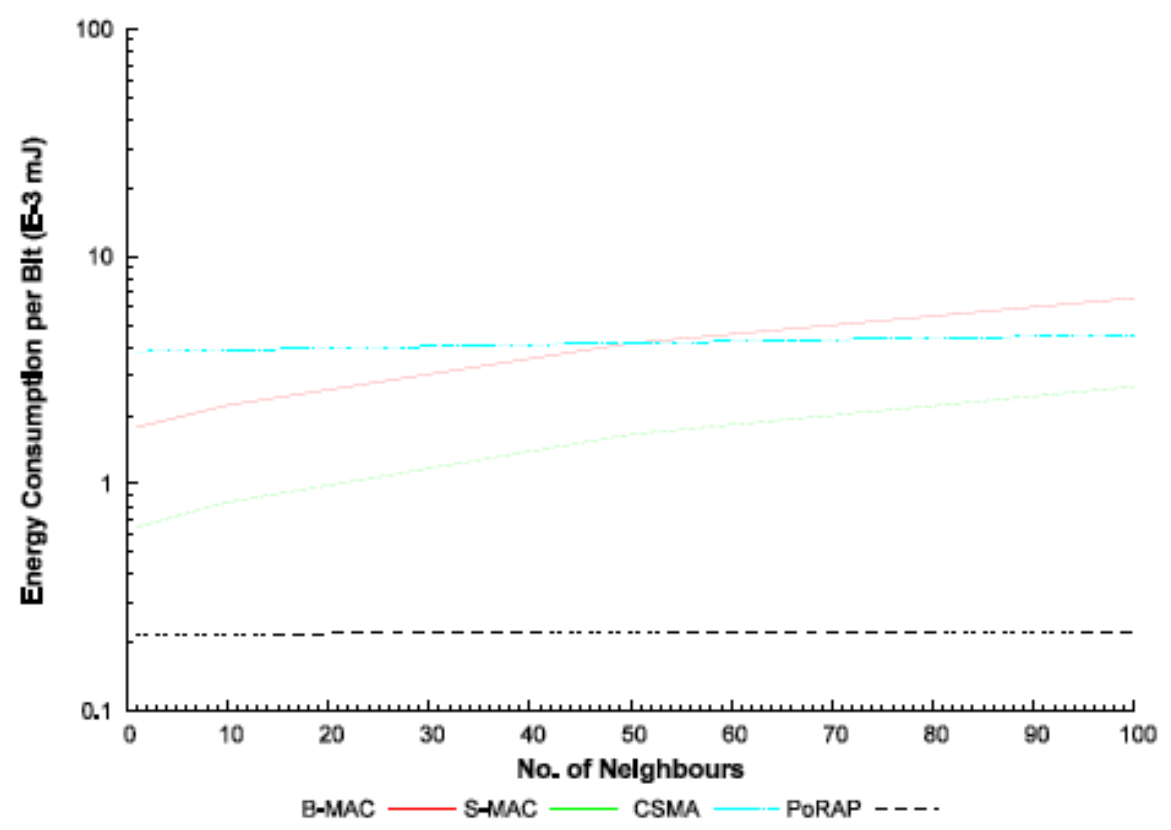

(b) 50s sampling period 


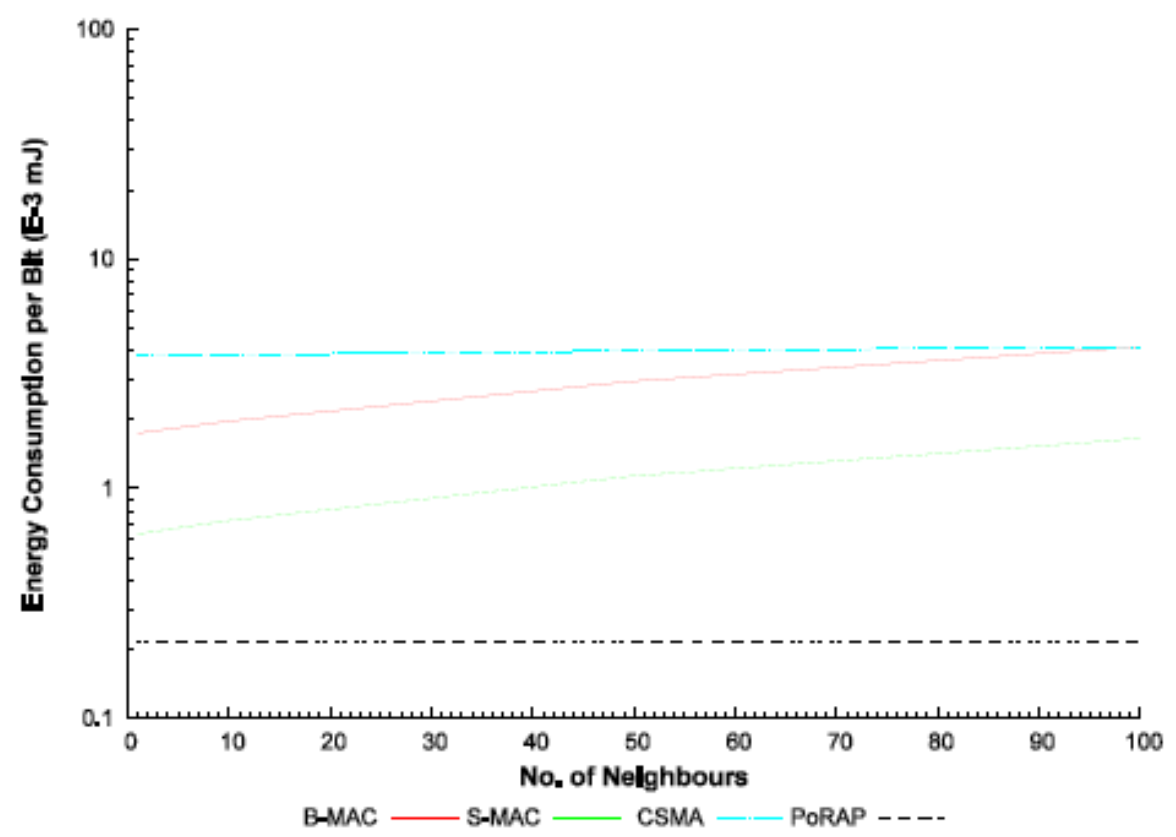

(c) $100 \mathrm{~s}$ sampling period

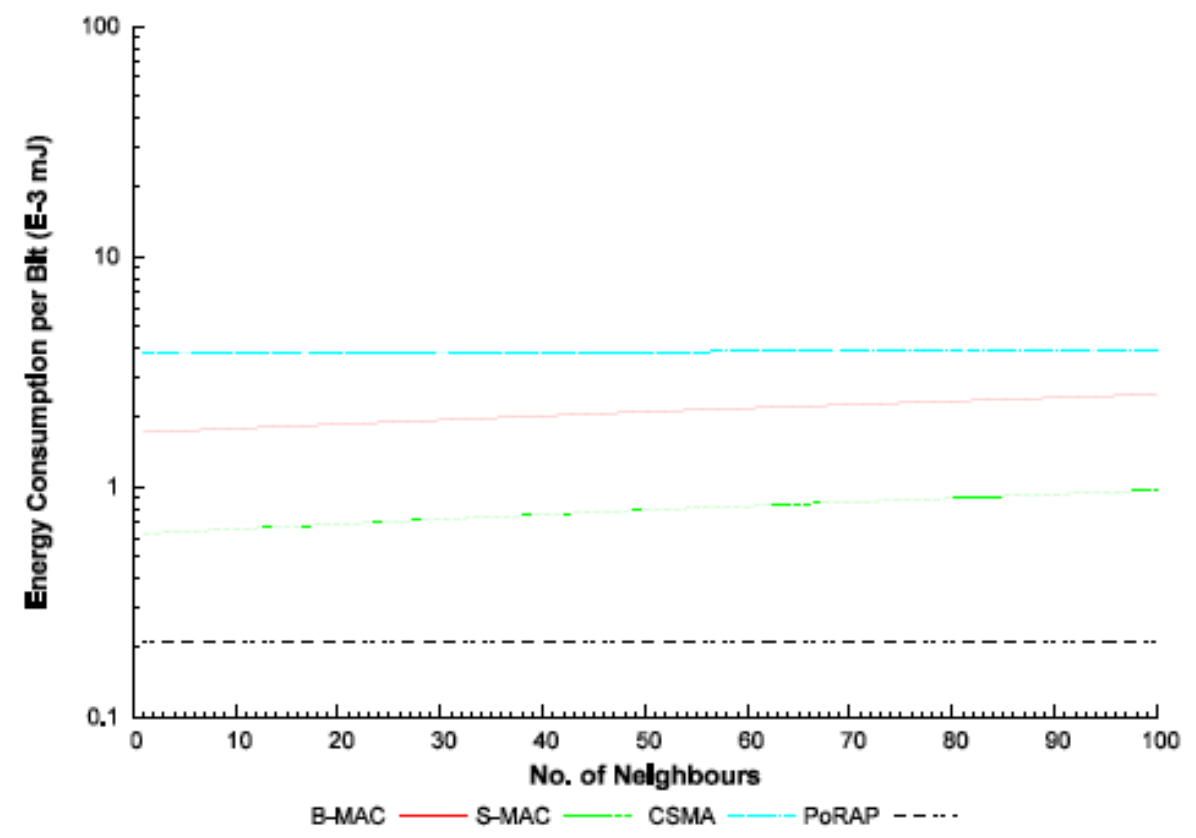

(d) 300s sampling period

Figure 5. Comparison in energy consumption at various sampling periods

According to Figure 5, energy consumption increases with the number of neighbours. It decreases with the less frequent sampling period. In the case of the multi-hop, more communications are required if there are more neighbours in the network. The sources are in sleep mode longer if they sample less frequent. Figure 5 demonstrates that PoRAP consumes the least amount of energy. Approximately 0.2 to $0.3 \times 10-3 \mathrm{~mJ}$ of energy is consumed per bit of data. PoRAP conserves 
more energy when the sampling period is smaller and the number of sources is higher. For example, at the 300 s sampling period and 50 sources, PoRAP consumes approximately $33 \%, 10 \%$ and 5\% of that required by S-MAC, B-MAC and CSMA, respectively.

The amount of saved energy by PoRAP due to amendments in check interval and active interval settings in B-MAC and S-MAC are shown in Table 1 and 2, respectively. The selected sampling period is 300 s. The average energy consumption is computed based upon the number of sources varying from 1 to 100 nodes.

Increases in the amount of conserved energy by PoRAP are observed in both Table 1 and 2 when check and active intervals are increased. The comparisons conducted in Table 1,2 and Figure 5 are based upon the parameter settings with respect to the GDI scenario where the frequency of data transmission is low. In order to investigate whether PoRAP is applicable to applications which require a high frequency of transmission, an experiment using one source is performed. The chosen check interval for B-MAC and active interval for S-MAC are $10 \mathrm{~ms}$ and $115 \mathrm{~ms}$, respectively. The results are shown in Figure 6.

Table 1. Comparison of energy consumption between B-MAC and PoRAP

\begin{tabular}{|c|c|c|c|}
\hline \multicolumn{2}{|c|}{ B-MAC } & \multirow[b]{2}{*}{$\begin{array}{l}\text { Average Energy } \\
\text { Consumption by } \\
\text { PoRAP } \\
\left(\times 10^{-3} \mathrm{~mJ}\right)\end{array}$} & \multirow[b]{2}{*}{$\begin{array}{c}\text { Saved Energy by } \\
\text { PoRAP (times) }\end{array}$} \\
\hline $\begin{array}{l}\text { Check Interval } \\
\text { (ms) }\end{array}$ & $\begin{array}{c}\text { Average } \\
\text { Energy } \\
\text { Consumption } \\
\left(\times 10^{-3} \mathrm{~mJ}\right)\end{array}$ & & \\
\hline 10 & 2.12 & & 9.1 \\
\hline 20 & 1.72 & & 7.2 \\
\hline 50 & 2.31 & 0.21 & 10.0 \\
\hline 100 & 3.90 & & 17.6 \\
\hline 200 & 7.31 & & 33.8 \\
\hline
\end{tabular}

Table 2. Comparison of energy consumption between S-MAC and PoRAP

\begin{tabular}{|c|c|c|c|}
\hline \multicolumn{2}{|c|}{ S-MAC } & \multirow{2}{*}{$\begin{array}{c}\text { Average } \\
\text { Energy } \\
\text { Consumption } \\
\text { by PoRAP } \\
\left(\times 10^{-3} \mathrm{~mJ}\right)\end{array}$} & \multirow[b]{2}{*}{$\begin{array}{c}\text { Saved Energy by } \\
\text { PoRAP (times) }\end{array}$} \\
\hline $\begin{array}{l}\text { Active Interval } \\
\text { (ms) }\end{array}$ & $\begin{array}{c}\text { Average } \\
\text { Energy } \\
\text { Consumption } \\
\left(\times 10^{-3} \mathrm{~mJ}\right)\end{array}$ & & \\
\hline 115 & 0.80 & & 2.8 \\
\hline 250 & 1.28 & & 5.1 \\
\hline 500 & 2.18 & 0.21 & 9.4 \\
\hline 750 & 3.08 & & 13.7 \\
\hline 1,000 & 3.98 & & 18.0 \\
\hline
\end{tabular}

Figure 6. Effects of sampling periods on average energy usage per second

According to Figure 6, PoRAP is capable of supporting high frequency transmission. The main reason is that a time slot is allocated to a source so it can transmit again without waiting. The CSMA consumes higher energy than B-MAC and S-MAC when the sampling period is longer than $1.5 \mathrm{~s}$ and $0.25 \mathrm{~s}$. In the case where a source sends every $0.1 \mathrm{~s}$, PoRAP uses $16 \%, 44 \%$ and $67 \%$ of the energy of B-MAC, S-MAC and CSMA, respectively. At the 1,000s sampling interval, PoRAP respectively requires $12 \%, 34 \%$ and $5.5 \%$ of the energy of B-MAC, S-MAC and CSMA. 


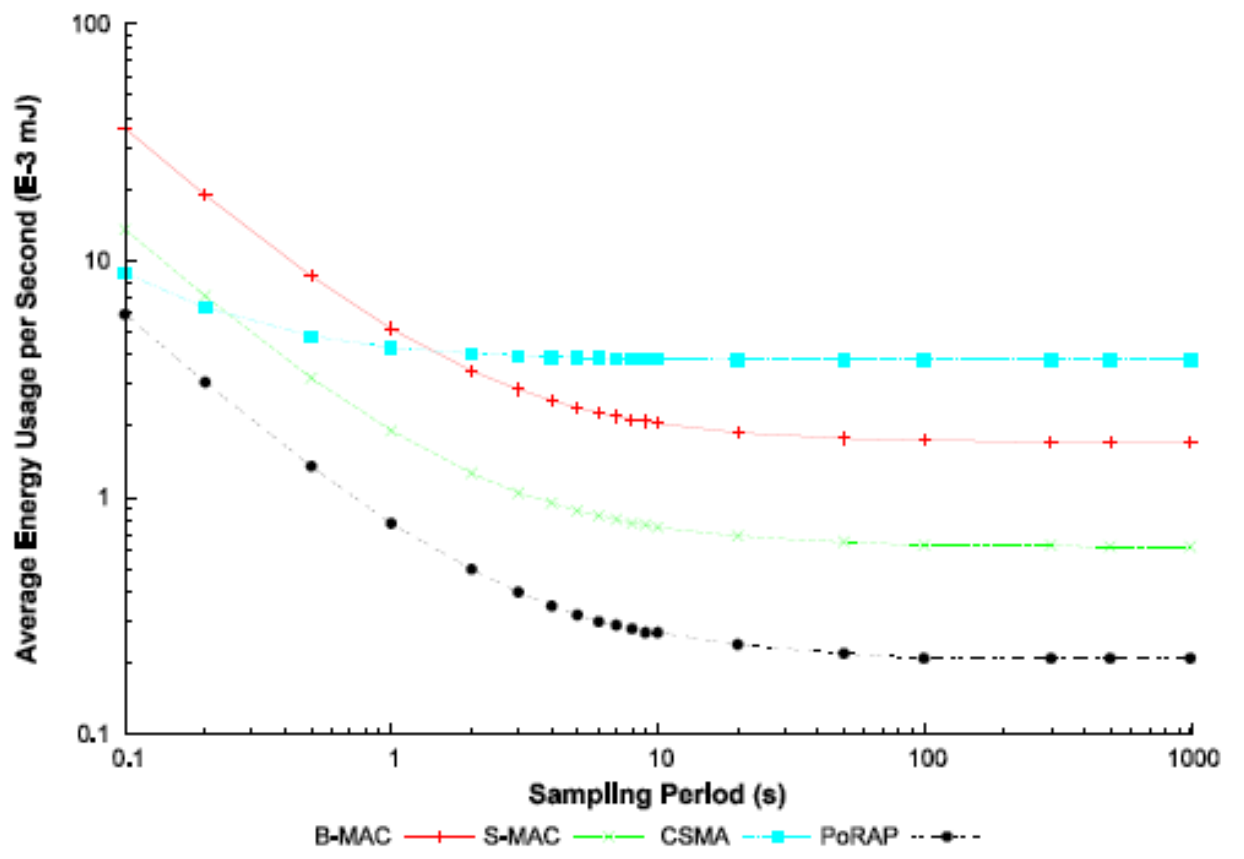

Figure 6. Effects of sampling periods on average energy usage per second

Previous results are based upon the assumption that the number of slots is equal to that of sources. However, there may be a case where the number of sources is unknown in advance. Further, the topology may be changed as some sources may leave or run out of energy. In such cases, the number of slots is higher than the number of sources. PoRAP is sometimes not applicable, for high duty cycle applications, as the source has to wait for the other slots to complete until the next communication cycle is started. Table 3 demonstrates the effects of the percentage of slot usage on minimum applicable sampling periods and corresponding average energy consumption in PoRAP. There is a single operating source in the network.

Table 3. Comparison of energy consumption at various sampling periods

\begin{tabular}{cccc}
\hline $\begin{array}{c}\text { Number of } \\
\text { Allocated Slots }\end{array}$ & Slot Usage (\%) & $\begin{array}{c}\text { Minimum Applicable } \\
\text { Sampling Period (s) }\end{array}$ & $\begin{array}{c}\text { Average Energy } \\
\text { Usage per Second } \\
\left(\times 10^{-3} \mathrm{~mJ}\right)\end{array}$ \\
\hline 100 & 1 & 3 & 0.40 \\
50 & 2 & 1.5 & 0.59 \\
20 & 5 & 0.6 & 1.17 \\
10 & 10 & 0.3 & 2.12 \\
5 & 20 & 0.2 & 3.08 \\
2 & 50 & 0.1 & 5.96 \\
1 & 100 & 0.1 & 5.96 \\
\hline
\end{tabular}

According to Table 3, a low duty cycle application is more efficient using PoRAP when the percentage of slot usage is high. However, PoRAP is not applicable if a source has to wait longer until the next cycle is started. Hence, a limitation of PoRAP arises when there is a high slot overhead because there are many sources in the network. Unlike PoRAP, the other protocols are applicable as they do not require slot allocations. At $20 \%$ of slot usage, PoRAP is not applicable at the $0.1 \mathrm{~s}$ sampling period. PoRAP applies when the source sends 5 packets every second (a $0.2 \mathrm{~s}$ 
sampling period) as it uses $16 \%, 43 \%$ and $48.5 \%$ of the energy of B-MAC, S-MAC and CSMA, respectively. A significant amount of energy can be saved by PoRAP when it is applicable.

In summary, several parameter settings such as data payload size and sampling interval in the Great Duck Island [6] and those in [4] are used in this comparative study. Longer communication durations result in a higher energy consumption. PoRAP is not significantly affected by the number of sources compared to S-MAC and B-MAC as intermediate nodes are not required for data forwarding. The main limitation of PoRAP is that it is not applicable to high duty cycle applications when the percentage of slot usage is low. However, it can conserve more energy than the other protocols when it can be used. The main reason is that it does not require multiple transmissions and receptions. Further, the sources are periodically switched to sleep mode to conserve energy.

Apart from considering energy consumption per bit per second, idle listening is an important source of energy wastage. CSMA uses the most amount of energy on idle listening, especially when the sampling period is high. Preamble is used in B-MAC for reliable data reception and its length is at least a check interval. Idle listening energy becomes significant for B-MAC for longer check intervals. Further comparison of required idle listening periods between B-MAC and PoRAP is determined.

According to [1], four durations between two consecutive data transmissions including 5 minutes, 10 minutes, 1 hour and 1 day, are studied to determine additional duration required in PoRAP for tackling the effects of clock drift and achieving time synchronisation. For B-MAC, a 1s period is and $10 \mathrm{~ms}$ check interval are used. This means that B-MAC has to conduct preamble communication for $10 \mathrm{~ms}$ every second. Hence, the node running B-MAC has to listen for the preamble for $(60 \times 10)$ or $600 \mathrm{~ms}$ within 1 minute. Table 4 compares the required idle listening periods between B-MAC and PoRAP. Note that a $32 \mathrm{Khz}$ timer is used in the comparison and there are 32 ticks in each millisecond.

Table 4. Comparison of required idle listening periods between B-MAC and PoRAP

\begin{tabular}{cccccc}
\hline \multirow{2}{*}{ Duration } & \multirow{2}{*}{ Ticks $\left(\times 10^{6}\right)$} & \multicolumn{3}{c}{ Required idle listening } & \multirow{2}{*}{ PoRAP/B-MAC } \\
\cline { 3 - 4 } & & \multicolumn{2}{c}{ B-MAC } & PoRAP & \\
\cline { 3 - 4 } & & $\left(\times 10^{3}\right) \mathrm{ms}$ & ticks & & \\
\hline 5 minutes & 9.8 & 3 & 96 & 63 & $66 \%$ \\
10 minutes & 19.6 & 6 & 192 & 84 & $44 \%$ \\
1 hour & 118 & 36 & 1,152 & 449 & $39 \%$ \\
1 day & 2,831 & 864 & 27,684 & 2560 & $9 \%$ \\
\hline
\end{tabular}

According to Table 4, PoRAP requires fewer ticks in all durations. A higher conservation in the idle listening period will be obtained if there are longer durations between transmissions. PoRAP uses only $9 \%$ of the ticks for accommodating time synchronisation compared to preamble communication in B-MAC when the source sends every day. One of the main reasons is that BMAC is specifically developed for the multi-hop wireless sensor networks where routing is necessary amongst sources. The sources have to check if there are packets addressed to them. Unlike the multi-hop, PoRAP is applied to direct communication and each source knows its communication schedule. The source is therefore often in the sleep mode.

\section{CONCLUSION}

Wireless sensor networks (WSNs) are becoming an important area of research and they have been implemented and deployed in various civil applications. Sensors are scattered over an area of 
interest and they are wirelessly connected. Hence, communication and power cables are not necessary. However, energy and resource constraint are major drawback of WSNs application in the real-world. Energy aware protocols have been developed for WSNs. This paper presents a comparative analysis between Power \& Reliability Aware Protocol (PoRAP) and B-MAC, SMAC and CSMA in terms of energy consumption.

According to the parameter space analysis, the check interval and active period are overheads of B-MAC and S-MAC, respectively. In B-MAC, the preamble length is at least a check interval. An active period is used in S-MAC for carrier sensing, hidden node avoidance and synchronisation between neighbours. The default active interval of S-MAC is $115 \mathrm{~ms}$. Idle listening is an important overhead in CSMA as the sources listen all the time. Control packet reception is considered as an overhead in PoRAP. The size of a control packet directly relates to the number of sources.

In the comparative study, an average energy usage per second is computed. The selected sampling period and data payload are 300s and 36 bytes which are the same as [4]. The chosen check interval of B-MAC is $10 \mathrm{~ms}$. PoRAP is not applicable to the applications which require a low duty cycle when the percentage of slot usage is low. This is because the source has to wait until all slots are completed to start a new communication cycle. A network consisting of a single source is used. In the case of $20 \%$ slot usage and 2s sampling period, PoRAP uses 16\%, $43 \%$ and $48.5 \%$ of the energy of B-MAC, S-MAC and CSMA, respectively.

PoRAP consumes approximately 0.2 to $0.3 \times 10-3 \mathrm{~mJ}$ for transmitting one bit of data. It consumes less energy when the sampling is less often and the number of sources is higher. PoRAP consumes approximately $33 \%$, almost $10 \%$ and almost $5 \%$ of that required by S-MAC, B-MAC and CSMA, respectively. A larger amount of conserved energy is achieved if the check and active intervals in B-MAC and S-MAC are increased. In the case where the $200 \mathrm{~ms}$ and $1,000 \mathrm{~ms}$ check and active intervals are respectively chosen in the B-MAC and S-MAC, PoRAP consumes approximately $3 \%$ and $5 \%$ of the energy required by such protocols.

Further analysis of the active period required by B-MAC for preamble communications and PoRAP for accommodating time synchronisation is conducted. The $10 \mathrm{~ms}$ check interval is used. As energy consumption directly relates to the active duration, the results demonstrate that at a 300 s sampling period and a 50source topology, up to approximately $10 \%$ of the energy can be conserved if PoRAP is used instead of B-MAC. The results demonstrate that PoRAP is applicable for the low duty cycle applications. The sources benefit more from PoRAP in terms of energy conservation compared to B-MAC, S-MAC and CSMA.

\section{REFERENCES}

[1] I. Khemapech, I. Duncan \& A. Miller, (2010) "Energy Preservation in Environmental Monitoring WSN", In The Proceedings of IEEE International Conference on Sensor Networks, Ubiquitous and Trustworthy, pp 312-319.

[2] L. Kleinrock \& F.A. Tobagi, (1975) "Packet Switching in Radio Channels: Part 1 - Carrier Sense Multiple-Access Modes and Their Throughput-Delay Characteristics", IEEE Computer Communication.

[3] W. Ye, J. Heidemann \& D. Estrin, (2003) "Medium Access Control with Coordinated, Adaptive Sleeping for Wireless Sensor Networks", USC/ISI Technical Report ISI-TR-567.

[4] J. Polastre, J. Hill \& D. Culler, (2004) "Versatile Low Power Media Access for Wireless Sensor Networks", In The Proceedings of ACM International Conference on Embedded Networked Sensor Systems. ACM Press.

[5] TinyOS, http://www.tinyos.net 
[6] A. Mainwaring, J. Polastre, R. Szewczyk, D. Culler, D \& J. Anderson, (2002) "Wireless Sensor Networks for Habitat Monitoring", In The Proceedings of ACM International Workshop on Wireless Sensor Networks and Applications. ACM Press.

[7] R. Lim, (2006) "Wireless Fire Sensor Network Demonstrator" M.S. thesis, Swiss Federal Institute of Technology, Zurich, Switzerland.

[8] Tmote Sky datasheet, http://www.eecs.harvard.edu/ konrad/projects/shimmer/references/ tmote-skydatasheet.pdf

[9] M. Mjeku \& N.J. Gomes, (2008) "Analysis of the Request to Send / Clear to Send Exchange in WLAN Over Fiber Networks”, Lightwave Technology, Vol. 26, pp 2531-2539.

\section{AUTHOR}

Ittipong Khemapech, is a lecturer in School of Science and Technology at University of the Thai Chamber of Commerce. He received B.Eng. in Civil Engineering and Ph.D. in Computer Science from University of St Andrews, UK. His research interests are structural engineering and in the area of wireless sensor networks with a special emphasis on energy aware protocol development.

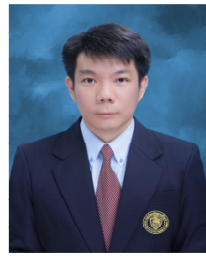




\title{
A METHOD TO IDENTIFY POTENTIAL AMBIGUOUS MALAY WORDS THROUGH AMBIGUITY ATTRIBUTES MAPPING: AN EXPLORATORY STUDY
}

\author{
Hazlina Haron ${ }^{1,2}$ and Abdul Azim Abd. Ghani ${ }^{3}$ \\ ${ }^{1}$ Faculty of Computer Science and Information Technology \\ Universiti Putra Malaysia \\ Serdang, Selangor Malaysia \\ delinn1612@yahoo.com \\ ${ }^{2}$ School of Computing, College of Arts and Sciences \\ Universiti Utara Malaysia \\ Sintok, 06010, Kedah, Malaysia \\ zlina1108@uum. edu . my \\ ${ }^{3}$ Faculty of Computer Science and Information Technology \\ Universiti Putra Malaysia \\ Serdang, Selangor Malaysia \\ azimefsktm. upm. edu.my
}

\begin{abstract}
We describe here a methodology to identify a list of ambiguous Malay words that are commonly being used in Malay documentations such as Requirement Specification. We compiled several relevant and appropriate requirement quality attributes and sentence rules from previous literatures and adopt it to come out with a set of ambiguity attributes that most suit Malay words. The extracted Malay ambiguous words (potential) are then being mapped onto the constructed ambiguity attributes to confirm their vagueness. The list is then verified by Malay linguist experts. This paper aims to identify a list of potential ambiguous words in Malay as an attempt to assist writers to avoid using the vague words while documenting Malay Requirement Specification as well as to any other related Malay documentation. The result of this study is a list of 120 potential ambiguous Malay words that could act as guidelines in writing Malay sentences.
\end{abstract}

\section{KEYWORDS}

Vagueness, Requirement Quality Attributes, Natural Language Processing, Malay Ambiguity, Ambiguity

\section{INTRODUCTION}

Requirement Specification is a document that acts as a medium between system developer and users. Users specified their systems' functional needs in a technical documentation. The specification would then be referred by system analysts in the process of developing the requested system. Requirement Specification usually uses natural language, due to its' flexibility and easy

David C. Wyld et al. (Eds) : CCSIT, SIPP, AISC, PDCTA, NLP - 2014

pp. 115-122, 2014. (C) CS \& IT-CSCP 2014

DOI : $10.5121 /$ csit.2014.4210 
to understand. However, natural language has its own disadvantages such as, tendencies to be prone to ambiguity and misinterpretation. It is often being misunderstood by people from various backgrounds and different levels of knowledge.

A requirement is said to be ambiguous when a same statement is being interpreted differently by different sets of people. A specification is affected by textual ambiguity when it provokes more than one way of reading a statement. Example, "the customer enters a card and a numeric personal code. If it is not valid then the ATM rejects the card". It is ambiguous because the word "it" could refer to two distinct objects. It could refer to either a card or a numeric personal code [1]. Words can be ambiguous in many ways. Linguistic ambiguity can be categorized into several main groups such as semantic, syntactic, pragmatic and lexical [2]. This has been agreed upon and then being enhanced into other types of ambiguity such as coordination ambiguity [3] and anaphoric ambiguity [4], [5].

One of the main reasons for ambiguity is the use of vague words. Words that are being used are not clear and usually lead to more than one meaning. Vagueness can be termed as not clearly expressed, imprecise, ill-defined and lacked expressions [6] . Vagueness shows a boundary of a word's meaning that is not clearly stated [7]. The usage of vague words reduces the level of clarity in a sentence. Vagueness can also be defined as ignorance and absence of knowledge [8]. A vague word can also be defined as a word that has multiple equally good possible candidates of the meaning. When a sentence reaches the 'borderline case' of truth which is neither true nor false, it is considered vague [9]. Malay words such as 'maksimum', 'automatik', 'segera', 'secepat mungkin', 'pantas', 'efisien', 'produktif', 'anggaran', 'kerap' are some of the adjectives considered vague. These words lead to uncertainty and multiple of interpretations and therefore, should be avoided.

FigureFigure 1 below depicts a conceptual view of Malay ambiguity and its' related elements gathered from open-interviews with Malay linguist experts.

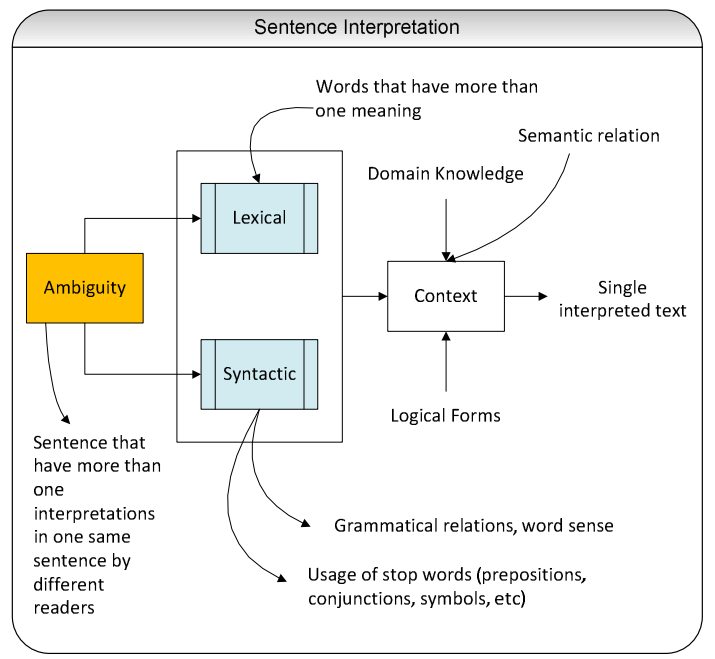

Figure 1: Conceptual view of Malay Ambiguity

\section{RELATED WORK}

Although various researches have focused on disambiguation techniques, not many highlighted how these ambiguous words originated. In addition, most previous researches focused on the 
English language. Due to limitation in scope, it is quite difficult to refer and construct Malay ambiguous words. Hence, this research is adopting the methods used in English and other languages' methods to suit our area of research.

\subsection{Vagueness Vs Ambiguity Issues}

A sentence must have a unique meaning in order to reflect one's perspective accurately. A sentence containing a vague word, would fail to impart its intended meaning. Vagueness is one of the many sources of ambiguity. For example, "Five piled stones are a heap" [10]. One can consider five piles of stones are a heap, while another might disagree with the statement as he/she may say ten piled stones are then a heap. Vagueness can impact ambiguity that lead to uncertainty and multiple interpretations (refer Figure 2). Vagueness and uncertainty are being distinguished, however, it correlates with one another [9]. They are complimentary but not parallel. Vagueness has a close similarity as semantic indeterminacy or it is termed as 'semantic nihilism' [10]. Therefore, many research concluded that to resolve vagueness, context involvement is necessary [8]. Context is crucial to ensure interpretation is unique in a sentence. [9] Vague can be assigned with different semantic value based on different possible situations, and each of the semantic values is called presification. Vague words leads to imprecise meaning, therefore it triggers ambiguity in a sentence. To disambiguate, we have to go back to its' roots of causal, by eliminating the vague words itself before any ambiguity can be detected.

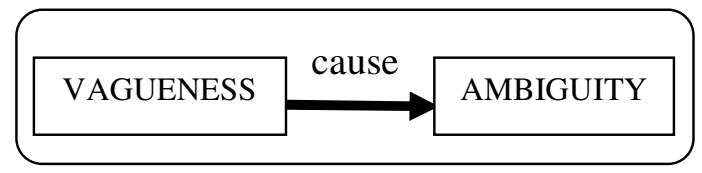

Figure 2: Relationship between Vagueness and Ambiguity

\subsection{Criterion of Ambiguous Words}

A dictionary of 100 ambiguous Arab words that has been developed, takes into consideration more than 10 word senses as the criteria [11]. These senses were extracted from the Arab dictionary. Chantree et al. extracted ambiguous sentences indicate coordination ambiguity and developed ambiguity threshold to set the ambiguity benchmark [12]. Amongst the factors involved in making sure readers understand what a sentence means are sentence length, ambiguous adjectives, adverbs and passive verbs [13]. A list of high potential English ambiguous words has been constructed in an Ambiguity Technical Report as a guideline to avoid ambiguous sentence [14]. Tjong et al. developed rules for clearer sentences in an attempt to avoid ambiguities [15]. These research proof that to begin an investigation to disambiguate an ambiguous sentence, one has to start by determining and identifying the vague words. These vague words could bring misconception and misinterpretation to the readers. As for the writers, they usually are not aware that they are even writing an ambiguous sentence in the first place.

Through previous literatures as guidelines, we have tabled out a criterion of potentially ambiguous words that acts as guidelines to extract the poor words as in Table 1.

Table 1: Criterion of ambiguous words (Malay)

\begin{tabular}{|l|l|}
\hline Criteria & Example (Malay words) \\
\hline $\begin{array}{l}\text { Words that have more than } \\
\text { one word classes }\end{array}$ & $\begin{array}{l}\text { Papar }(\mathrm{adj}, \mathrm{kk}), \text { amat }(\mathrm{kk}, \mathrm{kt}), \text { alam }(\mathrm{kn}, \\
\mathrm{kk}) \text {, abstrak }(\mathrm{kk}, \mathrm{kn})\end{array}$ \\
\hline $\begin{array}{l}\text { Words have more than one } \\
\text { meaning }\end{array}$ & Perang, semak, alam, akan, \\
\hline
\end{tabular}




\begin{tabular}{|l|l|}
\hline $\begin{array}{l}\text { Vague adjectives, adverbs and } \\
\text { verbs }\end{array}$ & $\begin{array}{l}\text { Efisien, mudah, pantas, segera, } \\
\text { lengkap, etc. }\end{array}$ \\
\hline Words that fall under proposed & Implicit - efisien \\
seven ambiguity attributes: & Connectives- beberapa \\
implicit, word class, weakness, & Weakness - anggaran \\
temporal, referential and & Temporal - bulanan \\
general specific variable. & Referential - sebelum, begini \\
& General specific variable - data itu \\
\hline
\end{tabular}

\section{PROPOSED METHODOLOGY}

We believed that to minimize and manage ambiguity, one has to go to the root cause. In this case, tracking and identifying the potential vague and ambiguous words are necessary before the process of ambiguity detection can take place. Hence, this strategy will be the first stage from overall of the research work.

\subsection{Ambiguity Attributes}

Table 2 below shows the structure of our proposed Ambiguity Attributes in an attempt to create a list of high potential ambiguous Malay words. These attributes are compiled based on several relevant quality attributes from previous literatures. It consists of six attributes most suitable with Malay words. The ambiguous Malay words are extracted based on these attributes from working RS and some have been translated from English using Dwibahasa Kamus Oxford Fajar [16]. Some of the word class attribute's words were extracted from Kamus Komprehensif Bahasa Melayu [17] for their part of speech (POS).

Table 2. Structure of Ambiguity Attributes

\begin{tabular}{|c|c|}
\hline Ambiguity Attributes & Description \\
\hline Implicit (IMP) : & \\
\hline i. General [18], [14] & $\begin{array}{l}\text { Subject or object in the sentence is generic rather } \\
\text { than specific. }\end{array}$ \\
\hline ii. Subjective [18] & Refers to personal opinion or feeling \\
\hline iii. Boundary [14] & $\begin{array}{l}\text { It has no definite boundary of true or false (or } \\
\text { between yes and no). }\end{array}$ \\
\hline iv. Unquantifiable [19] & Non-quantifiable \\
\hline \multicolumn{2}{|l|}{ Connectives (CON): } \\
\hline i. Adjective[14] & $\begin{array}{l}\text { Word belonging to one of the major form classes } \\
\text { in any of numerous languages and typically } \\
\text { serving as a modifier of a noun to denote a quality } \\
\text { of the thing named, to indicate its quantity or } \\
\text { extent, or to specify a thing as distinct from } \\
\text { something else }\end{array}$ \\
\hline ii. Adverb [14] & $\begin{array}{l}\text { Word belonging to one of the major form classes } \\
\text { in any of the numerous languages, typically } \\
\text { serving as a modifier of a verb, an adjective, } \\
\text { another adverb, a preposition, a phrase, a clause, or } \\
\text { a sentence, expressing some relation of manner or }\end{array}$ \\
\hline
\end{tabular}




\begin{tabular}{|c|c|}
\hline & $\begin{array}{l}\text { quality, place, time, degree, number, cause, } \\
\text { opposition, affirmation, or denial, and in English } \\
\text { also serving to connect and to express comment on } \\
\text { clause content }\end{array}$ \\
\hline iii. Verb [14] & $\begin{array}{l}\text { Word that characteristically is the grammatical } \\
\text { centre of a predicate and expresses an act, } \\
\text { occurrence, or mode of being, that in various } \\
\text { languages is inflected for agreement with the } \\
\text { subject, for tense, for voice, for mood, or for } \\
\text { aspect, and that typically has rather full descriptive } \\
\text { meaning and characterizing quality but is } \\
\text { sometimes nearly devoid of these especially when } \\
\text { used as an auxiliary or linking verb }\end{array}$ \\
\hline iv. Dangling Else [14] & $\begin{array}{l}\text { The requirement has no other exit when one case is } \\
\text { not met (Exception case) }\end{array}$ \\
\hline v. Preposition [12],[20] & $\begin{array}{l}\text { Connective words. A function word that typically } \\
\text { combines with a noun phrase to form a phrase } \\
\text { which usually expresses a modification or } \\
\text { predication }\end{array}$ \\
\hline Temporal [19],[14] & $\begin{array}{l}\text { Words that has time/duration type that invites } \\
\text { multiple interpretation. Un-boundary timing or } \\
\text { duration }\end{array}$ \\
\hline $\begin{array}{l}\text { Referential (REF) [14], [19], } \\
{[4],[5],[21]}\end{array}$ & $\begin{array}{l}\text { Sentence that contains more than one requirement } \\
\text { in a sentence. Sentence contains explicit references } \\
\text { to (not numbered sentences, not defined, not } \\
\text { described, no glossary) }\end{array}$ \\
\hline Variable (VAR) [14] & $\begin{array}{l}\text { Common word that invites vague interpretation } \\
\text { and understanding. Too generic. }\end{array}$ \\
\hline Weakness (WN) [18] & Sentence that contains weak main verb \\
\hline
\end{tabular}

\subsection{Process of creating Malay Ambiguous Lexicons}

Figure 3 below depicts the overall process of creating potential ambiguous Malay words repository. Data from sample documents are filtered based on certain criteria. Potentially ambiguous words that have been successfully extracted will undergo testing and verification process before being saved in a repository called Malay Ambiguous Words. The detailed step by step process is described below. 


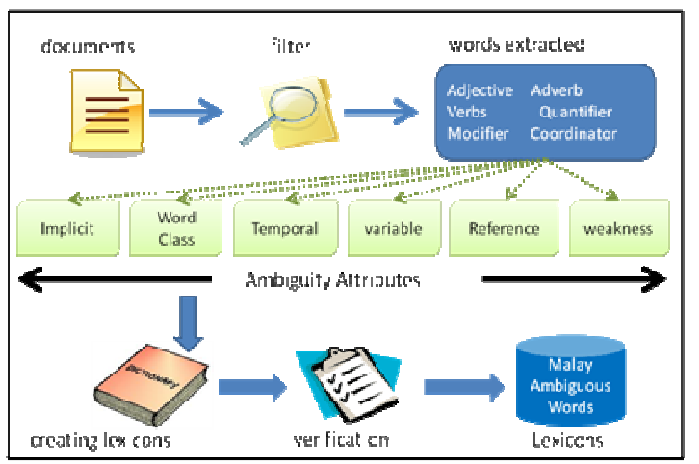

Figure 3. Overall Process of Identifying Ambiguous Words

Step 1: We collected samples of Malay Requirement Specifications from companies as our source of training data. Potentially ambiguous words were extracted from the sentences based on criterion as in Table 1.

From literatures, we constructed six ambiguity attributes that are at most relevant and appropriate with our scope (refer Table 3). Based on these attributes, we mapped the extracted potential Malay ambiguous words with the ambiguity attributes to confirm characteristics of vagueness. By filtering using the above criteria, the list of words considered potentially ambiguous are also referred to as ambiguous candidates. They are kept in a repository to be further analysed using contextual-based detection technique.

Step 3: The identified potentially ambiguous Malay words will undergo a verification process to ensure genuine ambiguity. The verification is expected to be done by Malay linguist experts.

Step 4: The verified words are stored in a database for the next phase of activities.

\section{DISCUSSION}

We have managed to collect 13 sets of Malay language Requirement Specifications from two domains; medical system and student information system. From these sources, a total of 2900 have been words eliminated. Examples of inappropriate words are such as English loanwords, words in short forms, double words such as 'rekod-rekod', 'kata nama khas (KNK)' and symbols such as full stops and other symbols. We then managed to extract 120 potentially ambiguous Malay words. Table below is the statistics of the words' mapping onto their appropriate Ambiguity Attributes.

Table 3. Words mapping based on Ambiguity Attributes

\begin{tabular}{|l|l|l|l|l|l|l|}
\hline & IMP & CON & T & REF & VAR & WN \\
\hline Tot & 51 & 41 & 11 & 27 & 22 & 21 \\
\hline$\%$ & 42.5 & 34.2 & 9.2 & 22.5 & 18.3 & 17.5 \\
\hline
\end{tabular}

From the statistic generated, the highest percentage of potential ambiguous Malay words falls under 'Implicit' category followed by 'Connectives' category and 'Referential'. The articulated data shows that potentially ambiguous Malay words most used are very generic, has a vague boundary, too subjective and reflects an unquantifiable criterion. These are the normal reason that triggers ambiguity. The list of ambiguous words is currently undergoing a verification process by Malay linguist experts. Two experts with the relevant background and expertise of the domain were selected from Faculty of Communication and Malay Language (FKBM), Universiti Putra Malaysia (UPM). 


\section{CONCLUSION}

In system requirement, linguistic ambiguity is often ignored or mistakenly unacknowledged. This leads to misunderstanding from both users and system developer's side, thereby contributing towards a failed system. The after effect of the situation could jeopardize system development cycle and project's time limitation as well as budgets. The Malay requirement specification environment still lacks in research that focussed on this situation. We have presented here a method to identify potential ambiguous Malay words and managed to construct a list of 120 potential commonly used ambiguous Malay words in a Malay requirement specification. This study is an attempt to assist writers to avoid using the high potential ambiguous words and promote greater clarity in sentence construction of documentation and significantly reduce misinterpretation by readers.

\section{ACKNOWLEDGEMENTS}

We would like to thank the Malay Linguist Experts from UPM, UM and UKM for their support and helpful comments. This work is supported and funded by $\mathrm{PhD}$ scholarship from Universiti Utara Malaysia and Universiti Putra Malaysia.

\section{REFERENCES}

[1] E. Kamsties and B. Paech, "Taming Ambiguity in Natural Language Requirements," in International Conference on System and Software Engineering and their Applications, 2000, pp. 1-8.

[2] D. M. Berry, "Ambiguity in Natural Language Requirements Document," Monterey Workshop 2007. 2007.

[3] F. J. Chantree, B. Nuseibeh, A. De Roeck, A. Willis, and F. of M. and C. Department of Computing, "Nocuous Ambiguities in Requirement Specifications," The Open University, UK, 2005.

[4] N. K. M. Noor, S. A. Noah, M. J. A. Aziz, and M. P. Hamzah, "Anaphora Resolution of Malay Text: Issues and Proposed Solution Model,” 2010 Int. Conf. Asian Lang. Process., pp. 174-177, Dec. 2010.

[5] N. K. M. N, M. J. Abd, S. Azman, and M. Noah, “" nya ' as anaphoric word : A proposed solution,” no. June, pp. 249-254, 2011.

[6] Merriam-Webster.com, "vague," http://www.merriam-webster.com. 2012.

[7] R. N. Shiffman, "A Model of Ambiguity and Vagueness in Clinical Practice Guideline Recommendations," in AMIA 2005 Symposium Proceedings , 2005.

[8] M. Michieka, "An Analysis of the Pragmatic Functions of Vague Language in Ekegusii," J. Lang. Technol. Entrep. Africa, vol. 3, no. 2, 2012.

[9] B. Bennet, "Modal Semantics for Knowledge Bases Dealing with Vague Concepts."

[10] D. Braun and T. Sider, "Vague, So Untrue," NOUS, vol. 2, pp. 133-157, 2007.

[11] L. Merhbene, A. Zouaghi, and M. Zrigui, "Ambiguous Arabic Words Disambiguation," 2010 11th ACIS Int. Conf. Softw. Eng. Artif. Intell. Netw. Parallel/Distributed Comput., pp. 157-164, Jun. 2010.

[12] F. J. Chantree, A. de Roeck, B. Nuseibeh, and A. Willis, "Identifying Nocuous Ambiguity in Natural Language Requirements," The Open University, UK, 2006.

[13] O. Ormandjieva, I. Hussain, and L. Kosseim, "Toward a text classification system for the quality assessment of software requirements written in natural language," Fourth Int. Work. Softw. Qual. Assur. conjunction with 6th ESEC/FSE Jt. Meet. - SOQUA '07, p. 39, 2007.

[14] R. Bender, “The Ambiguity Review Process.” Bender RBT Inc., 2003.

[15] S. F. Tjong, M. Hartley, and D. M. Berry, "Extended Disambiguation Rules for Requirements Specifications," 2007.

[16] J. M. Hawkins, "Kamus Dwibahasa Oxford Fajar," Kamus Dwibahasa Oxford Fajar. Oxford Fajar Sdn Bhd, 2007.

[17] A. Othman, "Kamus Komprehensif Bahasa Melayu," Kamus Komprehensif Bahasa Melayu. Oxford Fajar Sdn Bhd, 2005. 
[18] F. Fabbrini, M.Fusani, S.Gnesi, and G.Lami, "The Linguistic Approach to the Natural Language Requirements Quality: Benefit of the use of an Automatic Tool," in Software Engineering Workshop, 2001. Proceedings. 26th Annual NASA Goddard, 2001, pp. 95-105.

[19] J. K. D. Sc and C. Eng, "A Prototype Tool for Improving the Wording of Requirements," 12th Annu. Int. Symp. NCOSE 2002, 2002.

[20] F. J. Chantree, A. Kilgarriff, A. de Roeck, and A. Willis, "Using a Distributional Thesaurus to Resolve Coordination Ambiguities," Department of Computing, Faculty of Mathematics and Computing, The Open University, UK, 2005.

[21] N. Karimah, M. Noor, S. A. Noah, M. Juzaiddin, A. Aziz, and M. P. Hamzah, "Malay Anaphor and Antecedent Candidate Identification: A Proposed Solution," Springer-Verlad Berlin Herdelb. 2012, pp. 141-151, 2012.

\section{AUTHORS}

Hazlina Haron is a $\mathrm{PhD}$ student in the Faculty of Computer Science and Information Technology, Universiti Putra Malaysia. She holds a B.Sc in Information Technology (1998) from Universiti Utara Malaysia and M.Sc in Computer Science (2006) from Universiti Putra Malaysia. Currently she is a tutor in Universiti Utara Malaysia. She had 8 years of experience as Software Engineer while working at Telekom Applied Business Sdn Bhd, a subsidiary of Telekom Malaysia Berhad and 3 years of experience as Assistant Manager doing Project Management Office at Telekom Malaysia Berhad. Her studies involve developing a technique best suited to detect ambiguity in Malay natural language. Her research areas include requirement engineering, natural language processing and ambiguity.

Abdul Azim Abd Ghani is a Professor in the Department of Software Engineering and Information Technology, Universiti Putra Malaysia. He received B.Sc in Mathematics/Computer Science from Indiana State University in 1984 and M.Sc in Computer Science from University of Miami in 1985. He received the Ph.D. in Software Engineering from University of Strathclyde in 1993. His research interests are software engineering, software measurement, software quality, and security in computing.
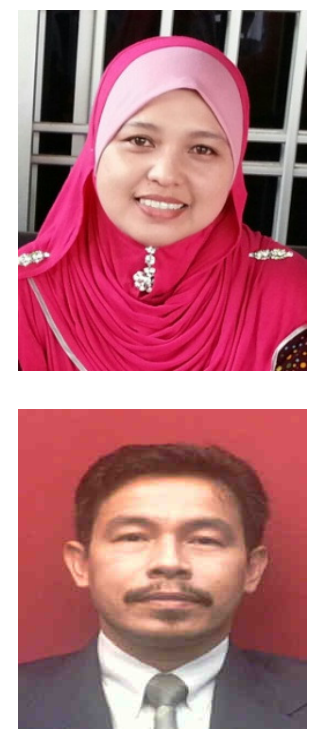


\title{
TOWARDS A SEMANTIC FOR UML ACTIVITY DIAGRAM BASED ON INSTITUTION THEORY FOR IT'S TRANSFORMATION TO EVENT-B MODEL
}

\author{
Aymen Achouri \\ Faculty of science of Tunis - University of Tunis \\ aymen_achouriehotmail.fr
}

\begin{abstract}
In this article, we define an approach for model transformation. We use the example of UML Activity Diagram (UML AD) and Event-B as a source and a target formalism. Before doing the transformation, a formal semantic is given to the source formalism. We use the institution theory to define the intended semantic. With this theory, we gain a algebraic specification for this formalism. Thus, the source formalism will be defined in its own natural semantic meaning without any intermediate semantic. Model transformation will be performed by a set of transformation schema which preserve the semantic expressed in the source model during the transformation process. The generated model expressed in Event-B language will be used for the formal verification of the source model. As a result, some model expressed in a precise formalism, the verification of this model can be seen as the verification of the Event-B model semantically equivalent to the source model. Then, in the present work we combine the institution theory, Event-Bmethod and graph grammar to develop an approach supporting the specification, the transformation and the verification of $U M L A D$.
\end{abstract}

\section{KEYWORDS}

Model transformation; Institution; UML Activity Diagram Institution; Transformation rules; Formal semantic

\section{INTRODUCTION}

Model Transformation is a critical process in software construction and development. As increasingly larger software systems are being developed, there is a tendency to have solid and effective tools to automatize the software development. The specification of a software can be formal and (or) graphical. As for graphical formalisms, we can mention as example the UML models, UML class diagram, UML activity diagram and interaction diagram as examples. For the formal ones, logic are increasingly used due to their mathematical basis. For example, Petri-net is used as a graphical and a formal specification formalism. Logic is the language of formalmethods like theoremproving and model checking. To facilitate and to make jointly graphical and formal language, there is a massive need to make generic techniques for the transformation of graphical models to formal notations. Also the use of the logic is difficult for non-familiar with logical 
concepts and specification. As a result, it is necessary to provide the possibility to make specification in a modeling level. The result is to use transformation engine so that we can to transform the specification (defined as a model conform to a specific formalism).

Stakeholders can begin with a graphical model (possibly with many system views), and with an automatic and correct transformation they cant to produce a specification in a formal logic. In the context of logic, institution theory is emerged as a cadre to study the multitude of logics and different relationships between it.

In our previous work [13], we used graph grammar to define an automatic transformation between UML AD and Event-B. Thanks to the notion of graph grammar, the automation aspect is given to the transformation. As for the semantic equivalence between source and target model is not proved. The reason is the absence of formal semantic for the source and the target formalism. As a first step, we use the institution theory in this paper to make the semantic needed for only the source formalism. In this paper, we take the example of UML Activity Diagram as a source formalism and Event-B as a target one.

The first contribution aims to give institutional presentation of UML AD. We can view this institutional presentation as a formal semantics of UML AD. This algebraic presentation of the source formalism will be a meta-level to study possible transformation to Event-B models. Also, it can be used to study some proprieties like model amalgamation, pushout and pullback of this formalism Those notions are important for the specification, composition specifications, and heterogeneous specification approaches. In addition, we define a set of rules that automatizes the generation of Event-B model from the defined semantic of UML AD. With the proposed solution, UML AD models are formalized in Institutions which make easier the translation into Event-B also the proof of correctness ans completeness of translation process.

The paper is organized as follows: in section 2 we present model transformation in general. Then in section 3, we define institution and we recall some important definitions. Section 4 shows how to prove that UML AD establish an institution. Section 5 defines the language of Event $\mathrm{B}$, the target formalism. Section 6 defines a set of rules for the transformation of UML AD to Event-B. Finally, the last section concludes our work.

\section{RELATED WORKS}

In the literature, the institution theory is well used and studied. We have three categories of works based on the institution theory.

The first category is interested in the use of institution theory and it's known concepts in the development of an heterogeneous specification approaches. This category consists of global theoretic logical based works. We begin with the approach of the heterogeneous specification in the tool cafeOBJ. This approach is based on a cube on eight logic and twelve projections (defined as a set of institution morphism and institution comorphism)[2]. This approach is based on the semantic based on Diaconescu's notion of Grothendieck institution [1]. Another approach is developed in the work of Till Mossakowski [15]. The heterogeneous logical environment developed by the author is formed by a number of logical systems formalized as institutions linked with the concepts of institution morphism and comorphism. 
The second category of works are specific to the use of institution theory in the specification of graphical formalism such UML diagrams. In this category, we mention the work present in [10] [17] [18] [19]. The approach defined by Cengarle et al. aims to define a semantic for UML class diagram, UML interactions diagram and OCL. Each diagram is described in it's natural semantic since the use of the algebraic formalization of each formalism. In addition to that relations between diagrams are expressed via institution morphism and comorphism. We note here that this approach is inspired by Mossakowski works in the heterogeneous institution setting.

The third category of works use this theory for an specific intention and precise cases of study. The work in [7] is a good candidate in this category where authors define a heterogeneous framework of services oriented system, using the institution theory. Authors (in [7]) aims to define a heterogeneous specification approach for service-oriented architecture (SOA). The developed framework consists of a number of specification of individual services written in a local logic and where the specification of their interactions is written in a global logic. The two logic are described via institution theory and an institution comorphism is used to link the two defined institution. This approach is inspired by the work of Mossakowski. Another work is developed in [11]where the authors propose to use institution to represent the logics underling OWL and Z. Then, they propose a formal semantic foundation to the transformation of OWL to Z via the use of institution comorphism. The foundations of this approach is also based on the works in [15].

Our proposed approach aims at first to give a semantic of UML AD via its representation as an institution. So that to consolidate our approach given [13]. Thus, with the defined semantic the transformation of UML AD model to an Event-B model can be semantically proven so that after the transformation we will have the two model semantically equivalent. It's clear that the approach we propose don't tackle the problematic of heterogeneous specification environment like [10] and in [15]. But we think that our work is a first attemps to support the use of a formal method like Event-B as target specification formalism since the Heterogeneous Tool Set [15] among the logic used Event-B is'nt present. The use of Event-B is argued with the following reasons:

- Event-B as a formal method support an interactive and an automatic theorem proving so that the resulted specification after the transformation process can be proved automatically. Event-B as a theorem prover is seeing a continuous improvement by industrial society.

- With the notion of refinement, we can to perform successive refinement to the Event-B model in order to arrive to a pseudo code written in language such Ada.

- Thanks to the notion of composition supported in Event-B, we can to define heterogeneous specification environment with different graphical formalism. And with the notion of composition system described with heterogeneous specification can be composed and so proved formally.

Our work is an inspired form [15]. We are devoted to use UML AD as a formalism for applications modelling. This formalismwill be represented as an institution. Which means that due the algebraic categorical presentation we gain to formal semantic of UML AD. 
The version of UML AD used in this paper is 2.0. In the literature, many approaches are proposed for the development of a formal semantic of UML AD. Recent works which addresses the newest version are the work of Harald Storrle in [5] [4] [12]. Storrle provide a formal definitions of the semantics of control-flow, procedure call, dataflow, and exceptions in UML 2.0 Activities. The defined semantic is inspired from Petri-net semantic. The choice of petri-net semantic by the authors is argued by the following reasons.

- The standard claims that in the version 2.0 of UML AD Activities are redesigned to use a Petri-like semantics instead of state machines.

- Thanks to the formal foundation adequateness of Petri-net to give a formal semantic for UML AD

- In addition, in [4] St"orrle have shown how standard Petri-net tools may be applied to verify properties of UML 2.0 activity diagrams, using a Petri-net semantics.

In our paper, we will not used any intermediate semantic for UML AD. We provide a formal semantic of UML AD with mathematical notions in term of categorical abstract presentation. Thus UML AD will be defined in it's own semantic. We gain from this categorical presentation the next benefit:

- From this categorical presentation of syntax and semantic of UML AD, we can to prove that UML AD can be written as an institution

- we can to use the defined institution for a heterogeneous specification tools like [10]

- Because we use Event-B as formal method for the verification of the UML AD we can to use the concepts of institution comorphism and institution morphism to transform UML AD to Event-B

\section{LOGIC AS AN INSTITUTION}

Institution is an abstract concept invented by Joseph Goguen and Rod Brustall because of the important variety of logics [3]. It offers an abstract theoretic presentation of logic in a mathematical way. An institution consists of notions of signatures, models, sentences, with a technical requirement, called the 'Satisfaction Condition', which can be paraphrased as the statement that 'truth is invariant under change of notation' [16]. The Satisfaction Condition is essential for reuse of specifications: it states that all properties that are true of a specification remain true in the context of another specification which imports that specification.

\section{Definition 1:}

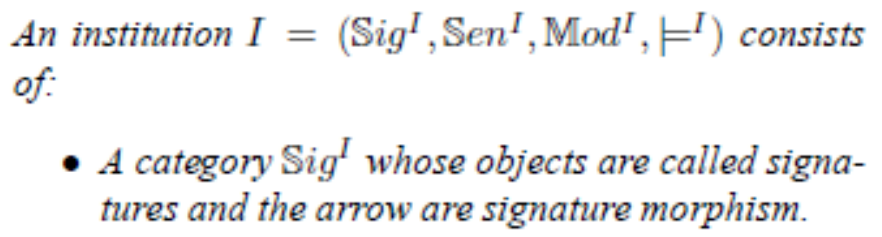


- A functor $\mathbb{S e n}^{I}: \mathbb{S i g}^{I} \rightarrow$ Set, this functor map each signature $\Sigma$ to the set whose elements are called sentences constructed over that signature. Also Sen map each signature morphism to function between sentences.

- A functor Mod $\mathbb{M}^{I}:\left(\mathrm{Sig}^{I}\right)^{o p} \rightarrow \mathbb{C}$ at, this functor map each signature $\Sigma$ to the category of models of this signature. Also Mod map each signature morphism to model homomorphism between models.

- A relation $\models_{\Sigma}^{I}$ giving for each sentences of a signature $\Sigma$ the models in which the sentences are true.

The relation $\models_{\Sigma}^{I}$ is called the satisfaction condition which can be interpreted like follows:

Given a signature morphism $\varphi: \Sigma \longrightarrow \Sigma^{\prime}$ in the institution

I.

For each model $M^{\prime} \in\left|\operatorname{Mod}\left(\Sigma^{\prime}\right)\right|$ and $e \in \operatorname{Sen}(\Sigma)$

$\operatorname{Mod}^{I}(\varphi)\left(M^{\prime}\right) \models \Sigma_{\Sigma}^{I} e \Rightarrow M^{\prime} \models_{\Sigma^{\prime}}^{I} \operatorname{Sen}^{I}(\varphi)(e)$

In the next section, we will prove that $\mathrm{UML} \mathrm{AD}$ can be represented by an institution. The institution of UML AD will be used as a formal semantic for this formalism.

\section{USING INSTITUTION FOR THE DESCRIPTION OF SOURCE FORMALISM}

\subsection{Graphical Formalism}

UML activity diagrams (UML AD) are graphical notation developed by OMG. It is used for the specification of workflow applications and to give details for an operation in software development. UML AD serve many purposes, during many phases of the software life cycle [4]. They are intended for being used for describing all process-like structures, (business processes), software processes, use case behaviors, web services, and algorithmic structures of programs. UML AD are thus applicable throughout the whole software life cycle, which means during business modeling, acquisition, analysis, design, testing, and operation, and in fact in many other activities. Thus, they are intended for usage not just by Software-Architects and SoftwareEngineers, but also by domain specialists, programmers, administrators and so on. The presentation of UML AD as an institution is inspired by the works in [10] [17] [18] [19]. The later work is devoted to define three institution for respectively UML Class diagram, UML Interactions Diagram and OCL. In our paper the semantic and the syntax of UML AD will be based on the works of H. Storrle. As we say in the previous section, the considered work is the more recent and relevant work in this context conformed with the standard. 
With the version 2.0 of UML $\mathrm{AD}$, the meta model for Activities has been redesigned from scratch. The main concept underlying Activity Diagrams is now called Activity [12]. The meta model defines six levels increasing expressiveness. The first level (Basic Activities) already includes control flow and procedurally calling of subordinate Activities by Activity Nodes that are in fact Actions. This paper is restricted to Basic Activities. Readers may refer to [4] [5] [12] for more details about the syntax and the semantic of UML AD.

To perform a model transformation between UML AD and Event-B we will use institution theory. Firstly, we represent UML AD as an institution. After we give the structure of Event-B model. Third, we determine a structure preserving mapping from the first institution to Event- B model.

\subsection{UML AD as an institution}

\subsubsection{The syntax of UML AD}

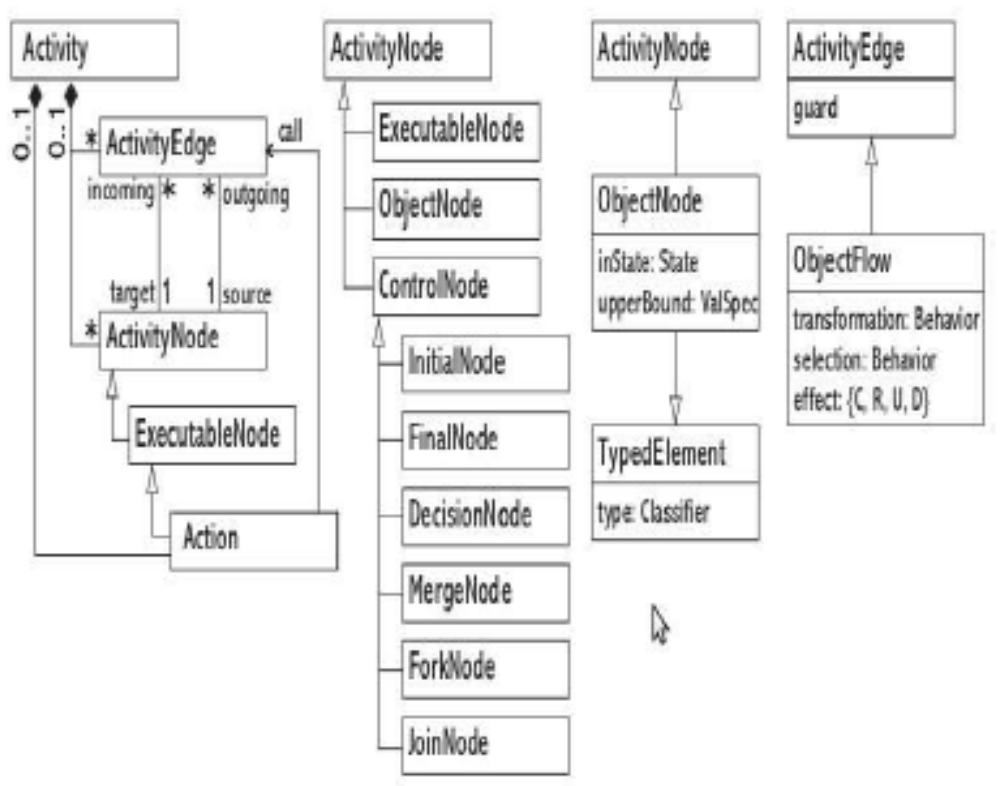

Figure 1. The diagram of the category of model and model homomorphism

Activity as defined in [5] is the coordination of elementary actions or it consists of one atomic action. Besides, given a class diagram, methods are functions that uses attributes of the considered class. Then, class diagram methods are functions or operations that changes the state of an object (defined as an instance of the considered class). In this two cases, we consider an activity as a method of a class in UML class diagram or we consider an activity as a coordination of one action or more. As result, we can to define an relation of hierarchy. This relation is defined between two activities Activity A and Activity B. 
An activity hierarchy $\mathbb{A}$ written as $\mathbb{A}=\left(A, \preccurlyeq_{A}\right)$ is a partial order with a set of activity names $A$ and a subclass relation $\preccurlyeq A \subseteq A \times A$.

Given an activity hierarchy $\mathbb{A}=\left(A, \preccurlyeq_{A}\right)$, a $A$-activity domain is a $A$-indexed family $\mathbb{N}=\left(N_{a}\right)_{a \in A}$ of sets of activity with $N_{a} \subseteq N_{a}$, if $a \preccurlyeq_{A} a^{\prime}$. we aim to prove that the Activity hierarchies can be formalized as a category which can be done via it's formalization as a Grothendieck construction and also as a monad. The two presentations of Activity hierarchies as Grothendieck construction and as a monad are shows in [19] with replacing class hierarchies with Activity hierarchies.

An UML AD signature consists of a pair $\Sigma=(\mathbb{A}, E)$ where $\mathbb{A}$ is the activity hierarchy and $E$ is the set of Activity Edges.

Given a signature $\Sigma=(\mathbb{A}, E)$ with $\mathbb{A}=\left(A, \preccurlyeq_{A}\right)$, we define a set $T$ of atomic formulas over $\Sigma$ by:

$T:=$ skip $\mid \operatorname{seq}(C, e, D) \wedge T$

with $\mathrm{e} \in E$ and $\mathrm{C}, \mathrm{D} \in A$,

Given UML AD signature $\Sigma_{1}=\left(\mathbb{A}_{1}, E_{1}\right)$ and $\Sigma_{2}=$ $\left(\mathbb{A}_{2}, E_{2}\right)$. we define a $\mathrm{UML} \mathrm{AD}$ signature morphism $\varphi: \Sigma_{1} \longrightarrow \Sigma_{2}$ as a morphism that maps Activity node names to Activity node names and maps Activity Edges to Activity Edges. We note here that Activity node can be one of the following node:

- $\quad$ EN: The set of Executable Nodes (i.e. elementary Actions);

- IN or FN : The Initial Nodes or the Final Nodes

- BN: the set of branch nodes, including both Merge Nodes and Decision Nodes

- $\mathrm{CN}$ : the set of concurrency nodes, subsuming Fork Nodes and Join Nodes;

- ON: the set of Object Nodes;

As for Activity Edges may be a pair AE, OF, where:

- AE: the set of plain Activity Edges between Executable Nodes and Control Nodes;

- OF: the set of Object Flows between Executable Nodes and Control Nodes on the one hand, and Object Nodes on the other.

Signature morphism extend to atomic formulas over $\sum_{1}$ as follows:

$\varphi($ skip $)=$ skip

$\varphi\left(\operatorname{seq}\left(C_{1}, e_{1}, D_{1}\right) \wedge \quad T_{1}\right)=\quad \operatorname{seq}\left(\varphi\left(C_{1}\right), \varphi\left(e_{1}\right), \varphi\left(D_{1}\right)\right) \wedge$

$\varphi\left(T_{1}\right)$ 


\subsection{The semantic of UML AD}

In Standard, The semantic of UML AD is determined by a path expressing the trace of the execution. For the execution, a token will move from initial Activity Node To final Activity Node [4]. Each AD Activity has its role in AD execution [12]. First of all, a token in The Initial Nodes means the beginning of the execution of UML AD. Then, the trace of the token will be defined by the outgoing edges of the initial AD. When a token arrive to an Executable Node, it will trigger the Action or the operation in this node. For Join Node, if there is a token offered on all incoming edges, then tokens are offered on the outgoing edge. A Fork Node means that, when an offered token is accepted on all the outgoing edges, duplicates of the token are made and one copy traverses each edge. In the case of Merge Node and Decision Node, every edge is associated to a condition determining the condition of the activation of the edge. For Merge Node, if there is a token offered to only one of the incoming edges where the condition is true (it is a sufficient condition), then token are offered on the outgoing edge of the Merge Node. A Decision Node means that, In the outgoing edge where the condition is true, an offered token will traverses this edge. A token that traverses a Object Node means the availability of the object (variable) needed to the execution of the coming activity.

Given a UML signature $\Sigma=(\mathbb{A}, E)$ with $\mathbb{A}=$ $\left(A, \preccurlyeq_{A}\right)$, a structure $I$ for $\Sigma$ is a triple $I=(\mathbb{N}, \mathbb{E}, \mu)$ where $\mathbb{N}=\left(N^{a}\right)_{a \in A}$ is an Activity domain for $\mathbb{A}, \mathbb{E}$ a domain of edges and $\mu: E \longrightarrow \mathbb{E}$ is an interpretation function for edges. Given a variable $\mathrm{C}$ a valuation $\beta$ for $\mathrm{C}$ in $I$ assigns values to variables. This means:

$\beta: C \longrightarrow N^{a}$

A sub-signature $\Sigma^{\prime}=\left(\mathbb{A}^{\prime}, E^{\prime}\right) \subseteq \Sigma$ with $\mathbb{A}^{\prime}=\left(A^{\prime}, \preccurlyeq A^{\prime}\right)$ induces a set of traces $T\left(\Sigma^{\prime}, I\right)$ defined as follows:

$T\left(\Sigma^{\prime}, I\right)=\left\{e_{1} . e_{2} . . e_{n} \quad \mid \quad i \in\{1, \ldots, n\}, e_{i}=\right.$ $\operatorname{seq}\left(C_{i}, e_{i}, D_{i}\right), C_{i}, D_{i} \in \mathbb{A}^{\prime}$ ande $\left._{i} \in E^{\prime}\right\}$

The set of $\mathbb{T}(I)$ of all traces is defined as :

$$
\mathbb{T}(I)=\left\{e_{1} \cdot e_{2} . . e_{n} \quad \mid \quad i \quad \in \quad\{1, \ldots, n\}, e_{i}=\right.
$$

$\operatorname{seq}\left(C_{i}, e_{i}, D_{i}\right)$ and $\left.C_{i}, D_{i}, e_{i} \in I\right\}$

The set $\Theta(T, \beta)$ of traces of an atomic formula $T$ over

$\Sigma$ in the structure $I$ under the valuation $\beta$ are inductively defined as follows:

$\mathrm{T}:=$ skip $\Longrightarrow \Theta(T, \beta)=\{\varepsilon\}$

$\mathrm{T}:=\operatorname{seq}(\mathrm{C}, \mathrm{e}, \mathrm{D}) \Longrightarrow \Theta(T, \beta)=\{\operatorname{seq}(\beta(C), \mu(e), \beta(D))\}$

$T:=$ skip $\mid \operatorname{seq}(C, e, D) \wedge T$

with $\mathrm{e} \in E$ and $\mathrm{C}, \mathrm{D} \in A$, 


\subsection{The satisfaction condition under the UML AD institution}

Let $\Sigma_{1}=\left(\mathbb{A}_{1}, E_{1}\right)$ and $\Sigma_{2}=\left(\mathbb{A}_{2}, E_{2}\right)$ be two UML $\mathrm{AD}$ signatures, an $\mathrm{UML} \mathrm{AD}$ signature morphism $\varphi: \Sigma_{1} \longrightarrow \Sigma_{2}$, two structure $I_{1}$ a $\Sigma_{1}$-structure and $I_{2}$ a $\Sigma_{2}$-structure defined as $I_{1}=\left(\mathbb{N}_{1}, \mathbb{E}_{1}, \mu_{1}\right)$ and $I_{2}=\left(\mathbb{N}_{2}, \mathbb{E}_{2}, \mu_{2}\right)$. Semantic invariance under the change of notation is formulated as $\Theta_{I_{2}}\left(\varphi\left(T_{1}\right), \beta_{2}\right)=\Theta_{I_{1}}\left(T_{1}, \beta_{1}\right)$ for any atomic formula $T_{1}$ over $\Sigma_{1}$. This can be shown by induction on the structure of $T_{1}$.

$\Theta_{I_{2}}\left(\varphi(\right.$ skip $\left.), \beta_{2}\right)=\{\varepsilon\}=\Theta_{I_{1}}\left(\varphi(\right.$ skip $\left.), \beta_{1}\right)$

$\Theta_{I_{2}}\left(\varphi(\operatorname{seq}(C, e, D)), \beta_{2}\right)$

$\Theta_{I_{2}}\left(\operatorname{seq}(\varphi(C), \mu(e), \varphi(D))(\right.$ skip $\left.), \beta_{2}\right)$

$\left\{\operatorname{seq}\left(\beta_{2}(\varphi(C)), \beta_{2}(\mu(e)), \beta_{2}(\varphi(D))\right\}\right.$

$\left\{\operatorname{seq}\left(\beta_{1}(C), \beta_{1}(e), \beta_{1}(D)\right\}=\Theta_{I_{1}}\left(T_{1}, \beta_{1}\right)\right.$

Also we have $T\left(\varphi\left(\Sigma_{1}\right), I_{2}\right)=T\left(\Sigma_{1}, I_{1}\right)$

\subsection{Signatures}

As shown in the example(fig??), the following elements or components can be captured visually. We have Noeud Initiale, A0, A1, A2, A3, A4 and Noeud Finale seven activity nodes. O is object node. We have also two control node AND SPLIT node and OR JOIN node. In addition to that two node one initial node and the other final node. Then, an itinerary between the activities showing a sequence of execution.

Institution in this context will be used to represent the concrete syntax thus the formal semantic of UML AD. To describe as an institution an UML AD, we identify the four elements of the mathematical definition of institution from the UML AD formalism.

We are conducted to define five predicates that represent different interconnections between UML AD objects. Those predicates are defined as follows:

- $\quad \operatorname{seq}(a, b)$ : A predicate that declare a sequence execution of $a$ and $b$ and an order means a before $b$.

- AND SPLIT(a,b,c): A predicate that declare a control node which is an and split node between the the activity node $\mathrm{a}, \mathrm{b}$ and $\mathrm{c}$.

- OR SPLIT(a,b,c): A predicate that declare a control node which is an or split node between the the activity node $\mathrm{a}, \mathrm{b}$ and $\mathrm{c}$.

- AND JOIN $(a, b, c)$ : A predicate that declare a control node which is an and join node between the the activity node $\mathrm{a}, \mathrm{b}$ and $\mathrm{c}$.

- $\operatorname{OR} \operatorname{JOIN}(\mathrm{a}, \mathrm{b}, \mathrm{c})$ : A predicate that declare a control node which is an or join node between the the activity node $\mathrm{a}, \mathrm{b}$ and $\mathrm{c}$. 
Let $\mathrm{Sig}^{\mathrm{UML}} \mathrm{AD}$ be the category of UML AD signatures. Sig $^{\mathrm{UML}} \mathrm{AD}$ is denoted by (AN,ON,CN), where AN is the set of Activity Node, ON is the set of Object Node and CN is the set of Control Node. The set CN represent also the list of predicates used in UML AD. For the example shown in figure 1, A0, A1, A2, A3, A4, O, Noeud Initiale, Noeud Fianle, and split, or join are the signatures.

\subsection{Sentences}

As for the sentences of the UML AD institution, we will define the concrete syntax. The abstract syntax can be studied by the notion of graph grammar [?]. The functor Sen is defined as follows :

Sen $^{\text {UML AD }}:$ Sig $^{\text {UML AD }} \rightarrow$ Set

Set is the set of sentences. It is constructed over the category of signatures. It expresses the existing information present in a graphical model of UML AD. Using the previous predicates, the sentences associated with a signature declares the clauses describing the execution of the activities over the elements of the signature. The five predicates defined previously represent $(\mathrm{CN})$ terms. which mean a syntactic structure $\sigma\left(\mathrm{t}_{1}, \ldots . . \mathrm{t}_{\mathrm{n}}\right)$ where $\sigma \in \mathrm{CN}_{8 \rightarrow 8}$ is an predicates symbol and $t_{1}, \ldots, t_{n}$ are $(C N)$ term. The set of sentences of UML AD contains the conjunction of the set of $(\mathrm{CN})$ terms.

The UML AD of ?? can be viewed by the following clauses(sentences):

$$
\left\{\begin{array}{l}
\text { seq }(\text { NoeudInitiale, } A 0) \wedge \\
\text { ANDSPLIT }(A 0, A 1, A 2) \wedge \\
O R J O I N(A 1, A 2, A 3) \wedge \\
\text { seq }(A 3, O, A 4) \wedge \\
\text { seq }(A 4, \text { NoeudFinale })
\end{array}\right.
$$

Given a signature of UML $\mathrm{AD}$ signatures $\varphi$ $:(\mathrm{AN}, \mathrm{ON}, \mathrm{CN}) \longrightarrow\left(\mathrm{AN}^{\prime}, \mathrm{ON}^{\prime}, \mathrm{CN}^{\prime}\right)$.

The translation $\operatorname{Sen}^{U M L A D}(\varphi)$ is defined as follows:

$$
\underset{\operatorname{Sen}}{\operatorname{Sen}^{U M L A D}(\varphi)\left(\rho_{2}\right)} \text {. }
$$

\section{Proposition 1:}

Sen $U M L A D$ is a functor defined as follows:

$\operatorname{Sen}^{U M L A D}: \operatorname{Sig}^{U M L A D} \rightarrow$ Set

As for the example (fig ??), Set = $\{\operatorname{seq}($ NoeudInitiale, $A 0), A N D S P L I T(A 0, A 1, A 2)$, ORJOIN $(A 1, A 2, A 3), \operatorname{seq}(A 3, O, A 4)$, seq $(A 4$, NoeudFinale $)\}$ 


\subsection{Models}

Given an UML AD signature(AN,ON,CN), a model M interprets:

- Each Activity Node as a name of concrete activity for example Al as the addition between $\mathrm{a}$ and $\mathrm{b}$,

- Each Object Node as a name of concrete object for example read real a,

- Each Control Node $\sigma \in C N_{w}$ as a function $M_{\sigma}: M_{\sigma} \rightarrow\{$ True $\}$ where w is the arity of the control node $M_{w}$ stand for $M_{s_{1}} \times \ldots \times M_{s_{n}}$ for $\mathrm{w}=s_{1} \times \ldots \times s_{n}$.

An (AN,ON,CN) model homomorphism h: $M \rightarrow M^{\prime}$ is a triple of functions such that:

- $\mathrm{h}$ is an $\mathrm{CN}$ algebra homomorphism $M \rightarrow M^{\prime}$ with $\mathrm{h}\left(M_{\sigma}(\mathrm{m})\right)=M_{\sigma}^{\prime}\left(h_{w}(m)\right)$ for each $\sigma \in C N_{w \rightarrow\{\text { True }\}}$ and for each $\mathrm{m} \in M_{w}$

- $h_{w}(m) \in M^{\prime}{ }_{w}$ for each $\mathrm{m} \in M_{w}$

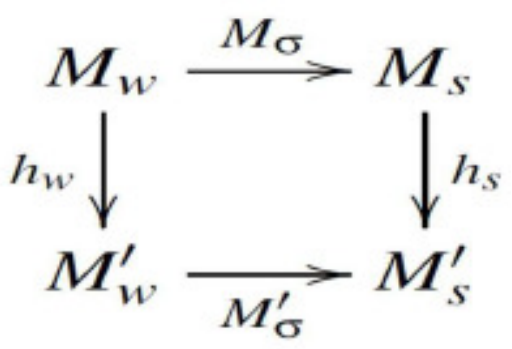

Figure 2. The diagram of the category of model and model homomorphism

\section{Proposition 2:}

(AN,ON,CN) models with (AN,ON,CN) model homomorphism for a category.

\section{Proposition 3:}

UML Activity Diagram form an Institution presented as below:

- Signatures are activity nodes names, control node names, objects node names. 
- Sentences are the conjunction of the predicates identifying the itinerary of the execution of activities. The predicates are $\operatorname{seq}\left({ }^{*}, *\right), \operatorname{AND} \operatorname{SPLIT}\left({ }^{*}, *, *\right)$, OR SPLIT $(* * * *)$, $\operatorname{AND} \operatorname{JOIN}(*, * *)$, OR $\operatorname{JOIN}(* *, *)$.

- Model interprets each signature as follows:

- Each activity as a name of concrete activity for example $A 1$ as the addition between a and $b$,

- Each object as a name of concrete objects for example read $a$,

- Each Control Node $\sigma \in C N_{w}$ as a function $M_{\sigma}: M_{\sigma} \rightarrow\{$ True $\}$ where $w$ is the arity of the control node $M_{w}$ stand for $M_{s_{1}} \times \ldots \times$ $M_{s_{n}}$ for $w=s_{1} \times \ldots \times s_{n}$.

In this section, we have presented a UML AD as an institution with signatures, sentences and models. This institution will be the kernel of our transformation to the target formalism which is Event-B. From this institution we can to conclude that the description is composed of a static part and a dynamic one. The static part appear in signatures and the dynamic part is the sentences of the institution. We mean by dynamic part the order of the execution of the activities.

\section{EVENT-B}

Event-B is the variant of the method B. It is a formal method used for the verification of reactive system developed by Jean-Raymond Abrial Event-B model is formed by a static part named contexts and dynamic part named machines. Like it mentioned in the example of an Event-B model(fig 3), the static part are carrier sets, constants, axioms, and theorems. As for the dynamic part, it consist of variables, invariants, theorems, variants, and events Here we recall some definition in order to construct the Event-B institution.

\subsubsection{Event-B language}

We give the structure of Event-B model as it is defined in Now we will describe the contents of each clause: 


\begin{tabular}{|l|}
\hline Model $\prec$ Model Identifier $\succ$ \\
Set \\
$\prec$ set identifier $\succ$ \\
Constants \\
$\prec$ constants identifier $\succ$ \\
axioms \\
$\prec$ label:predicate $\succ$ \\
theorem \\
$\prec$ label:predicate $\succ$ \\
Variables \\
$\prec$ variables identifier list $\succ$ \\
Invariant \\
$\prec$ label: predicate $\succ$ \\
Events \\
$\prec$ event list $\succ$ \\
End \\
\hline
\end{tabular}

Figure 3. The structure of an Event-B model.

The clause Model stand for the name of the model.

This name will replace $\prec$ Model Identifier $>$.

-The clause Set mean the set used in the model. The list of sets will be written in the model.

-The clause Constants list the list of the constants.

-The clause axioms list the predicates used in the model.

-The clause theorem lists the various theorems which have to be proved within the static part.

-The clause Variables contains the list of the variables used in the dynamic part of the model.

-The clause Invariant list the invariants that must be proved by the list of events of the model.

-The clause Events contains the definition of event of the model.

\section{THE TRANSFORMATION OF AN UML AD INSTITUTION TO AN EVENT-B MODEL}

The transformation of UML AD to Event-B model will be ensured by a set of transformation rules. In fact, this transformation will rules will behave on the algebraic definition of UML AD. To perform the transformation, we will begin by the transformation of the dynamic part of UML $\mathrm{AD}$ to to the dynamic part of Event-B. The dynamic part of UML AD is the sentences of UML $\mathrm{AD}$ institution. As for Event-B, the dynamic part is the set of events presents in the Event-B model.

\subsection{Sentences of UML AD to set of Event-B model}

We present here a set of transformation rules between the set of sentences of UML AD and the set of Event-B events. The table below give the set of the transformation rules.

For more details of how to make the transformations rules, readers are invited to read our previous work. 
Table 1. Transformation rules of the dynamic part.

\begin{tabular}{|c|c|}
\hline $\begin{array}{l}\mathrm{UML} \quad \mathrm{AD} \\
\text { sentences }\end{array}$ & Event-B event \\
\hline $\operatorname{seq}(a, b)$ & $\begin{array}{l}\text { Eventseq1=SELECT stateN1=a \& } \\
\text { G0a THEN stateN1:=b } \| \text { S0b END }\end{array}$ \\
\hline $\begin{array}{l}\text { AND } \\
\operatorname{SPLIT}(a, b, c)\end{array}$ & $\begin{array}{l}\text { Eventand_split1=IF } \quad \text { G0b \& } \\
\text { stateN1B1=a THEN stateN1B1:=b } \\
\text { || S0b ELSIF G0c \& stateN1B2=a } \\
\text { THEN stateN1B2:=c } \| \text { S0c END }\end{array}$ \\
\hline $\begin{array}{l}\text { OR } \\
\operatorname{SPLIT}(a, b, c)\end{array}$ & $\begin{array}{lll}\text { Eventor_join2=IF } & \text { G0c } & \text { \& } \\
\text { stateN1B1=a THEN } & \text { S0c } & \| \\
\text { stateN2:=c ELSIF } & \text { G0c } & \text { \& } \\
\text { stateN1B2=b THEN } & \text { S0c } & \| \\
\text { stateN2:=c END } & & \end{array}$ \\
\hline $\begin{array}{l}\text { AND } \\
\text { JOIN }(a, b, c)\end{array}$ & $\begin{array}{l}\text { Eventand_join1=IF } \quad \text { G0c \& } \\
\text { stateN1B1=a \& stateN1B2=b } \\
\text { THEN } \quad \text { stateN1B1:=c } \| \\
\text { stateN1B1:=c }\end{array}$ \\
\hline $\begin{array}{l}\text { OR } \\
\text { JOIN }(a, b, c)\end{array}$ & $\begin{array}{lll}\text { Eventor_join } 2=\text { IF } & \text { G0c } & \text { \& } \\
\text { stateN1B1=a THEN } & \text { S0c } & \| \\
\text { stateN2:=c ELSIF } & \text { G0c } & \text { \& } \\
\text { stateN1B2=b THEN } & \text { S0c } & \| \\
\text { stateN2:=c END } & & \end{array}$ \\
\hline
\end{tabular}

\subsection{Sentences and Signatures of UML AD to the static part of Event-B model}

After obtaining the dynamic part of the Event-B model, we will, in this section, define how to get the static part of Event-B model from the sentences and the signatures of UML AD institution. Below we give an overview of how to get the static part:

- Model $\prec$ Model Identifier $>$

The name of the model can to be given arbitrary.

- Variables $<$ Variables list $>$

The list of the variables can be identified from the event defined in the previous sections.

- Invariant $<$ label: predicate $>$

Invariant show the set of each variable. It can be also identified from the set of event defined in the previous section.

- Assertions $\prec$ Assertions list $>$

The assertions clause is the conjunction of the guard of the event of the Event-B model. The guard will be identified fromthe set of events defined in the previous section. 
- Initialization $\prec$ Initialization list $>$

As an example, we take the UML ad model of the figure ?? and perform the transformation rules. Transformation rules will acts on the institution of UML AD. 4 represent the transformation of UML AD of fig ??.

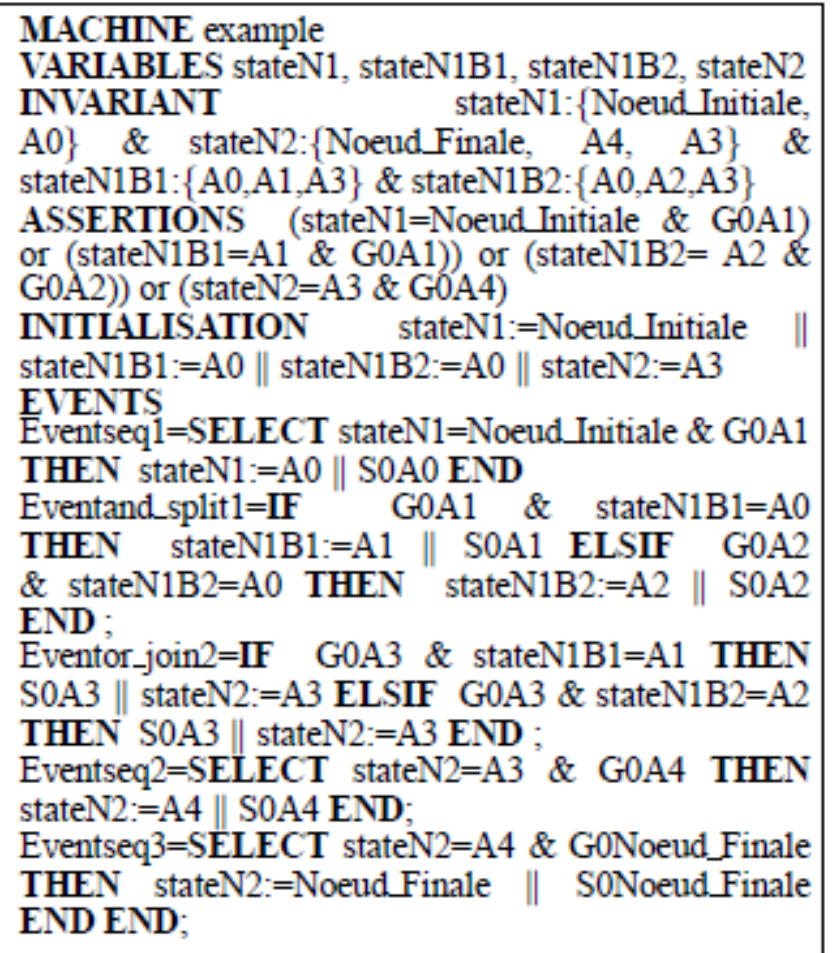

Figure 4. Event-B model: the result of the UML AD transformation.

\section{CONCLUSIONS}

We have presented in this paper a formal approach for the transformation of UML AD to Event-B model. We use institution as a language for the description of UML AD. Thus, we give an institutional definition of UML AD. The institution of UML AD is constructed under the institution of First Order Logic. As for the target formalism which is Event-B, we define properly the syntax of an Event-B model adapted to UML AD models. For the transformation, we give a set of transformation rules of UML AD sentences to Event-B models. As for the future work, we aim to prove that Event-B can be represented as an institution. As a consequence, we will have two different institution describing each formalism. Institution morphism and comorphism will be a solid and effective notions to link and make transformation from one formalism to the other. Also refinement can be another future goal to move from one institution to the other. In our works, institution is considered as meta level to fulfill model transformation. As for the development of an engine of model transformation between the UML AD and Event-B, we aims to enrich our previous developed tool mentioned in [?]. We will add the formal semantic of UML $\mathrm{AD}$ developed in this work to our tool. 


\section{REFERENCES}

[1] R. Diaconescu, Grothendieck institutions, Applied Categorical Structures, 10, 1994, 167-174.

[2] R. Diaconescu and K. Futatsugi, Logical Foundations of CafeOBJ, Theoretical Computer Science, 285, 289-318.

[3] J. Goguen and G. Rosu, Institution Morphisms, Formal Aspects Computing, 13, 2002, 274-307.

[4] H. Storrle, Structured nodes in UML 2.0 activities, Nordic J. of Computing, 11, 2004, 279-302.

[5] H. Storrle, Semantics and Verification of Data Flow in UML 2.0 Activities, Electronic Notes Theoretic Computer Science, 127, 2005, 35-52.

[6] J.-R.Abrial, Modeling in Event-B: System and Software Engineering (Cambridge University Press, 2010).

[7] A.Knapp, G.Marczynski, M.Wirsing and A.Zawlocki, A heterogeneous approach to service-oriented systems specification, SAC, 2010, 2477-2484.

[8] M. Codescu, F. Horozal,M. Kohlhase, T.Mossakowski, F. Rabe and K. Sojakova, Towards Logical Frameworks in the Heterogeneous Tool Set Hets, WADT, 2010, 139-159.

[9] M. A. Martins, A. Madeira, R. Diaconescu and L. S. Barbosa, Hybridization of Institutions, CALCO, 2011, 283-297.

[10] M. V. Cengarle, A. Knapp, A. Tarlecki and M. Wirsing, A Heterogeneous Approach to UML Semantics, Concurrency, Graphs and Models, 2008, 383-402.

[11] D. Lucanu, Institution Morphisms for Relating OWL and Z, The 17th International Conference on Software Engineering and Knowledge Engineering, 2005.

[12] H. St"orrle and J. H. Hausmann and U. Paderborn, Towards a formal semantics of UML 2.0 activities, German Software Engineering Conference, 2005, 117-128.

[13] L. J. Ben Ayed, A. Ben Younes and A. Achouri, Using AToM3 for the Verification of Workflow Applications, ICSOFT (2), 2010, 32-39.

[14] Vitolins, Valdis and Kalnins, Audris, Semantics of UML 2.0 Activity Diagram for Business Modeling by Means of Virtual Machine, the Ninth IEEE International EDOC Enterprise Computing Conference, 2005, 181-194.

[15] T. Mossakowski, Heterogeneous Specification and the Heterogeneous Tool Set, Habilitation thesis, Universitaet Bremen, 2005.

[16] R. Diaconescu, Institution-independentModel Theory (Birkh“auser Basel, 2008).

[17] M.V.Cengarle , UML 2.0 Interactions: Semantics and Refinement, Technical report, Institut fr Informatik, Technische Universitt Mnchen, 2004.

[18] M.V.Cengarle and A.Knapp , An Institution for UML 2.0 Interactions, Technical report, Institut fr Informatik, Technische Universitt Mnchen, 2008.

[19] M.V.Cengarle , An Institution for UML 2.0 Static Structures, Technical report, Institut fr Informatik, Technische Universitt Mnchen, 2008. 


\title{
AN EXPLICIT TRUST MODEL TOWARDS BETTER SYSTEM SECURITY
}

\author{
Orhio Mark Creado ${ }^{1}$, Bala Srinivasan ${ }^{2}$, Phu Dung Le ${ }^{3}$, and Jefferson $\operatorname{Tan}^{4}$ \\ ${ }^{1}$ Caulfield School of Information Technology, Monash University, \\ Melbourne, Victoria, Australia \\ mark. creado@monash.edu \\ ${ }^{2}$ Clayton School of Information Technology, Monash University, \\ Melbourne, Victoria, Australia \\ srini@monash.edu \\ ${ }^{3}$ Caulfield School of Information Technology, Monash University, \\ Melbourne, Victoria, Australia \\ phu.dung. le@monash.edu \\ ${ }^{4}$ IBM Research - Australia, Melbourne, Victoria, Australia \\ jeffetan@aul.ibm.com
}

\begin{abstract}
Trust is an absolute necessity for digital communications; but is often viewed as an implicit singular entity. The use of the internet as the primary vehicle for information exchange has made accountability and verifiability of system code almost obsolete. This paper proposes a novel approach towards enforcing system security by requiring the explicit definition of trust for all operating code. By identifying the various classes and levels of trust required within a computing system; trust is defined as a combination of individual characteristics. Trust is then represented as a calculable metric obtained through the collective enforcement of each of these characteristics to varying degrees. System Security is achieved by facilitating trust to be a constantly evolving aspect for each operating code segment capable of getting stronger or weaker over time.
\end{abstract}

\section{KEYWORDS}

System Security, Trusted Computing, Trust Framework .

\section{INTRODUCTION}

Trust is an implicit commodity in the world today. We inherently trust our financial institutions, service providers, and even other motorists without any second thought. However, although synonymous, being trusted and being trustworthy are very different [1]. Trust as a human construct is extremely pliable; but this is not the same case when considering computing systems. Computing systems execute code that performs operations which produce usable outputs. Each instruction can be considered to be a singular operation. Therefore, a computing system can only be trusted and secure depending on the next instruction it executes. So how can a computing system rely on securing itself from itself? [2].

In this paper we propose a novel concept to alleviate the ambiguity of trust levels associated with executing code so as to ensure better overall system security. To achieve this goal, we primarily David C. Wyld et al. (Eds) : CCSIT, SIPP, AISC, PDCTA, NLP - 2014 pp. 139-151, 2014. @ CS \& IT-CSCP 2014

DOI : $10.5121 /$ csit.2014.4212 
define trust in terms of a computing architecture; wherein, trust is no longer defined as a singular attribute but instead as a combination of characteristics which can collectively determine the overall trust level for any operating code. The aim behind this paradigm is to represent trust as an evolving concept within a computing system capable of growing stronger or weaker over time based on past operating performance. In our opinion, this is a significant step away from current models which advocate trust to be a binary outcome based on superficial constructs such as a valid username and password.

The rest of this paper is structured as follows. Section 2 briefly covers some of the relevant literature in the area. Section 3 defines the explicit trust model architecture along with its main component - the trust engine, outlining the various trust categories and trust levels within each category and concludes with a real world application of the proposed model's theory. Section 4 evaluates our proposal with a formal analysis of security vs. performance and provides some real world tangibility by using an asset centric threat model documenting some of the attacks pertinent to system security and how the proposed model aims at resolving them. Section 5 concludes our work and provides directions for future research.

\section{BACKGROUND}

Trust, as a concept, traces its roots back as a psychological and sociological construct. In computing, its definition cannot be applied completely; as machines tend to be programmatic intelligence, the task of quantifying malicious intent becomes more challenging [3]. Bevan [4] proposes that with human-computer interactions there remain many variations of trust and trust levels; but not all of these levels can be accounted for in human-human interactions. Yet, computing systems have aimed to satisfy only a few of these variations at any one time, so how can security be achieved if only partial trust can be achieved?

Trust in computing has been an active area of research for a very long time. One of the most prominent implementations of trusted computing has been the Trusted Computing Group's (TCG) Trusted Platform Module (TPM) [5,6]; which used a cryptographically secure hardware to perform trusted software operations. A well-known implementation of this hardware platform was observed in Microsoft's Next Generation Secure Computing Base (NGSCB) [7-11]. The drawback of this approach was that it tried to facilitate for a trusted area within an otherwise insecure environment.

Alternative solutions include the implementation of microkernels as proposed by Setapa [12], and Heiser et. al. [13]. A good example of a hybrid approach between hardware and software policy has been proposed in the work of Nie et. al. [14]. The drawback with some of these approaches is that microkernels can be vulnerable during the boot phase of a computing system, and relying on hardware based solutions to implement security models is equivalent to no security if it is possible to compromise the actual hardware [15].

Trust can be defined as a concept with multiple characters [16], the challenge of implementing trust in computing has been the subjectiveness of the term 'trust' in relation to the user. This means that any operating code executed on a system can behave differently at different times depending on the user, the operating environment variables, and the desired outcome being sought. The determination of being trustable is still an open concern with human interactions, so why should computing systems be any different? Real world implementations aren't quite as simplistic, so as to be able to always consistency and accurately reduce the outcome of trust and security to a binary result. It isn't feasible, or possible, to account for all the possible scenarios which must have trusted operations defined. Modern day computing systems and their operations are never static, so why should the definition of trusted operations and trust in computing be? 


\section{ExPlicit Trust MODEL}

Trust within operations in today's digital age is of paramount importance. Trust enforcing mechanisms with binary outcomes have become a single point of failure leading to the exploitation or compromise of a system. This section elaborates our proposal for explicit trust.

\subsection{Security as a Combination of Characteristics}

The more trusted the executing operations within a computing system, the more secure is the computing system. With this analogy, the explicit trust model defines a set of characteristics, each with its own set of properties, which can collectively determine the trust associated with all operating code within a computing system. It is important to note that although achieving absolute trust is not possible, it is quite possible to achieve near absolute trust through the correct enforcement of each of the identified characteristics and properties. The defined characteristics are as follows:

- Invulnerable

Invulnerability can be achieved through the reduction in the number of exploitable errors in operating code. This can be practically envisioned through the definition of secure programming languages, through secure coding practices, and through rigorous application testing. Furthermore, all code should be implicitly defined to handle all errors and be responsible for proper allocation and deallocation of resources. Properties include:

- Defined Bounds: Ensuring that all input parameters comply with expected inputs, errors which exploit programming language vulnerabilities for input data types can be prevented.

- Handled Exceptions: Ensuring that all output parameters produced comply with expected outputs, errors which exploit programming language vulnerabilities for output handling can be prevented.

- Integrity

Integrity can be achieved by having accountability standards in place for all operating code. As all usable code serves a specific purpose and has an author; a publicly verifiable metric, such as digital signatures, associated with the operating code should be provided so as to ascertain its ownership and ensure its authenticity to perform its intended purpose. Properties include:

- Accountability: Ensuring that all operating code must have a valid and publicly verifiable digital signature which can uniquely identify the owner/author of that operating code and can also uniquely identify the integrity of the code.

- Verification

Being verified can be achieved through rigorous state management by the operating system. Virtualization technology employs similar aspects which facilitate the management of system state. By preventing unauthorized changes in system states, undesirable states of operation arising from unexpected exceptions in operating code can be prevented. Properties include:

- Managed States: Ensures that all operations executing one instruction at a time do not forget the operating state of the calling instruction/process parent thereby ensuring the correct completion of instructions from start to finish. 
- Trustworthy

Being trustworthy can be achieved through the proper definition of a calculable trust metric associated with any operating code. Initially assigned based on the credibility of its owner and post that, based on historical performance based on correct execution calculable via deterministic trust algorithms. Properties include:

- Trust Levels: Ensures that all operating code must have a defined trust level which indicates its level of trustworthiness to the system and upon each execution is recalculated and modified accordingly based on the outcome of that execution.

\subsection{Identifying Trust Categories and Trust Levels}

To facilitate for evolving trust, the proposed model also defines a set of trust categories and underlying trust levels associated with each category. The purpose of these categories is to allow the trustworthiness associated with all operating code to either increase or decrease based on historical performance; thereby implicating higher trustworthiness for correct successful operations and lower trustworthiness for incorrect unsuccessful operations. The following trust categories and underlying trust levels have been defined:

\section{- Functional Trust}

This category outlines the basic trust requirements for all operations within a computing system. All operating system code, user application code, and network services code must have a trust level associated with this category. To allow for application scalability, the standardized constructs which outline the fundamental operations for each application can be application specific. Defines the following trust levels:

- Operational Trust - Is required for system level operations, such as Systemsystem communication and high priority OS operations.

- Verifiable Trust - Is the basic requirement for all operational code executed by the system or user to be verifiable and accountable.

- Denied Trust - Is defined for operational components which are not verifiable and accountable; such as malicious operations aimed at exploiting or compromising the computing system, thereby completely preventing their execution on the computing system.

- Transactional Trust

This category is defined for operational components to constantly evolve their trust levels by serving as an intermediary between two functional trust levels. Trust levels under this category are deterministically calculable based on past historical operations over time. Defines the following trust levels:

- Transitional Trust - Intermediate between verifiable and operational trust, facilitates evolution of trust for operations with good historical performance.

- Untrustable Trust - Intermediate between verifiable and denied trust, facilitates evolution of trust for operations with detrimental historical performance. However, to support versatility and scalability, this trust level allows operational components which do not meet all the verification and accountability standards, but without significant operating history to deny execution, to execute within a constrained operating environment. 


\subsection{Defining the Trust Architecture}

This section aims to integrate the defined concepts of the proposed security characteristics in conjunction with the proposed trust levels so as to define the explicit trust model's trust architecture. Traditionally computing systems allow for three types of execution modes: System, User, and Guest. Whilst beneficial, these modes do not define any level of granularity between each and often overlap based on the nature of operations. The proposed model advocates the requirement for a trust level to be associated with the operating code rather than the execution mode of the computing system.

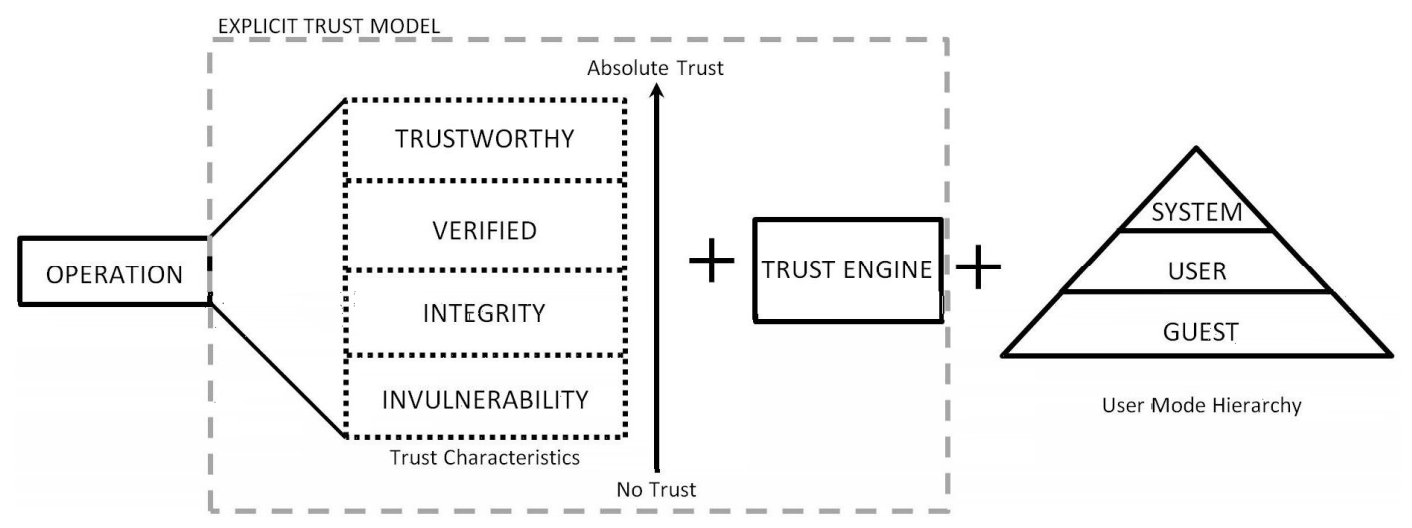

Figure 1. Explicit Trust Model Architecture

Fig. 1 provides a conceptual definition of the proposed trust architecture. The architecture mandates that each operation must be able to satisfy each of the security characteristics by fulfilling their underlying properties. In a realistic scenario these characteristics could be satisfied only to a certain degree and therefore would allow the deterministic calculation of a trust level on a scale from no trust to absolute trust. For this purpose, the architecture defines a trust engine component which acts as an intermediary and facilitates the calculation and determination of the associated trust levels with each operation prior to execution. The last stage of the process is the execution of operating code under one of the execution modes facilitated by the operating system.

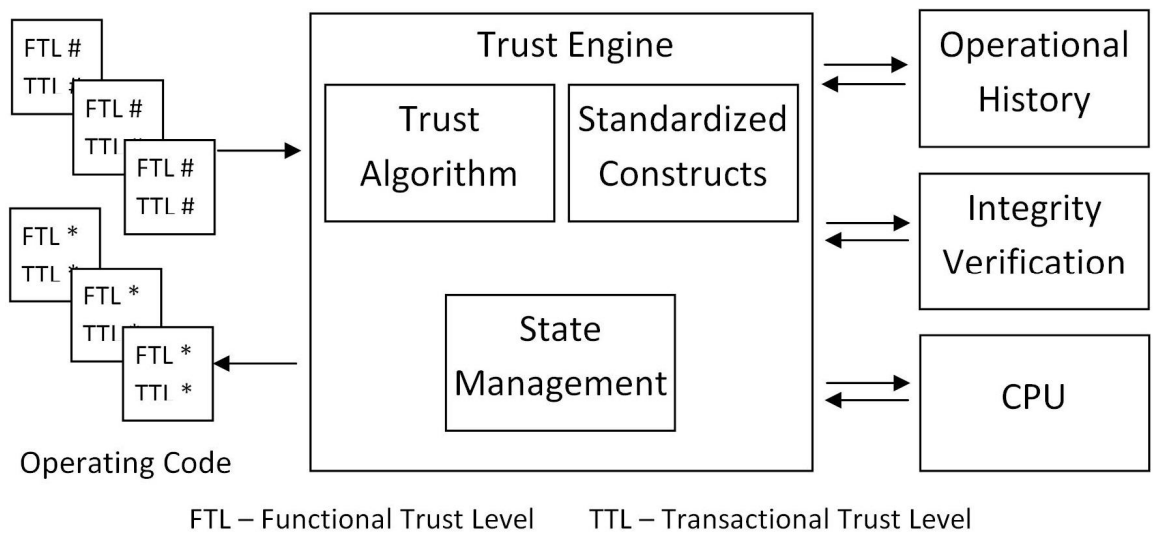

Figure 2. Trust Engine Architecture

The proposed trust engine is the most integral part of the explicit trust model, and Fig. 2 outlines a conceptual definition of the explicit trust model's trust engine architecture. The proposed 
workings of the trust engine will be defined; its practical implementation, at this stage, is out of scope of this paper.

The trust engine serves as the common link which integrates the various security characteristics with the defined trust levels applicable to operating code. This is facilitated by specifying two calculable trust metrics for each block of operating code; the first being its functional trust level and the second being its transactional trust level. Prior to executing any operating code the trust engine facilitates the following process:

- Verifies its associated functional trust level.

- Verifies its associated integrity signature.

- Determines operating mode and passes instructions for execution.

- On completion of execution, it verifies the state management registry to ensure correct execution.

- Depending on execution outcome, it updates the operation history registry.

- Executes the trust algorithm to deterministically calculate a new transactional trust level based on the operational history.

- Lastly, updates the operating code with a new functional and/or transactional trust level metric.

We now define the various components of the trust engine architecture which facilitate the inner working process of the trust engine. The included components are:

- Operating Code - Defines the basic set of operating instructions which need to be executed on a computing system.

- Trust Algorithm - Deterministic algorithm which takes into account the number of historical executions, correct executions, incorrect executions, owner trust metric, and other key inputs to determine a trust metric for any operating code.

- Standardized Constructs - Defined as an optional customizable add on to the model which would facilitate user or application specific trust requirements.

- Integrity Verification - Verifies the integrity signature of the operating code against the hash of the operating code and the owner's public key.

- Operational History - This registry stores aggregated historical operations for all operating code resident within the computing system serving as input to the trust algorithm's calculation.

- State Management - This registry monitors the execution of processes and forking of parent processes to ensure desirable states of operation and complete execution of instructions from start to finish.

- CPU - Facilitates for the processing and execution of operating code instructions; and accepts required inputs and produces any applicable outputs.

\subsection{Integrating The Explicit Trust Model}

This section aims to further the readers understanding of a possible real world application of the explicit trust model. Fig. 3 illustrates the step by step process of executing operating code based on the associated trust level. 


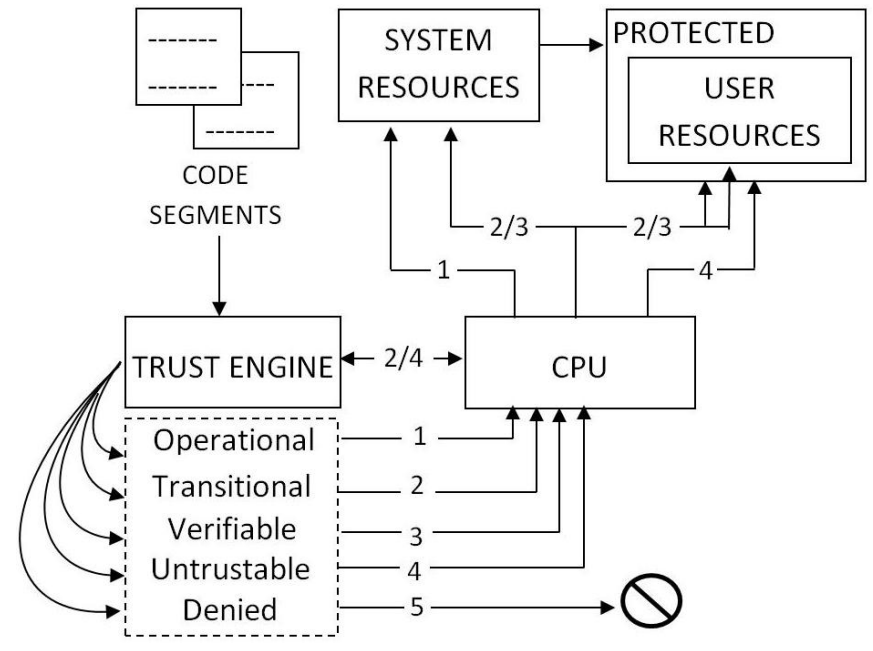

Figure 3. Operational Trust Levels

As observed in Fig. 3, all operations within a computing system can be reduced to operating code making the explicit trust model universally applicable to all operations, users, or even components within a computing system. By associating a trust level with each object it facilitates for a model whereby past operations dictate future access; thereby allowing for an evolving level of trust.

System code is initially owned by its author/owner and once installed on the system changes ownership to the system thereby preventing all future modifications of system level operating code by any user. Updates to system level code would require verification of the original owner in order to allow for modifications of system level code files. By assigning an operational trust level, all operating code within this trust level definition would have access to all system and user level resources.

System and user code which belongs to third parties are always defined with a verifiable trust level provided they have correct integrity signatures. The transactional trust level allows this operating code to evolve to an operational trust level wherein access to system resources might be required in order to perform system level operations. The transactional trust level determines the access to protected user resources and/or system resources.

System and user code which consistently encounter errors or detrimentally affect system state are categorized with a denied trust level wherein all operating code with this trust level is not allowed to be executed on the computing system. To facilitate for operating code without sufficient operating history and/or without verification signatures the untrustable trust level allows for execution of these instructions within a protected environment wherein system level access is completely restricted.

\section{Evaluation}

In this section we proposal a more formal evaluation of the proposed model for both security and performance. For real world tangibility, we also provide a concise asset centric threat analysis of the model with the emphasis on the computing system. Lastly we conclude the section with an objective discussion of the proposed theory. 


\subsection{Security vs. Performance Analysis}

Let us assume that an operating code $C$ is comprised of $n$ lines of operating code, which is a total of $n . k .[0]$ operations; such that

$$
C \equiv n \equiv n \cdot k \cdot[0]
$$

represents the total number of operations for $n$ lines of code where

$$
k=\sum_{i=1}^{n}[0]_{n_{i}} / n
$$

is the coefficient of the number of instructions per line of code.

Eq. (1) therefore represents the default number of instructions to be executed without any additional security enforcing properties.

The explicit trust model calculates security of the model using a probabilistic approach due to the inverse relationship between security and performance. We reduce both metrics to the number of operations being performed in order to deterministically evaluate the additional overhead. Keeping this mind we can represent the following:

$$
\text { Perf }=n \cdot k \cdot[O] \quad, \quad \operatorname{Sec}=1-\frac{1}{n \cdot k \cdot[O]}
$$

where security is calculated as the probability of finding a single error in any operating code subtracted from the probability of code execution.

Each security enforcing characteristic within the explicit trust model can further be enforced with the addition of additional lines of code to the basic set of operating instructions. We can transform Eq. (1) for each property to represent the total number of additional instructional overhead as follows:

$$
c+P_{i}=\frac{n}{l_{i}}=\frac{n \cdot k \cdot[0]}{l_{i}}
$$

where $l$ is the additional number of lines of code added to $n$ for property $P_{i}$

Accounting for each of the defined characteristics within the explicit trust model, we can transform Eq. (2) as follows:

Perf $=\left\{1+\frac{1}{l_{1}}+\frac{1}{l_{2}}+\frac{1}{l_{3}}+\frac{1}{l_{4}}\right\}, n \cdot k \cdot[0] \quad, \operatorname{Sec}=1-\left[\frac{1}{n \cdot k_{0}[0]} \cdot\left\{1+l_{1}+l_{2}+l_{3}+l_{4}\right\}\right]$

where each of the properties of Invulnerability, Integrity, Verification, and Trustworthy have been numerically represented.

Fig. 4 outlines the trade-off between performance and security for the proposed model. The graph depicts the deterministic curve which defines the increase in security with a slight decrease in performance. Since all operating code must be executed in order to be functional, the depicted graph is directly based on the number of operations irrespective of the size of the executing code; thereby facilitating for the evaluation of the additional overhead required in terms of ascertaining additional levels of security for the minimal trade-off in performance.

Furthermore, the evaluation methodology provides for an objective overview of a deterministic vs. probabilistic model; due to the nature of computing systems wherein performance degradation is the direct result of increased operations. However, lapses in security should be based on a 
probabilistic model; as the mere existence of a vulnerability does not imply exploitation without other key factors being supportive as well.

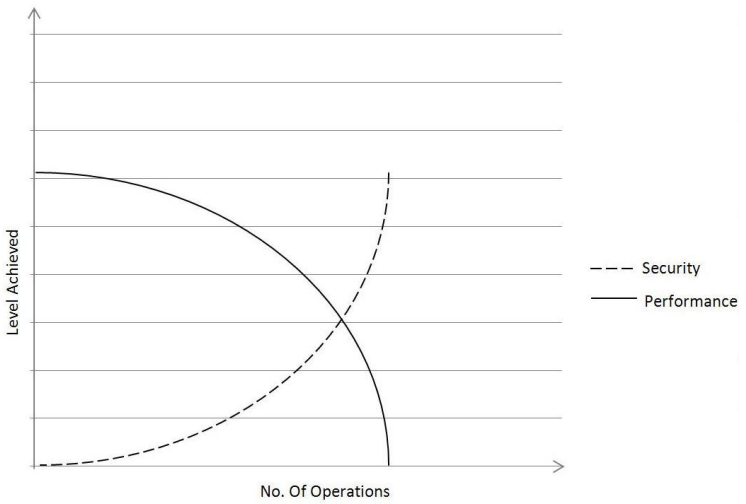

Figure 4. Performance vs. Security Trade-off

Lastly, the proposed work allows for the determination of optimums so as to maintain the balance between security and performance to ensure the usability of a computing system without compromising user friendliness. Furthermore, the abstraction of security enforcing characteristics away from the end user ensures that security does not remain as an optional add-on within a computing system.

\subsection{Asset Centric Threat Model}

In this section we aim to provide the reader with some real world tangibility by proposing the possible feasibility of the proposed model and its application towards preventing real world threats affecting modern day computing systems.

\subsubsection{Attack Process Flow}

Computing systems are processing stations for data - performing operations, and producing desired output or errors. Abstracted within this simplistic view is the attack path used to compromise the system. All attacks must exploit specific inputs so as to compromise a system. Figure 5 graphically outlines the perceived vs. actual process flow of an attack as it happens within a computing system.

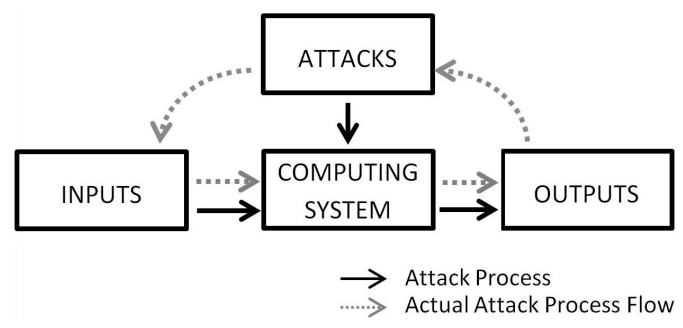

Figure5. Attacker's Process Flow

\subsubsection{Threat Identification and Mitigation}

With regards to the defined attack process flow, we isolate and outline the various interacting component for the proliferation of trust within a computing system pertinent to our threat model: 
- Inputs - Are classified as data needing to be processed by the execution of some code. The issuer of the data or instruction, whether internal or external, is irrelevant to the operation and is therefore not an input.

- Outputs - Post execution of any instruction, the computing system is capable of producing the following outputs: data, errors, or other processes.

- Attacks - The following threats, applicable to system security, have been identified: service disruption, privilege escalation, data theft/manipulation, system corruption, protocol exploitation.

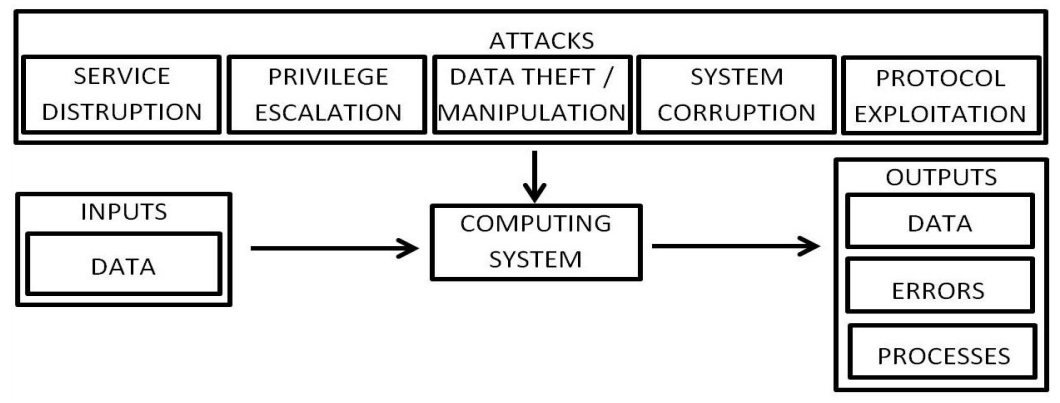

Figure 6. Computing System Threat Model

We identify five main attacks which target computing systems specifically, and represent them in Fig 6 to represent how they relate to our attack process flow. For conciseness we represent the threat mitigation process for these attacks in Fig 7 without taking into account the threat trees for each attack.

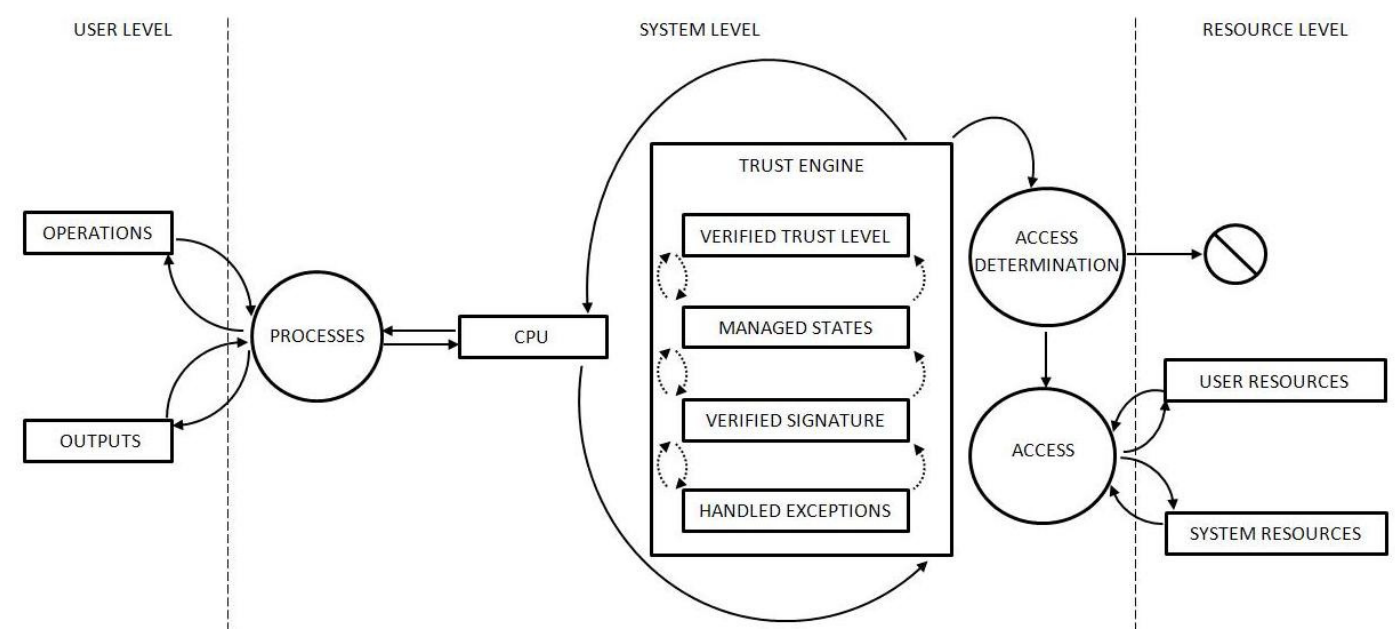

Figure 7. Explicit Trust Threat Mitigation Model

The proposed analysis segments the attack process into three stages: User, System, and Resource. Whilst majority of the actual process might happen at the system level, most attacks target exploiting the resource level by gaining access at the user level. The prevention of these attacks is proposed via the means of the trust engine's trust level determination process which can determine if each of the underlying security enforcing properties is satisfied. By defining a linear progression of characteristics for each operation within the explicit trust model, the trust engine facilitates for a semi-hierarchical approach towards the fulfilment of trust properties to ensure overall system security. The following are broad definitions of these attacks and their mitigations within the proposed model: 
- Service Disruption

Are targeted towards disrupting basic operations; examples include denial-of-service and distributed denial-of-service. The exploitation is targeted towards the communication protocol used and the requirement to acknowledge and respond to all incoming requests. These attacks can be prevented by the integrity and verification properties of the explicit trust model. By validating dual authentication and ensuring state management for all processes these attacks can be circumvented to ensure that all systems communicating with the server can be identified and be held accountable for their actions.

- Privilege Escalation

Are targeted towards gaining unauthorized access; an example is a buffer overflow attack. The exploitation is targeted towards finding and exploiting coding flaws by passing modified inputs to overwrite memory registers. These attacks can be prevented by the invulnerability, integrity, verification, and trustworthy properties of the explicit trust model. By ensuring that all programming code has proper error handling and resource utilization code in place and by ensuring that all code has an identifiable owner who can be trusted via means of a trust metric associated with the application code. Furthermore, proper state management to ensure instructions finish in order can further prevent these types of attacks.

- Data Theft / Manipulation

Are targeted towards stealing user data or information; examples include viruses, trojans, spyware/malware etc. The exploitation is targeted towards covert exploitation under the pretence of some other legitimate operation. These attacks can be prevented by the integrity and trustworthy properties of the explicit trust model. Any operating code through covert channels would not be signed with any integrity signature; and furthermore, any default trust metrics associated with these would only be at the verifiable level, which would allow only protected execution thereby thwarting any system level exploitation.

\section{- System Corruption}

Are targeted towards rendering a system unusable; an example is bios corruption. The exploitation is targeted towards overwriting the master boot record thereby rendering the next start-up unable to load. These attacks can be prevented by the integrity and trustworthy properties of the explicit trust model. Any system level changes would require the original author's verification of the operating code. Code affecting the boot load process would ideally be required to have vendor integrity and trustworthy metrics assigned.

\section{- Protocol Exploitation}

Are targeted towards exploiting vulnerabilities in communication protocols; examples include ping of death, certificate forging, session hijacking, scripting etc. The exploitation is targeted towards system modification, disruption, or compromise. These attacks can be prevented by the verification property of the explicit trust model. By ensuring that all processes have a managed state of execution, any variations can be trapped and terminated so as to prevent undesirable states of operations. 


\subsection{Discussion}

One of the biggest challenges in theoretical computer science is the evaluation of a proposal so as to ascertain the viability of the idea. Our approach to resolve this has been to provide a different perspective to the reader from a conceptual viewpoint with links to practical applications. Although some of the proposed concepts might seem like existing open challenges in the computing industry the proposed work targets resolving them from a more fundamental point of view which is the underlying source of the vulnerability rather than trying to propose a fix for any specific vulnerabilities. By adopting this approach, our goal remains to propose a model which can be independent of the underlying platform, operating system, application, or component.

For conciseness of this paper, many proposed concepts specifically in the threat model's asset centric approach have been condensed; nonetheless, most of these are implementable via modifying the operating code for most commodity programs and signature verification is currently handled by most operating systems. By reducing our proposal to the most fundamental unit of operating code we allow for the definition of security enforcing characteristics by modifying the existing code. The mammoth task of fixing real world systems is perhaps out of scope of the proposed work; but the argument remains is that if we could fix existing issues we wouldn't still have them. The very fact that vulnerabilities still exist within computing systems points to the fact that the underlying infrastructure might need changing and although perhaps already in the works by big vendors for the not so distant future, this paper has aimed at providing a more conceptually sound, yet practically realisable model to further the state of secure computing.

\section{CONCLUSION}

If the existing paradigms for ensuring trust and security within computing systems were adequate, we wouldn't have as many vulnerabilities and exploitations of systems happening all over the world. Identity theft wouldn't be an issue, man-in-the-middle attacks wouldn't exist, and financial crime would be non-existent. However, that would be an ideal world scenario, but for now there exists a need for our computing infrastructure to evolve to the next level rather than patch existing technology with band-aid solutions which sometimes introduce new vulnerabilities in the process. In this paper we have proposed a novel approach towards promoting system security by ensuring trusted operations through the proliferation of trust explicitly. We reduce higher order systems to the basic fundamental units of operating code so as to be able to define a linear set of properties which collective define trust as a combination of individual characteristics, rather than viewing it as a singular attribute. Through this approach, we define a process for the deterministic calculation of trust levels based on the degree of satisfaction of each of the properties underlying each of the identified characteristics. By rendering trust as a deterministic metric calculable based on past historical performance, we facilitate for a paradigm of evolving trust within a computing system which can evolve to grow stronger or weaker over time depending on past executions. Furthermore, we evaluate our proposal for the trade-off between security and performance by alleviating the ambiguity between the deterministic vs. probabilistic approach by reducing both aspects to the number of instructions executed we are able to provide a more viable benchmark for comparison which is logically sound.

In our opinion, there remains a large void for secure operations within computing systems with the growing diversity of devices and platforms. Through the incorporation of the proposed model it remains feasible to define security at the core of all operations within a computing system rather than as an add-on aspect dependent on the user. Our plans for continued work in this area include defining a framework for secure computing which is capable of incorporating trust as a fundamental component of its operation. We also have plans to publish our idea of a practical way to realize the proposed model within a computing system. Also in the works include the 
development of a deterministic trust algorithm which is capable of providing a calculable metric as a trust level using statistical and probabilistic models based on past operational history.

\section{REFERENCES}

[1] M. Abadi, "Trusted Computing, Trusted Third Parties, and Verified Communications," in IFIP International Federation for Information Processing. vol. 147, Y. Deswarte, F. Cuppens, S. Jajodia, and L. Wang, Eds., ed: Springer US, 2004, pp. 290-308.

[2] S. Bratus, P. C. Johnson, A. Ramaswamy, S. W. Smith, and M. E. Locasto, "The cake is a lie: privilege rings as a policy resource," in Proceedings of the 1st ACM workshop on Virtual machine security, Chicago, Illinois, USA, 2009, pp. 33-38.

[3] D. Trcek, "A formal apparatus for modeling trust in computing environments," Mathematical and Computer Modelling, vol. 49, pp. 226-233, January 2009.

[4] C. R. Bevan, "Human to Computer Trust in Urban Pervasive Computing," PhD Thesis, University of Bath, 2011.

[5] Trusted Computing Group. (2012, 29 Nov 2012). Trusted Platform Module Specifications in Public Review. Available: http://www.trustedcomputinggroup.org/resources/trusted_platform_module_specifications_in_public_ review

[6] Trusted Computing Group. (2007, Dec 28). TCG Specification Architecture Overview. Available: http://www.trustedcomputinggroup.org/files/resource_files/AC652DE1-1D09-3519ADA026A0C05CFAC2/TCG_1_4_Architecture_Overview.pdf

[7] M. Abadi and T. Wobber, "A Logical Account of NGSCB," in Lecture Notes in Computer Science. vol. 3235, D. Frutos-Escrig and M. Nunez, Eds., ed: Springer Berlin Heidelberg, 2004, pp. 1-12-.

[8] Microsoft. (2003, 29 Nov 2012). NGSCB - Trusted Computing Base And Software Authentication. Available: www.microsoft.com/resources/ngscb/documents/ngscb_tcb.doc

[9] Microsoft. (2003, 29 Nov 2012). Security Model for the Next-Generation Secure Computing Base. Available: http://www.microsoft.com/resources/ngscb/documents/ngscb_security_model.doc

[10] Microsoft. (2003, 29 Nov 2012). NGSCB - Hardware Platform for the Next-Generation Secure Computing Base. Available: http://www.microsoft.com/resources/ngscb/documents/FNGSCBhardware.doc

[11] M. Peinado, Y. Chen, P. England, and J. Manferdelli, "NGSCB: A Trusted Open System," in Lecture Notes in Computer Science. vol. 3108, H. Wang, J. Pieprzyk, and V. Varadharajan, Eds., ed: Springer Berlin Heidelberg, 2004, pp. 86-97-.

[12] S. Setapa, M. A. M. Isa, N. Abdullah, and J. L. A. Manan, "Trusted computing based microkernel," in Computer Applications and Industrial Electronics (ICCAIE), 2010 International Conference on DOI 10.1109/ICCAIE.2010.5771164, 2010, pp. 1-4.

[13] G. Heiser, J. Andronick, K. Elphinstone, G. Klein, I. Kuz, and L. Ryzhyk, "The road to trustworthy systems," in Proceedings of the fifth ACM workshop on Scalable trusted computing, Chicago, Illinois, USA, 2010, pp. 3-10.

[14] X.-W. Nie, D.-G. Feng, J.-J. Che, and X.-P. Wang, "Design and Implementation of Security Operating System Based on Trusted Computing," in Machine Learning and Cybernetics, 2006 International Conference on DOI - 10.1109/ICMLC.2006.258997, 2006, pp. 2776-2781.

[15] B. Parno, "Bootstrapping trust in a "trusted" platform," in Proceedings of the 3rd conference on Hot topics in security, San Jose, CA, 2008, pp. 1-6.

[16] J.-P. Steghofer, R. Kiefhaber, K. Leichtenstern, Y. Bernard, L. Klejnowski, W. Reif, T. Ungerer, E. Andre, J. Hahner, and C. Muller-Schloer, "Trustworthy Organic Computing Systems: Challenges and Perspectives," in Lecture Notes in Computer Science. vol. 6407, B. Xie, J. Branke, S. M. Sadjadi, D. Zhang, and X. Zhou, Eds., ed: Springer Berlin Heidelberg, 2010, pp. 62-76. 


\title{
ENERGY EFFICIENT LOAD BALANCED ROUTING PROTOCOL FOR WIRELESS SENSOR NETWORKS
}

\author{
Alghanmi Ali Omar and ChongGun Kim* \\ Department of Computer Engineering, Yeungnam University, South Korea \\ alghanmia88@gmail.com, cgkimeyu.ac.kr
}

\begin{abstract}
Due to the enormous applications of wireless sensors, the research on wireless sensor networks remains active throughout the past two decades. Because of miniaturization of sensor nodes and their limited batteries, the energy efficiency and energy balancing are the demand in-need to extend the life time of sensor networks. This study proposes an energy-aware directional routing protocol for stationary wireless sensor network. The routing algorithm is non-table driven, destination aware and packet forwarder nodes are selected on the basis of admissible heuristic logical distance, and packet forwarding direction is also determined in very simplistic method. The algorithm is designed for 1-hop, 2-hop and '2-hop \& 1-hop combine' communication method. The energy balancing mechanism of this paper is based on two state thresholds and simulation result shows its superiority over the existing directional routing protocols of wireless sensor networks.
\end{abstract}

\section{KEYWORDS}

Wireless Sensor Network, Routing Protocol, Energy Efficient, Load Balancing, Directional Routing, Stationary Topology .

\section{INTRODUCTION}

Wireless sensor network (WSN) is an emerging communication technology for environmental monitoring and target tracking. It has numerous applications and placements from public to military usages and from underwater to space shuttle placements. A wireless sensor networks consists a number of sensor nodes, those are wirelessly communicated to each other and cooperatively pass data towards the base station to accomplish their dispensed responsibilities. The sensor nodes of WSN is small in size, and it consists of tiny dimension of battery for power supply, small memory chip to store data and routing table, and radio interface to send and receive signals. As the sensor nodes have limited battery power, it is not feasible or possible to recharge the batteries of sensors, such as the sensors of underwater sensor networks, battle field sensors, natural disaster prevention and monitoring sensors and implantable bio-sensors. So, energy efficient communication methods are indispensable for WSNs [2].

Routing and data disseminations are the focal causes of energy consumption of WSNs. For effective routing protocols efficient data dissemination can reduce unbalanced energy consumption of sensor nodes in a significant amount. The nodes of sensor networks can be stationary with respect to environment or can be mobile with dynamic environmental perspective [11]. As a sensor node has small memory capacity, so the large routing table driven routing procedures are not suitable for WSNs. Thus, this paper proposed an energy balanced non-table driven routing protocol for stationary WSNs.

David C. Wyld et al. (Eds) : CCSIT, SIPP, AISC, PDCTA, NLP - 2014

pp. 153-167, 2014. (C) CS \& IT-CSCP 2014

DOI : $10.5121 /$ csit.2014.4213 
Sensor nodes of a wireless sensor networks generally work as a unit of a system to complete certain obligations. Shutdown of any sensor nodes from the network creates data deficiency, and as a result the whole sensor network produces an erroneous result, incorrect and imperfect vision of environment and network is became paralyzed. In some applications, like in biomedical sensor networks, the consequence of paralyzed network cases death penalty. A routing method, which ensures balanced consumption of energy among the sensor nodes of the WSNs is essential.

\section{RELATED WORKS}

Energy efficiency is not only an issue of wireless sensor networks, it also a challenging issues in all forms of networks to meet the green communication requirements. Efficient routing protocol ensures the efficient and energy-aware communication in wireless sensor networks. Routing protocols of wireless sensor networks have been studied in reference [1] with introducing some challenges and future directions. Partial differential equation based geographical routing is proposed in reference [2]. The model is dependent on a central node, which collects the position information, residual energy information and then determines the routing path based on the proposed algorithm. The proposal is based on centralized control unit, which is not suitable for the WSNs, where there is no central node. A cluster [3][4] based and threshold sensitive routing protocol is presented in reference [5], where the authors consider power availability, nodes position, and reachability factors to determine the routing path by using cluster head. Though this proposal achieved energy efficiency but the proposal didn't concentrate on networking life time.

A hybrid routing protocol for WSNs is presented in reference [6], which allows a comprehensive information retrieval of environmental analysis and facilitate users to query of past, present and future data. This is also an application specific and cluster based routing protocol, which is focused on efficient path finding by maintaining energy-efficiency but not concerning about network life time. Some other cluster based routing protocol also proposed in reference [7] and [8]. Greedy perimeter stateless routing approach for wireless networks is proposed in reference [9], where it considers the position of source and destination to send data packets, they also presented better results than shortest-path and ad-hoc routing protocols in respect of routing protocol overhead, packet delivery rate and path length. They didn't consider energy efficiency and energy balancing issues in their routing protocols. The security gaps, and possible attacks of wireless sensor networks routing are studied in reference [10], the study presented the countermeasures and challenges of designing routing protocol with ensuring security of the data packets travelling through huge nodes of WSNs.

Power efficient topologies for sensor networks are presented in reference [11], where the authors proposed directional source aware routing protocol (DSAP) and deploy it in different 2D and 3D static network topologies to study power efficient network topology. Though the presented DSAP minimizes the energy consumption of the nodes of considered networks, but DSAP could not ensure energy balancing among the nodes of WSNs.

\section{SYSTEM MODEL}

The system model of the proposed energy efficient and load balanced routing protocol (EELBRP) for wireless sensor network is discussed in this section. Wireless sensors are deployed in various patterns based on application requirements. This paper considers that the deployment of sensor nodes follows two-dimension (2D) topology as on DSAP. The neighbour nodes are defined on the basis of 1-hop communication model and 2-hop communication model. 


\subsection{1-Hop Communication Model}

In 1-hop communication model, shown in figure 1, a sensor node has maximum eight neighbours within direct communication range. Between the node and the neighbours nodes can participate in transmissions, receptions and forwarding of data. When a node transmits packet to a neighbour node other eight neighbours can overhear the packet. In transmission, a node expenses energy for running transmitter circuits $\left(E_{T x_{-} \text {circuit }}\right)$ and also expenses energy for sending packets to distance d1 that is one hop distance amplification energy $\left(E_{T x_{-} a m p l i f i e r}\right)$ cost. Thus, to transmit $b$ bits of packet to its 1-hop neighbour, transmitter node expenses total $E_{T x_{-} \text {tcost }}$ energy by equation (1).

$$
E 1_{T x_{-} \text {trost }}(b)=E 1_{T_{\text {m_circuit }}} * b+E 1_{\text {Tx_amplifier }} * b * d_{1}^{2}
$$

As all the 1-hop neighbour nodes from the sender, overhear the $b$ bits, $n_{l}$ shows the number of neighbours except the receivers. The total overhearing energy cost of all neighbour nodes $\left(E_{R x_{-} \text {tcost }}\right)$ is equivalent to the total energy consumption of $n_{1}$ receiver circuits ( $E_{R x_{-} \text {circuit }}$ ) as formulated in equation (2).

$$
E_{\text {Ris_tegst }}(b)=n_{1} * E_{\text {lix_circuit }} * b
$$

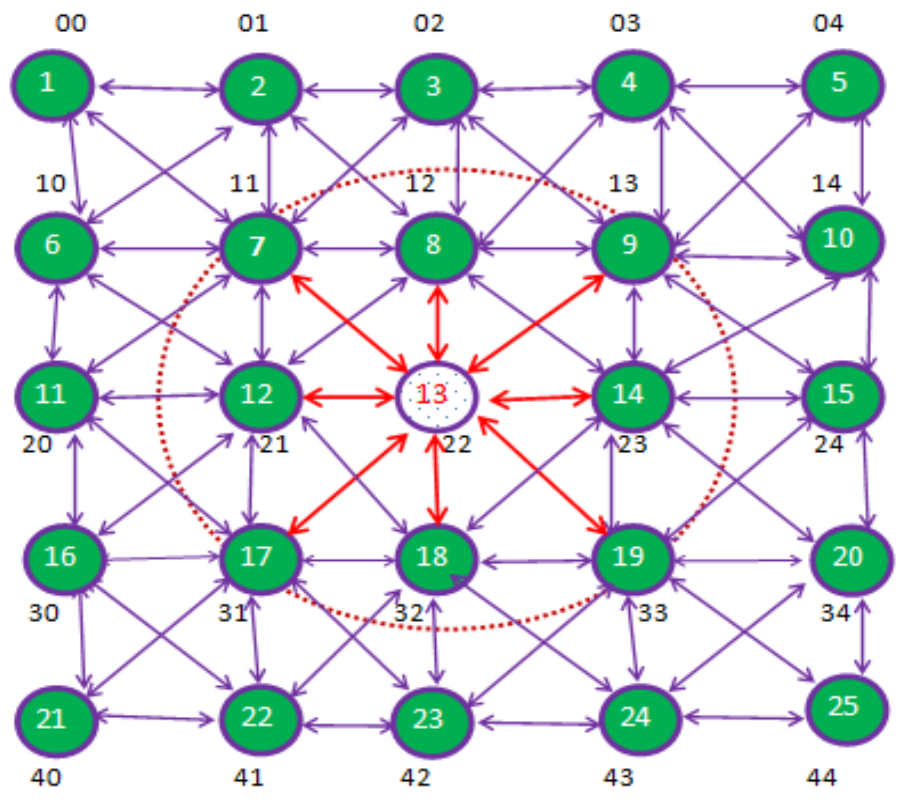

Figure 1. 1-Hop communication model for EELBRP

\subsection{2-Hop Communication Model}

The 2-hop communication model for the proposed EELBRP is shown at figure 2, where sensor node has maximum twenty neighbours. When any node transmits packet other neighbours can overhear the packet.

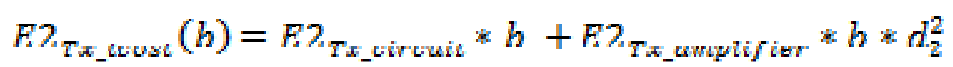

In case of transmission, a node not only expenses energy for running transmitter circuits $\left(E_{T x_{-} \text {circuit }}\right)$ but also expenses energy for sending packets to distance $d_{2}$ that is amplification energy ( $E_{T x_{-} \text {amplifier }}$ ) cost to transmit packet over $d_{2}$ distance. Thus, to transmit $b$ bits of packet to any of its 2-hop neighbours, the transmitter node expenses total $E_{T x_{-} \text {tcost }}$ energy by equation (3). 
In 2-hop communication model, all the 1-hop neighbour nodes $n_{l}$, and the nodes situated far from distance $d_{1}$, also distance $d_{2}$ nodes $n_{2}$; overhears the $b$ bits of packet, so the total overhearing energy cost of all neighbour nodes $\left(E 2_{R x_{-} t c o s t}\right)$ is equivalent to the total energy consumption of $\left(n_{1}\right.$ $+\mathrm{n}_{2}$ ) receiver circuits ( $\left.E_{R x_{-} \text {circuit }}\right)$ as formulated in equation (4).

$$
E 2_{\text {Rx wuse }}(b)=\left(n_{1}+n_{2}\right) * E_{\text {Rxx wir will }} * b
$$

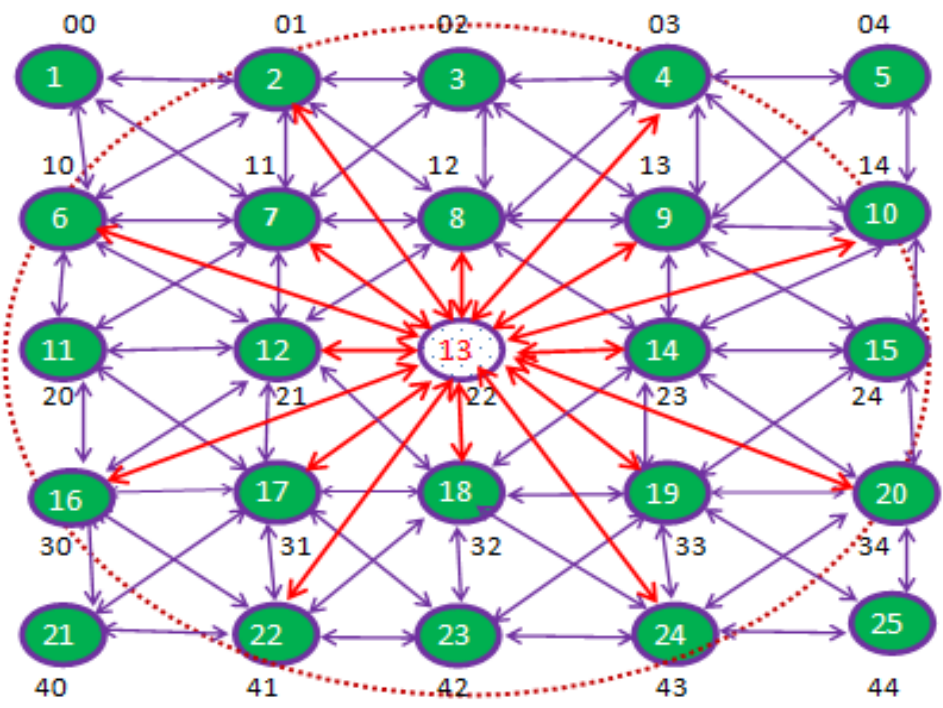

Figure 2. 2-Hop communication model for EELBRP

\section{Routing Protocol for Energy EFficiency ANd LoAd Balancing}

In this section, the detail discussion of the proposed EELBRP is presented.

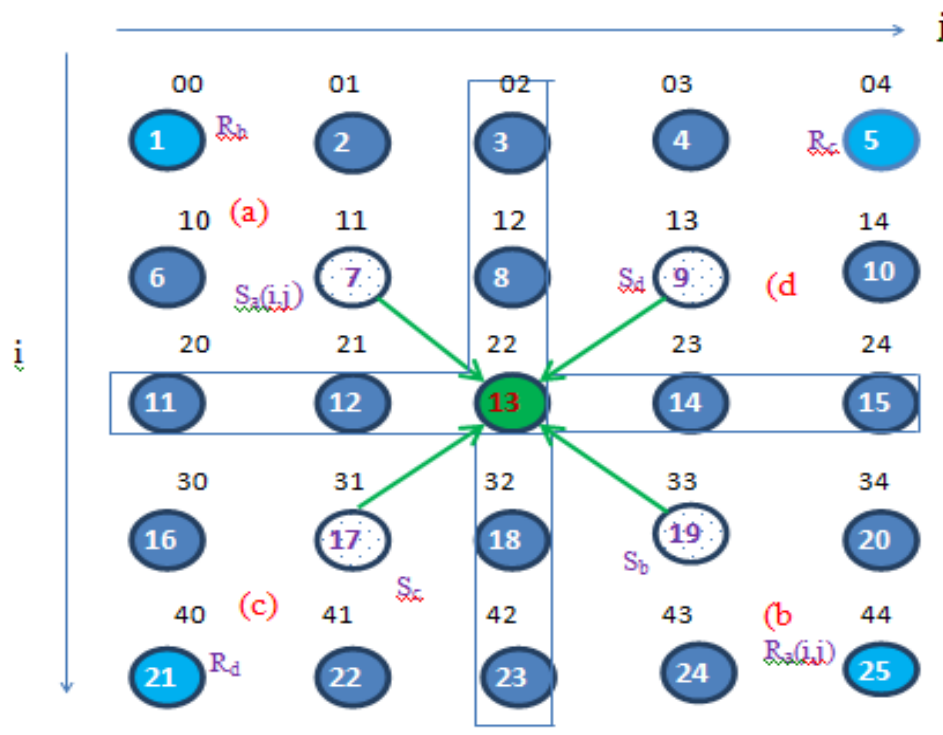

Figure 3. Direction of destination node from source node in 1-hop communication model

The proposed EELBRP is a directional routing protocol, corresponding to the direction of receiver node; it selects the forwarder nodes from a feasible set of forwarders. 


\subsection{Routing Procedure for 1-Hop communication Model}

Consider the case (a) of figure 3 , where the sender node $S_{a}$ is 7 and destination node $R_{a}$ is 25 . To find out the direction of the receiver node, the Cartesian coordinates of sender node $S_{a}(i, j)$ and the destination node $R_{a}(i, j)$ is compared according to algorithm 4.1.

In algorithm 4.1, two direction variables are D1 and D2, and L, R, D, U represent of left, right, down and up respectively. Based upon the determined values D1 and D2, the feasible set of forwarder are selected and called as the adjacent list for 1-hop communication Adi_PR1 and that is Adj_PR1_a $=\{\mathrm{D} 1 \mathrm{R} 1, \mathrm{R} 1, \mathrm{D} 1, \mathrm{U} 1 \mathrm{R} 1, \mathrm{D} 1 \mathrm{~L} 1\}=\{13,8,12,3,11\}$ is for case $(\mathrm{a})$, where $\mathrm{u} 1=\mathrm{i}-1$, $\mathrm{d} 1=\mathrm{i}+1, \mathrm{~L} 1=\mathrm{j}-1$, and $\mathrm{r} 1=\mathrm{j}+1$.

Similarly, the adjacent list of prioritized nodes for 1-hop communication of case (b), (c), (d) is defined respectively as

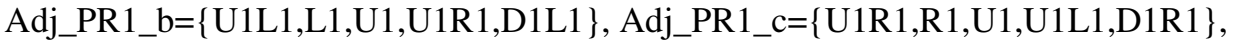
Adj_PR1_d= $\{$ D1L1,L1,D1,D1R1,U1R1 $\}$.

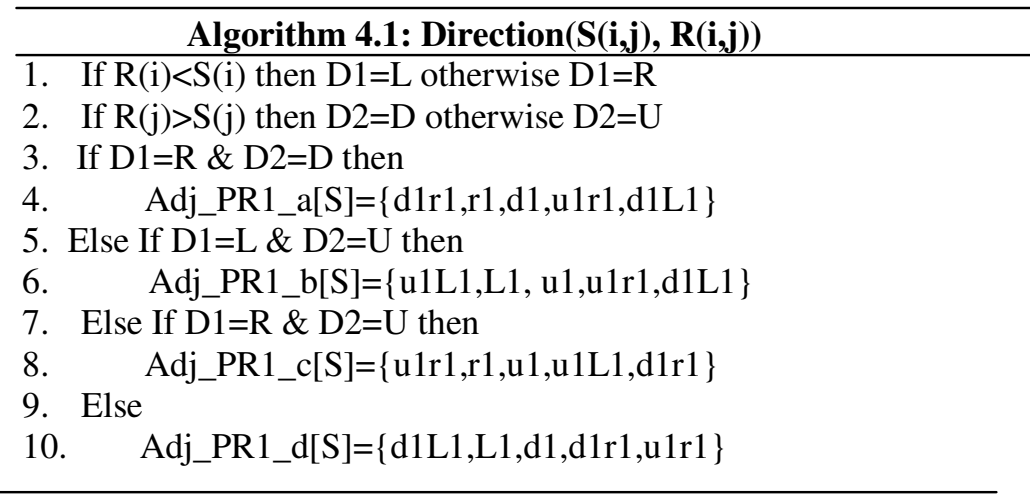

Among the feasible set of forwarder of Adj_PR1, the best suitable forwarder is selected based upon the logical distance or air distance or admissible heuristic distance from probable forwarder to the destination, which is determined in algorithm 4.2. As the assumed WSNs deployed using 2D and stationary topology, each and every node has a logical Cartesian coordinates to find out logical distance $L d$ using equation number (5)

$$
\operatorname{Ld}[v] \leftarrow \sqrt{(R(i)-v(i))^{2}+\left(R(j)-v(j)^{2}\right.}
$$

Where $R(i, j)$ is the logical coordinate of receiver node and $v(i, j)$ is the logical coordinate of feasible forwarder.

\begin{tabular}{l}
\hline Algorithm 4.2: $\operatorname{Relax}(\mathbf{R}, \mathbf{v})$ \\
\hline $\operatorname{Ld}[\mathrm{v}] \leftarrow \sqrt{\left(R(0)-v(i)^{2}+\left(R()-v(j)^{2}\right.\right.}$ \\
\hline
\end{tabular}

After finding the logical distances from list of feasible forwarder nodes to receiver nodes, the node with minimum distance is selected as the most suitable forwarder, maintain a minimum priority queue of suitable forwarders. The routing method is presented in algorithm 4.3 is an alias of Dijkstra's algorithm, where $T$ stands for topology or given WSNs , N[T] represents the nodes of the topology $T$, and variable Route gradually stores the routing path from sender to receiver. 


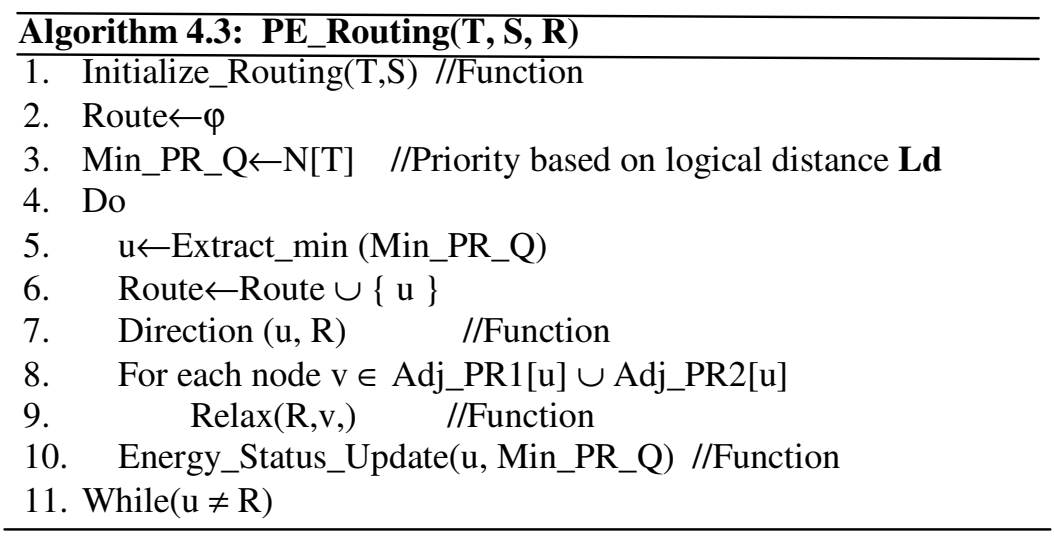

Algorithm 4.3, consists a procedure for initialization of nodes residual energy variable Res_energy as maximum energy of the batteries of sensor nodes at starting time. Logical distances from sender to all other nodes are also initialized as infinite at the beginning of routing procedure in algorithm 4.4 .

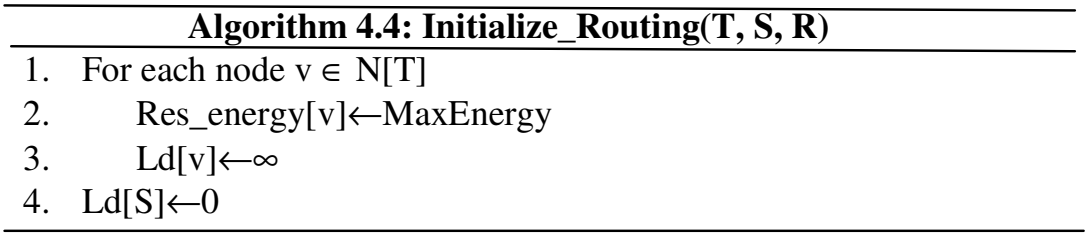

Algorithm 4.3, also consists an energy status updating function for dynamic updates of sensor nodes residual energy after sending and receiving of data packets. For changing the energy status of receiver (forwarder) nodes, all the 1-hop neighbor nodes (Adj1) are considered because of the broadcasting nature of WSNs. Algorithm 4.5 is designed with the energy model formulated in equation (1) and (2).

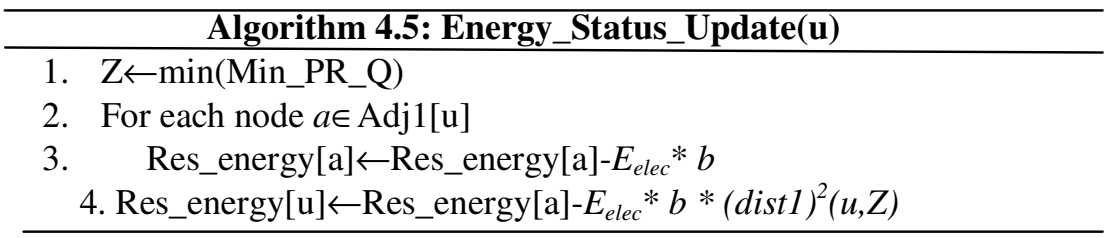

\subsection{Routing Procedure for 2-Hop\& 1-hop Combine Communication Model}

The routing procedure for 2-hop \& 1-hop combine communication model of proposed EELBRP is little bit different from 1-hop communication model. In this combine communication model sender node always tries to send packets to its one hop neighbours first for forwarding. The direction of destination node will need to be determined here also in combine model. The procedure of determining the direction of receiver node is defined in algorithm 4.6.

Consider the case (a) of figure 4, where sender node $S_{a}$ is 7 and receiver node $R_{a}$ is 25 , we follow almost same procedure to determine the direction as we discussed in section 4.1, but the feasible set of forwarders are also included not only the 1-hop but also the 2-hop nodes, which we call the adjacent list of prioritized nodes for 2-hop communication Adi_PR2 and that is

Adj_PR2_a $=\{d 2 \mathrm{r} 1, \mathrm{r} 2 \mathrm{~d} 1, \mathrm{~d} 2, \mathrm{r} 2\}=\{18,14,17,9\}$, where $\mathrm{d} 2=\mathrm{i}+2, \mathrm{r} 2=\mathrm{j}+2, \mathrm{u} 2=\mathrm{i}-2$, and $\mathrm{L} 2=\mathrm{j}-2$. Similarly, the adjacent list of prioritized nodes for 2-hop communication of case (b), (c), (d) is 


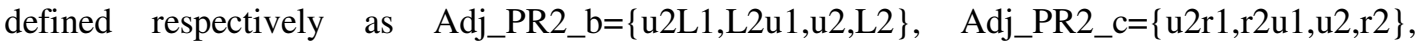
Adj_PR2_d $=\{$ d2L1,L2d1,d2,L2 $\}$.

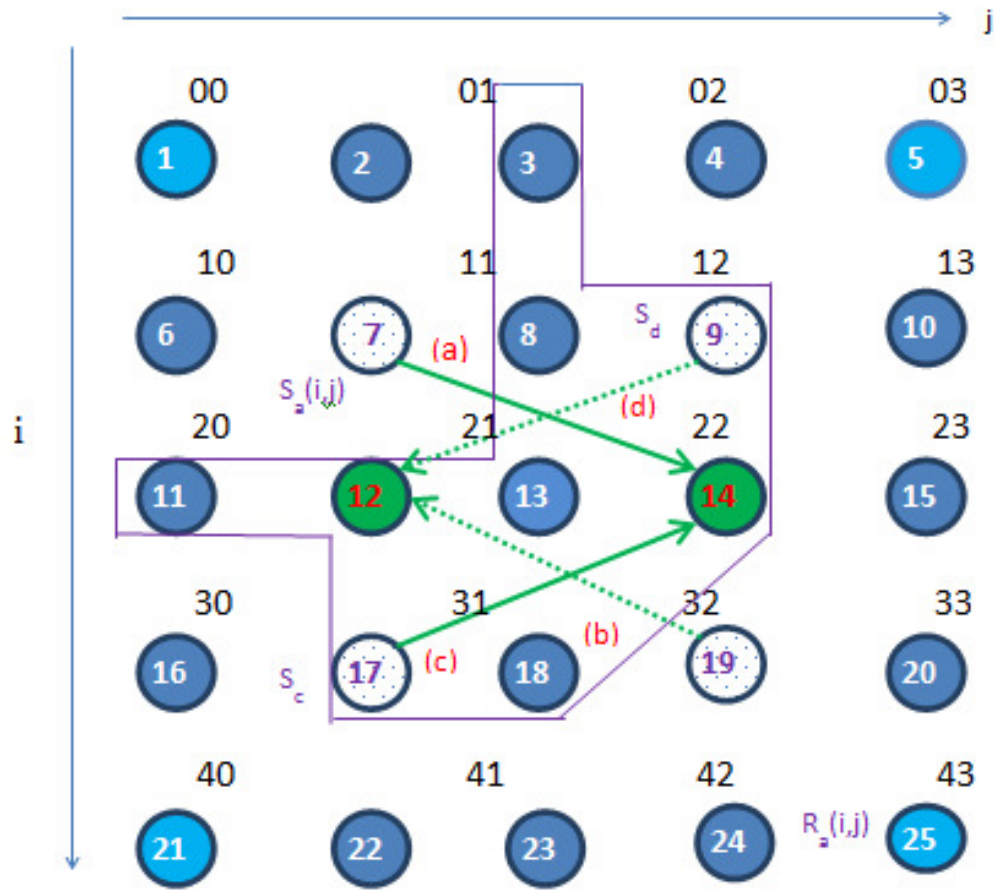

Figure 4. Direction of destination node corresponding to source node in 2-hope \& 1-hop combine communication model

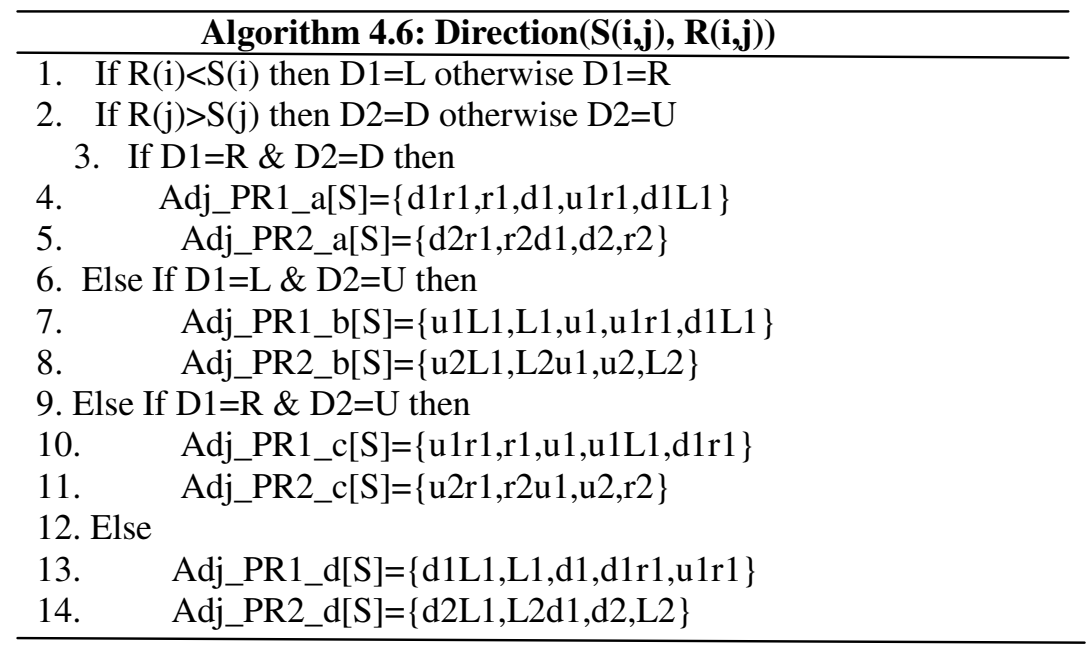

Among the feasible set of forwarders of Adj_PR1 and Adj_PR2, the best suitable forwarder is selected based upon the logical distance from probable forwarder to destination, which is determined in algorithm 4.7, the logical distance of destination node from 1-hop feasible forwarders will get higher priority as their distance is customized with negative sign. 


\begin{tabular}{|c|}
\hline Algorithm 4.7: Relax $(R, v)$ \\
\hline 1. IF $\mathrm{v} \in$ Adj_PR2[u] \\
\hline 2. $\quad \mathrm{Ld}[\mathrm{v}] \leftarrow\left(\sqrt{ }(R(i)-v(i))^{2}+(R(j)-v(j))^{2}\right)$ \\
\hline 3. Else \\
\hline $\mathrm{Ld}[\mathrm{v}] \leftarrow-\sqrt{(R(i)}-v(i))^{2}+(R(j)-v(j))^{2}$ \\
\hline
\end{tabular}

Among the determined logical distances, the feasible forwarder node with minimum distance is selected as the suitable forwarder towards the receiver. Algorithm 4.8 presents the suitable forwarder selection procedure considering both of the feasible set of forwarders of Adj_PR1 and Adj_PR2 at line number 8 .

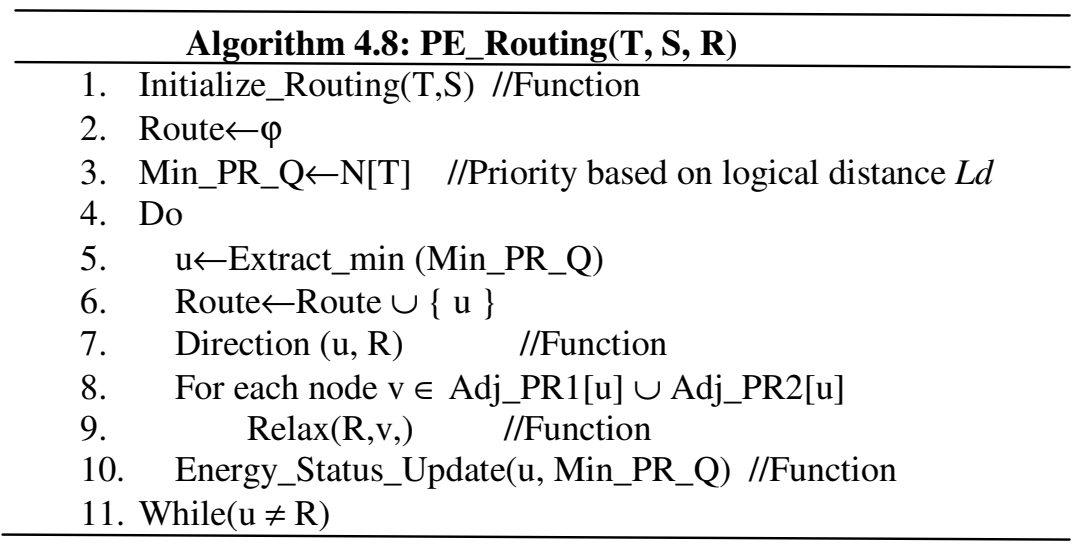

Algorithm 4.8 also calls the energy status updating procedure, which is presented in algorithm 4.9. For changing the energy status of receiver (forwarder) nodes, all the 1-hop neighbor nodes (Adj1) and 2-hop neighbor nodes (Adj2) are considered because of the broadcasting nature of WSNs. Algorithm 4.9 is designed with the energy model formulated in equation (3) and (4).

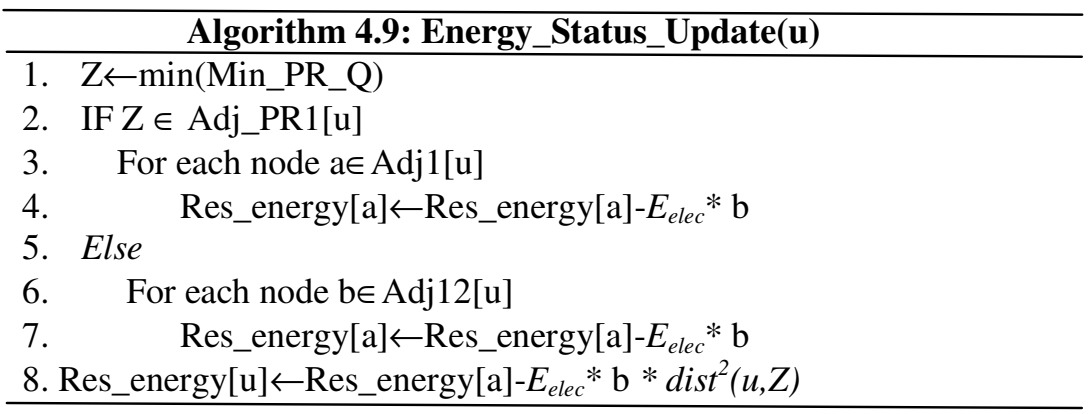

\subsection{Routing Procedure for 2-Hop\& 1-Hop Combine Communication Model with Energy-Awareness}

To design an energy aware and energy balanced routing protocol the 2-hop \& 1-hop combine communication model of figure 4 is used. As the Relax function is the controlling function of suitable forwarder node selection, the Relax function is designed accordingly in algorithm 4.10 to select forwarders in energy-efficient manner. If residual energy of the prioritized neighboring node of sender is greater than the threshold-1 (th1) then just follow 2-hop \& 1-hop combine routing procedure to select forwarders, but if residual energy of all of the prioritized neighboring node of sender is less than the threshold-1 (th1) but greater than threshold-2 (th2) then determine energy ratio of each of the node, and update logical distance variable based on energy ratio. So, in such case the routing algorithm 4.8 tries to balance energies of the whole network between 
threshold-2. Finally, if the residual energy of the entire prioritized neighboring node of sender is less than the threshold-2 then algorithm determines, which node consumes less energy i.e which node has much residual energy and update logical distance accordingly. By this procedure, the node with highest residual energy among feasible forwarder list Adj_PR1 and Adj_PR2 placed in the 1st position of the minimum priority queue as well as selected as the best suitable forwarder for balancing energies among its neighbors.

\begin{tabular}{|c|c|}
\hline \multicolumn{2}{|c|}{ Algorithm 4.10: Relax $(R, v)$} \\
\hline 1. IF Res_e & energy $[v]>$ th1 \\
\hline 2. $\quad \mathrm{IF} v \in$ & $E$ Adj_PR2[u] \\
\hline 3. & $\mathrm{Ld}[\mathrm{v}] \leftarrow-\left(\sqrt{(R(x)-v(i))^{2}+(R(y)-v(j))^{2}}\right)$ \\
\hline Else & \\
\hline 5. & $\mathrm{Ld}[\mathrm{v}] \leftarrow \sqrt{(R(x)-v(i))^{2}+\left(R(y)-v(j)^{2}\right.}$ \\
\hline 6. Else IF & th2<Res_energy $[\mathrm{v}]<$ th 1 \\
\hline 7. & $\mathrm{Ld}[\mathrm{v}] \leftarrow \frac{\text { mgsidual Engrgy [v] }}{\text { initinl }}$ \\
\hline 8. Else & \\
\hline 9. & Ld[v] $\leftarrow$ Initial_Energy[v]-Res_Energy[v] \\
\hline
\end{tabular}

\section{SIMULATION RESULTS}

To evaluate the performance of the proposed EELBRP algorithm, the MATLAB R2010a simulation tools are used with $\mathrm{C}++$ simulation program to analyze the energy efficiency and balancing status. The simulation scenario is presented in Table 1 .

Table 1. Simulation scenario for performance study of EELBRP algorithm

\begin{tabular}{|l|l|l|}
\hline Simulation Parameters & Symbols & Values \\
\hline Topology & $2 D$ & $\begin{array}{l}\text { 8 neighbors within } 1 \text { hop } \\
20 \text { neighbors within } 2 \text { hop }\end{array}$ \\
\hline Number of nodes & $n \times n$ & $11 \times 11$ \\
\hline $\begin{array}{l}\text { Horizontal (or vertical) } \\
\text { distance between two nodes }\end{array}$ & $d_{1}$ & 0.5 meters \\
\hline Packet size & $b$ & 512 bits \\
\hline Total number of packets & $P$ & 10,000 \\
\hline Transmitter circuitry energy & $E_{T x_{-} \text {circuit }}$ & $50 \mathrm{~nJ} / \mathrm{bit}$ \\
\hline Transmitter amplification energy & $E_{T x_{-} \text {amplifier }}$ & $100 \mathrm{pJ} / \mathrm{bit} / \mathrm{m}^{2}$ \\
\hline Receiver circuitry energy & $E_{R x_{\_} \text {circuit }}$ & $50 \mathrm{~nJ} / \mathrm{bit}$ \\
\hline Threshold-1 & th1 & $53 \mathrm{~J}$ \\
\hline Threshold-2 & th2 & $25 \mathrm{~J}$ \\
\hline
\end{tabular}

The performance of proposed EELBRP is studied using two essential performance metric of wireless sensor networks i.e energy efficiency and network lifetime. Figure 5 and figure 6 shows the performance of the EELBRP algorithm without using energy balancing procedure. Between these two figures, the figure 5 shows the residual energies of each of the $11 \mathrm{X} 11=121$ nodes of the wireless sensor networks after handling 10,000 packets of 512 bits and following 1 hop communication method. The total energy consumption is 4228.360266 Joules and residual energy is balancing between 27 to 95 Joules. 
Whereas, figure 6 shows the residual energies of each nodes of the wireless sensor networks after handling same number of packets of 512 bits and following 2 hop communication method. The total energy consumption is 5082.707723 Joules and residual energy is balancing between

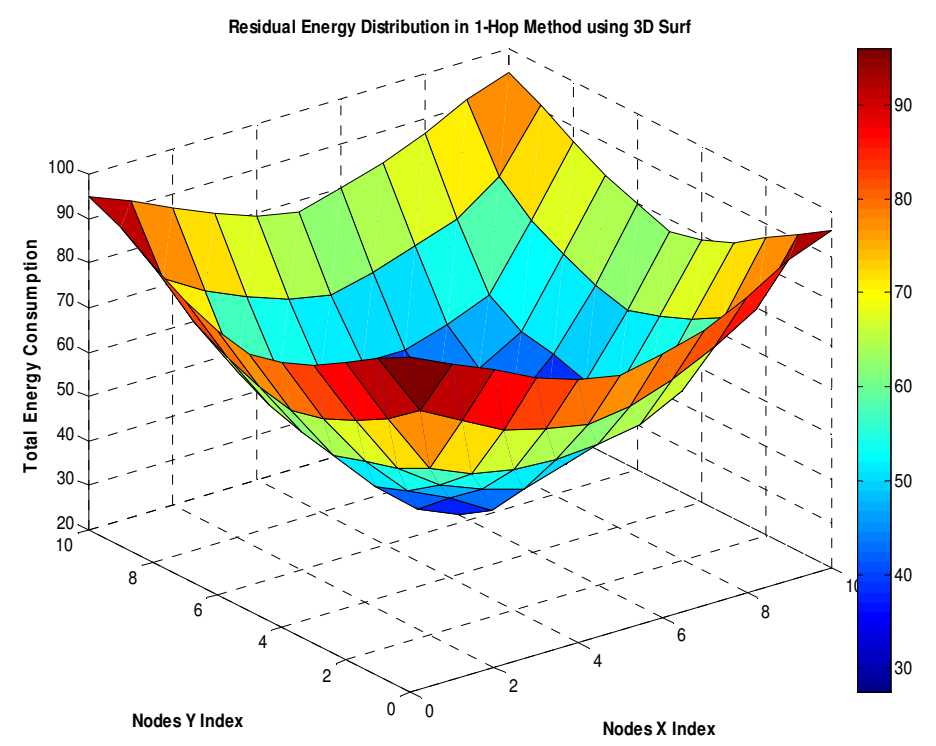

Figure5. Residual Energy of each nodes using EELBRP (1-Hop case)

21 to 90 Joules. In comparison between 1-hop and 2-hop communication method, following the EELBRP without energy balancing procedure, 1-hop communication method consumes less energy than 2-hop communication method. In comparison of energy balancing performance, in both 1-hop and 2-hop cases the energy difference remains around 70 Joules, which means some of the network nodes dies very firstly then other nodes, and network become paralyzed with short period of time i.e performance of EELBRP without energy balancing procedure is not impressive in respect to network lifetime.

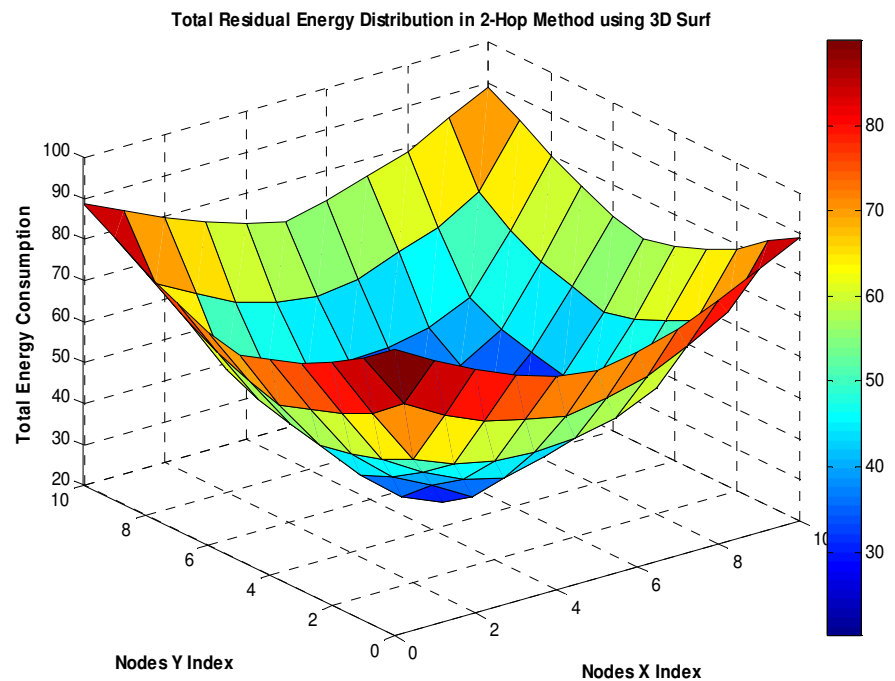

Figure 6. Residual Energy of each nodes using EELBRP (2-Hop Case) 
Figure 7 shows the residual energy distribution the nodes of the wireless sensor networks following the proposed EELBRP with energy balancing procedure, where 1-hop and 2-hop combine communication model is used rationally to improve network lifetime. The total energy consumption is 4660.138730 Joules and residual energy is balancing between 65 to 92 Joules. In comparison to 1-hop communication method, it consumes little bit more energy but in comparison to 2-hop communication method, it consumes less energy.

But in comparison of energy balancing performance, the residual energy difference among the sensor nodes of the network is 27 Joules, which means the EELBRP with energy balancing procedure enhances network lifetime significantly with sacrificing limited total network energy.

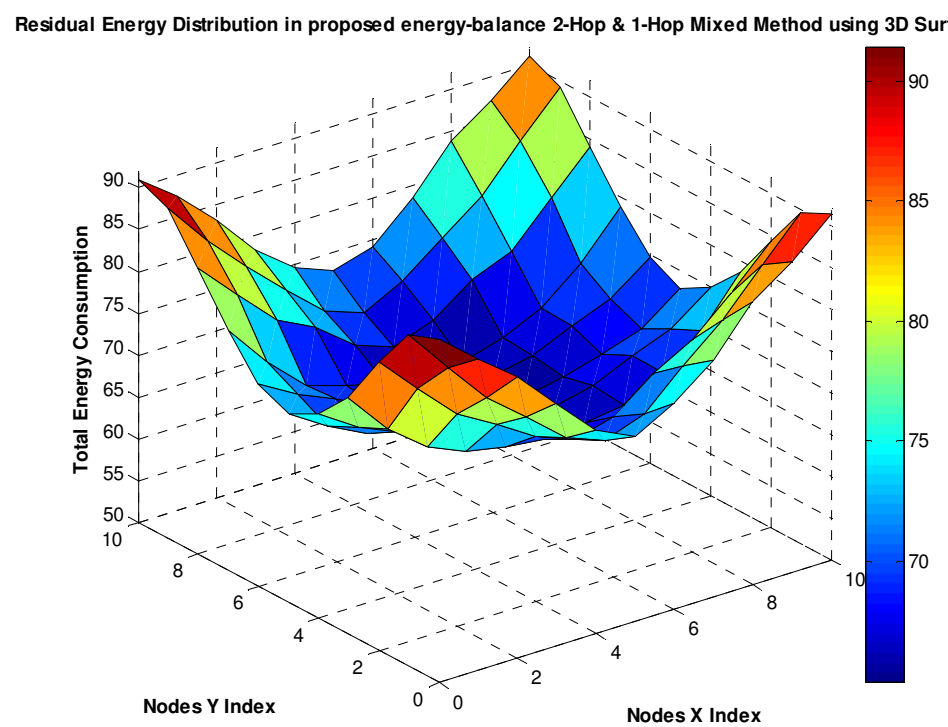

Figure 7. Residual Energy of each nodes using EELBRP (2-Hop \& 1-Hop combine with energy balancing procedure, where threshold $1=53$ Joules and threshold $2=25$ Joules)

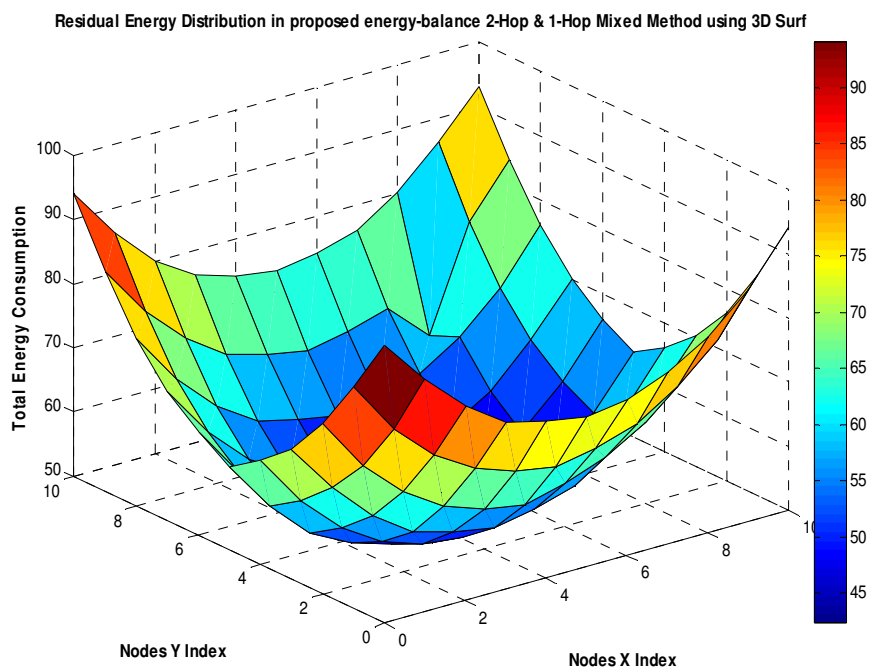

Figure 8. Residual Energy of each nodes using EELBRP (2-Hop \& 1-Hop combine with energy balancing procedure, where threshold $1=40$ Joules and threshold $2=5$ Joules) 
The performance of the EELBRP with energy balancing procedure is also evaluated using different threshold values. Figure 7 9 shows the network energy performance graph of various threshold conditions, where the threshold 1 with 53 joules and threshold 2 with 25 joules clearly shows their suitability and justification of threshold selection in figure 7.

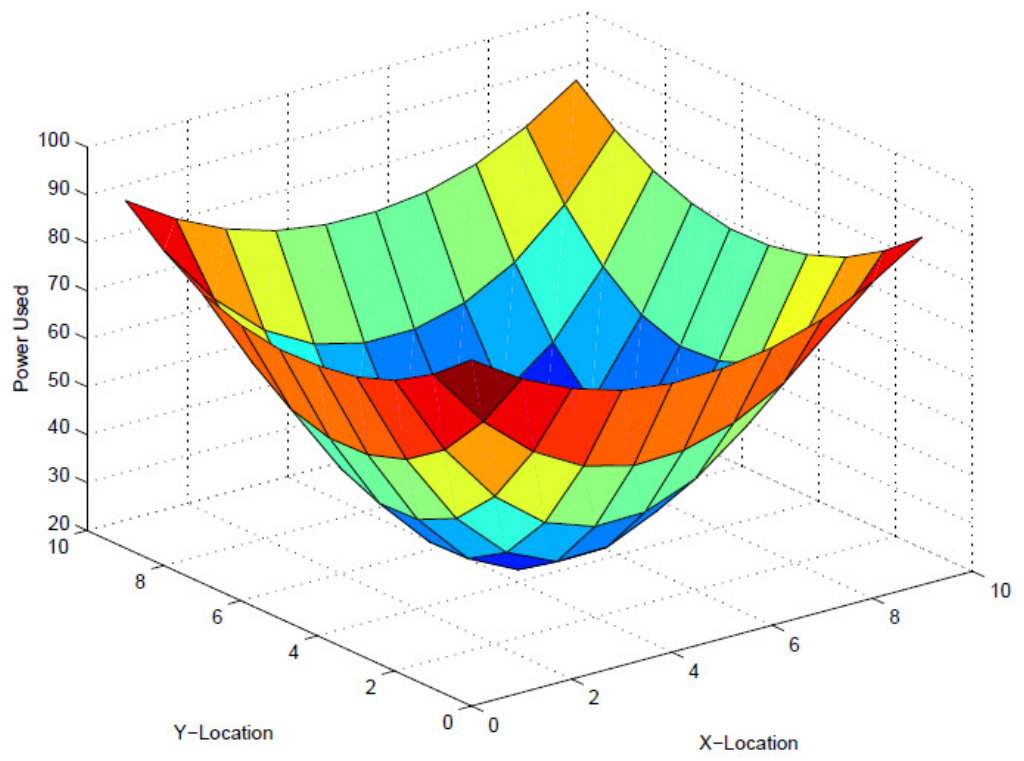

Figure 9. Residual Energy of each nodes using EELBRP (2-Hop \& 1-Hop combine with energy balancing procedure, where threshold $1=60$ Joules and threshold $2=35$ Joules)

Table 2. Comparison of Total Energy Consumption among 2-hop, 1-hop \& "2-hop + 1-hop combine with energy balancing" method

\begin{tabular}{|c|c|c|c|c|}
\hline $\begin{array}{l}\text { Threshold } 1 \text { and } \\
\text { threshold } 2\end{array}$ & $\begin{array}{c}\text { Energy } \\
\text { Consumption }\end{array}$ & $\begin{array}{c}\text { 2-hop } \\
\text { communication } \\
\text { Method }\end{array}$ & $\begin{array}{c}\text { 1-hop } \\
\text { communication } \\
\text { method }\end{array}$ & $\begin{array}{l}\text { Proposed } \\
\text { EELBRP } \\
\text { with energy } \\
\text { balancing }\end{array}$ \\
\hline \multirow[t]{2}{*}{$40 \mathrm{~J}$ and $5 \mathrm{~J}$} & $\begin{array}{c}\text { Total } \\
\text { Consumption }\end{array}$ & $5082.70772 \mathrm{~J}$ & $4228.36026 \mathrm{~J}$ & $\begin{array}{r}4357.4121 \mathrm{~J} \\
\text { Less } \\
\text { consumption } \\
\end{array}$ \\
\hline & $\begin{array}{l}\text { Residual } \\
\text { Energy } \\
\text { distribution }\end{array}$ & 21 90 & 27 95 & $\begin{array}{r}42 \sim 94 \\
\text { Imbalance } \\
\text { energy } \\
\end{array}$ \\
\hline \multirow[t]{2}{*}{$53 \mathrm{~J}$ and $25 \mathrm{~J}$} & $\begin{array}{c}\text { Total } \\
\text { Consumption }\end{array}$ & $5082.70772 \mathrm{~J}$ & $4228.36026 \mathrm{~J}$ & $\begin{array}{r}4660.1387 \mathrm{~J} \\
\text { Moderate } \\
\text { consumption } \\
\end{array}$ \\
\hline & $\begin{array}{l}\quad \text { Residual } \\
\text { Energy } \\
\text { distribution }\end{array}$ & 21 90 & 27 95 & $\begin{array}{r}65 \sim 92 \\
\text { Balanced } \\
\text { energy }\end{array}$ \\
\hline \multirow[t]{2}{*}{$60 \mathrm{~J}$ and $35 \mathrm{~J}$} & $\begin{array}{c}\text { Total } \\
\text { Consumption }\end{array}$ & $5082.70772 \mathrm{~J}$ & $4228.36026 \mathrm{~J}$ & $\begin{array}{l}\text { 4983.0121J } \\
\text { Higher } \\
\text { consumption } \\
\end{array}$ \\
\hline & $\begin{array}{l}\text { Residual } \\
\text { Energy } \\
\text { distribution }\end{array}$ & $21 \sim 90$ & $27 \sim 95$ & $\begin{array}{l}\text { 69 94 } \\
\quad \text { Balanced } \\
\text { energy }\end{array}$ \\
\hline
\end{tabular}

The performance study of EELBRP algorithm is summarized in table 2, where the proposed EELBRP with energy balancing strategy shows its energy balancing power with moderate energy 
consumption. The 2-hop and 1-hop communication method using EELBRP without energy balancing procedure shows similar energy consumption disregarding threshold values because EELBRP without energy balancing procedure has no option of selecting energy threshold values.

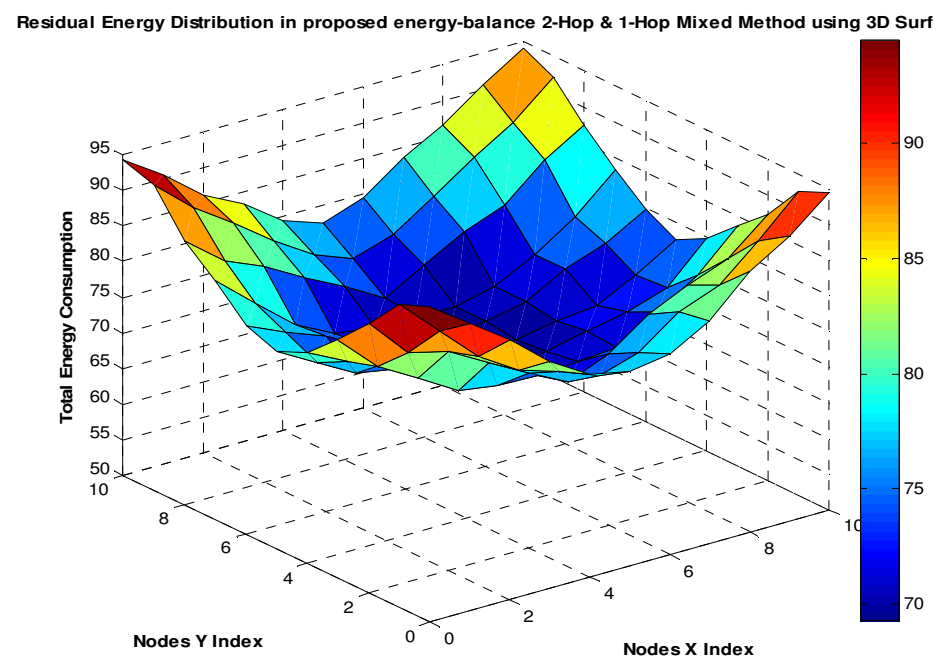

Figure 10. Residual energy of each node using DSAP method

The performance of proposed EELBRP algorithm is also compared with the existing benchmarked DSAP routing algorithm, because DSAP also directional and concerned about stationary network topologies like EELBRP. Following 1-hop communication and using same simulation scenario of EELBRP simulation study, the residual energy graph is presented in figure 10. The figure shows that DSAP balancing residual energy distribution between 20 to 89 Joules and the total energy consumption is 4612.215061 Joules, whereas EELBRP balanced energy between 27 to 95 and consumes 4228.360266 joules of energy shown in figure 5 .

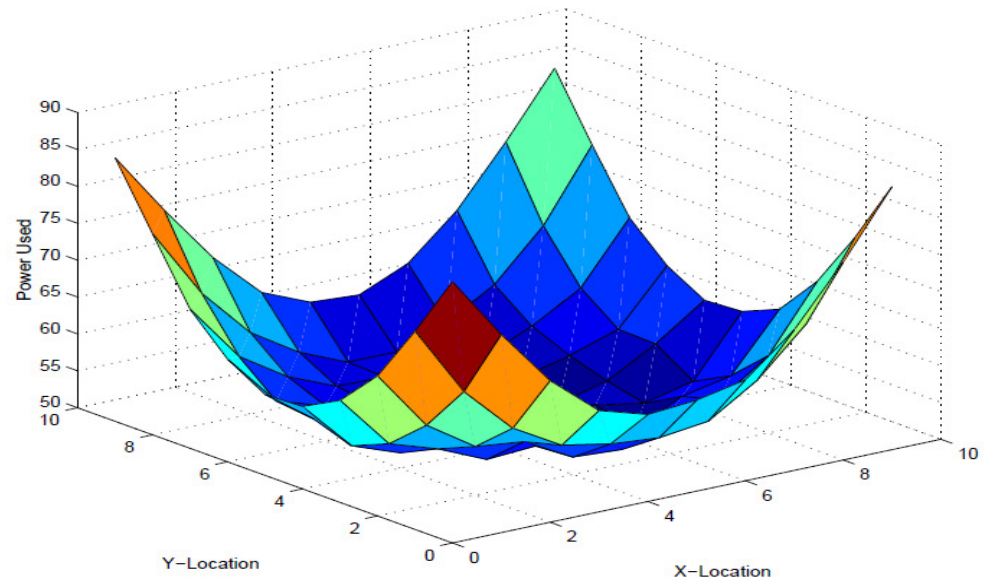

Figure 11. Residual energy graph of power-aware DSAP method

The main cause behind the lower performance of DSAP is the weakness in determination of directional values (DV) i.e considering networks connection similar like wired networks rather than using the broadcasting nature of wireless networks effectively. Conversely, EELBRP 
determine the logical distance from destination node to feasible forwarder nodes and select the best forwarder to forward data packets towards the destination node.

The performance of power-aware DSAP is also presented in figure 11. The residual energy distribution the nodes of the wireless sensor networks following power-aware DSAP is 50 to 83 and total energy consumption is 4978.735009 Joules. On the other hand, the proposed EELBRP with energy balancing procedure keeps lower bound of residual energy to 65 and upper bound to 92, so energy is more balanced in EELBRP and it enhances the network lifetime as well while maintaining less energy consumption 4660.138730 Joules than power-aware DSAP.

\section{Conclusions}

Development of energy aware and energy balanced routing protocol for stationary wireless sensor networks is the major significant contribution of this study. Considering the ability of dynamic energy changing capability of sensor nodes, the presented routing protocol is simulated in 1-hop, 2-hop and "2-hop \& 1-hop combine" communication method. The proposed EELBRP shows improved performance by accepting and combining with energy balancing and energy efficiency perspectives. As the proposed routing protocol is directional, the use of directional antenna surely reduces the energy consumption of the network in a significant rate.

\section{ACKNOWLEDGEMENTS}

This work has been funded by the BK21+ program of the National Research Foundation (NRF) of Korea.

\section{REFERENCES}

[1] Al-Karaki, Jamal N., and Ahmed E. Kamal. "Routing techniques in wireless sensor networks: a survey." Wireless Communications, IEEE 11.6 (2004): 6-28.

[2] Kalantari, Mehdi, and Mark Shayman. "Energy efficient routing in wireless sensor networks." Proc. of Conference on Information Sciences and Systems. 2004.

[3] Mary Wu, InTaek Leem, Jason J. Jung, and ChongGun Kim. "A resource reuse method in cluster sensor networks in ad hoc networks." In Intelligent Information and Database Systems, pp. 40-50. Springer Berlin Heidelberg, 2012.

[4] Mary Wu, Byung Chul Ahn Kim, and Chong Gun,. "A channel reuse procedure in clustering sensor networks." Applied Mechanics and Materials 284, pp: 1981-1985, 2013.

[5] Manjeshwar, Arati, and Dharma P. Agrawal. "TEEN: A Routing Protocol for Enhanced Efficiency in Wireless Sensor Networks." IPDPS. Vol. 1. 2001.

[6] Manjeshwar, Arati, and Dharma P. Agrawal. "APTEEN: A Hybrid Protocol for Efficient Routing and Comprehensive Information Retrieval in Wireless Sensor Networks." ipdps. Vol. 2. 2002.

[7] Heinzelman, Wendi Rabiner, Anantha Chandrakasan, and Hari Balakrishnan. "Energy-efficient communication protocol for wireless microsensor networks."System Sciences, 2000. Proceedings of the 33rd Annual Hawaii International Conference on. IEEE, 2000.

[8] Heinzelman, Wendi B., Anantha P. Chandrakasan, and Hari Balakrishnan. "An application-specific protocol architecture for wireless microsensor networks." Wireless Communications, IEEE Transactions on 1.4 (2002): 660-670.

[9] Karp, Brad, and Hsiang-Tsung Kung. "GPSR: Greedy perimeter stateless routing for wireless networks." Proceedings of the 6th annual international conference on Mobile computing and networking. ACM, 2000.

[10] Karlof, Chris, and David Wagner. "Secure routing in wireless sensor networks: Attacks and countermeasures." Ad hoc networks 1.2 (2003): 293-315.

[11] Salhieh, Ayad, et al. "Power efficient topologies for wireless sensor networks. "Parallel Processing, International Conference on, 2001.. IEEE, 2001. 


\section{AUTHORS}

\section{*Corresponding Author:}

ChongGun Kim received the B.E and the M.E degree in Electronic Engineering from YeungNam University, Korea in 1981 and 1987 respectively. He received $\mathrm{Ph} . \mathrm{D}$ degree in Computer Science and Information Mathematics from University of ElectroCommunications, Tokyo, Japan, in 1991. He has been serving as a Professor at the Department of Computer Engineering, Yeungnam University since March 1991. In his doctoral work, he was engaged in research on load balancing of distributed computing systems. He was a visiting scholar of Virginia Tech., USA and UCSC, USA in 1996 and 2003. His current research interests include Computer Networks, Wireless Sensor Networks, Network Security, and Distributed Computing Systems.

Alghanmi Ali Omar received the B.S. degree in Computer Science from Kyung Hee University, Korea in 2012. He received King Abdullah Scholarship from Riyadh, Saudi Arabia for his Bachelor studies in Computer Science. Currently, he is pursuing his master degree in Computer Engineering at Yeungnam University in Korea. He also received King Abdullah Scholarship for his Master studies in Computer Engineering. His areas of research interests include Wireless Sensor Networks, Network Security, Information Security, and Energy Balancing Mechanism.
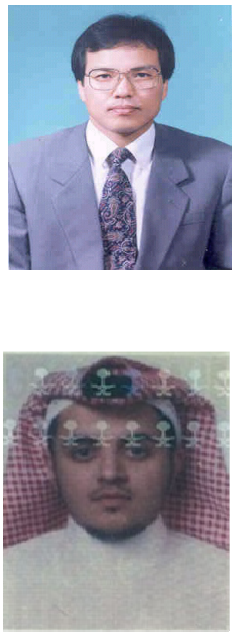
INTENTIONAL BLANK 


\title{
USER PREFERRED COLOR COMBINATION DESIGN USING INTERACTIVE GENETIC ALGORITHM
}

\author{
Tad Gonsalves and Ayumi Kawai \\ Department of Information \& Communication Sciences, \\ Sophia University, Tokyo, Japan \\ t-gonsalesophia.ac.jp \\ a2-kawai@nri.co.jp
}

\begin{abstract}
Selecting the right combination of colors in designing a product is often a difficult task. In this paper, the authors propose a decision support system for modeling the color combination design suited for personal preference using interactive Genetic Algorithm (iGA). iGA is different from the traditional GA in that it leaves the evaluation of the objective function to the personal preferences of the user. The iGA interactive system is capable of creating an unlimited number of color combination options taking into consideration the preferences of the user. The user chooses and indicates his/her preferences and directs the process of optimizing the color combination. The final outcome of this user-system interaction is a color-combination design which the user might have not even imagined before he/she began interacting with the system. Users find the system efficient, user-friendly and responding in real-time without causing any user fatigue.
\end{abstract}

\section{KEYWORDS}

Interactive Genetic Algorithm, kansei engineering, optimization, user-preference .

\section{INTRODUCTION}

A lot of design engineering is dedicated to the creation and mixing of colors. Engineering firms devote a lot of their time and resources studying the effects that colors produce on the customers. Kansei engineering, a branch of engineering developed in Japan, concerns itself with the design and manufacture of commercial products taking into consideration the preferences and feelings of the users and customers. Kansei (literally, "emotional / affective engineering") aims at the development or improvement of products and services by translating customer's psychological feelings and needs into product's design domain [4]. Kansei has been used in the design of digital cameras [1], color mapping [2], cars, computers, etc. [4]. Implementing the Kansei is quite a subjective task. Therefore, a decision support system which can model the designs automatically and present a plan of the various designs without heavily biasing the design is necessary. Interactive Genetic Algorithms (iGAs) are optimization techniques used to estimate customers' Kansei [17]. Interactive Genetic Algorithm (iGA) differs from the conventional Genetic Algorithm (GA) in the evaluation of the fitness function. In the conventional GA, the objective function is numerically defined and the algorithm cycles through the stipulated number of generations, while in iGA the evaluation of the objective function is left to the subjective

David C. Wyld et al. (Eds) : CCSIT, SIPP, AISC, PDCTA, NLP - 2014

pp. 169-174, 2014. (C) CS \& IT-CSCP 2014

DOI : $10.5121 /$ csit.2014.4214 
judgment of the user. The termination criterion, too, is in the hands of the user. The aim of GA is to numerically optimize the objective function. In contrast, the aim of iGA is to "optimize" a given design so that it approximates the subjective feelings and judgment of the user. iGA has been used to in designing the layout and lighting of rooms [3], fashions [5], web sites [8], hearing aids [10], and music composition [13], all according to the users' preferences.

This paper deals with the application of iGA in designing color combination of products taking into consideration the user's tastes and preferences. When it comes to the choice of colors or the choice of combination of colors, the user does not normally have a clear idea of the colors. What the user can do is use the color-palette that is available in Microsoft software products and produce a color-combination product of his own choice. However, this would be left entirely to the subjective judgment and creative ability of the user. The result would be a color-combination object designed from a limited number of options - the options being created by the user himself/herself. But in the iGA interactive system, it is the system that creates an unlimited number of options taking into consideration the preferences of the user. The system suggests options based on the preferences indicated by the user. The user then chooses and indicates his preferences and directs the process of optimizing the color combination. The final outcome of this user-system interaction is color-combination design which the user might have not even imagined before he/she began interacting with the system.

This paper is organized as follows: Section 2 describes the interactive Genetic Algorithm. Section 4 presents the iGA system interface along with the internal representation of iGA. Section four presents the various simulation scenarios and results. The paper ends with a short conclusion indicating the direction of future research using iGA.

\section{INTERACTIVE GENETIC ALGORITHM}

The basic difference between GA and iGA can be explained as follows: In the conventional GA, the algorithm automatically cycles through the generations every time producing better fit individuals and stops when the pre-defined number of generations is reached. The user cannot intervene in the evolutionary process from the start to finish. However, in the case of iGA, it is the intervention of the user that drives the evolutionary process.

The application of the iGA is to assist the user in designing the color combination of his/her liking that we propose in this paper consists of the following steps (Figure. 1):

Generation: A population consisting of $\mathrm{N}$ number of individuals is randomly generated. The genes of the individual chromosomes are controlled in such a way that the phenotype colors produced by them are in the range of the initially selected by the user.

Display: The different color combinations and the background produced by the above chromosomes is displayed to the user as shown in Figure. 2 and Figure. 3.

Evaluate: This step is what distinguishes the iGA from the conventional GA. In conventional GA the fitness function is quantified and clearly defined. GA evaluates the solutions based on their fitness function. In iGA program, the evaluation of the fitness of the individual solutions is left to the subjective judgment of the user.

Selection: In the iGA system, there is usually an interface through which the user interacts with the system. The system depicts a feasible number of choices on the interface. From these, the user selects items of his/ her preference usually by using the interface radio buttons and check boxes. 
Crossover: iGA works on the solutions selected by the user and making cross-overs or recombinations to produce new solutions ("offspring") which are closer to the user's preference than the solutions of the previous generation.

Mutation: Some of the newly produced solutions in the above step are subjected to (minor) random mutations. Mutations produce variation in the iGA population and prevent premature convergence of the algorithm.

Termination criterion: This step, too, is very different from the conventional GA. There is no objective pre-defined termination criterion. It rests on the subjective judgment of the user. The user normally terminates the interactive program when he/she is satisfied by the design suggested by the interactive system.

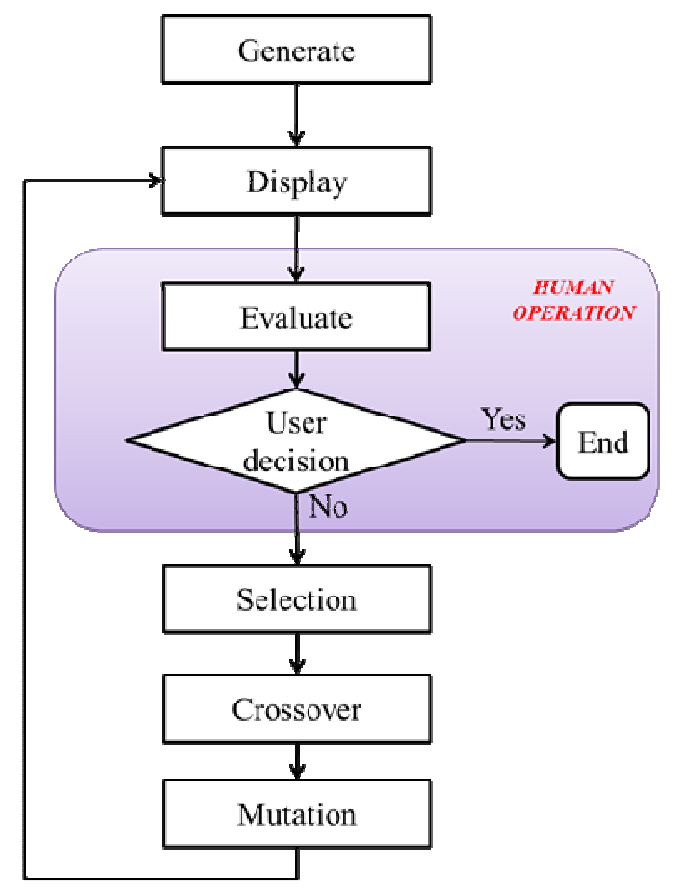

Figure 1. The iGA process

\section{IGA SYSTEM INTERFACE AND IGA}

The interface of the color combination decision support system is shown in Figure. 2 and Figure. 3. It consists of four different objects and a background. The user sets in motion the interactive process by choosing a color for each of the four objects and the background. Several radio buttons are provided on the interface. The user accordingly selects whether the color of a given object and/or the background is to be made lighter or darker or more vivid in the next iGA generation.

The color-combination choices indicated by the user on the interface represent a "solution" in the iGA representation. The aim of the iGA system is to optimize this combination taking into consideration the lighter/darker/vividness of the user selected colors. For this, the system evaluates the fitness of the current solution. It then generates the next solution by incorporating the user's preferences and with a certain amount of randomness. The user further selects the color combination suggested by the system. The user-iGA interaction cycles continue till the user is satisfied by the color combination suggested by the system. If at any time the user is not satisfied 
with the suggestions made by the system, the use can roll-back by pressing the "Back" button. He /she can then proceed from a particular color combination of his/her preference recorded in the history of the interaction.

\section{SIMULATION RESULTS}

The following sub-sections describe two important results: user interaction with the system to produce color combination of his/her liking and the user response about the user-friendliness, efficiency and real-time response of the proposed iGA system.

\subsection{User interaction with iGA system}

One of the convenient uses of the iGA system is to produce tiny variations in the original color combination design indicated by the user. As shown in Figure. 2 the system suggests slight variations in colors, not too far away from the original colors. This procedure can be repeated many times to obtain a desired color combination centered round a theme.

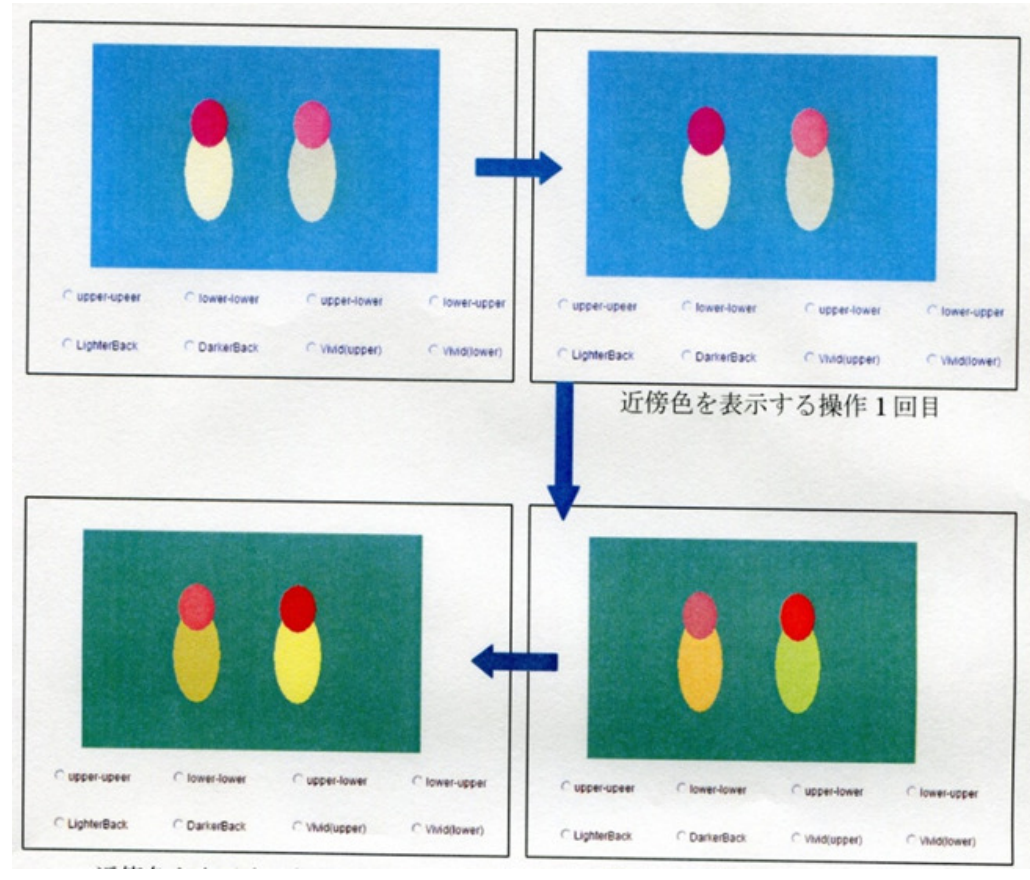

Figure 2. Slight variations in color combination design
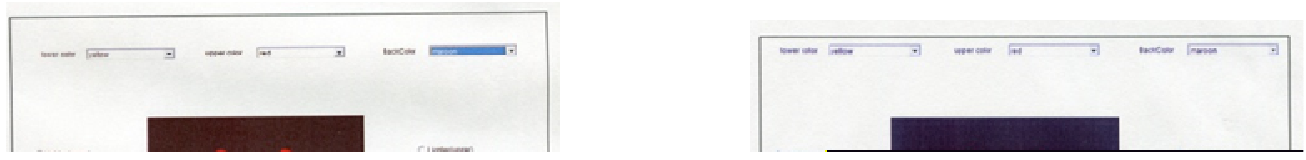

Figure 3. Optimal color combination design produced by the user 
Fig. 4 shows the ideal use of the iGA system. The user chooses different colors for the four distinct objects in the foreground. He/she also chooses the color of the background. In Fig. 4 the user has selected light yellow and red color variations on the foreground objects and maroon color for the background. The user then requests the system to produce lighter objects against a darker background. The final result is obtained after about 5-6 iterations.

\subsection{User feedback}

The author also conducted a small-scale questionnaire with ten volunteers. The results are tabulated in Table 1. On the whole the users find the system easy to use and user friendly. Most of the users could determine a color combination they had in mind within 5-6 cycles of interaction with the system. One of the well-known shortcomings of an interactive system driven by Evolutionary Algorithms is the user fatigue. The user has to interact with the system repeatedly until he/she arrives at an acceptable solution. Several studies have been conducted to measure the user fatigue and ameliorate the situation [11]. In the proposed iGA color combination interactive system all the users' fatigue score was very low. Moreover, they were satisfied with the real-time response of the system.

Table 1: User Questionnaire response

\begin{tabular}{|c|c|c|c|c|}
\hline & Excellent & Good & Fair & Poor \\
\hline $\begin{array}{c}\text { Attainment of preferred color } \\
\text { combination }\end{array}$ & 7 & 3 & 0 & 0 \\
\hline $\begin{array}{c}\text { System accuracy and } \\
\text { efficiency }\end{array}$ & 8 & 2 & 0 & 0 \\
\hline $\begin{array}{c}\text { User-friendliness } \\
\text { Real-time response }\end{array}$ & 8 & 3 & 1 & 0 \\
\hline User-fatigue control & 7 & 2 & 0 & 0 \\
\hline
\end{tabular}

\section{CONCLUSIONS}

In this paper, the author has proposed a decision support system of modeling color combination designs suited for personal preferences by using the interactive Genetic Algorithm (iGA). iGA is an extended version of GA in which the evaluation of the objective function is left to the subjective judgment of the user. The user interacts with the iGA system to design a color combination of his/her preference. The system can produce remarkable color combinations ranging from a slight variation to the original design suggested by the user to highly sophisticated designs that have never been imagined by the user. Simulation experiments show a variety of color combination designs produced by the user interacting with the system. The results of the user questionnaire show that the system is user-friendly, efficient, responds in real-time and does not cause any user-fatigue.

\section{REFERENCES}

[1] Chien-Cheng Chang, Factors influencing visual comfort appreciation of the product form of digital cameras. International jpurnal of Industrial Ergonomics 38 (2008) 1007-1016. 
[2] Y. Fukada, K. Sato, Y. Mitsukura, M. Fukumi, A Design of the Mapping Method of Color and KANSEI, RISP International Workshop on Nonlinear Circuits and Signal Processing (NCSP), pp.7376, China, 2007.

[3] Yosuke Fukada, Keiko Sato, Yasue Mitsukura and Minom Fukumi, The Room Design System of Individual Preference with IGA, International Conference on Control, Automation and Systems,Oct. 2007, Seoul, Korea.

[4] M. Iguchi, Kansei Information Processing, Japan, Ohmsha, 1994.

[5] H. S. Kim and S. B. Cho, "Application of interactive genetic algorithm to fashion design," Engineering Applications of Artificial Intelligence, vol. 13, no. 6, pp. 635 - 644, 2000.

[6] Chih-Chin Lai, andYing-Chuan Chen, A User-Oriented Image Retrieval System Based on Interactive Genetic Algorithm, IEEE Transactions on Instrumentation and measurement, vol.60, No. 10, pp. 3318-3325.

[7] Ali. M. Malkawi, Ravi S. Srinivasan, Yun K. Yi, Ruchi Choudhary, "Decision Support and Design Evolution: Integrating Genetic Algorithms, CFD and Visualization," Automation in Construction, pp.33-44, 2005.

[8] A. Oliver, N. Monmarche, G. Venturini, Interactive Design of Web Sites with a Genetic Algorithm, Proceedings of the IADIS International Conference WWW/Internet, pp.355-362, Lisbon, Portugal.

[9] K. Sato, Y. Mitsukura, M. Fukumi, "Designing of the Color KANSEI Information Map Considering the Individual Characteristic by Using Multivariable Analysis," SICE-ICASE International Joint Confer ence, FP23-4, pp.3706-3710, Busan, Korea, 2006.

[10] H. Takagi, "Application of interactive evolutionary computation to optimal tuning of digital hearing aids," in Int'l Conf. on Soft Computing, IIZUKA'98, World Scientic, 1998.

[11] Takagi, H. ; Pallez, D., Paired comparison-based Interactive Differential Evolution, World Congress on Nature \& Biologically Inspired Computing, 2009. NaBIC 2009.

[12] Sun Yan, Wang Wanliang and Liu Xiaojian, An improved evaluation method for interactive genetic algorithms and its application in product design, 2010 IEEE Fifth International Conference on BioInspired Computing: Theories and Applications (BIC-TA).

[13] N. Tokui, H. Iba, Music Composition with Interactive Evolutionary Computation, in Proc. Generative Art 2000, International Conference on generative Art, Milan, Italy, 2000. 


\title{
PROCESS-DRIVEN SOFTWARE DEVELOPMENT METHODOLOGY FOR ENTERPRISE INFORMATION SYSTEM
}

\author{
Kwan Hee $\operatorname{Han}^{1}$ and Yongsun $\mathrm{Choi}^{2}$ \\ ${ }^{1}$ Department of Industrial \& Systems Engineering, \\ Engineering Research Institute, \\ Gyeongsang National University, Jinju, Korea \\ hankh@gnu.ac.kr \\ ${ }^{2}$ Department of System Management \& Engineering, Inje University, Korea \\ yschoidinje.ac.kr
}

\begin{abstract}
In today's process-centered business organization, it is imperative that enterprise information system must be converted from task-centered to process-centered system. Traditional software development methodology is function-oriented, in which each function manages its own data and it results in redundancy because data that belongs to one object are stored by several functions. Proposed in this paper is a process-driven software development methodology, in which business process is a major concern and workflow functionalities are identified and specified throughout the entire development life cycle. In the proposed methodology, the development process, modeling tools and deliverables are clarified explicitly. Proposed methodology can be a guideline to practitioners involved in enterprise software development, of which workflow is an essential part.
\end{abstract}

\section{KEYWORDS}

Business Process, Software Engineering, Enterprise Information System, Object-Oriented, System Development Methodology

\section{INTRODUCTION}

Nowadays the unpredictability of market changes, the growing product complexity and continuous pressure on costs force enterprises to develop the ability to respond and adapt to change quickly and effectively. To cope with these challenges, most enterprises are struggling to change their existing business processes into agile, product- and customer-oriented structures to survive in the competitive and global business environment. In today's dynamic business environment, the ability to improve business performance is a quintessential requirement for all enterprises [1].

David C. Wyld et al. (Eds) : CCSIT, SIPP, AISC, PDCTA, NLP - 2014

pp. 175-186, 2014. (C) CS \& IT-CSCP 2014

DOI : $10.5121 /$ csit.2014.4215 
Therefore, many enterprises have recently been adopting enterprise information systems such as ERP (Enterprise Resource Planning), WFM (Workflow Management) and PLM (Product Life cycle Management) system to attain their performance goals.

As business environment is being changed, substantial change is being also occurred in the development of enterprise information system. This is mainly due to the change of management stricture as well as change of software development methodology. First of all, many enterprises recognized that it is difficult to survive by Taylor's 'scientific management' under the rapid change of business environment. Therefore, to cope with these challenges, the concept of business process management (BPM) has been recently proposed. BPM is the identification, understanding, and management of business processes linked with people and systems and across organizations [2]. It is an approach to transform traditional functional organization to marketoriented process organization. So, it is needed that enterprise information system must be converted from task-centered to process-centered system. In other words, it is necessary to transfer from existing function-oriented information system supporting individual task to workflow management system integrating entire business process for specific customer satisfaction [3]. Therefore, workflow automation is emerging as an essential part of information system beyond supporting transactional task in the recent enterprise information system development.

With respect to software development methodology, 'software crisis' was occurred due to the ever-increasing complexity of software structure and rapid increase of software development and operational cost. To overcome this crisis, in the area of system development methodology, objectoriented development methodology is widely used as an alternative of existing structured system development methodology that revealed difficulties of systematic integration of each phase's deliverables and efficient development phase transition. Another limitation of existing software development methodology is that there is no common modeling method for developer and end user.

In summary, to align with current process-centered approach of enterprises, integrated software development methodology is needed to deal with workflow automation for efficient business process management and object-oriented modeling for systematic software design simultaneously.

The objective of this paper is to propose a process-driven object-oriented software development methodology by integrating business process modeling and information system modeling which were separately modeled in the conventional development of enterprise information system. Within proposed methodology, it is suggested that UML (unified modeling Language) is suitable to the business process modeling as well as software modeling.

The rest of the paper is organized as follows: Section 2 reviews current practices and limitations of typical software development methodologies. Section 3 describes a proposed process-driven software development framework. Finally, the last section summarizes results and suggests directions for future research. 


\section{LiMitATIONS OF CURRENT SOFTWARE DEVELOPMENT METHODOLOGIES}

Typical software development process for enterprise information system is as follows (Figure 1): (1) Systems analysis, (2) Systems design and (3) Systems implementation and operation.

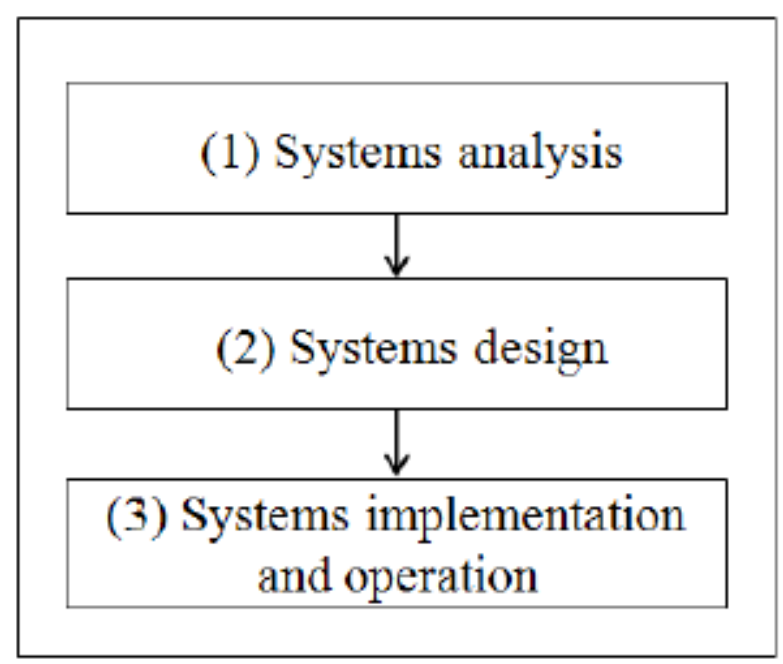

Figure 1. Conventional software development process

Business process is a collection of interrelated work tasks, initiated in response to an event, which achieves a specific result for the customer of the process [4]. Enterprise system consists of organizations performing these business activities.

Therefore, in order to develop enterprise information system, business process analysis is a prerequisite task. Current object-oriented software development process often omits this requirement or deals with this task in the system design phase (Phase-2) slightly.

The task of business process analysis is usually performed by business analysts under the name of business process modeling or workflow modeling during business process reengineering projects. Major modeling tools include IDEF0 [5], EPC [6] and Petri net [3], [7]. As described above, in spite of close relationship between business process modeling and software system modeling, these two tasks are typically performed separately in recent software development practices.

UML (Unified Modeling Language) is a de facto standard modeling tool for object-oriented software development, which was announced by OMG (Object management Group) in November, 1997. The current version of UML is 2.3 (May 2010). It was being rapidly adopted because of its graphical notation, which is readily understood, and a rich set of semantics for capturing key features of object-oriented systems.

Moreover, it was addressed that UML is also appropriate for business process modeling as well as information system modeling by virtue of its expressiveness, user-friendliness, and integration 
capability with information systems [8], [9]. Recently, there are considerable active approaches to UML-based business process modeling [8], [9], [10].

However, UML did not define development process since it is only a modeling tool intended for the purpose of software development. As an object-oriented development using UML, Unified process (UP) [11], [12] is widely adopted beyond OMT [13], Booch [14], Ericsson and objectory methodologies. The characteristics of UP are as follows: 1) it is a use case-centered process. 2) It is an architecture-centered evolutionary incremental approach. However, there are not a few substantial difficulties in applying UP to real software development projects in spite of its strengths such as software productivity increase, reusability increase and natural mapping to real world.

\section{PROCESS-DRIVEN SOFTWARE DEVELOPMENT METHODOLOGY}

Proposed process-driven software development methodology is based on the facts found in current development practices as follows: 1) it is difficult to transfer the deliverables of business process modeling to the process of enterprise information system development. 2) Though UML is used as a de facto standard modeling tool, there is no explicit guidelines about which UML diagram must be used appropriately in the specific development phase.

Since software development methodology is a systematic approach to successfully develop software system, so it must provide process, notation, and tools for software development in a consistent and integrated way. The process of proposed development methodology consists of 6 phases as follows (Figure 2):

(1) Business process analysis

(2) User Requirements analysis

(3) Structure Design

(4) Architecture Design

(5) Detail Design

(6) Implementation and Operation 


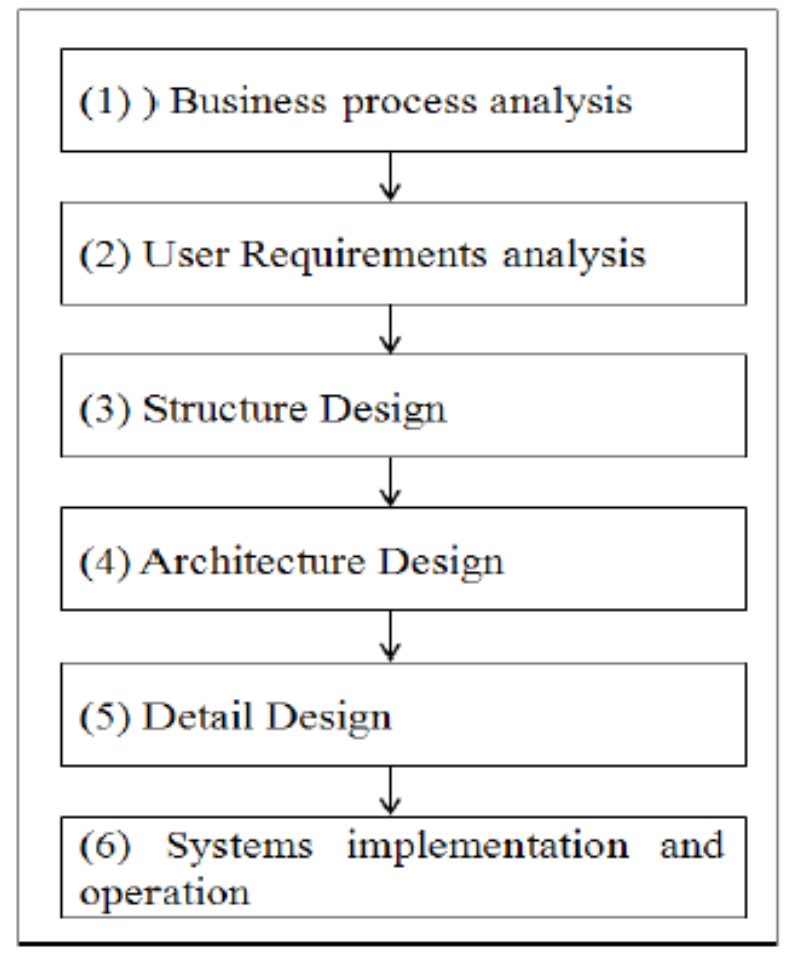

Figure 2. Process-driven software development process

Following subsections describe the framework of each development phase. This framework consists of 3 elements as follows: 1) development process, 2) modeling tool, 3) deliverables (called 'model')

\subsection{Business Process Analysis Phase}

Proposed methodology starts with business process analysis. The main purpose of this phase is to better understand the key mechanisms of existing business and to show the structure of an innovated business.

A business is a complex system, consisting of a hierarchical organization of departments and their functions. Thus, business process analysis focuses on the core business tasks and its key mechanisms. The central concept used for business process analysis is the business process, which describes activities within the business and how they relate to and interact with the resources in the business to achieve a goal for the process.

Before designing new processes, it should be clear which performance aspects of the business process are targeted for improvement. In most circumstances, it would be ideal if a redesign of a business process decreases the time required to handle customer needs, decreases the required cost of executing the business process, improves the quality of service delivered, and improve the ability to change the business process to react to variation.

The UML diagram used in this phase is an activity diagram. Among UML diagrams, the activity diagram is most suitable for business process modeling because of the following features: 1) it 
can describe more easily dynamic behaviors of business process and events triggering the process. 2) It can represent various workflow routing types such as sequential routing, join, split, iteration, and parallel routing suggested by WfMC (Workflow Management Coalition).

Deliverables in this phase are 'as-is' process and 'to-be' process model. As-is process model describes current aspects of business processes, which is a target of information system, whereas to-be process model describes desirable innovative process after installing new enterprise information system. Information systems are key enables to achieving process innovation or BPR (Business Process Reengineering).

In the activity diagram for describing business process, business object, which is an input and output of certain activity, must be specified explicitly. Business objects are categorized and specified as physical, document, drawing, data set objects by using UML stereotype notation. A swim-lane is divided by actor or organizational unit. During to-be process analysis, more attention is needed to the cross-swim-lane activity flow and the potential parallel processing activities.

\subsection{User Requirements Analysis}

The main purpose of this phase is to describe what a new system should do or what an existing system already does. A 'functional model', which is a deliverable, is built through an iterative process mainly by discussion between the system developers and end users. The actual work required to create a functional model involves defining the system, finding actors and the use cases, describing the use cases, defining the relationships between uses cases, and finally validating the model. The UML use case diagram and use case description is used in this phase.

In this phase, user requirements are elicited from to-be process model, and must be converted to functional requirements of information system. To do this, business activities within a process requiring the service of information system should be identified from the to-be process model. Typical activities requiring information system service are as follows: 1) information storage, retrieval, and organizing, 2) information processing, transformation and delivery, 3) decision making, 4) communication, and 5) hardware control.

In other words, use case defines service specifications, which is provided by enterprise information system to business processes.

There are informational requirements for each process, which describes the "what" factor. It creates or modifies objects. Objects represent things that are described both by properties and by the method that can be applied to them. They are specified at the structure design phase in more detail.

Functional model describes what is required of a system by defining how it will be used by external actors. An actor, which is usually but not necessarily human, causes the system to perform its functions by means of use cases.

A business use case may be thought of as a collection of related process steps, and actors may be thought of as the organization roles that execute the process steps. Because a business process 
defines how a purpose is to be achieved, a business use case should also be directed to satisfy a business purpose.

Most modern enterprise information system requires workflow functionalities since the workflow loop is the basis of all business processes. Workflow is defined as the automation of a business process, in whole or part, during which documents, information or tasks are passed from one participant to another for action, according to a set of procedural rules [15].

Therefore, in this phase, workflow type must be determined for the business process automation. There are two cases when developing enterprise information system as follows: 1) concurrent development of new transaction processing system and workflow system, 2) additional development of workflow system on the existing information system.

With respect to the degree of coupling between systems, there are two types as follows [16]: 1) autonomous workflow management system, 2) embedded workflow management system. Autonomous system, which is independent from other information system, executes workflows by invoking application software when needed. Embedded system can only be operated within its environmental system such as ERP and PDM (Product Data Management) system.

Though the workflow type selection is dependent to enterprise operating environment, autonomous workflow management system is advantageous when there exist several heterogeneous information system within enterprise.

\subsection{Structure Design}

The main purpose of this phase is to describe the internal structure of information system satisfying user requirements in terms of domain concepts called class. By doing this, we can understand the features of generated data from new information system.

The UML class diagram is used in this phase, and deliverable is a 'structure model'. In this phase, major task is to identify responsible classes for functionalities of each use case described in the functional model, and to establish a relationship between classes undertaking same responsibilities. Once basic classes responsible for use case are identified, then associated classes are investigated sequentially. Major candidates of basic class are business objects identified in the business process analysis phase.

In general, class is categorized into 3 types such as entity, boundary and control class [12]. In this paper, control class is further extended to 2 subtypes of logic control class and workflow class. Former class deals with business logic, and the latter deals with the flow of business process.

Boundary class is in charge of interactions between information system and external actor. According to the characteristics of information processing requirements, boundary class is further classified into data-centric and document-centric class. If it is data-centric, boundary class is converted into general input/output screen in the detail design phase. If it is document-centric, it is designed as electronic form.

Entity class deals with static and persistent information required by enterprise when performing business activities. 
Control class is in charge of executing business rules and sequencing and coordinating business activities. Logic control sub-class has a responsibility of detecting exceptional situation, checking the condition of business rules and settling the attribute value and so on. Workflow control class performs sequencing and coordination of business activities. In other words, it enables and control access to knowledge and information while coordinating all the roles in a business process.

The establishment of structure model having well-defined workflow functionalities is very essential in today's modern process-oriented organization. Workflow is a key enabler of BPR or PI (Process Innovation). The major benefits of workflow management are as follows:

1) Improved efficiency: automation of many business processes results in the elimination of many unnecessary steps.

2) Better process control: improved management of business processes is achieved through standardizing working methods and the availability of audit trails.

3) Improved customer service: consistency in the processes leads to greater predictability in levels of response to customers.

4) Flexibility: software control over processes enables their redesign in line with changing business needs.

5) Business process improvement: focus on business processes leads to their streamlining and simplification.

\subsection{Architecture Design}

Information system architecture is a description of entire resources including hardware, software and network, and their relationships for fulfilling organization's information needs. In particular, the term software architecture intuitively denotes the high level structures of a software system.

It can be defined as the set of structures needed to reason about the software system, which comprise the software elements, the relations between them, and the properties of both elements and relations [17].

The main purpose of this phase is to describe the basic structure of software system and relationship between software elements, and to suggest guidelines to the detail design phase.

UML class diagram and UML deployment diagram are used in this phase, and deliverable is an 'architecture model'. Software architecture is represented by UML class diagram, and hardware/network architecture is represented by UML deployment diagram. In the modern clientserver computing, overall software structure is mainly designed as a hierarchical structure [18]. Currently, 3-tier architecture is widely used in developing enterprise information system [19].

In the layered pattern, sub-system is represented by hierarchical layer responsible for system functionalities as follows: 1) interface layer is for user interface, 2) application layer for business logic, 3) storage layer for persistent data management.

Recently, interface layer is subdivided to presentation layer and interface layer to reflect various presentation media such as web browser and smart phone. Storage layer can be extended further to service layer for common functionalities such as message handling, security check, and access control. 


\subsection{Detail Design}

The main purposes of this phase are as follows:

1) To describe how internal entities interact with each other, and when their state is changed.

2) To refine structure model by specifying operations and attributes of classes.

UML sequence diagram, communication diagram, state diagram and class diagram are used in this phase, and deliverables are 'dynamic behavior model' and 'detail design structure model'. UML sequence diagram or communication diagram is used to represents how internal entities exchange messages with each other when performing their duties. UML state diagram is used to represent state change of internal entities.

Whereas functional model focuses on functional view, and structure model focuses on internal structural view, detail design model focuses on dynamic view according to passage of time.

In this phase, main tasks are to identify the specific operations of each domain entity and their state change. During defining operations for undertaking responsibilities of domain entity in response to external events, if temporal sequence of each operation is important, UML sequence diagram is used.

Otherwise, if collaboration relationship between domain entities is important, UML communication diagram is suitable. For identifying state changes of domain entity, UML state diagram is used.

After specifying operations of domain objects in the dynamic behavior model, 'structure model' is refined to 'detail design structure model' on which operations of each entity is added and specified.

Besides, a class identified as boundary class in the structure model is also refined to reflect user interface design. It is designed as input/output screen or electronic form. If persistent storage is required, additional classes for the database implementation are also added to detail design structure model.

In summary, Figure 3 shows the development process, UML diagrams and deliverables of proposed process-driven software development methodology. 


\begin{tabular}{|c|c|c|}
\hline Development Process & UMLModeling Tool & Deliverables \\
\hline $\begin{array}{l}\text { (1) Business process } \\
\text { analysis }\end{array}$ & -activity diagram & $\begin{array}{l}\text {-'as-is' process model } \\
\text { - 'to-be' process model. }\end{array}$ \\
\hline $\begin{array}{l}\text { (2) User Requirements } \\
\text { analysis }\end{array}$ & $\begin{array}{l}\text {-use case diagram } \\
\text {-use case description }\end{array}$ & -functional model \\
\hline (3) Structure Design & -class diagram & -structure model \\
\hline (4) Architecture Design & $\begin{array}{l}\text {-class diagram } \\
\text {-deployment diagram }\end{array}$ & -architecture model \\
\hline (5) Detail Design & $\begin{array}{l}\text {-sequence diagram, } \\
\text {-communication diagram } \\
\text {-state diagram } \\
\text {-class diagram }\end{array}$ & $\begin{array}{l}\text {-dynamic behavior model } \\
\text {-detail design structure } \\
\text { model }\end{array}$ \\
\hline
\end{tabular}

Figure 3. Tool \& deliverables of process-driven software development methodology

\section{CONCLUSIONS}

External environment of enterprise are rapidly changing brought about majorly by global competition, cost and profitability pressures, and emerging new technology. Current environment and their impact on the organizations are that product's lifetime is shorter, product has customer specific versions, open market requires cost/lead-time reduction, and organizations become more internationalized, think globally. To cope with these challenges, most enterprises are transforming their organization from functional and hierarchical one to process-centered one for customer satisfaction. To support process-centered organization, enterprise information system must be developed in process-driven way.

Traditional software development methodology is function-oriented, in which each function manages its own data and it results in redundancy because data that belongs to one object are stored by several functions. Additionally, workflow automation is emerging as an essential part of information system beyond supporting transactional task in the recent enterprise information system development.

Proposed in this paper is a process-driven object-oriented software development methodology, in which business process is a major concern, and workflow functionalities are identified and designed throughout the entire development life cycle. In the proposed methodology, the development process, modeling tools and deliverables are clarified explicitly. 
Therefore, proposed methodology can be a guideline to practitioners involved in enterprise software development, of which workflow is an essential part.

However, Usefulness of proposed methodology must be validated in real development projects, which is one of further research in near future.

\section{ACKNOWLEDGEMENTS}

This work was supported by the Nuclear Power Core Technology Development Program of the Korea Institute of Energy Technology Evaluation and Planning (KETEP) granted financial resource from the Ministry of Trade, Industry \& Energy (MOTIE), Republic of Korea (No. 20131510101690: Development of a public monitoring system and competence enhancement technologies for strengthen Korean safety culture in nuclear organizations).

\section{REFERENCES}

[1] Han K. H, Kang J. G. and Song M. (2009), "Two-stage process analysis using the process-based performance measurement framework and business process simulation," Expert Systems with Applications, vol. 36, no. 3, part 2, pp. 7080-7086.

[2] Debevoise T. (2005), Business process management with a business rules approach, Roanoke, VA, USA: Business Knowledge Architects.

[3] Aalst W. M. P. (1998), "The application of Petri nets to workflow management," The Journal of Circuits, Systems and Computers, vol. 8, no.1, pp. 21-66, 1998.

[4] Sharp A., McDermott P. (2001), Workflow Modeling, Boston: Artech House.

[5] National Institute of Standards and Technology (1993), Integration definition for function modelingdraft federal information processing standards publication 183, NIST, USA.

[6] Scheer A. W. (1998), Business process engineering, Berlin, Germany: Springer-Verlag.

[7] Han K. H., You S. K. and Kim B. H. (2009), "Qualitative and Quantitative Analysis of Workflows Based on the UML Activity Diagram and Petri Net," WSEAS Transactions on Information Science and Applications, vol. 6, no. 7, pp. 1249-1258.

[8] Eriksson H. E., Penker M (2000), Business modeling with UML, New York: John Wiley \& Sons, Inc.

[9] Marshall C. (2000), Enterprise modeling with UML, Reading, MA: Addison Wesley.

[10] Hruby P. (1998), "Structuring specification of business system with UML," OOPSLA'98 Business Object Workshop IV, Vancouver, British Columbia, Canada.

[11] Kruchen P. (1998), The rational unified process: an introduction, Reading, MA: Addison-Wesley.

[12] Jacobson I., Booch G. and Rumbaugh J. (1999), The unified software development process, Reading, MA: Addison-Wesley.

[13] Rumbaugh J. et al (1991)., Object-oriented modeling and design, Englewood Cliffs, New Jersey: Prentice Hall..

[14] Booch G. (1994), Object-oriented design with application, Benjamin/Cummings. Redwood city, California:

[15] WfMC (1999), Workflow Management Coalition Terminology \& Glossary, Document Number WFMC-TC-1011, Workflow management Coalition, 1999.

[16] Muehlen, R. A. (2000), "Workflow classification: embedded \& autonomous workflow management systems," Workflow Management Coalition.

[17] Clements P., Bachmann F., Bass L., Garlan D., Ivers J., Little R., Merson P., Nord R. and Stafford J. (2010), Documenting Software Architectures: Views and Beyond, Second Edition. Boston: AddisonWesley.

[18] Larman C. (2005), Applying UML and patterns, Third Edition, New Jersey: Prentice Hall PTR.

[19] Shuttle (1995), Three tier computing architectures and beyond, Gartner Group. 


\section{AUTHORS}

Kwan Hee Han is a professor in the department of industrial \& systems engineering, engineering research institute of Gyeongsang National University in Korea. His research interests focus on simulation modeling, virtual manufacturing, business process management and process mining, etc.

Yongsun Choi is a professor in the department of system management \& engineering of Inje University in Korea. His research interests include process modelling and analysis, decision support systems, internet of things, multiple criteria optimization, etc.
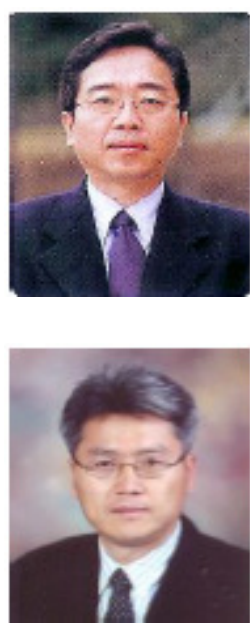


\title{
DEVELOPMENT AND EVALUATION OF A WEB BASED QUESTION ANSWERING SYSTEM FOR ARABic Language
}

\author{
Heba Kurdi, Sara Alkhaider, Nada Alfaifi \\ Department of Computer Science, Al Imam Muhammad Ibn Saud Islamic \\ University \\ Riyadh, SA \\ hakurdi@imamu.edu.sa, csce.t.sara@gmail.com, \\ n.alfaify@hotmail.com
}

\begin{abstract}
Question Answering (QA) systems are gaining great importance due to the increasing amount of web content and the high demand for digital information that regular information retrieval techniques cannot satisfy. A question answering system enables users to have a natural language dialog with the machine, which is required for virtually all emerging online service systems on the Internet. The need for such systems is higher in the context of the Arabic language. This is because of the scarcity of Arabic QA systems, which can be attributed to the great challenges they present to the research community, including the particularities of Arabic, such as short vowels, absence of capital letters, complex morphology, etc. In this paper, we report the design and implementation of an Arabic web-based question answering system, which we called "JAWEB", the Arabic word for the verb "answer". Unlike all Arabic questionanswering systems, JAWEB is a web-based application, so it can be accessed at any time and from anywhere. Evaluating JAWEB showed when compared to ask.com, the well-established web-based QA system, JAWEB provided 15-20\% higher recall. These promising results give clear evidence that JAWEB has great potential as a QA platform and is much needed by Arabicspeaking Internet users across the world.
\end{abstract}

\section{KEYWORDS}

Question Answering system, Natural Language Processing, Arabic language tools.

\section{INTRODUCTION}

Question-answering is a challenging task in general. It involves state-of-the-art techniques in various fields, such as Information Retrieval (IR), Natural Language Processing (NLP), Artificial Intelligence (AI) and software technologies. The main goal of a question-answering system, is to give a precise answer to users' queries in natural language [1] [2]. This has the great advantage of helping users to get desired answers without searching large pools of information. Unlike search engines, such as Google and Yahoo, which allow a user to retrieve webpages or documents that are partially relevant to given keyword(s), while leaving the job of excluding irrelevant hyperlinks and locating desired passages to users, QA systems provide the user with highly relevant information in answer to their questions at the passage or sentence level [3]. QA systems categorize questions into three main groups: first, fact-seeking questions, i.e. factoid questions,

David C. Wyld et al. (Eds) : CCSIT, SIPP, AISC, PDCTA, NLP - 2014

pp. 187-202, 2014. (C) CS \& IT-CSCP 2014

DOI : $10.5121 /$ csit.2014.4216 
asking about (who, when, where, what and how much) which relate to different types of entities: person, location, organization, time and quantity. This type of question is the most common form of questions and therefore is the focus of this work. Second is list questions (e.g. List all the countries of the European Union), asking about multiple pieces of information with a common relation between them. Third is why-type questions (e.g. Why is the sky blue?) which seeks explanations of causes for a certain phenomenon or event. The second and third categories are less common in QA systems and require more advanced NLP and AI techniques and were therefore excluded from this study.

Based on the knowledge domain, QA systems classify questions as either closed-domain questions, which ask about information related to a specific domain (for example medical or sport, etc.), or open-domain questions which ask about information in any knowledge discipline. They require wider knowledge and advanced techniques to extract the most relevant answers [4]. The latter is the focus of this paper.

A QA system consists of three main components: a question-classification component, an information-retrieval component, and an answer-extraction component. The questionclassification component plays a primary role as it categorizes questions based on their keywords: who, when, where, what and how much. The information-retrieval component searches for relevant answers in information sources by looking for matching keywords. Finally, the answerextraction component selects the most relevant answers and ranks them accordingly. The quality of a QA system heavily depends on the effectiveness of this module as it makes the decision about the final list of answers to be presented to the user [5] [6].

Although the systems have common core components, they differ vastly in the techniques utilized by each component. This can be attributed mainly to the supported language(s) by the system, since each language has special features that affect how it should be analyzed, searched and retrieved.

Arabic is among the most popular languages with nearly 300 million speakers across the globe. It is a Semitic language. These are well known for their non-concatenative morphology, in which roots consist of an isolated set of constants, rather than syllables or words. This feature among many others has rated NLP and IR as grand challenging tasks for the Arabic language, despite some attempts such as [7]-[15]. Consequently, the huge Arabic content on the Internet is still underutilized [16]. Important emerging applications, question-answering systems for online services in particular, are simply not available in Arabic, despite their abundance in many other languages, especially English and other Latin-based languages. The challenging features of the Arabic language, sacristy of Arabic QA systems and lack of web-based Arabic QA systems are the main drivers for this paper. It adds several contributions to this important field, including comprehensively surveying Arabic QA systems and a comparing between them, developing and evaluating of JAWEB, the first Arabic web-based QA system, and extending the Arabic Corpus provided in [17].

The remaining of this paper is organized as follows: In section 2, the challenges of the Arabic language are highlighted. Section 3 reviews related work in QA systems. The system architecture and functional components of JAWEB are introduced in section 4 while the implementation process is described in section 5. In section 6, the experimental results are presented and discussed. Finally, section 7 concludes the paper and indicates future work. 


\section{Challenges OF ARabic language}

Arabic is highly inflectional and derivational. This results in sparseness of terms in text, which leads to inefficiency in many statistical IR and NLP techniques. The absence of diacritics in Modern Standard Arabic also adds a lot of ambiguity to Question Analysis and Answer Extraction [16].

Many aspects are involved in the slow progress of Arabic NLP and IR, compared to the accomplishments in English and other languages. These aspects, as highlighted in [18], include:

1. Arabic has a complex morphology. It is highly derivational and inflectional, which extremely complicates morphology analysis.

- Arabic is a derivational language, to find a word in an Arabic dictionary, we start by extracting the root and then we search for the root in the dictionary.

- Arabic is an inflectional language, which means that the construction of a word involves finding the root and adding affixes (prefix, infix and suffix) to it.

2. The correct meaning of an Arabic word can be ambiguous in the absence of diacritical marks (short vowels); so a written word may hold different meanings.

3. Arabic does not use capital letters which makes differentiating between named entities and other words difficult.

4. Numerals are written form left to right, while alphabets are written from right to left. This makes editing Arabic text difficult when both numbers and letters are presented on the same line.

5. The most used encodings for Arabic text, UTF-8 and Unicode, present many problems when processing Arabic texts.

6. Lack of Arabic corpora, lexicon and electronic dictionaries.

\section{RELATED WORK}

Question-Answering systems present a good solution for textual information retrieval and knowledge sharing and discovery. This is why a large number of QA systems have been developed especially in English, which might be related to the language features and the maturity of research in the countries speaking it. The situation is less bright for the Arabic language, despite of its wide spread. Although research in the field of Arabic QA systems has already started [7]-[15], it is slow progressing and has limited results. Computerized tools and resources in general are lacking in Arabic [16], which has reflected negatively on the number of Arabic QA systems. The list includes:

- AQSA (1993) [19] seems to be the first QA system in the language. It is a knowledge-based QA system that extracts answers from structured data only. It uses the frames technique to present the knowledge from the radiation domain. However, no published evaluation is available for the system.

- QARAB (2004) [20] is a stand-alone (non-web-based) QA systems that uses IR and NLP techniques to extract answers from a collection of Arabic newspaper texts. It provides 
answers to factoid questions but does not support other types of questions, such as how or why.

- ArabiQA (2007) [21] is a stand-alone Arabic QA system that deals with factoid questions, using Named Entity Recognition techniques and Java Information Retrieval System (JIRS) for Arabic text. It is designed specifically for Arabic factoid questions. However, system implementation has not been completed yet.

- QASAL (2009) [22] is a stand-alone Arabic QA system for factoid questions which uses the NooJ platform [24] as a linguistic development environment. The system takes advantage of some linguistic techniques from IR and NLP to process Arabic text documents containing answers to factoid questions. Published work based on this systems does not include experimental results or performance metrics. The overall functionality of the system is limited to the amount of available tools developed for the Arabic language by the NooJ Arabic modules.

- ArQA (2011) [18] is a stand-alone QA system that provides answers to factoid questions expressed in Arabic. This system has a pipeline architecture with four modules: question processing, passage retrieval, answer extraction and answer validation modules. Each module is the result of combining several IR and NLP techniques and tools to improve validity of returned answers.

- AQuASys (2011) [17]: a stand-alone Arabic QA system for factoid questions. It extensively utilizes NLP techniques to analyses questions and retrieves answers from an Arabic corpus that has been developed by the authors. Retrieved answers are scored and presented based on their relevance.

The survey above indicates clearly the lack of web-based QA systems, therefore JAWEB has been developed as a web-based application to bridge this gap providing ubiquitous accessed at any time and from anywhere.

\section{JAWEB ARCHITECTURE}

JAWEB is an Arabic web-based QA system that focuses on factoid questions. It accepts questions related to any named entity, including person, location, organization, time etc. After analyzing the question, important information is extracted to retrieve the most relevant answers from an Arabic corpus. The system is composed of four components: user interface, question analyzer, passage retrieval and answer extractor. The user interface runs at the client side while all other modules run at the server side. As illustrated in Figure 1, each component is structured of several modules with a distinct task carried by each.

\section{User Interface}

The user interface is implemented as a simple webpage with an input form that accepts Arabic factoid questions expressed in natural language. The question handler module validates entered questions and passes them to the server side. Later on, when the answers are retrieved from the corpus, the answer-viewer module formats and presents them in descending order of relevance; the desired answer is displayed at the top, followed by a list of candidate answers. 


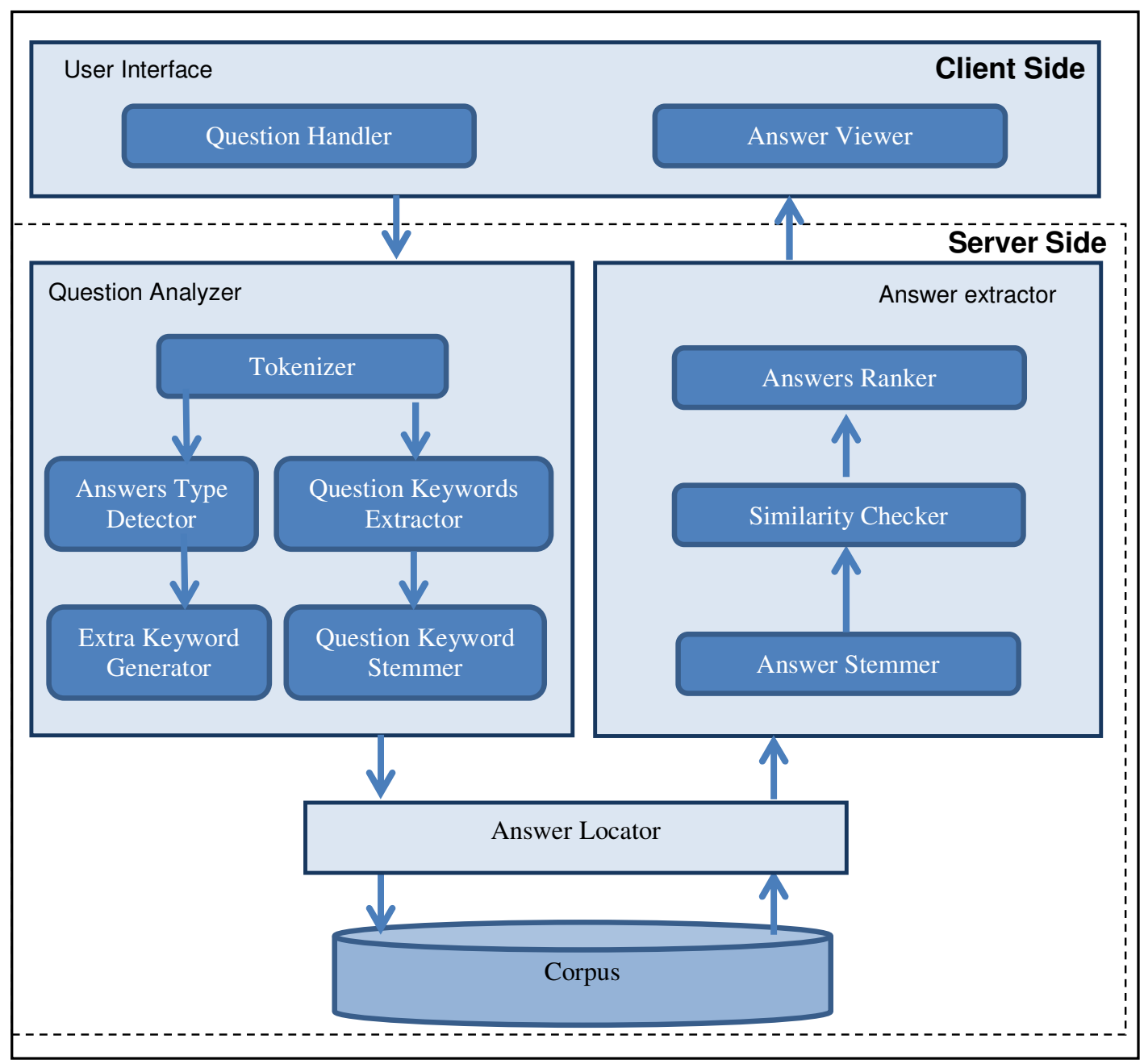

Figure 1: JAWEB system detailed architecture

\section{Question-Analyzer}

This component aims at identifying the type of the question. It has five modules: tokenizer, answer-type detector, question keyword extractor, extra keywords generator and question words stemmer. The user enters a question in a web-based form at the client side. The question is sent to the server side, where the tokenizer breaks the question into separate words. Afterwards, the answer-type detector identifies the category of the answer, which can be time, organization, person, location etc., based on the interrogative nouns (منى who, من what, أين where and when).The question keywords are the rest of the words in the question, after excluding stopwords and interrogative nouns; they are identified by the question keywords extractor module. The extra keywords generator produces synonyms of the keywords to expand the range of related answers. For example, for a question like: منى استقلت تونس (When has Tunisia become

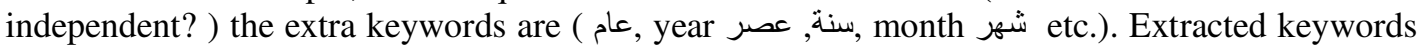
are stemmed, if necessary, so affixes are removed by the question keywords stemmer module to ease computing similarity with the keywords in the extracted answers later. 


\section{Answers Retriever}

This component searches for potential answers and retrieves them from the corpus. It locates all sentences that contains a pattern that matches any word from the list of question keywords and extra keywords.

\section{Answers Extractor}

This component is responsible for selecting the most relevant answers from the potential answers list. It is composed of three modules. The first module is the answer keywords stemmer which gets roots of the keywords in the retrieved answer to ease checking similarity between a user question and potential answers, a task carried out by the second module, which is the answersimilarity checker. Similarity is measured by counting the number of matching keywords between the question and each retrieved answer. Based on this, the third module, answers ranker, sorts answers in descending order of their relevance. The most relevant answer is considered as the desired answer while the rest of answers are candidate answers.

\section{SYSTEM IMPLEMENTATION}

JAWEB has been built based on AQuASys [17] and as a natural extension to it. It provides a web interface to the system, an additional support for Arabic language presentation in web browsers, an extended corpus as well as an extensive evaluation and testing framework. The user interface was developed as a JSP webpage that accepts Arabic factoid questions. It provides a user-friendly interface where a user can type his/her questions. The question should begin with an interrogative noun: ( منى, أين, من, ما, كم الكمية، كم العددية)

Dreamweaver, an Adobe proprietary web development application, was used to design the interface. The servlet and java classes on the server side were implemented using NetBeans IDE. The server was a GlassFish Server 3.1.2, which is an open-source application server project started by Sun Microsystems for the Java EE platform and now sponsored by Oracle Corporation. This server ran on $2.50 \mathrm{GHz}$ CPU and an internal memory of $4 \mathrm{~GB}$.

An extended version of the Arabic corpus developed by [17], containing 39,660 words with size of $457 \mathrm{~KB}$, was used as the information pool to retrieve answers. The Arabic Khoja's stemmer [23] was adopted for keywords stemming. The system was tested thoroughly to insure correctness of obtained results and then an extensive evaluation have been carried out to compare JAWEB performance with a well established web-based QA system that supports Arabic Language, Ask.com [10], as described in the following section.

\section{Performance evlauation}

The end objective of JAWEB is to develop a web-based QA system that answers Arabic factoid questions with high accuracy and speed. To evaluate this system, three performance measures were considered:

- RECALL [32]: the ratio of the number of relevant sentences retrieved to the total number of relevant sentences in the corpus. It is usually expressed as a percentage and calculated as:

$$
R E C A L L=A / A+B * 100 \%
$$

Where $A$ : number of retrieved relevant answers, $B$ : number of relevant answer not retrieved 
- PRECISION [32]: the ratio of the number of relevant sentences retrieved to the total number of sentences retrieved. It is usually expressed as a percentage and calculated as:

$$
\text { PRECISION }=A / A+C * 100 \%
$$

Where $A$ : number of retrieved relevant answers, $C$ : number of retrieved irrelevant answers

- RESPONSE TIME: the time from when a user hits the search button until a response is displayed on the browser.

To have clear insight into JAWEB performance, we used a well-established QA system that supports the Arabic Language, Ask.com [10], as a benchmark. Ask.com is a famous web-based question answering system with multilingual support. It accepts colloquially-expressed questions and retrieves hyperlinks to webpages that contain similar keywords to those in the questions.

We ran several experiments with each of the supported type of factoid questions to get answers form Ask.com and JAWEB. Snapshots of answers to some example questions, listed in Table 1, are presented in Figure 2 - Figure 7. All figures illustrate that JAWEB has consistently given the right and most accurate answer as the first responses which can be attributed to the use of the stemmer (in both question and answer modules), the extra keywords generator and the similarity checker which has resulted in accurate ranking of retrieved answers . In contrast, Ask.com provided the correct answer in the second or third hyperlinks.

Comparisons between Ask.com and JAWEB in terms of RECALL, PRECISION and RESPONSE $T I M E$ are depicted in the graphs in Figure 8, Figure 9 and Figure 10, respectively. It is important to note that having no access to the knowledge base of Ask .com and in order to be able to calculate the RECALL and PRECISION, we have only considered the first five retrieved pages, which may slightly affect the accuracy of the experiments.

Figure 8 shows that RECALL of JAWEB was always higher than that of Ask.com and maintained at $100 \%$, as the former has successfully retrieved all relevant answers from the corpus. The JAWEB PRECISION was admittedly less than ask.com, as shown in Figure 9, but it should be noted that it performed as well as the famous website, scoring over $90 \%$ in precision, despite the large difference in corpus sizes. In addition, even at its worst, for a question type that ask.com had the lowest precision too, JAWEB was not less precise than $70 \%$. Regardless, the precision can easily be improved as the project launches, when feedback from users is attained and the corpus is grown in size and capacity. We believe that this is clear evidence that JAWEB has great potential as a QA platform and is much needed by Arabic-speaking Internet users across the world.

Naturally, ask.com was faster than our system, as illustrated in Figure 10, thanks to the use of high-power server CPUs for ask.com, as opposed to JAWEB $2.50 \mathrm{GHz}$. Again, if the project launches, the response time is expected to improve dramatically, when servers that are more powerful are provided.

Table 1: Experimental questions

\begin{tabular}{|c|c|c|c|}
\hline No. & Type & Arabic & English \\
\hline Q1 & Who & إمن هو محمد طنجة؟ & Who is Muhammad Tangier? \\
\hline Q2 & When & منى توحدت المملكة العربية السعودية؟ & When was Kingdom of Saudi Arabia united? \\
\hline Q3 & What & ماهي الأهرامات المصرية؟ & What are Egyptian pyramids? \\
\hline Q4 & Where & أين تقع المملكة العربية السعودية؟ & Where is the Kingdom of Saudi Arabia located? \\
\hline Q5 & How much & كم تبلغ درجة حر ارة القشرة الأرضية؟؟ & How much is the temperature of the Earth's crust? \\
\hline Q6 & How many & 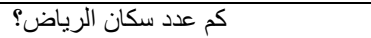 & How many residents are there in Riyadh? \\
\hline
\end{tabular}




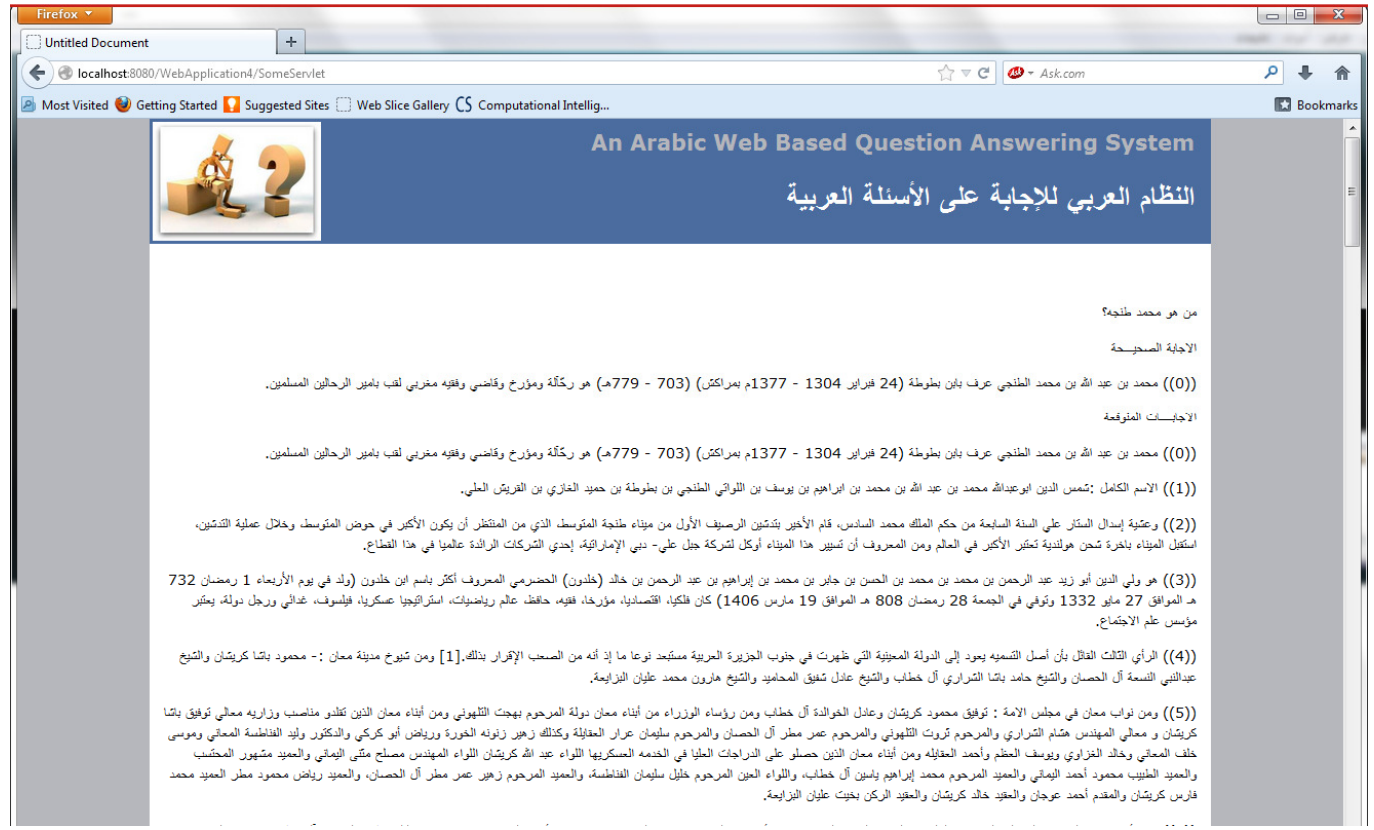

Figure 2.a: JAWEB answers for a who question

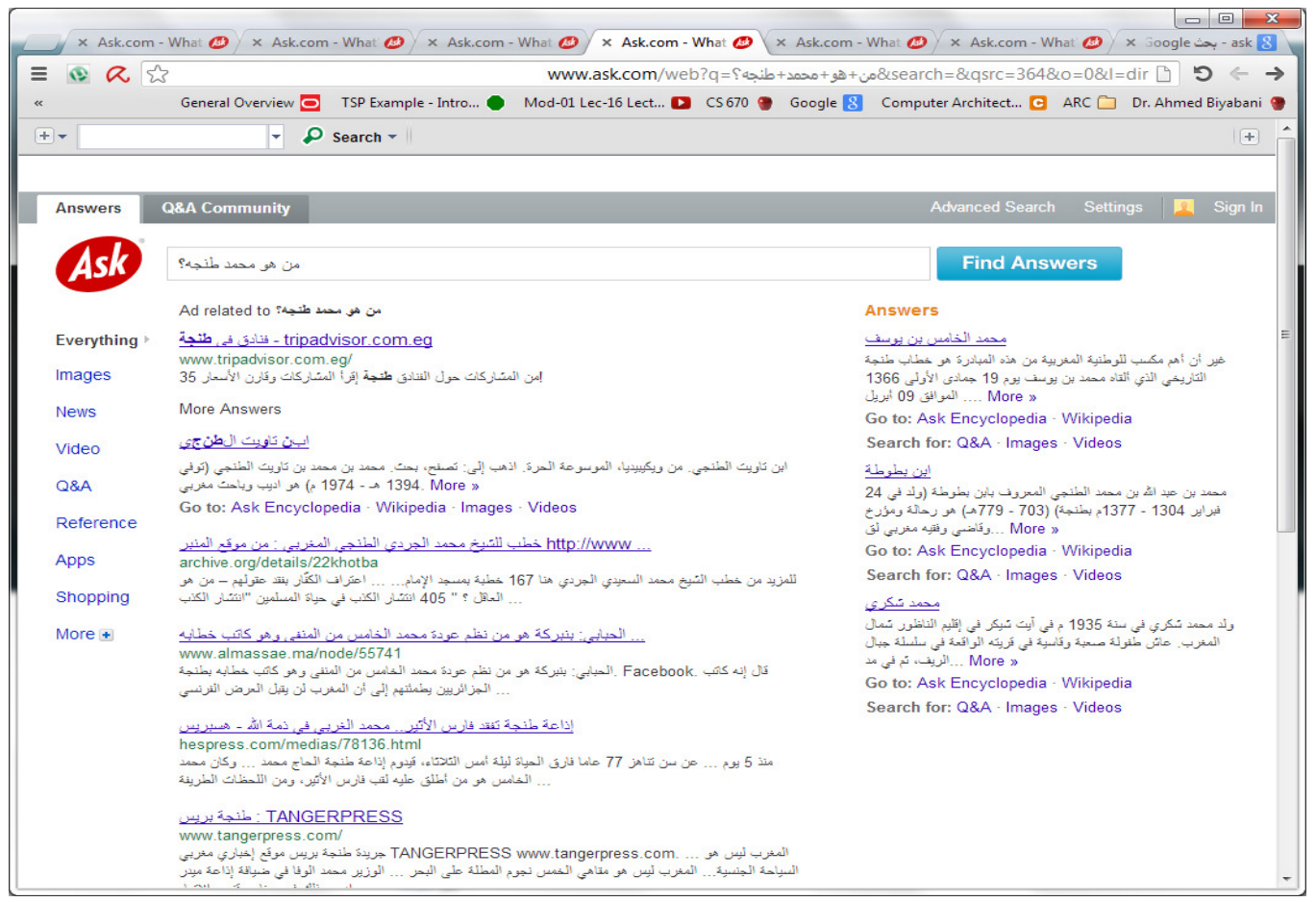

Figure 2.b: Ask answers for a who question 


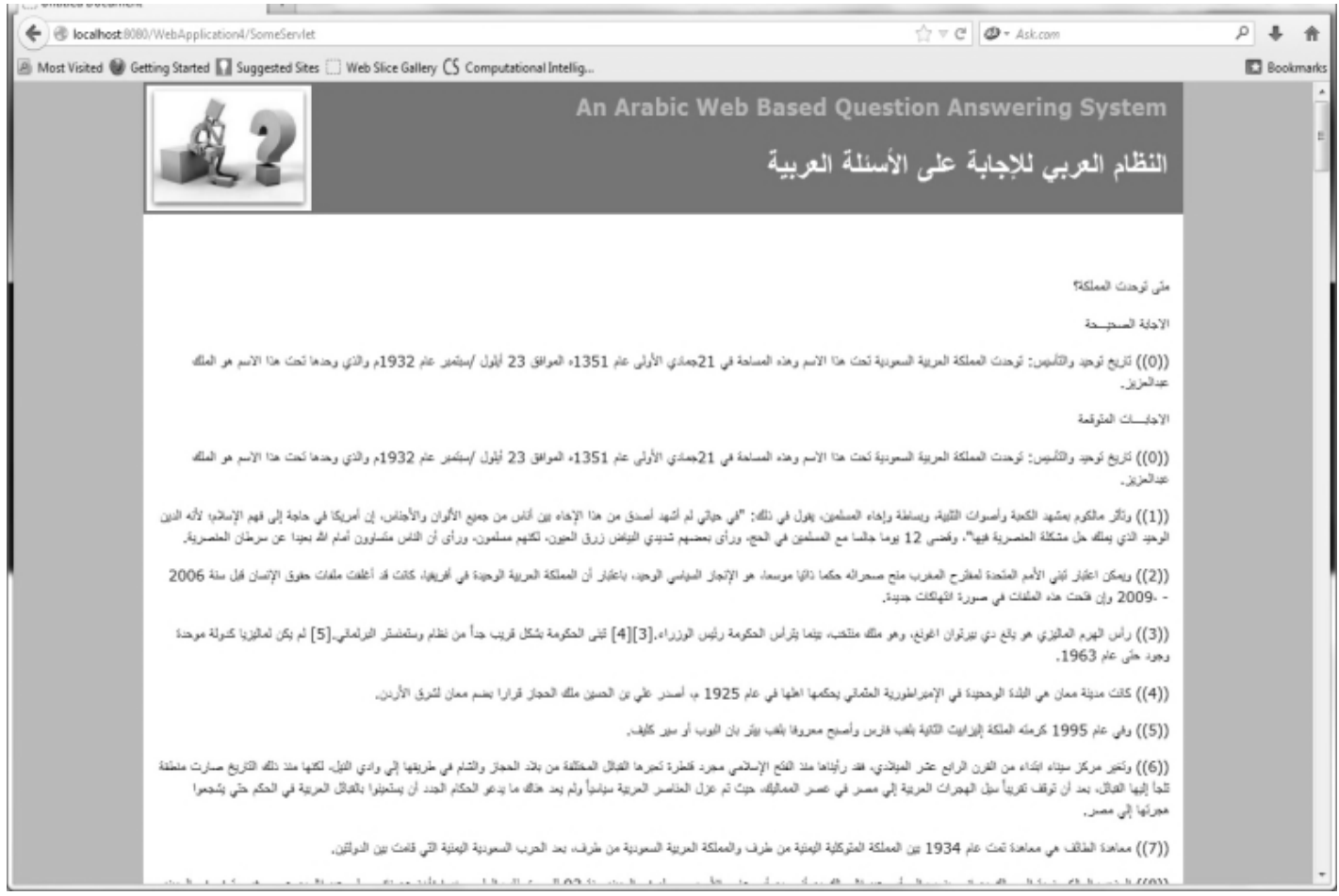

Figure 3.a: JAWEB answers for a when question

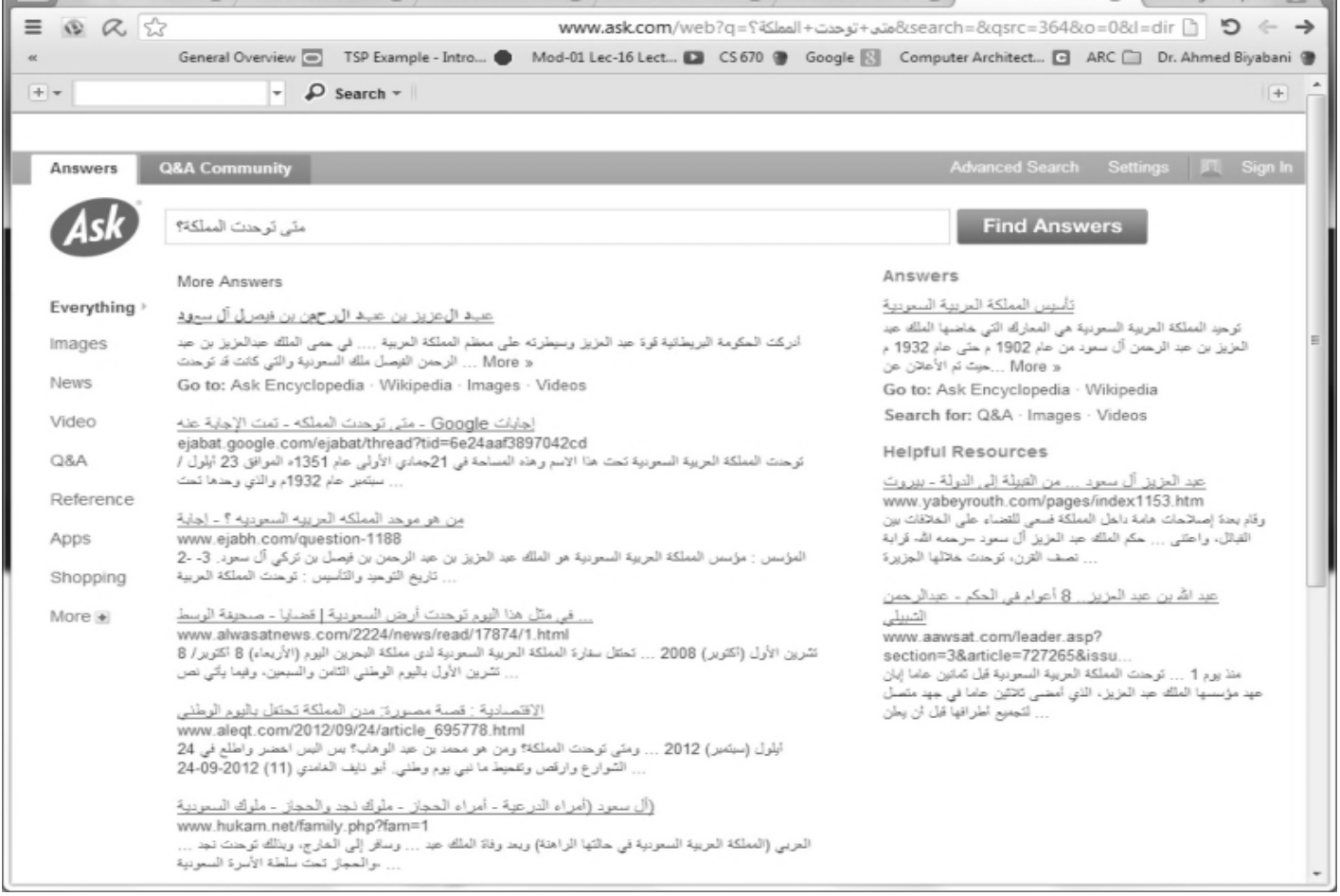

Figure 3.b: Ask.com Answers for when question 


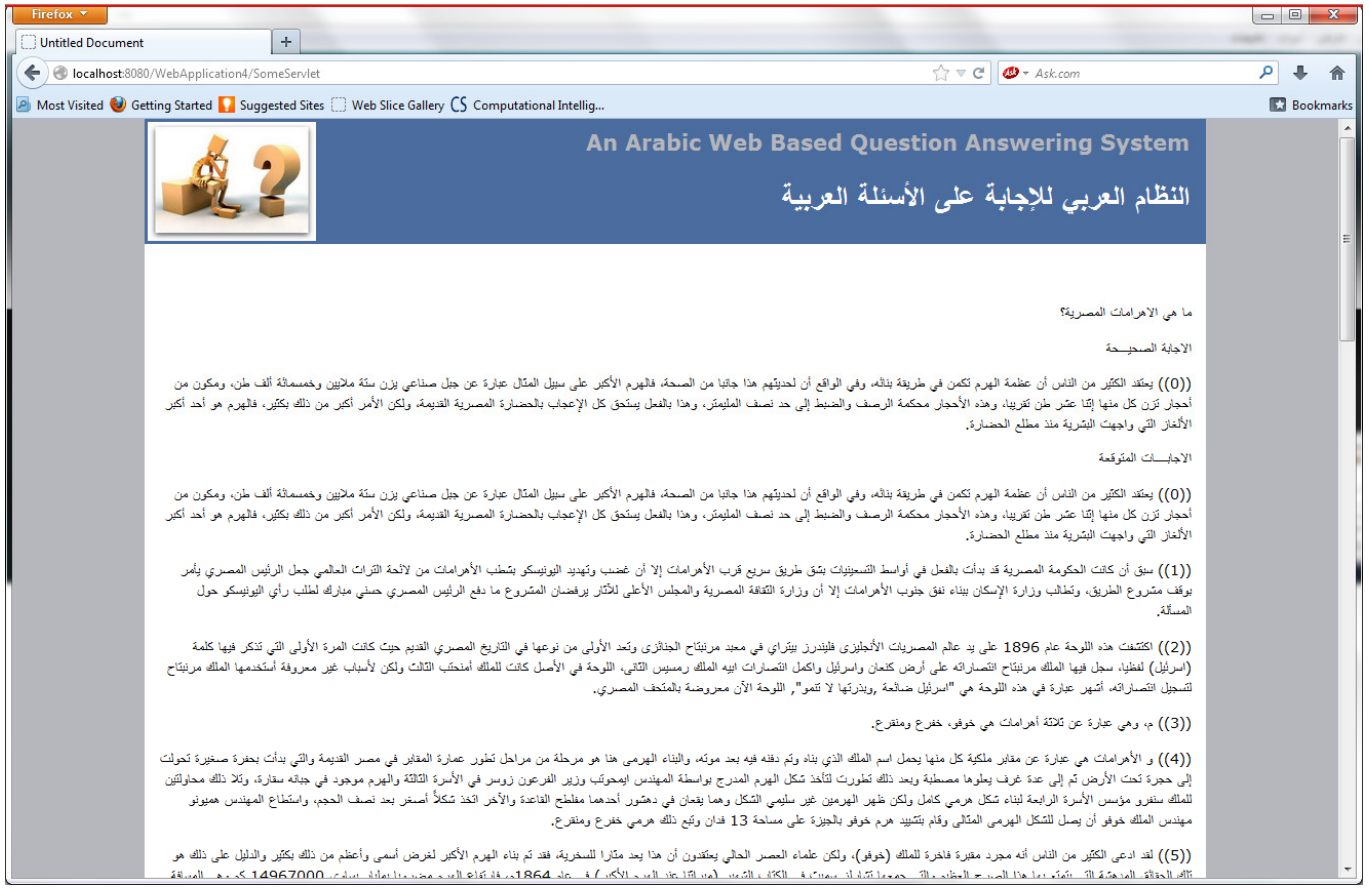

Figure 4.a: JAWEB answers for a what question

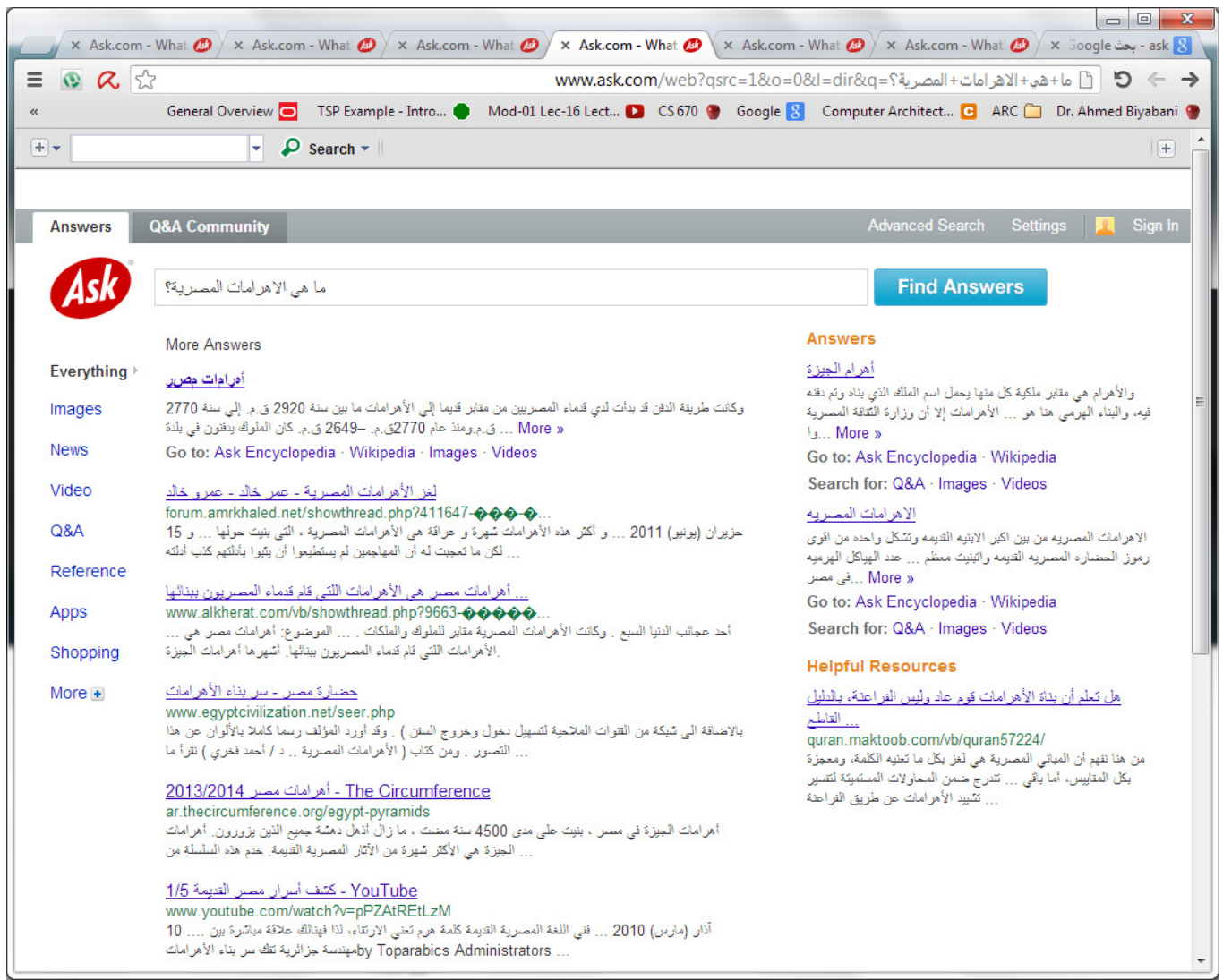

Figure 4.b: Ask answers for a what question 


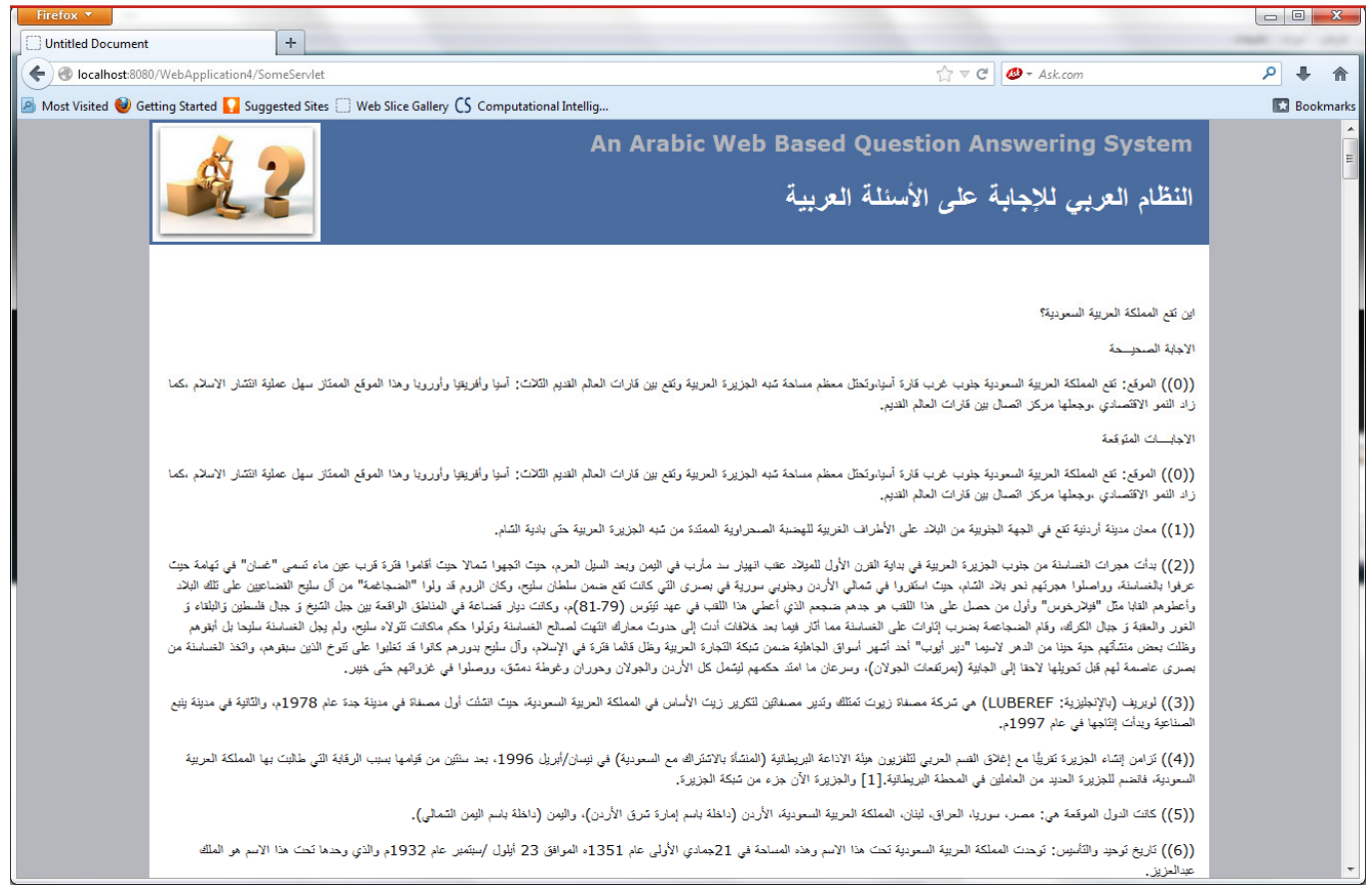

Figure 5.a: JAWEB answers for where question

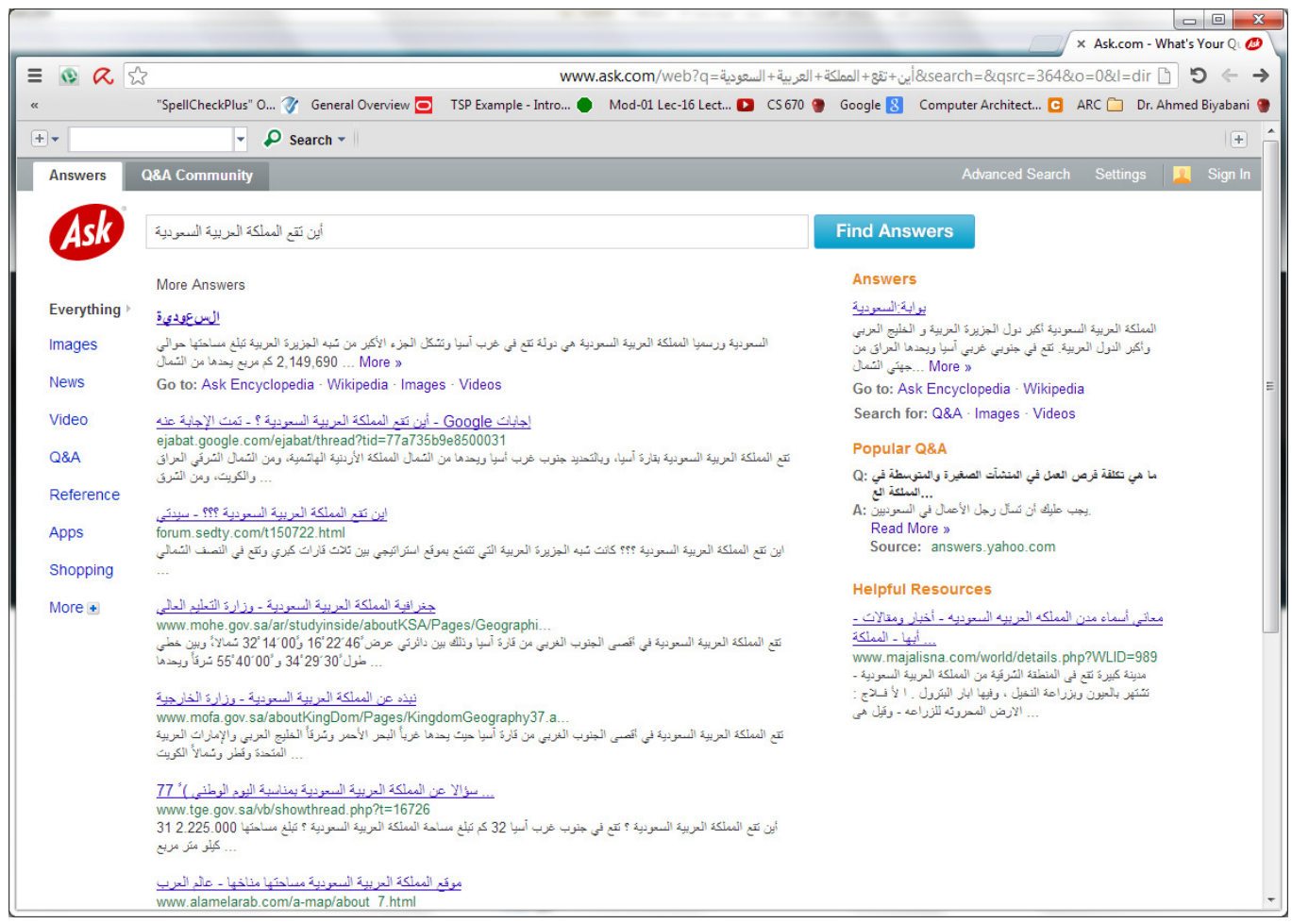

Figure 5.b: Ask answers for a where question 


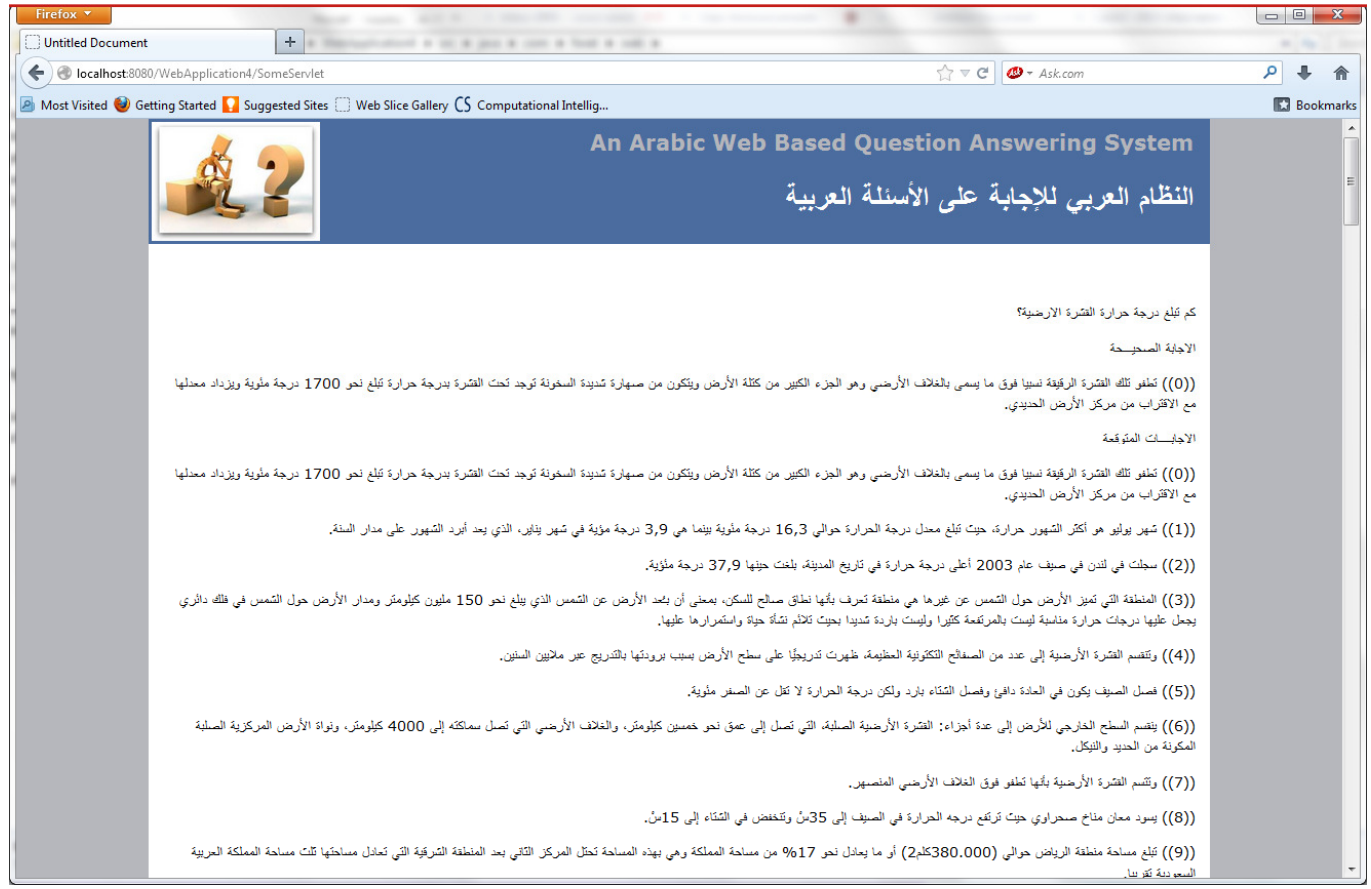

Figure 6.a: JAWEB answers for a how much question

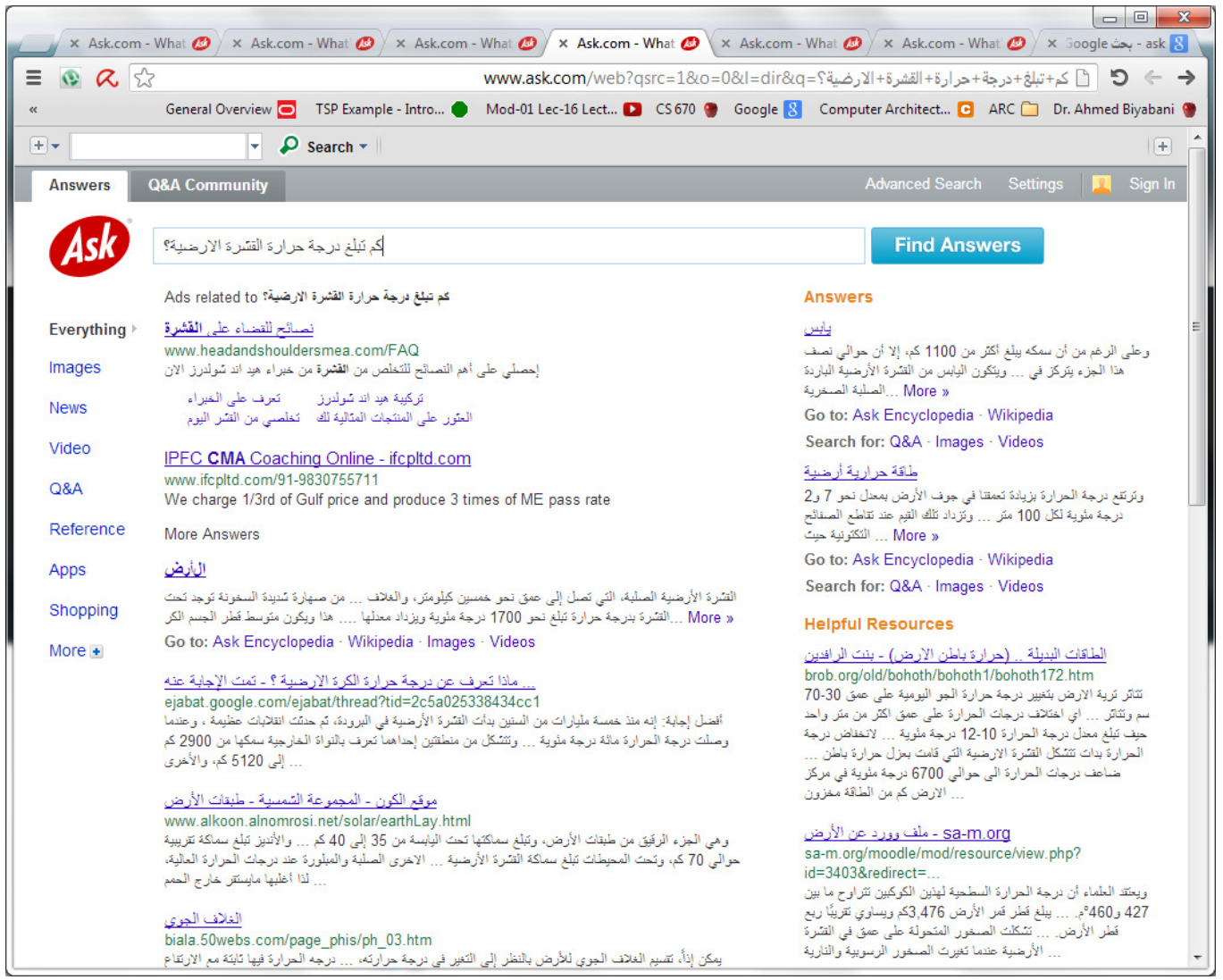

Figure 6.b: Ask answers for a how much question 


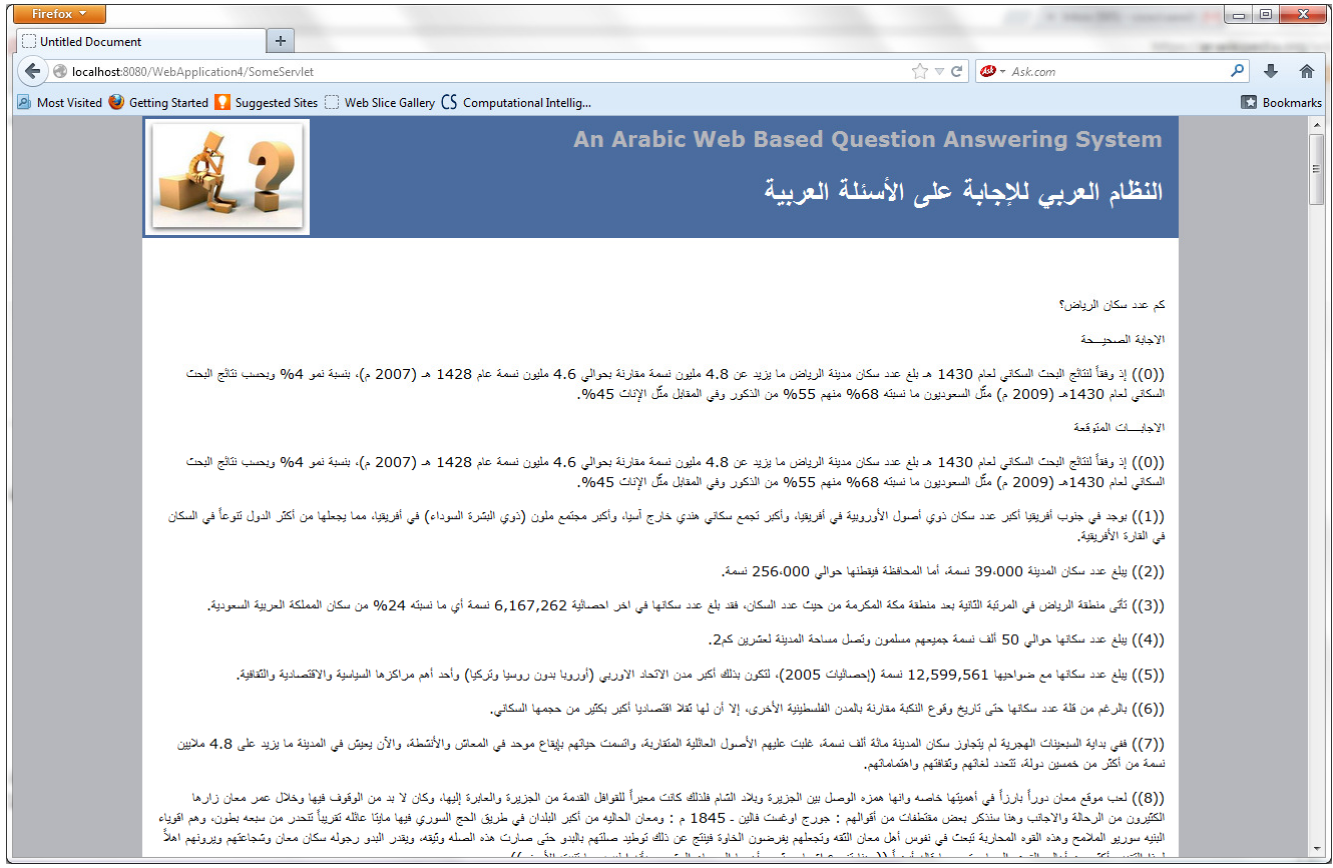

Figure 7.a: JAWEB answers for a how many question

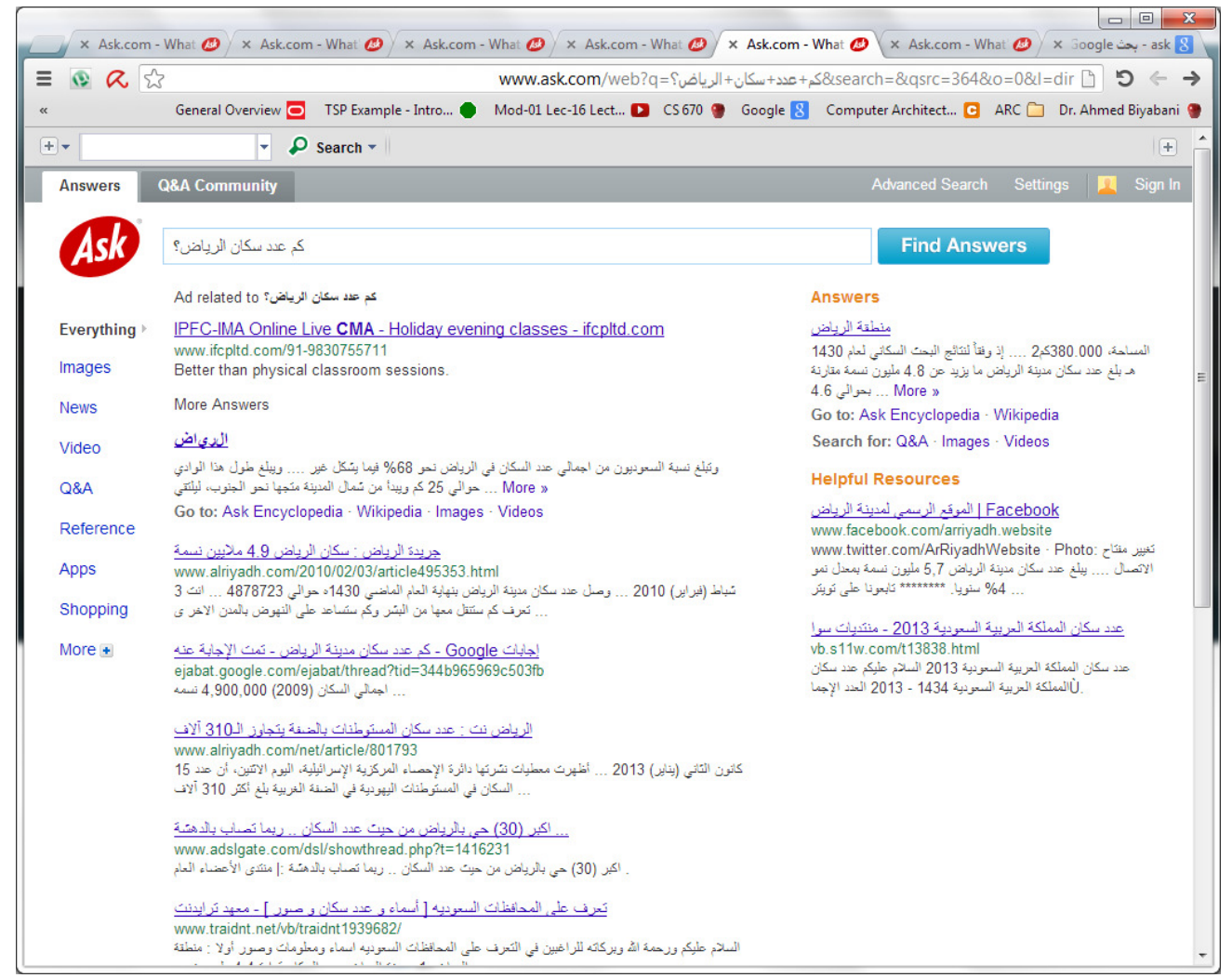

Figure 7.b: Ask answers for a how many question 


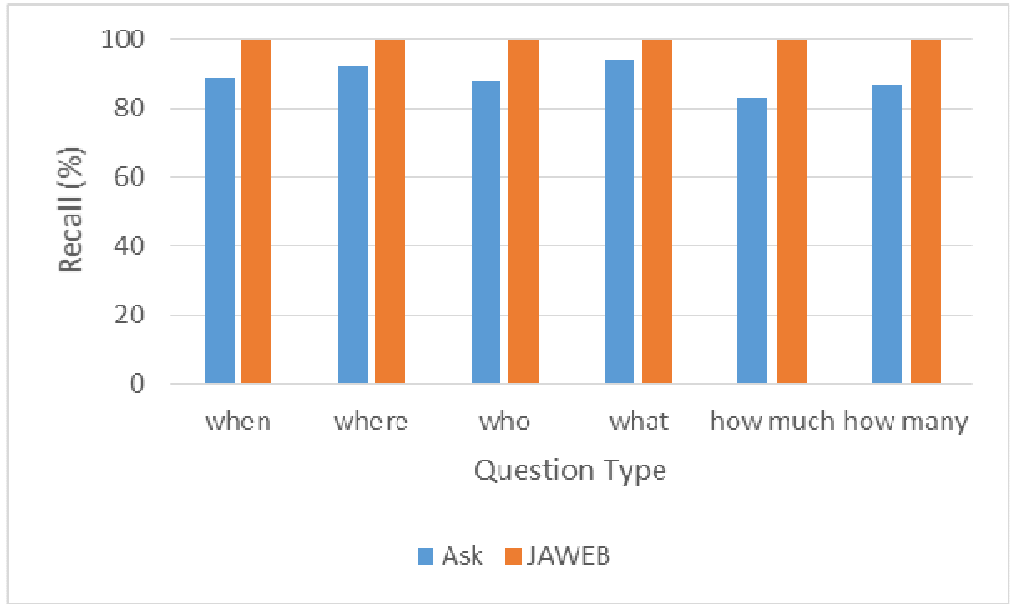

Figure 8: Comparison of the Recall

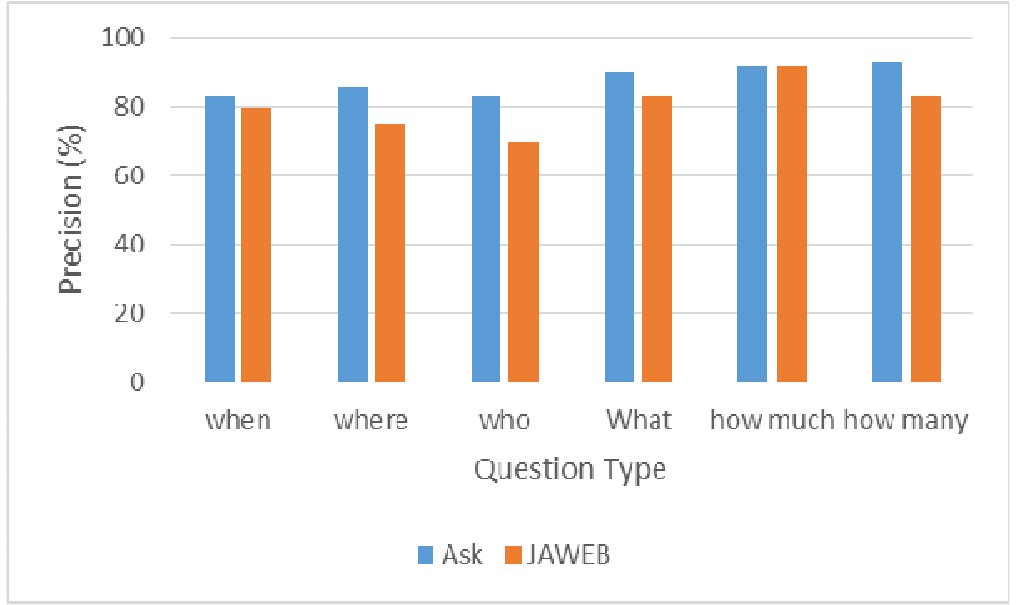

Figure 9: Comparison of the Precision

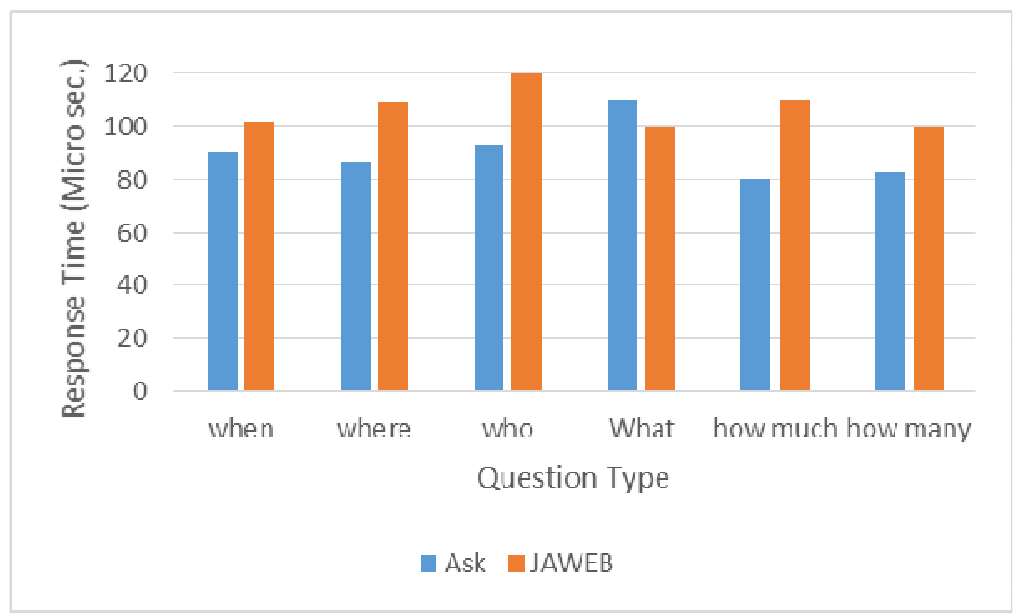

Figure 10: Comparison of the performance-Time 


\section{CONCLUSION \& FUTURE WORK}

JAWEB is a web-based Arabic question answering application system. It takes native Arabic questions from the end user and processes them in the server. The processing consists of three modules: question analysis, passage retrieval, and answer extraction. JAWEB finds and extracts accurate answers for the user. It also retrieves the most relevant potential answers and ranks them on the web page.

The evaluation experiment shows the high recall performance of the system, which is attributable to stemming. In the future, we expect to improve user interaction to incorporate user feedback for more precise results. We also plan on using the Arabic Named Entity Recognition to provide more accurate answers. In addition, we will consider the "why" and "how" questions, which require deep linguistic and semantic analysis.

\section{ACKNOWLEDGEMENTS}

This work was partially funded by the Long-Term Comprehensive National Plan for Science, Technology and Innovation of the Kingdom of Saudi Arabia, grant number 11- INF1895-08.

We would like to thank Dr. Sman Bekhti and his colleagues for their valuable support and comments based on their Arabic QA system AQUASYS. We would also like to thank Dr. Shereen Khoja for providing us with the Arabic stemmer package that she has developed.

\section{REFERENCES}

[1] R. Alshalabi "Experimenting with a Question Answering System for the Arabic Language", Computers \& the Humanities, Vol. 38, pp. 397-415, Nov 2004.

[2] K. Arai and A. Handayani "Question Answering System for an Effective Collaborative Learning", International Journal of Advanced Computer Science and Applications, Vol. 3, pp.60-64,

[3] A. Allam and M. Haggag, "The Question Answering Systems: A Survey," International Journal of Research and Reviews in Information Sciences (IJRRIS), Vol. 2, No. 3, September 2012.

[4] D. Hai, L. Kosseim, "The Problem of Precision in Restricted-Domain Question Answering. Some Proposed Methods of Improvement", In Proceedings of the ACL 2004 Workshop on Question Answering in Restricted Domains, Barcelona, Spain, Publisher of Association for Computational Linguistics, July 2004, PP.8-15.

[5] A. Lamjiri, "A Syntactic Candidate Ranking Method for Answering Non-copulative Questions," Ph.D. thesis, Concordia University, 2007.

[6] P. Walke, S. Karale, "Implementation approaches for various categories of question answering system," In Proceedings of IEEE Conference on Information \& Communication Technologies (ICT), 2013, pp .402,407, 11-12 April 2013.

[7] R. Alshalabi, "Pattern-based stemmer for finding Arabic roots," Information Technology Journal, Vol. 4, pp. 38-43, 2005.

[8] R. Grishman, B. Sundheim, “Message Understanding Conference-6: A Brief History,” In Proceedings of International Conference on Computational Linguistics, 1996.

[9] A. Borthwick, “A Maximum Entropy Approach to Named Entity Recognition”. PhD thesis, New York University, 1999.

[10] Y. Benajiba, P. Rosso , J. Ruiz, "ANERsys: An Arabic Named Entity Recognition system based on Maximum Entropy", In Proceedings of CICLing, 2007

[11] M. Maamouri, A. Bies, T. Buckwalter, "The Penn Arabic Treebank: Building a Large-scale Annotated Arabic Corpus," In Proceedings of Arabic Language Resources and Tools, 2004.

[12] S. Mesfar, "Standard Arabic formalization and linguistic platform for its analysis," In Proceedings of The Challenge of Arabic for NLP/MT Conference, London, England, pp. 84-95, 2006.

[13] A. Kabbaj, K. Bouzoubaa, "Amine Platform Page on SourceForge", http://amineplatform.sourceforge.net/, checked Aug. 2nd, 2013 
[14] JM Gómez, D. Buscaldi, P. Rosso, E. Sanchis, "JIRS Language-Independent Passage Retrieval System A Comparative Study," In Proceedings of 5th International Conference on Natural Language Processing, ICON-2007, Hyderabad, India, January 4-6, 2007

[15] W. Black, S. Elkateb, H. Rodriguez, M. Alkhalifa, P. Vossen, A. Pease, C. Fellbaum "Introducing the Arabic WordNet project," In Proceedings of 3rd International WordNet Conference (GWC-06), 2006.

[16] A. Ezzeldin, M. Shaheen "A Survey of Arabic question answering: challenges, tasks, approaches, tools, and future trends", In Proceedings of The International Arab Conference on Information Technology, 2012, pp. 280-287.

[17] S. Bekhti, A. Rehman, M. Al-Harbi, T. Saba "AQuASys: an Arabic Question Answering System Based on Extensive Question Analysis and Answer Relevance Scoring," International Journal of Academic Research, Vol. 3, pp.45-54, July 2011.

[18] O. Badawy, M. Shaheen and A. Hamadene "ARQA High-Performance Arabic Question Answering System”, In Proceedings of Arabic Language Technology International Conference, 2011, pp. 129136.

[19] F. Mohammed, K. Nasser, H. Harb "A knowledge-based Arabic Question Answering System (AQAS)," In Proceedings of ACM SIGART Bulletin, 1993, pp. 21-33.

[20] B. Hammo, H. Abu-Salem, S. Lytinen. "QARAB: A Question Answering System to Support the Arabic Language". In Proceedings of the workshop on computational approaches to Semitic languages, 2002, pp. 55-65, Philadelphia.

[21] Y. Benajiba, P. Rosso, A. Lyhyaoui "Implementation of the ArabiQA Question Answering System's components.", In Proceedings of Workshop on Arabic Natural Language Processing, 2nd Information Communication Technologies Int. Symposium, ICTIS, 2007.

[22] W. Brini, M. Ellouze, S. Mesfar, L. Belguith "An Arabic Question-Answering system for factoid questionns," In Proceedings of IEEE International Conference on Natural Language Processing and Knowledge Engineering, 2009.

[23] S. Khoja, "Stemming Arabic Text,"” http://zeus.cs.pacificu.edu/shereen/research.htm, checked may 6th, 2013.

[24] http://www.nooj4nlp.net/pages/introduction.html, checked may 6th, 2013.

\section{AUTHORS}

Dr. Heba Kurdi received her B.Sc. and MSc. degrees in computer science from King Saud University, Riyadh, Saudi Arabia, in 1999, and 2003 respectively. In 2010, she got her PhD in wireless networks and communications from the School of Engineering and design, Brunel University, UK, where she also contributed as a visiting researcher in Summer 2011. She is currently an assistant professor at Al Imam Muhammad Ibn Saud Islamic University, Riyadh, SA. She has many publications in international conferences and journals and she is also a frequent reviewer for several computer science journals. Her research interest includes grid and cloud computing, and Bio-inspired Engineering.

Sara Alkhaider received her B.Sc. in Computer Science from Princess Noura University, Riyadh, Saudi Arabia, in 2009. She is currently an MSc student of Computer Science at Al Imam Muhammad Ibn Saud Islamic University, Riyadh, Saudi Arabia,. Her research interest includes web technologies, information retrival.

Nada Al Faifi is an MSc student at the Computer Science Department in Al Imam Muhammad Ibn Saud Islamic University, Riyadh, Saudi Arabia. She has received her BSc. Degree in Computer sciences from the same university. Her research interest includes semantic web and artificial intellegince. 


\title{
COHERENCE ENHANCEMENT DIFFUSION USING ROBUST ORIENTATION ESTIMATION
}

\author{
Mohammad A. U. Khan ${ }^{1}$, Tariq M. Khan ${ }^{2}$, Wadee Al-Halabi ${ }^{3}$, Hiba

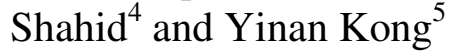 \\ ${ }^{1,4}$ Department of Electrical and Computer Engineering, \\ Effat University, Jeddah, Saudi Arabia. \\ mohammad_a_khan@yahoo.com \\ ${ }^{2,5}$ Department of Engineering, Macquarie University, Sydney, Australia \\ tariq045@gmail.com \\ ${ }^{3}$ Department of Computer Science, King Abdulaziz University, Saudi Arabia \\ wsalhalabi@kau.edu.sa
}

\begin{abstract}
In this paper, a new robust orientation estimation for Coherence Enhancement Diffusion (CED) is proposed. In CED, proper scale selection is very important as the gradient vector at that scale reflects the orientation of local ridge. For this purpose, a new scheme is proposed in which pre calculated orientation, by using orientation diffusion, is used to find the correct true local scale. From the experiments it is found that the proposed scheme is working much better in noisy environment as compared to the traditional Coherence Enhancement Diffusion.
\end{abstract}

\begin{abstract}
KEYWORDS
Fingerprint Image Enhancement, Coherence Enhancement Diffusion Directional Filter Bank (DFB), Decimation free Directional Filter Bank (DDFB).
\end{abstract}

\section{INTRODUCTION}

Biometric technology deals with identifying humans with their traits or characteristics. It deals with measurable albeit distinctive characteristics which can be used to label and to a large extent describe individuals [1]. Examples include, face recognition, DNA, palm print, hand geometry, retina, iris recognition and fingerprint. Out of these many biometric techniques, fingerprint identification is the most prevailing one which has been successfully used in numerical applications in the past [2]. It has been observed that every human being has a unique and immutable fingerprint [3]. Individually is hidden in the pattern of ridges and valleys on the fingerprint surface. Ridges are found to terminate themselves at some locations and split at others. These are referred to as ridge ending and bifurcation respectively. The locations corresponding to these ridge endings and bifurcations are known as minutiae points. Identifying human fingerprint means locating correctly these minutia points.

With the advancement in computer technology, need to automatic fingerprint identification of fingerprint scans arises. Reliable extraction of minutiae is one of the critical step for an automatic fingerprint identification system (AFIS). We can forsee the importance of the quality of input David C. Wyld et al. (Eds) : CCSIT, SIPP, AISC, PDCTA, NLP - 2014 
fingerprint scan in determining the reliability of the overall automatic fingerprint identification system (AFIS) [1]. The fingerprints are obtained with fingerprint scanners that may not provide a perfect scan in all circumstances. The presence of dirt or oil on the surface of the finger may result in blurred scan. Furthermore, due noise present in scanner electronics, the fingerprint scans may not be of clear quality. Mostly, the noise in fingerprint image manifest itself in terms of cuts or interpolated ridge lines. For automatic fingerprint identification system (AFIS) to work reliability, these broken ridge lines need to be restored vis some enhancement process [4].

A fingerprint enhancement process generally consists of estimating local image parameters, which in turn are used to tune a contextual filter that moves from one location to another on the surface of fingerprint, smoothing noise on the way. Contextual filters can be conveniently defined in the framework of partial differential equations [5]. A major development in PDE based image diffusion was introduced earlier by Perona and Malik [6]. On the basis of their study, number of diffusion filters have been proposed [7, 8, 9, 10, and 11]. Most of these techniques are based on a scalar diffusivity parameter which guides the diffusion flux moving along the orthogonal to the gradient direction. These techniques are linear and isotropic in nature, and have same common disadvantages, like edge blurring. To improve the performance of these isotropic diffusion Joachim Weickert [12] proposed a non-linear isotropic diffusion, which replaces scalar diffusivity parameter with diffusion matrix to provide greater flexibility in dealing with oriented structure on point to point basis. This technique is well known as Coherence Enhanced Diffusion (CED). The analysis of the CED technique can be carried out by an eigen decomposition of the diffusion matrix, which results in eigenvalues and eigenvectors. The eigenvalues dictates the amount of smoothing, while the eigenvectors are responsible for steering these smoothing in proposer directions. Though eigenvalues are essential part of coherence enhanced diffusion, a clean computation of eigenvectors without noise may be ultimately responsible for evolving the whole diffusion process. We believe that a CED in its current status lacks this clean steering process.

The impact of steering diffusion in wrong directions may distort some genuine minutia points while creating fake ones as the same time. We propose to relieve the stress in providing clean eigenvectors in the form of embedding an orientation diffusion block in the CED process. Thus letting CED to worry only about proper diffusion speeds at each point. Thus CED will provide the diffusion of noisy fingerprints with diffusivity parameters that align themselves with clean directions at each diffusion step.

The research presented in this paper is concerned with finding reliable orientations and then finding a way to embed them in the conventional CED algorithm. Orientation diffusions [13] has been there for a while, that provides a framework for diffusing directions alone with noise reduction at each step. We adapted the method to be used with the conventional CED scheme to boost its performance in noisy images. The rest of the paper is organized as follow. Section II details the Coherence Enhanced Diffusion. In section III a summary of orientation diffusion method is discussed. Section IV describes how the pre-computed orientation is used in Coherence Enhancement Diffusion. In Section V testing and validation is presented and finally the paper is concluded in section VI.

\section{COHERENCE ENHANCED DIFFUSION}

Weickert [14] considered a general class of anisotropic diffusion process. The process utilizes a diffusion matrix depending on the local image structure via the so-called second-moment matrix (or structure tensor). The second-moment matrix is a well-established tool for texture analysis $[15,15,17$, and 18]. In [14], Weickert investigated the use of second-moment matrix information to design anisotropic diffusion scale-space, which enhances the coherence of the ridge-like 
texture in fingerprint. The method is generally referred to as Coherence Enhanced Diffusion (CED), and is summarized in the following sections.

Consider an input fingerprint image $L(x, y)$. The anisotropic scale-space for the image $L(x, y)$ can be found by implementing the heat equation, given by:

$\partial_{t} L=\operatorname{div}(D \nabla L)$

where $D$ is the $2 \times 2$ diffusion matrix, adopted to the local image structure, via a structural descriptor, called the second-moment matrix $\mu$, defined as:

$\mu=\left(\begin{array}{ll}\mu_{11} & \mu_{12} \\ \mu_{12} & \mu_{22}\end{array}\right)=\mu=\left(\begin{array}{cc}L_{x}^{2} & L_{x} L_{y} \\ L_{x} L_{y} & L_{y}^{2}\end{array}\right)$

where $L_{x}{ }^{2}, L_{x} L_{y}$ and $L_{y}{ }^{2}$ represents the Gaussian derivative filters, in x-and-y directions. The matrix is a symmetric $2 \times 2$ matrix, with two dominant eigen values $\lambda_{\min }$ and $\lambda_{\max }$, given by:

$\lambda_{\text {min }}=1 / 2\left(s_{11}+s_{12}+\alpha\right)$
$\lambda_{\text {max }}=1 / 2\left(s_{11}+s_{12}-\alpha\right)$

Where

$\alpha=\sqrt{\left(s_{11}-s_{22}\right)^{2}+4 s_{12}^{2}}$

Let us now define the diffusion speeds $c_{u}$ and $c_{v}$ as

$$
\begin{aligned}
& c_{u}=\max \left(0.01,1-e^{-\left(\lambda_{\min }-\lambda_{\max }\right)^{2}} / k^{2}\right) \\
& c_{v}=0.01
\end{aligned}
$$

The diffusion matrix $D$ with component is given by:

$$
\begin{aligned}
& d_{11}=1 / 2\left(\left(c_{u}-c_{v}\right)+\frac{\left(c_{u}-c_{v}\right)-\left(s_{11}-s_{22}\right)}{\alpha}\right) \\
& d_{12}=\frac{\left(c_{u}-c_{v}\right) s_{12}}{\alpha} \\
& d_{22}=d_{11}=1 / 2\left(\left(c_{u}-c_{v}\right)-\frac{\left(c_{u}-c_{v}\right)-\left(s_{11}-s_{22}\right)}{\alpha}\right)
\end{aligned}
$$


Once the diffusion matrix is constructed, we are set to start the evaluation process using Eq. 1 with input image $L(x, y)$ as the starting point. In the process of evaluation, the image got enhanced ridge pattern, as shown in Fig. 1

The diffusion matric $\left(\begin{array}{ll}d_{11} & d_{12} \\ d_{12} & d_{22}\end{array}\right)$ can be decomposed using eigen decomposition, into eigenvalues $c_{u}$ and $c_{v}$ along with eigen directions. By plotting these eigen directions on top of the enhanced image it can be observed that these directions need to be corrected for better evolution. However, their cleaning process for eigen directions need to be embedded in the CED process such that each diffusion step is proceeded in the clean direction. For cleaning directions at each step, we take the help of orientation diffusion process as suggested in [19].

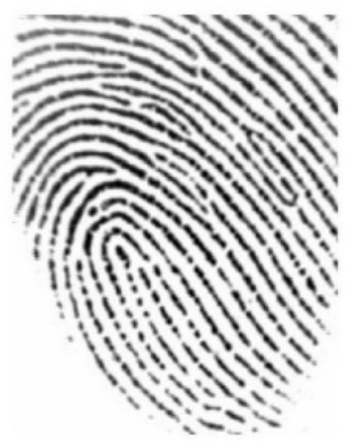

(a)

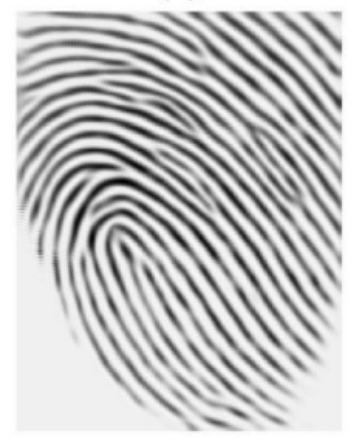

(c)

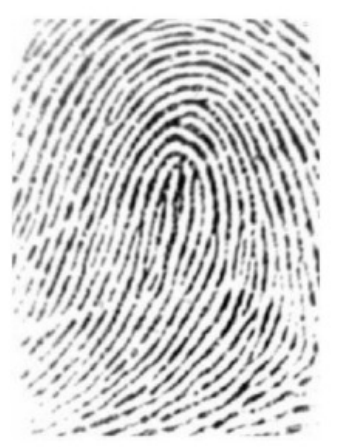

(b)

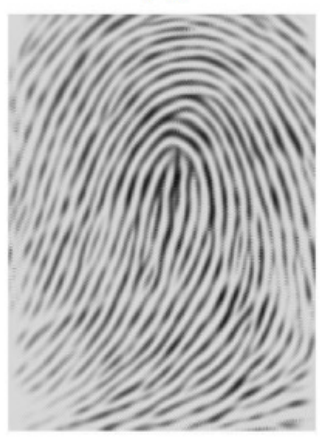

(d)

Fig. 1. a) and b) are two noisy images from FVC database. c) and d) shows the results of CED enhancement of a) and b) respectively.

\section{ORIENTATION DIFFUSION}

Orientation filed estimation is considered as first step moving towards enhancement process as suggested in [1]. But in noisy environment, this provide a challenge. In research literature, different techniques have been in use for orientation estimation in noise-degraded images. The notable examples can be cited in [20], where a filter bank based approach is used, and [21], where waveform projection, and [22] with spectral estimation. To increase the degree of accuracy for the initial coarse estimation, mathematical modelling using Bayesain network [23] and complex polynomial can be proposed. Later, [24] introduces an enhanced gradient-based approach for reliable orientation estimation. In this scheme, dominant orientation of a base block from its four overlapping neighbourhoods is estimated and finally a best estimate is selected from least noise- 
affected neighbourhood. However, gradients are unl-reliable and error-prone on fingerprint images affected by noise like scares, smudges, dryness or wetness of finger [25]. Recently, DFB has [26] also been used to provide reliable orientation. However, we feel that orientation diffusion provide a natural coupling for our purpose. The reason for this natural coupling stems from the fact that orientation diffusion provides a gradually decrease in noise levels present in orientations as opposed to a step process adopted by DFB based orientations.

According to [19], the correct way of diffusing orientation is to adopt a non-linear diffusion function in the form as given below:

$\theta(x, t+1)=\theta(x, t)+\lambda \sum_{n \in N(x)} \sin (\theta(n, t)-\theta(x, t))$

where $N(x)$ is the set of all 8 neighbours around $\mathrm{x}$ location. Also $\theta$ has to be in the range of $[-\pi, \pi]$.

The discrete implementation of orientation diffusion, as described by Eq. 7 on a cropped noisy pattern of the fingerprint image shown in Fig. 4. The initial condition of diffusion process was calculated from the gradient of the image intensities, i.e, $\theta_{\text {initial }}=\tan ^{-1}\left(\frac{g_{y}}{g_{x}}\right)+\frac{\pi}{2}$. The boundary condition are set to zero gradients.

The results of running the diffusion may be seen in Fig. 2. We notice the desirable effect of smoothing orientations on the way. The two singularities of orientations that ate present in the fingerprint would be difficult to detect in the original image using a local gradient operator. We found that this orientation diffusion inspired by simple physical systems, can de-noise an orientations data effectively, by eating out already existing print-singularities with new generation of singularities, Thus, it does support the casuality property of diffusion.

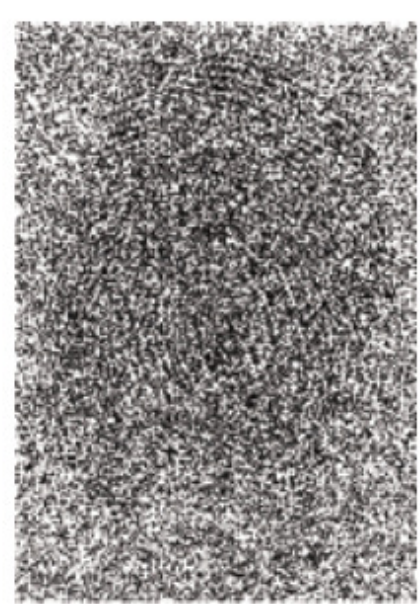

a

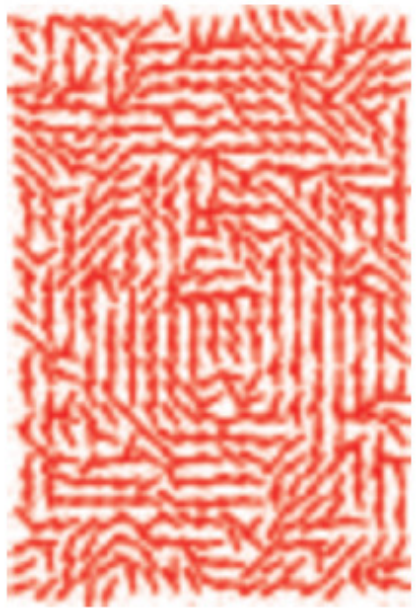

b

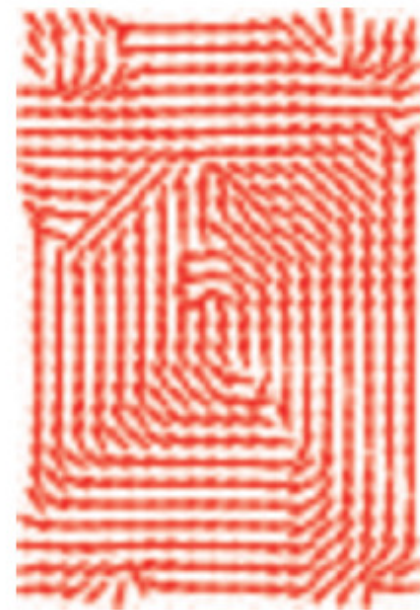

C

Fig. 2. a) sample noisy image, b) CED Orientation, and c) Orientation enhanced by orientation diffusion 


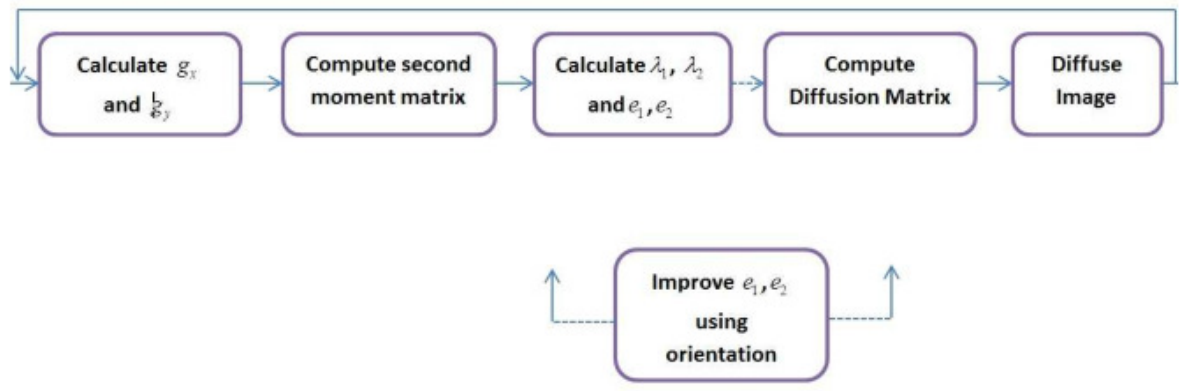

Fig. 3. Orientation Fed CED block diagram

\section{ORIENTATION FED COHERENCE ENHANCED DIFFUSION}

As we discussed in section II, the CED process starts by taking an input image, then calculate its gradients $g_{x}$ and $g_{y}$ respectively. From $g_{x}$ and $g_{y}$, it computes the second moments matrix $\left(\begin{array}{ll}s_{11} & s_{12} \\ s_{12} & s_{22}\end{array}\right)$ to depict the rotation in local structure. The second moment matrix can be decomposed to provide us with eigen values $\lambda_{1} \& \lambda_{2}$ and also the eigen directions $e_{1} \& e_{2}$. In the conventional design, these lambdas and eigenvectors can be used in the construction of diffusion matrix used in diffusion process.

However, in our proposed scheme, we keep intact the lambdas, but take $e_{1} \& e_{2}$ direction for further cleaning. For this purpose, we feed $e_{1} \& e_{2}$ into our orientation diffusion module for further de-noising. Once cleaned, we feed this cleaned $e_{1} \& e_{2}$ back to our module of computing diffusion matrix. And thus the process continues.

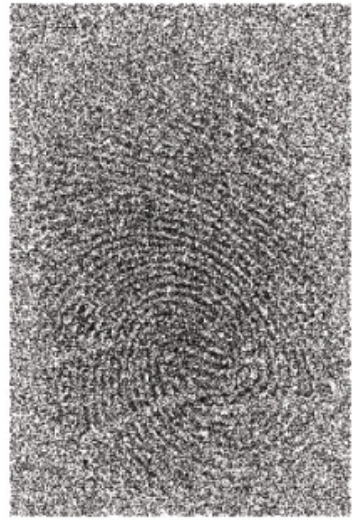

a

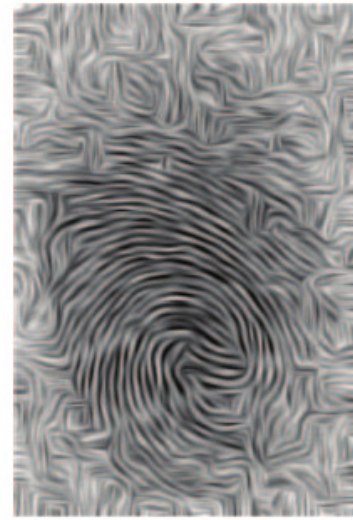

b

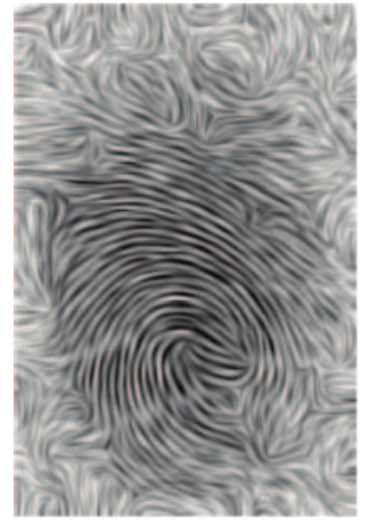

C

Fig. 4. a) sample noisy image, b) CED enhanced image, and c) Orientation Fed CED 


\section{TESTING AND VALIDATION}

Statistically, the set of minutiae denoted by symbol $M$ were algorithmically obtained and evaluated against the set of minutiae marked by an expert denoted by symbol $F$ and the minutiae are classified into two types: termination and bifurcation. Each minutiae point $m$ compartmentalized into one of the following classes:

- Correct: The correlation between minutiae points generated through the algorithm and those obtained from the expert is perfect position, i.e $\left(m \in F \cap M\right.$ and type $_{F}(m)=$ type $\left._{M}(m)\right)$

- False: The expert did not mark the location corresponding to the one obtained through the algorithm, i.e $(m \in M, m \notin F)$

- Missing: The minutiae location has been marked by the expert but couldn't be seen within the algorithmically generated image, i.e $(m \notin M, m \in F)$

- Misclassified: The minutiae location marked by the expert could be is also the one found by the method with the exception that one has been classified as bifurcation and other has been classified as termination, i.e $\left(m \in F \cap M\right.$ and type $_{F}(m) \neq$ type $\left._{M}(m)\right)$

Compartmentalization of all the minutiae set is followed by the performance measurement which is computed statistically. This is done on minutiae obtained from the algorithm as well as minutiae marked by the experts which makes the performance measurement relative to either total number of minutiae found algorithmically or the total number of minutiae found by the expert markings. The measures relative to minutiae marked by the expert which detects the aptitude of the technique to detect correct minutiae are as follows:

- Missing minutiae is defined as $\frac{M i s \sin g}{|F|}$

- False minutiae is defined as $\frac{\text { False }}{|F|}$

- Misclassified minutiae is defined as $\frac{\text { Missclassified }}{|F|}$

- Correct minutiae is defined as $\frac{\text { Correct }}{|F|}$

- Total error which is the summation of false positive, false negative and misclassified errors is defined as $\frac{\text { Mis } \sin g \cup \text { False } \cup \text { Missclassified }}{|F|}$

- Classification rate for the purpose of enhancing the graphical visualization is defined as Correct

Missclassified 
Large number of missing minutiae could be tolerated to certain extent but the large number of false minutiae would lead to larger noise rate within the mapping stage. On the other hand, prominence of classification error is based on the manner in which the mapping algorithm deals with the types of minutiae. Using the techniques described above, the database of true minutiae was generated, by using the sample images given in Fig. 6 which is used to evaluate the orientation and scale-matched filters to produce improved input image along with the techniques described above.

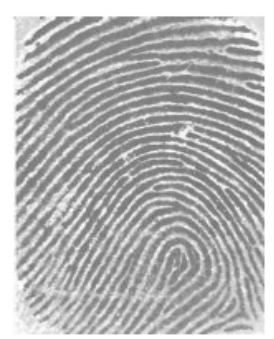

(Image 1)

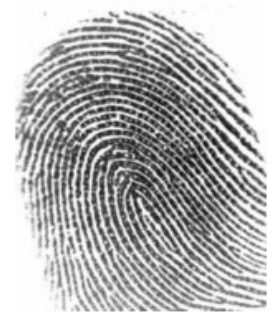

(Image5)

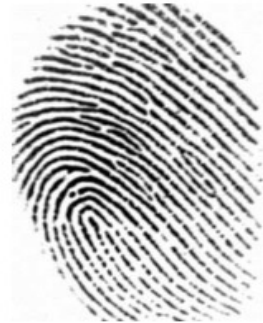

(Image2)

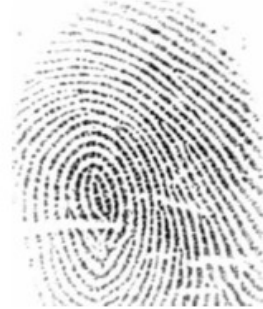

(Image6)

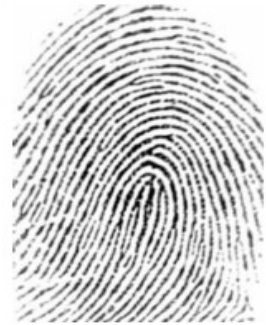

(Image 3)

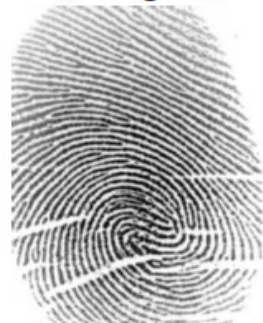

(Image7)

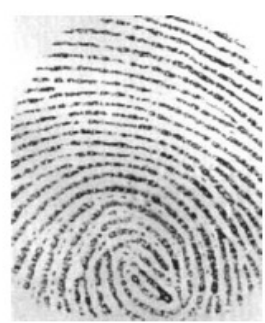

(Image4)

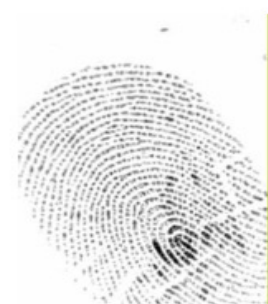

(Image8)

Fig. 5. Sample images used for evaluation measure

The table compares the result obtained using the above method.

Table1. This table shows the evaluation measure comparing minutiae of proposed algorithm

\begin{tabular}{|c|c|c|c|c|}
\hline Proposed & Correct & False & Missing & Misclassified \\
\hline Image1 & 21 & 4 & 6 & 4 \\
\hline Image2 & 18 & 8 & 8 & 5 \\
\hline Image3 & 21 & 19 & 9 & 1 \\
\hline Image4 & 31 & 5 & 5 & 1 \\
\hline Image5 & 20 & 12 & 20 & 3 \\
\hline Image6 & 10 & 15 & 20 & 4 \\
\hline Image7 & 16 & 11 & 27 & 1 \\
\hline Image8 & 6 & 2 & 30 & 2.5 \\
\hline Mean & 17.875 & 9.5 & 15.625 & \\
\hline
\end{tabular}


Table2. This table shows the evaluation measure comparing minutiae of [12] CED Enhancement algorithm

\begin{tabular}{|c|c|c|c|c|}
\hline [12]CED & Correct & False & Missing & Misclassified \\
\hline Image1 & 19 & 8 & 6 & 6 \\
\hline Image 2 & 17 & 9 & 7 & 5 \\
\hline Image3 & 20 & 21 & 6 & 3 \\
\hline Image4 & 24 & 5 & 10 & 4 \\
\hline Image5 & 21 & 17 & 19 & 5 \\
\hline Image6 & 8 & 16 & 22 & 2 \\
\hline Image7 & 15 & 10 & 26 & 3 \\
\hline Image8 & 5 & 3 & 30 & 2 \\
\hline Mean & 16.125 & 13.25 & 15.75 & 3.75 \\
\hline
\end{tabular}

The average number of minutiae points present in the image marked by the expert and the ones marked by the algorithm for all the eight images was taken, which represented \$F\$. This was computed to be 15 using which some performance measures were computed. For the proposed method, classification error which is calculated as misclassified over correctly detected minutiae was found to be

$\frac{\text { Missclassified } / F \cap M}{|F|} * 100=0.89 \%$

For the CED method, classification error which is calculated as misclassified over correctly detected minutiae was found to be

$\frac{\text { Missclassified } / F \cap M}{|F|} * 100=1.45 \%$

In Fig7, the bar chart shows the performance evaluation measure of both schemes.

\section{CONCLUSIONS}

Coherence enhancement diffusion filters are the one which have control on the size/shape of the filter but, these use derivative for rotation matrix which is not considered as a reliable method. Therefore, in this work presents a new and a reliable method for orientation estimation is proposed to explicitly calculate the orientation field of a fingerprint image. By using this orientation new local scales for Diffusion matrix are adaptively calculated. The experiments conducted on the noisy images showed promising results of the proposed scheme. 


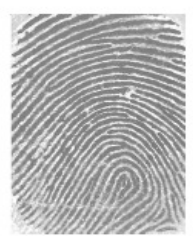

(a1)

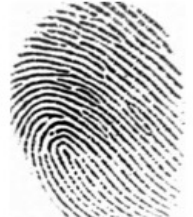

(a2)

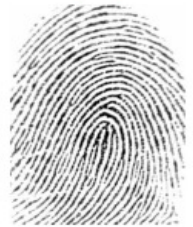

(a3)

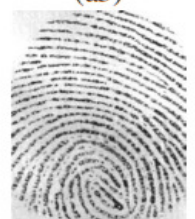

(a4)

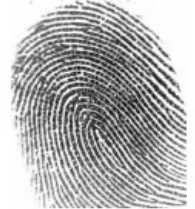

(a5)

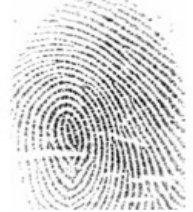

(a6)

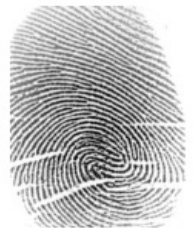

(a7)

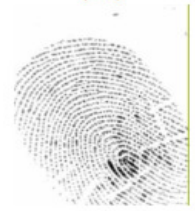

(a8)

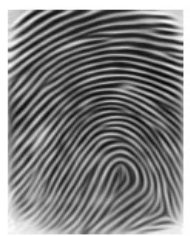

(b1)

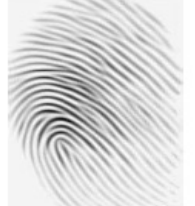

(b2)

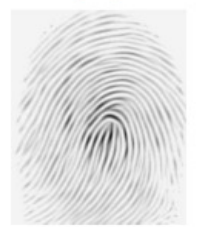

(b3)

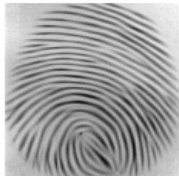

(b4)

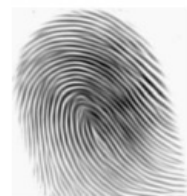

(b5)

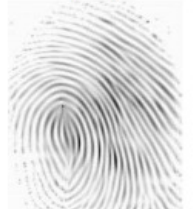

(b6)

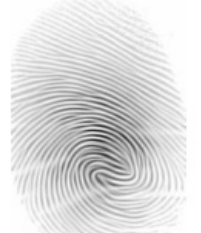

(b7)

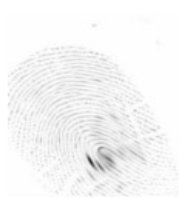

(b8)

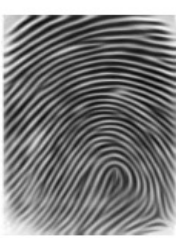

(c1)

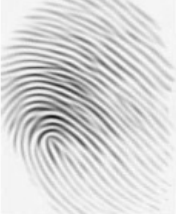

(c2)

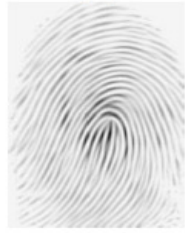

(c3)

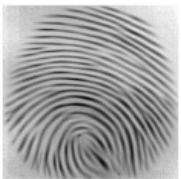

(c4)

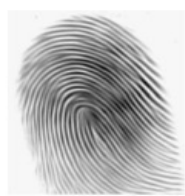

(c5)

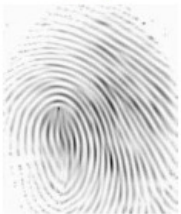

(c6)

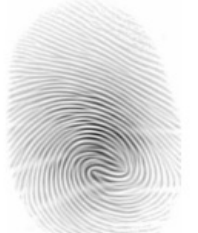

(c7)

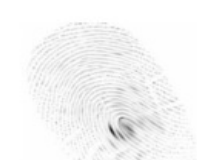

(c8)

Fig. 6. Sample images used for evaluation measure 


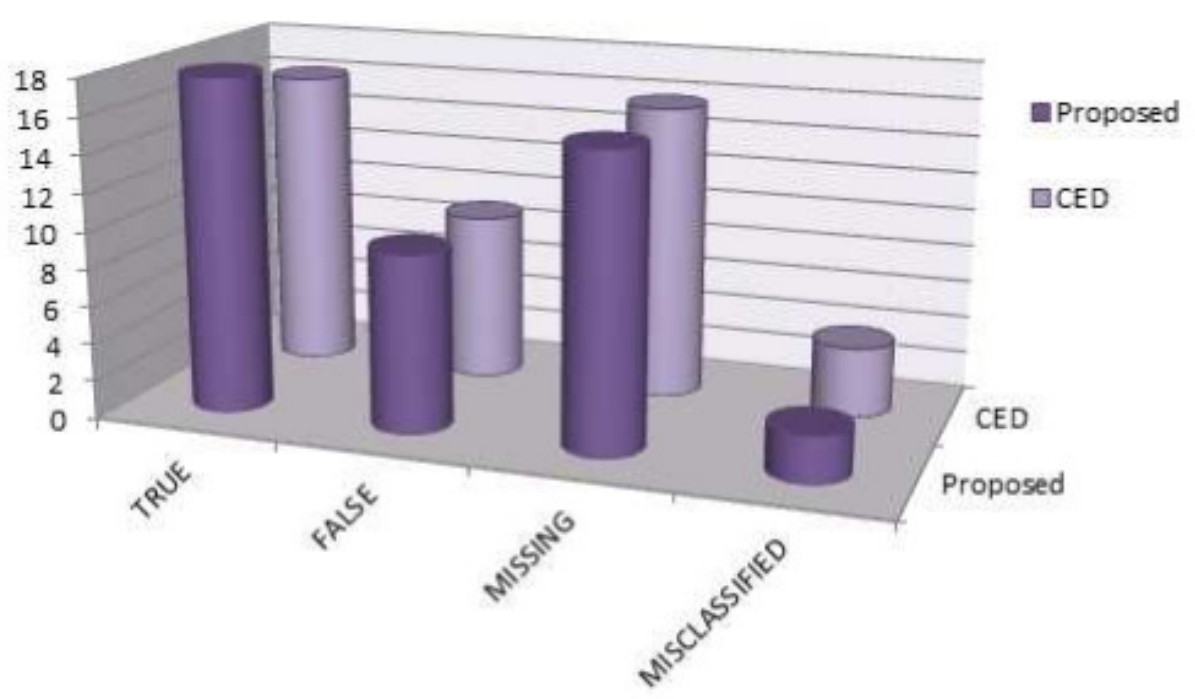

Fig. 7. Performance Evaluation Measure of both schemes

\section{REFERENCES}

[1] L. Hong, Y. Wan, and A. Jain, "Fingerprint image enhancement: Algorithm and performance evaluation," IEEE Transactions on Pattern Analysis and Machine Intelligence, vol. 20, pp. 777789, 1998.

[2] M. A. Khan amd A. Khan, T. Mahmmod, M. Abbas, and N. Muhammad, "Fingerprint image enhancement using principal component analysis (pca) filters," in International Conference on Information and Emerging Technologies (ICIET), 2010.

[3] Anil K. Jain, S. Prabhakar, and Sharath Pankanti., Can identical twins be discriminated based on fingerprints?, Michigan State University, De- partment of Computer Science, Michigan State University, East Lansing, Michigan, October 2000., technical report msu-cse-00-23, edition.

[4] M.A.U. Khan, T. M. Khan, and S. A. Khan, "Coherence enhancement diffusion using multi-scale dfb," in 7th International Conference on Emerging Technologies, 2011.

[5] J Weickert, "A review of nonlinear diffusion filtering," cale-Space Theory in Computer Vision, vol. 1252, pp. 3-28, 1997.

[6] P Perona and J Malik, "Scale-space and edge detection using anisotropic diffusion.," IEEE Trans Mach Intell., vol. 12(7), pp. 629639, 1990.

[7] L Alvarez, P-L Lions, and J-M Morel, "Image selective smoothing and edge detection by nonlinear diffusion,” II SIAM J Numer Anal, vol. 29, pp. 845866, 1992.

[8] G Sapiro, "Tannenbaum, affine invariant scale-space," International Journal of Computer Vision, vol. 11, pp. 25-44, 1993.

[9] L Rudin, S Osher, and E Fatemi, "Nonlinear total variation based noise removal algorithms," Modeliz Mat Traitement d Images, p. 149179, 1992.

[10] HM Salinas and D Cabrera Fernandez, "Comparison of pde-based nonlinear diffusion approaches for image enhancement and denoising in optical coherence tomography," IEEE Transactions on Medical Imaging, vol. 26, pp. 761-771, 2007.

[11] D Fang, Z Nanning, and X Jianru, "Image smoothing and sharpening based on nonlinear diffusion equation.," Signal Processsing, vol. 88(11), pp. 28502855, 2008.

[12] J Weickert, “Coherence-enhancing diffusion filtering," Int. J. Computer Vision,, vol. 31, pp. 111-127, 1999.

[13] Muhammad A. K. Khan and Wadee Alhalabi, "Robust multi-scale orientation estimation: Spatial domain vs fourier domain," in Inter- national Conference on Communications, Signal Processing, and their Applications, 2013. 
[14] Joachim Weickert, Anisotropic Diffusion in Image Processing, ECMI Series, Teubner-Verlag, Stuttgart, Germany, 1998.

[15] K. Ito and K. Kunisc, Augmented Lagrangian methods for nonsmooth, convex optimization in Hilbert spaces, Preprint No. 409/1994, Fachbere- ich Mathematik (3), Technische Universitat Berlin, Strae des 17. Juni 136, 10623 Berlin, Germany, 1994.

[16] R. Illner and J. Tie, "On directed diusion with measurable background," Math. Meth. Appl. Sci., p. 681690, 1993.

[17] O. Faugeras and R. Keriven, "On projective plane curve evolution," in ICAOS 96: Images, wavelets and PDEs, Lecture Notes in Control and Information Sciences, 1996, p. 6673.

[18] G.-H. Cottet and M. El Ayyadi, "Nonlinear pde operators with memory terms for image processing," in Proc. IEEE Int. Conf. Image Processing (ICIP96, Lausanne, Sept. 1619, 1996), 1996, p. 481483.

[19] P. Perona, “Orientation diffusions," IEEE Trans. on Image Process., vol. 7, pp. 457-467, 1998.

[20] A.K. Jain, S. Pankanti, and L. Hong, "A multichannel approach to fingerprint classification," IEEE Trans. Pattern Anal. Machine Intell, vol. 21, pp. 348359, 1999.

[21] B.G. Sherlock, D.M. Monro, and K. Millard, "Fingerprint enhancement by directional fourier filtering," IEE Proc. Vision, Image Signal Process., vol. 141, pp. 8794., 1994.

[22] C.L. Wilson, G.T. Candela, and C.I. Watson, "Neural network fingerprint classification," J. Artif. Neural Networks, vol. 1, pp. 203-228, 1994.

[23] S.C. Dass, "Markov random field models for directional field and singularity extraction in figerprint images," IEEE Trans. Image Process, vol. 13, pp. 13581367, 2004.

[24] Yi Wang, Jiankun Hu, and Fengling Han, "Enhanced gradient-based algorithm for the estimation of fingerprint orientation fields," Applied Mathematics and Computation, vol. 185, pp. 823833, 2007.

[25] Gottschlich C, P. Mihailescu, and A. Munk, "Robust orientation field estimation and extrapolation using semilocal line sensors,” IEEE Trans. Inf. Forensics Sec., vol. 4, pp. 802811, 2009.

[26] P. T. H. Truc, M. A. U. Khan, Y. K. Lee, and T. S. Kim, "Vessel enhancement filter using directional filter bank," Computer Vision and Image Understanding, vol. 113, pp. 101-112, 2009.

\section{AUTHORS}

\section{Dr Yinan Kong}

Dr Kong leads the VLSI Research Group at Macquarie. He is also currently the Director of Higher Degree Research, Department of Engineering, Macquarie University, Sydney, Australia. Dr Kong obtained his PhD from the University of Adelaide, Australia.

During his research at the VLSI Research Group at Macquarie University and Centre for High Performance Integrated Technologies and Systems (CHiPTec) at the University of Adelaide, Dr Kong focused on digital VLSI design at an arithmetic level for specific applications like cryptography and DSP. He achieved the hardware construction of a 1024-bit RSA cryptosystem at only $0.4 \mathrm{~ms}$ per decryption, the fastest ever FPGA implementation of RSA. In the meantime, he developed Bachelor Degree of Computer Engineering at Macquarie Engineering as well as postgraduate teaching in embedded computer system design and computer arithmetic with hardware focused.

\section{Tariq Mahmood Khan}

Tariq Mahmmod Khan is interested in both Digital Image Processing (with an emphasis on biometrics) and VLSI. He is currently involved in the hardware implementation of multi-modal biometrics project, led by Yinan Kong. The main goal of this project is to develop a fast processor for different design approaches. The aim is to test these approaches on a multi-modal biometric system consisting fingerprint, iris and face. Prior to starting his $\mathrm{PhD}$ in Engineering at Macquarie University, he received a degree in $\mathrm{BS}$ Computer Engineering from COMSATS Institute of Information \& Technology, Islamabad Pakistan and MSc in Computer Engineering from University of Engineering \& Technology, Taxila Pakistan.

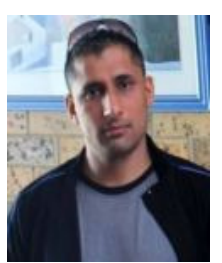




\title{
A FAST PU MODE DECISION ALGORITHM FOR H.264/AVC TO HEVC TRANSCODING
}

\author{
Jiunn-Tsair Fang ${ }^{1}$, Zong-Yi Chen ${ }^{2}$, Tsai-Ling Liao ${ }^{2}$, and Pao-Chi Chang ${ }^{2}$ \\ ${ }^{1}$ Department of Electronic Engineering, \\ Ming Chuan University, Taoyuan, Taiwan \\ fang@mail.mcu.edu.tw \\ ${ }^{2}$ Department of Communication Engineering, \\ National Central University, Jhongli, Taiwan \\ pcchang@ce.ncu.edu.tw
}

\begin{abstract}
H.264/AVC has been widely applied to various applications. However, a new video compression standard, High Efficient Video Coding (HEVC), had been finalized recently. In this work, a fast transcoder from H.264/AVC to HEVC is proposed. The proposed algorithm includes the fast prediction unit $(P U)$ decision and the fast motion estimation. With the strong relation between H.264/AVC and HEVC, the motion vectors (MVs), residuals, and modes from each coding block of H.264/AVC can be reused to predict the current encoding PU of HEVC. Furthermore, the $M V$ variance from $H .264 / A V C$ is calculated to decide the search range of $P U$ and also to reduce the prediction mode. Simulation results show that the proposed method can save up to $53 \%$ of the encoding time and maintains the rate-distortion $(R-D)$ performance for HEVC.
\end{abstract}

\section{KEYWORDS}

H.264/AVC, HEVC, PU, Fast Algorithm, Transcoding

\section{INTRODUCTION}

H.264/AVC is currently one of the popular video compression standards [1]. However, with increasingly demands on the high quality of video services, previous video compression standards are no longer satisfied. The new video compression standard, High Efficient Video Coding (HEVC) [2], had been finalized in April 2013. During the transfer to HEVC, transcoders become practical tools continuing to serve users. A fast transcoder from H.264/AVC to HEVC focusing on the prediction unit $(\mathrm{PU})$ is proposed.

Video transcoding refers to converting the video content from one format into another. For the transcoder of H.264/AVC to HEVC, the video bit-stream of H.264/AVC is fully decoded, and some parameters are extracted. These parameters are mainly motion vectors (MVs), residuals, and modes from each coding block. With strong relation between these two standards, these extracted parameters can be reused for HEVC, and the HEVC encoder does not need to create the related parameters for the prediction. Figure 1 shows the typical fast transcoder architecture. The proposed method is to build up the relation from these extracted parameters of H.264/AVC to predict the PU of HEVC. Then, the original procedure to predict PU by HEVC encoder can be skipped, and therefore the encoding time of HEVC is reduced.

David C. Wyld et al. (Eds) : CCSIT, SIPP, AISC, PDCTA, NLP - 2014

pp. 215-225, 2014. (C) CS \& IT-CSCP 2014

DOI : $10.5121 /$ csit.2014.4218 
Most related works for the transcoding focus on the fast mode decision and fast motion estimation because the time saving is significant. For the fast mode decision, the relationship between the input block and the current block was explored in [3]. Jing et al. determined the optimal coding mode for the re-encoding process [4]. Zhang et al. determined the best PU by the proposed power spectrum model [5]. As for the fast motion estimation, MV refinement was proposed to have a better MV predictor [3]. Garrido-Cantos et al. proposed to reduce the motion search range for H.264/SVC with the information of H.264/AVC [6]. In [7], the authors proposed a fast mode decision algorithm for HEVC to H.264/AVC intra frame transcoding.

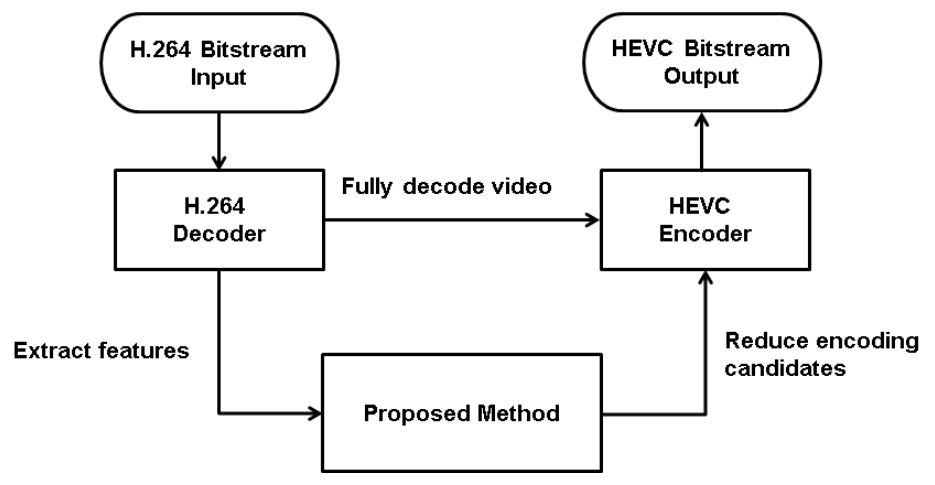

Figure 1. Typical fast transcoder architecture

Although promising results have been accomplished, their transcoders are applied for the same coding size between two standards. The coding unit (CU) sizes in depths 0 and 1 of HEVC are larger than the macroblock (MB) size of H.264/AVC. Thus, to design the transcoding from H.264/AVC to HEVC, various procedures are required for the different coding size prediction. A fast mode decision algorithm was proposed to explore the relationship between the input block and the current block for each depth of CU recently [8]. However, the PU was not considered in their method. Thus, a fast transcoding from H.264/AVC to HEVC focusing on PU is proposed.

The proposed algorithm consists of the fast mode decision and the fast motion estimation. Because of various coding sizes between H.264/AVC and HEVC, depths 0 and 1 and depths 2 and 3 of $\mathrm{CU}$ are applied various methods. The MVs, residuals, and modes information from H.264/AVC are reused for the PU encoder. As for the fast motion estimation, the MV variance from H.264/AVC is calculated to decide the search range of PU and also to reduce the prediction mode.

The rest of this paper is organized as follows. In Section 2, the proposed transcoding method is described. Section 3 shows the simulation results, and the conclusion is in Section 4.

\section{PROPOSED METHOD}

The proposed algorithm consists of the fast mode decision and the fast motion estimation. Before discussing the proposed algorithm, the preprocessing of the MV normalization is described.

\subsection{Motion Vector Normalization}

The MV extracted from various temporal references or various spatial sizes of H.264/AVC must be normalized to be the parameter predictor for HEVC. H.264/AVC applies multiple frames for the prediction, and the extracted MV may come from various temporal references. The MV normalization, denoted as $m v_{\text {norm }}$, is derived using (1): 


$$
m v_{n o r m}=\frac{1}{\alpha} m v_{n-\alpha},
$$

where $n$ is the current frame and $n-\alpha$ is the reference frame. Equation (1) shows that the MV normalization is to divide the frame number between the reference frame and the current frame. In other words, the extracted MV from the reference frame has a larger weighting if the reference frame is closer to the current frame.

H.264/AVC applies variable block sizes for the prediction, and the extracted MV may come from various sizes of the block. The MV normalization is to compare the area of the reference block with the area of the $4 \times 4$ block, which is derived using (2):

$$
m v_{\text {norm }}=\frac{\text { area of the reference block }}{\text { area of the } 4 \times 4 \text { block }} m v \text {. }
$$

Equation (2) shows that the extracted MV from the reference block has a larger weighting if the reference block has a larger area.

\subsection{Fast Mode Decision Algorithm}

In the proposed transcoder, the MVs, residuals, and modes information from H.264/AVC are reused to predict the PU of HEVC. However, the CU sizes in depths 0 and 1 of HEVC are larger than the MB size in H.264/AVC. Thus, the proposed fast mode algorithms for the transcoder to predict PU in depths 0 and 1 and PU in depths 2 and 3 are different.

\subsubsection{Fast Mode Decision Algorithm for Depth 0 and 1}

The proposed method for the transcoder to predict PU in depths 0 and 1 is described as follows. For the $2 \mathrm{~N} \times 2 \mathrm{~N}$ mode of PU, if all MVs in H.264/AVC are the same, the corresponding blocks in $\mathrm{HEVC}$ can be merged into a $2 \mathrm{~N} \times 2 \mathrm{~N}$ mode of $\mathrm{HEVC}$. For $2 \mathrm{~N} \times \mathrm{N}$ and $\mathrm{N} \times 2 \mathrm{~N}$ modes of $\mathrm{PU}$, the $\mathrm{MV}$ variance is defined as:

$$
V A R=\frac{1}{N} \sum_{n=1}^{N}\left(M V_{n}-\mu_{M V}\right)^{2},
$$

where $N$ is the total number of MVs in the reference block, and $\mu_{M V}$ is the average value of these MVs. According to (3), the calculations of the MV variances of the block $2 \mathrm{~N} \times \mathrm{N}$ and block $\mathrm{N} \times 2 \mathrm{~N}$ are as follows:

$$
\begin{aligned}
& \overline{V A R}_{2 N \times N}=\frac{1}{2}\left(V A R_{2 N \times N_{(U)}}+V A R_{2 N \times N_{(D)}}\right) \\
& \overline{V A R}_{N \times 2 N}=\frac{1}{2}\left(V A R_{N \times 2 N_{(L)}}+V A R_{N \times 2 N_{(R)}}\right),
\end{aligned}
$$

where $\overline{V A R}_{2 N \times N}$ is the average MV variance of upper and bottom blocks of the mode $2 \mathrm{~N} \times \mathrm{N}$, and $\overline{V A R}_{N \times 2 N}$ is the average $\mathrm{MV}$ variance of left and right blocks of the mode $\mathrm{N} \times 2 \mathrm{~N}$. The block segment is drawn in Figure 2. If the $\overline{V A R}_{X 2 N \times N} \geq 2 \times \overline{V A R}_{X N \times 2 N} \cap \overline{V A R}_{Y 2 N \times N} \geq 2 \times \overline{V A R}_{Y N \times 2 N}$, the mode $2 \mathrm{~N} \times \mathrm{N}$ in $\mathrm{PU}$ is skipped. On the other hand, if $\overline{V A R}_{X N \times 2 N} \geq 2 \times \overline{V A R}_{X 2 N \times N} \cap \overline{V A R}_{Y N \times 2 N} \geq 2 \times \overline{V A R}_{Y 2 N \times N}$, the mode $\mathrm{N} \times 2 \mathrm{~N}$ in PU is skipped. This is because a reference block with a larger MV variance is with less chance to be the candidate for the prediction. 

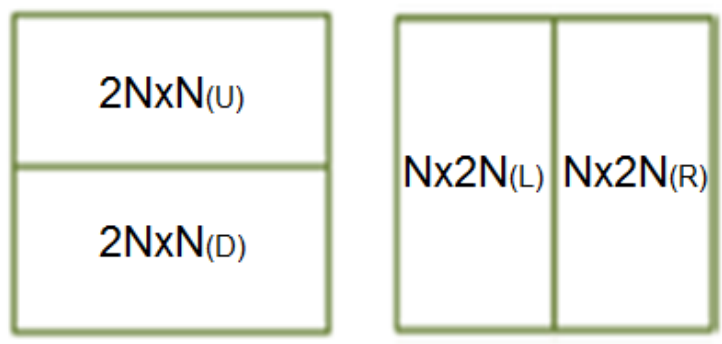

Figure 2. The block segment of the blocks $2 \mathrm{~N} \times \mathrm{N}$ and $\mathrm{N} \times 2 \mathrm{~N}$

For the asymmetric motion partition (AMP) mode in HEVC, the residual of each MB in H.264/AVC is applied to determine the candidate of the prediction. The residual is denoted as the sum of all the coefficients in the MB, derived using (4):

$$
\text { residual }=\sum_{i} \sum_{j}\left|\operatorname{coeff}\left(x_{i}, y_{j}\right)\right|
$$

where $\left(x_{i}, y_{j}\right)$ is the coefficient position of the $x$-axis and $y$-axis in an $\mathrm{MB}$, respectively. If the residual of $2 \mathrm{~N} \times \mathrm{N}_{(\mathrm{U})}$ is greater than the residual of $2 \mathrm{~N} \times \mathrm{N}_{(\mathrm{D})}$, the $2 \mathrm{~N} \times \mathrm{nD}$ mode is skipped. Otherwise, the $2 \mathrm{~N} \times \mathrm{nU}$ mode is skipped. As the residual of the $\mathrm{N} \times 2 \mathrm{~N}_{(\mathrm{L})}$ is greater than the residual of $\mathrm{N} \times 2 \mathrm{~N}_{(\mathrm{R})}$, the $\mathrm{nR} \times 2 \mathrm{~N}$ mode is skipped. Otherwise, $\mathrm{nL} \times 2 \mathrm{~N}$ mode is skipped. This is because a reference block with a larger residual implies that the block is with more content details and has a worse prediction, and the chance to adopt a mode with a worse prediction in H.264/AVC to PU is relatively low.

For the intra mode, it is selected only if the inter prediction has a poor prediction. Thus, if a $2 \mathrm{~N} \times 2 \mathrm{~N}$ block contains no any intra mode in H.264/AVC, the intra mode in HEVC is skipped.

\subsubsection{Fast Mode Decision Algorithm for Depth 2 and 3}

As for the depths 2 and 3, the MB size in H.264/AVC is the same as the CU size in HEVC. The proposed transcoder for fast algorithms in depths 2 and 3 are described as follows, respectively.

- $\quad$ Depth 2 fast mode decision algorithm:

$>$ If $\mathrm{H} .264 / \mathrm{AVC}$ is skip $=>$ just do $2 \mathrm{~N} \times 2 \mathrm{~N}$

$>$ If $\mathrm{H} .264 / \mathrm{AVC}$ is $16 \times 16=>$ do $2 \mathrm{~N} \times 2 \mathrm{~N}, 2 \mathrm{~N} \times \mathrm{N}$ and $\mathrm{N} \times 2 \mathrm{~N}$

$\rightarrow$ If residual $=0=>$ just do $2 \mathrm{~N} \times 2 \mathrm{~N}$

If $\mathrm{H} .264 / \mathrm{AVC}$ is $16 \times 8 \Rightarrow>$ do $2 \mathrm{~N} \times 2 \mathrm{~N}, 2 \mathrm{~N} \times \mathrm{N}, 2 \mathrm{~N} \times \mathrm{nU}$ and $2 \mathrm{~N} \times \mathrm{nD}$

$\rightarrow$ If residual $=0=>$ just do $2 \mathrm{~N} \times 2 \mathrm{~N}$ and $2 \mathrm{~N} \times \mathrm{N}$

$>$ If $\mathrm{H} .264 / \mathrm{AVC}$ is $8 \times 16 \Rightarrow$ do $2 \mathrm{~N} \times 2 \mathrm{~N}, \mathrm{~N} \times 2 \mathrm{~N}, \mathrm{~nL} \times 2 \mathrm{~N}$ and $\mathrm{nR} \times 2 \mathrm{~N}$

$\rightarrow$ If residual $=0=>$ just do $2 \mathrm{~N} \times 2 \mathrm{~N}$ and $\mathrm{N} \times 2 \mathrm{~N}$

$>$ If H.264/AVC is subMB $=>$ skip intra mode

$>$ If $\mathrm{H} .264 / \mathrm{AVC}$ is intra16 $=>$ do $2 \mathrm{~N} \times 2 \mathrm{~N}$ and intra

$>$ If $\mathrm{H} .264 / \mathrm{AVC}$ is intra $4=>$ do $2 \mathrm{~N} \times 2 \mathrm{~N}, 2 \mathrm{~N} \times \mathrm{N}, \mathrm{N} \times 2 \mathrm{~N}$ and intra mode

- $\quad$ Depth 3 fast mode decision algorithm:

If H.264/AVC is skip or $16 \times 16=>$ just do $2 \mathrm{~N} \times 2 \mathrm{~N}$

$>$ If H.264/AVC is $16 \times 8,8 \times 16$ or $8 \times 8=>$ skip intra

$\rightarrow$ If residual $=0=>$ just do $2 \mathrm{~N} \times 2 \mathrm{~N}$

If $\mathrm{H} .264 / \mathrm{AVC}$ is $8 \times 4=>$ skip intra $\mathrm{N} \times \mathrm{N}$

$\rightarrow$ If residual $=0=>$ skip $\mathrm{N} \times 2 \mathrm{~N}$ and intra mode 
If $\mathrm{H} .264 / \mathrm{AVC}$ is $4 \times 8 \Rightarrow>$ skip intra $\mathrm{N} \times \mathrm{N}$

$\rightarrow$ If residual $=0=>$ skip $2 \mathrm{~N} \times \mathrm{N}$ and intra mode

If $\mathrm{H} .264 / \mathrm{AVC}$ is $4 \times 4=>$ skip intra $\mathrm{N} \times \mathrm{N}$

$\rightarrow$ If residual $=0=>$ skip intra mode

If $\mathrm{H} .264 / \mathrm{AVC}$ is intra $16 \Rightarrow>$ do $2 \mathrm{~N} \times 2 \mathrm{~N}$ and intra $2 \mathrm{~N} \times 2 \mathrm{~N}$

$\rightarrow$ If residual $=0=>$ just do $2 \mathrm{~N} \times 2 \mathrm{~N}$

If $\mathrm{H} .264 / \mathrm{AVC}$ is intra4 $=>$ do $2 \mathrm{~N} \times 2 \mathrm{~N}$ and intra

\subsection{Fast Motion Estimation Algorithm}

In general, the procedure of the motion estimation spends the most encoding time. Two methods are applied for the proposed transcoder to reduce the search range of the motion estimation. One is to apply the adaptive motion search, and the other is to adjust the search range by the transition probability of the current encoding PU.

An adaptive motion search is proposed to reduce the search range. The reference search range (refSR) for each PU is defined using (5):

$$
\text { refSR }=\max _{i}\left(\max \left(\left|\mathrm{MV}_{\mathrm{X}_{i}}-\mathrm{AMVP}_{\mathrm{X}} \mathrm{I}, \mathrm{MVV}_{\mathrm{Y}_{i}}-\mathrm{AMVP}_{\mathrm{Y}}\right|\right)\right) .
$$

Equation (5) shows that to predict each PU, the search range is replaced by the refSR which is the maximum difference between the advance motion vector prediction (AMVP) of HEVC and each MV from H.264/AVC. Comparing with the fixed search range by HEVC, the proposed refSR is more flexible and effective. This is because the refSR adopts both information of MVs from H.264/AVC and HEVC. If the refSR is small, the MV prediction is with high accuracy. So, the search range can be small. On the other hand, if the refSR is large, the prediction is with low accuracy. The maximum difference between the predicted MVs as the search range shown in (5) can cover the worst condition. The difference between the H.264/AVC MV and the HEVC AMVP with respect to the $x$ and $y$ direction is drawn Figure 3 .
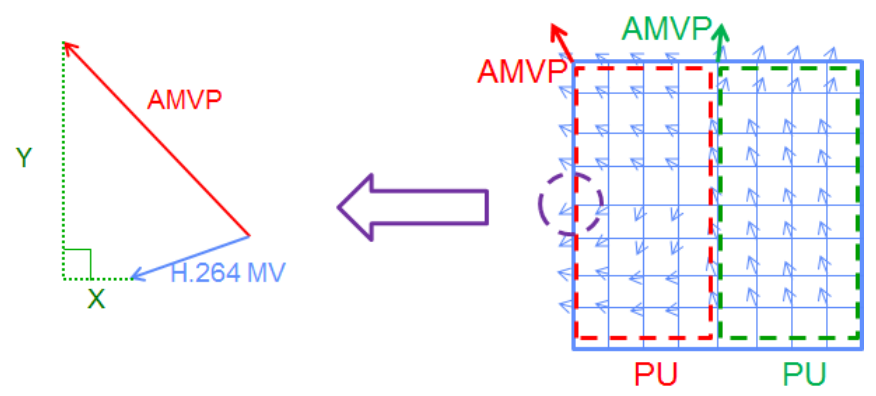

Figure 3. The difference between H.264/AVC MV and HEVC AMVP

In addition to the refSR, the mode transition probability from H.264/AVC to HEVC is also considered. If a PU in HEVC is with high probability to occur, the search range can be longer. Otherwise, the search range can be shorter if this PU rarely occurs.

For depths 2 and 3, the transition probability is directly measured from the mode in H.264/AVC to the PU in HEVC because the MB size in H.264/AVC is the same as the CU size in HEVC. Tables 1 and 2 show the transition probability of depths 2 and 3, respectively. The left column in Table 1 or 2 lists each mode in H.264/AVC and the above row lists the final PU by the HEVC 
encoder. For each row, it shows the transition probability of a mode in H.264/AVC to a PU in HEVC. The sum of the transition probability of each row is 1 .

Table 1. The transition probability for depth 2 .

HEVC

\begin{tabular}{|l|l|l|l|l|l|l|l|l|l|}
\hline & $16 \times 16$ & $16 \times 8$ & $8 \times 16$ & $16 \times 4$ & $16 \times 12$ & $4 \times 16$ & $12 \times 16$ & intra16 \\
\hline skip & 0.9772 & 0.0064 & 0.0082 & 0.0007 & 0.0013 & 0.0023 & 0.0012 & 0.0026 \\
\hline $16 \times 16$ & 0.8264 & 0.0395 & 0.0464 & 0.0189 & 0.0158 & 0.0222 & 0.0183 & 0.0108 \\
\hline $16 \times 8$ & 0.5681 & 0.2551 & 0.0514 & 0.0393 & 0.0315 & 0.0196 & 0.0174 & 0.0177 \\
\cline { 2 - 10 } \\
\cline { 2 - 10 } & $8 \times 16$ & 0.5427 & 0.0425 & 0.2778 & 0.0173 & 0.0155 & 0.0447 & 0.0352 & 0.0243 \\
\cline { 2 - 10 } & $8 \times 8$ & 0.3983 & 0.1674 & 0.1820 & 0.0491 & 0.0457 & 0.0670 & 0.0613 & 0.0291 \\
\cline { 2 - 9 } & 0.3528 & 0.1674 & 0.1771 & 0.0819 & 0.0745 & 0.0622 & 0.0557 & 0.0283 \\
\hline & $4 \times 8$ & 0.3342 & 0.1568 & 0.1827 & 0.0468 & 0.0399 & 0.1066 & 0.0972 & 0.0358 \\
\hline $4 \times 4$ & 0.3109 & 0.1706 & 0.1819 & 0.0619 & 0.0597 & 0.0936 & 0.0892 & 0.0323 \\
\hline intra16 & 0.6367 & 0.0073 & 0.0108 & 0.0032 & 0.0010 & 0.0014 & 0.0027 & 0.3369 \\
\hline intra4 & 0.2657 & 0.0414 & 0.0443 & 0.0054 & 0.0091 & 0.0237 & 0.0269 & 0.5835 \\
\hline
\end{tabular}

Table 2. The transition probability for depth 3 .

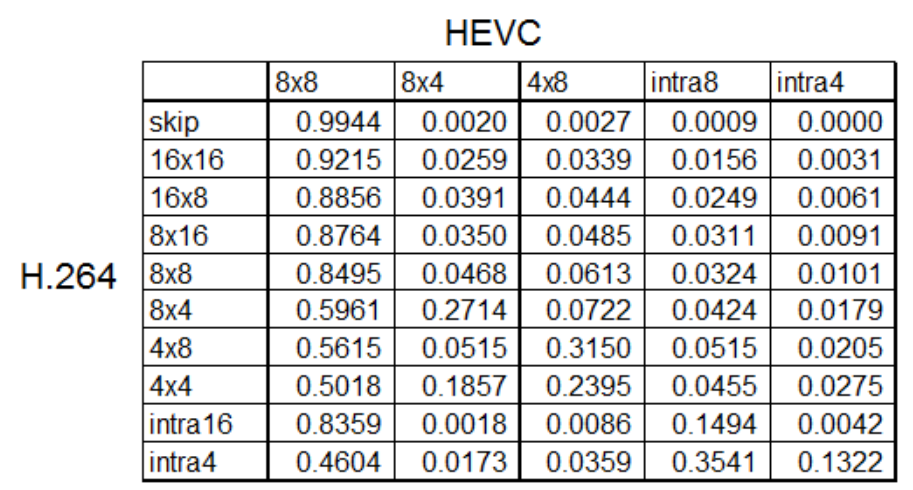

For depths 0 and 1 of CU, the CU size in HEVC is large than the MB size in H.264/AVC. If a block has been encoded as a subMB by H.264/AVC, the chance for this block to be merged into a larger size of $\mathrm{CU}$, encoded by HEVC, in depths 0 or 1 is low. In other words, if the current encoding CU contains more subMBs, already encoded by H.264/AVC, the chance for this CU to be encoded by HEVC in depths 0 and 1 is lower. The subMB denotes as a block with an $8 \times 8$ size or less. Thus, the transition probability can be obtained by counting the number of subMB within a CU. Counting the subMB is based on each $8 \times 8$ block, where an $8 \times 8$ block or all the smaller blocks within an $8 \times 8$ block are counted by 1 .

Tables 3 and 4 show the transition probability for depths 0 and 1, respectively. The left column in Table 3 or 4 lists the number of subMB for the current encoding CU, and the above row lists the final PU by the HEVC encoder. For each row, it shows the transition probability of the PU. The sum of the transition probability of each row is 1 . 
Table 3. The transition probability for depth 0 .

HEVC PU mode

\begin{tabular}{|c|c|c|c|c|c|c|c|c|c|}
\hline \multirow{6}{*}{ SubMB } & & $64 \times 64$ & $64 \times 32$ & $32 \times 64$ & $64 \times 16$ & $64 \times 48$ & $16 \times 64$ & $48 \times 64$ & intra64 \\
\hline & 0 & 0.6661 & 0.1002 & 0.1417 & 0.0172 & 0.0106 & 0.0163 & 0.0328 & \begin{tabular}{|l}
0.0151 \\
\end{tabular} \\
\hline & $1-4$ & 0.2508 & 0.2675 & 0.2930 & 0.0307 & 0.0233 & 0.0452 & 0.0786 & 0.0108 \\
\hline & $5-8$ & 0.1544 & 0.2897 & 0.3683 & 0.0337 & 0.0296 & 0.0500 & 0.0565 & 0.0179 \\
\hline & $9-12$ & 0.1379 & 0.3040 & 0.3894 & 0.0172 & 0.0259 & 0.0402 & 0.0439 & 0.0415 \\
\hline & $13-16$ & 0.0455 & 0.3482 & 0.4442 & 0.0417 & 0.0720 & 0.0152 & 0.0000 & 0.0333 \\
\hline
\end{tabular}

Table 4. The transition probability for depth 1 .

HEVC PU mode

\begin{tabular}{|c|c|c|c|c|c|c|c|c|c|}
\hline \multirow{6}{*}{ SubMB } & & $32 \times 32$ & $32 \times 16$ & $16 \times 32$ & $32 \times 8$ & $32 \times 24$ & $8 \times 32$ & $24 \times 32$ & intra32 \\
\hline & 0 & 0.6195 & 0.0979 & 0.1159 & 0.0343 & 0.0273 & 0.0440 & 0.0371 & 0.0240 \\
\hline & 1 & 0.3201 & 0.1640 & 0.2084 & 0.0619 & 0.0626 & 0.0836 & 0.0752 & 0.0242 \\
\hline & 2 & 0.2106 & 0.1787 & 0.2346 & 0.0765 & 0.0722 & 0.1037 & 0.1018 & 0.0219 \\
\hline & 3 & 0.1662 & 0.1845 & 0.2342 & 0.0938 & 0.0795 & 0.1152 & 0.1078 & 0.0188 \\
\hline & 4 & 0.1471 & 0.1859 & 0.2507 & 0.0840 & 0.0653 & 0.1250 & 0.1278 & 0.0141 \\
\hline
\end{tabular}

The search range of a PU is adjusted by a weighting factor which is assigned based on the PU transition probability. The assignment is listed in Table 5.

Table 5. The weighting table to the search range.

\begin{tabular}{|c|c|}
\hline Probability & Weighting, $\boldsymbol{W}$ \\
\hline $\mathrm{P} \leq 0.1$ & $\mathbf{0 . 6}$ \\
\hline $0.1<\mathrm{P} \leq 0.3$ & $\mathbf{0 . 8}$ \\
\hline $0.3<\mathrm{P} \leq 0.5$ & $\mathbf{1}$ \\
\hline $\mathrm{P}>0.5$ & $\mathbf{1 . 2}$ \\
\hline
\end{tabular}

Finally, the search range is defined using (6):

$$
\text { Search Range }=\min (W \times \text { refSR, original } S R) .
$$

The final search range is refSR multiplied by the weighting, but it cannot be greater than the default value, 64, of HEVC.

\section{EXPERIMENTAL RESULTS}

Simulations were taken by both JM, the software of H.264/AVC, version17.2 [9] with a QP value of 24, and HM, the software of HEVC, version 9.2 [10] with QP values 24, 27, 31, and 35. The input sequences were IPPP...P with different classes, and the test zone (TZ) fast search was applied in HEVC [10]. The test hardware was a PC with Intel I7-3770, 3.4GHz CPU, 16G RAM, and Windows 7 the professional system. Experimental results include the fast mode decision, fast motion estimation, and the overall performance. The overall performance is also compared with the performance proposed by [8]. 


\subsection{Results for the Fast Mode Decision Algorithm}

Table 6 lists the performance for the fast mode decision. The proposed method saves 32-35\% of the encoding time from the test sequences under four various QPs. In particular, the E class of sequences can save more encoding time because their contents are homogeneous, with smooth contents. Thus, larger size of blocks or more skip modes can be adopted by the proposed method.

Table 6. Experimental results for fast mode decision.

\begin{tabular}{|c|c|c|c|c|c|c|}
\hline & \multicolumn{3}{|c|}{$\mathrm{QP}=24$} & \multicolumn{3}{|c|}{$\mathrm{QP}=27$} \\
\hline Sequence & $\begin{array}{c}\triangle \mathrm{PSNR} \\
(\mathrm{dB})\end{array}$ & $\begin{array}{c}\triangle \text { Bitrate } \\
(\%)\end{array}$ & $\begin{array}{c}\triangle \text { EncTime } \\
(\%)\end{array}$ & $\begin{array}{c}\triangle \mathrm{PSNR} \\
(\mathrm{dB})\end{array}$ & $\begin{array}{c}\triangle \text { Bitrate } \\
(\%)\end{array}$ & $\begin{array}{c}\triangle \text { EncTime } \\
(\%)\end{array}$ \\
\hline ClassC_BasketballDrill & -0.041 & $1.64 \%$ & $-35.71 \%$ & -0.047 & $1.78 \%$ & $-34.57 \%$ \\
\hline ClassC_RaceHorses & -0.020 & $1.26 \%$ & $-27.35 \%$ & -0.030 & $1.49 \%$ & $-25.30 \%$ \\
\hline ClassD_BasketballPass & -0.026 & $1.28 \%$ & $-29.14 \%$ & -0.035 & $1.26 \%$ & $-28.38 \%$ \\
\hline ClassD_BlowingBubbles & -0.019 & $0.44 \%$ & $-27.14 \%$ & -0.018 & $0.52 \%$ & $-26.40 \%$ \\
\hline ClassE_vidyo1 & -0.042 & $0.41 \%$ & $-49.00 \%$ & -0.034 & $0.90 \%$ & $-48.79 \%$ \\
\hline ClassE_vidyo4 & -0.033 & $0.73 \%$ & $-43.89 \%$ & -0.040 & $0.71 \%$ & $-43.18 \%$ \\
\hline \multirow[t]{2}{*}{ Averge } & -0.030 & $0.96 \%$ & $-35.37 \%$ & -0.034 & $1.11 \%$ & $-34.44 \%$ \\
\hline & \multicolumn{3}{|c|}{$\mathrm{QP}=31$} & \multicolumn{3}{|c|}{$\mathrm{QP}=35$} \\
\hline Sequence & $\begin{array}{c}\triangle \mathrm{PSNR} \\
(\mathrm{dB})\end{array}$ & $\begin{array}{c}\triangle \text { Bitrate } \\
(\%)\end{array}$ & $\begin{array}{c}\triangle \text { EncTime } \\
(\%)\end{array}$ & $\begin{array}{c}\triangle \text { PSNR } \\
(\mathrm{dB})\end{array}$ & $\begin{array}{c}\triangle \text { Bitrate } \\
(\%)\end{array}$ & $\begin{array}{c}\triangle \text { EncTime } \\
(\%)\end{array}$ \\
\hline ClassC_BasketballDrill & -0.050 & $1.91 \%$ & $-33.27 \%$ & -0.070 & $1.62 \%$ & $-32.50 \%$ \\
\hline ClassC_RaceHorses & -0.042 & $1.72 \%$ & $-23.61 \%$ & -0.043 & $1.73 \%$ & $-21.39 \%$ \\
\hline ClassD_BasketballPass & -0.047 & $1.55 \%$ & $-27.79 \%$ & -0.087 & $1.91 \%$ & $-27.18 \%$ \\
\hline ClassD_BlowingBubbles & -0.033 & $0.62 \%$ & $-24.74 \%$ & -0.033 & $0.88 \%$ & $-23.27 \%$ \\
\hline ClassE_vidyo1 & -0.038 & $0.60 \%$ & $-48.98 \%$ & -0.031 & $0.74 \%$ & $-49.41 \%$ \\
\hline ClassE_vidyo4 & -0.049 & $0.74 \%$ & $-43.18 \%$ & -0.046 & $1.03 \%$ & $-43.35 \%$ \\
\hline Averge & -0.043 & $1.19 \%$ & $-33.60 \%$ & -0.052 & $1.32 \%$ & $-32.85 \%$ \\
\hline
\end{tabular}

\subsection{Results for the Fast Motion Estimation Algorithm}

Table 7 lists the performance of the fast motion estimation. The proposed method saves approximately to $15 \%$ of the encoding time and maintains the R-D performance from the test sequences. In particular, the test sequence RaceHorses in class $C$ has better performance than other sequences. This is because this sequence is a high motion sequence, and the proposed adaptive search range can be more effective than the method of fixed search range. On the other hand, the test sequence Vidyo 1 in class $\mathrm{E}$ is a low motion sequence, the TZ search in HEVC can quickly find the best MV so that the time saving by the proposed method is reduced. 
Table 7. Experimental results for fast motion estimation.

\begin{tabular}{|c|c|c|c|c|c|c|}
\hline & \multicolumn{3}{|c|}{$\mathrm{QP}=24$} & \multicolumn{3}{|c|}{$\mathrm{QP}=27$} \\
\hline Sequence & $\begin{array}{c}\triangle \mathrm{PSNR} \\
(\mathrm{dB})\end{array}$ & $\begin{array}{c}\triangle \text { Bitrate } \\
(\%)\end{array}$ & $\begin{array}{c}\triangle \text { EncTime } \\
(\%)\end{array}$ & $\begin{array}{c}\triangle \mathrm{PSNR} \\
(\mathrm{dB})\end{array}$ & $\begin{array}{c}\triangle \text { Bitrate } \\
(\%)\end{array}$ & $\begin{array}{c}\triangle \text { EncTime } \\
(\%)\end{array}$ \\
\hline ClassC_BasketballDrill & -0.007 & $0.25 \%$ & $-15.70 \%$ & -0.008 & $0.38 \%$ & $-16.94 \%$ \\
\hline ClassC_RaceHorses & -0.001 & $0.34 \%$ & $-22.39 \%$ & -0.006 & $0.46 \%$ & $-22.67 \%$ \\
\hline ClassD_BasketballPass & -0.018 & $0.41 \%$ & $-17.14 \%$ & -0.017 & $0.25 \%$ & $-16.98 \%$ \\
\hline ClassD_BlowingBubbles & -0.006 & $0.11 \%$ & $-12.34 \%$ & -0.005 & $-0.21 \%$ & $-13.37 \%$ \\
\hline ClassE_vidyo1 & -0.011 & $-0.05 \%$ & $-7.78 \%$ & -0.008 & $-0.11 \%$ & $-7.46 \%$ \\
\hline ClassE_vidyo4 & -0.008 & $-0.04 \%$ & $-15.73 \%$ & -0.012 & $0.09 \%$ & $-15.70 \%$ \\
\hline \multirow[t]{2}{*}{ Averge } & -0.009 & $0.17 \%$ & $-15.18 \%$ & -0.009 & $0.14 \%$ & $-15.52 \%$ \\
\hline & \multicolumn{3}{|c|}{$\mathrm{QP}=31$} & \multicolumn{3}{|c|}{$\mathrm{QP}=35$} \\
\hline Sequence & $\begin{array}{c}\triangle \text { PSNR } \\
(\mathrm{dB})\end{array}$ & $\begin{array}{c}\triangle \text { Bitrate } \\
(\%)\end{array}$ & $\begin{array}{c}\triangle \text { EncTime } \\
(\%)\end{array}$ & $\begin{array}{c}\triangle \mathrm{PSNR} \\
(\mathrm{dB})\end{array}$ & $\begin{array}{c}\triangle \text { Bitrate } \\
(\%)\end{array}$ & $\begin{array}{c}\triangle \text { EncTime } \\
(\%)\end{array}$ \\
\hline ClassC_BasketballDrill & 0.002 & $0.47 \%$ & $-16.95 \%$ & -0.033 & $-0.16 \%$ & $-16.29 \%$ \\
\hline ClassC_RaceHorses & -0.025 & $0.42 \%$ & $-24.48 \%$ & -0.020 & $0.40 \%$ & $-23.87 \%$ \\
\hline ClassD_BasketballPass & -0.030 & $0.01 \%$ & $-16.12 \%$ & -0.042 & $0.33 \%$ & $-14.84 \%$ \\
\hline ClassD_BlowingBubbles & -0.005 & $0.31 \%$ & $-13.64 \%$ & -0.024 & $0.49 \%$ & $-13.47 \%$ \\
\hline ClassE_vidyo1 & -0.010 & $-0.28 \%$ & $-7.31 \%$ & 0.002 & $-0.38 \%$ & $-7.02 \%$ \\
\hline ClassE_vidyo4 & -0.004 & $-0.41 \%$ & $-14.66 \%$ & 0.001 & $0.15 \%$ & $-13.25 \%$ \\
\hline Averge & -0.012 & $0.09 \%$ & $-15.53 \%$ & -0.019 & $0.14 \%$ & $-14.79 \%$ \\
\hline
\end{tabular}

\subsection{Results for the Overall Performance}

Table 8 lists the overall performance. The proposed algorithm can save 43-45\% of the encoding time. Comparing with [8], the proposed method performs better in the encoding time but increases the bitrate a little. In particularly, as the QP is closer to the original QP by H.264/AVC, the proposed method saves much of the encoding time. Therefore, as these two transcoding standards are with the closer QP assignment, the proposed algorithm can be more effective.

\section{CONCLUSIONS}

In this work, a fast transcoder from H.264/AVC to HEVC focusing on the PU is proposed. The proposed method consists of the fast mode decision and the fast motion estimation. Various fast mode algorithms are applied to the transcoder for depths 0 and 1, and depths 2 and 3, respectively. As for the fast motion estimation, adaptive search range with the mode transition probability is proposed. Experimental results show the algorithms of the fast mode decision and the fast motion estimation can save $32-35 \%$ and $15 \%$ of the encoding time, respectively. The overall encoding time can be reduced up to $53 \%$. In other words, more than half of the encoding time can be saved under the acceptable R-D performance. 
Table 8. Experimental results for overall algorithm.

\begin{tabular}{|c|c|c|c|c|c|c|}
\hline & \multicolumn{3}{|c|}{ Reference [8] } & \multicolumn{3}{|c|}{ Proposed Method } \\
\hline Sequence & $\begin{array}{c}\triangle \mathrm{PSNR} \\
(\mathrm{dB})\end{array}$ & $\begin{array}{c}\triangle \text { Bitrate } \\
(\%)\end{array}$ & $\begin{array}{c}\triangle \text { EncTime } \\
(\%)\end{array}$ & $\begin{array}{c}\triangle \mathrm{PSNR} \\
(\mathrm{dB})\end{array}$ & $\begin{array}{c}\triangle \text { Bitrate } \\
(\%)\end{array}$ & $\begin{array}{c}\triangle \text { EncTime } \\
(\%)\end{array}$ \\
\hline ClassB_Cactus & -0.030 & $0.38 \%$ & $-35.67 \%$ & -0.028 & $0.83 \%$ & $-44.41 \%$ \\
\hline ClassB_ParkScene & -0.021 & $0.16 \%$ & $-32.02 \%$ & -0.032 & $0.65 \%$ & $-44.97 \%$ \\
\hline ClassC_BasketballDrill & -0.045 & $0.97 \%$ & $-36.75 \%$ & -0.046 & $1.89 \%$ & $-46.57 \%$ \\
\hline ClassC_RaceHorses & -0.019 & $0.94 \%$ & $-41.24 \%$ & -0.019 & $1.69 \%$ & $-43.83 \%$ \\
\hline ClassD_BasketballPass & -0.054 & $1.59 \%$ & $-39.02 \%$ & -0.037 & $1.53 \%$ & $-42.09 \%$ \\
\hline ClassD_BlowingBubbles & -0.063 & $1.63 \%$ & $-33.50 \%$ & -0.020 & $0.67 \%$ & $-36.41 \%$ \\
\hline ClassE_vidyo1 & -0.062 & $-0.39 \%$ & $-43.43 \%$ & -0.057 & $0.30 \%$ & $-53.27 \%$ \\
\hline ClassE_vidyo4 & -0.042 & $-0.07 \%$ & $-43.61 \%$ & -0.038 & $0.98 \%$ & $-53.73 \%$ \\
\hline QP24 Averge & -0.042 & $0.65 \%$ & $-38.16 \%$ & -0.035 & $1.07 \%$ & $-45.66 \%$ \\
\hline ClassB_Cactus & -0.015 & $0.44 \%$ & $-37.16 \%$ & -0.028 & $1.15 \%$ & $-43.64 \%$ \\
\hline ClassB_ParkScene & -0.022 & $0.20 \%$ & $-33.55 \%$ & -0.036 & $0.82 \%$ & $-44.02 \%$ \\
\hline ClassC_BasketballDrill & -0.028 & $0.97 \%$ & $-37.48 \%$ & -0.050 & $2.22 \%$ & $-45.90 \%$ \\
\hline ClassC_RaceHorses & -0.029 & $1.09 \%$ & $-41.94 \%$ & -0.035 & $2.20 \%$ & $-43.45 \%$ \\
\hline ClassD_BasketballPass & -0.050 & $1.60 \%$ & $-39.52 \%$ & -0.051 & $1.61 \%$ & $-41.52 \%$ \\
\hline ClassD_BlowingBubbles & -0.068 & $1.03 \%$ & $-34.17 \%$ & -0.027 & $0.74 \%$ & $-36.25 \%$ \\
\hline ClassE_vidyo1 & -0.023 & $0.23 \%$ & $-45.84 \%$ & -0.042 & $1.13 \%$ & $-53.28 \%$ \\
\hline ClassE_vidyo4 & -0.026 & $-0.02 \%$ & $-45.98 \%$ & -0.047 & $0.79 \%$ & $-53.53 \%$ \\
\hline QP27 Averge & -0.033 & $0.69 \%$ & $-39.46 \%$ & -0.040 & $1.33 \%$ & $-45.20 \%$ \\
\hline ClassB_Cactus & -0.016 & $0.87 \%$ & $-38.26 \%$ & -0.048 & $1.27 \%$ & $-42.88 \%$ \\
\hline ClassB_ParkS & -0.025 & $0.25 \%$ & $-34.98 \%$ & -0.042 & $0.89 \%$ & $-43.04 \%$ \\
\hline ClassC_BasketballDrill & -0.029 & $1.08 \%$ & $-38.67 \%$ & -0.054 & $2.35 \%$ & $-44.99 \%$ \\
\hline ClassC_RaceHorses & -0.043 & $1.18 \%$ & $-42.41 \%$ & -0.059 & $2.33 \%$ & $-42.72 \%$ \\
\hline ClassD_Basketba & -0.046 & $1.07 \%$ & $-40.43 \%$ & -0.066 & $1.78 \%$ & $-40.68 \%$ \\
\hline ClassD_BlowingBubbles & -0.059 & $1.35 \%$ & $-35.20 \%$ & -0.037 & $1.12 \%$ & $-35.25 \%$ \\
\hline ClassE_vidyo1 & -0.027 & $-0.32 \%$ & $-48.39 \%$ & -0.060 & $0.83 \%$ & $-53.40 \%$ \\
\hline ClassE_vidyo4 & -0.024 & $0.06 \%$ & $-47.58 \%$ & -0.056 & $1.07 \%$ & $-53.20 \%$ \\
\hline QP31 Averge & -0.034 & $0.69 \%$ & $-40.74 \%$ & -0.053 & $1.46 \%$ & $-44.52 \%$ \\
\hline ClassB_Cactus & -0.017 & $1.21 \%$ & $-39.16 \%$ & -0.054 & $1.75 \%$ & $-42.10 \%$ \\
\hline ClassB_ParkScene & -0.017 & $0.50 \%$ & $-36.27 \%$ & -0.043 & $0.84 \%$ & $-41.96 \%$ \\
\hline ClassC_BasketballDrill & -0.062 & $0.98 \%$ & $-39.21 \%$ & -0.092 & $2.31 \%$ & $-44.17 \%$ \\
\hline ClassC_RaceHorses & -0.052 & $1.12 \%$ & $-42.82 \%$ & -0.063 & $2.42 \%$ & $-41.29 \%$ \\
\hline ClassD_BasketballPass & -0.081 & $0.87 \%$ & $-41.36 \%$ & -0.109 & $2.12 \%$ & $-39.43 \%$ \\
\hline ClassD_BlowingBubbles & -0.051 & $0.99 \%$ & $-35.73 \%$ & -0.044 & $1.41 \%$ & $-34.05 \%$ \\
\hline ClassE_vidyo1 & -0.015 & $0.30 \%$ & $-49.58 \%$ & -0.042 & $0.94 \%$ & $-53.60 \%$ \\
\hline ClassE_vidyo4 & -0.013 & $0.17 \%$ & $-48.52 \%$ & -0.051 & $1.18 \%$ & $-52.74 \%$ \\
\hline QP35 Averge & -0.039 & $0.77 \%$ & $-41.58 \%$ & -0.062 & $1.62 \%$ & $-43.67 \%$ \\
\hline
\end{tabular}

\section{REFERENCES}

[1] Advanced Video Coding, ITU-T Rec. H.264 and ISO/IEC 14496-10 (MPEG-4 AVC), Version 13, Mar. 2011.

[2] JCT-VC, "High Efficiency Video Coding (HEVC) Text Specification Draft 10,” JCTVC-L1003, 12th JCTVC meeting, Geneva, Jan. 2013.

[3] E. Peixoto and E. Izquierdo, "A Complexity-Scalable Transcoder from H.264/AVC to the New HEVC Codec," in Proc. IEEE International Conference on Image Processing (ICIP), Sep. 2012, pp. 737-740.

[4] X. Jing, W. C. Siu, L. P. Chau, and A. G. Constantinides, "Efficient Inter Mode Decision for H.263 to H.264 Video Transcoding Using Support Vector Machines," in Proc. IEEE International Symposium on Circuits and Systems (ISCAS), May 2009, pp. 2349-2352.

[5] D. Zhang, B. Li, J. Xu, and H. Li, "Fast Transcoding from H.264/AVC to High Efficiency Video Coding," in Proc. IEEE International Conference on Multimedia \& Expo. (ICME), July 2012, pp. 651-656. 
[6] R. Garrido-Cantos, J. D. Cock, J. L. Martínez, S. V. Leuven, and P. Cuenca, "Motion-Based Temporal Transcoding from H.264 AVC-to-SVC in Baseline Profile," IEEE Trans. Consum. Electron., vol. 57, no. 1, pp. 239-246, Feb. 2011.

[7] J. Zhang, F. Dai, Y. Zhang, and C. Yan, "Efficient HEVC to H.264/AVC Transcoding with Fast Intra Mode Decision," in Proc. the 19th International Conference on Multimedia Modeling, Jan. 2013, Lecture Notes in Computer Science vol. 7732, 2013, pp. 295-306.

[8] Z. Y. Chen, C. T. Tseng, and P. C. Chang, "Fast Inter Prediction for H.264 to HEVC Transcoding," in Proc. the 3rd International Conference on Multimedia Technology (ICMT), Guangzhou, China, Nov. 2013, Atlantis Press, pp. 1301-1308.

[9] Joint Video Team software JM17.2. Available at: https://iphome.hhi.de/suehring/tml/download/

[10] HM Reference Software 9.2. Available at: https://hevc.hhi.fraunhofer.de/svn/svn_HEVCSoft-ware 


\title{
OFF-LINE SYSTEM FOR THE RECOGNITION OF HANDWRITTEN ARABIC CHARACTER
}

\author{
Ahmed Sahlol $^{1}$ and Cheng Suen ${ }^{2}$ \\ ${ }^{1}$ Department of Computer Teacher preparation, \\ Damietta University, Damietta, Egypt \\ asahloleencs.concordia.ca \\ ${ }^{2}$ Department of Computer Science, Concordia University, Canada \\ parmidireencs. concordia.ca
}

\begin{abstract}
Recognition of handwritten Arabic text awaits accurate recognition solutions. There are many difficulties facing a good handwritten Arabic recognition system such as unlimited variation in human handwriting, similarities of distinct character shapes, and their position in the word. The typical Optical Character Recognition (OCR) systems are based mainly on three stages, preprocessing, features extraction and recognition.

In this paper, we present an efficient approach for the recognition of off-line Arabic handwritten characters which is based on structural, Statistical and Morphological features from the main body of the character and also from the secondary components. Evaluation of the accuracy of the selected features is made. The system was trained and tested with CENPRMI dataset. The proposed algorithm obtained promising results in terms of accuracy (success rate of $100 \%$ for some letters at average 88\%). In Comparable with other related works we find that our result is the highest among others.
\end{abstract}

\section{KEYWORDS}

Handwritten Arabic Characters, Feature extraction, Secondary component

\section{INTRODUCTION}

The estimated number of historical Arabic manuscripts exceeds three millions [1], so we translate images of typewritten or handwritten text into machine-editable text encoded in a standard encoding scheme (ASCII). Handwritten text recognition of such as Arabic text is an active research problem [2].

Now OCR systems have expanded to recognize Latin alphabets, Japanese Katakana syllabic characters, Kanji (Japanese version of Chinese) characters, Chinese characters, Hangul characters, etc.

Work on Arabic OCR started in 1970s [3]. The first published work on Arabic OCR dates back to 1975 [5]. The first Arabic OCR system was made available in 1990s [6]. The recognition of Arabic handwriting presents some unique challenges and benefits to the researchers [7]. Although more than three decades have passed, there has been a lack of effort in the recognition of Arabic handwritten texts compared to the recognition of texts in other scripts [6].

David C. Wyld et al. (Eds) : CCSIT, SIPP, AISC, PDCTA, NLP - 2014

pp. 227-244, 2014. (C) CS \& IT-CSCP 2014

DOI : $10.5121 /$ csit.2014.4219 
The main problem encountered when dealing with handwritten Arabic characters is that characters written by different persons representing the same character are not identical but can vary in both size and shape. Unlimited variation in human handwriting styles similarities of distinct character shapes, character overlaps, and interconnections of neighboring characters. In addition, the mood of the writer and the writing situation can have an effect on writing styles.

\section{- Arabic Writing System}

Arabic is written from right to left and is always cursive. It has 28 basic characters. Thus, roughly the alphabet set can expand to 84 different shapes according to the position of the letter (beginning, middle, end or isolated) as well as according to the style of writing (Nasekh, Roqa'a, Farisi and others).

A character is drawn in an isolated form when it is written alone and is drawn in three other forms when it is written connected to other characters in the word. For example, the character Ain has four forms: isolated, initial, medial and final. See Fig. 1.

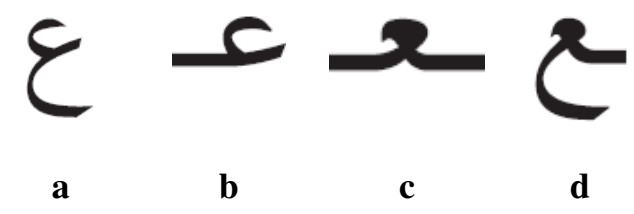

Figure 1. Different forms of Ain character: a. isolated, b. initial c. medial d. final

The secondary components are character components that are disconnected from the main body. Sixteen Arabic letters have from one to three secondary components (dots).

Additionally, some characters like alif (أ), kaaf (ك) can have a zigzag-like stroke called Hamza (ء). Those dots and Hamzas are called secondaries and they are located above the character primary part as in Alif (أ), or below like Baa (ب), or in the middle like Jeem (ج).

The type and position of the secondary components are very important features of Arabic letters. In the below Table 1 we see letters can be only distinguished only by their secondary components. For example, Tah (b) A1 and Thah (ظ) A2 differ only by the number of dots above the main body, also Seen (س) B1 and Sheen (ش) B2.

Another important kind of variations in drawing the secondary components appears mostly in drawing two or three dots. As shown in table1 Sheen (ش) B2, B3 and B4, the three dots come in three variations: isolated, connected and linked to the character, respectively. Also Taa (ت) C1, $\mathrm{C} 2$ can be drawn in two variations: two isolated dots or one short horizontal dashed line.

Another kind of differences between characters depended only on the position of the two dots; see Taa (ت) C1, Yaa (ي) D1 and Taa (ت) C2, Yaa (ي) D2 respectively.

There is also another classification challenge; that some characters which contain secondary components can also be written without those secondary components depending on "Roqa'a" writing styles. For example Yaa (ي) can be drawn with two isolated dots D1 or with short dashed line D2 or without any dots D3. Another example is Alif (أ) which can be drawn with hamza E1(default) or without it E2. 
One recognition difficulty is due to some writers' styles which can join the secondary components of isolated and final forms with main body curves. Table 1 shows some examples: Samples F1 and F2 show how the one dot of isolated Geem (ج) is joined to the main character body.

Another difficulty in recognizing the secondary components comes due to quickly writing, as writers draw them connected to the main body. For example, Samples H2, H3 show hamza connected to Kaf's (5) body.

Table 1: variations in drawing character secondary components

\begin{tabular}{|c|c|c|c|c|}
\hline & 1 & 2 & 3 & 4 \\
\hline A & $b$ & b & & \\
\hline B & $\omega^{\mu}$ & $\ddot{\ddot{u}}$ & $\hat{\sim}$ & $\sim$ \\
\hline $\bar{C}$ & i" & 4 & & \\
\hline D & ي & $\circlearrowleft$ & $c s$ & \\
\hline $\mathrm{E}$ & s & 1 & & \\
\hline $\bar{F}$ & Z & $\bar{\sigma}$ & & \\
\hline $\mathrm{H}$ & s & (3) & e) & \\
\hline
\end{tabular}

Considerable work has been undertaken in the area of Arabic character recognition, their targeted in various ways to improve accuracy and efficiency but with limited success, this is due to the nature of Arabic characters and to the problems mentioned above.

El-Dabi et al. [8] presented a recognition system for typed Arabic text, which involves a statistical approach for character recognition. El-Sheikh et al [9] proposed algorithms to recognize Arabic handwritten characters; this system assumes that characters result from a reliable segmentation stage, thus, the position of the character is known a priori. Four different sets of character shapes have been independently considered (initial, medial, final, and isolated). Each set is further divided into four subsets depending on the number of strokes in the character. El-Khaly et al. [10] discussed an algorithm for the machine recognition of optically captured Arabic characters and their isolation from the printed text. Moment-invariant descriptors are investigated for the purpose of recognition of individual characters. Sabri Mohmoud [11] has used Fourier and contour analysis for the recognition of Arabic characters with acceptable recognition rates. The features of an input character are compared to the models' features using a distance measure. The model with the minimum distance is taken as the class representing the character.

If we look at a character as image from which we can extract much useful information, such information can be structural features such as loops, branch-points, endpoints, and dots or statistical which include pixel densities, histograms of chain code directions, moments, and Fourier descriptors. Many approaches and techniques have been proposed like [12] [13] [14] [15] [16] used loops, dots, curves, relative locations, height, sizes of parts of characters, loop positions and types, line positions and directions and turning points. Others like [17] [18] [19] used statistics from moments of horizontal and vertical projection. Histogram of slopes along contour is used by [20]. 
Artificial Neural Networks (ANNs) are the common seed in most if not all classifiers recognition. In this paper we use Neural Networks as a classifier like [21] which proposed a system for the recognition of handwritten Arabic characters recognition. Also Sherif and Mostafa [22] [23] presented a parallel design for back propagation Neural Networks approach in order to accelerate the computation process. But another kind of Neural network called Learning Vector Quantization (LVQ) was used in [24] for handwritten Arabic character recognition. While others [25] [26] used SVM (support vector machine) as a classifier for Arabic numeral.

In this paper, we propose a novel approach for extracting statistical, morphological and topological features of handwritten Arabic characters. We apply this technique in extracting moment features and show that this technique provides better feature sets that give higher recognition accuracies.

This paper is organized in nine sections. Section 2 describes the Binarization Algorithm used to convert grayscale image to be binary image, Section 3 introduces the normalization technique used for reducing the shape variation between the images. Section 4 describes some kind of noise removal Algorithms which have an important contribution during the classification stage. Section 5 illustrates different kind of feature extraction techniques used to extract useful features from character images. Section 6 describes the Classification phase; which analyzes the used classifier including its architecture, training and testing phase. Section 7 provides experimental results included classification accuracy and analysis. Finally, Section 8 describes the main conclusions and future work.

\section{MATERIALS AND METHODS}

The goal of this work is to develop a system that recognizes off-line Arabic handwritten characters. Features needed for the recognition process include different kinds of features. Neural network is then used to classify the characters based on the features that were extracted from the input character.

\subsection{Binarization}

Our purpose of this step is to convert the input image to binary image based on threshold.

Binary images may contain numerous imperfections. In particular, the binary regions produced by simple thresholding are distorted by noise and texture. Morphological image processing pursues the goals of removing these imperfections by accounting for the form and structure of the image.

Replace all pixels in the input image with luminance greater than level with the value 1 (white) and replace all other pixels with the value 0 (black).

Computes a global threshold (level) that can be used to convert an intensity image to a binary image is a normalized intensity value that lies in the range $[0,1]$.

We use Otsu's method [27], which chooses the threshold to minimize the intraclass variance of the black and white pixels.

Otsu's thresholding method involves iterating through all the possible threshold values and calculating a measure of spread for the pixel levels at each side of the threshold, i.e. the pixels that either fall in foreground or background. The aim is to find the threshold value where the sum of foreground and background spreads is at its minimum. 
The algorithm assumes that the image to be thresholded contains two classes of pixels (e.g. foreground and background), then calculates the optimum threshold separating those two classes so that their combined spread (intra-class variance) is minimal.

we use Otsu method not only because it is a global binarization technique but also its short running time; the Mean running time for the Otsu's binarization method was 2.0 secs and this is one of the lowest running time (Original Sauvola algorithm [28] takes 12.6 secs).

\subsection{Slant Correction}

We make slant Correction for every character image to eliminate any slant in each character. The basic idea is to locate near-vertical strokes in the character and estimate the average slant of the character from these strokes. Then, the slant in a character is corrected by applying a shear transformation to the character.

\subsection{Normalization.}

Normalization is to regulate the size, position, and shape of character images, so as to reduce the shape variation between the images of same class. Denote the input image and the normalized image by $\mathrm{f}(\mathrm{x}, \mathrm{y})$ and $\mathrm{g}(\mathrm{x} \square, \mathrm{y} \square)$, respectively, normalization is implemented by coordinate mapping

$$
\left\{\begin{array}{l}
x^{\prime}=x^{\prime}(x, y), \\
y^{\prime}=y^{\prime}(x, y) .
\end{array}\right.
$$

we denote the width and height of the original character by W1 and H1, the width and height of the normalized character by W2 and $\mathrm{H} 2$, and the size of the standard (normalized) plane by L As seen in Figure 3. The standard plane is usually considered as a square and its size is typically $32 \times$ 32 or $64 \times 64$, among others. We define the aspect ratios of the original character (R1) and the normalized character (R2) as

$$
R_{1}=\frac{\min \left(W_{1}, H_{1}\right)}{\max \left(W_{1}, H_{1}\right)}
$$

and

$$
R_{2}=\frac{\min \left(W_{2}, H_{2}\right)}{\max \left(W_{2}, H_{2}\right)}
$$

which is always considered in the range of $[0,1]$.

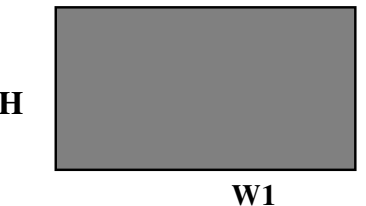

Figure 2. a. Original character

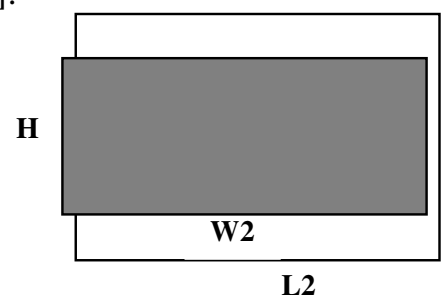

b. normalized character filled in standard plane.

We use Linear Backward mapping method [29] where:

$$
\begin{aligned}
& x=x^{\prime} / \alpha \\
& y=y^{\prime} / \beta
\end{aligned}
$$


and $\alpha$ and $\beta$ denote the ratios of scaling, given by:

$$
\begin{gathered}
\alpha=W_{2} / W_{1} \\
\beta=H_{2} / H_{1}
\end{gathered}
$$

Where W1 and H1 are the horizontal span and vertical span of the strokes of the original character (size of minimum bounding box).

\subsection{Noise removal}

\subsubsection{Statistical Noise removal}

In addition to enhancement of character image by contrast and dynamic range modification, a character image can also be enhanced by reducing degradations that may be present. This area of image enhancement overlaps with image restoration.

Median filtering [30] is a nonlinear process useful in preserving edges in an image while reducing random noise.

The median is calculated by first sorting all the pixel values from the surrounding neighborhood into numerical order and then replacing the pixel being considered with the middle pixel value. (If the neighborhood under consideration contains an even number of pixels, the average of the two middle pixel values is used). Figure 4 shows the process of Median filtering.

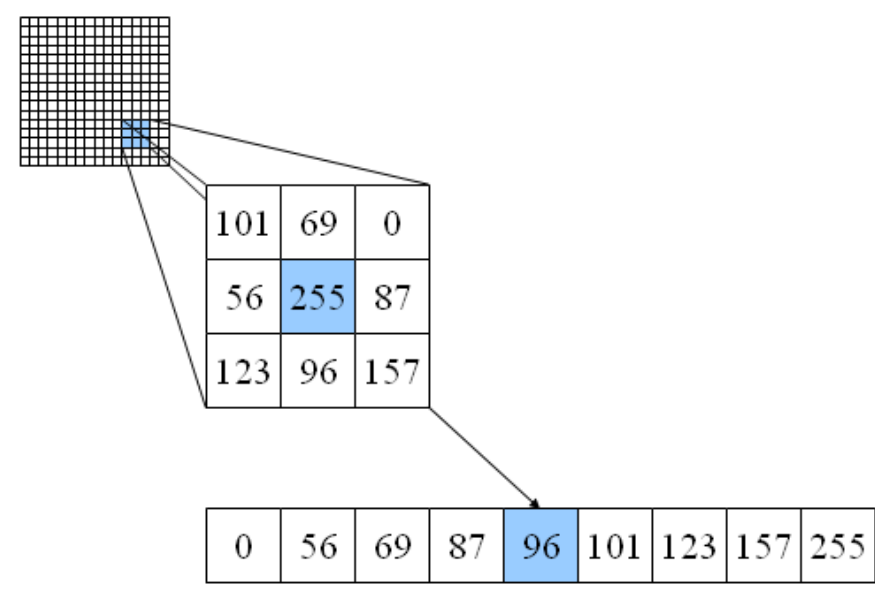

Figure 3. Median filtering process

A template of size $3 \times 3,5 \times 5,7 \times 7, \ldots$ etc is applied to each pixel. The values within this template are sorted and the middle of the sorted list is used to replace the template central pixel.

Median filtering can preserve discontinuities in a step function and can smooth a few pixels whose values differ significantly from their surroundings without affecting the other pixels [31]. In this paper, several filters were tested however a $3 \times 3$ median filter was chosen because it gave us the best result. The median filter is used to reduce noise in an image by considering each pixel within its neighboring pixels to decide whether or not it is representative of its surroundings. Then, it replaces the pixel value with the median of the values of the neighboring pixels. 


\subsubsection{Morphological noise removal}

Fills isolated interior pixels (individual 0s that are surrounded by 1s), such as the center pixel in this pattern.

For each pixel $\mathrm{p}$ in the binary image $\mathrm{I}$, check the two neighbors (as shown in the below figure) and decide whether $\mathrm{P}$ to be 0 or 1 if $\mathrm{B}(\mathrm{p})=4$, where $\mathrm{B}(\mathrm{p})$ is the number of non-zero neighbors of p.

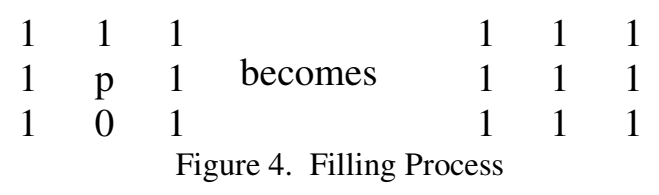

Bridges unconnected pixels, that is, sets 0-valued pixels to 1 if they have two nonzero neighbors that are not connected.

For each pixel $\mathrm{p}$ in the binary image I, check the two neighbors (as shown in the below figure) and decide whether $\mathrm{P}$ to be 0 or 1 if $\mathrm{B}(\mathrm{p} 1)>=2$, where $\mathrm{B}(\mathrm{p})$ is the number of non-zero neighbors of $\mathrm{p}$.

\begin{tabular}{|c|c|c|c|c|c|}
\hline 1 & 0 & 0 & & 1 & 1 \\
\hline 1 & $\mathrm{p}$ & 1 & becomes & 1 & 1 \\
\hline 0 & 0 & 1 & & 0 & 1 \\
\hline
\end{tabular}

Remove isolated pixels (individual 1s that are surrounded by $0 \mathrm{~s}$ ), such as the center pixel in this pattern.

For each pixel $\mathrm{p}$ in the binary image I, check the two neighbors (as shown in the below figure) and decide whether $\mathrm{P}$ to be 0 or 1 , if $\mathrm{B}(\mathrm{p})=0$, where $\mathrm{B}(\mathrm{p})$ is the number of non-zero neighbors of p.

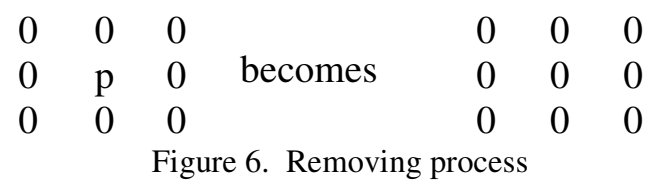

Another kind Morphological operation used in this paper is Dilation:

\section{- Dilation}

It is an operation that grows or thickens objects in a binary image The specific manner and extent of this thickening is controlled by a shape referred to as a structuring element. This Morphological technique exposes an image to a small shape or template; the structuring element is positioned at all possible locations in the image and it is compared with the corresponding neighborhood of pixels. Some operations test whether the element "fits" within the neighborhood, while others test whether it "hits" or intersects the neighborhood. Figure 8 shows how dilation works . 


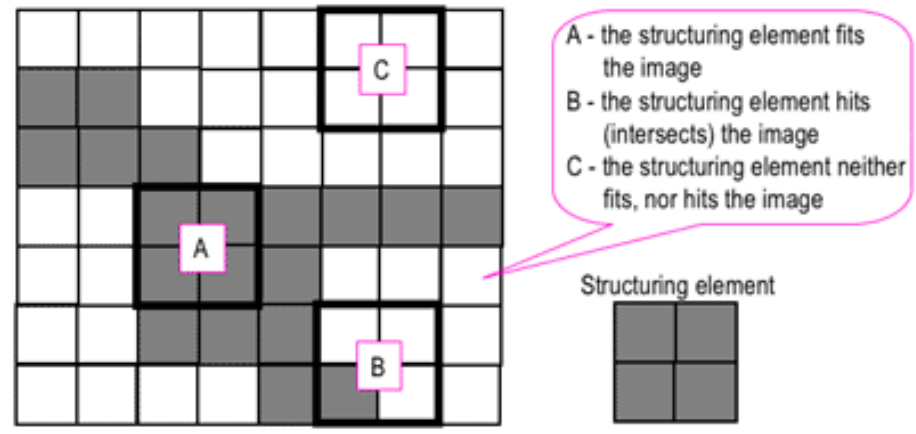

Figure 7. Exposing an image to a structuring element

(white and grey pixels have zero and non-zero values, respectively).

A morphological operation on a binary image creates a new binary image in which the pixel has a non-zero value only if the test is successful at that location in the input image.

The dilation of A by B is defined by:

$$
A \oplus B=\bigcup_{b \in B} A_{b}
$$

The dilation is commutative, also given by:

$$
A \oplus B=B \oplus A=\bigcup_{a \in A} B_{a}
$$

If $\mathrm{B}$ has a center on the origin, then the dilation of $\mathrm{A}$ by $\mathrm{B}$ can be understood as the locus of the points covered by B when the center of B moves inside A. The dilation of a square of side 10, centered at the origin, by a disk of radius 2 , also centered at the origin, is a square of side 14 , with rounded corners, centered at the origin. The radius of the rounded corners is 2 .

The dilation can also be obtained by: $\quad A \oplus B=\left\{z \in E \mid\left(B^{g}\right)_{z} \cap A \neq \varnothing\right\}$ where $\varnothing$ is the empty set and $B$ is the st

$$
A \oplus B=\left\{z \in E \mid\left(B^{g}\right) \ldots A \neq \varnothing\right\}
$$

$$
B^{s}=\{x \in E \mid-x \in B\}
$$

In this work we create a square of $2 \times 2$ of ones as a structuring element. Example of badly and for good dilation see fig. 9 .

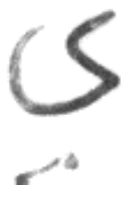

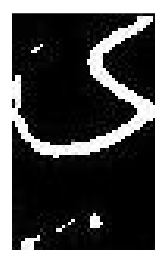

b

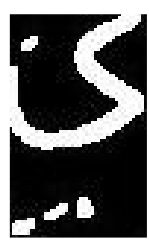

c

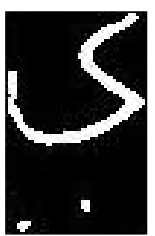

d

Figure 8. Dilation for Yaa character a. Original image b. primary Preprocessed c. badly Dilated d.

\subsection{Feature Extraction} perfect Dilated

\subsubsection{Structural Features:}

\subsubsection{Upper and Lower profile.}

The upper and lower profiles capture the outlining shape of a connected part of the character. Upper (or lower) connected part profile is computed by measuring the distance (pixel count) of each column group from the top (or, bottom) of the bounding box of the connected part to the closest ink pixel in that column group. 
Algorithm: Upper (or Lower) Profile

Input: binary preprocessed character image

Output: feature vector, F, representing upper (or, lower) profile.

1. Read the image into a two-dimensional array.

2. Divide the width into g column groups.

3. for each column group

Compute the distance from the top (or, bottom) of the bounding box of the connected part to the closest ink pixel in that group by counting the number of white pixels.

Get the ratio of distance to the number of black pixels of each group. Figure 10 shows the upper and lower profiles of character "Faa".

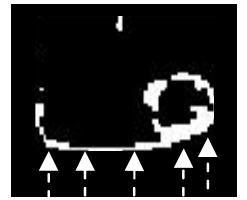

a

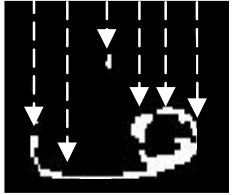

b

Figure 9. a. Upper profile b. Lower profile for character "Faa"

\subsubsection{Horizontal and Vertical projection profiles.}

Projection profile based feature extraction method delivers excellent results even in the absence of some important preprocessing steps such as smoothing and thinning. In fact, in this type of feature extraction it will be disadvantageous to apply the thinning process because there will be a huge loss of important information related to the count and position of white pixels present in the character image.

Vertical profile is the sum of white pixels perpendicular to the y axis. It is computed by scanning the character column wise along the $y$-axis and counting the number of white pixels in each column.

Similarly, for horizontal projection profile is sum of black pixels but it is perpendicular to the $\mathrm{x}$ axis. The character is traced horizontally along the $\mathrm{x}$-axis. The row wise sum of number of white pixels present in each row. See fig. 10.

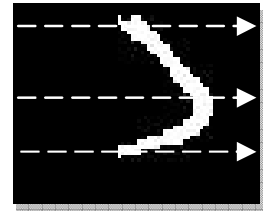

a

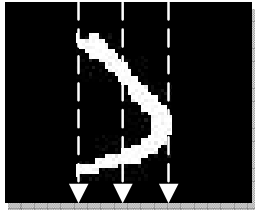

b

Figure 10. Character Daal projection profile a. horizontally b. vertically 


\subsubsection{Statistical Features:}

\subsubsection{Connected components.}

We consider the important "middle ground" between the individual foreground pixels and the set of all foreground pixels. This leads to the notion of connected components, also referred to as the following objects:

A pixel $\mathrm{p}$ at coordinates $(\mathrm{x}, \mathrm{y})$ has two horizontal and two vertical neighbors whose coordinates are $(\mathrm{x}+1, \mathrm{y}),(\mathrm{x}-1, \mathrm{y}),(\mathrm{x}, \mathrm{y}+1)$ and $(\mathrm{x}, \mathrm{y}-1)$. This set of 4 neighbors of $\mathrm{p}$ denoted $\mathrm{N} 4(\mathrm{p})$ is illustrated in figure 11, where the four diagonal neighbors of $\mathrm{p}$ have coordinates $(\mathrm{x}+1, \mathrm{y}+1),(\mathrm{x}+1, \mathrm{y}-1),(\mathrm{x}-$ $1, y+1)$ and $(x-1, y-1)$ respectively.

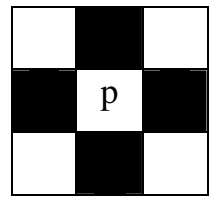

a

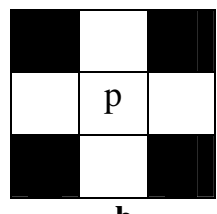

b

Figure 11. a. Pixel $\mathrm{p}$ and its 4 neighbors b. Pixel $\mathrm{p}$ and its diagonal neighbours

Connected component is an important feature because most of Arabic characters contain one or more connected components like (أ, ب, ت, ث, خ, ذ, ض, غ, ق, effective for distinguishing among those characters which contain connected components and others which don't contain connected components.

\subsubsection{Topological features:}

\subsubsection{End points.}

Another feature that is useful is the number of end points in the character. Those points have only one neighbor and the other three neighbors are noise.

Algorithm: Get end points of a character image

Input: binary preprocessed character image

Output: feature vector, $\mathrm{F}$, representing the most common end point of the character.

1. Read the character image, preprocess it (Binarization, normalization,...)

2. Dilate the image to join all its parts. Dilation essentially just adds pixels around existing white pixels.

3. Shrink the image to points (removes pixels so that objects without holes shrink to a point, and objects with holes shrink to a connected ring halfway between each hole and the outer boundary).

4. Find the pixel that is still white, which $i$ is the row and $j$ is the column of the white pixel in image such that image $(\mathrm{i}, \mathrm{j})$ is equal to 1 . All the other pixels should be 0 in the image.

5. Gather $i$ and $j$ in one vector " $G$ ".

6- Normalized each of the vector elements using Min-max method [31]:

Normalized value $=$ each value-min(all vector values) $) /(\max ($ all vector values $)-\min ($ all vector values).

7- Determine the most repeated values by calculating differences between adjacent elements.

8- Finally, determine the Most frequent values in the character array .

9- Put all the end points for the same character in a feature vector.

Notes about the algorithm: 
-At the 2nd stage "Dilation". We did it twice because we used Otsu method in binarization. -At the 3rd stage "shrinking". We shrank the character image until we reach the fewest number of white pixels. The below figure describes all the stages for the above algorithm.

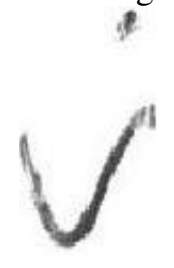

a

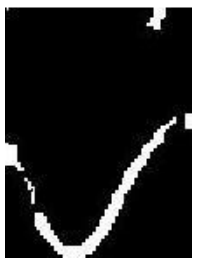

b

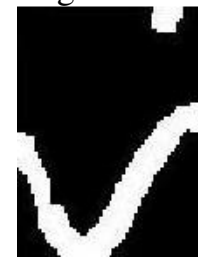

c

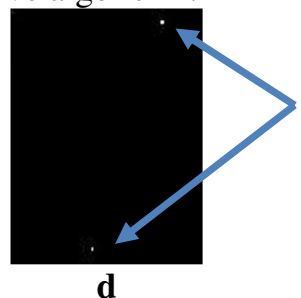

d

Figure 12. End point stages for Zaay character

a. original

b. preprocessed

c. dilated

d. end points

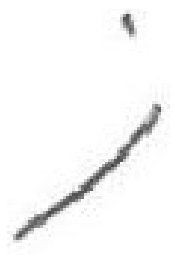

a

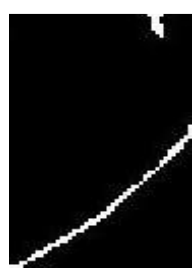

b

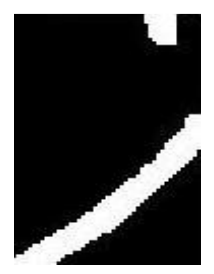

c

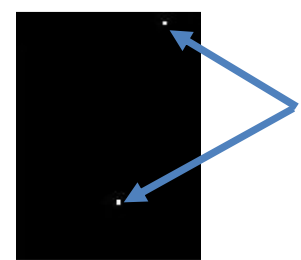

d

Figure 13. End point stages for another Zaay character $\quad$ a. original $\quad$ b. preprocessed $\quad$ c. dilated d. end points

It is obvious that the outer boundary points (end points) of the two Zaay characters are so close although they do not have the same shape. When we check the coordinates (step 4) of the end points of the two characters we find that the two characters have exactly the same coordinates of one from the two end points.

\subsubsection{Pixel Ratio.}

The character area is the total number of white (foreground) and black pixels (background) of the character. Pixel ratio is: (the number of white pixels / the number of black pixels).

\subsubsection{Height to Width Ratio.}

Since different people write same characters in different sizes, the absolute width and height are not reliable features for Arabic handwritten characters. However, some Arabic characters are wider than others. Therefore, the aspect ratio (height/width ratio) of the character is a useful feature.

\section{- Feature Normalization}

The simplest normalization technique is the Min-max normalization method [31]. Min-max normalization is best suited for the case where the bounds (maximum and minimum values) of the scores produced by a matcher are known. In this case, we can easily shift the minimum and maximum scores to 0 and1, respectively. However, even if the matching scores are not bounded, we can estimate the minimum and maximum values for a set of matching scores and then apply the min-max normalization. 
We use this method because it has one of the best recognition performances. Also it is efficient but sensitive to outliers in the training data, but if the parameters of the matching score distribution is known this method would suffice.

When the minimum and maximum values are estimated from the given set of matching scores Min-max normalization retains the original distribution of scores except for a scaling factor and transforms all the scores into a common range $[0,1]$.

\subsection{Classification}

The classification stage is the decision making part of the recognition system.

The Feed Forward Neural Network (back propagation) (2-NN) [32] is one of the most powerful classifiers. The number of hidden units of ANN should be selected to be high enough to model the problem at hand but not too high to overfit. The number of hidden units is selected to have the best performance on the validation set.

\subsubsection{Network Architecture}

The input vectors are defined as a matrix called alphabet (133x1848), representing 1848 samples (66 character image x 28 "number of Arabic alphabet") of 133 elements (feature). The target vectors are also defined with variable called target. Each target vector is a 28 -element vector with a 1 in the position of the letter. It represents, and 0's everywhere else. For example, the letter "I)" is to be represented by a 1 in the first element (as "|l” is the first letter of the alphabet), and 0's everywhere else. It is then required to identify the letter by responding with a 28 -element target vector. The 28 elements of the target vector each represent a letter. To operate correctly, the network should respond with a 1 in the position of the letter being presented to the network. All other values in the output vector should be 0 .

The neural network needs 133 inputs and 28 neurons in its output layer to identify the letters. The network is a two-layer network. The log-sigmoid [33] transfer function at the output layer was picked because its output range ( 0 to 1$)$ is perfect for learning to output Boolean values. The function generates outputs between 0 and 1 as the neuron's net input goes from negative to positive infinity see Fig.14.

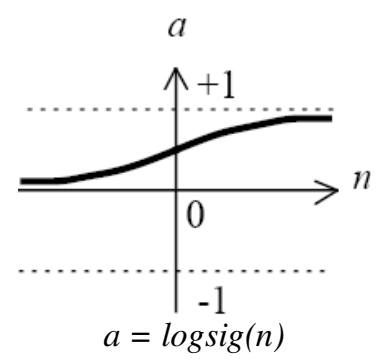

Figure 14. Log-sigmoid transfer function.

In building the network, the data was divided randomly into two categories. Training data consisted of $80 \%$ of the data (1540 characters" 55 samples of each of the 28 character"). The remaining $20 \%$ of the data was assigned to the testing data (308 characters" 11 samples of each of the 28 character"). Fig.15. shows the network architecture. 


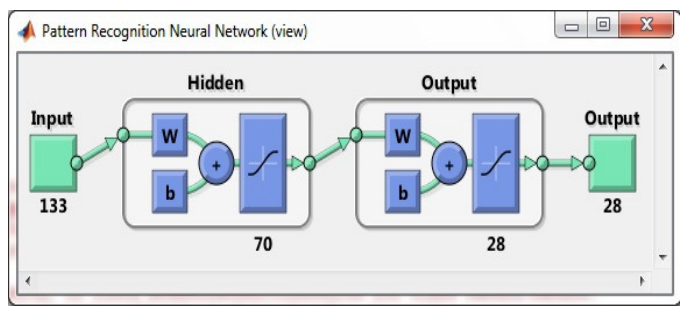

\subsubsection{Network Training}

Figure 15. Network architecture

To create a network that can handle noisy input vectors (characters) it is best to train the network on both ideal and noisy characters.

The network is trained to output a 1 in the correct position of the output vector and to fill the rest of the output vector with 0 's.

Back propagation training method is followed. This method was selected because of its simplicity and because it has been previously used on a number of pattern recognition problems. The method uses in this work called principle of gradient descent [34].

This function is useful because the conjugate gradient algorithms have relatively modest memory requirements. Memory is important when working with large networks, and yet it is much faster than other algorithms [35].

The gradient descent updates the network weights and biases in the direction in which the performance function decreases most rapidly, the negative of the gradient. One iteration of this algorithm can be written as:

$$
\mathbf{x}_{k+1}=\mathbf{x}_{k}-\alpha_{k} \mathbf{g}_{k}
$$

where $\mathbf{x}_{k}$ is a vector of current weights and biases, $\mathbf{g}_{k \text { is the current gradient, and }} \alpha_{k \text { is the }}$ learning rate. This equation is iterated until the network converges. The scaled conjugate gradient algorithm [34] is based on conjugate directions, but does not perform a line search at each iteration.

For evaluating the performance we choose to use the mean square error mse; the average squared error between the network outputs and the target outputs t. It is defined as follows:

$$
F=m s e=\frac{1}{N} \sum_{i=1}^{N}\left(e_{i}\right)^{2}=\frac{1}{N} \sum_{i=1}^{N}\left(t_{i}-a_{i}\right)^{2}
$$

In this paper we stop the network training is if the sum of squared error falls below 0.001 .

\subsubsection{Testing the Network}

After many trials of eliminating, adding and modifying features and also adjusting network hidden layers (start with 20 then 35 ...70 neurons) after 70 neurons network performance does not increase. A network of 70 neurons in hidden layer was able to predict about $88 \%$ of the input characters correctly ( $96 \%$ in the training phase and $88 \%$ in the testing phase). 


\section{RESULTS AND DISCUSSIONS}

The test set size used in the experiments is 308 characters ( 11 different sample of each of the 28 character), Experimental results are presented in Table 2.

Table 2: Recognition rates for Arabic letters in this system.

\begin{tabular}{|c|c|c|c|c|c|}
\hline Character & $\begin{array}{c}\text { Recognition } \\
\text { Percentage }\end{array}$ & Character & $\begin{array}{c}\text { Recognition } \\
\text { Percentage }\end{array}$ & Character & $\begin{array}{c}\text { Recognition } \\
\text { Percentage }\end{array}$ \\
\hline i & $96 \%$ & j & $80 \%$ & ق & $61 \%$ \\
\hline ب & $87 \%$ & س & $78 \%$ & 5 & $81 \%$ \\
\hline ت & $69 \%$ & ش & $88 \%$ & J & $96 \%$ \\
\hline$\dot{H}$ & $76 \%$ & ص & $100 \%$ & 5 & $92 \%$ \\
\hline ج & $100 \%$ & ض & $72 \%$ & ن & $100 \%$ \\
\hline$\tau$ & $94 \%$ & b & $91 \%$ & o & $97 \%$ \\
\hline$\dot{\tau}$ & $100 \%$ & ظ & $83 \%$ & 9 & $100 \%$ \\
\hline د & $83 \%$ & $\varepsilon$ & $66 \%$ & ي & \\
\hline ذ & $88 \%$ & $\dot{\varepsilon}$ & $99 \%$ & & \\
\hline J & $89 \%$ & ف & $100 \%$ & & \\
\hline
\end{tabular}

The achieved results are very promising. Experimental results showed that the proposed method give a recognition rate of about $88 \%$ for all letters although we get success rate of $100 \%$ for some letters.

As compared to the previous works we find that Aburas [36] system achieved 70\% of recognition rate, also Khedher [37] system achieved $73.4 \%$ of recognition rate, Taani [38] system achieved $75.3 \%$ of recognition rate. We achieved $1 \%$ increase in recognition over the Abandah [39] system. All of those systems work on isolated handwritten Arabic characters.

In this work Statistical and structural features were combined from the main body or the boundary with other features to get high recognition accuracy.

These features include the letter form, secondary type (Hamza,dots) and number of those secondary components. The results shown in the previous table illustrate that higher recognition accuracies are achieved using the proposed feature extraction technique. Extracting features from the main body and secondary components provides more valuable features that exploit the recognition potential of the secondary components of handwritten Arabic letters (comparison between ض ض ص ص ش $\omega$ and. These results also confirm the importance of the secondary components of the handwritten Arabic letters.

After many trials of eliminating, adding and modifying features and also adjusting neural network hidden layers

Some notes on the features:

-We tried many kind of features one example is a feature which extracts the Number of holes in the character because a lot of handwritten Arabic letters contain holes which can be very

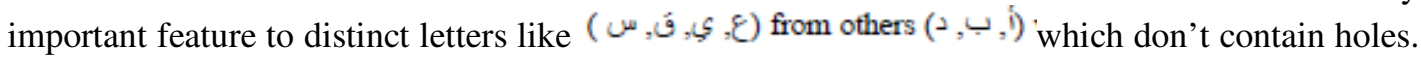
Unfortunately this feature doesn't perform well and doesn't give me dependable results because the variation in writing Arabic handwritten characters, so we eliminate this feature from my feature list. 
-Another kind of feature which has been examined is secondary component position. Unfortunately because of the variety of secondary components may take different positions and it is highly depending on writing styles, so this feature doesn't give me dependable results so we decide not to use this feature.

Our database of handwritten Arabic samples is CENPRMI [40] dataset. It includes Arabic off-line isolated handwritten characters. The database contains 11620 characters (about 332 samples for each character). These characters were written according to 12 different templates by 13 writers, with each template adopted by 5-8 writers. The 11620 character samples are divided into three groups 198, 67 and 67 for training, validating and testing purposes respectively. Table 3 shows collection of samples of isolated Arabic characters.

Table 3: Variation in characters from dataset

\begin{tabular}{|c|c|c|c|c|}
\hline Letter & Variatic & & & \\
\hline ن ن & $\sim$ & 3 & ن & $j$ \\
\hline س & $\mathrm{s}^{\mu}$ & & 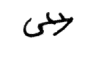 & $\omega$ \\
\hline ش & $\dot{\jmath}$ & $\ddot{\ddot{g}}$ & ن̈ & $\hat{v}$ \\
\hline ك & c) & 3 & e) & \\
\hline$\dot{\varepsilon}$ & $\dot{z}$ & < & $\varepsilon$ & $\varepsilon$ \\
\hline ف & هن & & G & 0 \\
\hline
\end{tabular}

As shown above, these samples show that loops are sometime introduced or filled and secondary objects are written in multiple styles, contains loops, under baseline, above baseline cavities, left and right directions, and different shapes and that makes the recognition process very hard.

We found that the Recognition rate is between $100 \%$ for easy to recognize letter forms and $61 \%$ for the hardest letter forms. Table 4 shows the ten letter forms that have the lowest classification rate.

Table 4: Worst 10 recognized characters

\begin{tabular}{|c|c|c|c|}
\hline No & Character & Recognition Rate & Often mistaken as \\
\hline 1 & ق & $61 \%$ & ف \\
\hline 2 & $\varepsilon$ & $66 \%$ & $\tau$ \\
\hline 3 & $ت$ & $69 \%$ & $j, \stackrel{ث}{ }$ \\
\hline 4 & ض & $72 \%$ & ظ, ص \\
\hline 5 & $\dot{H}$ & $76 \%$ & ف ف, ق \\
\hline 6 & 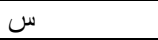 & $78 \%$ & ص \\
\hline 7 & $j$ & $80 \%$ & $J$ \\
\hline 8 & ك & $81 \%$ & $\tau, \varepsilon$ \\
\hline 9 & د & $83 \%$ & ك \\
\hline 10 & ظ & $83 \%$ & ش ,ر \\
\hline
\end{tabular}

These ten letters are always drawn with loops or drawn with loops in some writing variations. There are substantial similarities among multiple Arabic letter form groups that have loops. Often 
the sole difference between such letters is a subtle difference in the loop's shape. Moreover, letters with secondaries tend to have low recognition accuracies because the variations in drawing the dots or hamzas give inaccuracies in extracting the secondary type feature. Moreover that multiple dots in some writing styles may be isolated (Naskh writing style) or continued dash (Rekaa writing style). After careful examination of the samples that were incorrectly recognized, we concluded that most of these samples are hard to recognize even by a human expert reader. However, we think that the door is open to search for extracting new features that capture subtle differences in loop shapes and secondary types.

\section{CONCLUSION}

This paper presents an approach for extracting features to achieve high recognition accuracy of handwritten Arabic characters.

We present some useful techniques during the preprocessing phase including binarization, normalization and some noise removing methods. Our algorithm extracts useful features not only from the main body, but also from the secondary components of the character. It also overcomes some of handwritten characters variations.

Selecting proper features for recognizing handwritten Arabic characters can give better recognition accuracies, therefore our feature included statistical, morphological and topological features.

Although, there are some challenges with some characters, but the overall recognition rate is perfect especially when compared to other handwritten Arabic characters systems.

After examining the recognition accuracy of each character we found that the recognition rate is

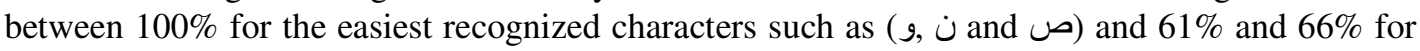
the hardest recognized characters such as (ق and $\varepsilon$ ), respectively.

We think that this is because those characters contain loops and also they can have different characteristics from one to other writing style. Characters with dots also tend to have low recognition accuracies because the variations in drawing the dots (dots can be linked to the main body of the character or even can be removed in some writing styles) give inaccuracies in extracting the secondary type feature.

Our future work includes increasing the efficiency of the proposed approach especially for the characters that were not recognized well by finding out other powerful feature, also including variations in writing the main body of the character and also the secondaries.

We hope also that we complete system for recognizing handwritten Arabic text passing through segmentation techniques for segmenting the words to characters.

\section{REFERENCES}

[1] M. S. Khorsheed, Automatic recognition of words in Arabic manuscripts, PhD thesis, University of Cambridge, 2000.

[2] N. Arica, Yarman-Vural. F, Optical Character Recognition for Cursive Handwriting, IEEE Trans Pattern Anal Mach Intell, 24(6), 2002, 801-813.

[3] B. Al-Badr and Mahmoud, S. A, Survey and bibliography of Arabic optical text recognition. Signal Process. 41, 1, 1995, 49-77. 
[4] A. Nazif, A system for the recognition of the printed Arabic characters. Master's thesis, Faculty of Engineering, Cairo University, 1975.

[5] V. Margner and H. El Abed, Databases and competitions: strategies to improve Arabic recognition systems. In Arabic and Chinese Handwriting Recognition, Lecture Notes in Computer Science, vol. 4768, Springer, 2008, 82-103.

[6] M. Cheriet, Visual recognition of Arabic handwriting: challenges and new directions. In Arabic and Chinese Handwriting Recognition, Lecture Notes in Computer Science, vol. 4768, Springer, 2008, 121.

[7] A. Amin, H. Bunke and P. Wang, Arabic character recognition, in Handbook of character recognition and document image analysis, Eds. World Scientific, 1997, 397-420.

[8] S. El-Dabi, R. Ramsis and A. Kamel, Arabic character recognition system: A statistical approach for recognizing cursive typewritten text, Pattern Recognition, Vol. 23, no. 5, 1990, 485- 495.

[9] T. S. El-Sheikh and S. G. El-Taweel, Real-time Arabic handwritten character recognition, Pattern Recognition, Vol. 23, no. 12 , 1990, 1323-1332.

[10] F. El-Khaly and M. A. Sid-Ahmed, Machine recognition of optically captured machine printed Arabic text, Pattern Recognition, Vol. 23, no. 11, 1990, 1207-1214.

[11] A.Sabri, Arabic character recognition using Fourier descriptors and character contour encoding, Pattern Recognition, Vol. 27, no. 6, 1994, 815-824.

[12] J. Ayman and M. Laheeb, Arabic Handwritten Characters Recognized by Neocognitron Artificial Neural Network, University of Sharjah Journal of Pure \& Applied Sciences, Vol. 3, No. 2, 2006.

[13] A. Amin, H. Al-Sadoun and S. Fischer, Hand- Printed Arabic Character Recognition System Using an Artificial Network. Pattern Recognition, 29, 1996, 663-675.

[14] A. Amin, Recognition of Hand-Printed Characters Based on Structural Description and Inductive Logic Programming. Pattern Recognition Letters, 24, 2003, 3187-3196.

[15] G. Olivier, H. Miled, K. Romeo \& Y. Lecourtier, Segmentation and Coding of Arabic Handwritten Words. Proc. 13th Int'l Conf.Pattern Recognition, 3, 1996, 264-268.

[16] I.S.I. Abuhaiba and P. Ahmed, Restoration of Temporal Information in Off-Line Arabic Handwriting, Pattern Recognition, 26, 1993, 1009-1017.

[17] I.S.I. Abuhaiba, S.A. Mahmoud and R.J. Green, Recognition of Handwritten Cursive Arabic Characters. IEEE Trans. Pattern Analysis and Machine Intelligence, 16, 1994, 664-672.

[18] M. Dehghan, K.Faez, M. Ahmadi and M. Shridhar, Handwritten Farsi (Arabic)Word Recognition: A Holistic Approach Using Discrete HMM. Pattern Recognition, 34, 2001, 1057-1065.

[19] H. Al-Yousefi and S.S. Udpa, Recognition of Arabic Characters. IEEE Trans. Pattern Analysis and Machine Intelligence, 14, 1992, 853-857.

[20] S. Abdelazeem and E. EL-Sherif, Arabic handwritten digit recognition. Int. J. Doc. Anal. Recog. 11, 3, 2008,127-141.

[21] G. Abandah and N. Anssari, Novel moment features extraction for recognizing handwritten Arabic letters. J. Comput. Sci. 5, 3, 2009, 226-232.

[22] K. Sherif and M. Mostafa, A Parallel Design and Implementation For Backpropagation Neural Network Using MIMD Architecture. IEEE, 1996, 1361- 1366.

[23] K. Sherif \& M. Mostafa, A Parallel Design and Implementation For Backpropagation Neural Network Using MIMD Architecture. IEEE, 1996, 1472- 1475.

[24] M. A. Ali, Arabic handwritten characters classification using learning vector quantization algorithm. In Image and Signal Processing, Lecture Notes in Computer Science, vol. 5099, Springer, 2008, 463470 .

[25] S. A. Mahmoud and S. O. Olatunji,. Automatic recognition of off-line handwritten Arabic (Indian) numerals using support vector and extreme learning machines. Int. J. Imaging 2, A09, 2009.

[26] S. A. Mahmoud and S. Owaidah, Recognition of off-line handwritten Arabic (Indian) numerals using multi-scale features and support vector machines. Arab. J. Sci. Engin. 34, 2B, 2009, 429-444.

[27] Otsu, N., "A Threshold Selection Method from Gray-Level Histograms," IEEE Transactions on Systems, Man, and Cybernetics, Vol. 9, No. 1, 1979, pp. 62-66.

[28] G. Lazzara and T. Géraud, Efficient multiscale Sauvola's binarization, Springer-Verlag Berlin Heidelberg, 2013.

[29] Cheriet. M, Character Recognition Systems, John Wiley, 2007, 36-38.

[30] S.L. Jae, Two dimensional signal and image processing, PRENTICE HALL PTR, Upper Saddle River, New Jersey. 07458, 1990.

[31] J. Hann, M.Kamber,. Data Mining: Concepts and Techniques, 3rd Edition. Morgan Kaufman Publishers, 2011. 
[32] S. Daniel, K. Vladimír, P. Jiri, Introduction to multi-layer feed-forward neural networks, ELSEVIER, Chemometrics and Intelligent Laboratory Systems 39, 1997, 43-62.

[33] P. CHANDRA, Sigmoidal Function Classes for Feedforward Artificial Neural Networks, Kluwer Academic Publishers, 2003, 185-195.

[34] M. F. Moller, A scaled conjugate gradient algorithm for fast supervised learning, Neural Networks, Vol. 6, 1993, 525-533.

[35] M. H. Beale, T. Hagan and B. Demuth, Neural Network Toolbox 7, User's Guide, by The MathWorks, Inc., 2010.

[36] A. A. Aburas and S. M. Rehiel, Off-line Omni-style Handwriting Arabic Character Recognition System Based on Wavelet Compression, Arab Research Institute in Sciences \& Engineering ARISER, 2007, 123-35.

[37] M. Z. Khedher, G. A. Abandah, and A. M. Al-Khawaldeh, Optimizing Feature Selection for Recognizing Handwritten Arabic Characters, World Academy of Science, Engineering and Technology 4, 2005, 81-84.

[38] A. T. Al-Taani and S. Al-Haj, Recognition of On-line Arabic Handwritten Characters Using Structural Features, Journal of Pattern Recognition Research, 2010, 23-37.

[39] G. A. Abandah, K. S. Younis and M. Z. Khedher, Handwritten Arabic Character Recognition Using Multiple Classifiers Based On Letter Form, Conf. on Signal Processing, Pattern Recognition, \& Applications Austria, 2008, 128-133.

[40] H. Alamri, J.Sadri, C.Y.Suen, N.Nobile, "A novel comprehensive database for Arabic off line handwriting recognition," The11thInternational Conference on Frontiers in Handwriting Recognition (ICFHR), pp. 664-669. 2008.

\section{AUTHORS}

Ahmed T. Sahlol Obtained Bachelor degree from Mansoura University, Egypt in 2004, 2 years Diploma from Mansoura University in 2006, Master degree from Mansoura University, Egypt in 2010. He is currently visiting researcher at Concordia University in Canada and he got a governmental scholarship to study his PhD. His research interests include pattern recognition, optical character recognition and image processing.

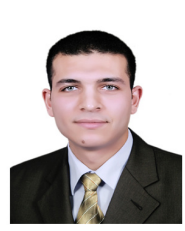

Ching Y. Suen is the Director of CENPARMI and the Concordia Chair on AI and Pattern Recognition. He has served as the Chairman of the Department of Computer Science and as the Associate Dean (Research) of the Faculty of Engineering and Computer Science of Concordia University. His research projects have been funded by the ENCS Faculty and the Distinguished Chair Programs at Concordia University, FCAR (Quebec), NSERC (Canada), the National Networks of Centres of Excellence (Canada) and the industrial sectors in various countries, including Canada, France, Japan, Italy, and the United States.

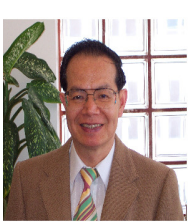




\title{
A NOVEL GLOBAL THRESHOLD-BASED ACTIVE CONTOUR MODEL
}

\author{
Nuseiba M. Altarawneh ${ }^{1}$, SuhuaiLuo $^{1}$, Brian Regan ${ }^{1}$, ChangmingSun $^{2}$ \\ ${ }^{1}$ School of Design Communication and IT, the University of Newcastle, \\ Callaghan NSW 2308, Australia \\ nuseiba.altarawneh@uon.edu.au, suhuai.luo@newcastle.edu.au, \\ brian.regan@newcastle.edu.au \\ ${ }^{2}$ CSIRO Computational Informatics, Locked Bag 17, \\ North Ryde, NSW 1670, Australia \\ changming. sunecsiro.au
}

\begin{abstract}
In this paper, we propose a novel global threshold-based active contour model which employs a new edge-stopping function that controls the direction of the evolution and stops the evolving contour at weak or blurred edges. The model is implemented using selective binary and Gaussian filtering regularized level set (SBGFRLS) method. The method has a selective local or global segmentation property. It selectively penalizes the level set function to be a binary function. This is followed by using a Gaussian function to regularize it. The Gaussian filters smooth the level set function and afford the evolution more stability. The contour could be initialized anywhere inside the image to extract object boundaries. The proposed method performs well when the intensities inside and outside the object are homogenous. Our method is tested on synthetic, medical and Arabiccharacters images with satisfactory results
\end{abstract}

\section{KEYWORDS}

Imagesegmentation, Active contour, Geodesic active contour, $C$-V model,Level set method, ZAC model.

\section{INTRODUCTION}

Active contour model (ACM) is one of the most successful techniques in solving image segmentation problems. The idea underlying the ACM is to evolve a curve or a surface defined within an image from some arbitrary initial shape towards its interior normal direction and stop it on the object boundary [1]. The parametric curve is associated with an energy function. During the deformation, the curve tries to minimize its energy so that the final curve possesses a local minimum when the contour is spatially aligned with the shape or the desired image features. Thus the problem of segmentation becomes an energy minimization problem [2]. To find the desired image features, parametric curves are initialized close to the desired feature and are forced to move toward the local minimum which will be on the desired features under the influence of internal and external forces. The internal forces are defined within the curve or surface to keep the model smooth during the deformation. The external forces are derived from the image data to move the curve toward the object boundary or the desired features within an image. The geodesic active contour models $[3,4]$ utilize the image gradient in order to construct an edge detector function. The objective of this function is to stop the contour evolution on the object boundary. The general edge detector function can be defined by a positive and decreasing function such as:

David C. Wyld et al. (Eds) : CCSIT, SIPP, AISC, PDCTA, NLP - 2014

pp. 245-254, 2014. (C) CS \& IT-CSCP 2014

DOI : $10.5121 /$ csit.2014.4220 
$g\left(\left|\nabla u_{0}\right|\right)=\frac{1}{1+\left|\nabla G_{\sigma}(x, y) * u_{0}(x, y)\right|}$

where $u_{0}$ is a given image in $\Omega$ and $G_{\sigma} * u_{0}$ denotes a smooth version of $u_{0}$ after convolving it with the Gaussian function. The values of $g\left(\left|\nabla u_{0}\right|\right)$ function will be positive in the homogenous regions and zero on the object boundary.

Due to its flexibility in allowing topological changes, the level set method has been extensively utilized in problems of curve evolution, especially the motion by mean curvature described by Osher and Sethian [5]. In the level set method, the evolution curve is represented implicitly via a Lipchitz function $\phi$, as $C=\{(x, y) \mid \phi(x, y)=0\}$. The zero level set of the function $\phi(t, x, y)$ represents the evolution curve $C$ at time $t$. The evolution of the curve $C$ in a normal direction with speed $F$ operates to solve the equation:

$\left\{\begin{array}{l}\frac{\partial \phi}{\partial t}=|\nabla \phi| F \quad \text { in }[0, \infty] \in R^{2} \\ \phi(x, y, 0)=\phi_{0}(x, y) \text { in } R^{2}\end{array}\right.$

where $\{(x, y) \mid \phi(x, y)=0\}$ represents the initial contour. A particular case is the motion by mean curvature in which $F=\operatorname{div}(\nabla \phi(x, y) /|\nabla \phi(x, y)|)$.It follows:

$$
\left\{\begin{array}{l}
\frac{\partial \phi}{\partial t}=|\nabla \phi| \operatorname{div}\left(\frac{\nabla \phi}{|\nabla \phi|}\right) \quad \text { in }[0, \infty] \mathrm{X} R^{2} \\
\phi(x, y, 0)=\phi_{0}(x, y) \text { in } R^{2}
\end{array}\right.
$$

Malladi et al.[6] proposed the following level set equation:

$$
\left\{\begin{array}{l}
\frac{\partial \phi}{\partial t}=|\nabla \phi|\left(-v+\frac{v}{\left(m_{1}+m_{2}\right)}\left(\left|\nabla G_{\sigma} * u_{0}\right|-m_{2}\right)\right) \\
\phi(x, y, 0)=\phi_{0}(x, y) \text { in } R^{2}
\end{array}\right.
$$

where $v$ isaconstant,and $m_{1}$ and $m_{2}$ arethemaximumandminimumvaluesoftheimage gradient $\left|\nabla G_{\sigma} * u_{0}\right|$, respectively . The evolving curve stops at the boundary ,i.e., points with the highest gradient. Caselles et al.[4] proposed a Geometric Active Contour model (GAC) based on the mean curvature motion:

$$
\left\{\begin{array}{l}
\frac{\partial \phi}{\partial t}=g\left(\left|\nabla u_{0}\right|\right)|\nabla \phi|\left(\operatorname{div}\left(\frac{\nabla \phi}{|\nabla \phi|}\right)+v\right) \quad \text { in }[0, \infty] \mathrm{X} R^{2} \\
\phi(x, y, 0)=\phi_{0}(x, y) \text { in } R^{2}
\end{array}\right.
$$

where $v$ is a constant. In GAC the curve moves in the normal direction with speed equal to $g\left(\left|\nabla u_{o}\right|\right)(\operatorname{div}(\nabla \phi /|\nabla \phi|)+v)$. The curve will stop the evolution when the function vanishes.

All the above ACMs are termed as edge-based models [7-10] because they utilize the image gradient as stopping criterion for the evolving curve.Edge-based models do not perform well in the presence of noise and in images with weak edges or without edges. In the case of a discrete 
gradient, the curve may pass through the edges because the function $g\left(\left|\nabla u_{0}\right|\right)$ never approaches zero at these points . These models possess a local segmentation property. They They are sensitive to the position of the initial contour as they are prone to the local minima and can only segment the desired object with a proper initial contour. As a result, these models fail to detect the boundaries when the initial contour is far from the boundary of the desired object. They also cannot detect the interior boundary without setting a proper initial contour inside the desired object. Region-based models represent another category of ACMs [11-16]. These models deploy statistical information inside and outside the contour in order to control its evolution. Region-based models are less sensitive to the position of the initial contour and they perform well in the presence of the noise and with images with weak edges or without edges. These models have a global segmentation property and can detect the interior and exterior boundaries at the same time, regardless of the position of the initial contour in the image. Chan and Vese [11] proposed a widely used regionbased model, namely the $\mathrm{C}-\mathrm{V}$ model. The $\mathrm{C}-\mathrm{V}$ model is based on Mumford-Shah segmentation techniques [13] and has been successfully implemented in a binary phase segmentations. The C-V model uses the statistical information inside and outside the contour with the aim of controlling the evolution. The $\mathrm{C}-\mathrm{V}$ model is formulated by minimizing the equation:

$$
F\left(c_{1}, c_{2}, C\right)=\mu \text { Length }(C)+v \text { Area }(\text { inside }(C))+\lambda_{1} \int_{\text {inside }}(C)\left|u_{0}(x, y)-c_{1}\right|^{2} d x d y+\lambda_{2} \int_{\text {outside }}(C)\left|u_{0}(x, y)-c_{2}\right|^{2} d x d
$$

where $u_{0}$ is a given image in $\Omega, \mu, v, \lambda_{1}, \lambda_{2}$ are positive parameters and $c_{1}, c_{2}$ are the average intensities inside and outside the curve $C$,respectively. With the level set method, one can assume:

$$
\left\{\begin{array}{l}
C=\{(x, y) \in \Omega: \phi(x, y)=0\} \\
\text { inside }(C)=\{(x, y) \in \Omega: \phi(x, y)>0\} \\
\text { outside }(C)=\{(x, y) \in \Omega: \phi(x, y)<0\}
\end{array}\right.
$$

$c_{1}$ and $c_{2}$ are solved as:

$$
\left\{\begin{array}{c}
c_{1}(\phi)=\frac{\int_{\Omega} u_{0}(x, y) H(\phi(x, y)) d x d y}{\int_{\Omega} H(\phi(x, y)) d x d y} \\
c_{2}(\phi)=\frac{\int_{\Omega} u_{0}(x, y)(1-H(\phi(x, y))) d x d y}{\int_{\Omega}(1-H(\phi(x, y))) d x d y}
\end{array}\right.
$$

where $H(\phi)$ refers to the Heaviside function and $\delta(\phi)$ is the Dirac function. The regularization version of $H$ and $\delta$ that were implemented in the $\mathrm{C}-\mathrm{V}$ model are:

$$
\left\{\begin{array}{l}
H_{\varepsilon}(z)=\frac{1}{2}\left(1+\frac{2}{\pi} \arctan \left(\frac{z}{\varepsilon}\right)\right) \\
\delta_{s}(z)=\frac{1}{\pi} \cdot \frac{\varepsilon}{\varepsilon+z}, z \in R
\end{array}\right.
$$

Thecorrespondingvariation level set formulation is then:

$$
\frac{\partial \phi}{\partial t}=\delta(\phi)\left[\mu \operatorname{div}\left(\frac{\nabla \phi}{|\nabla \phi|}\right)|\nabla \phi|-v-\lambda_{1}\left(u_{0}-c_{1}\right)^{2}+\lambda_{2}\left(u_{0}-c_{2}\right)^{2}\right]
$$


where $\mu$ controls the curve smoothness during the deformation, $v$ is a constant to increase the propagation speed, and $\lambda_{1}$ and $\lambda_{2}$ control the image forces inside and outside the contour $C$, respectively. The values of $\delta \varepsilon(z)$ tend to be near zero, if $\varepsilon$ is too small . In this case, extraction of the desired object may fail if the initial contour starts far from the desired object. The final contour location may not be accurate if $\varepsilon$ is large [12]. Zhang et al.[12] proposed an new regionbased active contour model, hereafter we refer to this model as Zhang et al. Active contour(ZAC) model . This model shares the advantages of the region based model as described in[11] as well as the edge based models in[3].

To this end, this study complements the work of Zhang et al. [12] by introducing a new edge stopping function (ESF). This paper is organized as follows: Section 2 reviews the ZAC model [12] and Section 3 describes our methodology. Section 4 shows some experimental results and finally the conclusion is made in Section 5.

\section{THE ZAC MODEL}

Zhang et al. [12] proposed a novel level set method termed as selective binary and Gaussian filtering regularized level set (SBGFRLS). This approach selectively penalizes the level set function to be a binary function. This is followed by using a Gaussian function to regularize it. The Gaussian filters smooth the level set function and afford the evolution more stability. SBGFRLS model reduces the computational coast of the re-initialization step which in turn makes it more efficient than the traditional level set methods [17]. It is worthwhile mentioning that the SBGFRLS method has the advantage of being a general and robust technique. It can be applied to the classical ACMs, such as GAC model [3] as well as C-V model [11]. The proposed model implemented with SBGFRLS approach has a property of selective local or global segmentation. A novel signed pressure force (SPF) [18] is proposed to control the direction of the evolution and to stop the evolving contour at weak or blurred edges. The ZAC model uses statistical information inside and outside the contour to formulate the SPF. The proposed SPF function is assigned with values in the range $[-1,1]$. It modulates the signs of the pressure forces inside and outside the region of interest as:

$$
\operatorname{spf}\left(u_{0}(x)\right)=\frac{u_{0}(x)-\frac{c_{1}+c_{2}}{2}}{\max \left(\left|u_{0}(x)-\frac{c_{1}+c_{2}}{2}\right|\right)}, x \in \Omega
$$

where $c_{1}$ and $c_{2}$ are defined in Eq. (8). The SPF assumes that if the intensities inside and outside the object are homogenous, it is instinctive that $\operatorname{Min}\left(u_{0}(\mathrm{x})\right)<=\mathrm{c}_{1}$ and $\operatorname{Max}\left(u_{0}(\mathrm{x})\right)<=\mathrm{c}_{2}$, accordingly,

therefore $\operatorname{Min}\left(u_{0}(\mathrm{x})\right)<\frac{\mathrm{C} 1+\mathrm{C} 2}{2}<\operatorname{Max}\left(u_{0}(\mathrm{x})\right)$. The SPF function has opposite signs around the object boundary in order to force the contour to shrink when it is outside the object and to expand when it is inside the object. The formulation of the level set function in the proposed method is given as:

$\frac{\partial \phi}{\partial t}=\operatorname{spf}\left(u_{0}(x)\right) \cdot \alpha|\nabla \phi|, x \in \Omega$

The ZAC model utilizes a binary function for the initialization of the level set function $\varphi$ instead of using the signed distance function as in the traditional level set method. The ZAC model deploys the image statistical information to stop the curve evolution on the desired object boundaries. This makes the ZAC model insensitive to noise and can perform well in the case of an object with weak edges or without edges. The ZAC model is capable of performing both local 
and global segmentation, in contrast to the $\mathrm{C}-\mathrm{V}$ model which can only handle global segmentation and extracts all the objects. The ZAC model has less computational complexity than the GAC and $\mathrm{C}-\mathrm{V}$ models.

\section{THE PROPOSED MODEL}

In this paper, we propose a new edge stopping function that controls the direction of the evolution and stops the evolving contour at weak or blurred edges. Our model is implemented using the SBGFRLS method, which grants it a selective local or global segmentation property. Our model adapts the same methodology of the ZAC model described by Zhang et al. [12]. Our novel modification stems from utilizing a new function termed the global threshold function (GTF) instead of using the SPF as in the original ZAC model. The GTF operates similarly to the SPF. Generally, both functions produce similar results. It controls the direction of the evolution and stops the evolving contour at weak or blurred edges. Our proposed model performs well when the intensities inside and outside the object are homogenous and in the binary segmentation phase, in an analogy to the ZAC model. The GTF has opposite signs around the object boundary in order to force the contour to shrink when it is outside the object and to expand when it is inside the object. The proposed GTF is assigned with values in the range $[-1,1]$ :

$$
g t f\left(u_{0}(x)\right)=\frac{u_{0}(x)-t}{\max \left(\left|u_{0}(x)-t\right|\right)}, x \in \Omega
$$

where $t$ is a threshold value computed automatically by using the global thresholding method[19]. The procedure of the proposed algorithm consists mainly of:

1. Initialisation of the level set function $\phi$ into a binary function.

2. Computing the value of $t$ using the global threshold method[19].

3. Computing the values of $g t f\left(u_{0}(x)\right)$ function according to Eq. (13).

4. Evolving the level set function $\phi$ according to Eq. (12).

5. If the local segmentation property is desired, let $\phi=1$ if $\phi>0$; otherwise $\phi=-1$.

6. In order to smooth the level set function, Gaussian filter is used to regularize it.

7. When the evolution of the level set function has converged, the procedure stops. Otherwise return to step4.

The contour could be initialized anywhere inside the image to extract all exterior and interior boundaries, even if the initial contour does not surround all the objects in the image. Our model entails all the advantages pertinent to the ZAC model. Our model gives a similar result to the ZAC model in less computational time because the values of $t$ and $0(())$ gtfux are computed only once. The ZAC model can extract objects with distinctive boundaries while interior intensities are not homogeneous. By contrast, our model extracts both the interior and exterior boundaries as shown in Figure 6.

\section{EXPERIMENTAL RESULTS}

In each experiment, we select values of $\rho, \varepsilon, \sigma, k$, and $s$ tobe1,1.5,1,5and1, respectively. The values of $\alpha$ and $t$ were set according to the images. Figure1 exhibits the global segmentation property of the $\mathrm{C}-\mathrm{V}$ model and our proposed model.The initial contour is initialized far from the objects, as shown in the first column of Figure1 .The second column shows the segmentation results of the $\mathrm{C}-\mathrm{V}$ model . As is displayed, the $\mathrm{C}-\mathrm{V}$ model fails to extract all the objects in the image. The third column shows the segmentation result of our model. Clearly, our model extracts 
accurately all the objects in the image regardless of the position of the initial contour, while the C$\mathrm{V}$ model may be trapped into the local minima and then result in unsatisfactory segmentation.
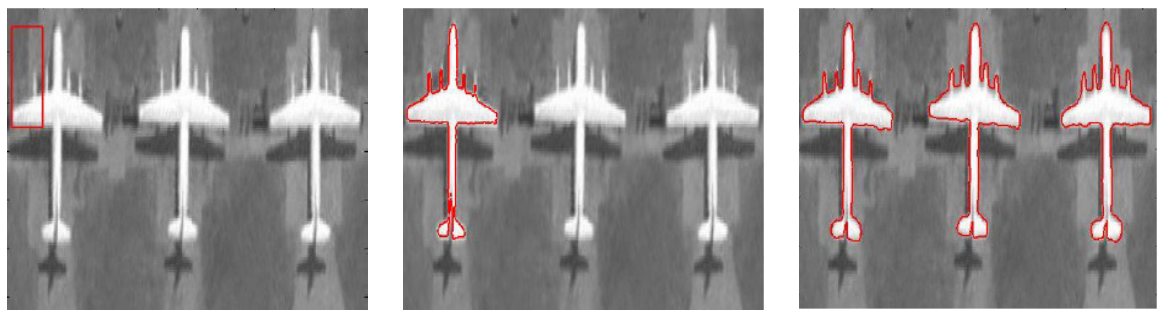

Figure 1. Comparisons of the global property between the C-V model and the proposed method. The first column shows the initial contour, the second column shows the segmentation results of the $\mathrm{C}-\mathrm{V}$ method and the third column shows the result of the proposed method with $t=172$ and $\sigma=20$. The original image is sourced from Zhang et al. [12].

Figure 2 presents segmentation results of the $\mathrm{C}-\mathrm{V}$ model and our proposed model in a magnetic resonance image of the left ventricle of a human heart. In an analogy to the ZAC model, our model also can selectively extract the desired object by setting the initial contour inside or surrounding the desired boundaries, while the $\mathrm{C}-\mathrm{V}$ model will extract all the objects. Furthermore, the evolution direction in our model can be controlled to obtain satisfactory segmentation results, while the $\mathrm{C}-\mathrm{V}$ model may get disordered results.
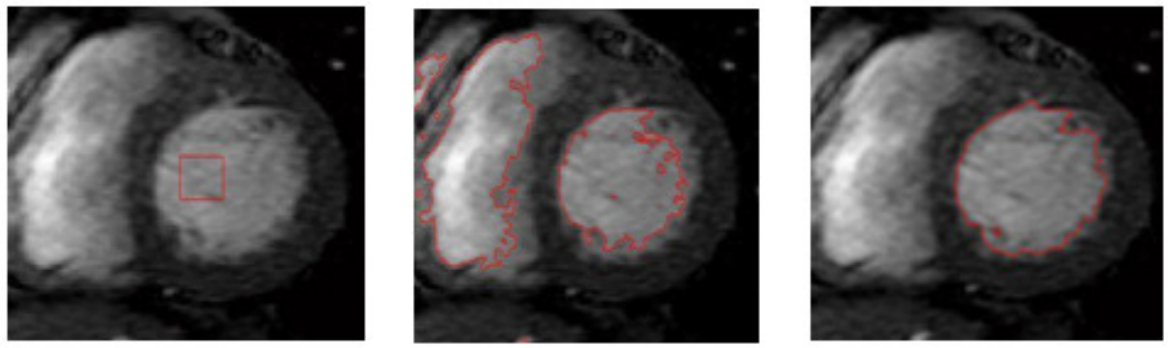

Figure 2. Segmentation results for a magnetic resonance image of the left ventricle of a

human heart. The first column shows the initial contour, the second column shows the segmentation results of the $\mathrm{C}-\mathrm{V}$ model and the third column shows the result of the proposed model with $t=111$ and $\alpha=5$.

Figure 3 demonstrates the local segmentation property of the proposed model in an MR image of a corpus callsum. As depicted in the image, our model gives satisfactory results. As our model is based on the ZAC model it can selectively extract the desired object by setting the initial contour inside or surrounding the desired boundaries with satisfactory segmentation results.

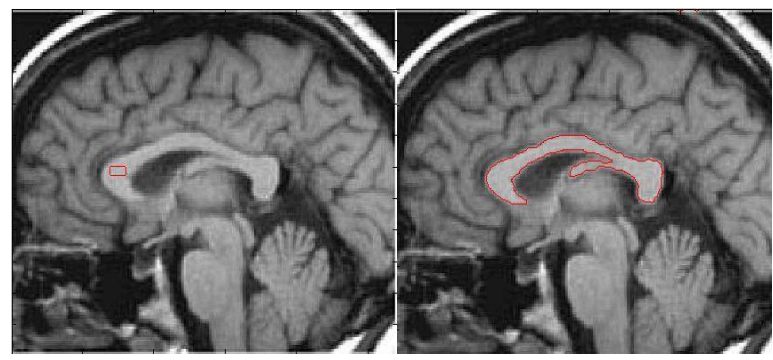

Figure 3. The first column shows the initial contour, the second column shows the segmentation result of the proposed method with $\alpha=10$. 
Figure 4 shows the local segmentation property of our proposed model.The initial contour resides near or surrounding the desired object , as shown in the first column of Figure 4 . The second column shows the segmentation result of our proposed model.
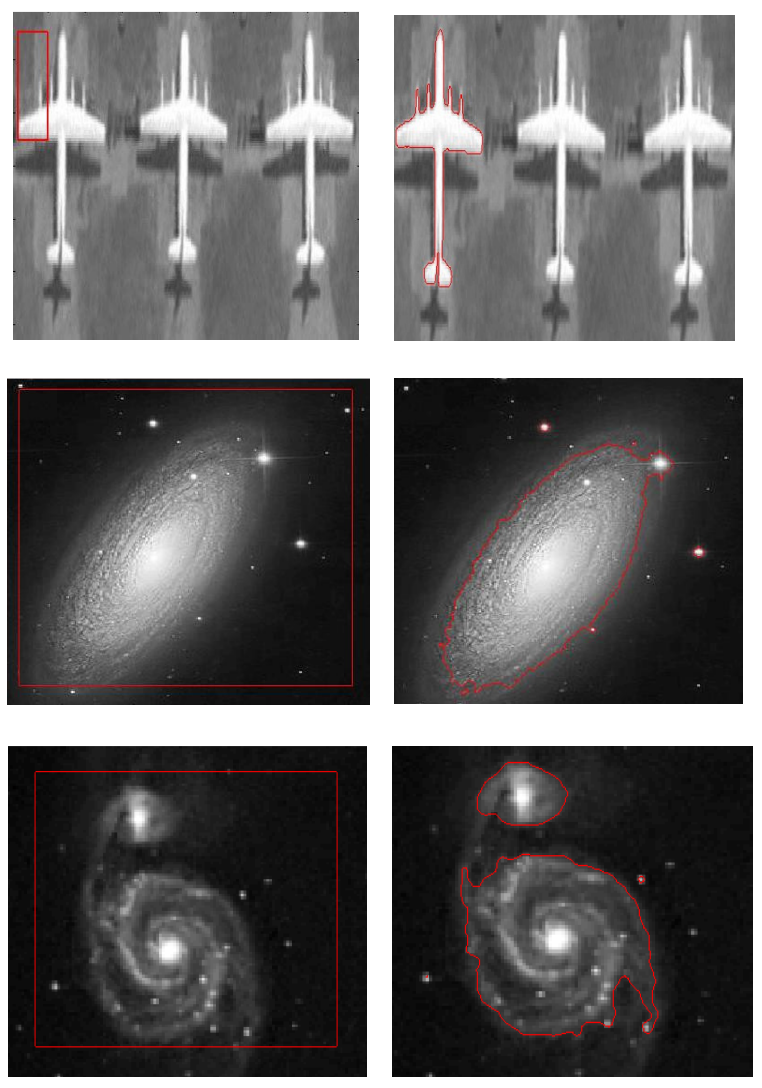

Figure4.Segmentationresultsfromourproposedmodel.Thefirstandsecondcolumn sshowtheinitialcontour, and segmentation results,respectively. $t$ Correspond to 172, 90 and 56.4071, for the first, second and third row, respectively and $\alpha$ $=20$. The original images are sourced from Zhang et al.[12].

Figure 5 shows the global segmentation results by our proposed model for noisy images .As it can be seen, despite of the presence of significant noise inherit in the image, our model performs well in detecting the desired object boundary.

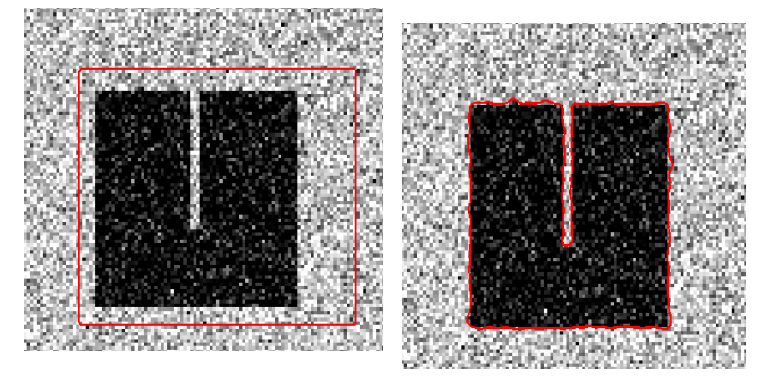

Figure5.Global segmentation results for a noisy image. The first column shows the initial contour, the second column shows the segmentation result of the proposed method with $t=114$.1 and $\sigma=10$. The original image is sourced from Zhang et al.[12]. 
Figure 6 shows the local segmentation property of the ZAC model and our proposed model. As shown in Figure 6, the ZAC model extracts objects with distinct boundaries whereas interior intensities are not homogeneous. In contrary, our model extracts the interior and the exterior boundaries. This represents the main shortcoming of our proposed model.
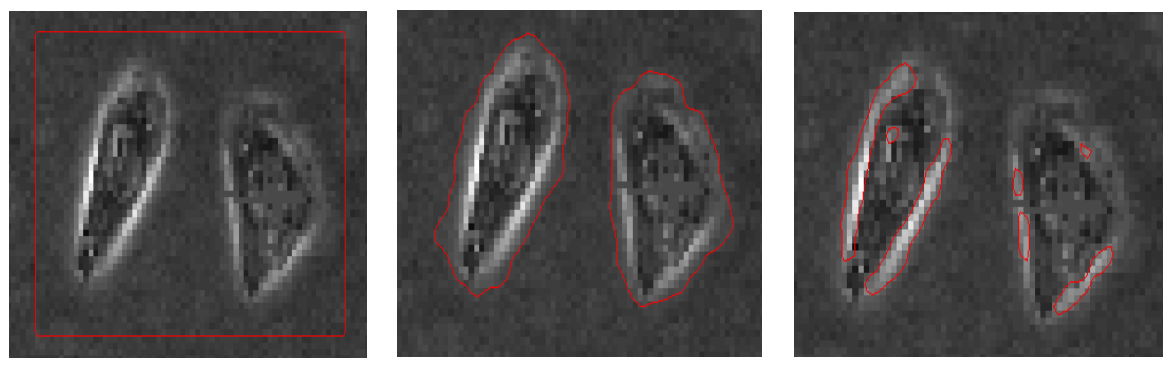

Figure 6.Local segmentation results for a real microscope cell image.The first column shows the initial contour, the second column shows the segmentation results of the ZAC method and the third column shows the result of the proposed method with $t=94.0314$. The original image is sourced from Zhang et al.[12].

Figure7 exhibits the performance of our proposed method in the case of Arabic-characters segmentation. As shown in this figure, our proposed model attains satisfactory segmentation results.
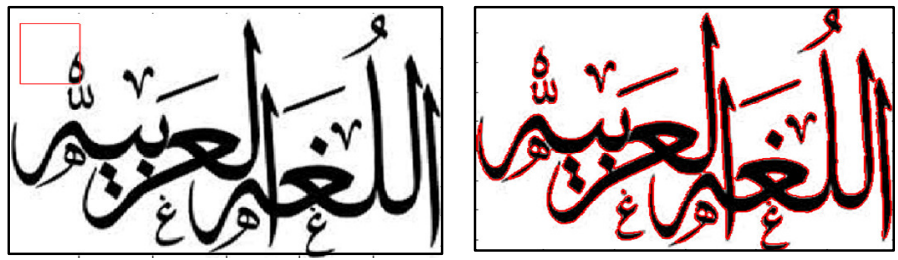

Figure7.Thefirstcolumnshowstheinitialcontour,thesecondcolumnshowsthesegm entationresultsoftheproposedmethodwith $t=141.3368$ and $\alpha=20$.

\section{CONCLUSIONS}

A novel global threshed-based active contour model with a new edge-stopping function is presented. The main merits of this approach consist in its ability to control the direction of the evolving contour and to stop it on the weak or blurred edges. Our model is implemented using the SBGFRLS method. We tested this method on several categories of images including synthetic, medical and Arabic-characters where a satisfactory performance is attained.

\section{REFERENCES}

[1] Kass, M., Witkin, A. \& Terzopoulos, D. (1988) "Snakes: Active contour models",International Journal of Computer Vision. Vol. 1, No.4, pp 321-331.

[2] Xu, C., Pham, D. L. \& Prince, J. L. (2000) "Image segmentation using deformable models",Handbook of Medical Imaging. Vol. 2, No., pp 129-174.

[3] Caselles, V., Kimmel, R. \& Sapiro, G. (1997) "Geodesic active contours",International Journal of Computer Vision. Vol. 22, No.1, pp 61-79.

[4] Caselles, V., Catté, F. , Coll, T. \& Dibos, F. (1993) "A geometric model for active contours in image processing",Numerische Mathematik. Vol. 66, No.1, pp 1-31.

[5] Osher, S. \& Sethian, J. A. (1988) "Fronts propagating with curvature-dependent speed: algorithms based on Hamilton-Jacobi formulations",Journal of Computational Physics. Vol. 79, No.1, pp 12-49. 
[6] Malladi, R., Sethian, J. A. \& Vemuri, B. C. (1995) "Shape modeling with front propagation: A level set approach",Pattern Analysis and Machine Intelligence, IEEE Transactions on. Vol. 17, No.2, pp 158-175.

[7] Zhu, G., Zhang, S., Zeng, Q. \& Wang, C. (2007) "Boundary-based image segmentation using binary level set method",Optical Engineering. Vol. 46, No.5, pp 050501-050501-3.

[8] Li, C., Xu, C., Gui, C. \& Fox, M. D. Level set evolution without re-initialization: a new variational formulation. in Computer Vision and Pattern Recognition, 2005. CVPR 2005. IEEE Computer Society Conference on. 2005. IEEE.

[9] Paragios, N. \& Deriche, R. (2000) "Geodesic active contours and level sets for the detection and tracking of moving objects",Pattern Analysis and Machine Intelligence, IEEE Transactions on. Vol. 22, No.3, pp 266-280.

[10] Xu, C. \& Prince, J. L. (1998) "Snakes, shapes, and gradient vector flow",Image Processing, IEEE Transactions on. Vol. 7, No.3, pp 359-369.

[11] Chan, T. F. \& Vese, L. A. (2001) "Active contours without edges",Image Processing, IEEE Transactions on. Vol. 10, No.2, pp 266-277.

[12] Zhang, K., Zhang, L., Song, H. \& Zhou, W. (2010) "Active contours with selective local or global segmentation: A new formulation and level set method",Image and Vision Computing. Vol. 28, No.4, pp 668-676.

[13] Mumford, D. \& Shah, J. (1989) "Optimal approximations by piecewise smooth functions and associated variational problems",Communications on Pure and Applied Mathematics. Vol. 42, No.5, pp 577-685.

[14] Li, C., Kao, C.-Y., Gore, J. C. \& Ding, Z. Implicit active contours driven by local binary fitting energy. in Computer Vision and Pattern Recognition, 2007. CVPR'07. IEEE Conference on. 2007. IEEE.

[15] Vese, L. A. \& Chan, T. F. (2002) "A multiphase level set framework for image segmentation using the Mumford and Shah model",International Journal of Computer Vision. Vol. 50, No.3, pp 271293.

[16] Ronfard, R. (1994) "Region-based strategies for active contour models",International Journal of Computer Vision. Vol. 13, No.2, pp 229-251.

[17] Osher, S. \& Fedkiw, R. (2003) Level set methods and dynamic implicit surfaces. Springer.

[18] Xu, C., Yezzi Jr, A. \& Prince, J. L. On the relationship between parametric and geometric active contours. in Signals, Systems and Computers, 2000. Conference Record of the Thirty-Fourth Asilomar Conference on. 2000. IEEE.

[19] Gonzalez, R. C. \& Richard, E. (2002) Digital Image Processing. Prentice Hall Press.

\section{AUTHORS}

NuseibaAltarawneh is a $\mathrm{PhD}$ student at the University of Newcastle, Australia. Her research interests include computer vision, image analysis, and pattern recognition. She obtained M.E. degrees in Computer science from the University of Jordan in 2009.

Dr. SuhuaiLuoreceived B.E. and M.E. degrees in Radio Engineering from Nanjing University of Posts and Telecommunications China in 1982 and 1987, respectively, and $\mathrm{PhD}$ degree in Electrical Engineering from the University of Sydney Australia in 1995.From 1995 to 2004, he worked as a senior research scientist with the Commonwealth Scientific and Industrial Research Organization Australia and the Bioinformatics Institute Singapore. He is currently a senior lecturer with the University of

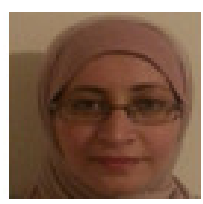

NewcastleAustralia. His research interest is in information technology and multimedia, including health informatics, machine learning, image processing, computer vision, and Internet-oriented IT applications.

Dr Brian Regan is a senior Lecturer in IT at the University of Newcastle. He is part of the Applied Informatics Research Group (AIR) with interests in health informatics, visualization and development methodologies.

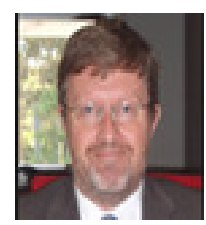


Dr.Changming Sun received the $\mathrm{PhD}$ degree in the area of computer vision from Imperial College London in 1992. Then, he joined CSIRO Computational Informatics, Australia, where he is currently a principal research scientist carrying out research and working on applied projects. His research interests include computer vision, image analysis, and pattern recognition. He has served on the program/organizing committees of various international conferences. He is an Associate Editor for EURASIP Journal on Image and Video Processing, a SpringerOne journal.

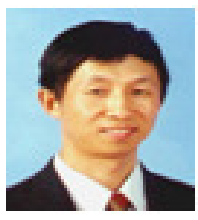




\title{
AUTOMATIC ESTIMATION OF LIVE COFFEE LEAF INFECTION BASED ON IMAGE PROCESSING TECHNIQUES
}

\author{
Eric Hitimana*1 and Oubong Gwun ${ }^{* 2}$ \\ *Department of Computer Science and Engineering, Chonbuk National \\ University, \\ Jeonju City, South Korea \\ ${ }^{1}$ hitimeric06@yahoo.fr, ${ }^{2}$ obgwunejbnu.ac.kr
}

\begin{abstract}
Image segmentation is the most challenging issue in computer vision applications. And most difficulties for crops management in agriculture are the lack of appropriate methods for detecting the leaf damage for pests' treatment. In this paper we proposed an automatic method for leaf damage detection and severity estimation of coffee leaf by avoiding defoliation. After enhancing the contrast of the original image using LUT based gamma correction, the image is processed to remove the background, and the output leaf is clustered using Fuzzy c-means segmentation in $V$ channel of $Y U V$ color space to maximize all leaf damage detection, and finally, the severity of leaf is estimated in terms of ratio for leaf pixel distribution between the normal and the detected leaf damage.
\end{abstract}

The results in each proposed method was compared to the current researches and the accuracy is obvious either in the background removal or damage detection.

\section{KEYWORDS}

Coffee rust, LUT, Background removal, Image segmentation, Color and luminance, Gamma correction

\section{INTRODUCTION}

A computer vision system is an attempt to replicate the human eye to brain assessment process, whereby the human eye is replaced by a digital camera and the human brain is replaced by a learning algorithm. The camera can record objective and consistent image data without substantial confounding noise [1].

Image processing has been proved to be effective tool for analysis in various fields and applications [2]. In evolution towards sustainable agriculture system it was clear that important contributions can be made by using emerging techniques. Precision agriculture was new and developing technology which leads to incorporate the advance techniques to enhance farm output and also enrich the farm inputs in profitable and environmentally sensible manner. With these techniques/ tools it was now possible to reduce errors, costs to achieve ecological and economical sustainable agriculture.

David C. Wyld et al. (Eds) : CCSIT, SIPP, AISC, PDCTA, NLP - 2014 
Coffee rust is the most economically important coffee disease in the world, and in monetary value, coffee is the most important agricultural product in international trade. Even a small reduction in coffee yields or a modest increase in production costs caused by the rust has a huge impact on the coffee producers, the support services, and even the banking systems in those countries whose economies are absolutely dependent on coffee export.

Infections occur on the coffee leaves. The first observable symptoms are small, pole yellow spots on the upper surfaces of the leaves. As these spots gradually increase in diameter, masses of orange urediniospores (or uredospores) appear on the under surfaces. The fungus sporulates through the stomata rather than breaking through the epidermis as most rusts do, so it does not form the pustules typical of many rusts. The powdery lesions on the undersides of the leaves can be orange-yellow to red-yellow to red-orange in color, and there is considerable variation from one region to another [3].

Coffee rust and other coffee pests cause premature defoliation, which reduces photosynthesis capacity and weakens the tree [4]. The most techniques used to avoid coffee rust and other pests that destroy the coffee leaves are pesticides and fungicides, but if they are not controlled well, they can cause ecosystem problems.

The detection of severity of infected leaves have been done by the farmers using naked eyes, which can contribute to many errors, and the precise ways are needed to be sure the amount of pesticides or fungicides to be applied while preserving ecosystem. Most leaf diseases destroy the leaf, so that it can be easy to detect the damage using image processing techniques, but in the case of coffee rust, there is only color change which acts as a special case.

From the shown problems, we propose a method for detecting the pests' attacks infection on image of coffee's leaf using image segmentation techniques.

In this paper, the novelty is that the images used are captured from the tree, to avoid defoliation.

Most researches about leaf disease detection [5], [6], [7], have been done, but they cut off the leaf and put it to the white background for easy processing, but our algorithm considers all leaves' images regardless the type of background.

As far as the system is concerned, our algorithm is made by three processes. Firstly, the captured coffee leaf is processed for contrast enhancement using LUT gamma correction algorithm by Sayaraman et al. [17]. Secondly, the enhanced image is processed to remove the unwanted background. At this stage, the captured image maybe having many surrounding leaves or branches of trees, the concern is to detect the main leaf to be processed separately. Lastly, the recovered leaf is segmented using fuzzy $\mathrm{C}$ mean clustering to detect the infected part of the leaf; the severity of infected leaf area is estimated by calculating the ratio of the infected pixel distributions to the normal leaf pixel distributions.

\section{RELATED WORKS}

In this section, we survey the related current researches on image processing in agricultures, such as background removal, infected leaf segmentation and area of leaf measurement.

\subsection{Leaf disease detection and classification}

As a rapid, nondestructive and objective method, image processing technology has been widely used in determination of some quality characteristics of agricultural products. Leaf disease detection and classification is a hot research in plant managements and taxonomy. 
Gloria D. et al. [5] proposed semi-automatic approach based on an initial pixel classification according to the chrominance feature from the $\mathrm{YCrCb}$ color space. It requires user-intervention to select a sample of pixels for training the color space classifiers. Thiago L. G. Souza et al. [6] automatically classified the main agents that cause damages to soybean leaflets. After extracting the contour of the damages, they are taken as a complex network, and trained using SVM.

C.P. Wijekoon, et al. [9] used Scion Image software to quantify a wide variety of fungal interactions with plant leaves. This software is responsible for measuring the change in leaf color caused by fungal sporulation or tissue damage. But it only deals with the detached, well placed and shadow free leaves. Qinghai $\mathrm{He}$ et al. [10] proposed the damage detection method by measuring the damage ratio in different color model after enhancing the leaf image, but the algorithm fails to handle the outdoor leaves for contrast and other noises. A.C. Nazare-JR. et al. [11] automatically quantified the damaged leaf area by handling the noises and recovering the leaf contour using computational geometry, but they recovered only the line segments but not the curved edges and they only detected the damaged/ destroyed parts, not the damage part in terms of color change, as we are considering the healthy leaf as the one responsible for photosynthesis process.

All of those methods only handle the detached, well placed, shadow free, with simple background leaves images, and thus they only did the simple image segmentation (thresholding) to remove the background noises. On the other hand, our proposed algorithm considers all types of leaves regardless any background, and can also detect all damaged leaf parts (in terms of destroying and color change).

\subsection{Background removal}

In still image object detection, many researchers proposed different algorithms for background removal for image segmentation purpose. Jeong-In Park et al. [18] suggested the variable order $n$ $\mathrm{x} \mathrm{m}$ dimensional vector, where the vectors are applied to the reduced objective image to remove the background. This method does not remove the actual background; rather it regenerates the filtered replica after replicating the background. It may look like the background is actually removed after it is applied to the image, but it is reconfigured with white color lines which are smoothly processed while retaining the background. This method have been proposed to overcome the computational time of code book, but compare to our method, this is still expensive in terms of computation depending on the size of objective image as it is dealing with image reduction and $\mathrm{n} \times \mathrm{m}$ dimensional vector processing.

In object extraction based on detecting salient regions [19], [20], [21], [22] they ended by segmenting the image to remove the background, but sometimes this method failed to detect the object in question. Guanqun Cao et al. [22] proposed a salient object extraction with opponent color boosting, the method is based on emphasis on color in an iso-salient color space and filtering by a DoG filter afterwards, but the detection is not accurate as it includes even other non objects. Our method has been compared with this one for object detection.

In most agriculture image processing applications, they applied a simple threshold or otsu's image segmentation [23] [11], [6] to separate the background and the foreground because the background is not complicated. Our proposed method for background removal provides an obvious accuracy to their papers as well. 


\subsection{Leaf Area Measurement}

Accurate and rapid non-destructive leaf area measurement/estimation is important in plant understanding and modeling ecosystem function. Utilizing the leaf area instruments, it is reliable and convenient to estimate leaf area using mechanical, digital or portable scanning planimeters [13], but the method is expensive and destructive i.e., means we need to cut off the leaf or some tools can not fit the whole leaf, so it need to be cut into different pieces and measure each piece separately and add together after.

Mahdi M. Ali et al. [14], after preprocessing the leaf and detecting the edge, they used digital vernier and compared the results with Li-Cor 3100 leaf area meter. The method is not destructive but still they used some devices to measure the area. Chaohui Lu et al. [8] captured the leaf and put on a hand normal panel, i.e. square (known area) drawn on a white paper, the image is processed using image processing techniques, and then the area is calculated through pixel number statistic. The accuracy can be affected by the geometric distortion of the panel. Sanjay B. Patil et al. [15], after processing the leaf image, the area is calculated by estimating the pixel statistic referencing to piece of coin (known area). The pixel count of the processed image depends on the distance between the camera and the object when the picture is taken.

In this paper, the proposed algorithm handles all leaf images regardless where and how the image was taken (background, environmental condition that can be affecting the contrast of image, etc), we ended by finding that to adopt one of the proposed method above, can lead us to many errors, because we did not care how far or close the image leaf were captured/shot.

To overcome those challenging, we decided to estimate the severity of the damaged area in percentage by calculating the ratio between the normal leaf pixel distributions (statistic) to the infected pixel distributions.

\section{PROPOSED METHOD}

This section describes the process of our proposed method step by step. Our algorithm consists of three steps: Image contrast enhancement, background removal and detection of the damaged area with its severity estimation. The figure 1 shows the overview of the system.

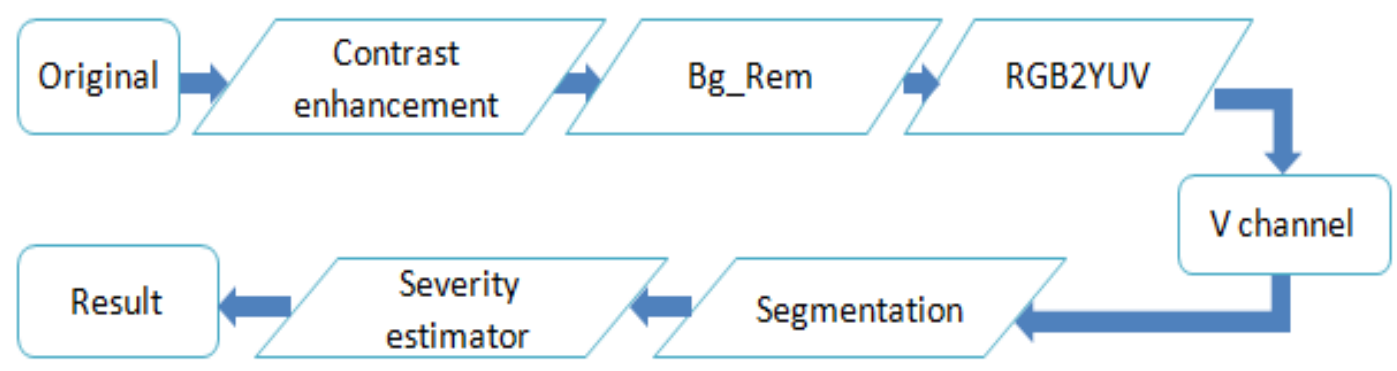

Figure 1: Framework of the proposed method

\subsection{Contrast enhancement}

The contrast enhancement process adjusts the relative brightness and darkness of objects in the scene to improve their visibility. As our method can handle any image regardless of its condition of shot, we decided to use LUT based gamma correction algorithm to deal with the image contrast enhancement. 


$$
\begin{aligned}
& L U T=M a x_{-} \text {int } \times\left(\frac{0: M a x_{-} \text {int }}{M a x_{-} \text {int }}\right)^{1 / \gamma} \\
& I^{\prime}=L U T(\text { double }(I))
\end{aligned}
$$

Look-Up Table (LUT) is formulated using the maximum intensity (Max_int, equals to 255), and the value of gamma depends on the input image.

The original brightness value of image I is mapped to I' by using the formulated LUT as shown on figure 2 .

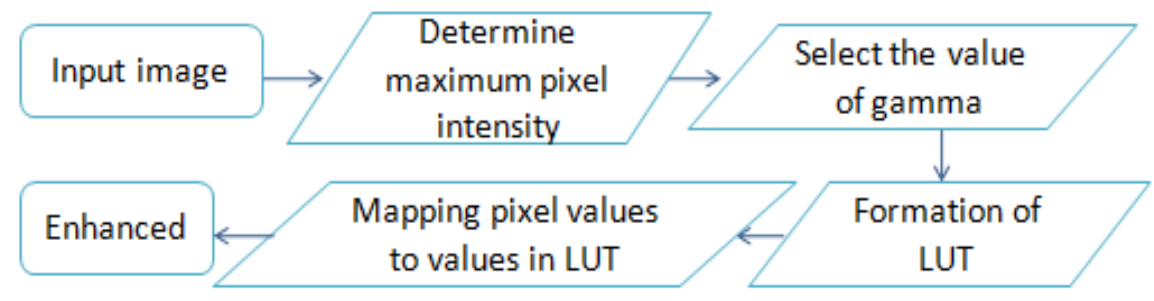

Figure 2: Image enhancement using gamma correction

We proposed a method to set the value of gamma automatically based on the characteristics of the original image.

1. Analyze the histogram of an input gray image,

2. Calculate the mean average intensity value

$$
I_{a v g}=\frac{1}{W x H} \sum_{i=1}^{W} \sum_{j=1}^{H} I(i, j)
$$

3. Normalize the value in range $[0,1]$

$$
r=\frac{I_{a v g}}{I_{\max }}
$$

4. Get the gamma value by using equation

$$
\gamma= \begin{cases}10 r-4 & \text { for } r>0.5 \\ 1 & \text { for } r=0.5 \\ r^{0.1}-0.4 & \text { for } r<0.5\end{cases}
$$

In our experimental results, there is no doubt to say that in both contrast conditions (low and high); the gamma value is selected efficiently. And this proposed automatic gamma value always enhances the image based on its average gray intensity value, this method is different from the adaptive gamma correction proposed by Shi-Chia Huang et al. [12] which requires some adjustable parameters and it always enhances the low contrast, because the value of gamma is always equal or less than 1 according to their equation $\gamma=1-c d f_{w}(l)$. The figure 3 and 4 show our results for contrast enhancement. 

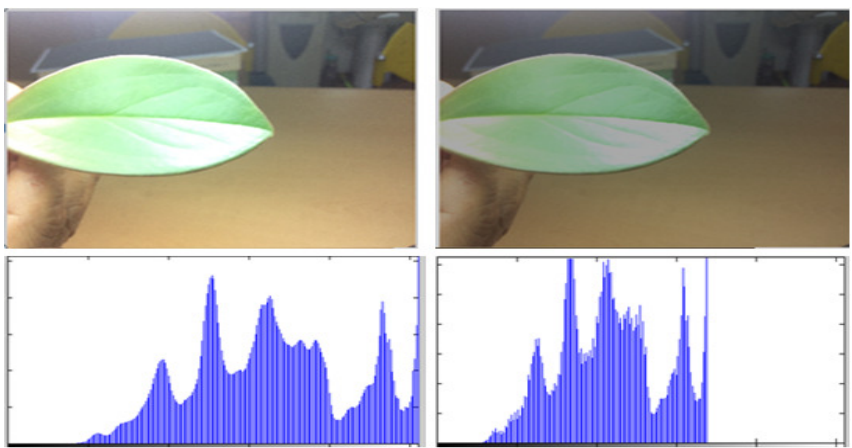

(b)

Figure 3: Result for contrast enhancement images and their gray value histograms: (a) original image of high contrast, (b) resulting image with adjusted contrast for better processing.
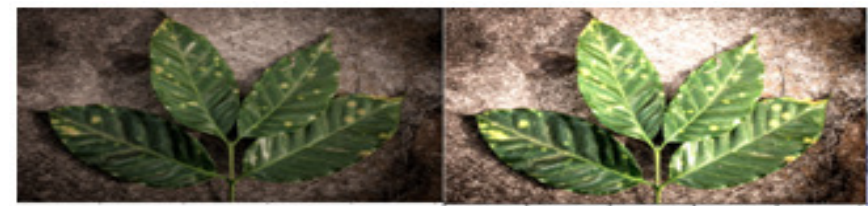

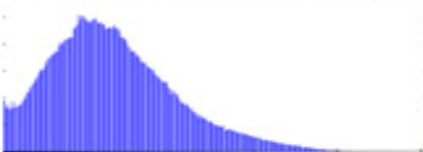

(a)

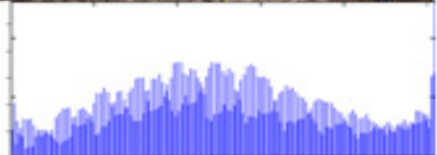

(b)

Figure 4: Result for contrast enhancement, images with their gray value histograms: (a) original image of low contrast, (b) resulting image with enhanced contrast for better processing.

\subsection{Background removal}

The leaf image processed maybe having some surrounding noises that can affect the accuracy of leaf damage detection. We decided to propose a method that can only keep the foreground object, i.e., leaf only.

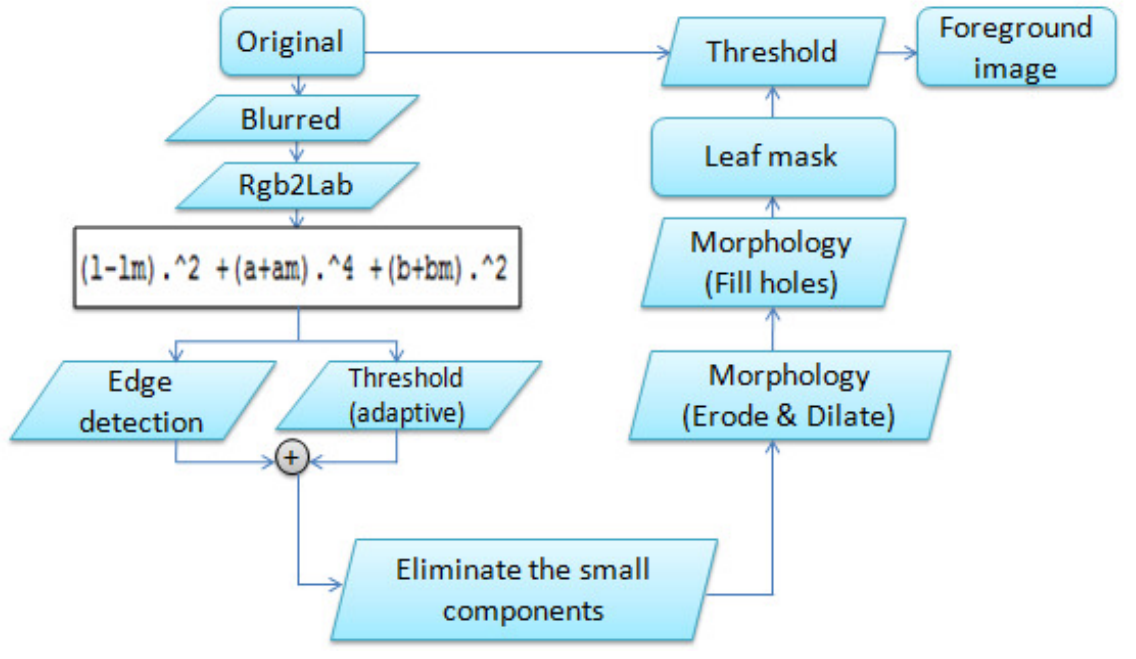

Figure 5: Proposed method for background removal 
The original image is blurred using Gaussian kernel to suppress the noise, and the image is converted to CIELab color space that have been proved as the most color space to detect the object based on the salient properties [17].

$$
O(x, y)=\left(I_{\mu l}-\tilde{I}_{l}\right)^{2}+\left(I_{\mu a}-\tilde{I}_{a}\right)^{4}+\left(I_{\mu b}-\tilde{I}_{b}\right)^{2}
$$

Where the $I_{\mu}$ is the arithmetic mean value of the image in each channel, $\tilde{I}$ is the corresponding image pixel vector value in the Gaussian blurred version (using a $5 \times 5$ separate binomial kernel) of the original image. The above proposed equation (6) can highlight the foreground object and suppress the background. The resulting image is threshold using the following adaptive threshold.

$$
T h_{r}=\frac{1}{W x H} \sum_{i=1}^{W} \sum_{j=1}^{H} O(x, y)
$$

The threshold image is combined by the boundary features detected using canny edge detector to adjust the overall structure of the object. The resulting image with different separate objects is judged to remains with the biggest object among the arrays using labeling method. Image erosion and dilation algorithms are applied to adjust the objects by using the disk element of fixed size. The output image is filled to recover the internal holes. The final image is a mask of the whole object within an image, and it is used as a threshold to segment the original image. The resulting output image at this stage is the image with background free as shown in the figure 6 below.

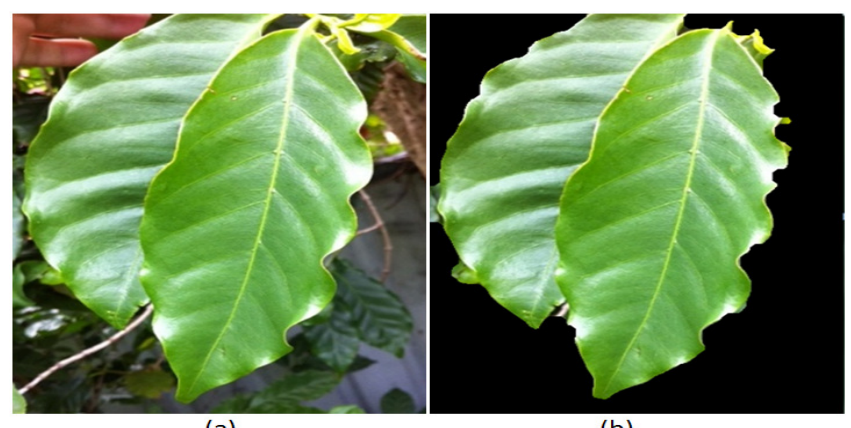

(a)

(b)

Figure 6: Result for background removal: (a) original image, (b) background free image

\subsection{Damage clustering}

At this stage, the leaf in question is available; the only problem is to detect the damage. In most proposed methods [11], [6], they tried to detect damage in gray image because their method only captured the destroyed part of the leaf as an infected leaf part. But our method considers a damaged part as all leaf areas that cannot contribute to the photosynthesis process.

In our case, we detected the damage leaf area in YUV color model, and our algorithm shows a good efficiency compared to other methods. And the other advantage for using YUV color model is that, the leaf veins are not mistaken as the damage. The $\mathrm{V}$ channel is clustered using Fuzzy CMean algorithm, where we only used two clusters. 


\subsection{Damage estimator}

In this paper, we decided to estimate the severity of the leaf damage, to allow the farmers being able to take into account their plant management (for pesticide or fungicides utilization).

After surveying different methods used for estimating leaf damages, we can say that most of them cannot give good results on our samples image database, we decided to estimate infection by calculating the percentage of the damaged pixels statistic to the normal real leaf pixels distribution.

$$
l_{\text {severity }}=\frac{l_{\text {inf ected }}}{l_{\text {normal }}} \times 100
$$

\section{EXPERIMENTAL RESULTS}

In order to validate and test our proposed method, we tested it to many type of leaves and have good results. We compared the results with other researches that have been done. Figure 7 shows the comparison of the proposed method for image background removal. Our proposed method was specific for leaf images, but it can also work better than other methods used for object detection.
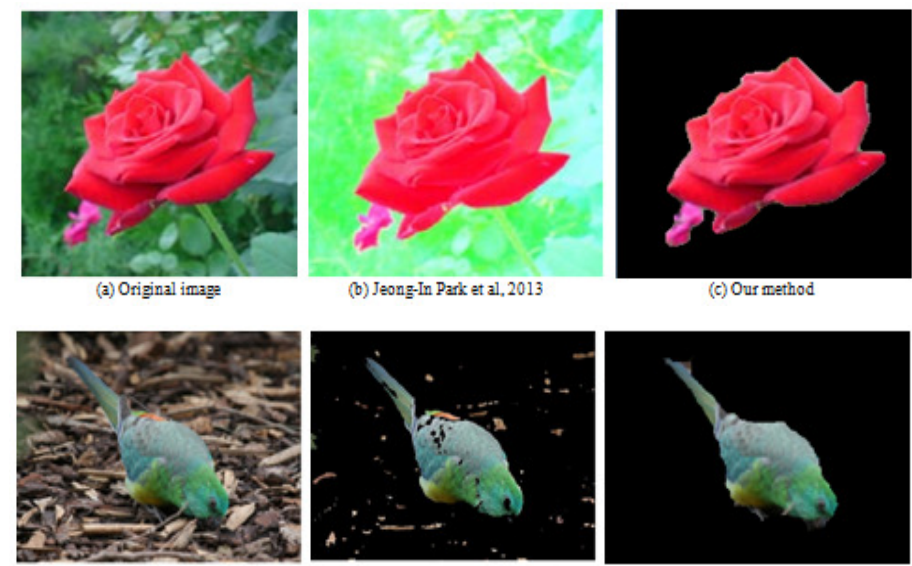

(a) Original imaga

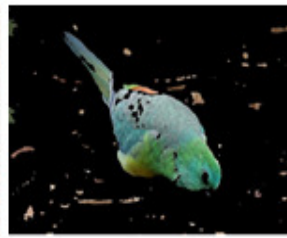

(b) Guanqun Cao et al, 2010

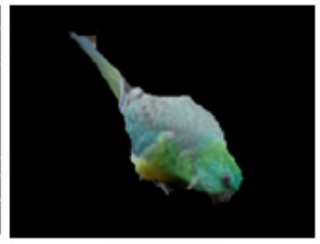

(c) Our method

Figure 7: Comparative results of our background removal method.

The figure 8 describes the comparison for our infected leaf detection. Our method for detecting damaged parts of leaf; it cares all part of leaf that cannot contribute to the photosynthesis process which is the main function of the leaf on the plant. Whereas the method of Nazare A.C [11], only considered the damaged leaf as the destroyed one, which is a wrong perception in terms of anatomical process of plants.

As we can see the last row in figure 8 , method of Nazare took the tested leaf as a healthy leaf, and according to anatomical concept of the plant, our method can come up with accurate leaf disease detection with estimation of $26,25 \%$. 


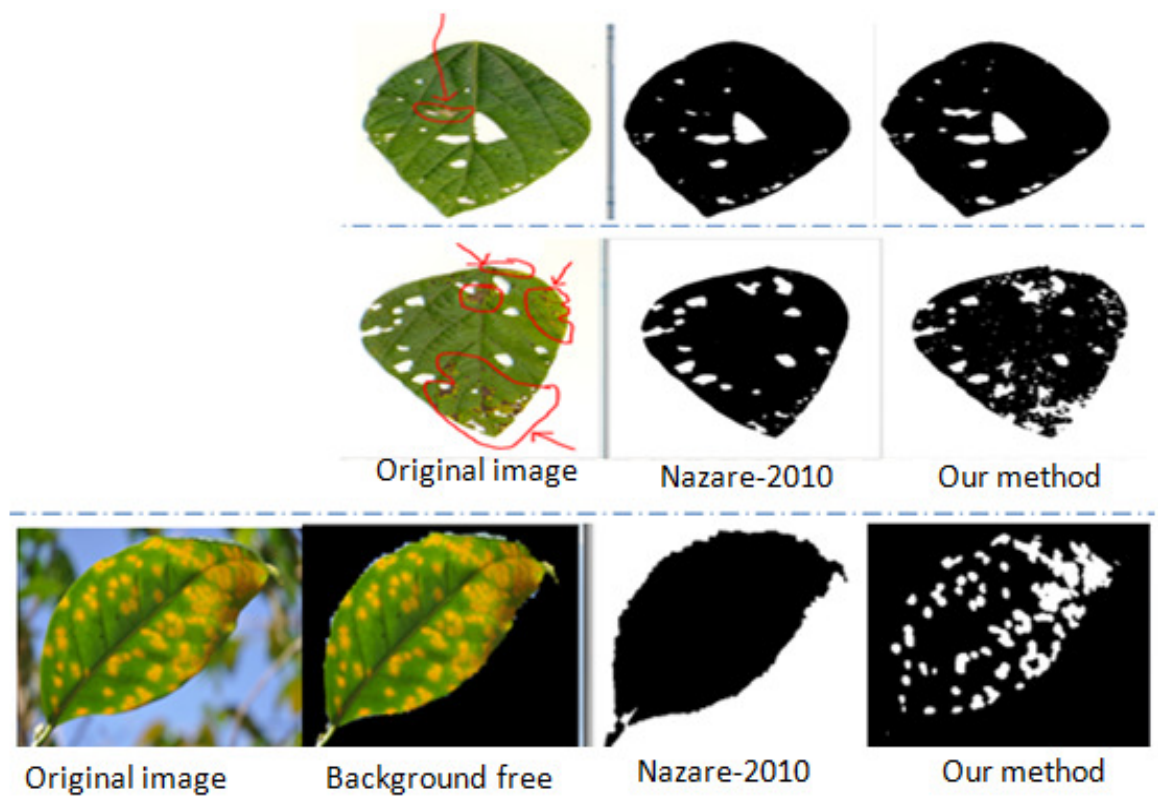

Figure 8: Infected damage detection comparison

The proposed method was applied to a big image database, and the detection was accurate and efficient. Figure 9 and 10 show more results, the former provides the estimate of $7 \%$, and the latter shows the damage at $22 \%$. This is an estimate, because we can see in figure 10 that the trunk was mistaken as an infection.

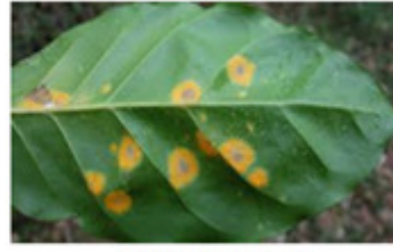

Input image

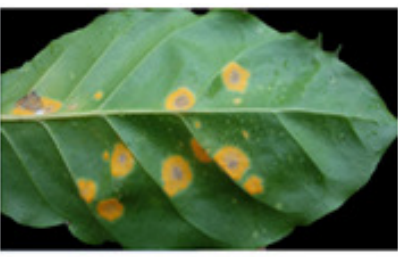

Background free

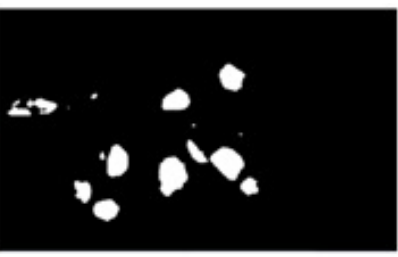

Detected damages

Figure 9: Detection with an estimation of $7 \%$

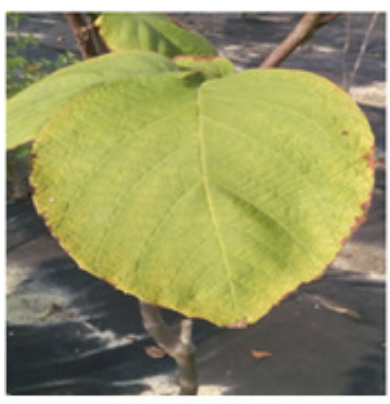

Input image

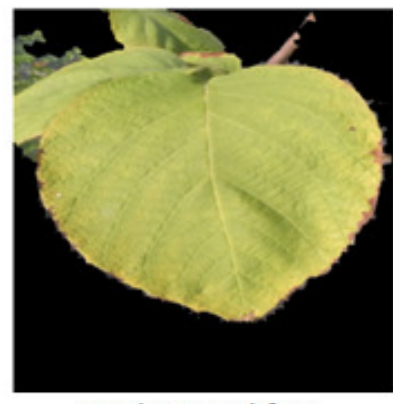

Background free

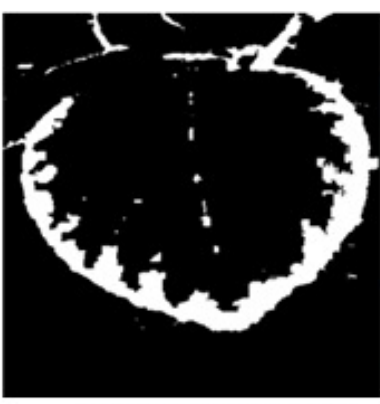

Detected damages

Figure 10: Detection with an estimation of $22 \%$

Nazare et al. evaluated their method by comparing with the manual segmented data by the expert in the area of Plant Science, and other proposed method of (Mura). And according to their results at that time, their method was better from others. 
The diagram below shows how accurate our method is compared by Nazare's method. From the same 27 tested images, our average detection was two times than Nazare's method (12.36 \pm 8.43 and $5.76 \pm 4.65$ respectively, i.e. $\mu \pm \delta$ for mean and standard deviation). The strong point for our method is that we can handle all leaf damages (destroyed and color change), it can be seen from the graph that for the last six leaves, which are infected by coffee rust as described in the introduction, Nazare's method considered them healthy, while are damaged already.

On other hand, it is also obvious that for the same destroyed leaf damages, the estimated values are almost the same, which shows that, our method works like their method and beyond for color change leaf damages.

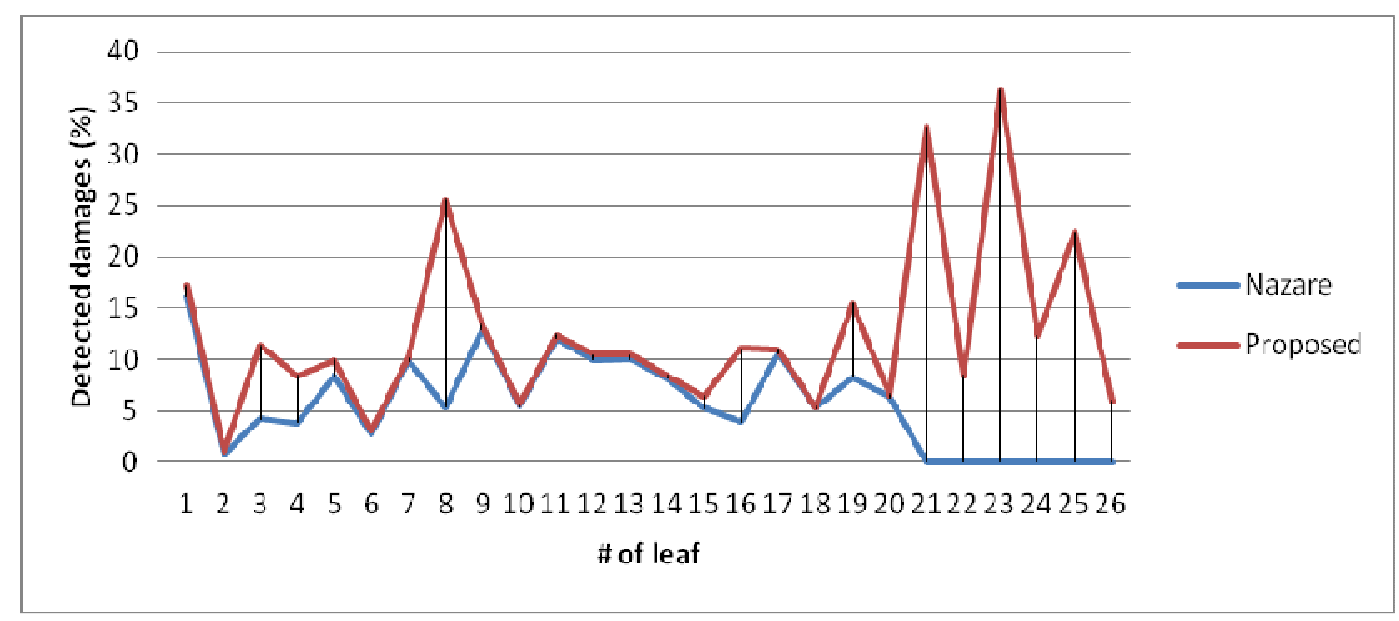

Figure 11: Comparative representation of our method and Nazare's method

\section{CONCLUSION}

In this paper, we proposed an automatic infected leaf detection algorithm that combines three processes: Image contrast enhancement, Image background removal, and estimation of detected infection. After adjusting the contrast by getting the value of gamma automatically, the system processes the original leaf image to keep the real leaf (foreground) by using the background removal method which is based on luminance and color. The background free image is then processed in YUV color model, i.e. on V channel, to maximize the detection of the leaf damage using the Fuzzy C-means Clustering.

The estimation of the severity of infected leaf was fast and quantitatively maximized all leaf damages compared to other methods and the necked eye process used by the farmers. It can help farmers to be sure which quantity of pesticides or fungicides their fields (coffee) require.

The proposed method was compared with some current researches, and it is obvious that it can over perform them either in background removal or in infection detection, even the method is fast; and avoids the defoliation done by all other methods surveyed. In the future, we are planning to upgrade our algorithm in real-time approach. 


\section{REFERENCES}

[1] Hasan M. Velioglu and Ozgur Sanglam, 'Evaluation of insect Damage on Beans using Image Processing Technology', 2012.

[2] Anup Vibhute and S K Bodhe 'Applications of Image processing in Agriculture: A Survey', International Journal of Computer Applications Volume 52- No 2, 2012.

[3] Robert H. Fulton, Richard A. Frederiksen, 'Coffee Rust in the Americas', The American Phytopathological Society, St. Paul, Minnesota, 1984

[4] Tropical Plant Diseases by Thurston, H.D, 1998. American phytopathological Society, St. Paul, Minnesota, p123-127

[5] Gloria Diaz, Eduardo R. Juan R. B. Norberto M., Recognition and Quantification of Area Damaged by Oligonychus Perseae in Avocado Leaves, 2009

[6] Thiago L., G,Souza, Eduardo S. M., Kayran Dos S, David M., Application of Complex Networks for automatic classification of damaging agents in Soybean Leaflets, 2011, IEEE International Conference in Image Processing

[7] C.P. Wijekoon, P.H Goodwin, T.Hsiang, Quantifying fungal infection of plant leaves by digital image analysis using Scion Image software, 2008, Journal of Microbiological Methods

[8] Chaohui et al Leaf Area Measurement Based on Image Processing, International Conference on Measurement Technology and Mechatronics Automation, 2010

[9] C.P. Wijekoon, P.H. Goodwin, T. Hsiang, Quantifying fungal infection of plant leaves by digital image analysis using Scion Image software, 2008

[10] Qinghai He, Benxue Ma, Duanyang Qu, Qiang Zhang, Xinmin Hou, Jing Zhao, Cotton Pests and Diseases Detection based on Image processing, June 2013, TELKOMNIKA pp.3445 3450

[11] A.C. Nazare-JR., D. Menotti and J.M.R neves and T. Sediyma, Automatic Detection of the Damaged Leaf Area in Digital Images of Soybean, IWSSIP 2010

[12] Shi-Chia Huang, Fan-Chieh Cheng, and Yi-Sheng Chiu, 'Efficient contrast enhancement using adaptive gamma correction with weighting distribution', 2012, IEEE Transaction on Image processing, pp:99

[13] Daughtry C, Direct Measurements of Canopy Structure. Rem. Sens. Rev. 5(1):45-60

[14] Mahdi M. Ali, Ahmedi Al-Ani, Derek Eamus and Daniel K.Y. Tan, A New Image processing based Technique for Measuring Leaf Dimensions, 2012, American-Eurasian J.Agric. \& Environ. Sci, pp 1588-1594

[15] Sanjay B. Patil and Shrikant K. Bodhe, Betel Leaf Area Measurement Using Image processing, 2011, IJCSE

[16] Radhakrishna Achanta, Sheila Hemami, Francisco Estrada, and Sabine Susstrunk, 'Frequency-Tuned Salient Region Detection', 2009, Computer Vision and Pattern Recognition, CVPR 2009, pp.15971604

[17] Jayaraman S., Veerakumar T., and Esakkirajan S. , Digital Image processing 2009, pp. 258-259

[18] Jeong-In Park and Jin-Tak Choi, 'A Background Removal Algorithm using the Variable Order n x m dimensional Vector', 2013, Proceedings, The 3rd International Conference on Circuits, Control, Communication, Electricity, Electronics, Energy, System, Signal and Simulation, 2013 (SERSC)

[19] Yiqun Hu, Xing Xie, Wei-Ying Ma, Liang-Tien Chia and Deepu Rajan, 'Salient Region Detection using Weighted Feaure Maps based on the Human Visual Attention Model.', 2005

[20] Ming-Ming Cheng, Gup-Xi Xhang, Niloy J.Mitra, Xiaolei Huang, Shi-Min Hu, 'Global Contrast based Salient Region Detection', 2011

[21] Jiang H., Wang J., Yuan Z., Liu T., Zheng N., Li S. 'Automatic Salient Object Segmentation Based on Context and Shape Prior', 2011

[22] Guanqun Cao, Faouzi Alaya Cheikh, Salient Region Detection with Opponent Color Boosting, 2010, Visual Information Processingb (EUVIP), 2rd European Workshop on, p13-p18

[23] N. Otsu, 'A threshold selection method from gray-level histogram', IEEE Transactions on System Man Cybernetics, vol. SMC-9, no.1, 1979, pp.62-66

[24] Li, Z., C. Ji and J. Liu, 2008, 'Leaf Area Calculation Based on Digital Image'. Computer and Computing Technologies in Agriculture, 259:1427-33. 


\section{AUTHORS}

\section{Eric Hitimana}

He received the BS degree in Computer Engineering and Information Technology from Kigali Institute of Science and Technology (KIST) Rwanda in 2010. He is graduating this coming February 2014 his MS degree in Computer Science and Engineering from Chonbuk National University, Rep. of Korea. He is an active research in Image processing

\section{Oubong Gwun}

He received the BS and MS degree in Electrical Engineering from Korea University in 1980, 1983 and the PhD degree in Interdisciplinary Graduate School of Engineering Sciences Kyushu University Japan in 1993. Now he is a professor of Chonbuk National University, Rep. of Korea. His interest area is Computer graphics, Image processing and Visualization.
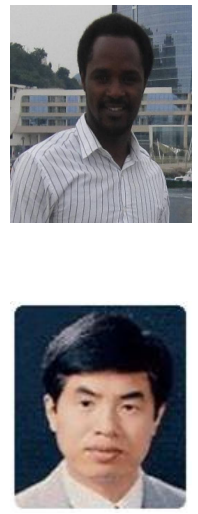


\title{
DAC FOR HIGH SPEED AND LOW POWER APPLICATIONS USING ABACUS
}

\author{
Shankarayya G. Kambalimath \\ Department of Electronics and Communication Engineering, \\ Basaveshwar Engineering College, Bagalkot - 587 102, Karnataka, India \\ kambalimathsgerediffmail.com
}

\begin{abstract}
This paper proposes a Chinese Abacus Digital-to-Analog Converter (DAC) for high speed and low power applications like audio and video applications. This circuit of DAC uses resister strings to get a good analog output. The designed DAC uses the algorithm of abacus. Instead of using binary code, here we use abacus code to control the switches. So the complexity and the area will be reduced automatically. The 8-bit DAC is comprised of 12 resistors and 24 NMOS switches. The 8-bit Abacus resistor DAC requires 12 resistors and 24 switches. The 8-bit resistor-string DAC requires 255 resistors and 256 switches. The most important advantages are that the numbers of both resistors and switches are all reduced effectively. The simulation environment uses 1 um process technology.
\end{abstract}

\section{KEYWORDS}

8-bit DAC, Chinese abacus \& DAC circuit

\section{INTRODUCTION}

Every real time signals are analog in nature, as more products perform calculations in the digital or discrete time domain, a device is required to convert from analog to digital known as ADC, after processing it should be in turn converted into analog as human beings can understand only analog signals and the device is digital to analog converter as DAC. Thus Data converters play an important role in an ever-increasing digital world [1]-[3].

Probably the most popular digital-to-analog converter application is converting stored digital audio and/or video signals. For example, stored digital information in MP3 format can be converted into music via a high-precision DAC. Most modern audio signals are stored in digital form (for example MP3s and CDs) and in order to be heard through speakers they must be converted into an analog signal. DACs are therefore founding applications in CD players, digital music players, and PC sound cards [4].

Specialist stand alone DACs can also be found in high-end hi-fi systems. This type normally take the digital output of a compatible CD player and convert the signal into an analog line level output that can then be fed into an amplifier to drive speakers. Similar type of digital-to-analog converters can be found in digital speakers such as USB speakers, and in sound cards. VOIP (Voice over IP) Phone, data transmission over the Internet is done digitally so in order for voice to be transmitted it must be converted to digital using an Analog to Digital computers and be converted into analog again using a DAC so the voice it can be heard on the other end [5]-[9].

David C. Wyld et al. (Eds) : CCSIT, SIPP, AISC, PDCTA, NLP - 2014

pp. 267-274, 2014. (C) CS \& IT-CSCP 2014

DOI : $10.5121 /$ csit.2014.4222 
This paper proposes an 8-bit Abacus resistor DAC. It is a modified version of resistor string DAC where instead of using Binary code to control switches here we are using Abacus code to do the same. So the complexity and area will be reduced. The 8-bit Abacus resistor DAC requires 12 resistors and 24 switches. The 8-bit resistor-string DAC requires 255 resistors and 256 switches. The most important advantages are that the numbers of both resistors and switches are all reduced effectively.

Rest of the paper is organized as follows. Section 2 introduces the operation of Chinese abacus, and binary to abacus module. The proposed Abacus DAC architecture is presented in section 3 . Section 4 explains the result analysis. Section 5 concludes our paper.

\section{CHINESE ABACUS}

The abacus also called a counting frame is a calculating tool that was in use centuries before the adoption of the written modern numeral system and is still widely used by merchants, traders and clerks in Asia, Africa, and elsewhere. Today, abaci are often constructed as a bamboo frame with beads sliding on wires, but originally they were beans or stones moved in grooves in sand or on tablets of wood, stone, or metal. The user of an abacus is called an abacist.

The Chinese abacus is typically $20 \mathrm{~cm}$ ( 8 in) tall and comes in various widths depending on the operator. It usually has more than seven rods. There are two beads on each rod in the upper deck and five beads each in the bottom for both decimal and hexadecimal computation. The suanpan can be reset to the starting position instantly by a quick jerk along the horizontal axis to spin all the beads away from the horizontal beam at the center. Suanpans can be used for functions other than counting. Unlike the simple counting board used in elementary schools, very efficient suanpan techniques have been developed to do multiplication, division, addition, subtraction, square root and cube root operations at high speed.

The binary abacus is used to explain how computers manipulate numbers. The abacus shows how numbers, letters, and signs can be stored in a binary system on a computer, or via ASCII. The device consists of a series of beads on parallel wires arranged in three separate rows. The beads represent a switch on the computer in either an 'on' or 'off' position.

\section{ABACUS DAC ARCHITECTURE}

The proposed Abacus DAC mainly consists of Binary to Abacus conversion block, Switch and Resistor block and Current source.

\subsection{Binary to Abacus conversion}

The designed DAC uses the algorithm of abacus. It uses Abacus code to control switches. Abacus is a tool used for mathematical manipulations. This Abacus tool can also be used to perform coding. The Abacus is made up of a set of unity elements representing the various decades of decimal numbers. 

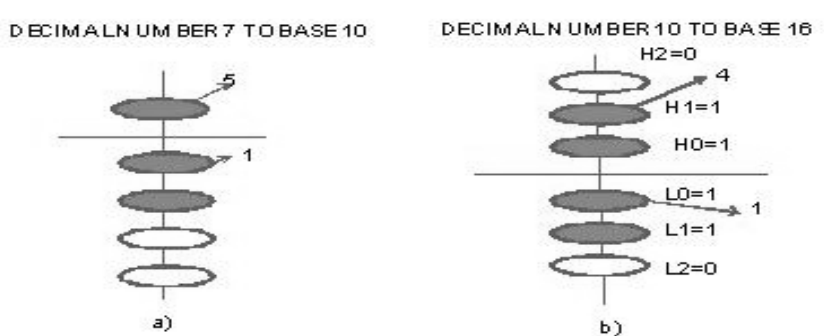

Figure 1. Abacus mapping

A basic column element of the abacus is depicted in figure 1 (a). The configuration represents the number seven. Each column element has one higher bead with a weight of five and four lower beads with a weight of one. In the 8-bit DAC design, each basic column element of this abacus scheme has three lower beads with a weight of one and three higher beads with a weight of four as shown in figure 1 (b). The basic element is able to represent decimal numbers in the range from 0 to 15 as depicted in figure 1 (b). Each weight value of the upper bead is four, and the value of lower bead is one. The binary to Abacus mapping table is as shown in the table 1 .

Table 1 Binary to abacus mapping table

\begin{tabular}{|c|c|c|c|c|c|c|c|c|c|}
\hline \multicolumn{4}{|c|}{ Binary Code } & \multicolumn{6}{|c|}{ Abacus Code } \\
\hline B3 & B2 & B1 & B0 & H2 & H1 & H0 & L2 & L1 & LO \\
\hline 0 & 0 & 0 & 0 & 0 & 0 & 0 & 0 & 0 & 0 \\
\hline 0 & 0 & 0 & 1 & 0 & 0 & 0 & 0 & 0 & 1 \\
\hline 0 & 0 & 1 & 0 & 0 & 0 & 0 & 0 & 1 & 1 \\
\hline 0 & 0 & 1 & 1 & 0 & 0 & 0 & 1 & 1 & 1 \\
\hline 0 & 1 & 0 & 0 & 0 & 0 & 1 & 0 & 0 & 0 \\
\hline 0 & 1 & 0 & 1 & 0 & 0 & 1 & 0 & 0 & 1 \\
\hline 0 & 1 & 1 & 0 & 0 & 0 & 1 & 0 & 1 & 1 \\
\hline 0 & 1 & 1 & 1 & 0 & 0 & 1 & 1 & 1 & 1 \\
\hline 1 & 0 & 0 & 0 & 0 & 1 & 1 & 0 & 0 & 0 \\
\hline 1 & 0 & 0 & 1 & 0 & 1 & 1 & 0 & 0 & 1 \\
\hline 1 & 0 & 1 & 0 & 0 & 1 & 1 & 0 & 1 & 1 \\
\hline 1 & 0 & 1 & 1 & 0 & 1 & 1 & 1 & 1 & 1 \\
\hline 1 & 1 & 0 & 0 & 1 & 1 & 1 & 0 & 0 & 0 \\
\hline 1 & 1 & 0 & 1 & 1 & 1 & 1 & 0 & 0 & 1 \\
\hline 1 & 1 & 1 & 0 & 1 & 1 & 1 & 0 & 1 & 1 \\
\hline 1 & 1 & 1 & 1 & 1 & 1 & 1 & 1 & 1 & 1 \\
\hline
\end{tabular}




\subsection{Switch and resistor design}

The Proposed DAC consists of switch and resistor block, which is as shown in figure 2. When digital input is 1, the circuit works on principle, to let to SW1 is ON, SW2 is OFF. The current pass through to be composed by the NMOS switch and resistor. Thus it contributes some voltage at output. When digital input is $0, \mathrm{SW} 1$ is OFF, SW2 is ON. The current pass through to be composed by the NMOS switch. Thus voltage contributed will be zero. The 8-bit DAC has 12 Switch and Resistor blocks, and completely series 12 switch and resistor circuit blocks.

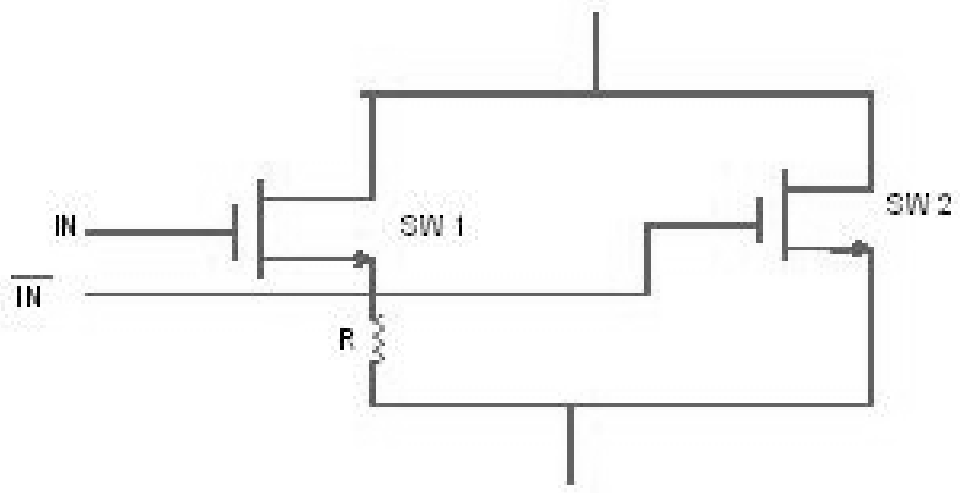

Figure 2. Switch and resistor block

\subsection{Current source}

In Beta multiplier circuit we are adding the resistor at the source side to make it supply independent and combination of NMOS and PMOS devices provide necessary biasing for the current mirror circuit. Figure 3 shows the Beta-multiplier reference for biasing current mirror circuit. In any self-biased circuit the unwanted one where zero current flows in the circuit. This unwanted state occurs when the gates of M1/M2 are at ground while the gates of M3/M4 are at VDD. When in this state, the gate of M5 is at ground and so it is off. The gate of M6 is somewhere between VDD and VDD - VTHP. M7, which behaves like an NMOS switch, turns on and leaks current into the gates of M1/M2 from the gates of M3/M4.

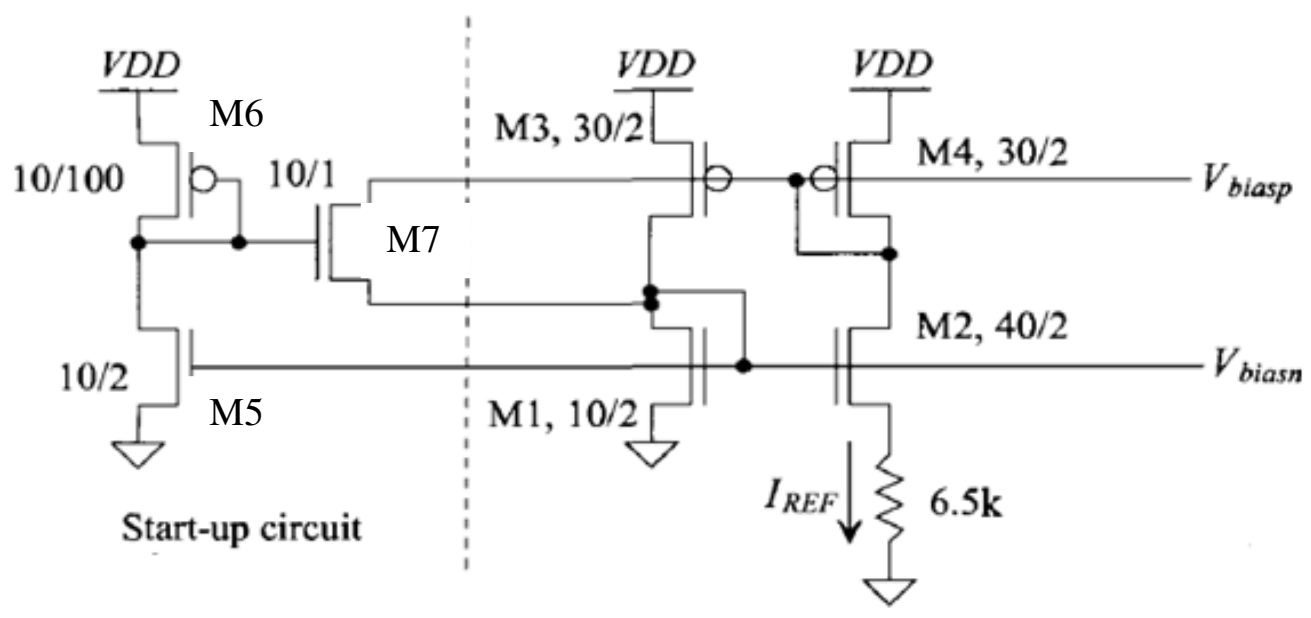

Figure 3. Beta-multiplier reference for biasing 
This causes the current to snap to the desired state and M7 to turn off. Note that during normal operation the start-up circuit should not affect the Beta-multiplier's operation. The current through M7 should be zero (or very small). Finally the proposed Abacus DAC consists of 12 switch and resistor blocks, two binary to Abacus mapping blocks and a current sources. 8-bit binary inputs from B0 to B7 are given as input to binary to Abacus conversion block which generates corresponding Abacus code. These generated Abacus code are in turn given to switch and resistor block to control the switches. Whenever Abacus code is 1 the respective switch and resistor block will contribute voltage at the output. The overall voltage will be sum of the voltage contributed by all 12 switch and resistor block. Thus digital domain is converted in to analog voltage.

\section{RESULT ANALYSIS}

To test the performance effectiveness of the proposed scheme, some of the simulation results analyzed are as follows: ideal 4-bit Abacus DAC output shown in figure 4, ideal 8-bit Abacus DAC output shown in figure 5, practical 8-bit Abacus DAC output shown in figure 6, differential non-linear error (DNL) is shown in figure 7, integral non-linear error (INL) is shown in figure 8 and offset error is shown in figure 9.

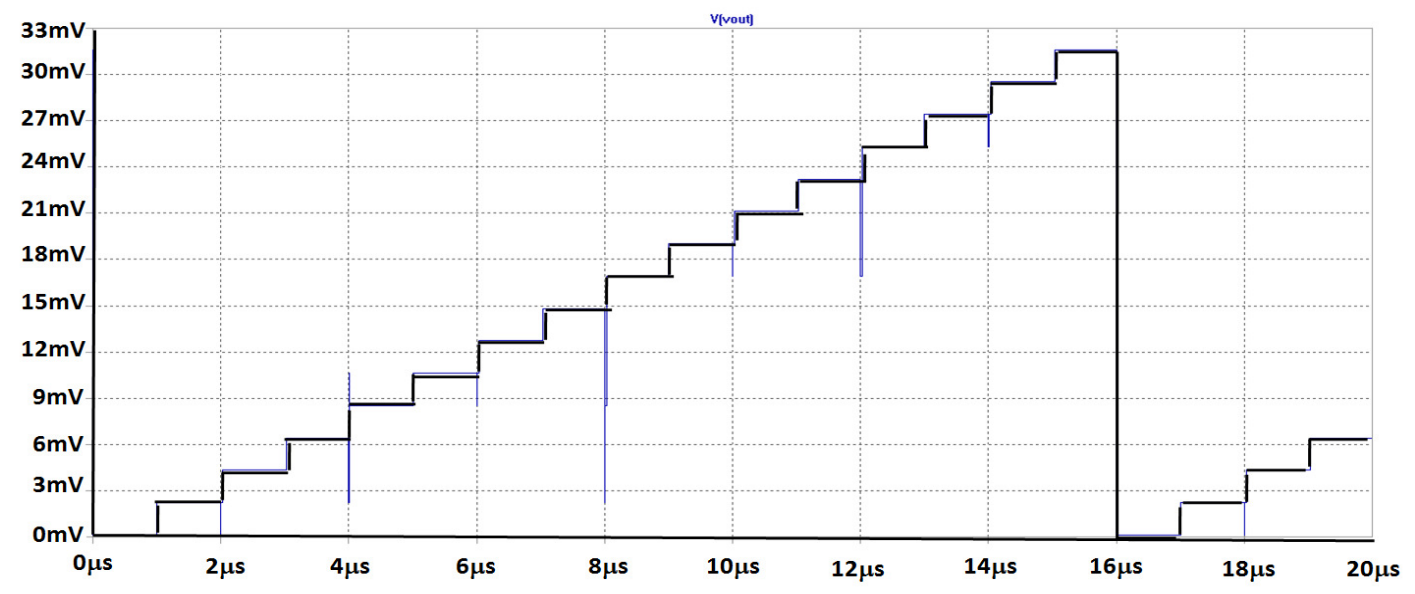

Figure 4. Ideal 4-bit Abacus DAC output

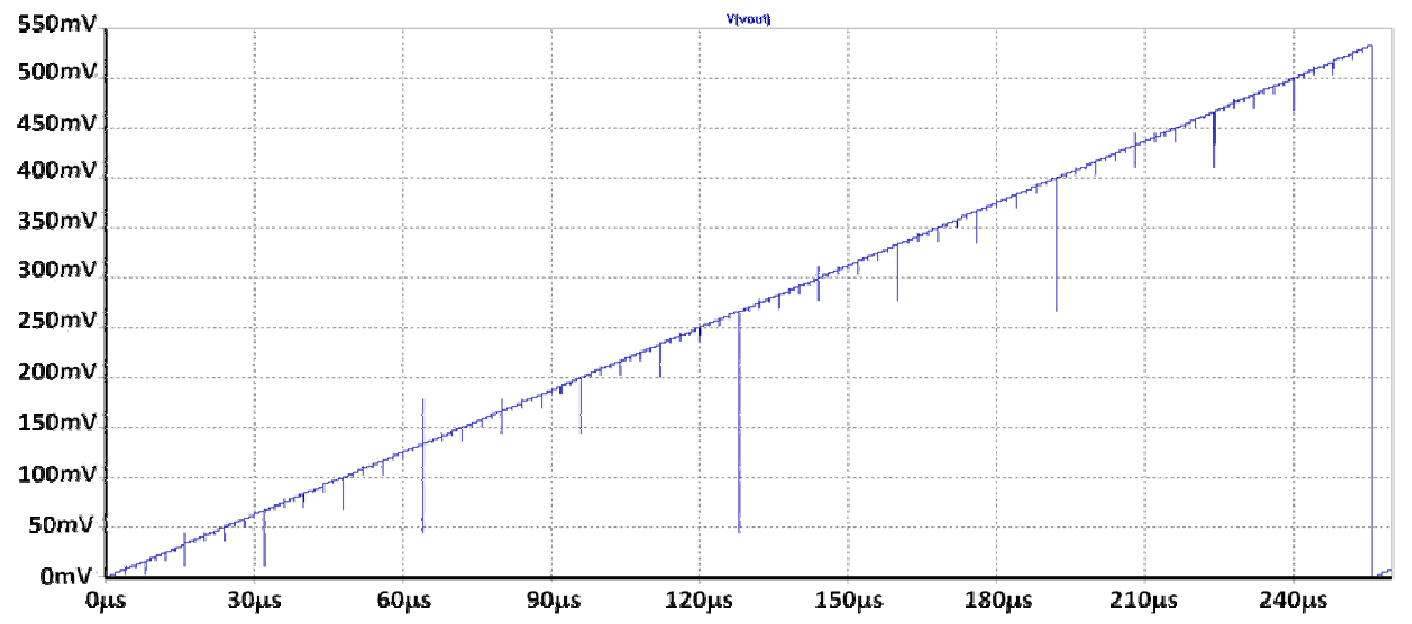

Figure 5. Ideal 8-bit Abacus DAC output 


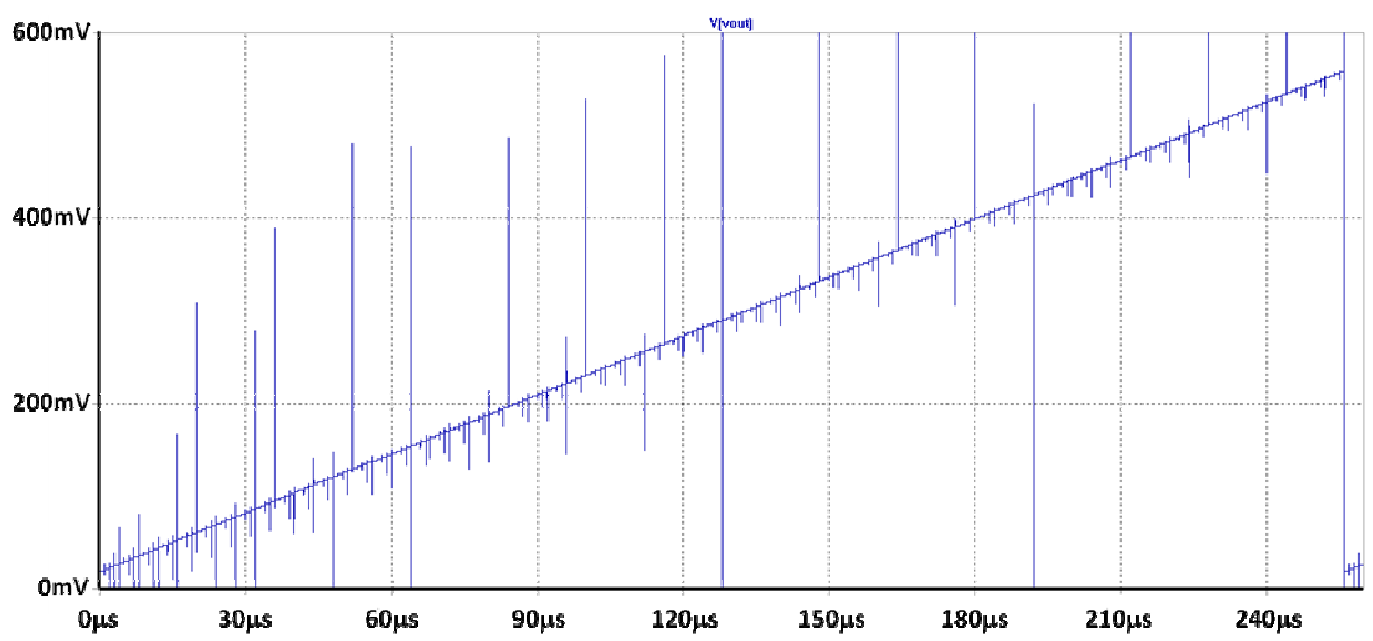

Figure 6. Practical 8-bit Abacus DAC output

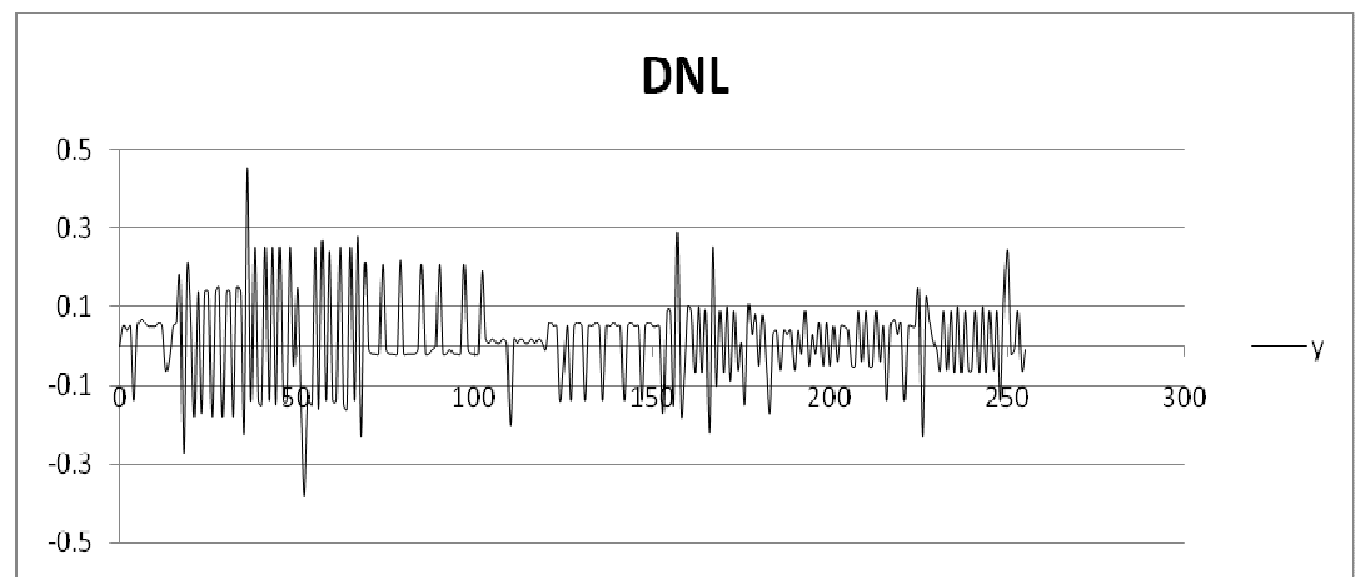

Figure 7. Differential non-linear error (DNL)

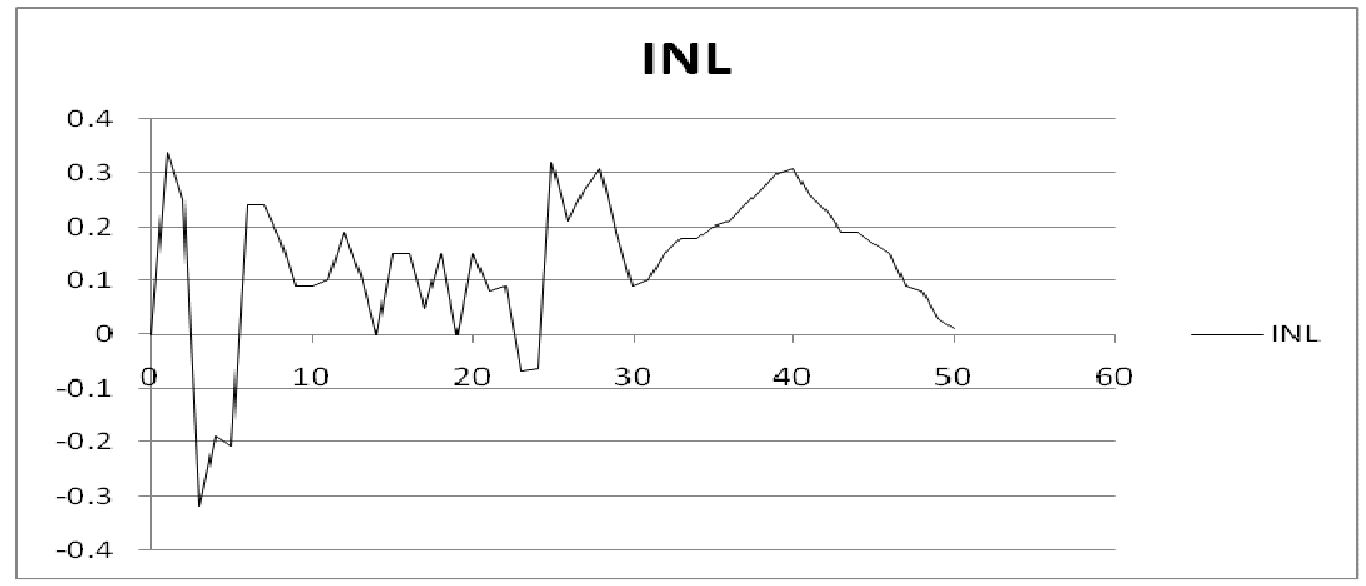

Figure 8. Integral non-linear error (INL) 


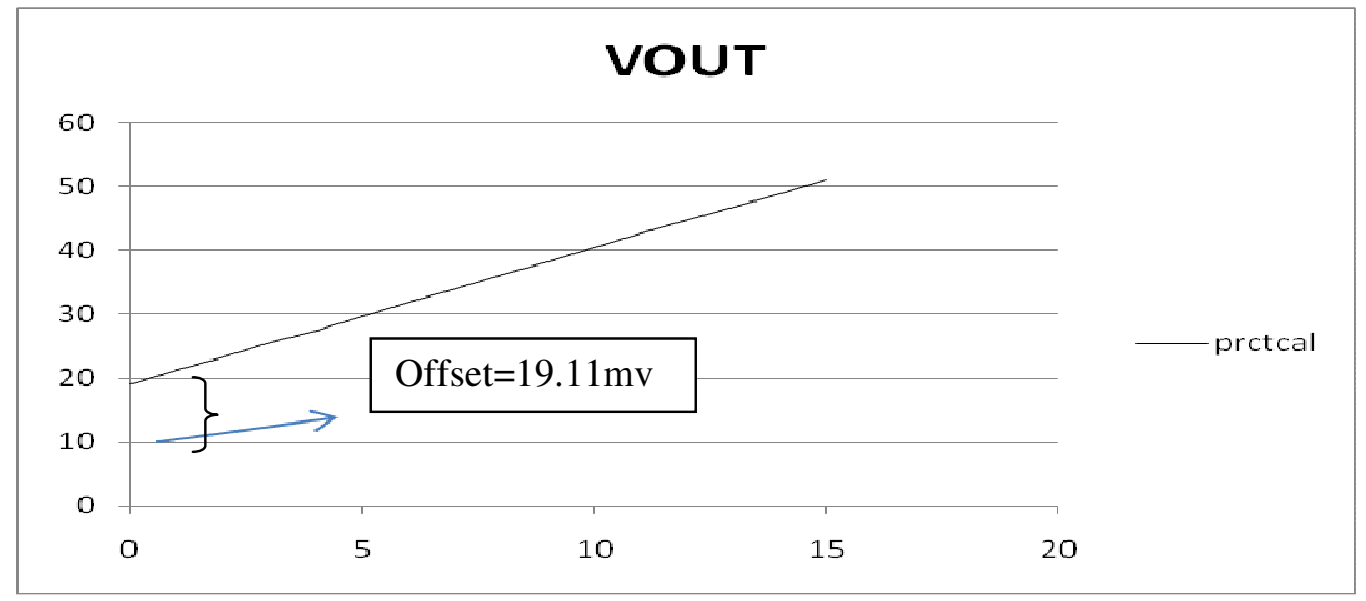

Figure 9. Offset error

\section{CONCLUSIONS}

The paper presents an 8-bit ABACUS digital to analog converter. The abacus algorithm is successfully adopted in the DAC design. Results show that this scheme reduces effectively the number of resistors of conventional resistor string DAC. The 8-bit digital to analog converter has low power consumption. $1 \mu \mathrm{m}$ process technology is used in the implementation of the proposed DAC. The simulation results of DNL and INL are below 0.35 LSB and 0.4 LSB, respectively.

\section{REFERENCES}

[1] Shun-He Huang, Chien-Hung Lin, Shu-Chung Yi and Jin-Jia Chen, "A Chinese Abacus DAC for Video Applications", Proc. Third International Conference on Intelligent Information Hiding and Multimedia Signal Processing (IIHMSP), pp. 507-510, November 2007.

[2] C.-C. Tsai, C.-H. Lai, W.-T. Lee and J.-O. Wu, "10 bit switched-current digital-to-analogue converter”, Proc. IEE Circuits Design Systems, pp. 287-290 June 2005.

[3] Miki, T., Nakamura, Y., Nakaya, M., Asai, S., Akasaka, Y., and Horiba, Y., “An 80-MHz 8-bit CMOS D/A Converter”, IEEE Journal of Solid-State Circuits, Volume 21, Issue 6, Dec 1986, pp. 983-988.

[4] Shu-Chung Yi, Kun-Tse Lee, Jin-Jia Chen, Chien-Hung Lin, Chuen-Ching Wang, Chin-Fa Hsieh, and Chih- Yung Lu, "The new architecture of radix-4 Chinese abacus adder", Proc. IEEE 36th International Symposium on Multiple-Valued Logic, May 2006.

[5] Zi-Yi Zhao, Jian-Hung Lin, Yu-Zhi Xie, Yen-Ju Chen, Yi-Jie Lin, and Shu-Chung Yi., "The novel Chinese abacus adder", Prcc. international symposium on VLSI design automation and test, 25-27 April 2007

[6] Huynh, J., Ngo, B., Pham, M., and He, L, "Design of a 10 Bit TSMC 0.25 $\mu$ m CMOS digital to analog converter", Proc. Sixth International Symposium on Quality Electronic Design, 21-23 March 2005, pp. 187-192.

[7] Moonsik Song, Bongsoon Kang, and Eurho Joe, "A 10-Bit 80MHz 3.0V For Video CMOS D/A Converter Applications", IEEE Transactions on Consumer Electronics, Volume 43, Issue 3, Aug. 1997, pp. 868-872.

[8] Leonard, J., Weste, N., Bodony, L., Harston, S., and Meaney, R., “A 66-MHz DSP augmented RAMDAC for smooth-shaded graphic applications", IEEE Journal of Solid-State Circuits, Volume 26, Issue 3, Mar 1991, pp. 217-228.

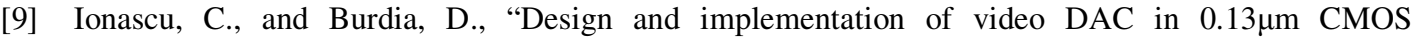
technology", IEEE SCS 2003. International Symposium on Signals, Circuits and Systems, Volume 2, 10-11 July 2003, pp.381-384. 


\section{AUTHOR}

Shankarayya G Kambalimath received the B.E. and M.E. degrees in Electronics and Communication Engineering from the Gulbaraga University and Bangalore University Karnataka, India in the year 1993 and 1999 respectively. He is currently pursuing the Ph.D. degree at the Visvesvaraya Technological University, Belgaum, Karnataka, India. His main research interests include VLSI and signal processing.

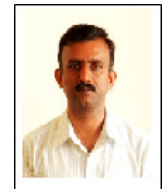




\title{
VISUAL SALIENCY MODEL USING SIFT AND COMPARISON OF LEARNING APPROACHES
}

\author{
Hamdi Yalın Yalı̧ç \\ ${ }^{1}$ Department of Computer Engineering, Hacettepe University, Ankara, Turkey \\ yalinyalicecs.hacettepe.edu.tr
}

\begin{abstract}
Humans' ability to detect and locate salient objects on images is remarkably fast and successful. Performing this process by using eye tracking equipment is expensive and cannot be easily applied, and computer modeling of this human behavior is still a problem to be solved. In our study, one of the largest public eye-tracking databases [1] which has fixation points of 15 observers on 1003 images is used. In addition to low, medium and high-level features which have been used in previous studies, SIFT features extracted from the images are used to improve the classification accuracy of the models. A second contribution of this paper is the comparison and statistical analysis of different machine learning methods that can be used to train our model. As a result, a best feature set and learning model to predict where humans look at images, is determined.
\end{abstract}

\section{KEYWORDS}

Image Processing, Computer Vision, Machine Learning Methods, Saliency Map, Eye-Tracking

\section{INTRODUCTION}

Understanding the region where people look on a scene can be useful in many applications such as graphics, design, advertising and human-computer interaction. For example, in nonphotorealistic rendering, different levels of details are proposed for different areas of the picture [2]. In this study, regions where people focus are processed in high-resolution, whilst for the other regions, low-resolution processing is performed. Auto-crop of pictures, thumbnails or previews from photos, relate partial displays of images on small screen mobile devices are examples of other fields of application.

Hardware solutions are also available for finding the region where people look at a scene. An observer sitting in front of a screen with an eye-tracking device has all the spots recorded at which he is looking at a scene. Special setups and multiple calibration steps are needed for working these tracking devices. Also this solution is relatively expensive and cannot be easily obtained. For this reason, the prediction of where people are looking is required independently from such tracking devices. As a solution, computer models are generated that calculate the salient point which attracts an observer's attention. These models analyze an image and extract a saliency map. One of the former studies is the model developed by Itti and Koch [3] and uses biologically inspired features like orientation, intensity and color. For each of the features, a saliency map is computed and combined into a single saliency map result which describes the

David C. Wyld et al. (Eds) : CCSIT, SIPP, AISC, PDCTA, NLP - 2014

pp. 275-282, 2014. (C) CS \& IT-CSCP 2014

DOI : $10.5121 /$ csit.2014.4223 
saliency of each pixel. Hou and Zhang [4] proposed a model which is independent of features or other forms of prior knowledge of objects. They analyzed the log spectrum of an image, extracted the spectral residual of an image in spectral domain, and proposed a method to construct the saliency map in spatial domain. Bruce and Tsotsos [5] presents a visual saliency model based on a first principle information theoretic formulation, named as Attention Based on Information Maximization (AIM) which performs better than the Itti model. Avraham et al. [6] uses a probabilistic model to mathematically estimate the most probable targets. Cerf at al. [7] improved on Itti's model by adding face detection. The most important characteristic of these studies is that models are derived mathematically, and not trained by some large eye-tracking dataset.

Kienze et al. [8] propose a model that learns saliency directly from human eye movement data. They only use low level features like previous studies. But the study by Ehinger et al. [9] shows that observers trying to search for pedestrians in a scene leads to a model combined of three sources: low-level features, target features and scene context. As a result of this study, while predicting human behavior, besides low-level features, high-level features which contain semantic information about the content of the scene must be used.

Our study is most closely related to the saliency model proposed by Judd et al. [1]. They used high and mid-level features as well as low levels. Another important contribution of their study is that they train the model using a supervised learning method (support vector machine) and observed positive contributions of the features in the result. To verify this, they needed real eyetracking data and they recorded viewer's eye fixations on images containing a large amount of data. They published this database for further studies and in our study, this publicly available database was used. Results of the different machine learning methods was compared and analyzed in order to obtain the most accurate model. In addition to the work of others, SIFT [17] feature is added to the feature set to improve the accuracy of the model.

In the following sections of this paper, dataset and data gathering protocol is analyzed, machine learning methods will be briefly explained and the feature set that was used to train the model is mentioned. Performance of different learning methods are compared and analyzed in the experimental results. Future work will also be discussed in the conclusion.

\section{DATASET AND LEARNING METHODS}

\subsection{Dataset Analysis}

Large ground truth data is required in the detection of where humans look in a scene. One of the largest studies in this field is the experiment of Judd et al. [1] practiced with 15 viewers using 1003 images. They collected 1003 random images from Flickr and LabelMe [10] (Figure 2) and full resolution images were displayed for 3 seconds to viewers in a dark room using a chin rest for a head stabilizer. An eye-tracker in front of them recorded their gaze path and fixation points (Figures 3 and 4). In order to guarantee a high-quality result, the camera's calibration was checked every 50 images. They provided a memory test at the end of each display to motivate and encourage users into paying attention, and asked them which of the images they had seen previously.

When we analyze the dataset, we noticed that viewers primarily looked at the living things. In the close up images, organs like the eye, nose and mouth were the most fixated points. But when people were located that bit further away, viewers searched for faces and limbs, such as a hand, or an arm. If a human does not exist in the scene, animals can also be said to attract the attention of people. Other important salient regions are objects like signs and boards which contain text. The 
fixations in the dataset have a bias towards the center because of the fact that photographers tend to place objects in the center of the scene.

\subsection{Machine Learning Methods}

In this study, different supervised learning methods are used while discovering the model of visual saliency and classification results of them are compared. The methods used are explained briefly in the section below.

\subsubsection{SVM (Support Vector Machines)}

This method tries to find the linear discriminant function (classifier) with the maximum margin between two classes. It is robust to outliners thus has strong generalization ability. With the help of the kernel function, which implicitly maps data to high-dimensional space, classification accuracy will increase. The main advantage of the method is its success with high-dimensional data.

\subsubsection{C4.5 (Decision Tree)}

This is an algorithm that constructs a simple depth-first decision tree. It uses information gain with feature selection and it is easy to implement. Classification of unknown records is considerably fast, but it is not suitable for large datasets because it needs to fit the entire data into memory.

\subsubsection{K-Nearest Neighbor}

This method is based on the principle of the nearest k-neighbor of the new data. Different computations can be used as a distance metric and the advantage of this classifier is that it doesn't require any training time. But testing time can be considerable and classifying unknown records is relatively expensive. It is also easily fooled in high dimensional spaces.

\subsubsection{Naive Bayes}

This is a statistical classifier which applies Bayes theorem. It assumes that attributes are independent from each other and is robust to isolated noise points and irrelevant attributes. However independence assumption may not hold for some attributes and it can cause a loss of accuracy.

\subsubsection{Adaboost}

It is a combination of classifiers that is constructed from the training data. It adaptively changes the distribution of training data with each iteration by focusing more on previously misclassified records. It can also be used to increase the classification accuracy of other methods.

\section{LEARNing OF THE ViSUAL SALIENCY MODEL}

\subsection{Features Used in Learning}

According to the results obtained by the analysis of the dataset, the features used in this study are listed below. For all images in the database, the features are extracted for each pixel and used for training of the model. MATLAB was used in image processing and feature extraction. 


\subsubsection{Low-level Features}

- Local energy of steerable pyramid filters [13] are used because they are physiologically conceivable and associated with visual attention. Pyramid sub-bands were computed in 4 orientations and 3 scales.

- Intensity, orientation and color contrast were considered as important features for saliency regions. 3 channels corresponding to these features were computed as described in the Itti and Koch [3] model.

- In addition to red, green and blue color channel values, and the probabilities of these channels, were also used.

- The probability of each color in a 3D color histogram was also a basic feature which was computed by the images filtered using median filter at 6 different scales.

\subsubsection{Mid-level Features}

Humans inherently look at the horizon line because most of the objects are on the earth's surface. Gist feature [14] was used to detect the horizon line.

\subsubsection{High-level Features}

Humans mostly looked at people and their faces as understood by the analysis of the database, the following high-level features were extracted:

- Viola Jones face detector [15],

- Felzenszwalb person and car detector [16].

\subsubsection{Central Priority}

Humans generally place the object of interest near the center of the image while taking pictures. For this reason, a feature which specifies the distance to the center for each pixel is used.

\subsubsection{SIFT Keypoints}

For objects in a scene, the interesting points on the object can be extracted to provide a feature description of them. This description can be used to identify the object in a test image. The SIFT [17] method can be used to detect these interesting points in an image. In this paper, the keypoint localization step of the method is used. Low-contrast keypoints are discarded and locations of the more interesting keypoints, which can be attractive to the human eye, are used. One contribution of this paper is the using of the position of interest points on an image as a feature and increasing the classification rate. Other local features, like SURF and GLOH were also experimented with, but since SIFT had a better effect on the model, it was SIFT that we decided to go with.

\subsection{Training Phase}

While training and testing the saliency model, machine learning methods described in Section 2.2 were used. Images in the database were divided up as $80 \%$ training and $20 \%$ testing. That means that 803 images were used for training and 201 for testing. 10 positively labeled pixels from the most 5\% salient locations and 10 negatively labeled pixels from the least 30\% salient locations were chosen from each image. Thus the examples are guaranteed to be both strongly positive and strongly negative. Any samples on the boundary between the two regions, and samples within 10 pixels of the image boundary were not selected. It is noted that selecting more than 10 samples as positive and negative did not increase the classification rate and contained redundant information. 
The dataset used to train the model consisted of 16,040 samples; the test dataset consisted of 4,020 samples. Every sample (point) has 34 continuous attribute and labeled as positive or negative. Obtained data was given as an input to the described learning methods for training and a more accurate model will be discussed. Details of the parameters used in the classifier training are explained below:

- In the model that uses SVM, all data were normalized and radial basis function (1) is used for kernel function (other kernels produce results similar to radial). $\gamma$ value is assigned to 0.8. Cost of misclassification didn't affect the result, so $c$ is assigned to 8 .

$$
k\left(x_{i}, x_{j}\right)=\exp \left(-\gamma\left\|x_{i}-x_{j}\right\|^{2}\right)
$$

- In the model that uses C4.5 decision tree, for spanning variables, the minimum number of objects (instances) in leaves $m=2$ and pruning confidence level ( $c f$ ) is assigned to $25 \%$.

- The number of nearest neighbors $(k)$ used in classification is 9 for the $\mathrm{kNN}$ method and it was observed that the value does not affect the result. In this model all attributes are normalized and Euclidean distance is used as a distance metric.

- In the NaiveBayes method, we used relative frequency as a prior in probability estimation. Size of LOESS window is 0.5 and LOESS sample points are set to 100.

- Adaboost method is used with SVM to improve the result obtained. Boosting with 10 SVM classifier is applied and weighted combination of weak learners is obtained.

\subsection{Comparison of Learning Methods}

In this section classification performance of machine learning methods will be discussed. While sampling, a 5-fold cross validation is used and how the specified model accurately worked in practice is shown.

Table 1. Evaluation results of the methods using same features

\begin{tabular}{|c|c|c|c|c|c|c|}
\hline \multirow{2}{*}{ Method } & \multicolumn{7}{|c|}{ Results (same features) } \\
\cline { 2 - 7 } & $\boldsymbol{C A}$ & Sens & Spec & AUC & Prec & Recall \\
\hline SVM [1] & 0,8801 & $\mathbf{0 , 8 8 2 5}$ & 0,8778 & $\mathbf{0 , 9 5 9 7}$ & 0,8786 & $\mathbf{0 , 8 8 2 5}$ \\
\hline C4.5 & 0,8410 & 0,8413 & 0,8407 & 0,8730 & 0,8409 & 0,8413 \\
\hline kNN & 0,8583 & 0,8407 & 0,8759 & 0,949 & 0,8695 & 0,8407 \\
\hline NaiveBayes & 0,8168 & 0,8209 & 0,8129 & 0,9029 & 0,8147 & 0,8209 \\
\hline AdaBoost & $\mathbf{0 , 8 8 2 1}$ & 0,8818 & $\mathbf{0 , 8 8 2 5}$ & 0,8818 & $\mathbf{0 , 8 8 2 3}$ & 0,8818 \\
\hline
\end{tabular}

Table 2. Evaluation results of the methods using same features and SIFT

\begin{tabular}{|c|c|c|c|c|c|c|}
\hline \multirow{2}{*}{ Method } & \multicolumn{5}{|c|}{ Results (same features+SIFT) } \\
\cline { 2 - 7 } & $\boldsymbol{C A}$ & Sens & Spec & AUC & Prec & Recall \\
\hline SVM & 0.9065 & $\mathbf{0 . 9 0 8 9}$ & 0.9091 & $\mathbf{0 . 9 8 8 4}$ & 0.9091 & $\mathbf{0 . 9 0 8 9}$ \\
\hline C4.5 & 0.8610 & 0.8656 & 0.8611 & 0.8971 & 0.8649 & 0.8659 \\
\hline kNN & 0.8820 & 0.8629 & 0.9059 & 0.9791 & 0.8991 & 0.8665 \\
\hline NaiveBayes & 0.8423 & 0.8405 & 0.8327 & 0.9282 & 0.8355 & 0.8482 \\
\hline AdaBoost & $\mathbf{0 . 9 0 8 5}$ & 0.9072 & $\mathbf{0 . 9 0 8 9}$ & 0.9099 & $\mathbf{0 . 9 0 8 7}$ & 0.9055 \\
\hline
\end{tabular}


Table 1 and 2 shows the evaluation results of the methods used in this study. As a key: $C A$ means classification accuracy, Sens means sensitivity, Spec means specificity, $A U C$ means area under ROC curve, Prec means precision, Recall as itself.

Values found in the evaluation results show how the models can be compared to human performance while looking at an image. The study [1] that our model is based on used the SVM model (Table 1) and reached $88 \%$ classification accuracy. Similarly in our study, the SVM model gives better results than other methods. The main reason for this performance is that our problem is based on a binary classification prediction (positive and negative points). Also the dataset contains high-dimensional data with large numbers, and this makes SVM more advantageous over the other methods.

C4.5 decision tree method produced lower accuracy results because it doesn't have a suitable structure for large datasets. In addition, it falls behind on computation time during training when compared to other methods. In this work, Random Forest method was also used, but because of the failure of the decision trees on this dataset and because the algorithm couldn't construct more than 10 trees due to computational time limits, this method has not been reviewed in this paper.

The model which uses kNN method produced a lower accuracy because the error rate increases in multi-dimensional space with this method. In addition, computational time of the testing stage is very high when compared to other methods. Evaluation results show that the NaiveBayes method is the least successful. Especially as dependencies between attributes (for example, color features and their probabilities are dependent) leads this method to failure.

The Adaboost method produced similar results with SVM; even better in some evaluations. That result is obvious because it used the successful method SVM, focusing more on previously misclassified samples iteratively and constructed a better model. Saliency maps obtained from some sample images using this model are shown in Figure 6.

Another comparison between learning methods is given in Figure 1 using ROC curve.

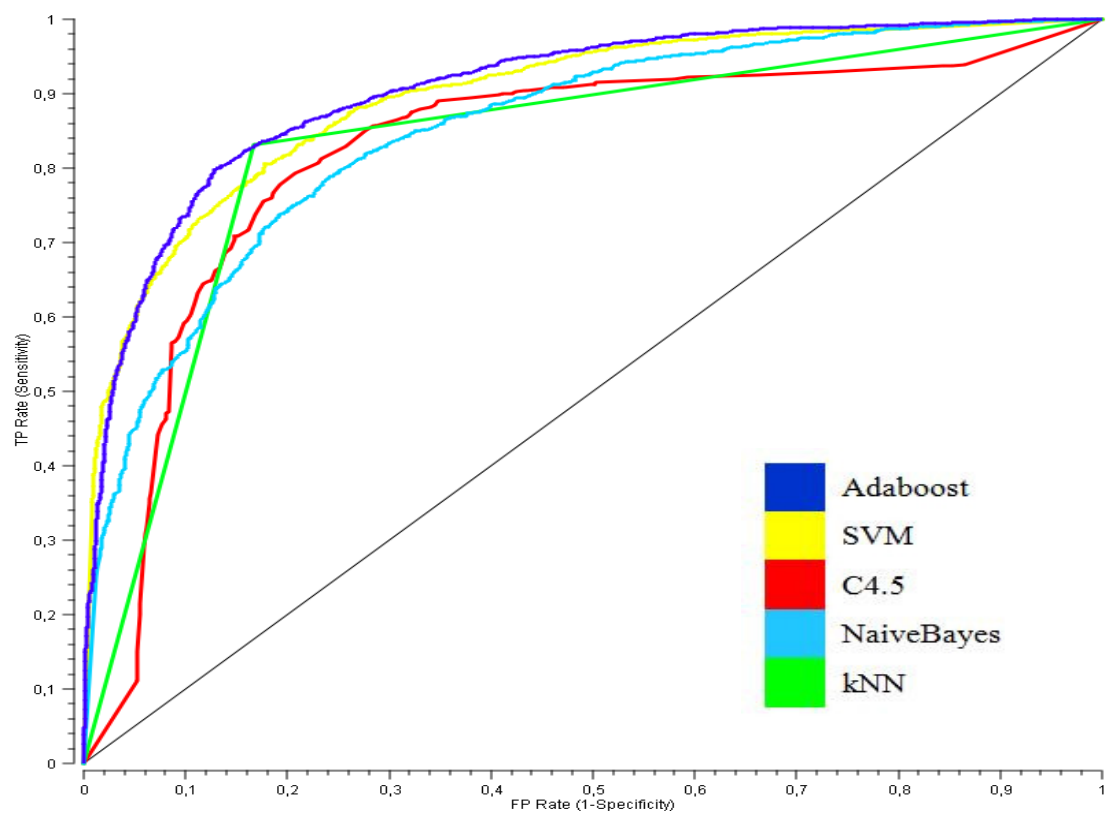

Figure 1. ROC curves of methods (Adaboost, SVM, kNN, C4.5, NaiveBayes) 


\section{CONCLUSIONS}

In this study, visual saliency models which try to predict where humans look at images are developed. For this purpose, a real eye-tracking database is used and different levels of features are extracted from the images. Using eye fixation points of 15 viewers, a ground truth saliency map (Figure 5) is uncovered with this information when different learning methods are trained and classification results are compared. As a conclusion of this study, machine learning methods that can be applied to this dataset are explained with reasons. Another contribution of this study is that the interest points extracted from the SIFT method are added to the feature set and better classification results $(\sim 90 \%)$ were then obtained over previous studies.

As a future piece of work, we expect to speed up the image processing step and feature extraction. However, training time of the model is not important because it can be done offline, but when a system wants to find the most salient points or regions on an image, this process must be handled quickly. If online saliency is successful, it can be integrated with many applications and can also be used in mobile systems.
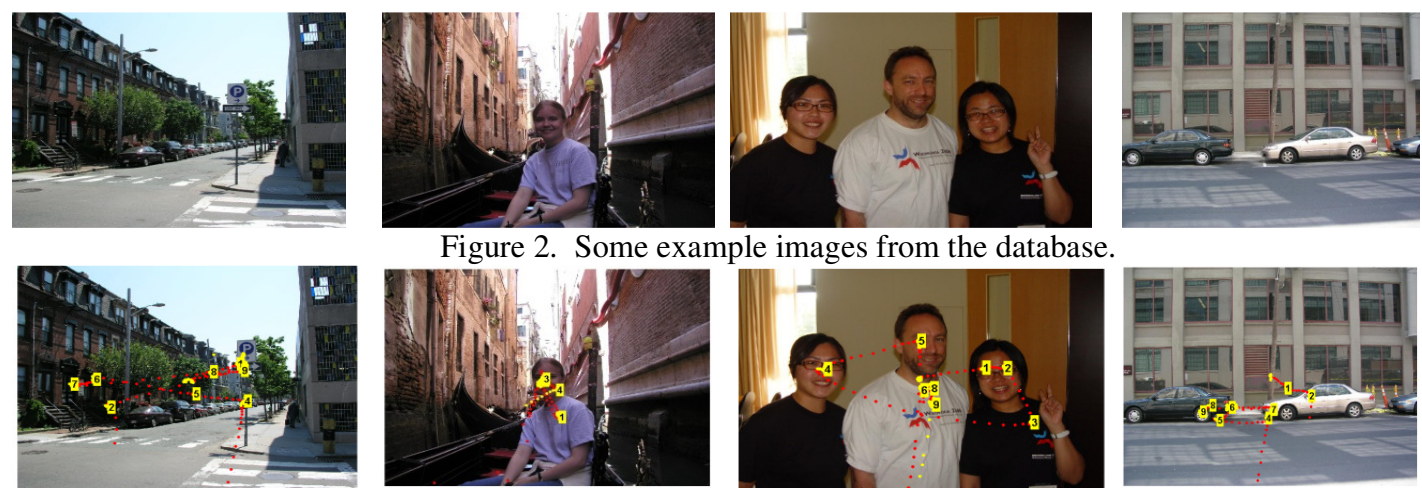

Figure 2. Some example images from the database.
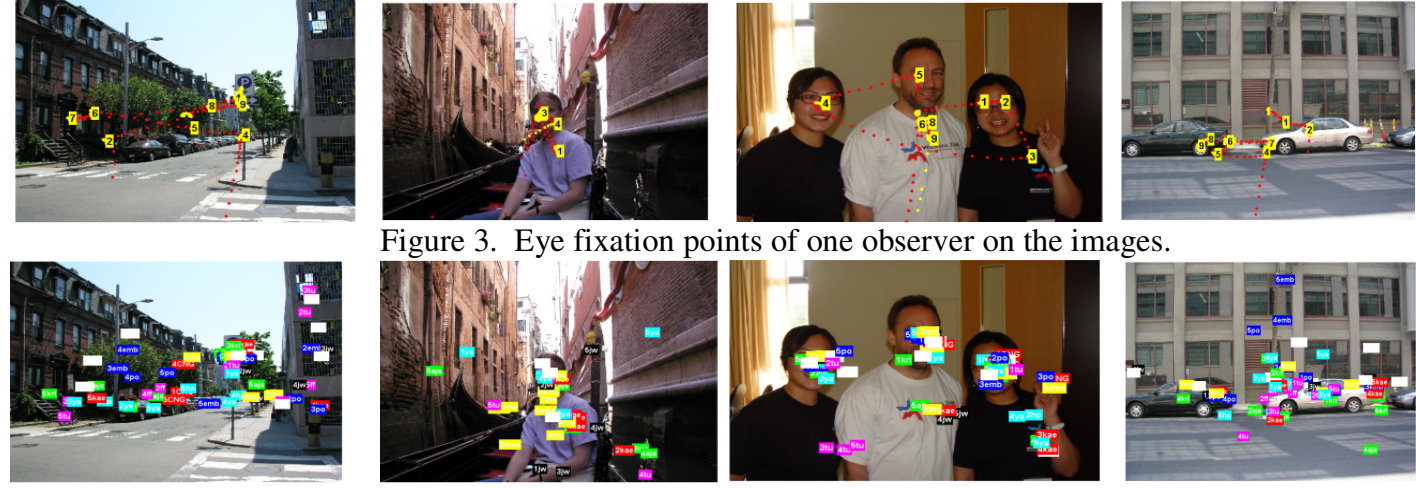

Figure 3. Eye fixation points of one observer on the images.

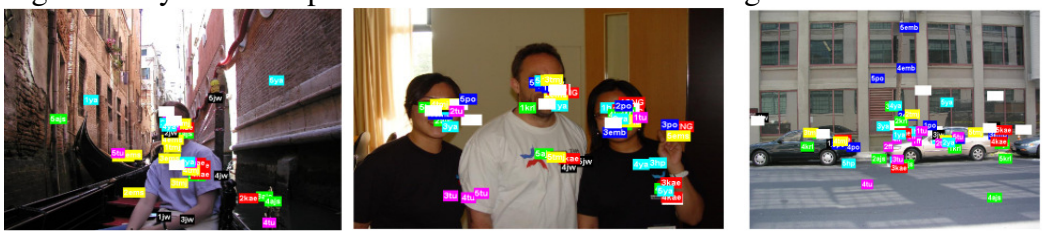

Figure 4. Eye fixation points of all observers on the images.
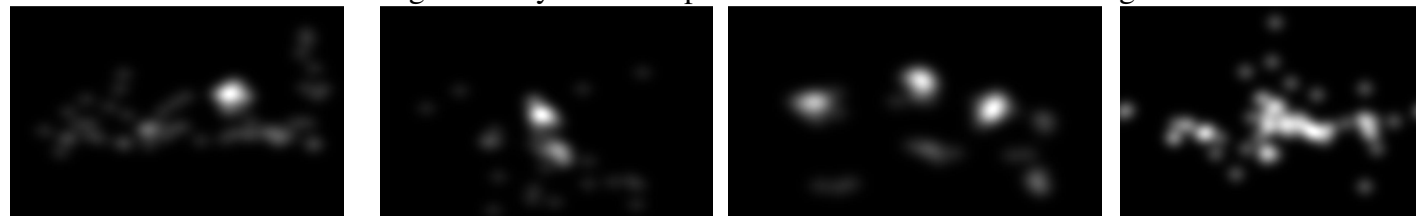

Figure 5. Continuous saliency map obtained from convolving Gaussian over fixation points of all observers.
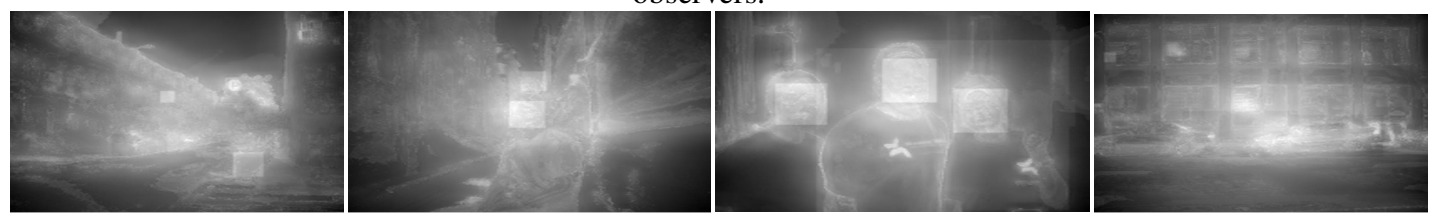

Figure 6. Saliency map obtained from Adaboost 


\section{REFERENCES}

[1] Judd, T., Ehinger, K., Durand, F., \& Torralba, A. (2009). "Learning to predict where humans look", IEEE International Conference on Computer Vision, pp 2106-2113, IEEE Computer Society.

[2] D. DeCarlo \& A. Santella, (2002) "Stylization and abstraction of photographs", ACM Transactions on Graphics, Vol. 21, No. 3, pp 769-776.

[3] L. Itti \& C. Koch, (2000) "A saliency-based search mechanism for overt and covert shifts of visual attention", Vision Research, Vol. 40, pp 1489-1506.

[4] X. Hou \& L. Zhang, (2007) "Saliency detection: A spectral residual approach", Computer Vision and Pattern Recognition, IEEE Computer Society Conference on, Vol 10, pp 1-8.

[5] N. D. B. Bruce \& J. K. Tsotsos, (2009) "Saliency, attention, and visual search: An information theoretic approach", Journal of Vision, Vol. 9, No. 3, pp 1-24.

[6] T. Avraham \& M. Lindenbaum, (2009) "Esaliency: Meaningful attention using stochastic image modeling", IEEE Transactions on Pattern Analysis and Machine Intelligence, Vol. 99, No. 1.

[7] M. Cerf, J. Harel, W. Einhauser, \& C. Koch, (2007),"Predicting human gaze using low-level saliency combined with face detection", In J. C. Platt, D. Koller, Y. Singer, and S. T. Roweis, editors, NIPS. MIT Press.

[8] W. Kienzle, F. A.Wichmann, B. Scholkopf, \& M. O. Franz, (2006) "A nonparametric approach to bottom-up visual saliency", In B. Scholkopf, J. C. Platt, and T. Hoffman, editors, NIPS, pp 689-696. MIT Press.

[9] K. Ehinger, B. Hidalgo-Sotelo, A. Torralba, \& A. Oliva, (2009) "Modeling search for people in 900 scenes: A combined source model of eye guidance", Visual Cognition.

[10] B. Russell, A. Torralba, K. Murphy, \& W. Freeman, (2005) "Labelme: a database and web-based tool for image annotation", MIT AI Lab Memo AIM-2005-025, MIT CSAIL, Sept. 2005.

[11] Cerf, M., Frady, E., \& Koch, C., (2009) "Faces and text attract gaze independent of the task: Experimental data and computer model", Journal of Vision, Vol. 9, No. 12, pp 1-15.

[12] Subramanian, R., Katti, H., Sebe, N., Kankanhalli, M., \& Chua, T. S., (2010) "An eye fixation database for saliency detection in images", In European Conference on Computer Vision, Vol. 6314, pp 30-43, Springer.

[13] E. P. Simoncelli \& W. T. Freeman, (1995) "The steerable pyramid: A flexible architecture for multiscale derivative computation", pp 444-447.

[14] A. Oliva \& A. Torralba, (2001) "Modeling the shape of the scene: A holistic representation of the spatial envelope", International Journal of Computer Vision, Vol. 42, pp 145-175.

[15] P. Viola \& M. Jones, (2001) "Robust real-time object detection", In International Journal of Computer Vision.

[16] P. Felzenszwalb, D. McAllester, \& D. Ramanan, (2008), "A discriminatively trained, multiscale, deformable part model", Computer Vision and Pattern Recognition 2008 IEEE Conference on, pp 18.

[17] Lowe, D. G., (2004) "Distinctive image features from scale-invariant keypoints", International journal of computer vision, Vol. 60, No. 2, pp 91-110.

\section{AUTHOR}

H. Yalın Yalıç was born in Ankara, Turkey in 1984. Yalıç received his B.Sc. degree from the Department of Computer Engineering, Çankaya University, Ankara, Turkey in 2007. He received his M.Sc. degree from the Department of Computer Engineering, Hacettepe University, Ankara, Turkey in 2010 and has continued with his Ph.D. education in the same department. He has worked as a Research and Teaching Assistant at Hacettepe University Computer Engineering Department since 2007. The author's major field of study is computer vision, image and video processing.

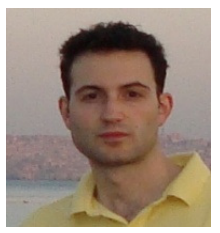




\title{
VIOLENT SCENES DETECTION USING MID-LEVEL VIOLENCE CLUSTERING
}

\author{
Shinichi Goto ${ }^{1}$ and Terumasa Aoki ${ }^{1,2}$ \\ ${ }^{1}$ Graduate School of Information Sciences, Tohoku University, Miyagi, Japan \\ s-goto@riec.tohoku.ac.jp \\ ${ }^{2}$ New Industry Creation Hatchery Center,Tohoku University, Miyagi, Japan \\ aokieriec.tohoku.ac.jp
}

\begin{abstract}
This work proposes a novel system for Violent Scenes Detection, which is based on the combination of visual and audio features with machine learning at segment-level. Multiple Kernel Learning is applied so that multimodality of videos can be maximized. In particular, Mid-level Violence Clustering is proposed in order for mid-level concepts to be implicitly learned, without using manually tagged annotations. Finally a violence-score for each shot is calculated. The whole system is trained ona dataset from MediaEval 2013 Affect Task and evaluated by its official metric. The obtained results outperformed its best score.
\end{abstract}

\section{KEYWORDS}

Multimedia Analysis, Video Processing, Machine Learning

\section{INTRODUCTION}

The amount of videos on the Internet has been rapidly increasing in recent years, which enables people to access them easily, and has given their lives entertainment. This situation also makes it possible for children to easily reach violent contents at the same time though it should be filtered. Because of the enormous number of them, however, it is almost impossible to give annotations on those videos manually to remove them. This makes it essential to develop the automatic classification system for violent videos. As a matter of fact, Technicolor [30] is proposing the need of a system that enables users to choose movies that are suitable for children in their families by providing a preview of violent segments beforehand in MediaEval [1]. MediaEval is a benchmarking workshop dedicated to evaluating algorithms for multimedia analysis and retrieval. They have started arranging Affect Task, which is intended to detect violent scenes in movies.

In spite of this situation, Violent Scenes Detection still has much difficulty because of its complexity, as well as its ambiguous definition. For instance, Chen et al. defines violence as "a series of human actions accompanying with bleeding" in [2], though Giannakopouloset al. only defines violent-related classes such as shots, fights and screams in [3].Or some papers have no enough description for the dataset used in detail according to the research in [4].

In this paper, we propose a novel system to detect violent scenes in movies, using a violent definition by MediaEval 2013 Affect Task, which is "physical violence accident resulting in human injury or pain." Our system is based on segment-level processing. First movies are

David C. Wyld et al. (Eds) : CCSIT, SIPP, AISC, PDCTA, NLP - 2014

pp. 283-296, 2014. (C) CS \& IT-CSCP 2014

DOI : $10.5121 /$ csit.2014.4224 
separated to segments, each of which has a fixed number of frames, and both of visual and audio feature vectors for each segment are extracted. Those feature vectors are used to train classifiers. In order to make the most use of multimodality of movies, Multiple Kernel Learning is applied for our system. In addition, Mid-level Violence Clustering is proposed in order for implicit violent concepts to be learned, without using annotations tagged manually by humans. Classifiers produce segment-level violence-scores, and finally they are converted to shot-level scores. Our system is trained and tested on a dataset from MediaEval 2013 Affect Task, and evaluated by its official metric MAP@100. We compare our results with results by participants in MediaEval. Moreover, an investigation for each mid-level violence cluster is performed for further understanding.

\section{RELATED WORK}

Compared with other related researches on video analysis such as event classification or action recognition, relatively few researches have been done for Violent Scenes Detection. Giannakopouloset al. [3] analyzed the effect of audio features on this task, such as energy entropy, zero crossing and so on. For each segment, those audio features are extracted, and fed as input for Support Vector Machine(SVM) without visual features. Bermejo et al., on the other hand, used only visual features [5]. Features such as Bag-of-Visual-Words (BoVW) [14], Space Time Interest Points (STIP)[21] and MoSIFT [22] are calculated for SVM. For a SVM kernel function, they compared Radial Basis Function (RBF), Chi-square and Histogram Intersection Kernel (HIK) [17], and reported that HIK obtained the best result. Chen et al. also used only visual information such as average motion vectors, camera motion, shot length and RGB values of pixels. They also proposed a shot-grouping algorithm for the further efficiency [2].

Though researches above use either visual or audio features, researches utilizing both of them have shown to improve results. The first try that utilized this multimodality is a work by Nam et al. at 1998 [6]. They characterized and indexed violence scenes in videos, proposing that violent signatures are represented as combination of multiple features. Their feature extraction is based on flame detection, blood detection and audio features such as energy entropy. Lin et al. [7] adopted PLSA to locate audio violence. PLSA is a probabilistic model utilizing the Expectation Maximization algorithm, which is often used in the field of natural language processing. For visual violence they used a linear weighted model fed with the results of violent event detections such as motion intensity, frame, explosion and blood. Finally two classifiers are built in a cotraining way. Penet et al. also utilized both modalities, comparing normal Early Fusion with Late Fusion [8]. Early Fusion concatenates features from both modalities before learning, while Late Fusion fuses the probabilities of both modalities. They reported that Late Fusion has more effectiveness. Derbas et al. [20] proposed Joint Audio-Visual Words representation, which constructs codebooks in the context of Bag-of-Words (BoW)by combining audio and visual features. They got the first in MediaEval 2013 without external data in terms of MAP@100 score. Instead of detecting violent scenes directly from low-level features, some works have used midlevel concepts such as Fire, Fights or Explosions given in MediaEval Affect Task [1]. Ionescu et al. proposed a frame-level violence prediction, applying a multi-layer perceptron in order to utilize these concepts [9][25].They put the first layer for the concept prediction, and the second layer for the violence prediction. They ranked first in MediaEval 2012 on $F_{1}$-score. In addition to those given concepts, Tanand Ngo [10]have utilized extra 42 violence concepts such as bomb or war from ConceptNet [23], which is composed of nodes representing concepts in the form of words or short phrases with their relationships. Their system trains those extra concepts using YouTube videos which they crawl additionally. Afterwards a graphical model of those concepts are generated, and Conditional Random Fields [31]refines it by using relationships in ConceptNet and co-occurrence information of concepts. Their MAP@100 result was the first place in 2013 Affect Task with external data. 


\section{VIOLENT SCENES DETECTION BASED ON MID-LEVEL CLUSTERING}

\subsection{Approach Overview}

The overview of our system, which is composed of the training process and the testing process, is displayed in Figure 1. For both processes, feature extraction and training/classification are based on the segment-level calculation. Here we define a segment as a bunch of 20 frames $(0.8$ seconds if FPS is 25).

First all training movies are separated to segments. Then both of visual and audio features are extracted for each segment (described in 3.2). Segments that are tagged as violent are gathered and separated $K(>0)$ clusters, each of which represents a concept or a combination of concepts that is led to violence in our assumption. This will be described in more detail in 3.3. For each cluster a classifier is trained by using Multiple Kernel Learning (MKL).

In the testing process, segmentation andfeature extraction are performed in the same way as the training process.Classifiers in all clusters evaluate each segment, producing violence-scores. Then scores are integrated to generate segment-level scores.Smoothing is applied in order to take the context of movies into account, and finally segment-level scores are converted to shot-level scores.

For our runs only violent and non-violent ground truth are used, and neither a high-level concept nor external data is used. The following sections explain our feature vectors and training system more precisely.

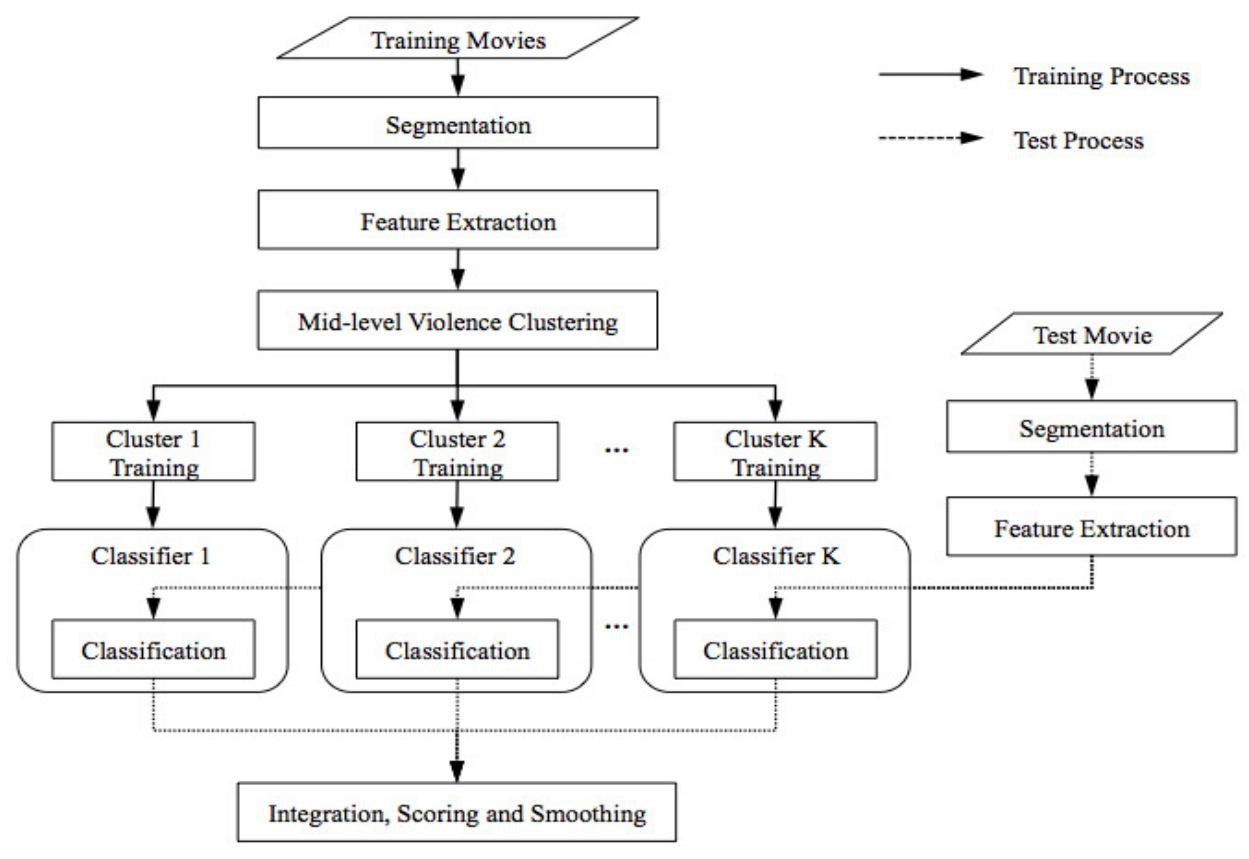

Figure 1. Approach Overview. 


\subsection{Features}

Recent works on violent scenes detection such as [10] and [24] have shown the effectiveness of using trajectory-based features as visual information, MFCC-based features as audio information. Similar to those researches, in total six feature spaces exist on our system. Trajectory, HOG, MBHx, MBHy and RGB histogram around trajectories are computed as visual features, and MFCC and delta-MFCC are calculated as audio features. For each segment these features are converted to the BoW form by using already generated codebooks. Codebooks are calculated by using randomly selected 100,000 features and k-means++ algorithm [33] before hand in each feature space respectively.

\subsubsection{Visual Features}

Trajectories, which can capture local motion of videos, are getting attentions especially in the field of action recognition. Wang et al. applied dense sampling, which is used in the field of image classification, to trajectories to improve their quality, and called them Dense Trajectories [11]. Except for those in homogeneous areas represented by a small value of the eigenvalue of the auto-correlation matrix, densely sampled feature points are tracked by calculating optical flows in each spatial scale until they reach the length of $L=15$ frames. Every frame newly sampled points are added if no tracked point is found in the neighborhood of each pixel.

For each trajectory, descriptors are extracted in its neighborhood. In addition to existing descriptors extracted along trajectories such as Histograms of Oriented Gradients (HOG) or Histograms of Oriented Optical Flow (HOF), they also proposed to extract Motion Boundary Histograms $(\mathrm{MBH})$, which was originally proposed in the field of human detection by Dalal et al. [12]. MBH represents the changes in the optical field, namely local motion information independent of camera motion by calculating the gradient of the optical flow. $\mathrm{MBH}$ is calculated separately along vertical direction (MBHx) and horizontal direction (MBHy).

Descriptors are calculated in $12(=2 * 2 * 3)$ subdivided volumes around trajectories and concatenated afterwards. For our system, HOG (96-dimension), MBHx (96-dimension) and MBHy (96-dimension) around trajectories are extracted. Also displacement vectors of trajectories are extracted for both of $\mathrm{x}$-direction and y-direction (30-dimension). Because of the frequent camera motion, HOF is expected to have poor contribution on our task, and therefore is removed. On the other hand, as color information is expected to be helpful just as blood or flame detections contributed to the results in some previous researches, 64-bin RGB histograms around trajectories are also calculated every 5 frame, which will be 192-dimension descriptor. Finally they are converted to the form of BoW for each segment, and 200-dimension trajectory, 400-dimension HOG, 200-dimension MBHx, 200-dimension MBHy, and 400-dimension RGB histogram are obtained. As parameters we used 32 for a neighbour range, 5 for a sampling step, and 6 for a spatial scale size.

\subsubsection{Audio Features}

Similar to Bag-of-Audio-Words in [13], MFCC (Mel Frequency Cepstrum Coefficients) and the $\log$ energy are first extracted every $10 \mathrm{~ms}$ with $5 \mathrm{~ms}$ overlap for audio features. The first derivative of MFCC and its energy are also calculated as delta-MFCC, which results in 26-d features in total. Then they are converted to 200-d BoW for each segment. 


\subsection{Mid-level Violence Clustering}

Before training classifiers by using features above, Mid-level Violence Clustering is applied. Figure 2 illustrates the construction process of mid-level clusters. Note substitutefigures are displayed for easy understanding instead of feature vectors.First whole violent segments in training movies are gathered. Then they are separated to $K(>0)$ clusters, each of which has similar segments. Then non-violent training segments are assigned to one of those clusters randomly, whose results construct clusters for mid-level violence classifiers. Practically we

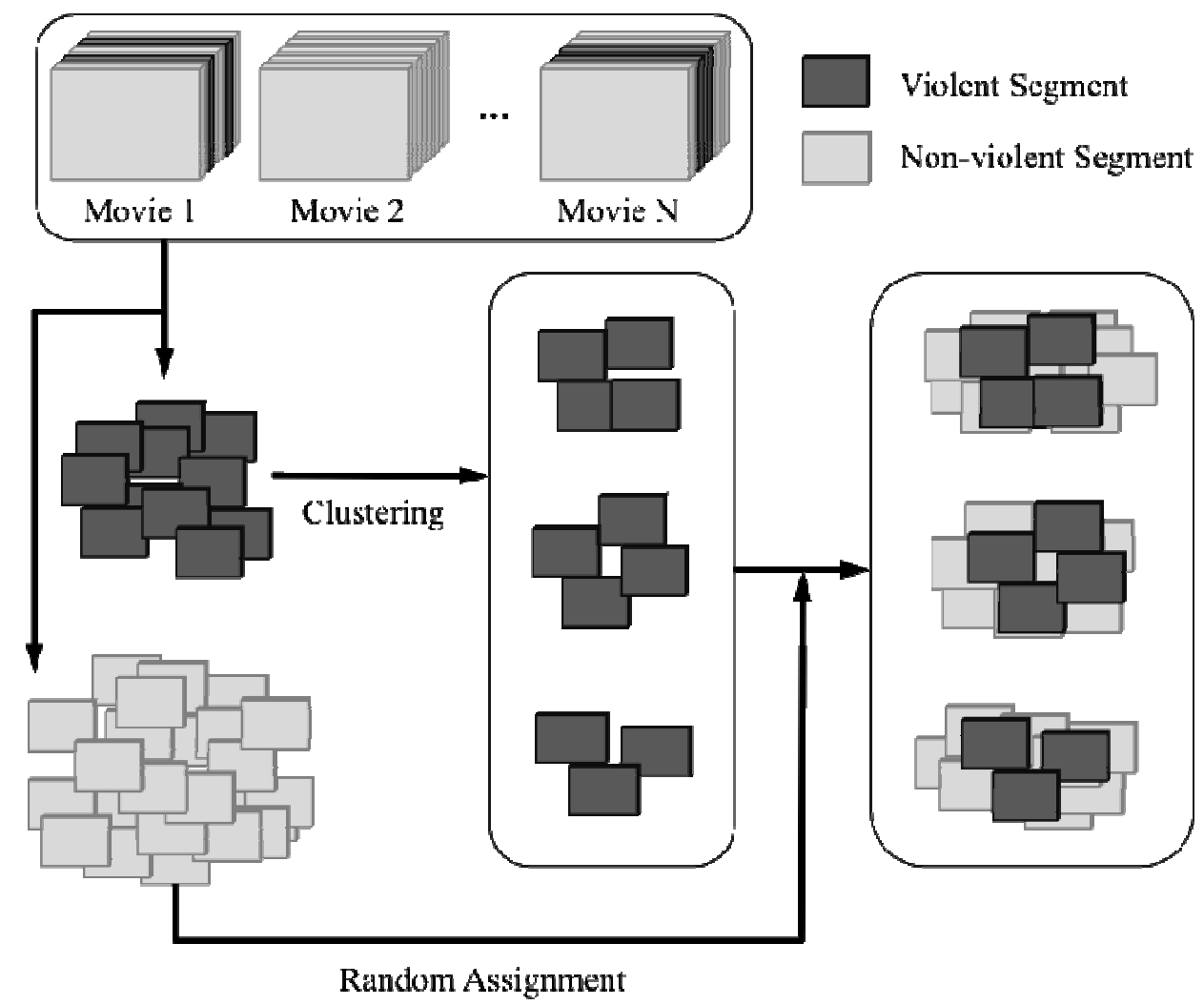

Figure 2. Mid-level Violence Clustering $(K=3)$.

need to handle 6 feature vectors while clustering. We simply concatenates them, and applied kmeans++ algorithm with Euclidean distance. This means Euclidian distance is used for the similarity among segments. Here we assume that in each cluster feature vectors for violent segments represent multiple mid-level concepts related to violence.

The reason why we design this clustering process is that the diversity of "violence" is huge: even if two segments are tagged as violent, their features might be largely different depending on their characteristics of "violence."For instance, although explosion scenes labelled as violent might have distinctive visual features, those of scream scenes might not similar even if they are also labelled as violent. A schematic example simplifies this problem in 2-dimension (see Figure 3a). There exist two classes (Class A and Class B), and what we want to do is to define a decision boundary that can classify new input points. As Figure 3a illustrates, data is noisy and this classification problem does not seem to be easily solved. Here let us suppose that Class A has two mid-level concepts, and those concepts can be separated to two clusters (Figure $3 \mathrm{~b}$ and Figure $3 c)$.In that case training and classifying Class $\mathrm{A}$ in each figure corresponds to training and 
classifying each mid-level concept in Class A, which is much easier problem than directly dealing with Figure 3a.

In violent scenes detection, as a few previous works such as [9] and [10] have shownmid-level concepts information can be helpful, it is appropriated to say that "violence" class has multiple mid-level concepts. Hence if those concepts are correctly clustered, training violence for one cluster corresponds to training one mid-level violent concept. In addition, being different from the problem in Figure 3, the number of feature points and dimensions are huge on our task.As 10 concepts are prepared in MediaEval, and as in [10] 52 concepts have been used, the number of mid-level concepts wouldbe much bigger, which is led to more complexity. Thus

$\bigcirc$ Class A

$\times$ Class B

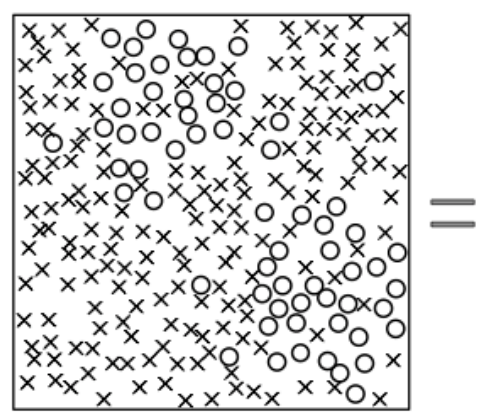

(a)

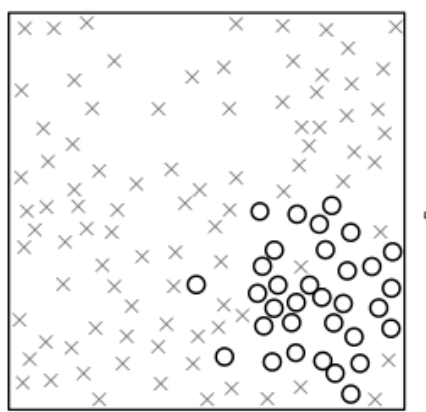

(b)

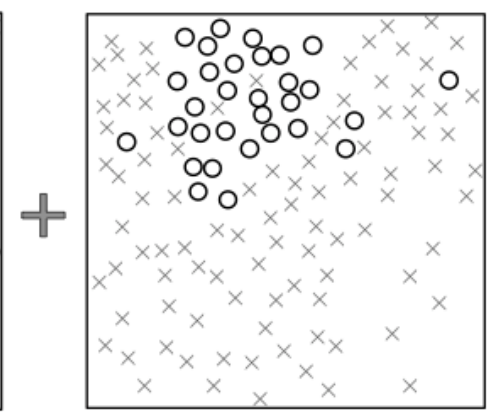

(c)

Figure 3. Example problem where mid-level clustering can be used $(K=2)$ : (a) the original problem, (b) first cluster, (c) second cluster. We assume two clusters exist in (a).

simplifying this problem is expected to be effective. The process of actual training, classification and integration are described in the following sections.

\subsection{Multiple Kernel Learning}

Support Vector Machine (SVM) has had a great contribution to the field of image classification over the last few years. In particular, BoVW with SVM [14] had succeeded extremely. In violence detection in movies, however, multiple feature spaces have to be handled, and then simply concatenating feature vectors and training classifiers, which is called Early Fusion, is not always a best way, according to the work by Cedric et al. [8].As they studied, when multimodal features exist, there are two available fusion schemes: namely Early Fusion and Late Fusion. Early Fusion concatenates features from both modalities before training, which means it can take the correlation of those feature spaces into account while training, though it deals with each feature space uniformly. On the other hand, on Late Fusion training itself is done in each feature space independently, and extracted probabilities from the testing process are fused afterwards. They compared Early Fusion with Late Fusion process when two modalities exist (visual and audio).Although they concluded that Late Fusion has more effectiveness, if more modalities exist just as our system, it has some drawbacks: 1) it cannot take correlations of multimodal features into account while training, 2) how to fuse probabilities needs to be decided manually beforehand.

To cope with this problem, and to maximize the multimodality of our features, we apply Multiple Kernel Learning (MKL), which can be regarded as a kind of Early Fusion, but aims at finding optimized weights for each feature space when multiple SVM kernels are applied [15]. This means MKL considers correlations of multiple feature spaces, but at the same time it considers differences of violent characteristics among multiple feature spaces. Fusing method does not have 
to be decided either. In MKL the whole kernel is composed of multiple sub-kernels, and is defined as the following equation:

$$
\boldsymbol{K}\left(\boldsymbol{x}_{i}, \boldsymbol{x}_{\boldsymbol{j}}\right)=\sum_{p} \beta_{p} \boldsymbol{K}_{p}\left(\boldsymbol{x}_{i}, \boldsymbol{x}_{\boldsymbol{j}}\right)
$$

where $\boldsymbol{K}_{p}$ are sub-kernels, and $\beta_{p}$ is a weight for $p$-thsub-kernel. In our case, kernels for Trajectory, HOG, MBHx, MBHy, RGB-histogram and Audio features are prepared.

When $N$ data points $\left(\boldsymbol{x}_{i}, y_{i}\right)\left(y_{i} \in\{ \pm 1\}, i=[1,2, \cdots, N]\right)$ are given and input features $\boldsymbol{x}_{i}$ is translated via $\Phi_{p}(\boldsymbol{x}) \mapsto \mathbb{R}^{D_{p}}, p=[1,2, \cdots, P]$ into $P$ feature spaces where $D_{p}$ denotes the dimensionality of $p$-th feature space,Bach et al. derived the dual for the MKL primary problem in [16], which represents its optimization problem as following:

$$
\begin{aligned}
\min & \boldsymbol{\gamma}-\sum_{i=\mathbf{1}}^{N} \alpha_{i} \\
\text { w.r.t. } & \boldsymbol{\gamma} \in \mathbb{R}, \alpha \in \mathbb{R}^{N} \\
\text { s.t. } & 0 \leq \alpha \leq 1 C, \sum_{i=\mathbf{1}}^{N} \alpha y_{i}=0 \\
& \frac{1}{2} \sum_{i, j=1}^{N} \alpha_{i} \alpha_{j} y_{i} y_{j} \boldsymbol{k}_{p}\left(\boldsymbol{x}_{i}, \boldsymbol{x}_{\boldsymbol{j}}\right) \leq \gamma, \forall p=1, \cdots, P
\end{aligned}
$$

where $C$ is a regularization parameter and we have one quadratic constraint per kernel.

For a sub-kernel, Histogram Intersection Kernel (HIK), which has been reported to perform well on histogram-based features [17], is adopted. HIK is defined as follows:

$$
\boldsymbol{K}_{\text {int }}(\boldsymbol{A}, \boldsymbol{B})=\sum_{i=1}^{m} \min \left(a_{i}, b_{i}\right)
$$

where $\boldsymbol{A}=\left[a_{1}, a_{2}, \cdots, a_{m}\right]$ and $\boldsymbol{B}=\left[b_{1}, b_{2}, \cdots, b_{m}\right]$. It measures the degree of similarity between two histograms.

MKL is applied to all $K$ clusters, resulting in generating $K$ classifiers.SHOGUN Toolbox [18] is used for our MKL implementation.

\subsection{Integration, Scoring and Smoothing}

After MKL, each segment in test movies is classified as violent or non-violent by $K$ classifiers. Suppose a movie has $N$ segments. For $k$-th classifier $(1 \leq k \leq K)$, classification results for all segments are obtained:

$$
\boldsymbol{C}_{k}=\left[c_{k, 1}, c_{k, 2}, \cdots, c_{k, N}\right]\left(c_{k, n} \in\{ \pm 1\}, 1 \leq n \leq N\right)
$$

where " +1 " represents violence, while " -1 " represents non-violence.

Let $d_{k, n}$ denote a"violence-score," which is a distance between a feature point of $n$-thsegment and a hyperplane of $k$-th classifier. $\boldsymbol{D}_{k}\left(=\left[d_{k, 1}, d_{k, 2}, \cdots, d_{k, N}\right]\right)$ are calculated as the result of MKL, and we define $\boldsymbol{S}_{k}$, scores by $k$-thclassifier as follows: 


$$
\boldsymbol{S}_{k}=\left[s_{k, 1}, s_{k, 2}, \cdots, s_{k, N}\right], \quad s_{k, n}=\left\{\begin{array}{r}
d_{k, n}\left(\text { if } c_{k, n}=+1\right) \\
0\left(\text { if } c_{k, n}=-1\right)
\end{array}\right.
$$

Scores by each classifier are integrated to produce pre-final scores $\boldsymbol{S}$ :

$$
\boldsymbol{S}=\left[s_{1}, s_{2}, \cdots, s_{N}\right], \quad s_{n}=\frac{\sum_{l=1}^{K} s_{l, n}}{K_{\text {vio }}}(1 \leq n \leq N)
$$

where $K_{v i o}$ is the number of classifiers whose $c_{k}$ is " +1 ,"that is, the number of classifiers which classify a target segment as violent.This means for each segment, if no cluster classifies it as violent, its violence-score is zero, while the mean value of violence-scores of classifiers which classify it as violent is assigned if $N$ clusters classify it as violent.

So far the context of movies are not taken into account. Hence scores are smoothed as a final step. Although in [24] the average value over a three-shot window is calculated, we adopt a moving average calculation so that the further neighbour segments are positioned, the fewer their effects are considered. Smoothed scores $\boldsymbol{S}$ are calculated by using pre-final scores $\boldsymbol{S}$ as follows:

$$
\dot{\boldsymbol{S}}=\left[\dot{s}_{1}, \dot{s}_{2}, \cdots, s_{M}\right], \quad \dot{s}_{\imath}=\frac{s_{i}+\sum_{m=1}^{M} \alpha^{m} \cdot\left(s_{i-m}+s_{i+m}\right)}{2 M+1}(0<\alpha<1)
$$

where $\alpha$ is a smoothing coefficient, $M$ is a neighbor range around a segment. We used 0.5 for $\alpha$ and 2 for $M$.

Scores for shots are calculated by converting segment-level scores after calculating frame-level scores. Because the numbers of frames in segments are consistent except for the final segment of the movie, frame-level scores are simply given as scores for segments which have those frames. Then for each shot scores for frames it has are summed and divided by the number of frames. This score is used as the final violence score for each shot. If this score is higher than a threshold, that shot is classified as violent. We choose 0.20 as a threshold.

\section{EXPERIMENT}

Our experiments are carried out following MediaEval 2013 Affect Task. Though in 2013 there are two subtasks and participants are allowed to submit multiple types of runs, in this paper we focus on shot-level classification with objective violence definition, which is "physical violence accident resulting in human injury or pain."Although there were participants who used additional data for their systems such as [10], our results are compared with systems without external data.

\subsection{Dataset}

Twenty-five movies are provided with shot boundary annotations. 18 are dedicated to the training process: Armageddon, Billy Elliot, Eragon, Harry Potter 5, I am Legend, Leon, Midnight Express, Pirates of the Caribbean 1, reservoir Dogs, Saving Private Ryan, The Sixth Sense, The Wicker Man, Kill Bill 1, The Bourne Identity, The Wizard of Oz, Dead Poets Society, Fight Club and Independence Day, and they are given with frame-level violence ground truth. 7 are dedicated to the test process: Fantastic Four, Fargo, Forrest Gump, Legally Blond, Pulp Fiction, The God Father 1 and The Pianist. Though in MediaEval 2013 participants were allowed to use prepared high-level concepts, our algorithm use only low-level features extracted from movies and shot boundary information. 


\subsection{Evaluation Criteria}

The official metric for the evaluation is the Mean Average Precision [19] at the 100 top ranked violent shots (MAP@100). Namely:

$$
\text { MAP@100 }=\frac{1}{100} \sum_{j=1}^{100} \frac{1}{m_{j}} \sum_{k=1}^{m_{j}} \operatorname{Precision}\left(R_{j k}\right)
$$

where $R_{j k}$ is the set of ranked list of violent shots from $1 s t$ to $k$-thfor the calculation of Average Precision of $j$-th shot.

\subsection{Overall Performance Evaluation}

Table 1 presents our results with results by participants in MediaEval 2013.For the number of clusters $K$ we tried 10,30, 50 and 100.Additionally a score when we construct clusters for each training movie is displayed. This is the case in which violence multiple mid-level concepts are mixed in each cluster. We call this as Training-movie Clustering in this table.

A result with $K=50$ shows the best score 0.558, and one can find Mid-level Violence Clustering scores outperform the best score 0.520 in MediaEval 2013. Also they are much higher than a score by training-movie clustering. As each cluster has multiple violent concepts

Table 1. Comparison between our results and results by participants in MediaEval 2013.

\begin{tabular}{|l|c|}
\hline \multicolumn{1}{|c|}{ Team (Run) } & MAP@ 100 \\
\hline LIG [20] & 0.520 \\
\hline FAR [25] & 0.496 \\
\hline Fudan [24] & 0.492 \\
\hline NII [28] & About 0.400 \\
\hline Technicolor [29] & 0.338 \\
\hline VISILAB [26] & 0.150 \\
\hline MTM [27] & 0.070 \\
\hline Mid-level Violence Clustering $(K=30)$ & 0.527 \\
\hline Mid-level Violence Clustering $(K=40)$ & 0.539 \\
\hline Mid-level Violence Clustering $(K=50)$ & $\mathbf{0 . 5 5 8}$ \\
\hline Mid-level Violence Clustering $(K=100)$ & 0.551 \\
\hline Training-movie Clustering & 0.487 \\
\hline
\end{tabular}

that might be largely different in Training-movie Clustering, this result supports our assumption that each cluster is supposed to have similar concepts in Mid-level Violence Clustering.

While a result seems to change a little depending on the number of clusters, even the high number of clusters (e.g. $K=100$ ) can keep a promising score. This is because when the number of clusters is big, some clusters have smaller violent segments assigned to themselves due to their anomalies. For instance, when we chose $K=100$, the smallest number of violent segments in a cluster was 2, although other clusters tended to contain 50-150violent segments. Even though this cluster could not find violent segments, it did not affect a final score either because our scoring equation (6) depends only on scores by classifiers that classify a target segment as violent.

Though our system achieves promising results, they are not high enough yet. The first point to be considered here is that our feature vectors might not be distinct enough. Although we have used only Trajectory-based features for visual information, they can be easily affected by camera 
motion. Even though features such as $\mathrm{MBH}$, which are proposed as robust to camera motion is extracted, they might be noisy if trajectories themselves are unreliable. Therefore some action against this problem is imperative.

The second reason is that thoughEuclidean distance is used for the similarity while Mid-level Violence Clustering, Histogram Intersection is used for sub-kernels in MKL, which might be inconsistent. To tackle this problem, two solutions can be considered: to perform clustering by using Histogram Intersection, or to apply sparse coding and max pooling [32] to whole feature vectors in order that linear kernel can be used for SVM.

Moreover, from our investigation, shots that contain frequent camera motion, multiple people and big sound tend to be miss-classified as violent. Loud sound such as abrupt brake by cars and crackers were also miss-classified. Meanwhile, common missed violent shots are violent scenes without sound, such as a scene in which a man is wringing other man's neck. Feature vectors that can detect these events are needed. Also in our system shot boundaries are not taken into account while constructing shots. Because shots often change in the middle of segments, feature vectors in those segments should be discarded.

\subsection{Discussion}

We still do not know how segments are classified by each cluster, or what kind of mid-level concepts is assigned to each cluster. Therefore we analyzed each cluster for its implicit

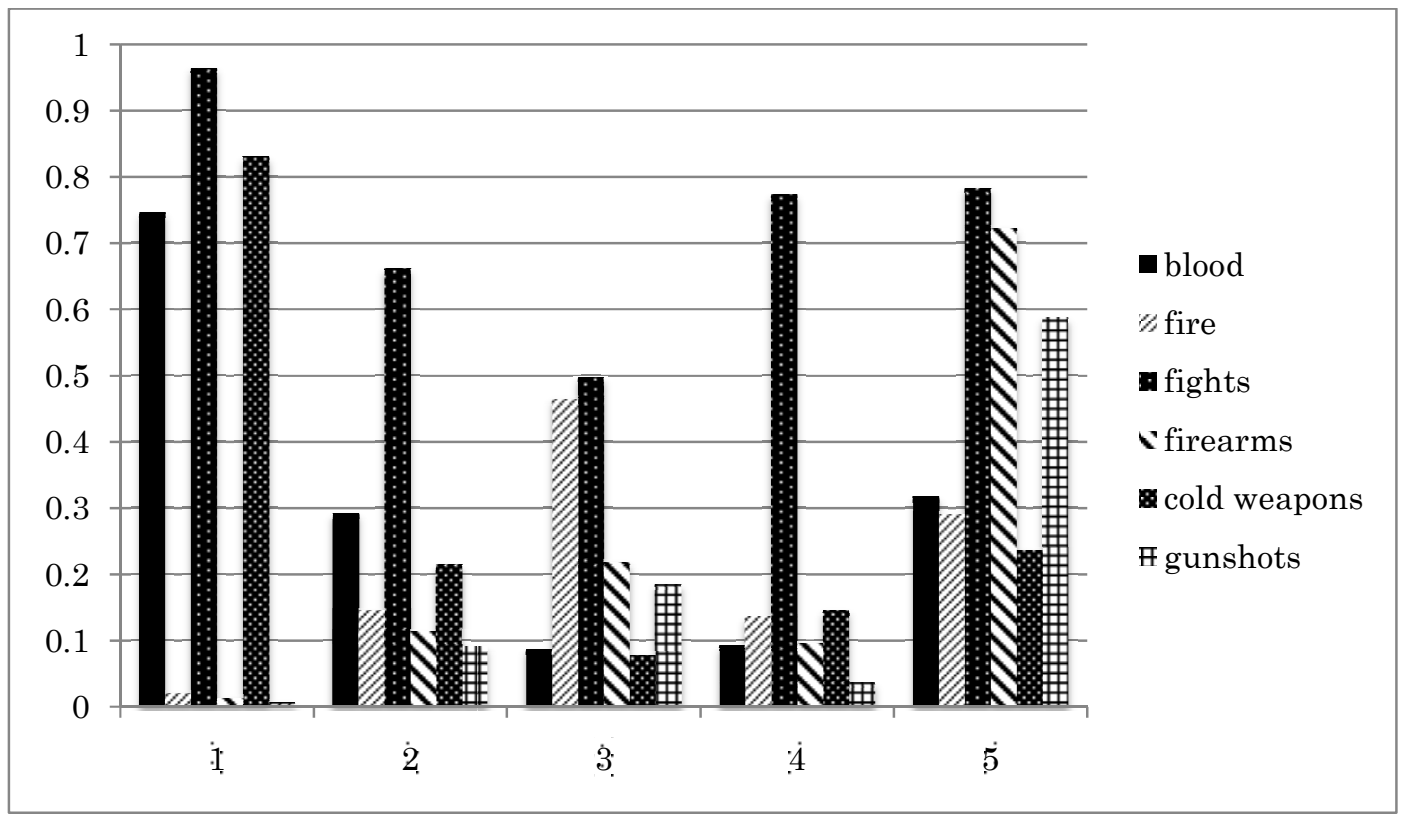

Figure 4. Ratios of segments annotated by each concept for clusters $(K=50)$. 




Figure 5. MKL weights for each cluster in Figure 4.

concept by examining the amount of violence-related concepts in it using the cluster number $K=50$. In MediaEval 2013, participants were also provided with mid-level concepts annotated at frame level by human assessors, which we did not use on our system. They consist of 7 visual concepts: presence of blood, presence of fire, fights, gory scenes, presence of firearms, presence of cold weapons, carchases, and 3 audio concepts: explosions, presence of screams, gunshots. It should be noted that these mid-level concepts are not always related to violencetags, and often multiple concepts are tagged in one frame. Since they are at frame-level, we converted them to segment-level annotations by simply tagging each segment if half of frames it contains are annotated. The ratio of segments annotated by each mid-level concept in each cluster is shown in Figure 4.Since it is in adequate to display ratios for all clusters and for all mid-level concepts in this figure due to the limit of the available spaces, only 5 representative clusters and 6 concepts are displayed. Additionally, optimized weights trained by MKL for each cluster are displayed in Figure 5.

By studying these figures one can find there are some correlations between concepts and weights. For instance, Cluster 1 has more segments annotated as blood and cold weapons, and it has higher weights for HOG and RGB feature space at the same time. It is safe to say that this cluster has segments in which fights with blood or cold weapons appear, just as in Kill Bill. Cluster 5, on the other hand, contains a high number of segments tagged as firearms and gunshots, and its weights for Audio is high. This leads to our presumption that Cluster 5 includes violent scenes of gunfire. On the other hand, the ratio of fights is high for all clusters. Though clusters and concepts in MediaEval are unrelated essentially, and so clusters do not always have to be distinctive in Figure 4 , this might be caused by the lack of distinctiveness of visual features.

\section{Conclusions}

In this paper, we proposed a novel system to detect violent scenes in movies by using Mid-level Violence Clustering and Multiple Kernel Learning with multimodal features. Our experiments proved clustering violence before training is effective, and mid-level concepts can be implicitly learned. Obtained results outperformed the best score in MediaEval 2013 Affect Task. In 
addition, we investigated relations between concepts of mid-level clusters and weights trained by Multiple Kernel Learning and found correlations. Future work is to find more appropriate feature vectors, as well as to adopt more suitable clustering method in the context of our system.

\section{ACKNOWLEDGEMENTS}

We acknowledge the MediaEval 2013 Affect Task: Violent Scenes Detection http:// www.multimediaeval.org / mediaeval2013/ for providing the dataset that has been supported, in part, by the Quaero Program http://www.quaero.org.

\section{REFERENCES}

[1] C. Demarty, C. Penet, M. Schedl, B. Ionescu, V.L. Quang,and Y. Jiang,"The Mediaeval 2013 Affect Task: Violent Scenes Detection," in MediaEval 2013 Workshop, Barcelona, Spain, Oct. 18-19 2013.

[2] Liang-Hua Chen, Hsi-Wen Hsu, Li-Yun Wang, and Chih-Wen Su, "Violence Detection in Movies," in Computer Graphics, Imaging and Visualization (CGIV), 2011 Eighth International Conference, 2011.

[3] Theodoros Giannakopoulos, Dimitrios Kosmopoulos, Andreas Aristidou, and Sergios Theodoridis, "Violence Content Classification Using Audio Features," in SETN'06 Proceedings of the 4th Helenic conference on Advances in Artificial Intelligence, pp. 502-507, 2006.

[4] Fillipe D. M. de Souza, Guillermo C. Chavez, Eduardo A. do Valle Jr., and Arnaldo de A. Araujo,"Violence Detection in Video Using Spatio-Temporal Features," in SIBGRAPI '10 Proceedings of the 2010 23rd SIBGRAPI Conference on Graphics, Patterns and Images, pp. 224230, 2010.

[5] E. Bermejo, O. Deniz, G. Bueno, and R. Sukthankar,"Violence Detection in Video Using Computer Vision Techniques," in CAIP'11 Proceedings of the 14th international conference on Computer analysis of images and patterns - Volume Part II, pp. 332-339, 2011.

[6] Jeho Nam, Masoud Alghoniemy, and H. Tewfik, "Audio-Visual Content-Based Violent Scene Characterization," in International Conference on Image Processing, Oct. 1998.

[7] Jian Lin and Weiqiang Wang,"Weakly-Supervised Violence Detection in Movies with Audio and Video Based Co-training," in PCM '09 Proceedings of the 10th Pacific Rim Conference on Multimedia: Advances in Multimedia Information Processing, pp. 930-935, 2009.

[8] Cedric Penet, Claire-Helene Demarty, Guillaume Gravier, and Patrick Gros, "Multimodal Information Fusion and Temporal Integration for Violence Detection in Movies," in ICASSP - 37th International Conference on Acoustics, Speech, and Signal Processing, 2012.

[9] Bogdan Ionescu, Jan Schluter, Ionut Mironica, and Markus Schedl,"A Naive Mid-level Conceptbased Fusion Approach to Violence Detection in Hollywood Movies," in ICASSP - 37th International Conference on Acoustics, Speech, and Signal Processing, 2012.

[10] Chun Chet Tan and Chong-Wah Ngo, "The Vireo Team at MediaEval 2013: Violent Scenes Detection by Mid-level Concepts Learnt from Youtube," in Working Notes Proceedings of the MediaEval 2013 Workshop, Barcelona, Spain, Oct. 18-19 2013, CEUR-WS.org, ISSN 1613-0073, http://ceurws.org/Vol-1043/mediaeval2013_submission_12.pdf.

[11] Heng Wang, Alexander Klaser, Cordelia Schmid, and Cheng-Lin Liu,"Action Recognition by Dense Trajectories,"in IEEE Conference on Computer Vision \& Pattern Recognition, pp. 3169-3176, Colorado Springs, United States, June 2011.

[12] N. Dalal,B. Triggs and C. Shmid, "Human detection using oriented histograms of flow and appearance," in European conference on computer vision, 2006.

[13] Stephanie Pancoast and Murat Akbacak,"Bag-of-audio-words Approach for Multimedia Event Classification," in INTERSPEECH'12, 2012.

[14] Gabriella Csurka, Christopher R. Dance, Lixin Fan, Jutta Willamowski, and Cedric Bray,"Visual Categorization with Bags of Keypoints," in Proc. of European Conference on Computer Vision (ECCV), pp. 1-22, 2004.

[15] S. Sonnenburg, G. Raetsch, C. Schaefer, and B. Schoelkopf,"Large Scale Multiple Kernel Learning," in Journal of Machine Learning Research, 2006.

[16] Francis R. Bach, Gert R. G. Lanckriet, and Michael I. Jordan, "Multiple Kernel Learning, Conic Duality, and the SMO Algorithm," in ICML '04 Proceedings of the twenty-first international conference on Machine Learning, 2004. 
[17] A. Barla, F. Odone, and A. Verri,"Histogram Intersection Kernel for Image Classification," in Proceedings of ICIP 2003, pp. 513-516, 2003.

[18] Soeren Sonnenburg, Gunnar Raetsch, Sebastian Henschel, Christian Widmer, Jonas Behr, Alexander Zien, Fabio de Bona, Alexander Binder, Christian Gehl, and Vojtech Franc, "The SHOGUN Machine Learning Toolbox," in Journal of Machine Learning Research, 11, pp. 1799-1802, June 2010.

[19] Christopher D. Manning, Prabhakar Raghavan and Hinrich Schutze,Introduction to Information Retireval,July 2008.

[20] Nadia Derbas, Bahjat Safadi and Georges Quénot,"LIG at MediaEval 2013 Affect Task: Use of a Generic Method and Joint Audio-Visual Words," in Working Notes Proceedings of the MediaEval 2013 Workshop, Barcelona, Spain, Oct. 18-19 2013, CEUR-WS.org, ISSN 1613-0073, http://ceurws.org/Vol-1043/mediaeval2013_submission_13.pdf.

[21] I. Laptev. "On Space-Time Interest Points,"in International Journal of Computer Vision, vol. 64, number 2/3, pp.107-123, 205.

[22] M. Chen and A. Hauptmann, "MoSIFT: recognizing Human Actions in Surveillance Videos,"in $C M U$ CS-09-161, 2009.

[23] H. Liu and P. Singh. "ConceptNet - a practical commonsense reasoning tool-kit,"in BT Technology Journal, vol. 22, pp. 211-226, Oct. 2004.

[24] Qi Dai, Jian Tu, Ziqiang Shi, Yu-Gang Jiang and Xiangyang Xue,"Fudan at MediaEval 2013: Violent Scenes Detection Using Motion Features and Part-Level Attributes," in Working Notes Proceedings of the MediaEval 2013 Workshop, Barcelona, Spain, Oct. 18-19 2013, CEUR-WS.org, ISSN 16130073, http://ceur-ws.org/Vol-1043/mediaeval2013_submission_6.pdf.

[25] Mats Sjöberg, Jan Schlüter, Bogdan Ionescu and Markus Schedl, "FAR at MediaEval 2013 Violent Scenes Detection: Concept-based Violent Scenes Detection in Movies," in Working Notes Proceedings of the MediaEval 2013 Workshop, Barcelona, Spain, Oct. 18-19 2013, CEUR-WS.org, ISSN 1613-0073, http://ceur-ws.org/Vol-1043/mediaeval2013_submission_10.pdf.

[26] Ismael Serrano, Oscar Déniz, Gloria Bueno,"VISILAB at MediaEval 2013: Fight Detection," in Working Notes Proceedings of the MediaEval 2013 Workshop, Barcelona, Spain, Oct. 18-19 2013, CEUR-WS.org, ISSN 1613-0073, http://ceur-ws.org/Vol-1043/mediaeval2013_submission_11.pdf.

[27] Bruno Do Nascimento Teixeira,"MTM at MediaEval 2013 Violent Scenes Detection: Through Acoustic-visual Transform," in Working Notes Proceedings of the MediaEval 2013 Workshop, Barcelona, Spain, Oct. 18-19 2013, CEUR-WS.org, ISSN 1613-0073, http://ceur-ws.org/Vol1043/mediaeval2013_submission_15.pdf.

[28] Vu Lam, Duy-Dinh Le, Sang Phan, Shin'ichi Satoh, Duc Anh Duong,"NII-UIT at MediaEval 2013 Violent Scenes Detection Affect Task," in Working Notes Proceedings of the MediaEval 2013 Workshop, Barcelona, Spain, Oct.18-19 2013, CEUR-WS.org, ISSN 1613-0073, http://ceurws.org/Vol-1043/mediaeval2013_submission_27.pdf.

[29] Cédric Penet, Claire-Hélène Demarty, Guillaume Gravier, Patrick Gros, “Technicolor/INRIA Team at the MediaEval 2013 Violent Scenes Detection Task," in Working Notes Proceedings of the MediaEval 2013 Workshop, Barcelona, Spain, Oct. 18-19 2013, CEUR-WS.org, ISSN 1613-0073, http://ceur-ws.org/Vol-1043/mediaeval2013_submission_31.pdf.

[30] Technicolor, http://www.technicolor.com, last accessed Nov. 2013.

[31] Hanna M. Wallach, "Conditional Random Fields: An Introduction,"Technical Report MS-CIS-04-21, Department of Computer and Information Science, University of Pennsylvania, 2004.

[32] Jianchao Yang, Kai Yu, Yihong Gong and Thomas Huang,"Linear Spatial Pyramid Matching using Sparse Coding for Image Classification,"in IEEE Conference on Computer Vision and Pattern Recognition(CVPR),2009.

[33] David Arthur and Sergel Vassilvitskii,"k-means++: The Advantages of Careful Seeding," in SODA '07 Proceedings of the eighteenth annual ACM-SIAM symposium on Discrete algorithms, pp. 10271035, 2007. 


\section{AUTHORS}

Shinichi GOTO received his B.Sc in Information and Intelligent Systems from Tohoku University in 2011. He is currently a M.Sc student at Tohoku University. His research interests include video processing, machine learning and web technologies.

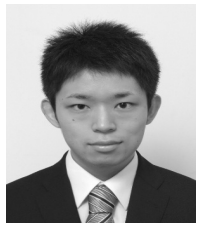

Terumasa AOKI is an associate professor at NICHe (New Industry Creation Hatchery Center), Tohoku University. He received his B.E, M.E and Ph.D degree from the University of Tokyo, in 1993, 1995 and 1998 respectively. He has received various academic excellent awards such as Young Scientist Award from MEXT (the Ministry of Education, Culture, Sports, Science and Technology in Japan, in 2007), Yamashita Award and the Best Education Award from IPSJ (Information Processing Society of Japan, in

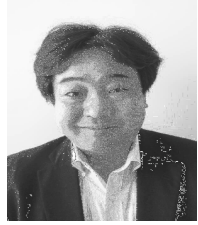
2001 and 2007 respectively), two Best Paper Awards from IIEEJ (the Institute of Image Electronics Engineers of Japan, in 2004 and 2009) etc. He is well known as the developer of DMD (Digital Movie Director) and NeoPoster etc. His current research topic is digital content technology, especially image processing, computer vision and computer graphics. 


\title{
HYBRID TECHNIQUE BASED ON N-GRAM AND NEURAL NETWORKS FOR CLASSIFICATION OF MAMMOGRAPHIC IMAGES
}

\author{
Pradnya Kulkarni ${ }^{1}$, Andrew Stranieri ${ }^{1}$, Siddhivinayak Kulkarni ${ }^{1}$, Julien \\ Ugon $^{1}$ and Manish Mittal $^{2}$ \\ ${ }^{1}$ Centre for Informatics and Applied Optimisation, Federation University \\ PO Box 663, Ballarat 3353, Victoria \\ p.kulkarni, a.stranieri, s.kulkarni,j.ugonefederation.edu.au \\ ${ }^{2}$ Lakeimaging, Ballarat, Victoria \\ mittalmelakeimaging.com.au
}

\begin{abstract}
Various texture, shape, boundary features have been used previously to classify regions of interest in radiological mammograms into normal and abnormal categories. Although, bag-ofphrases or n-gram model has been effective in text representation for classification or retrieval of text, these approaches have not been widely explored for medical image processing. Our purpose is to represent regions of interest using an n-gram model, then deploy the n-gram features into a back-propagation trained neural network for classifying regions of interest into normal and abnormal categories. Experiments on the benchmark miniMIAS database show that the n-gram features can be effectively used for classification of mammograms into normal and abnormal categories in this way. Very promising results were obtained on fatty background tissue with $83.33 \%$ classification accuracy.
\end{abstract}

\section{KEYWORDS}

N gram, Bag of Phrases, Neural Network, Mammograms, Image Processing

\section{INTRODUCTION}

Breast cancer is one of the major causes of death. Early detection is possible with the help of mammography [1]. There are vast amount of mammographic images generated every day. Detection of abnormality is a difficult and demanding job for the radiologists as the features of the abnormality are obscured or can be similar to those in a normal breast. There are always interobserver variations among the radiologists due to difference in their experience level. There are also intra-observer variations in reading mammograms caused by fatigue and stress. Although a computer program may never be able to achieve the level of knowledge and cognitive capability of a radiologist, it is better at doing certain tasks repetitively and consistently. Computer aided detection systems can thus be used as a second opinion for the radiologists to improve their interpretation accuracy. 
Computer aided detection systems usually use segmentation techniques to locate the region of interest, get the features and use the features to discover if the region is normal or abnormal. Different texture features such as histograms, co-occurrence matrix, gabor filters and wavelet transforms have been previously used for classification of regions of interest [2][3][4]. Classification of regions of interest is also useful for image retrieval task where a radiologist looks for similar past cases.

In the field of information retrieval, the bag-of-words concept was originally used in text retrieval systems. The document can be represented with the histograms of the words present in it. Forming phrases by grouping $\mathrm{n}$ sequential words, we get a bag-of-phrases model of a document. Thus n-gram is composed of $n$ sequential words. The use of n-grams is already proven to be very efficient in the natural language processing [5]. The same concept can be applied to the image retrieval or classification problem. An image can be represented with the help of n-grams or bagof-visual-phrases model. Pedrosa [6] shows that the n-gram representation can improve the retrieval performance over bag-of-words model for medical as well as non-medical image datasets.

The purpose of this paper is to extract $n$-gram features for the regions of interest in the miniMIAS dataset and use these features along with a back-propagation neural network to classify the regions into normal and abnormal categories. The paper is organised as follows. Section 2 details the literature review, research methodology is described in Section 3, Section 4 demonstrates experimental results and analysis of these results by varying $n$, effect of increasing grey levels and effect of background tissue on classification accuracy and Section 5 concludes the paper.

\section{LITERATURE REVIEW}

For detection of mammographic masses, the methods reported to date mainly utilize morphological features to distinguish a mass from the normal mammographic background [7]. Linear discriminant analysis is used in [8], where $76 \%$ sensitivity and $64 \%$ specificity was achieved on the mammographic dataset acquired from Michigan hospitals. Spatial grey level dependence matrix (SGLD) features along with artificial neural network were used to classify the regions of interest from miniMIAS dataset into normal and abnormal categories [9]. In this case 119 regions were used for the training (jack and knife method) and the remaining 119 regions of regions were used for testing purpose. Radial basis function neural network (RBFNN) provided classification accuracy of $78.15 \%$ whereas the Multilayer perceptron neural network (MPNN) provided classification accuracy of $82.35 \%$. In [10] Wong tried to classify mammographic regions of interests into mass or non-mass using texture features and artificial neural network. Four significant Grey level co-occurrence matrix (GLCM) features (correlation (0), angular second moment $(0)$, inverse difference moment (0) and correlation (45)) were used on the miniMIAS dataset. Using leave-one-out resampling on 50 ROIs, classification accuracy of $86 \%$ was achieved. An effort to find the most distinguishing textural features between normal and abnormal ROIs for miniMIAS dataset can be seen in work [11]. Using the multivariate $t$ test they found that the ASM, Correlation, Sum_Var and Diff_Entropy have the discriminating power for GLCM of distance 1, whereas Angular second moment is the only discriminating feature for GLCM of distance 3 .

Nithya experimented on a DDSM database of mammography to classify the ROIs into normal and cancerous categories using artificial neural network [12]. Their method used textural features (Correlation, Energy, Entropy, Homogeneity and sum of Square variance) to train a neural network with 200 mammograms (100 normal, 100 cancer) and tested this with 50 mammograms (25 normal, 25 cancer). Mass classification using gabor filter features and SVM for classification is reported in [13]. 
A bag of words model used effectively in text retrieval is further extended as Bag-of-visual-words for image classification and retrieval [14][15][16][17][18][19][20]. Researchers have taken various approaches for representing an image with the bag-of-words model. It has been used to analyse mammographic images in [21]. In this method using histogram intersection and SVM a classification accuracy of $73.3 \%$ was achieved. Wang reports that this bag-of-visual-words approach is successfully applied for classifying the breast images into BI_RADS categories [21]. This approach has also shown to be useful in lung image classification [23]. The bag of words model has proven to be useful for classification (88\% classification accuracy) of normal versus microcalcifications in mammographic images [24]. This approach is also been shown useful in the classification of focal liver lesions on CT images in [25]. In [26] raw intensities without normalization are used as local patch descriptors. The raw patches are then sampled densely with the stride of one pixel in the liver lesion region to form the BoW representation.

Extension of a bag-of-visual-words model is a bag-of-visual-phrase model also called n-gram model. This model has been investigated on various medical and non-medical datasets [6]. They used scale invariant Fourier transform (SIFT) features by identifying keypoints and then represented the image as a Bag of Visual Phrases model. This work shows that the bag-of-visualphrases model is more efficient in representing image semantics and improves the retrieval performance.

\section{RESEARCH METHODOLOGY}

This section describes various stages used for extracting n-gram features and classifying mammographic images into two classes: a) Normal and b) Abnormal. Figure 1 shows each component of the proposed technique.

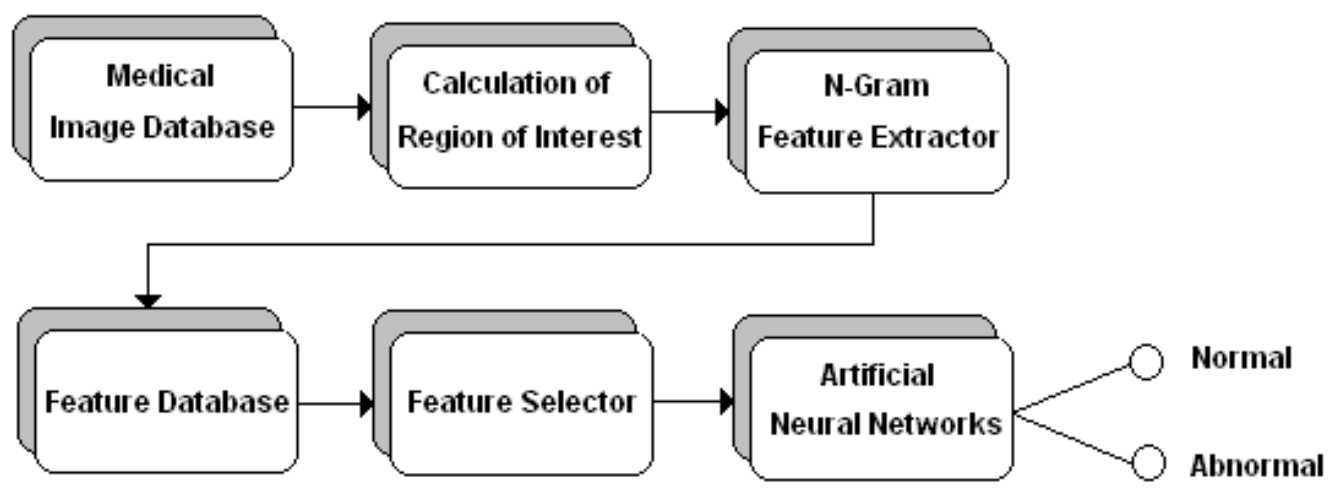

Figure 1. Block diagram of the proposed technique of n-gram feature extractor and ANN classifier

\subsection{Calculation of Region of Interest}

We are using benchmark miniMIAS database [27]. In this database abnormality is specified with the help of $\mathrm{x}$ and $\mathrm{y}$ coordinates of the centre of abnormality and a radius of circle enclosing the abnormality. Normal mammograms are also present. Equal size regions of $140 \times 140$ pixels around the abnormality are extracted. Figure 2 shows an example image with extraction of region of interest from a mammogram. For normal mammograms regions of $140 \times 140$ pixels are cropped from the centre of the mammogram. 


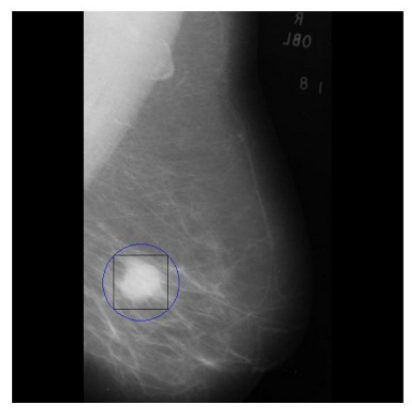

Figure 2: Example ROI extraction containing a circumscribed mass.

\subsection{Grey scale reduction}

The images are in portable grey map format. Each pixel is represented with a grey level between 0 to 256. For reducing computational complexity, the images are reduced to $\mathrm{G}$ number of grey level bins. To reduce the images to $\mathrm{G}$ number of grey level bins, the $\mathrm{i}^{\text {th }}$ bin is composed of grey levels illustrated by equation (1).

$$
\text { Bin } i=(i-1) * \text { binsize to }(i * \text { binsize })-1
$$

Where

$$
\text { binsize }=\frac{\text { highest greylevel-lowest greylavel }}{\text { number of bing }}
$$

\section{3 $\mathrm{N}$ gram feature extractor}

In text retrieval context, Word n-grams are a phrases formed by a sequence of $n$-consecutive words. E.g. 1-gram representation is composed of words such as \{mass, network, reporting\}. On the other hand, 2-gram is represented by sequence of two words for example \{benign mass, neural network, structured reporting\}. Phrases formed by $\mathrm{n}$ consecutive letters provide more complete representation of the document thereby increasing the retrieval performance. Character $\mathrm{n}$-grams are phrases formed by $\mathrm{n}$ consecutive characters. For example, the 3 -grams in the phrase "the fox" are "the, he_, e_f, _fo, fox"; the four grams are "the_f, he_f, e_fo, _fox". Algorithms for generating and counting n-grams are fast and efficient.

For our experiments, we consider grey scale intensity of a pixel is equivalent to one visual character. We can then represent the image in terms of sequence of n-consecutive intensity levels. We call this representation visual character n-gram model. One gram features would thus be similar to the histograms of each grey levels present in an image. Two gram features would consider every possible combination of the two grey levels adjacent to each other. This would closely relate to co-occurrence matrix features with an adjaceny distance of 1 . We restrict experiments in this study to three and four gram features.

The number of possible phrases is dependent on the grey level bins used to represent the image and can be calculated with the following equation.

$$
\text { num of possible phrases inn gram }=(\text { num of greylevel bins })^{n}
$$

Thus with the images reduced to 8 grey level bins we have 8 one gram features, 64 two gram features, 512 three gram features and 4096 four gram features for every region of interest 


\begin{tabular}{l}
\multicolumn{7}{c}{ Window 2 } \\
\begin{tabular}{|c|c|c||c|c|c|c|c|}
\hline \hline 105 & 100 & 102 & 100 & 103 & 101 & 102 & 103 \\
\hline \hline 100 & 98 & 101 & 99 & 100 & 100 & 103 & 99 \\
\hline 99 & 99 & 99 & 99 & 102 & 103 & 100 & 99 \\
\hline 99 & 98 & 99 & 103 & 103 & 102 & 101 & 99 \\
\hline
\end{tabular}
\end{tabular}

Figure 1: Sliding window for calculating three gram features

We then use sliding window of size $\mathrm{n}$ so that we are looking at phrase formed by $\mathrm{n}$ consecutive visual characters at a time. The count of how many times the visual phrase is repeated in an image is stored as its $\mathrm{n}$ gram features. Figure 3 shows how a sliding window of size 3 pixels is used to calculate 3 gram features of an image.

\subsection{Feature Normalization and Feature Database}

After calculating the n-gram features we normalize the features by dividing the count with the largest count in the available features so that feature values range between zero to one. The normalized features are stored in the feature database. For improving the classification accuracy, we selected the features which clearly distinguish between normal and abnormal ROIs.

\subsection{Artificial Neural Network Classifier}

Artificial neural networks have been used effectively for classification of images. Multilayer perceptron and radial basis function network have been used for classification of mammograms [9]. Different neural network algorithms are characterised by learning method and architecture of the network. The supervised learning neural network is efficient to learn the extracted n-gram features from normal and abnormal images. Supervised neural network is used for classifying the images into two categories (normal and abnormal). The input neurons are varied according to the number of features calculated for $\mathrm{n}$ - grams.

The normalized features are given as input to the neural network. The corresponding outputs were set to 1 depending upon normal or abnormal ROI features present at the input. Classification accuracy is calculated with the following equations, where $n_{a b}$ is the number of correctly classified abnormal ROIs, $n_{n}$ is the number of correctly classified normal ROIs, $N_{a b}$ is the total number of abnormal ROIs present and $N_{n}$ is the total number of normal ROIs.

$$
\begin{aligned}
& \text { Abnormal Classification Accuracy }=n_{a b} / N_{a b} \\
& \text { Nornal Classification Accuracy }=n_{n / N_{n}} \\
& \text { Overall Classification Accuracy }=\frac{n_{a b}+n_{n}}{N_{a b}+N_{n}}
\end{aligned}
$$

\section{EXPERIMENTAL RESULTS AND ANALYSIS}

This section details various experiments and analysis of the results conducted on Mini-MIAS benchmark medical image database. 


\subsection{Mini-MIAS Database and Image Pre-processing}

The Mammographic Image Analysis Society, UK has provided Mini- MIAS as benchmark medical image database for research purposes [27]. The mammograms have been reduced to 200 micron pixel edge and clipped or padded so that every image is $1024 \times 1024$ pixels. Abnormality area or region of interest (ROI) is specified with the help of $x, y$ image coordinates of centre of abnormality and the radius of a circle enclosing the abnormality. Information about the type of abnormality such as calcification, mass (circumscribed, speculated or ill-defined), architectural distortion or asymmetry is specified in the database. Type of Background tissue such as Fatty, Fatty-glandular, Dense-glandular has also been mentioned for each mammogram. Images of normal mammograms are also present in the database. The images are in portable grey map format which can be directly read using matlab software.

For our experiment we cropped a square of size $140 \times 140$ pixels around the centre of the abnormality. For normal mammograms a square of size $140 \times 140$ pixels was cropped from the centre of the mammogram. Example abnormal and normal ROIs are shown in Figure 4.
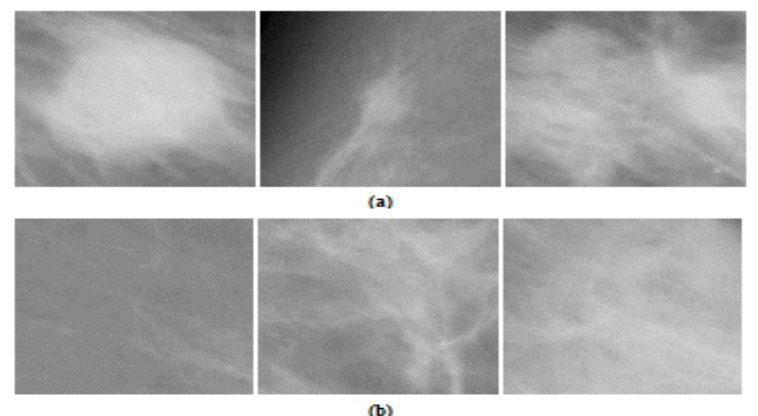

Figure 4 (a): Abnormal ROIs and (b): Normal ROIs

\section{2 $\mathrm{N}$ gram feature extraction}

The regions of interests were reduced in grey scale levels for reducing computational complexity. The n-gram features are then calculated from the grey scale reduced regions of interests. However, all possible phrases may not be present in a corpus and hence the vocabulary of visual phrases reduces significantly reducing the computational complexity of the system. Table 1 shows the number of possible visual phrases and the actual phrases present in the corpus with different grey level bins used. The features which are helpful in clearly distinguishing between normal and abnormal ROIs are selected.

Table 1: Number of possible and actual phrases for 3 gram representation

\begin{tabular}{|c|c|c|}
\hline $\begin{array}{c}\text { Grey } \\
\text { levels }\end{array}$ & $\begin{array}{c}\text { Number of possible } \\
\text { visual phrases }\end{array}$ & $\begin{array}{c}\text { Number of visual } \\
\text { phrases present in the } \\
\text { corpus }\end{array}$ \\
\hline 8 & 512 & 35 \\
\hline 16 & 4096 & 82 \\
\hline 24 & 13824 & 137 \\
\hline
\end{tabular}




\subsection{Classification using artificial neural network}

The selected n-gram features are used as inputs for the neural network. The output of the neural network then shows the classification of regions into normal and abnormal categories. Various experiments are carried out to see the effect of changing $n$, background tissues and grey scale levels. Learning rate, momentum and number of iterations are varied to get the maximum output accuracy. The optimum results were obtained with the following settings for six input neurons and 2 output neurons as shown in Table 2 .

Table 2: Neural network parameters

\begin{tabular}{|l|l|}
\hline Neural Network Parameters & Values \\
\hline Learning Rate & 0.3 \\
\hline Momentum & 0.7 \\
\hline Iterations & 3000 \\
\hline
\end{tabular}

\subsection{Effect of number of words in $n$ grams on classification accuracy}

To see the effect of increasing the number of words in a visual phrase, we used 102 regions of interests with fatty background tissue. We used 3 fold cross validation technique to test the classification accuracy. Out of 102, 69 images were used for training and 33 images were used for testing purposes in each fold. To analyse the effect of increasing the grey level bins, three gram features were used. The following table shows that classification accuracy did not increase significantly with the increase in the number of grey level bins used.

Table 1: Effect of increase in number of grey level bins on classification accuracy

\begin{tabular}{|c|c|c|c|}
\hline & \multicolumn{3}{|c|}{ Classification accuracy } \\
\hline $\begin{array}{c}\text { Number of grey } \\
\text { level bins }\end{array}$ & Abnormal & Normal & Overall \\
\hline 8 & $76.19 \%$ & $66.66 \%$ & $69.56 \%$ \\
\hline 16 & $76.19 \%$ & $68.75 \%$ & $71.01 \%$ \\
\hline 24 & $76.19 \%$ & $64.58 \%$ & $68.11 \%$ \\
\hline
\end{tabular}

Hence we reduce the ROIs to 8 grey level bins. Table 4 shows the effect of increasing $n$ on the classification accuracy. 
Table 4: Effect of number of words in n-gram on classification accuracy

\begin{tabular}{|c|c|c|c|c|}
\hline & \multicolumn{3}{|l|}{ Classification accuracy } \\
\hline $\begin{array}{c}\text { Number of consecutive } \\
\text { visual words in a } \\
\text { phrase }\end{array}$ & $\begin{array}{c}\text { Number of } \\
\text { possible } \\
\text { phrases }\end{array}$ & Abnormal & Normal & Overall \\
\hline Onegram & 8 & $76.19 \%$ & $64.58 \%$ & $68.11 \%$ \\
\hline Twogram & 64 & $76.19 \%$ & $64.58 \%$ & $68.11 \%$ \\
\hline Threegram & 512 & $80.95 \%$ & $66.66 \%$ & $73.81 \%$ \\
\hline Fourgram & 4096 & $85.71 \%$ & $70.83 \%$ & $78.27 \%$ \\
\hline
\end{tabular}

\subsection{Effect of background tissue change on classification accuracy}

There are three types of background tissues in mammograms. To analyse the effect of background tissue on classification accuracy, we used 33 training pairs and 12 testing pairs with 3 fold cross validation technique. We found that there are six pure sequence three gram features which distinguish between normal and abnormal regions quite effectively. These six pure sequence three gram features are used as inputs to the neural network. Table 5 shows the results.

Table 5: Effect of background tissue on classification accuracy

\begin{tabular}{|l|l|l|l|}
\hline & \multicolumn{3}{|l|}{ Classification accuracy } \\
\hline Background tissue & Abnormal & Normal & Overall \\
\hline Fatty & $83.33 \%$ & $83.33 \%$ & $83.33 \%$ \\
\hline Fatty Glandular & $50.00 \%$ & $61.90 \%$ & $58.62 \%$ \\
\hline Dense Glandular & $50.00 \%$ & $50.00 \%$ & $50.00 \%$ \\
\hline
\end{tabular}

\section{CONCLUSION AND FUTURE RESEARCH}

A new feature extraction technique called visual character n-gram is proposed for classification of mammograms into normal and abnormal categories. The regions of interests were reduced in grey scale levels to reduce computations complexity. A back-propagation neural network was used for classification purpose. Three fold cross validation technique was used to test the classification accuracy. Number of experiments was conducted for extracting n-gram features and classification of these features using neural networks. Very promising results were obtained on fatty background tissue with $83.33 \%$ classification accuracy on Mini-MIAS benchmark database. Although, direct comparisons with other neural network and co-occurance matrix approaches reported in the literature are not immediately possible because each study deploys different image sets and ROI, however the classification accuracies obtained here are promising and warrant further examination. As the number of visual words in a phrase increases, significant improvement in overall classification accuracy is observed. Results are analysed by changing various parameters. Future research will incorporate investigating distinguishing n-gram features for improving classification accuracy for fatty glandular and dense glandular background tissue. 
We would like to use n-gram text features of the associated radiology reports and investigate effect of fusion of n-gram image features and n-gram text features to improve classification accuracy.

\section{REFERENCES}

[1] Bovis, K., Singh, S., Fieldsend, J., \& Pinder, C. (2000) "Identification of masses in digital mammograms with MLP and RBF nets." Proceedings of the IEEE-INNS-ENNS International Joint Conference (IJCNN), Vol 1, pp. 342-347.

[2] Müller, H., Michoux, N., Bandon, D., \& Geissbuhler, A. (2004) "A review of content-based image retrieval systems in medical applications-clinical benefits and future directions." International Journal of Medical Informatics, Vol. 73, No. 1, pp. 1-23.

[3] Cheng, H. D., Shi, X. J., Min, R., Hu, L. M., Cai, X. P., \& Du, H. N. (2006) "Approaches for automated detection and classification of masses in mammograms." Pattern Recognition, Vol. 39, No. 4, pp. 646-668.

[4] Akgül, C. B., Rubin, D. L., Napel, S., Beaulieu, C. F., Greenspan, H., \& Acar, B. (2011) "Contentbased image retrieval in radiology: current status and future directions." Journal of Digital Imaging, Vol. 24, No. 2, pp. 208-222.

[5] Suen,C. (1979) "n-gram statistics for natural language understanding and text processing," IEEE Transactions on Pattern Analysis and Machine Intelligence, Vol. 1, No. 2, pp. 164-172.

[6] Pedrosa, G. V., \& Traina, A. J. (2013) "From Bag-of-Visual-Words to Bag-of-Visual-Phrases using nGrams." In $26^{\text {th }}$ Graphics, Patterns and Images (SIBGRAPI), pp. 304-311.

[7] Chan, H. P., Wei, D., Helvie, M. A., Sahiner, B., Adler, D. D., Goodsitt, M. M., \& Petrick, N. (1995) "Computer-aided classification of mammographic masses and normal tissue: linear discriminant analysis in texture feature space." Physics in medicine and biology, Vol. 40, No. 5, pp. 857-876.

[8] Petrosian, A., Chan, H., Helvie, M., Goodsitt, M., Adler, D. (1994) "Computer-aided diagnosis in mammography: classification of mass and normal tissue by texture analysis." Physics in Medicine and Biology, Vol. 39, No. 12, pp. 2273-2288.

[9] Christoyianni, I., Dermatas, E., \& Kokkinakis, G. (1999) "Neural classification of abnormal tissue in digital mammography using statistical features of the texture." In Electronics, Circuits and Systems, Proceedings of ICECS'99, Vol 1, pp. 117-120.

[10] Wong, M., Nguyen, H., \& Heh, W. (2006) "Mass Classification in Digitized Mammograms Using Texture Features and Artificial Neural Network." Journal of Pattern Recognition, Vol. 39, No. 4, pp. 646-668.

[11] Wei, C. H., Li, C. T., \& Wilson, R. (2005) "A general framework for content-based medical image retrieval with its application to mammograms.” In Proceedings of SPIE, Vol. 5748, pp. 135-142.

[12] Nithya, R., \& Santhi, B. (2011) "Classification of normal and abnormal patterns in digital mammograms for diagnosis of breast cancer." International Journal of Computer Applications, Vol. 28, No. 6, pp. 21-25.

[13] Hussain, M., Khan, S., Muhammad, G., Berbar, M., \& Bebis, G. (2012) "Mass detection in digital mammograms using gabor filter bank.” In IET Conference Image Processing (IPR 2012), pp. 1-5.

[14] Caicedo, J., Cruz-Roa, A., and Gonzalez, F. (2009) "Histopathology image classification using bag of features and kernel functions," International Conference on Artificial Intelligence in Medicine, Lecture Notes in Computer Science, Vol. 5651, pp. 126-135.

[15] Jégou,H., Douze, M. and Schmid, C. (2010) "Improving bag-of-features for large scale image search," International Journal of Computer Vision, Vol. 87, No. 3, pp. 316-336.

[16] Nister, D. and Stewenius,H. (2006) "Scalable recognition with a vocabulary tree," in IEEE Conference on Computer Vision and Pattern Recognition (CVPR), pp. 2161-2168.

[17] Philbin, J., Chum, O., Isard,M., Sivic, J. and Zisserman, A.(2007) "Object retrieval with large vocabularies and fast spatial matching," IEEE Conference on Computer Vision and Pattern Recognition (CVPR), pp. 1-8.

[18] Rahman, M., Antani, S. and Thoma,G.(2011) "Biomedical cbir using "bag of keypoints" in a modified inverted index," International Symposium on Computer-Based Medical Systems, pp. 1-6.

[19] Sivic, J. and Zisserman, A. (2003) "Video google: A text retrieval approach to object matching in videos," International Conference on Computer Vision, Vol. 2, pp. 1470-1477. 
[20] Wang, J., Li, Y., Zhang,Y., Xie,H. and Wang,C.(2011) "Boosted learning of visual word weighting factors for bag-of-features based medical image retrieval,"TTe)s691(m)-.3h1( )-83.882(r)-0.881(,)-19.66(C)TJ 208.ho 


\title{
NATURAL LANGUAGE PROCESSING THROUGH DIFFERENT CLASSES OF MACHINE LEARNING
}

\author{
Harsh Jain and Keshav Mathur \\ Department of Information Science and Engineering, \\ BMS College of Engineering, Bangalore, India \\ harshjain30@gmail.com \\ keshavmathur@outlook.com
}

\begin{abstract}
The internet community has been benefitting tremendously from the works of various researchers in the field of Natural Language Processing. Semantic orientation analysis, sentiment analysis, etc. has served the social networks as well as companies relying on user reviews well. Flame identification has made the internet less hostile for some users. Spam filtering has made the electronic mail a more efficient means of communication. But with the incessant growth of the internet, NLP using machine learning working on massive sets of raw and unprocessed data is an ever-growing challenge. Semi-supervised machine learning can overcome this problem by using a large set of unlabeled data in conjunction with a small set of labeled data. Also, focusing on developing NLP systems that can contribute to developing a unified architecture could pave the way towards General Intelligence in the future.
\end{abstract}

\section{KEYWORDS}

Semantic Orientation, Social Networks, E-mail, Sentiment Analysis, Unsupervised Machine Learning

\section{INTRODUCTION}

Natural Language Processing (NLP) is a field of Artificial Intelligence that deals with the interactions between natural languages and computers. The purpose of NLP is to enable machines to process an input in natural or human language and derive meaning from it.

Modern NLP algorithms are based on machine learning and are designed to automatically learn rules for generating an output from a given input through the analysis of large corpora of labelled or unlabelled examples. Many different classes of machine learning algorithms have been applied to NLP tasks. They can be organized based on the type of input for training in the following way:

- Supervised learning algorithms, where the input comprises of labelled examples. The output for previously unseen inputs is generated by using a general function or mapping from inputs to outputs created with the help of the training data.

- Unsupervised learning algorithms, where the input comprises of unlabelled examples. The desired output is unknown and produced by discovering structure in the data.

- Semi-supervised learning, which uses a combination of both labelled and unlabelled examples.

David C. Wyld et al. (Eds) : CCSIT, SIPP, AISC, PDCTA, NLP - 2014

pp. 307-315, 2014. (C) CS \& IT-CSCP 2014

DOI : $10.5121 /$ csit.2014.4226 
With the internet boom and increasing popularity of social networks, analysis of the written word has become more significant. Tools that can perform various tasks related to the internet, such as identifying spam, analysing the semantic orientation of words and sentiment analysis, have become highly useful. In this paper, various supervised, unsupervised and semi-supervised machine learning approaches for performing such tasks have been outlined. It also identifies their application and possible improvements in the domain of social networks.

\section{DISCUSSIONS}

According to UNESCO as well as BBC, there are about 7,000 different languages in the world. With copious amounts of documents produced in a many languages every day, a mechanism that could relate documents in different languages and indicate their similarity would serve as a great tool. A language-independent supervised approach to controlled vocabulary keyword assignment using the EUROVOC thesaurus, whose multi-lingual nature allows cross-language document comparison was presented by Ralf Steinberger [1]. In the first step, which is keyword assignment, if a certain word occurs significantly more often in a given text than it occurs, on average, in a large selection of 'normal' texts (the reference corpus), it was identified as a keyword. Both the documents are lemmatised for this. Lists of stop words and multi-word expressions were also included. Then, using the EUROVOC thesaurus, frequent words were mapped to associates, which were multiplied by their keyness or relevance to the document to generate ranks and identify the descriptors of the document. Although the author could not conduct extensive tests on his algorithm, the initial tests conducted on the corpus gave satisfactory results. One inherent drawback of automated keyword generation is that there is no standard way of testing the accuracy of the results produced by such an algorithm, since it is well-known that no set generated manually can be claimed as the right set for a document. Also, the corpus that was used by the author pertains to a very specific legalistic and administrative sublanguage. This algorithm with some optimization has vast potential for use in cross-language document search and comparison.

When it comes to application of NLP to the web and social networks, mechanisms to identify the semantic orientation of words are particularly useful. In a supervised approach by Hatzivassiloglou and McKeown [2], it was proposed that there are constraints on the semantic orientations of conjoined adjectives. Using corpus data, the hypothesis was verified and the results were found to be extremely significant statistically, except for a few cases where the sample was small. Next, these constraints were used to construct a log-linear regression model automatically. This model, with the combination of supplementary morphology rules, predicted whether two conjoined adjectives are of same or different orientation. An appreciable accuracy of $82 \%$ was achieved. Several sets of adjectives were classified according to the links inferred in this way and labelled as positive or negative. A remarkable $92 \%$ accuracy was achieved on the classificationtas $\mathrm{k}$ for reasonably dense graphs and $100 \%$ accuracy on the labelling task. But in the classification of adjectives, the number of times the method accurately classified a test set dropped when the data was sparser, ranging from $92 \%$ to $78 \%$.A strong point of this method is that decisions on individual words are aggregated to provide decisions on how to group words into a class and whether to label the class as positive or negative.

Hatzivassiloglou and McKeown's work was extended by Hatzivassiloglou with Janyce M. Wiebe [16]. Subjectivity in natural language refers to aspects of language used to express opinions and evaluations. This paper studied the effects of dynamic adjectives, semantically oriented adjectives and gradable adjectives on a simple subjectivity classifier and established that they are strong predictors of subjectivity. Since the mere presence of one or more adjectives is useful in predicting that a sentence is subjective, this paper investigated the effects of additional lexical semantic features of adjectives. It considered two such features: semantic orientation, which 
represents an evaluative characterization of a word's deviation from the norm for its semantic group; and gradability, which characterizes a word's ability to express a property in varying degrees. For gradability, two indicators were used: grading modifiers such as very and inflected forms of adjectives. In the experiment all adjectives with a frequency of 300 or more from the 1987 Wall Street Journal corpus (21 million words) were extracted; producing a list of 496 words. 453 of the 496 adjectives (91.33\%) were assigned gradability labels by hand (using the designations of the Collins COBUILD dictionary), while the remaining 53 words were discarded. The method automatically assigned labels to the entire set of 453 adjectives, using 4-fold crossvalidation with resulted precision of $94.15 \%$, recall of $82.13 \%$, and accuracy of $88.08 \%$ for the entire adjective set. In the experiment they measure the precision of a simple prediction method for subjectivity: a sentence is classified as subjective if at least one member of a set of adjectives $\mathrm{S}$ occurs in the sentence and objective otherwise. By varying the set $\mathrm{S}$ (e.g., all adjectives, only gradable adjectives, only negatively oriented adjectives, etc.), it is noted that all sets involving dynamic adjectives, positive or negative polarity, or gradability are better predictors of subjective sentences than the class of adjectives as a whole. This kind of analysis could help efforts to encode subjective features in ontologies. The authors had begun to incorporate the features developed into systems for recognizing flames and mining reviews in Internet forums. They were seeking ways to extend the orientation and gradability methods so that individual word occurrences, rather than word types, are characterized as oriented or gradable. They also planned to incorporate the new features in machine learning models for the prediction of subjectivity and test their interactions with other proposed features.

Wiebe also independently came up with a supervised approach for subjectivity [17].The author claims that subjectivity tagging is about distinguishing sentences that are used to present opinions and evaluations from sentences used to present factual information. Her previous work on subjectivity (Wiebe, Bruce, \& O'Hara 1999; Bruce\&Wiebe 2000) had established a relation between subjectivity and the presence of adjectives in a sentence. This paper identified higher quality adjective features using the results of a method for clustering words according to distributional similarity (Lin 1998), seeded by a small amount of detailed manual annotation. These features were then further refined with the addition of lexical semantic features of adjectives, specifically polarity and gradability, which could be automatically learned from corpora. For the experiment a corpus of 1,001 sentences of the Wall Street Journal Treebank Corpus (Marcus et al. 1993) was manually annotated with subjectivity classifications and also the strength of subjective elements was manually rated on a scale of 1 to 3 . The experiment, with a simple adjective feature where a sentence is subjective if one adjective is found, had a precision of 55.8\%. With the improvements in adjective features using Distributional Similarity where a sentence is labelled as subjective if an adjective from the seed sets is found, it had a precision of $61.2 \%$ (a significant improvement). Further improvements can be made with addition of lexical semantic features of adjectives (polarity and gradability). Both features used together showed an accuracy of $71 \%$, displaying together they are more precise. Previous work on subjectivity had been focused on many applications like recognizing flames, mining Internet forums for product reviews, and clustering messages by ideological point of view. Wiebe's approach in this paper was directed at supplementing such endeavors by developing a repository of potential subjective elements to enable us to exploit subjective language. The adjectives learned by the experiment were being incorporated into a system for recognizing flames in Internet forums. In addition; the author planned to apply the method to a corpus of Internet forums, to customize knowledge acquisition to that genre.

Hatzivassiloglou's, McKeown's and Wiebe's work was based on supervised machine learning and required training data. Peter D. Turney [3]presented a paper in 2002 that proposed an unsupervised learning algorithm for semantic orientation. It also wasn't limited to just adjectives. The general strategy in this paper was to infer semantic orientation from semantic association. The semantic orientation of a given word was calculated from the strength of its association with 
some chosen positive words, minus the strength of its association with some chosen negative words. This general strategy is called SO-A (Semantic Orientation from Association). This paper also examined SO-PMI-IR (Semantic Orientation from Point-wise Mutual Information and Information Retrieval) and SO-LSA (Semantic Orientation from Latent Semantic Analysis).The experiments suggested that the SO-PMI-IR can reach the same level of accuracy as Hatzivassiloglou and McKeown's, given a sufficiently large corpus. The results also hinted that SO-LSA is able to use data more efficiently than SO-PMI-IR, and SO-LSA might surpass the $80 \%$ accuracy attained by SO-PMI-IR, given a corpus of comparable size.

PMI-IR was proposed, and compared with LSA, by Turney in his previous work [6].In this paper, PMI-IR was presented as a new unsupervised algorithm for recognizing the closest synonym of a given problem word from a given option set. It achieved this by using PMI algorithm that analyzed statistical data collected by Information Retrieval (IR). The performance was evaluated by testing it on 80 synonym test questions from the Test of English as a Foreign Language (TOEFL) and 50 synonym test questions from a collection of tests for students of English as a Second Language (ESL). It was then compared to that of Latent Semantic Analysis (LSA) on the same question sets. LSA is a statistical algorithm based on Singular Value Decomposition (SVD). A variation on this algorithm has been applied to information retrieval, where it is known as Latent Semantic Indexing (LSI).[5] The paper also evaluated four different versions of PMI-IR, using four different kinds of queries to the Alta Vista search engine. The first described cooccurrence as words being present in the same document, second one used the NEAR operator, the third removed the problem of antonyms being rated same as synonyms and the fourth one took context into account.

The PMI-IR algorithm, like LSA, is based on the concept of co-occurrence. The core idea is that "a word is characterized by the company it keeps". There are many well-known lexical databases that include synonym information. But these have a problem of sparse data and the need for extensive manual labor for each language. Several hybrid approaches have been proposed which combine statistical and lexical information. PMI-IR addresses the sparse data problem by using a huge data source: the Web.

The results with the TOEFL questions showed that PMI-IR (in particular for the query which removes the antonym problem) can score almost 10\% higher than LSA. However, the interpretation of the results was difficult, due to two factors: (1) PMI-IR was using a much larger data source than LSA. (2) PMI-IR was using a much smaller chunk size than LSA. A future work that emerges from this is seeing how LSA would perform for such a large data source like the one used for PMI and also test the hypothesis by Landauer and Dumais [4] who claim that mutual information analysis would obtain a score of about $37 \%$ on the TOEFL questions, given the same source text and chunk size as they used for LSA. PMI-IR may prove to be suitable as a tool to aid in the construction of lexical databases and automatic keyword extraction. It might also be useful for improving IR systems.

Turney's algorithm [3] could have many potential applications, such as filtering "flames" for newsgroups, improving Tong's system for generating sentiment timeline[7], in the analysis of survey responses to open ended questions, in an automated chat system to help decide whether a positive or negative response is most appropriate or to classify reviews.

Turney used his PMI-IR algorithm in an approach for classification of reviews [8]. The simple unsupervised learning algorithm took as input a written review and generated a classification (thumbs up or thumbs down) as the output. The classification was based on the average semantic orientation of the phrases in a review that contained adjectives or adverbs. The first step was to use a part-of-speech tagger to identify phrases in the input text that contain adjectives or adverbs. The second step was to evaluate the semantic orientation of each extracted phrase. This was done 
using the PMI-IR algorithm. A phrase was considered to have a positive semantic orientation when it had good associations(e.g., "subtle nuances") and a negative semantic orientation when it had bad associations (e.g., "very cavalier"). The final step was the classification of a review as recommended(thumbs up) or not recommended(thumbs down) based on the average semantic orientation of all the extracted phrases.

The algorithm was evaluated on 410 reviews from Epinions (all from unique users) from four different domains (automobiles, banks, movies, and travel destinations).It achieved an average accuracy of $74 \%$ ranging from $84 \%$ for automobile reviews to $66 \%$ for movie reviews.

Turney's algorithm is useful in labelling an input text as positive or negative. But to some domains in the online community, this is not enough. Companies around the world have turned to the internet to obtain reviews for their products. They require much more information than just the overall sentiment about the topic. For example, a smart phone manufacturing company might want to analyse its public discussion forums and people's reviews on its social network fronts to find out the specific features of the phone that are favoured by its users. This makes the tools for extraction of sentiments about a given topic almost indispensable to this community. A "Sentiment Analyzer" was proposed by Jeonghee Yi et al [9]. Sentiment Analyzer (SA) extracted sentiment (or opinion) about a subject from online text documents. The authors believe that although the overall opinion about a topic is useful, it is only a part of the information of interest. So, instead of classifying the sentiment of an entire document about a subject, SA detected all references to the given subject, and determined sentiment in each of the references. The paper anticipated shortcomings of the purely statistical approaches and showed that the analysis of grammatical sentence structures and phrases based on NLP techniques mitigated some of the shortcomings. To extract the feature terms form a document, only the nouns were selected from the document. Then, one of the two feature term selection algorithms, one based on the mixture language model by Zhai and Laffertry [10]; and the other based on the likelihood-ratio test by Dunning [11], were applied. The extracted feature terms by each of the algorithms were manually examined by two human subjects and their precision and accuracy were tabulated. The Likelihood Test method consistently performed better than the Mixture Model algorithm. The next step was identifying the sentiment phrase and the assignment of the sentiment to a subject. For this, $S A$ used sentiment terms defined in the sentiment lexicon and sentiment patterns in the sentiment pattern database. The sentiment lexicon contained the sentiment definition of individual words collected from various sources. The sentiment pattern database contained sentiment extraction patterns for sentence predicates. For each sentiment phrase detected, $S A$ determined its target and final polarity based on the sentiment pattern database. $S A$ first identified the Ternary-expression for the statement, and tried to find matching sentiment patterns. Once a matching sentiment pattern was found, the target and sentiment assignment were determined as defined in the sentiment pattern.SA consistently demonstrated high quality results of $87 \%$ for review articles, 86 $\sim 91 \%$ (precision) and $91 \sim 93 \%$ (accuracy) for the general web pages and news articles. The results on review articles were comparable with the state-of-the-art sentiment classifiers, and the results on general web pages were better than those of the state of the art algorithms by a wide margin. The more advanced sentiment patterns required a fair amount of manual validation. In the future, full parsing could provide better sentence structure analysis, thus better relationship analysis.

Most social networks allow the users to communicate verbally. Although, a lot of them let the users decide whether something is inappropriate for public discussions (flagging), an intelligent system could help the system determine basic conspicuous inappropriate content. Such messages could contain the use of abusive language. Some of them could be directed towards a person, perhaps in a private message, not using any abusive language, but of hostile nature. These messages could be identified and filtered through supervised machine learning, thus reducing the need for user involvement in the process. Ellen Spertus presented aprototype system 
"Smokey"[12], which analyses the syntax and semantics of each sentence for 47 pre-listed features and builds a vector accordingly. It then combines the vectors for the sentences within each message. For training, a set of 720 messages was used by Quinlan's C4.5 decision-tree generator to determine the feature-based rules used to categorize the messages as "flame" or "okay". The test set consisting of 460 messages was then categorized by the system, as well as manually for accuracy analysis. Smokey was able to correctly categorize $64 \%$ of the flames and $98 \%$ of the non-flames in the test. There are certain limitations that this system was unable to overcome, such as recognizing sarcasm and innuendo and making sense of complex sentences and mistakes in grammar, punctuation, and spelling. In the future, this system could learn from dictionaries and thesauri, user feedback, or proximity to known insults. It could also benefit from morphological analysis, spelling and grammar correction and analysing logical parse trees of sentences.

A system like Smokey could also be used to prioritize mail and help in the identification of spam mail. Internet subscribers world-wide are unwittingly paying an estimated $€ 10$ billion a year in connection costs just to receive "junk" emails, according to a study undertaken for the European Commission [13]. Many machine learning approaches have already been suggested to help identify and get rid of spam emails. A lot of them rely on the keyword-based approach, where certain keywords found in spam emails are used to identify other spam emails. But there is an inherent flaw in using keyword-based approach: if the keywords that are considered for a mail to be marked as spam are known, spammers could actively work to avoid their inclusion in their mails. It could be seen as an "arms race", where the spammers continuously identify and avoid the keywords that the anti-spam systems consider and the system engineers continuously try to find and add new keywords to stay one step ahead of the spammers. Ion Androutsopoulos et al investigated the performance of two machine-learning algorithms, the Naïve Bayesian approach and a memory-based classification approach in the context of anti-spam filtering, and demonstrated how both are better than the keyword-based filter [14]. An experiment was conducted for the same, which used a benchmark corpus consisting of both spam as well as legitimate mails. An important note was the cost of a mistake: a legitimate mail marked as spam is much more undesirable than a spam passing as legitimate. This difference factor was denoted by $\lambda$. In the Naive Bayesian approach, the corpus was pre-processed in which Baye's theorem and the theorem of total probability were employed. In the memory-based classification approach, a variant of the simple $k$-nearest-neighbour $(k$-nn) algorithm was used. The experiment used the algorithm implemented in the TiMBL software. Next, formulae were derived for parameters like Weighted Accuracy and Weighted Error, as well as Total Cost Ratio, which were used to compare a filter's performance with the baseline (when no filter is used). $\lambda$ was varied creating three different scenarios and in each scenario, the number of selected attributes was varied between 50 and 700. In all three scenarios, the two aforementioned approaches performed better than the keyword-based approach, and except the scenario in which $\lambda$ was very high, also better than the no filter approach. In the future, alternative learning methods for the same task could be examined, including attribute-weighted versions of the memory-based algorithm. Alternative attribute selection techniques including term extraction methods to move from word to phrasal attributes can also be explored.

Md. Saiful Islamet al [15] in a study investigated the possibilities of modelling spammer behavioural patterns instead of vocabulary as features for spam email categorization, as they believe that keyword-based approach will eventually be less successful as spammers will try and circumvent the filters that such models will employ. The three well-known machine learning algorithms; Naïve Bayes, DTI and SVMs; were experimented to model common spammer patterns. Common spammer techniques were listed and a model was developed exploiting machine learning algorithms to capture common spammer patterns. 21 such patterns were extracted from each of the 1000 mails consisting of equal number of spam and legitimate mails. Accuracy, Precision and Recall were calculated for each of the three methods using the corpus 
and it was found that Naïve Bayesian classifier is more efficient than the other two classifiers. Building a perfect data set free from noise or imperfection remains a continuous challenge for spam filtering techniques as noise adversely affect the classifier's performance. Also, most training models of the classifier have limitations on their operations .Naïve Bayes has the advantage of incremental inclusion and/or exclusion of features and DTIoffers best expressive power. So, natural progression will be combining these two MLalgorithms in multi-core architecture, running both classifier simultaneously indifferent cores to minimize time and applying voting mechanism to increase positivity, which will give best opportunity to model spammer common patterns. Multi-classifier based spam filters exploiting spammer behavioural patterns can also be developed.

The very big challenge in pattern recognition task and machine learning process is to train a discriminator using labeled data. However, real world problems have largely raw and unprocessed data and labeling them becomes almost impossible. Semi-supervised learning overcomes this problem by using a small set of labeled data along with a huge set of unlabeled data to train the discriminator.

In a paper, Shafigh Parsazad et al [18] proposed an evolutionary approach called Artificial Immune System (AIS) to determine which data is better to be labeled to get the high quality data. The human immune system was an inspiration for this paper because it is robust, adaptable and automatic. Immune system consists of white blood cells which are responsible for detection and elimination of Antigens. These are called Anti bodies. The immune system has a memory to save its work. In the paper, a modified version of the aiNet algorithm proposed by Younsi (called aiNetC) is used. aiNetC works on the principle that more the similarity between the antibody and the antigen more is the strength of connection between them. The measure of similarity between antibody and antigen cells is called Affinity. An affinity threshold in antibody detection process called Network Affinity Threshold (NAT) is defined. If the affinity of a given antibody and antigen is lower than NAT, it's assumed the antibody recognized the antigen. It iterates over all antigens till all are recognized or a number of generations reached and after this antibodies too close to each other are eliminated. The main purpose of aiNetC is clustering. The algorithm proposed by the authors modifies aiNetC so that instead of clustering the data it tries to describe them with very few antibodies. They argue that if these antibodies have labels, the accuracy of the clustering will be greatly improved. Another advantage of such a method in semi-supervised learning is that labeling the data will not be random and it is done with the lowest possible information that can be provided. For experimentation, two semi-supervised learning algorithms were included: semisupervised KMeans as the clustering algorithm and semi-supervised support vector machines as the classification algorithm. Five datasets were used. First of all random set of labels were generated and fed into these algorithms. Then the algorithm was used for analyzing the data and all datasets were fed to aiNetC algorithm. After analyzing, aiNetC algorithm recommended some data to be labeled by the user to achieve the best result. This labeled data was again fed to the learning algorithms as information that we have from dataset. Result of this learning was measured. Experimental results showed the vast improvements in the results.

Most NLP systems focus on individual NLP tasks and very few have of them have characteristics that could contribute to developing a unified architecture. In a paper [19], Ronan Collobert and Jason Weston defined a rather general convolutional network architecture and described its application to many well-known NLP tasks including part-of-speech tagging, chunking, namedentity recognition, learning a language model and the task of semantic role-labeling (SRL) and all these tasks were integrated into a single system which was trained jointly. The main focus of this paper is SRL. Complex tasks like this require large number of complex features which make traditional NLP systems slow and not desirable for large applications. The authors advocated a deep neural network (NN) architecture, trained in an end-to-end fashion. The input sentence is 
processed by several layers of feature extraction. They showed how multi-task learning and semisupervised learning significantly improve the performance of this task in the absence of handengineered features. Multitask learning (MTL) is the procedure of learning several tasks at the same time with the aim of mutual benefit. A NN automatically learns features for the desired tasks in the deep layers of its architecture, thus sharing deep layers in these NNs would improve features produced by these deep layers, and hence improve generalization performance. The MTL network jointly trains supervised tasks on labeled data and unsupervised tasks on unlabeled data because unlabeled data is abundant and freely available on the web. The experimental results showed that the deep NN could be applied successfully to various tasks such as SRL, NER, POS, chunking and language modeling. They also demonstrated that learning tasks simultaneously can improve generalization performance. In particular, when training the SRL task jointly with the language model their architecture achieved state-of-the-art performance in SRL without any explicit syntactic features. This was an important result, given that the NLP community considers syntax as a mandatory feature for semantic extraction. In the experiment NER error was not considered and future work would include more thorough evaluations of these tasks.

\section{CONCLUSION}

With the exponential growth of internet, it's becoming increasingly important for all organizations relying on it in some way to make the most of the information they have at their disposal. Since the internet is grounded in natural language, NLP has become a very important topic. The internet community has been benefitting tremendously from the works of various researchers in this field. But, with the internet's growth has come a hyper exponential growth in the amount of data that humans are producing each year. The fifth annual IDC Digital Universe study released on June 28,2011 [19] stated that the world's information was doubling every 2 years. It projected that the world would be generating 50 times the amount of information by the end of the decade. Although there are well-developed algorithms in both the supervised and unsupervised classes of machine learning for various NLP tasks, NLP using machine learning working on massive sets of raw and unprocessed data is an intricate challenge. Semi-supervised machine learning can simplify this problem by using a large set of unlabeled data in conjunction with a small set of labeled data and could be the way forward for NLP in the future. Another useful approach would be to focus on developing NLP systems in such a way that they can contribute to developing a unified architecture. Such architecture would be necessary for generic semantic tasks and could pave the way towards General Intelligence in the future.

\section{REFERENCES}

[1] Ralf Steinberger. Cross-lingual Keyword Assignment

[2] Vasileios Hatzivassiloglou and Kathleen R. McKeown. 2007. Predicting the Semantic Orientation of Adjectives

[3] Peter D. Turney. May 16, 2002. Unsupervised Learning of Semantic Orientation from a HundredBillion-Word Corpus

[4] T.K. Landauer, S.T. Dumais: A Solution to Plato's Problem: The Latent Semantic Analysis Theory of the Acquisition, Induction, and Representation of Knowledge. Psychological Review, 104 (1997) 211240.

[5] S. Deerwester, S.T. Dumais, G.W. Furnas, T.K. Landauer, R. Harshman: Indexing by Latent Semantic Analysis. Journal of the American Society for Information Science, 41 (1990) 391-407.

[6] Peter D. Turney: Mining the Web for Synonyms: PMI-IR versus LSA on TOEFL.

[7] R.M. Tong. An operational system for detecting and tracking opinions in on-line discussions. Working Notes of the ACM SIGIR 2001 Workshop on Operational Text Classification (pp. 1-6). New York, NY: ACM. (2001)

[8] Peter D. Turney. Thumbs Up or Thumbs Down? Semantic Orientation Applied to Unsupervised Classification of Reviews 
[9] Jeonghee Yi, Tetsuya Nasukawa, Razvan Bunescu, Wayne Niblack. Sentiment Analyzer: Extracting Sentiments about a Given Topic using Natural Language Processing Technique

[10] C. Zhai and J. Lafferty. 2001. Model-based feedback in the language modeling approach to information retrieval. In Proc.of the 10th Information and Knowledge Management Conf.

[11] T. E. Dunning. 1993. Accurate methods for the statistics of surprise and coincidence. Computational Linguistics, 19(1).

[12] Ellen Spertus. Smokey: Automatic Recognition of Hostile Messages

[13] Data protection: "Junk" E-mail Costs Internet Users 10 Billion a Year Worldwide -Commission Study. In: http://europa.eu/rapid/pressReleasesAction.do?reference=IP/01/154,last accessed on 15thNov, 2013

[14] Ion Androutsopoulos, Georgios Paliouras, Vangelis Karkaletsis, Georgios Sakkis, Constantine D. Spyropoulosand Panagiotis Stamatopoulos. Learning to Filter Spam E-Mail: A Comparison of a Naïve Bayesian and a Memory-Based Approach

[15] Md. Saiful Islam, Abdullah Al Mahmud, Md. Rafiqul Islam. Machine Learning Approaches for Modelling Spammer Behaviour

[16] Vasileios Hatzivassiloglou, Janyce M. Wiebe. Effects of Adjective Orientation and Gradability on Sentence Subjectivity

[17] Janyce M.Wiebe. Learning Subjective Adjectives from Corpora

[18] Shafigh Parsazad, Ehsan Saboori, Amin Allahyar.Data Selection for Semi-Supervised Learning

[19] Ronan Collobert, Jason Weston. A Unified Architecture for Natural Language Processing: Deep Neural Networks with Multitask Learning

\section{ACKNOWLEDGEMENTS}

The authors wish to thank the Accendere KMS Research team for the guidance and mentoring which made this work successful. 


\title{
3D MODELLING, SIMULATION AND PREDICTION OF FACIAL WRINKLES
}

\author{
Sokyna Al-Qatawneh ${ }^{1}$, Ali Mehdi ${ }^{2}$, and Thamer Al Rawashdeh ${ }^{3}$ \\ ${ }^{1}$ Department of Multimedia Systems, Faculty of Science and IT, Al-Zaytoonah \\ University of Jordan, Amman, Jordan, S . Qatawneh e zuj . edu • jo \\ ${ }^{2}$ Department of Computing and Iinformatics, Faculty of IT, Saudi \\ ElectronicUniversity, Riyadh, Saudi Arabia, A.Mehdi@seu.edu.sa \\ ${ }^{3}$ Department of Computer Science, Faculty of Science and IT, Al-Zaytoonah \\ University of Jordan, Amman, Jordan, Thamer. R@zuj . edu. jo
}

\begin{abstract}
Ageing is a natural phenomenon and it is part of our daily life process; the Facial Ageing (FA) process has been of a great interest to many researchers and some firms like airports and police departments; this is due to the fact that the face appearance changes as people age resulting in difficulties identifying certain individuals. In this paper, two-dimensional wrinkle maps will be used in the design of a three-dimensional system for the purpose of facial wrinkles simulation and prediction. Our findings will challenge many commercial softwares in the innovation of the techniques in setting solid grounds to generate real-time $3 D$ wrinkles that can be used later for various reasons that may include security, 3D facial ageing simulation systems, facial animation, etc. The $2 D$ binary wrinkles will be mapped on the corresponding $3 D$ face models using the generated outlined images. NURBS curves will then be projected on those wrinkles to form a three-dimensional wrinkle map. The coloured wrinkle map, as well as some parameters, will be combined together in an algorithm to predict the appearance of the individual wrinkles in every age group that are divided into decades, starting from the age of 20. The novelty of the adopted procedure in comparison to the previous works, is the new elements that have been integrated and collaborated to boost accuracy and to generate a more realistic outcome.
\end{abstract}

\section{KEYWORDS}

Facial Aging; Facial Wrinkles; Wrinkle Maps; 3D Wrinkle Modelling.

\section{INTRODUCTION}

Facial Ageing (FA) process has been of a great interest to many researchers and some firms like airports and police departments; this is due to the fact that the face appearance changes as people age resulting in difficulties identifying certain individuals. FA research is a form of Facial Recognition (FR) and the main reason for facial recognition is delivered from the forensic needs to identify victims or suspects[1]. The human brain has the ability and capacity to quickly distinguish between known and unknown faces. The biggest advantage of using the face (over 
other parts of the human body) in many applications is that wherever a person may be, the face is also there.

Moreover, the face carries a significant number of features that identify individuals such as gender, emotional state, ethnic origin, age etc. The available facial ageing simulation and prediction technologies lack in simulation accuracy, especially when simulating the appearance of wrinkles. This might be due to the fact that wrinkles appear in different locations on human faces and the amount of change in their development also differs. Failing to simulate the wrinkles in real-time can have a direct impact on the ageing prediction accuracy as wrinkles play a vital role as a major ageing feature.

One of the most popular 2D facial ageing simulation systems is the April Age Progression Software 'age-me'[2]. On the other hand, FaceGen [3] is a widely known 3D face generator modelling software that includes an embedded ageing system. Both softwares are available for commercial purposes and share the disadvantage of generating wrinkles based on assumptions and no real data is considered. Our findings will challenge both commercial softwares in the innovation of the techniques in setting solid grounds to generate real-time $3 \mathrm{D}$ wrinkles that can be used later for various reasons that may include security, 3D facial ageing simulation systems, facial animation, etc.

This work will commence by generating a 3D face model for any given individual. 2D binary wrinkles will be mapped on the corresponding 3D face models using the generated outlined images. NURBS curves will then be projected on those wrinkles to form a three-dimensional wrinkle map. The coloured wrinkle map, as well as some parameters, will be combined together in an algorithm to predict the appearance of the individual wrinkles in every age group that are divided into decades, starting from the age of 20.

In the following section a review of related literature will be presented, while section 3 explainsthe work methodology, in which the 3D models are acquired, as well as the 3D face mesh processing, texture mapping and wrinkles construction are discussed. The results of the simulations and predictions are presented in section 4. Finally, section 5 will conclude the findings on the 3D modelling, simulation and prediction of facial wrinkles.

\section{RELATED WORK}

In addition to the fact that facial wrinkle appearance is a vital sign of ageing, it also plays an important feature in presenting FE; therefore, it became the focus of a number of FE research.Yin and Basu [4] presented a partial texture updating method for facial expression synthesis with facial wrinkles. Based on the observation of the wrinkle appearance and the change along with performed expressions, they extracted the partial texture information, then used the texture dissimilarity between the neutral expression and the active expression to extract the active texture for the expression representation.

For expressive facial animations, Li et al. [7] developed a method for modelling dynamic facial wrinkle. They started with structured facial mesh, then divided the face region into wrinkle subregions, and created some wrinkle lines with their algorithm in these sub-regions. Some key nodes were labelled on the facial mesh; these key nodes affect wrinkle lines if wrinkle lines located the sub-regions that abut on the key nodes. With the algorithm, motion of wrinkle lines 
was produced by movement of the key nodes. Consequently, wrinkle lines derived facial mesh to model expressive wrinkles.

From expressions on a 3D face model, Antini et al. [8] proposed a framework for threedimensional face representation and matching for identification purposes. Basic traits of a face were encoded by extracting curves (wrinkles) of salient ridges and ravines from the surface of a dense mesh. A compact graph representation was then extracted from these curves through a modelling technique that was capable to quantitatively measure spatial relationships between curves in a three-dimensional space. Therefore, face recognition was obtained by matching 3D graph representations of faces.

\section{METHODOLOGY}

\subsection{D Face Creation}

The construction of 3D face models using 2D face photographs, such as the work in [9], has become the focus of study on 3D face modelling, in which generated textures will affect directly the sense of reality of the generated 3D face models[10] and it is indeed one of the most difficult problems in the fields of computer graphics and computer vision.

On the other hand, 3D scanners are becoming widely employed for the task of 3D face generation. However, in this work, the 3D face models are generated from the neutral face of any individual using FaceGen for its simplicity and relative low cost.

The 2D face image is marked (Figure 1) for feature point extraction then processed. The method is based on a 3D morphable face model that encodes shape and texture in terms of model parameters. The stages of this process are shown in Figure 2, where the 3D face model as well as the facemask (texture) are exported.

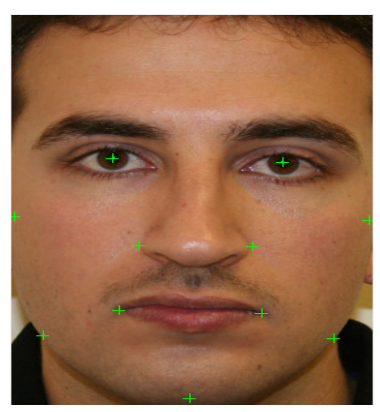

Figure 1: Marked 2D face image

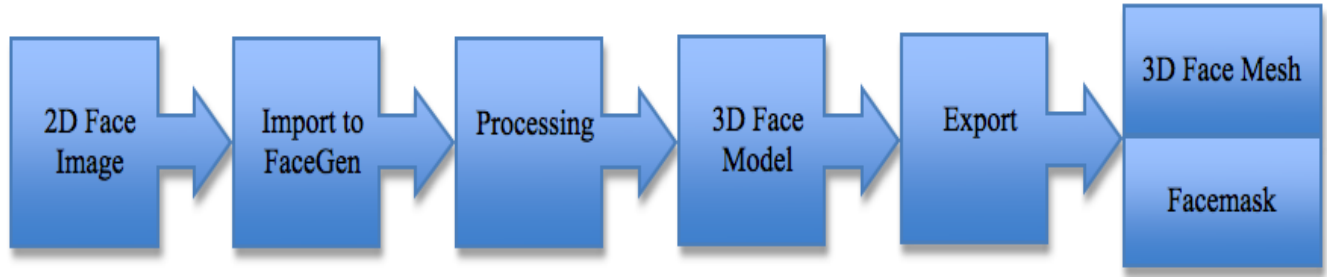

Figure 2: The process of generating and exporting the 3D face model 
The process of the image takes about $10-15$ minutes depending on the speed of the system. The result of the 3D face model is displayed in Figure 3 while the exported materials are shown in Figure 4.

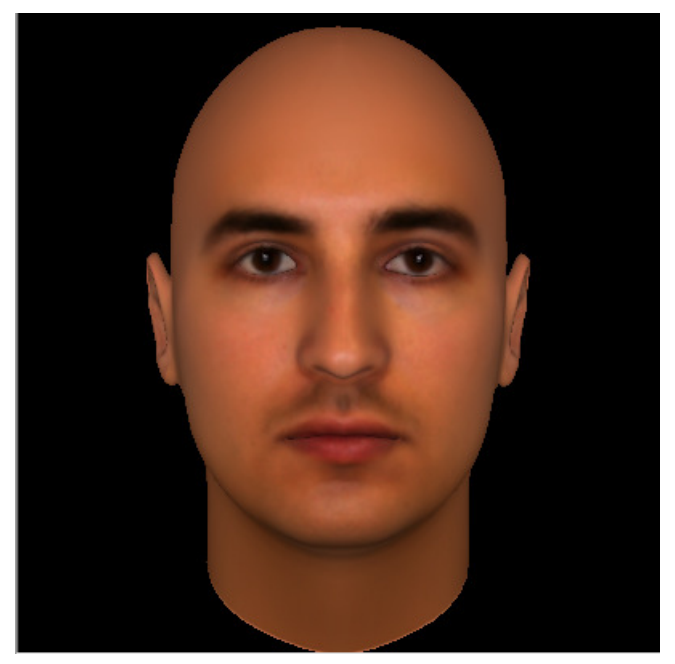

Figure 3: The 3D face model

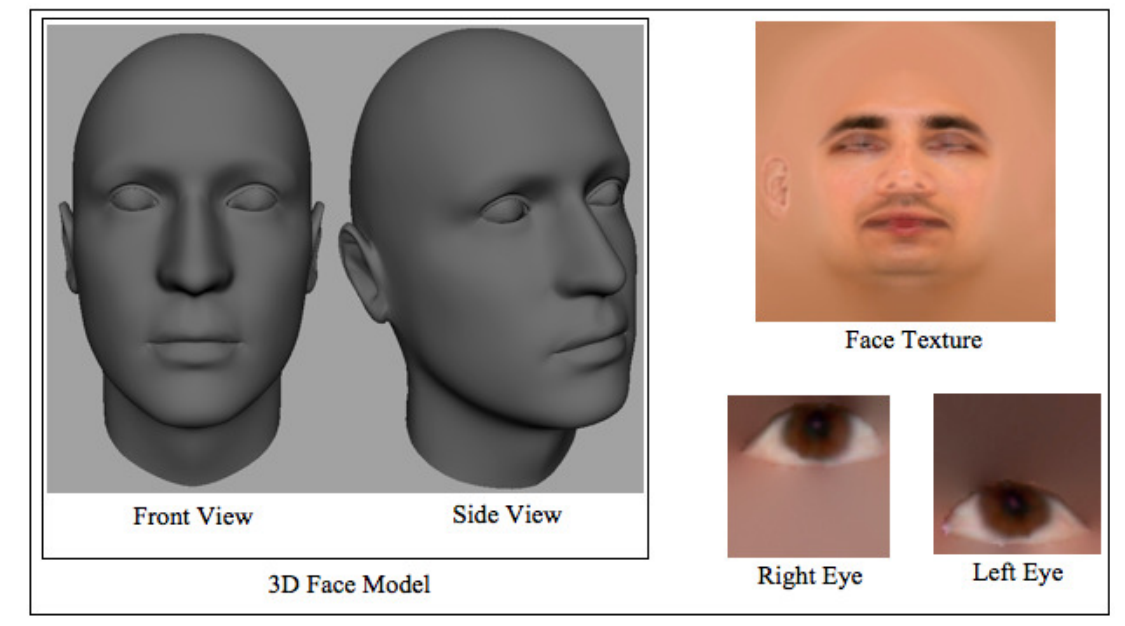

Figure 4: Exported items

\subsection{The 3D Face Mesh}

The generated face models contain simple mesh; in the modelling process of wrinkles, we take into consideration the flow of the facial muscles, so that the edge loops are in the direction of the natural flow of the human muscles (as described in Figure 5). 


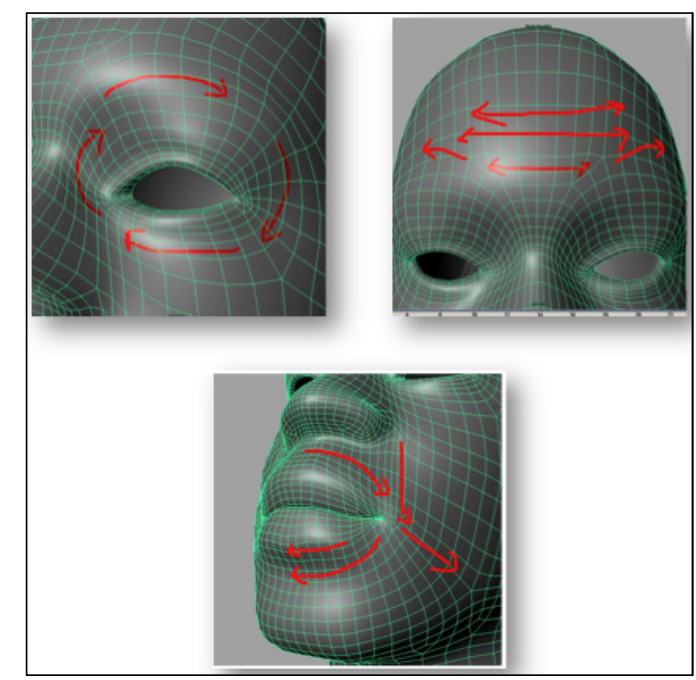

Figure 5: Facial muscle flow

The 3D face has to be processed further for optimisation; this process involves increasing the mesh by adding edge loops in order to boost the smoothness of the wrinkles later in the simulation. This method might cause the program to have a slow loading time due to the addition of more geometry to the model.

An alternative method to create wrinkles can be implemented by generating a so-called normal map $[11,12]$. In 3D computer graphics, normal mapping is a technique used for faking the lighting of bumps and dents. It is used to add details without using more polygons,

We fabricated our own normal map (an example is shown in Figure 6); the depressions make the surface look deeper, which gives the illusion of wrinkles. In our work, accuracy and realistically in terms of the wrinkle locations are top priorities; hence, using normal maps will be inadequate. For this reason, the higher resolution 3D face polygons will be used in this work despite the contingency of a slow loading time.

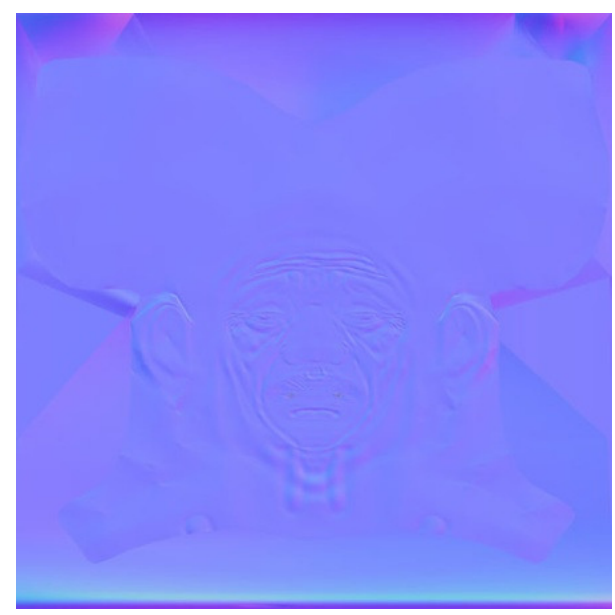

Figure 6: Our generated normal map 


\subsection{Texture Mapping}

Texture mapping is the method for adding detail or colour to a computer-generated graphics or 3D Models. In this section, the texture that is generated in Figure 4 is considered using a UV space. The UV mapping is the process of texturing a 2D image on a 3D object. In contrast to $\mathrm{X}, \mathrm{Y}$ and $\mathrm{Z}$, which are the coordinates for the original 3D object in the modelling space, another set of coordinates is required to describe the surface of the mesh, so the letters $U$ and $V$ are used. The Figure 7 shows an example of our 3D faces that is opened up to be a UV map.



Figure 7: (a) Our 3D face and its UV map; (b) Textured 3D head model

The eyes are textured in the same manner but on a smaller scale.The final texturing result is presented in the figure 8 .

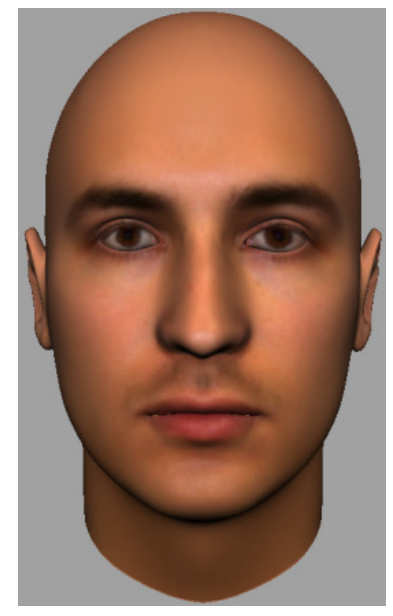

Figure 8: Final texture 


\subsection{Wrinkles Construction}

The generated binary wrinkle maps are mapped on their corresponding 3D face models; Figure 9 illustrates an example. Wrinkles are then constructed on the 3D models by the projection of the NURBS curves.

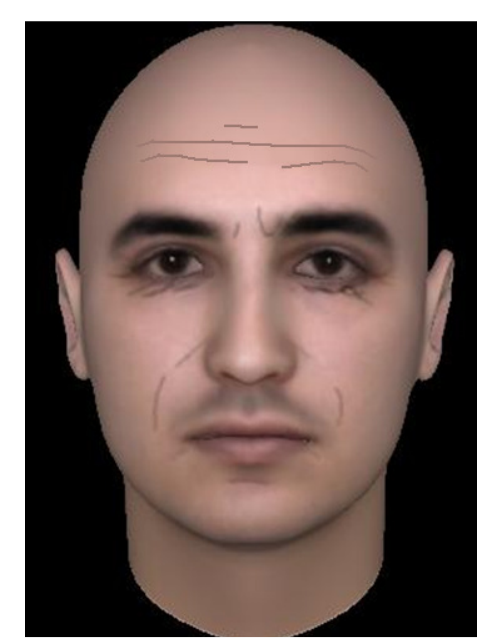

Figure 9: The binary wrinkle map is textured on the 3D face model

Upon the projection of the NURBS curves; the curves are modelled to enhance the look of the 3D wrinkle maps (Figure 10). This is performed by observing the original images in order to add or fill gaps that might be present in the binary wrinkle maps due to detection failure.

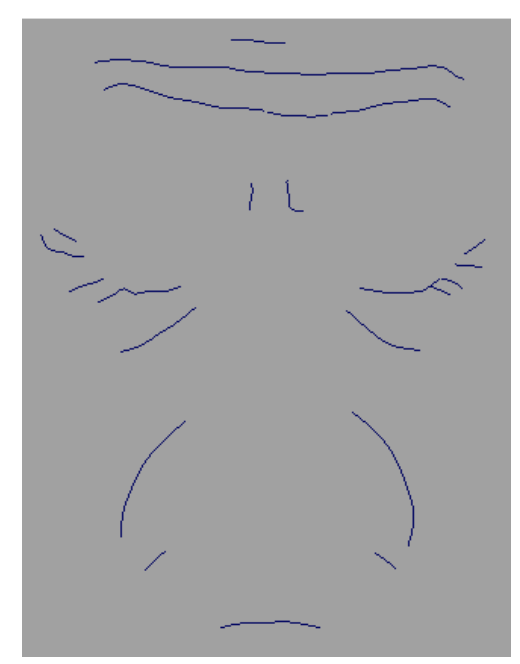

Figure 10: An enhanced 3D wrinkle map 


\subsection{Wrinkles Simulation and Prediction}

For the tasks of simulation and prediction, three elements (for any individual) are considered: the binary wrinkle map, the general development of the wrinkles over the decades and the coloured wrinkle map.

The binary map acts as a benchmark that displays the full facial wrinkles and their locations. While the coloured map will work within each age group, i.e. the wrinkles that have the highest intensity values will appear first and so on. Finally, the findings will be displayed in a form of a graph to study the behaviour of the wrinkles as age progresses.

\section{RESULTS}

Some sample results are presented in figure 11 and compared to the simulation results that are generated by the commercial software FaceGen. Our results are presented on the left hand side while FaceGen results are presented on the right hand side.

Our method doesn't only work on the standard head models that are taken from FaceGen, but it accepts 3D face scans as well. Figures 12 are generated results using a 3D face scan for Person S and also compared to the generated results from FaceGen:

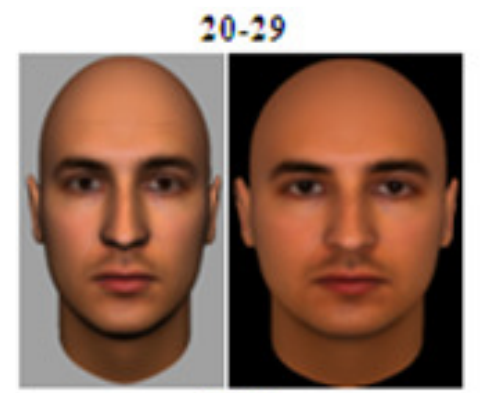

$30-39$

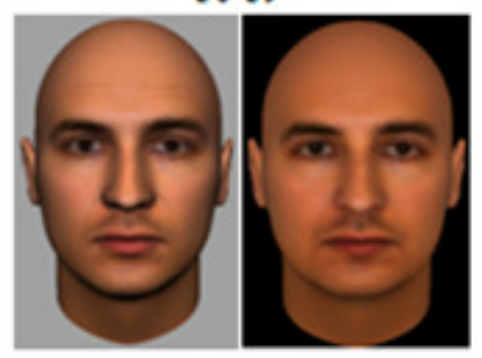

$40-49$

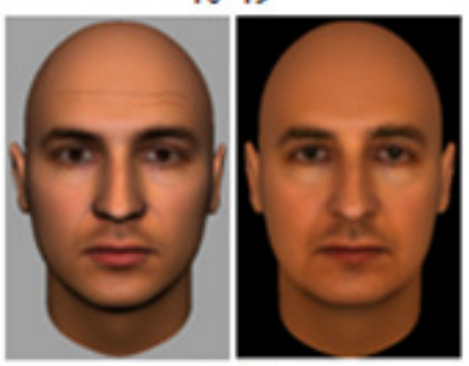

$50-59$

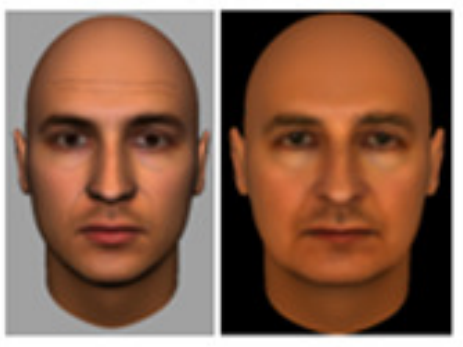

Figure 12: A comparisons between our proposed system results(on the left hand side) and FaceGen results (on the right hand side).

\section{DISCUSSION AND CONCLUSION}

In this work, novel techniques were used in the tasks of facial wrinkles simulation and prediction. Various parameters were utilised in order to develop this unique facial wrinkle pattern formation 
system, which include the generated wrinkle maps for any given individual along with published literature on the general development of the facial wrinkles over the years.

The provided system has the privilege of using real-time data that has high potential to boost the accuracy. Moreover, the system was compared to other available popular technologies and proved competence.
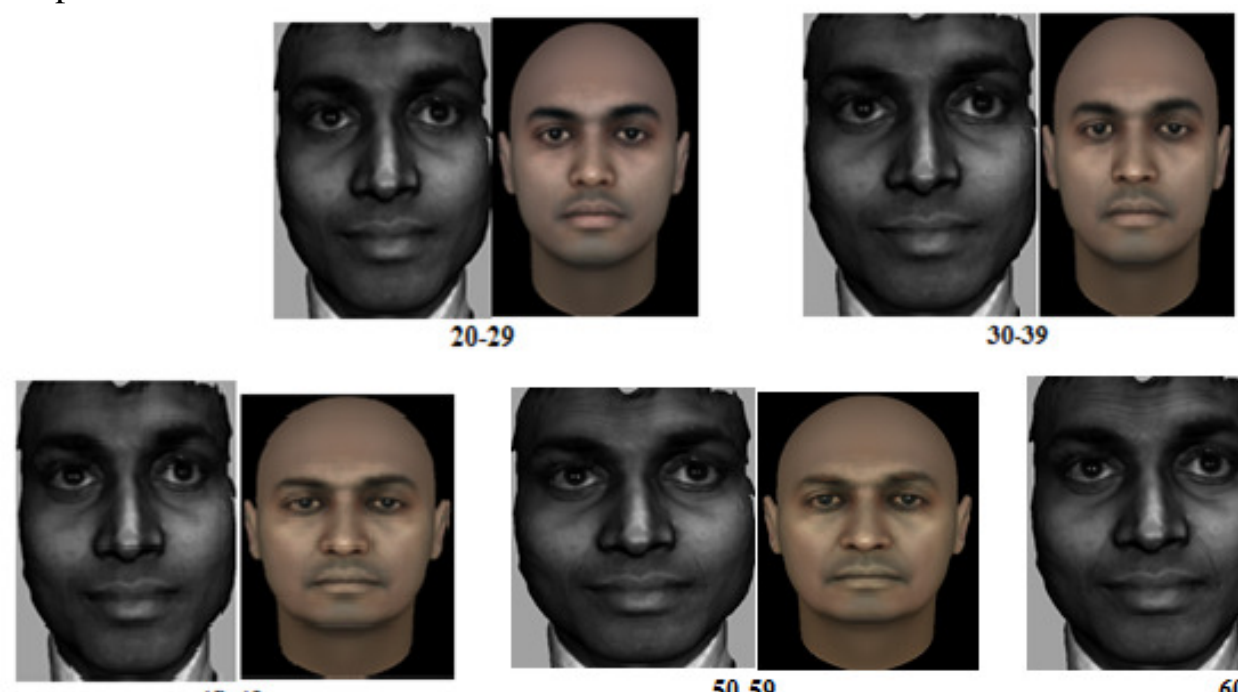

40-49

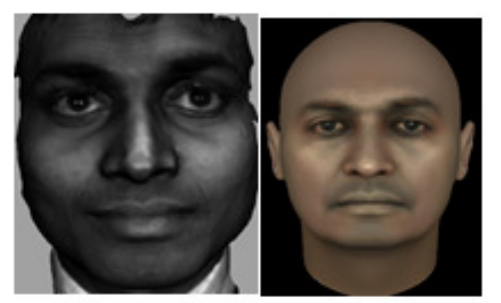

$50-59$

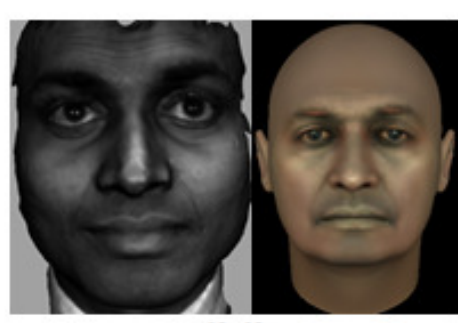

$60-69$

Figure 12: A comparisons between generated results using a 3D face scan results (on the left hand side) and FaceGen results (on the right hand side).

In comparison to FaceGen, it was seen from their results that their system could only display common facial wrinkles; Better results than FaceGen were obtained by using the age-me software. This software uses 2D images and performs the age predictions using 2D approach. The generated wrinkles in this software do not match the actual wrinkle maps of the individuals, which shows that either some assumptions were made in the generation of wrinkles based on data estimation or wrinkles were randomly produced. Though, both cases jeopardise the accuracy of wrinkles formation.Furthermore, the developed system has the distinctive advantage of its ability to accept standard (mean) 3D head models as well as 3D face scans.

\section{REFERENCES}

[1] Clement, J., and Marks, M., "Computer Graphic Facial Reconstruction". 2005, USA: Elsevier.

[2] April Age Progression Software. [cited 17/01/2011]; Available from: http://www.age-me.com/.

[3] FaceGen. [cited 31/03/2010]; Available from: http://www.facegen.com/.

[4] Yin, L., and Basu, A., "Generating Realistic Facial Expressions with Wrinkles for Model-Based Coding". Computer Vision and Image Understanding, November 2001. Vol. 84(Issue 2): p. 201-240.

[5] Zhang, Y., "Muscle-Driven Modeling of Wrinkles for 3D Facial Expression", in IEEE international conference on multimedia and expo. 2008, IEEE: Hannover. p. 957-960.

[6] Tian, Y., Kanade, T. and Cohn, J. , "Recognizing action units for facial expression analysis", in IEEE Transactions on Pattern Analysis and Machine Intelligence. 2001, IEEE. p. 97-115.

[7] Li, M., Yin, B., Kong, D. and Luo, X. , "Modeling Expressive Wrinkles of Face For Animation", in Proc. of Fourth International Conference on Image and Graphics 2007, IEEE. p. 874-879. 
[8] Antini, G., Berretti, S., Del Bimbo, A., and Pala, P., "3D Face Identification Based on Arrangement of Salient Wrinkles", in IEEE International Conference on Multimedia and Expo. July 2006: Toronto, Ont. p. 85-88.

[9] Lin, W., Weijun, H., Rui, C., and Xiaoxi, W., "Three-Dimensional Reconstruction of Face Model Based on Single Photo", in Proceedings of the International Conference on Computer Application and System Modeling 2010, IEEE: Taiyuan. p. 674.

[10] Wang, H., Zheng, Q., and Sun, Y., "A Method of Generating Global View Texture Images in 3D Face Modeling", in The sencond International Conference on Information Science and Engineering (ICISE). December 2010, IEEE: Hangzhou, China. p. 3435.

[11] Cohen, J., Olano, M., Manocha, D., "Appearance-Preserving Simplification", in Proceedings of the 25th annual conference on Computer graphics and interactive techniques. 1998, ACM: New York, USA.

[12] Yang, B., Pan, Z., "A Hybrid Adaptive Normal Map Texture Compression Algorithm", in Proceedings of the 16th International Conference on Artificial Reality and Telexistence--Workshops. 2006, IEEE: Hangzhou. 


\title{
NEURAL NeTWORK APPROACH TO RAILWAY STAND LATERAL SKEW CONTROL
}

\author{
Peter Mark Beneš ${ }^{1}$, Matouš Cejnek ${ }^{1}$, Jan Kalivoda ${ }^{2}$ and Ivo Bukovsky ${ }^{1}$ \\ ${ }^{1}$ Department of Instrumentation and Control Engineering, Czech Technical \\ University, Prague, Czech Republic \\ \{PeterMark. Benes; Matous. Cejnek; Ivo.Bukovsky\} @fs.cvut.cz \\ ${ }^{2}$ Department of Automobiles, Internal Combustion Engines and Railway \\ Vehicles, Czech Technical University, Prague, Czech Republic \\ Jan.Kalivoda@fs.cvut.cz
}

\begin{abstract}
The paper presents a study of an adaptive approach to lateral skew control for an experimental railway stand. The preliminary experiments with the real experimental railway stand and simulations with its 3-D mechanical model, indicates difficulties of model-based control of the device. Thus, use of neural networks for identification and control of lateral skew shall be investigated. This paper focuses on real-data based modelling of the railway stand by various neural network models, i.e; linear neural unit and quadratic neural unit architectures. Furthermore, training methods of these neural architectures as such, real-time-recurrentlearning and a variation of back-propagation-through-time are examined, accompanied by a discussion of the produced experimental results.
\end{abstract}

\section{KEYWORDS}

Roller Rig, Linear Neural Unit, Quadratic Neural Unit, Real-Time-Recurrent-Learning (RTRL), Back-Propagation-Through-Time (BPTT)

\section{INTRODUCTION}

An ongoing problem, which is currently under research in the railway industry, is that of lateral skew control of railway carriage wheel sets with Independently Rotating Wheels (IRW). In particular, the control of position of the leading wheel set of a railway carriage bogie, such to achieve central positioning of the wheel set, with respect to the span of the rail track. Or furthermore, to control the lateral deviation of the wheel set to follow a given desired set point. The primary necessity for such control arises from the need to improve behaviour of IRW wheel sets, as currently under study at CTU, on an experimental railway stand (roller rig). Particularly, for minimisation of wheel flange and rail head wearing as well as lateral forces and, furthermore, optimal stability at higher speeds of the wheel set. To date, various methods for control of the wheel set lateral position of IRW wheel sets have been under investigation, with both mechanical and electrical means of control [2], each featuring their own, individual drawbacks.

At CTU, the latest study features active control of the roller rig through manipulation of the yaw torque of the rig wheel set [1], via a state feedback and cascade PID control for a linearization of the model of the CTU roller rig. However, the results from this initial experimentation shows, that

David C. Wyld et al. (Eds) : CCSIT, SIPP, AISC, PDCTA, NLP - 2014 
such method is not suitable for real time control. Thus, this paper aims to investigate the possible use of a neural network approach for lateral control of such railway wheel sets. The suitability of application to this problem is motivated by promising theoretical studies of higher-order neural units (HONUs), especially the quadratic neural unit for engineering problems for [3]-[7]. These studies are focused on the use of supervised learning based approaches for polynomial structured neural units, also known as a class of HONUs, for adaptive identification and control of real engineering systems. Further motivation arises from the successful implementation of a quadratic neural unit controller (Neuro-controller), for control of a bathyscaphe system located in the automatic control laboratories of CTU [12]. Where, here, such controller adhered more closely to the desired behaviour of the system than the conventionally used PID controller. An extension on this result can be recalled in the work [11]. Where, further study was made via introduction of a new software for adaptive identification and control, along with further testing on both a theoretical system and the previously mentioned bathyscaphe system. Given this, in this paper we aim to investigate use of neural network (NN) approaches in the following manner. We will begin by explaining in more depth the problem behind the recently employed state feedback and cascade PID control of the linearization of the CTU roller rig. The proceeding section will then describe the principles and control schemes behind the various experimented methods, for adaptive identification and control. Following this, an experimental analysis of the various approaches of $\mathrm{NN}$, focusing firstly on adaptive identification of the CTU roller rig system and then to test the various NN methods for control. The final component of this paper will then be, to analyse and compare the produced experimental results, with a conclusion to be drawn at the end.

\section{PROBLEM DESCRIPTION}

This section aims to describe in more depth, the functionality behind the previously introduced experimental railway stand (CTU roller rig). More importantly, for the scope of this paper, we will elaborate on the necessity for control of such roller rig and the issues of conventional linear PID control or state feedback control, for controlling the lateral skew.

\subsection{Experimental Setup of the Roller Rig at CTU}

The below figure (Figure 1) depicts the experimental setup of the CTU roller rig, along with the 3 -D model for design and simulation purposes. This rig features 5 motor drives, two pairs of $0.5 \mathrm{~m}$ diameter rollers are independently driven, via the largest of the set of drives (FM1 \& 2). To simulate both straight and curved tracks, similar to those present on real railway networks. A central servo motor (FM4) was introduced, to yaw the lower roller pair for replicating curved track motion. This setup however, assumes simulation of a rail pair without effects of rail buckling. For manipulation of the wheel set yaw, a separate servo motor, central to the wheel set (FM3) is installed. For the scope of this paper, this is the most crucial component, as it is the action of this servo motor that is used for control actuation for the lateral skew of the wheel sets. Where, the discussed control setups in this paper will analyse control via manipulation of this servo motor torque. Finally, a fifth drive FM5 is located between the front roller set, as a control differential. 

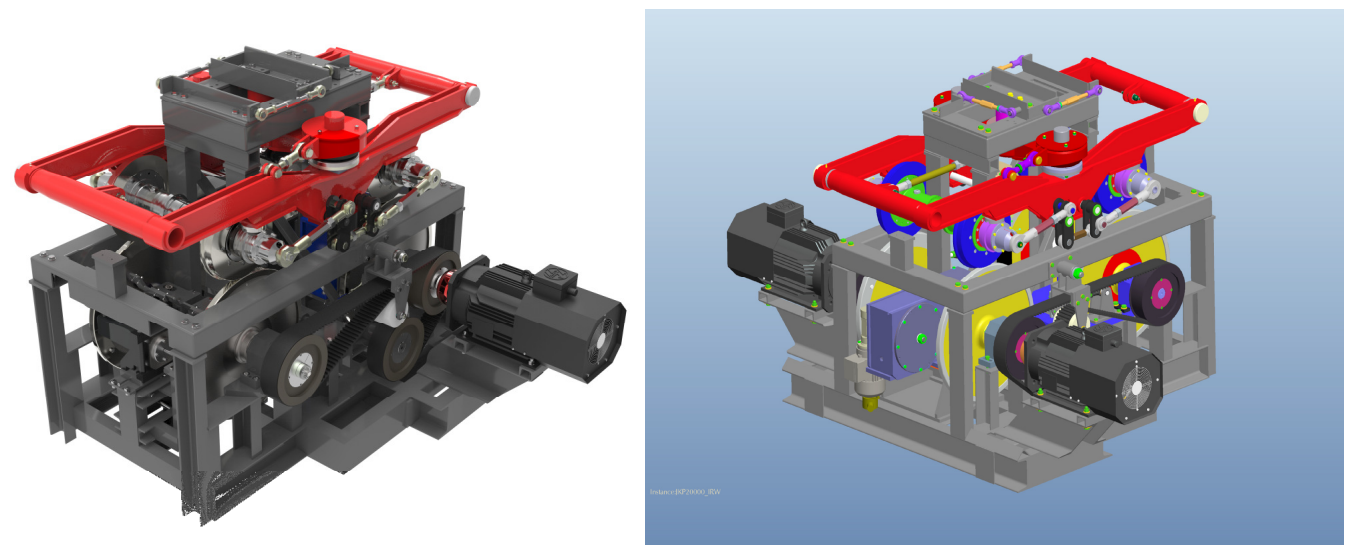

Figure 1: CTU Roller Rig (real left, PTC Pro/Engineer 3-D model on the right)

\subsection{Problem of the State Feedback and Cascade PID Control}

In the work [1], the method of lateral skew control via state feedback with cascade PID setup is introduced. In this presented setup, a linearization of the roller rig is used, via translation to a state space representation, achieved via a $12 \times 12$ matrix of dynamics. However, according to theory, this linearization of the roller rig system appeared to be uncontrollable and unobservable via analysis by common linear algebra and control approaches.

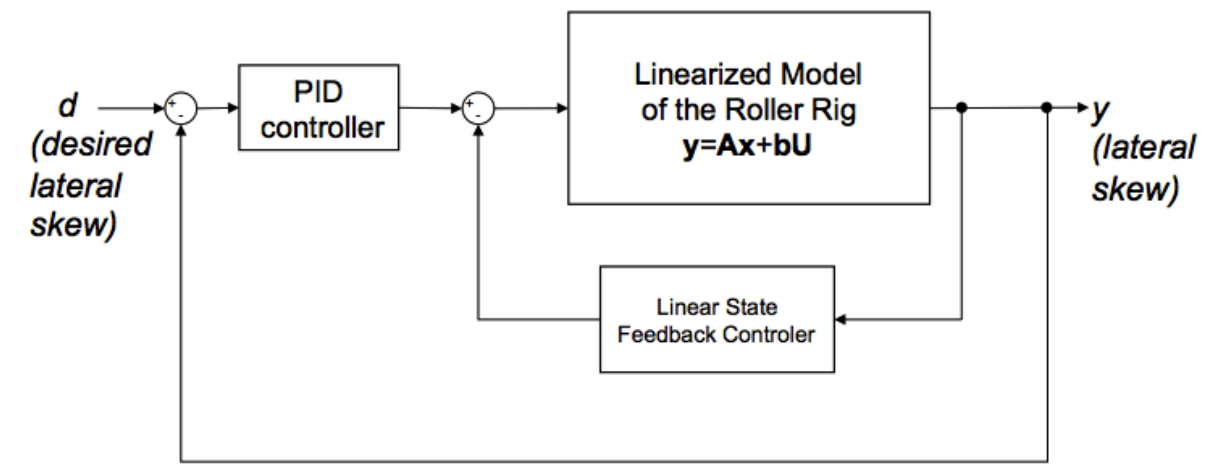

Figure 2: Control loop on linearization of the roller rig with State feedback + Cascade PID control

The above figure (Figure 2), depicts the control scheme for this setup. After numerous testing, it was found that a simulation step of approximately < 1E-6 was necessary, in order to achieve stability of the continuous control loop, which cannot be used for practical application. Hence, it is for this reason that an alternative approach is necessary for the lateral skew control of such railway wheel set. Thus, this paper aims on investigating the possibility of adaptive identification and control of the stand, based on measured data. For the purposes of this paper the data will be generated via a 3-D simulation model via software SIMPACK, linked via SIMAT toolbox with MATLAB Simulink, to provide real time simulation of the roller rig as a plant system for the investigated control approaches. Then, this data will be used as the training data for neural network models, to simplify otherwise complex simulation models and to allow a constant sampling for real time usability, furthermore, investigation into the potentials for real-time neuralnetwork based control. 


\section{APPLIED METHODS}

In this section, the various $\mathrm{NN}$ approaches, aimed for adaptive identification and control of the previously introduced lateral skew problem, will be described. These approaches are based on the well know Gradient Descent (GD) method used as a tool for defining the learning rule of the applied neural units. These applied neural units (adaptive models) are trained via two methods, which will be focused on in this paper. These methods are namely, the method of incremental training or Real Time Recurrent Learning (RTRL) [8] applicable for dynamic adaptive models or a batch form of training, which is a variation of the back propagation through time or (BPTT) training [10], as an extension of RTRL training in combination with the famous LevenbergMarquardt equation.

\subsection{Preliminaries}

This subsection aims to review the important theories from works [11]-[13], behind the GD method and structures of the neural units used within this paper. Firstly, we will begin by introducing the famous GD algorithm for the linear and quadratic neural units. To understand this, we must begin with the polynomial models of the linear and quadratic (LNU and QNU) neural units respectively, as follows

$$
\begin{gathered}
y=\sum_{i=0}^{n} x_{i} w_{i}=w_{0} \cdot x_{0}+w_{1} \cdot x_{1}+\ldots+w_{n} \cdot x_{n}=\mathbf{w} \cdot \mathbf{x} \\
y=\sum_{i=0}^{n} \sum_{j=0}^{n} x_{i} x_{j} w_{i, j}=w_{0,0} x_{0} x_{0}+w_{0,1} x_{0} x_{1}+\ldots+w_{n, n} x_{n}{ }^{2}=\text { rowx.colW }
\end{gathered}
$$

Where, $y$ represents the output of the LNU (1) and QNU (2) respectively. In regards to the LNU, $\mathbf{w}$ stands for an updatable vector of neural weights and $\mathbf{x}$, represents the input values of the engineering system in the case of a purely static model and in the sense of a dynamic model, a combination of inputs of the real system and neural model outputs. Looking at equation (2), rowx is a long-vector representation of the utilised input vector. Where, colW represents a weight matrix of the quadratic neural unit in general. From this, we may then understand the GD algorithm as applied to such neural units.

$$
\begin{gathered}
w_{i+1}=w_{i}+\mu \cdot e(k) \cdot \frac{\partial y(k)}{\partial w_{i}} \\
\operatorname{colW}(\mathrm{k}+1)=\operatorname{col} \mathbf{W}(k)+\mu \cdot e(k) \cdot \frac{\partial y(k+1)}{\partial \operatorname{col} \mathbf{W}}
\end{gathered}
$$

Here, equation (3) \& (4) depicts the GD algorithm for both the LNU and QNU respectively. Here the output of the GD algorithm is to incrementally over sample-by-sample, update the neural weights such to adaptively teach the LNU or QNU model, to learn the engineering system. Here, $\mu$ represents the learning rate of the GD algorithm. This is analogical to humans where, setting $\mu$ relatively high, corresponds to faster learning of the human and consequently, means the less detail the human can remember and digest from their learning. Furthermore, setting this parameter to a smaller value, corresponds to a slowing rate of learning i.e. the human may remember the information learned, quite well, but less information overall about the subject. The next parameter is $e(k)$ ( $k$ representing the number of the sample), this represents the current error between the real and calculated output of the model. The final term $\frac{\partial y(k)}{\partial w_{i}}$, corresponds to the partial derivatives of the neural unit output, with respect to the individual neural weights. 
Regarding the QNU in equation (4), we see that the GD algorithm is analogical, with exception of updating the matrix of neural weights as opposed to a vector in the sense of the LNU.

Till now, the structures of GD for LNU and QNU were reviewed in the format of RTRL or sample-by-sample method of learning. Where, the neural weights are updated over each new sample of the engineering system data. However for certain engineering processes, it is more advantageous to use the BPTT batch method of training these neural weights, over runs of the algorithm rather than over each sample. This is because the RTRL method focuses on the contemporary governing law of the system as opposed the BPTT which focuses more so on the main governing law of the input and outputs of the engineering system. The BPTT method is achieved via an extension of the RTRL method with combination of the famous LevenbergMarquardt equation. It is also important to note that this method is more preferable in cases where the data may be affected by noise. The following equation denotes the weight update rule for the BPTT method;

$$
\Delta \mathbf{w}=\left(\mathbf{J}^{T} . \mathbf{J}+\frac{1}{\mu} . \mathbf{I}\right)^{-1} . \mathbf{J}^{T} . \mathbf{e}
$$

Here, the neural weights are updated over each run of the algorithm (or batch trained) in the following way $w=w+\Delta w$. Here equation (5) describes the change, necessary for the update of the batch trained weights. Where, $\mathbf{J}$ represents the Jacobian matrix of derivatives for the neural unit. This may be the complete partial derivatives of the neural model with respect to each neural weights, or in practical applications it seems useful to simply introduce this Jacobian matrix as the input vector or matrix itself, being $\mathbf{x}$ and colx for LNU and QNU respectively. Furthermore, it is important to note that $\operatorname{col} \mathbf{x}=\mathbf{r o w} \mathbf{x}^{\mathrm{T}}$.

Often in such adaptive neural units, it is apparent that a modification of the normalised learning rate may be used to solve issues associated with instability of learning. In practise, it is possible to employ the simplified normalised learning rate as presented in the work [11], as follows;

$$
\eta=\frac{\mu}{\mathbf{x}(\mathrm{k}) \mathbf{x}(\mathrm{k})^{T}+1}
$$

Where, equation (6) represents the normalised learning rate in the sense of LNU. This is analogically represented in the QNU, by replacement of $\mathbf{x}(\mathrm{k})$ (i.e. each $\mathrm{k}$ th sample of the input vector $\mathrm{x})$ with $\operatorname{col} \mathbf{x}(\mathrm{k})$. It should be noted that the above representation holds for RTRL training for dynamic adaptive models, where the algorithms used in this paper for BPTT method of training, take the learning rate $\mu$ itself.

\subsection{Adaptive Identification and control}

The previous section focused on usage of neural units in the sense of adaptive identification of a real engineering system. In this subsection, we extend on these neural units as a method of control, with brief review from works [11]-[13], and extension as applied to the lateral skew problem, which is indeed our focus within this paper. 


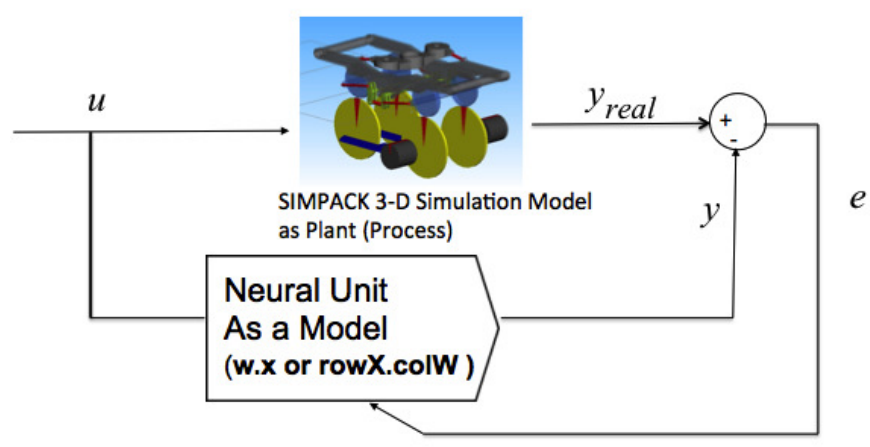

Figure 3: Adaptive Identification with supervised learning of neural networks (where, w.x=LNU, row X.colW=QNU)

The above figure (Figure 3), depicts the identification scheme for the previously reviewed neural network architectures. Where, for the scope of this paper a simulation of the real roller rig will be used for data generation of the investigated neural network approaches for control. $u$, represents the input data of the roller rig, in our case this is the yaw torque of the servo motor system for manipulating the lateral skew of the wheel set. The output $y_{\text {real }}$, is the simulated output from the real time 3-D SIMPACK model and $y$, being the output of the neural unit, and the difference being the error, $e$.

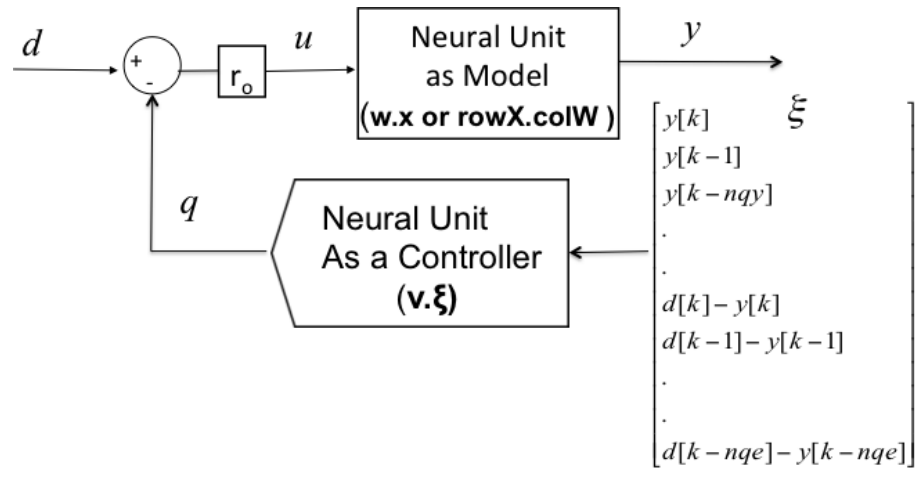

Figure 4: Adaptive control loop for experimental study of a neural network controller (where, w.x=LNU, rowX.colW=QNU)

The above (Figure 4), shows an extension of the discussed neural architectures for application to lateral skew control of the roller rig system. Here, once the neural unit as a model is identified, it may be utilised as the foundation for a neural network based control setup. For the scope of this paper, we will propose an offline tuned control scheme, as the goal here, is to investigate the potentials of applying a neural network based control, for this application. However, the extension to online control is indeed the ultimate aim of our research to this problem, beyond this paper. Figure 4, depicts use of a second neural unit as a controller. Similarly to the previously mentioned architectures, as a controller here too the neural unit may take shape to that of the LNU or QNU adaptive models. However, in this case the adaptive neural weights are tuned differently to that of those used for the neural unit as a model and hence, depicted as $v$. Analogically, should a QNU structure be applied, these neural weights would be represented in a matrix form. Further to this, the above figure (Figure 4) introduced a new vector $\xi$. This vector comprises of a combination of outputs from the neural unit as a model and the difference between the desired behaviour (in our case desired yaw torque or lateral skew of the roller rig) and the 
output of the neural model. v. $\xi$ or collectively, the variable $q$, thus serves as a manipulator for the newly feed samples of the neural unit as an identified model. Here the GD algorithms are employed in the following manner to achieve sample-by-sample adaptation of the neural weights for the controller, as follows;

$$
v_{i+1}=v_{i}+\mu . \operatorname{ereg}(k) \cdot \frac{\partial y(k)}{\partial v_{i}}
$$

Where $v_{i}$, are adaptable neural weights of the neural unit as a controller and $e_{r e g}(k)$ is the error between the desired value of the real system (in our case the roller rig, where the desired value will be denoted as $d$ ) and the real system output value at sample $k . \frac{\partial y(k)}{\partial v_{i}}$, is the partial derivative of the output of the neural unit as a model, with respect to the individual adaptive neural weights of the neural unit as a controller. An extension of this weight update scheme for BPTT training would result in the following form $v=v+\Delta v$, where the change of neural weights for each batch would be analogical to equation (5).

\section{EXPERIMENTAL ANALYSIS}

This section aims to analyse the previously reviewed methods of adaptive identification and control, via the introduced neural network architectures. Particularly, we aim to investigate the applicability of the discussed neural architectures for this problem of the lateral skew control of railway wheel sets. In this section, we will simulate our results using simulation data of the previously described 3-D SIMPACK model (at mid-range speed) linked with MATLAB Simulink in real time simulation. The data was produced with 0.001 sampling over a 10 second interval. First, we will analyse the results for identification of the roller rig as a system, via the different methods of the neural network models, followed by an extension of control, where the various combinations of neural network architectures will be tested.
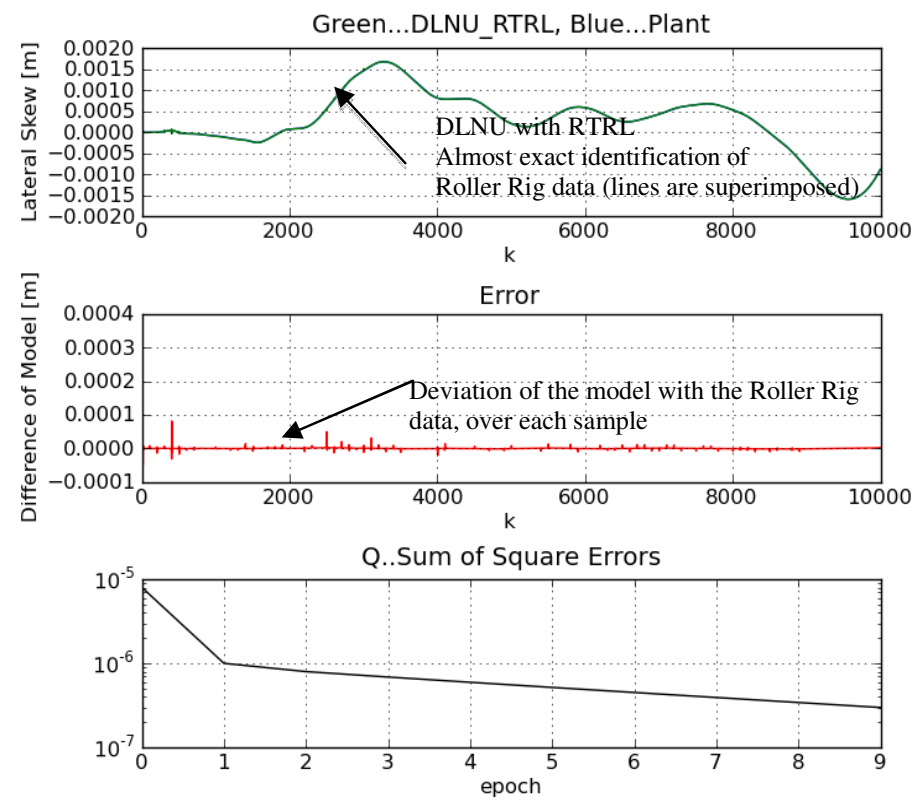

Figure 5: Testing of the adaptive identification, where the plant (roller rig) is represented by blue colour and green being the neural model. Trained by DLNU with RTRL Training; $\mu=1$, epochs $=10$, For $n y=3$ (previous samples of model output) and $n u=5$ (previous samples of real system input) 
The above figure (Figure 5) and following figure (Figure 6), illustrates the adaptive identification process of the roller rig system data. We can note, that both methods of the dynamic LNU or (DLNU) and dynamic QNU or (DQNU), for RTRL learning methods, achieved almost exact identification. Where, the DQNU performed slightly faster in terms of convergence to minima of sum of square errors, as opposed to the DLNU.
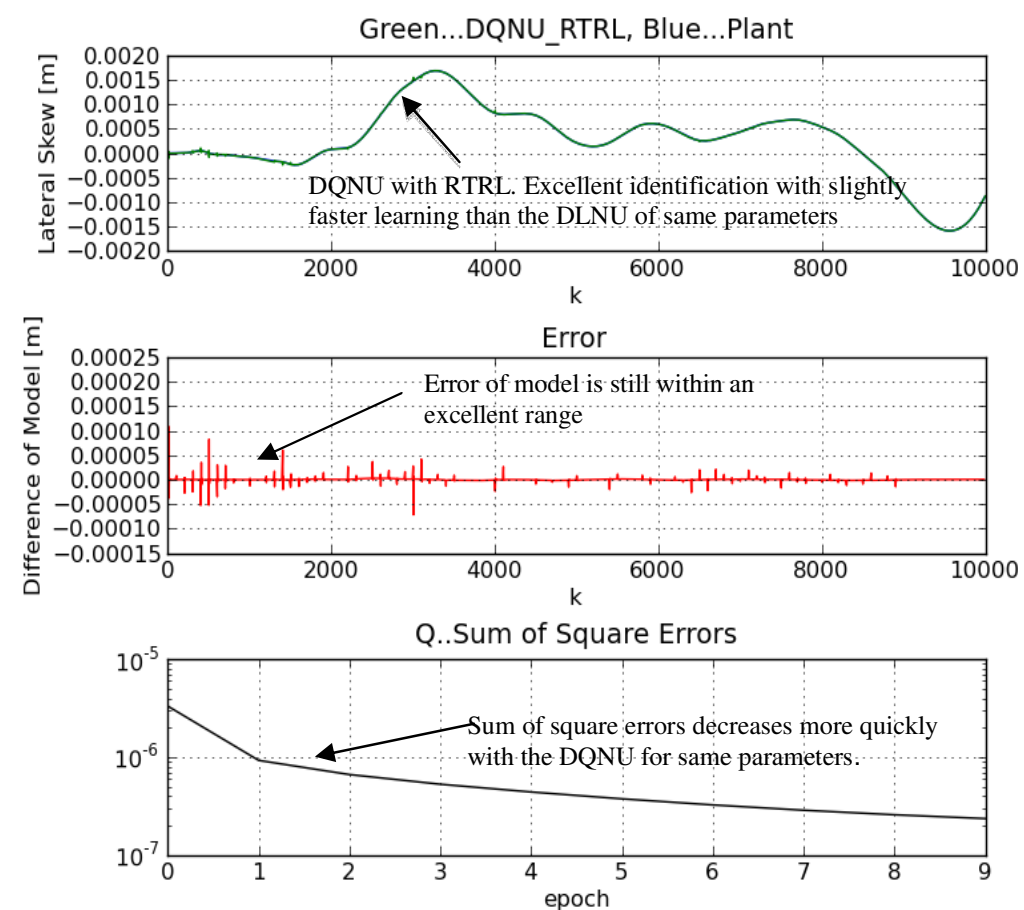

Figure 6 : Testing of the adaptive identification, where the plant (roller rig) is represented by blue colour and green being the neural model. Trained by DQNU with RTRL Training; $\mu=1$, epochs $=10$, For $n y=3$ (previous samples of model output) and $n u=5$ (previous samples of real system input) 


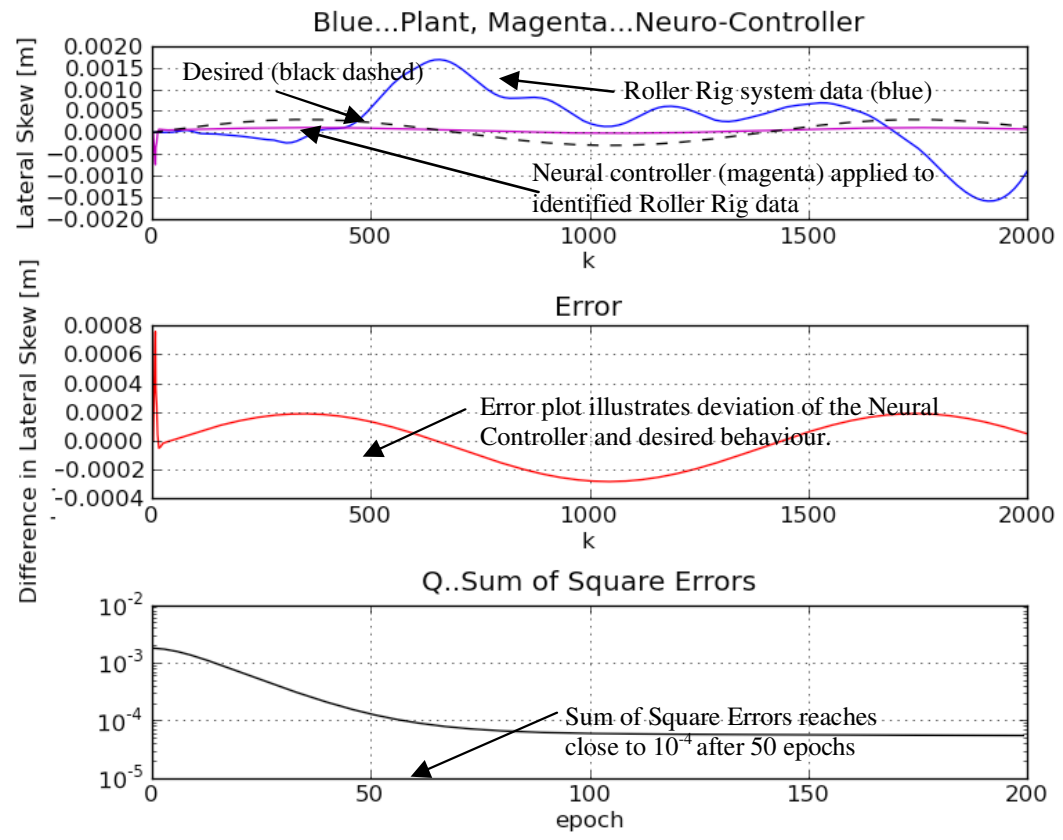

Figure 7: Testing of the adaptively tuned control loop, where the roller rig is represented by trained DLNU with constant and previously trained parameters (Figure 5) and the adaptive feedback controller is trained via LNU, after 10 epochs Adaptive Identification (of DLNU with RTRL) + Neural Unit Controller for lateral skew control of roller rig system - Using LNU with Incremental training $\mu=0.1$, Data re-sampling $=$ each $5^{\text {th }}$ sample, epochs $=200$, for $n q y=0$ (previous samples of neural model output) and $n q e=2$ (previous samples of difference of the neural model output and desired behaviour of the system.
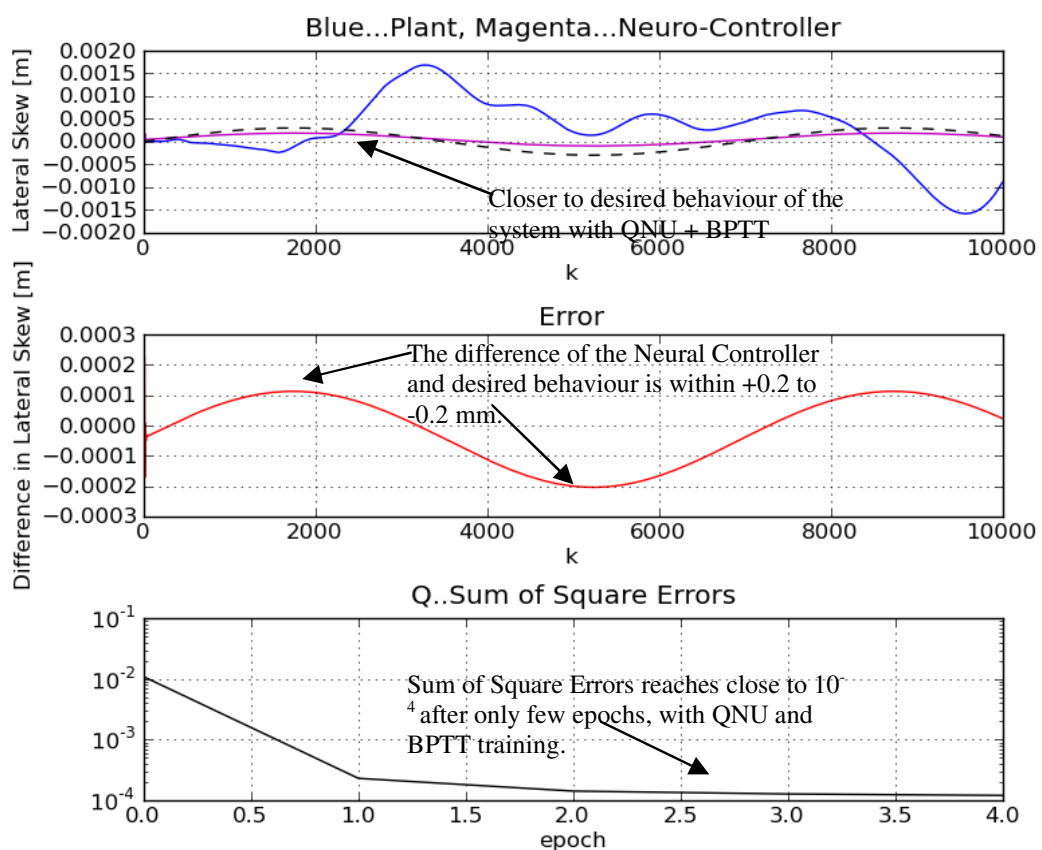

Figure 8: Testing of the adaptively tuned control loop, where the roller rig is represented by trained DQNU with constant and previously trained parameters (Figure 6) and the adaptive feedback controller is trained via QNU, after 10 epochs Adaptive Identification (of DQNU with RTRL) + Neural Unit Controller for lateral skew control of roller rig system -

Using QNU with BPTT Training $\mu=0.00818$, epochs $=5$, for $n q y=0$ and $n q e=3$ 
Here, Figure $7 \&$ Figure 8 show the application of the various neural units for control of the lateral skew. The black dotted line, illustrates the chosen desired behaviour of the roller rig lateral skew. It was found that the BPTT training method used in combination with the QNU had the best performance. Providing closer control to the desired behaviour, as compared with the incremental training methods of both LNU and QNU architectures.

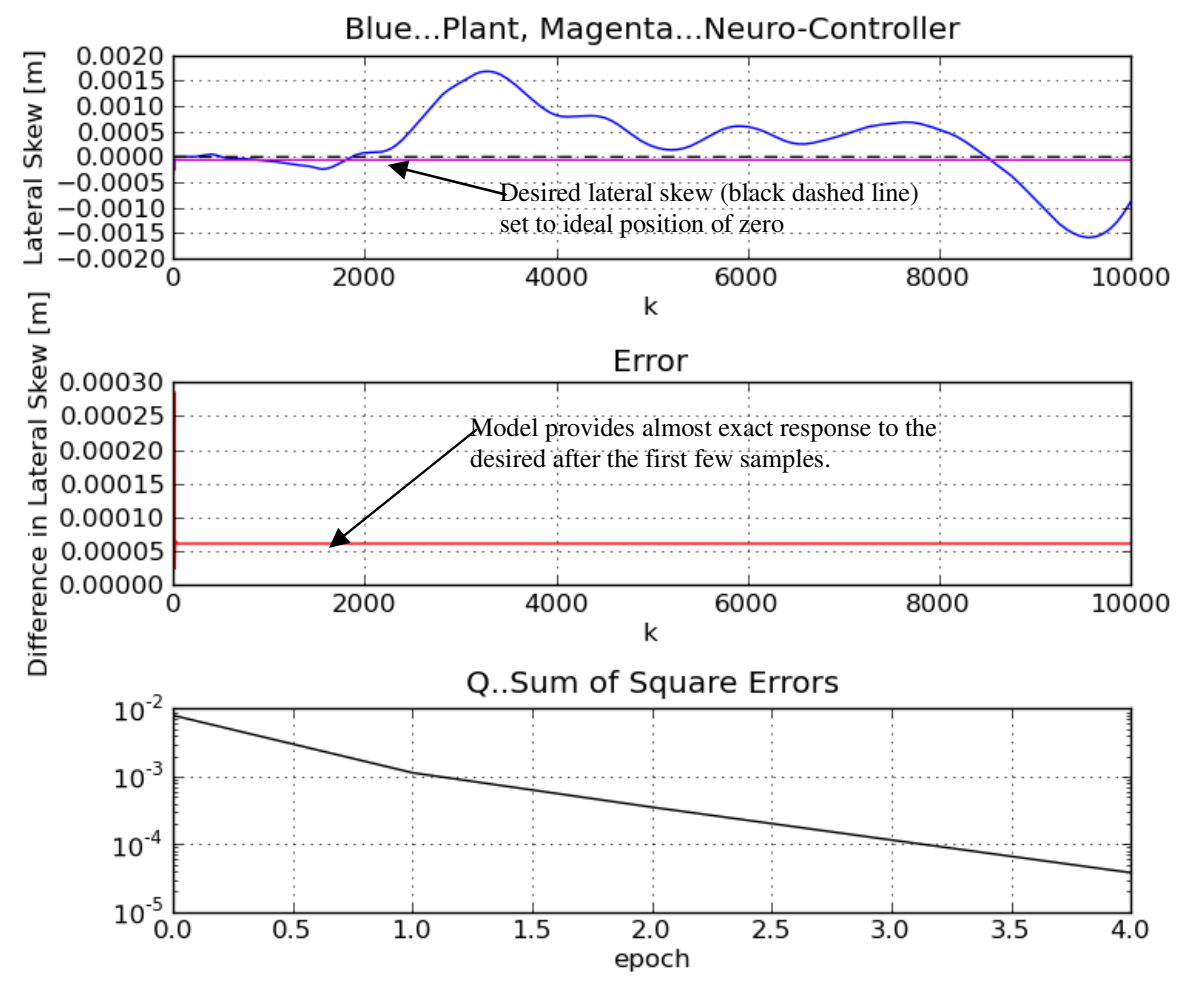

Figure 9: Testing of the adaptively tuned control loop, where the roller rig is represented by trained DQNU with constant and previously trained parameters (Figure 6) and the adaptive feedback controller is trained via QNU,

after 10 epochs Adaptive Identification (of DQNU with RTRL) + Neural Unit Controller for lateral skew control of roller rig system - Using QNU with BPTT Training $\mu=0.001$, epochs $=5$, for $n q y=3$, nqe $=3$

Figure 9 depicts a theoretical test of the roller rig system under an idealised case of zero lateral skew. This is included in our results to investigate the capability of the used neural controller. From this figure, we see that after several small samples at the beginning of the controller's application the controller provides almost exactly zero lateral skew. This result however, must be reasoned with what is really possible in terms of actuation of the real system and real conditions of the rig, such to achieve this ideal of a result.

\section{DISCUSSION OF EXPERIMENTAL RESULTS}

In the previous section, the experimental results of the various neural network architectures for adaptive identification and control were presented. From the first set of figures, regarding the adaptive identification for the roller rig system, it was found that the most suitable learning method for both the DLNU and DQNU was the RTRL training method. Here, relatively few epochs were adequate for both architectures to identify the system data, with almost exact 
identification. The DQNU model was slightly faster in learning the behaviour of the system, thus, was used as the identified neural unit as a model, in the control section. The extension of these various architectures for control showed more distinguishable results. The application of both LNU and QNU with incremental learning for the neural controller was only possible when a higher number of epochs were run, or higher learning of the model with a small learning rate. Few runs of the algorithm were unable to match the desired behaviour even moderately. However, after the first few samples of application as in Figure 7, reasonable behaviour of the controller was achieved. The combination of identification via the DQNU followed by extension of a QNU trained by BPTT on the neural controller, showed to follow more closely to the desired behaviour of the roller rig system than the other tested combinations. Where, the setup of the QNU with BPTT neural controller featured input vector $\xi$, being comprised only of several errors or differences of the desired behaviour, with the output of the neural unit as a model. Further to this, the training was substantially quicker than the incremental training method, showing that only several epochs were sufficient for the model to achieve its optimal behaviour with respect to the desired of the system.

In the above results, two forms of the desired behaviour were used. The first was under proposal that the lateral derivation of the wheel set would be within a small tolerance range, which is most realistic in real application of such control. The presented desired data is relative to the small range of lateral skew in the simulation data. Where, within this exampled range, we could be sure the wheel flanges would not contact the rails of the real roller rig. The desired behaviour is however relative to the setup of the wheel sets and rails, thus the desired deviation and lateral skew outputs may indeed vary for real railway applications. The second was an idealised situation where no lateral skew at all would occur. This is principally unrealistic for the neural controller to achieve zero lateral skew, due to a combination of natural factors on real railway wheel sets, however for demonstrational purposes, we may note the capability of the neural controller, providing almost exact behaviour of the idealised lateral skew, as applied on the identified roller rig system.

\section{CONCLUSION}

Referring back to the original control loop depicted in Figure 1 (as presented in work [1]), of the Simulink model of the linearization of the roller rig system and state feedback with cascade PID controller. We can recall that a minimum sampling of $<1 \mathrm{E}-6$ seconds for numerical stability was necessary, which is not possible for practical application. After investigation of the presented neural network approaches, we may conclude that it has promising potential for real application on controlling the lateral skew of IRW railway wheel sets. Now, we note that the achieved sampling can be within order of 0.005 (5E-3) seconds, for adequate functionality of the neural network based, adaptive identification and control system as applied to the roller rig. The tested models in this paper were DLNU and DQNU for adaptive identification models and LNU or QNU architectures as a controller. The experimental results of these architectures showed that both DLNU and DQNU, precisely approximated the complex SIMPACK model of the roller rig system. This result however could vary when using more or, real training data measured from the rig itself. In terms of control of the lateral skew, the QNU architecture showed better results to keep the lateral skew within close limits to the desired behaviour of the system, which is also in general consensus with our previous findings on using QNU for control. We also can note the significantly faster tuning of the control loop via QNU as compared to the LNU, more particularly, the BPTT training method as applied to the controller compared to the incremental method. Where, more desirable control was shown, in much fewer epochs or runs of the controller algorithm. Thus, this case study shows that with proper tuning of the investigated neural units, application to the real system is theoretically possible, to achieve adequate control 
for this investigated problem. Thus, real application of such adaptive control loop of neural network based architecture, is indeed the very next step of our research.

\section{ACKNOWLEDGEMENTS}

The authors would like to acknowledge the following grant for support during this work: SGS12/177/OHK2/3T/12SGS12/177/OHK2/3T/12, Non-conventional and Cognitive Signal Processing Methods of Dynamic Systems.

And also the Technology Agency of Czech Republic. Project No: TE01020038 "Competence Center of Railway Vehicles".

\section{REFERENCES}

[1] J. Kalivoda, P. Bauer (2013) "Curving Behaviour of 2-Axle Bogie with Independently Rotating Wheels - Simulations and Scaled Roller Rig Tests"- 23rd International Symposium on Dynamics of Vehicles on Roads and Tracks, Qingdao, China, August 19th-23rd.

[2] S. Bruni, R. M. Godall, T.X. Mei, H. Tsunashima (2007) "Control and monitoring for railway vehicle dynamics", Vehicle System Dynamics, vol. 45 issue 7-8, pp. 743-779

[3] Gupta, M., M., Bukovsky, I., Homma, N., Solo M. G. A., Hou Z.-G. (2012): "Fundamentals of Higher Order Neural Networks for Modeling and Simulation“, in Artificial Higher Order Neural Networks for Modeling and Simulation, ed. M. Zhang, IGI Global,.

[4] Bukovsky, I., Bila, J., Gupta, M., M, Hou, Z-G., Homma, N. (2010): "Foundation and Classification of Nonconventional Neural Units and Paradigm of Nonsynaptic Neural Interaction" in Discoveries and Breakthroughs in Cognitive Informatics and Natural Intelligence within the series of the Advances in Cognitive Informatics and Natural Intelligence (ACINI), ed. Y. Wang, IGI Publishing, Hershey PA, USA, ISBN: 978-1-60566-902-1, pp.508-523.

[5] Bukovsky, I., Homma, N., Smetana, L., Rodriguez, R., Mironovova M., Vrana S. (2010): "Quadratic Neural Unit is a Good Compromise between Linear Models and Neural Networks for Industrial Applications", ICCI 2010 The 9th IEEE International Conference on Cognitive Informatics, Tsinghua University, Beijing, China, July 7-9.

[6] Bukovsky I., S. Redlapalli and M. M. Gupta (2003): Quadratic and Cubic Neural Units for Identification and Fast State Feedback Control of Unknown Non-Linear Dynamic Systems, Fourth International Symposium on Uncertainty Modeling and Analysis ISUMA 2003, IEEE Computer Society, , Maryland USA, ISBN 0-7695-1997-0, p.p.330-334

[7] Rodriguez, R., Bukovsky, I., Homma, N.: "Potentials of Quadratic Neural Unit for Applications", in International Journal of Software Science and Computational Intelligence (IJSSCI) ,vol 3, issue 3, IGI Global, Publishing, Hershey PA, USA ISSN.

[8] Bukovsky, I., Lepold, M., Bila J. (2010): "Quadratic Neural Unit and its Network in Validation of Process Data of Steam Turbine Loop and Energetic Boiler”,WCCI 2010, IEEE Int. Joint. Conf. on Neural Networks IJCNN, Barcelona,Spain.

[9] R. J. Williams and D. Zipser (1989) "A learning algorithm for continually running fully recurrent neural networks," Neural Computation, vol. 1, pp. 270-280.

[10] P. J.Werbos (Oct. 1990) "Backpropagation through time: What it is and how to do it" Proc. IEEE, vol. 78 , no. 10 , pp. $1550-1560$.

[11] Peter M. Benes (2013): Software Application for Adaptive Identification and Controller Tuning Student's Conference STC, Faculty of Mechanical Engineering, CTU in Prague.

[12] Ladislav Smetana (2008): "Nonlinear Neuro-Controller for Automatic Control Laboratory System", Master's Thesis, Czech Tech. Univ. in Prague,

[13] Laboratory System Batyscaphe, Automatic Control Laboratory, Dpt. of Instr. and Cont. Eng., FME, Czech Technical University in Prague, online http://vlab.fsid.cvut.cz/en/ulohy/batyskaf.php, 2010. 


\section{Short Biography of the Authors}

Peter Mark Beneš received his Bachelor's degree with honours at Czech Technical University (CTU) in Prague in 2012. Currently he is a Master's student, with expected PhD studies to follow in 2014. His research focuses on non-conventional neural networks for adaptive identification and control of industrial systems including hoist mechanisms and skew control of rail-based mechanisms as such cranes and railway vehicles. Peter's work has been awarded in local and international student competitions also with an industrial BOSCH award in 2013.

Matouš Cejnek received his Bachelor's degree at Czech Technical University (CTU) in Prague in 2012. Currently he is a Master's student at Czech Technical University in Prague. His research focuses on nonconventional neural networks for adaptive systems and novelty detection in time series and biomedical applications. Matous's work has been awarded in local and international student competitions 2013.

Jan Kalivoda graduated from Czech Technical University (CTU) in Prague where he received his Master's degree with honours in 1996 and Ph.D. in the field of Machines and Equipment for Transportation in 2006. Currently he is a teacher and active researcher in the Department of Automotive, Combustion Engine and Railway Engineering at CTU. His research interests include MBS models of railway vehicles, Mechatronics for railway vehicles, Active control of railway vehicle suspensions and wheel sets.

Ivo Bukovsky graduated from Czech Technical University in Prague (CTU) where he received his Ph.D. in the field of Control and System Engineering in 2007 and became associate professor since 2013. His research interests include higher-order neural networks, adaptive evaluation of time series and systems, multi-scale-analysis approaches, control and biomedical applications. He was a visiting researcher at the University of Saskatchewan (2003), at the University of Manitoba in Canada (2010), and he was a visiting professor at Tohoku University (2011) 
Computer Science \& Information Technology (CS \& IT)

INTENTIONAL BLANK 


\title{
ANOTHER ADAPTIVE APPROACH TO NOVELTY DETECTION IN TIME SERIES
}

\author{
Matouš Cejnek, Peter Mark Beneš, Ivo Bukovsky \\ Department of Instrumentation and Control Engineering, \\ Czech Technical University, Prague, Czech Republic \\ \{Matous.Cejnek; PeterMark.Benes; Ivo.Bukovsky\} @f s.cvut.cz
}

\begin{abstract}
This paper introduces a novel approach to novelty detection of every individual sample of data in a time series. The novelty detection is based on the knowledge learned by neural networks and the consistency of data with contemporary governing law. In particular, the relationship of prediction error with the adaptive weight increments by gradient decent is shown, as the modification of the recently introduced adaptive approach of novelty detection. Static and dynamic neural network models are shown on theoretical data as well as on a real ECG signal.
\end{abstract}

\section{KEYWORDS}

Novelty Detection, Time Series, Gradient Descent, Neural Networks, ECG

\section{INTRODUCTION}

In principle, novelty carried through measured samples of data may be evaluated either via probability based approaches as exampled in [1] or, via learning system based approaches as in the work [2]. The first of these streams, i.e. probabilistic, is represented by the statistical approaches of novelty measures and by probabilistic approaches for evaluation of entropy. The Sample Entropy (SampEn) and the Approximate Entropy (ApEn) are very typical and very relevant examples to be mentioned [3]-[4]. These approaches are closely related to the multi-scale evaluation of fractal measures, where further case studies utilizing SampEn, ApEn, and Multiscale Entropy (MSE) can be found in [5]-[7]. Further to this, probabilistic entropy approach to the concept shift (sometimes the concept drift) detection in sensory data is reported in [8]. The second of the mentioned streams is represented by the utilization of learning systems, such as neural networks and fuzzy-neural systems, and this is also the main area of focus for the presented work in this paper. During the last three decades of $20^{\text {th }}$ century, the works that were focused in regards to learning systems are that of [9]-[12], and for incremental learning approach can be referenced for example also the work [13]. Then, a particularly focused approach toward the utilization of learning systems has been rising with works [14]-[17]. Where, nonlinear estimators and learning algorithm were utilized for the fault detection via the proposed utilization of a fault function that evaluates behaviour of residuals of a learning system. Currently, significant research that shall also be referenced is adaptive concept drift detectors, proposed in [18]. Some readers might also see some analogies of the proposed approach in this paper to the Adaptive Resonance Theory [19]. Another approach to novelty detection is based on utilization of adaptive parameters of incrementally learning models (neural networks), i.e. the Adaptation Plot

David C. Wyld et al. (Eds) : CCSIT, SIPP, AISC, PDCTA, NLP - 2014

pp. 341-351, 2014. (C) CS \& IT-CSCP 2014

DOI : $10.5121 /$ csit.2014.4229 
[20] that has been recently enhanced with multi-scale approach [21]. A most recent method is the Learning Entropy, i.e., a multi-scale approach to evaluation of unusual behaviour of adaptive parameters of a learning model is introduced in [22]. This paper however introduces another, different approach to novelty detection, that is neither based on statistical approaches, nor is it based on evaluation of error residual. Contrary to [21], this new approach operates only on parameter space of incrementally learning systems, and it does not use the multi-scale approach for detection sensitivity. Here the method introduced shows one of the possibilities how to detect perturbations within measured data in every new sample.

\section{USED METHODOLOGY}

The method of detection proposed in this paper, utilises an adaptive prediction model. It is interesting to note how promising such method of detection works when used with a static linear adaptive model on non-linear data. Thus, for demonstrational purposes of this method, we will use a linear based adaptive model. The adaptation technique as applied to the predictive model, is based on the classical Gradient Descent algorithm. The employed linear adaptive model initially features a vector of random numbers as initial neural adaptive weights, which over each sample, are incrementally trained to model the real data, and an input vector comprised from previous samples of the real data, as follows;

$$
y(k+1)=\sum_{i=0}^{n} w_{i} x_{i}=\mathbf{w} \cdot \mathbf{x}
$$

Where, here, $\mathbf{w}$ denotes the row vector of all neural weights and $\mathbf{x}$ represents the vector of inputs. For the scope of this paper, the structure of this input vector for the linear predictive model is as follows;

$$
x(k)=\left[\begin{array}{lllll}
1 & y_{r}(k) & y_{r}(k-1) & \ldots & y_{r}(k-10)^{T}
\end{array}\right]
$$

Where, $\mathrm{x}_{0}=1$ allows for neural bias in case of the linear predictive model and $y_{r}$ represents measured values of the time series. The input vector was chosen to feature ten previous samples of the measured time series. Prior to prediction, the input vector was standardized via equation (3), in order to acquire better stability of the adaptive algorithm.

$$
y_{r}=\frac{y_{r}-\operatorname{mean}\left(y_{r}\right)}{3 \times \operatorname{std}\left(y_{r}\right)}
$$

For further improvement of the model stability, we provide adaptation of the learning rate $\mu$ via Normalized Least Squares method [23]. The equation (4) describes how this adaptation is performed. The Learning Rate adaptation is utilized before calculation of every sample. From equation (4) it is obvious that the normalized learning rate is smaller than the default learning rate in dependency on input $\mathbf{x}(\mathrm{k})$ for every sample;

$$
\eta=\frac{\mu}{1+\mathbf{x}(k)^{T} \times \mathbf{x}(k)}
$$

Where, $\eta$ serves as a substitution for $\mu$ For novelty detection estimation in every new sample in discrete time $\mathrm{k}$, we use the product of absolute values of the prediction error and adaptive weight changes according to equation (5), as follows;

$$
N D(k)=\left[\left|e(k) \times \Delta w_{i}(k)\right| ; i=0, . ., 10\right]
$$

Equation (5), thus presents the main principle behind this introduced method of novelty detection of every new sample according to temporary system dynamics. This method is simultaneously using prediction error and changes of adaptive weights ( $(\mathrm{k})$ and $\Delta \mathrm{w}_{\mathrm{i}}(\mathrm{k})$, respectively). This is the main theoretical result of this work. In the following sections we will investigate functionality of this method on real and artificial ECG with sampling of $256 \mathrm{~Hz}$ frequency. 


\section{EXPERIMENTAL ANALYSIS}

In this section, we will demonstrate the newly introduced method via computer simulations created in programming language Python 2.7 [24].The numerical algorithms were realized via python library Numpy [25]. The simulation was performed on a personal computer and was faster than real time measuring of an ECG signal. Thus, this justifies that such implementation is applicable for online realization.

\subsection{Artificial ECG}

In this subsection, we will demonstrate usage of the newly introduced method on an artificial ECG signal both, with and without noise, before eventually testing on a real ECG signal (of 256 $\mathrm{Hz}$ frequency), in the following subsection. For demonstrational purposes in this section, noise was added to the signal to highlight the ability of this introduced method, in detection of unexpected samples within the data. Especially where, the predictive model features lower prediction accuracy, comparative to data without noise. The reason why we test this method on an artificial signal, is to emphasize how well the detection works on perturbed data, if the signal doesn't contain any complicated phenomena. The artificial ECG time series used in our paper was created by a serially repeated pattern of a real ECG signal, sampled over one period. Thus, this artificial time series is an ideally periodic signal.

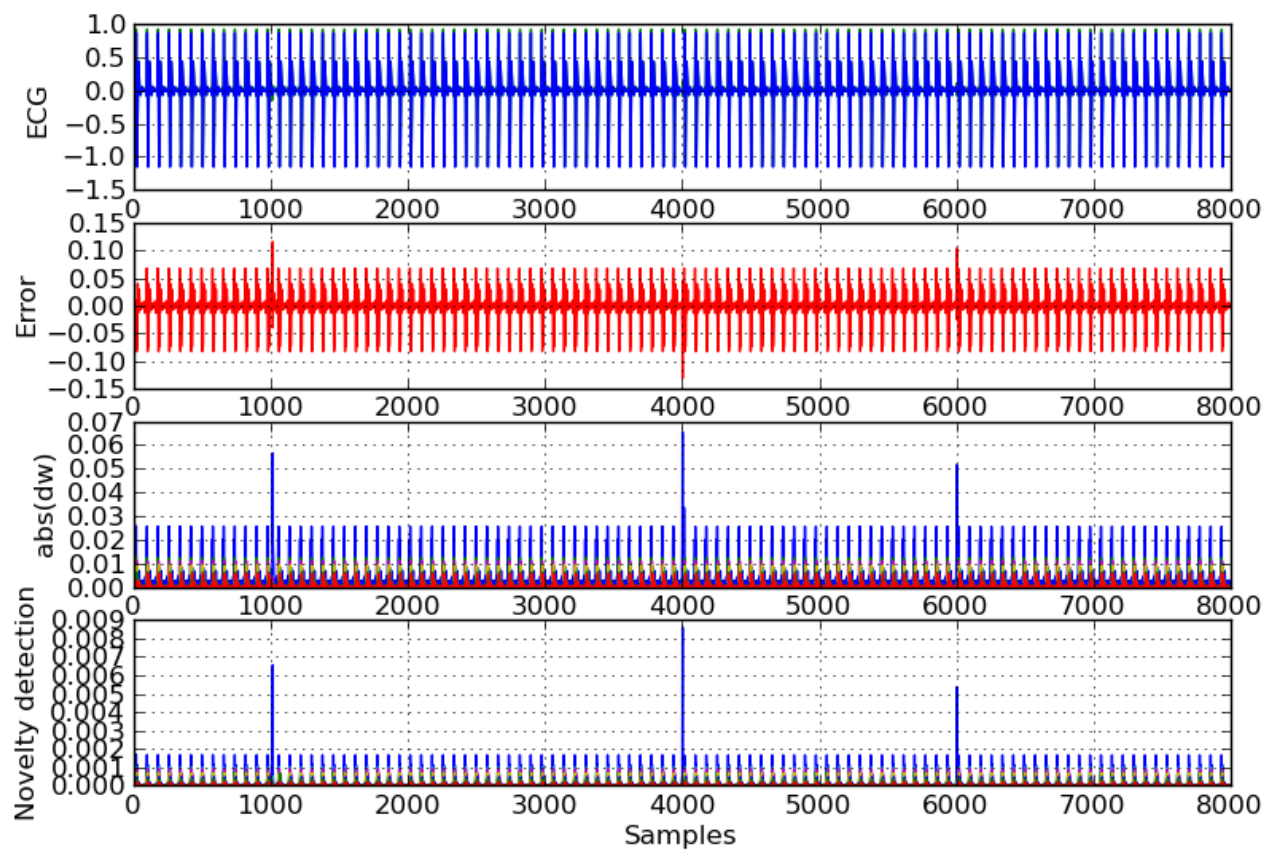

Figure 1. Novelty Detection used on artificial ECG without noise (for details refer to Figure 2) 

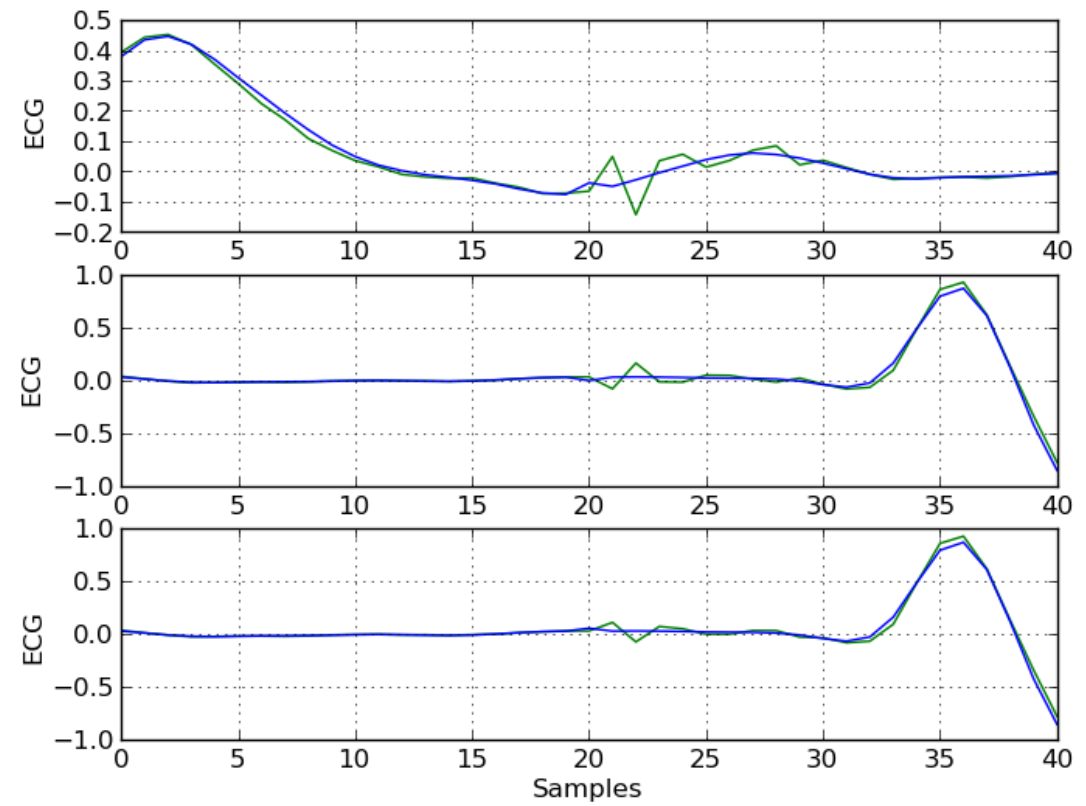

Figure 2. Details of prediction in areas of introduced perturbations in artificial ECG without noise (blue signal with perturbation, green - predicted signal)

Regarding the length of the pre-training data for the predictive model, we chose to take the first 2000 samples of the artificial data (ref. Figure 1). For sufficient training, 100 epochs was adequate. Figure 2, depicts the details of all perturbations included in the artificial ECG signal without noise. The size of the introduced perturbation is $0.03 \mathrm{mV}$. As we can see on Figure 2, these perturbations are small in comparison with the amplitude of the signal. Looking at the behaviour of the used predictive model, here we see the model tries to relearn immediately when the prediction error and weight adaptation increases (Figure 1). The return of the predictive model to previous prediction accuracy takes approximately 20 following samples. In Figure 1, it is possible to see the prediction error in specific places of a single period. These errors are caused by insufficient prediction ability of the simple, linear predictive model. Furthermore, in Figure.1, these errors are not detected as new data by the applied novelty detection method. Figure 3, shows the simulation of the artificially created ECG with the addition of noise. Here again, three perturbations were introduced to the data. These perturbations are located on the same positions as the signal without noise. This introduced noise was implemented via a generator of pseudorandom numbers, composed as a vector of random numbers in range from 0 to 0.01 , added via the following equation (6);

$$
y_{r}(k)=y_{r}(k)+\text { rand }, \text { rand } \in\langle 0,0.01\rangle, k=1000,4000,6000 .
$$

Figure 3 displays the real signal together with the simulated. Here in the first graph, it is possible to see the difference between the signals within the region of peaks of the amplitude. For the used predictive model, it is much more difficult to learn the pattern of the signal with added noise in comparison to that without noise. The errors and absolute values of the weight increments (Figure 3 ) are not entirely dependent on the periodicity of the signal. In the plot of error on Figure 3, it is not possible to see the perturbations clearly, as in the plot of prediction error without noise on Figure 2. In the graph of absolute error of weight increments, we can more evidently see the location of the perturbations. On the graph of novelty detection (Figure 3) the perturbation locations are even more evidently seen and, this is because a huge part of the models periodic 
errors, are filtered. Furthermore, these errors have no impact on the detection of unexpected samples in the data.

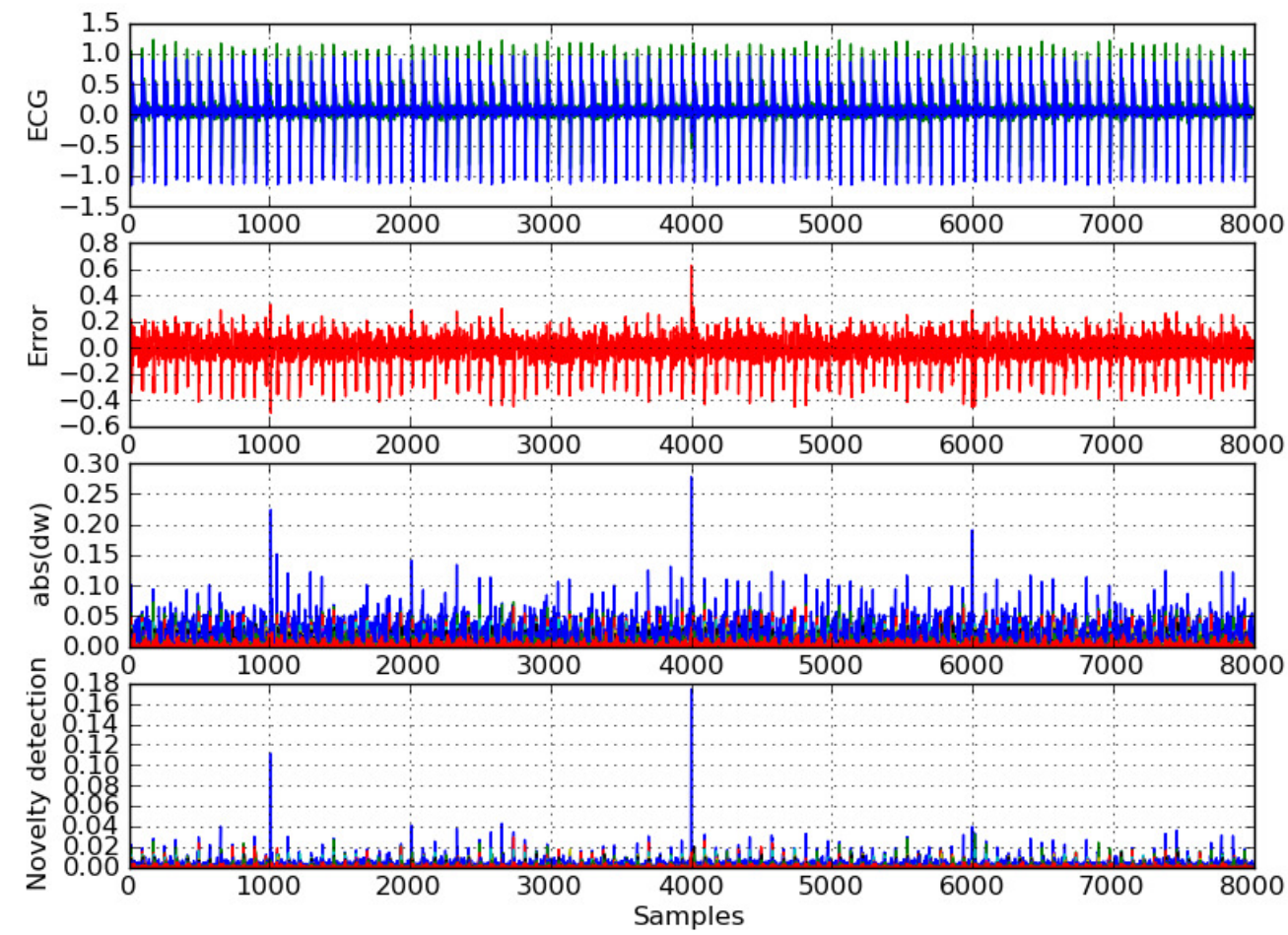

Figure 3. Novelty Detection used on artificial ECG with noise (details on Figure 4)
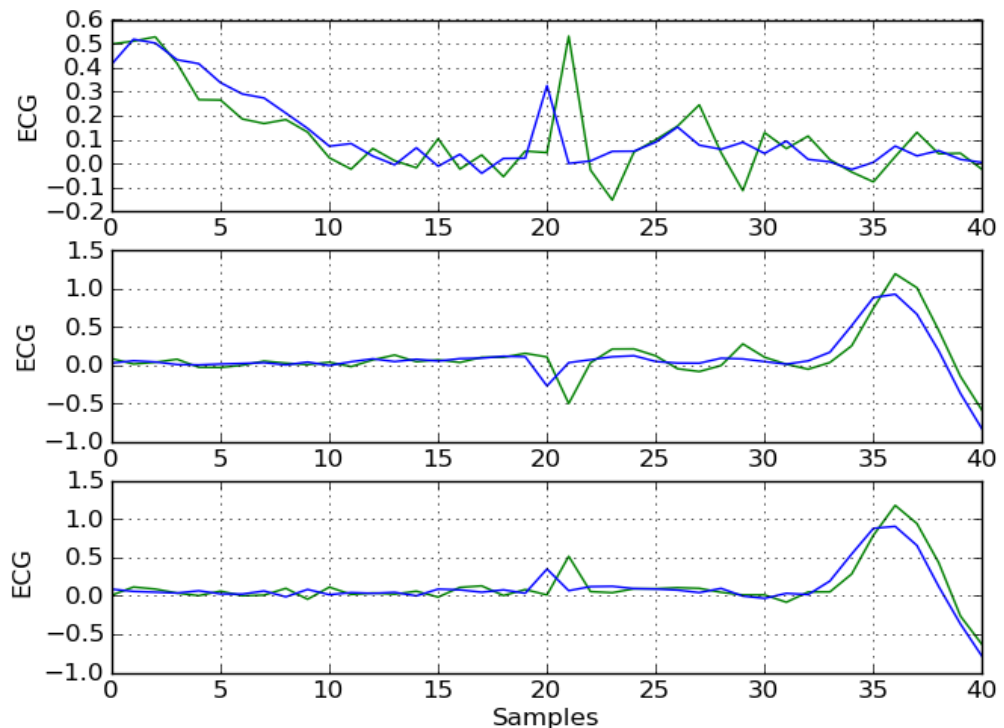

Figure 4. Details of prediction in areas of introduced perturbations in artificial ECG with noise (blue signal, green - predicted signal)

Figure 4 shows the details of three perturbations included into the artificial signal containing noise. Here, it is clearly shown that the prediction error of the signal with noise and also in the un- 
perturbed section of the signal, could reach substantially high values. The adaptive model again immediately reacts to the introduced perturbation and tries to relearn the data signal. The time in which the model needs for regaining normal accuracy after meeting a perturbation is hard to estimate, because the prediction error is strongly dependant on the level of noise present in the data.

\subsection{Real ECG Signal}

In this paper we also demonstrate the introduced method on an ECG signal. The used time series was measured by an internal cardio-defibrillator with $256 \mathrm{~Hz}$ frequency (the measured data was obtained courtesy of [26]). This signal was chosen because it contains spontaneous ventricular tachycardia, which is a rare phenomenon to measure. In this subsection, this introduced method of novelty detection will be demonstrated via a real ECG signal, which features a healthy, sinus like structure followed, by a ventricular tachycardia (arrhythmia). Later, we will show that this technique works in both in the sinus like region and also in the arrhythmic region of this signal. For demonstration, we will choose the same linear neural unit as the one previously used for prediction of the artificial ECG signal. The size of data chosen for learning of the predictive model is 1000 samples. In order to achieve sufficient pre-learning of the used neural unit with such amount of data, less than 500 epochs is adequate for achieving optimal results. Using any larger size of epochs doesn't seem to significantly improve the accuracy. Figure. 5 clearly depicts which part is the healthy ECG signal and which part represents the arrhythmia. In the introduced novelty detection, it is possible to detect the start of the arrhythmia signal, approximately 1000 samples before the arrhythmia is introduced (the shape of the period before the arrhythmia looks the same, but the scale of amplitudes starts varying). In the first graph of Figure 5, we can see how the measured signal is practically equivalent with the predicted signal. The included perturbations are not clearly seen in this graph. However, these perturbations are located in the samples of discreet time $1000,3000,5000$. On the graph of the prediction error, it is possible to see the perturbations quite well and even more so in the graph of absolute values of adaptive weights. However, looking on the graph of novelty detection, these perturbations are even more evidently pronounced. Moreover, the periodic errors are suppressed in region of the healthy ECG and arrhythmia signal. It is important to notice that the suppressing of the periodic error, is not that dominant in the onset of arrhythmia, unlike in other parts of the data. On Figure 6, we can see the prediction models seem to be quite accurate, but in some parts of the ECG period, there is always some small inaccuracy. These inaccuracies are mostly located in the positions of global minima's and maxima's, for a given period on the measured signal. The included perturbations are significantly small in size (0.04 - approximately $2 \%$ of the amplitude of healthy ECG signal). Furthermore, the adaptive model immediately reacts with re-learning of the ECG data, where by achieving the previous model accuracy, takes approximately 5-10 following samples. 

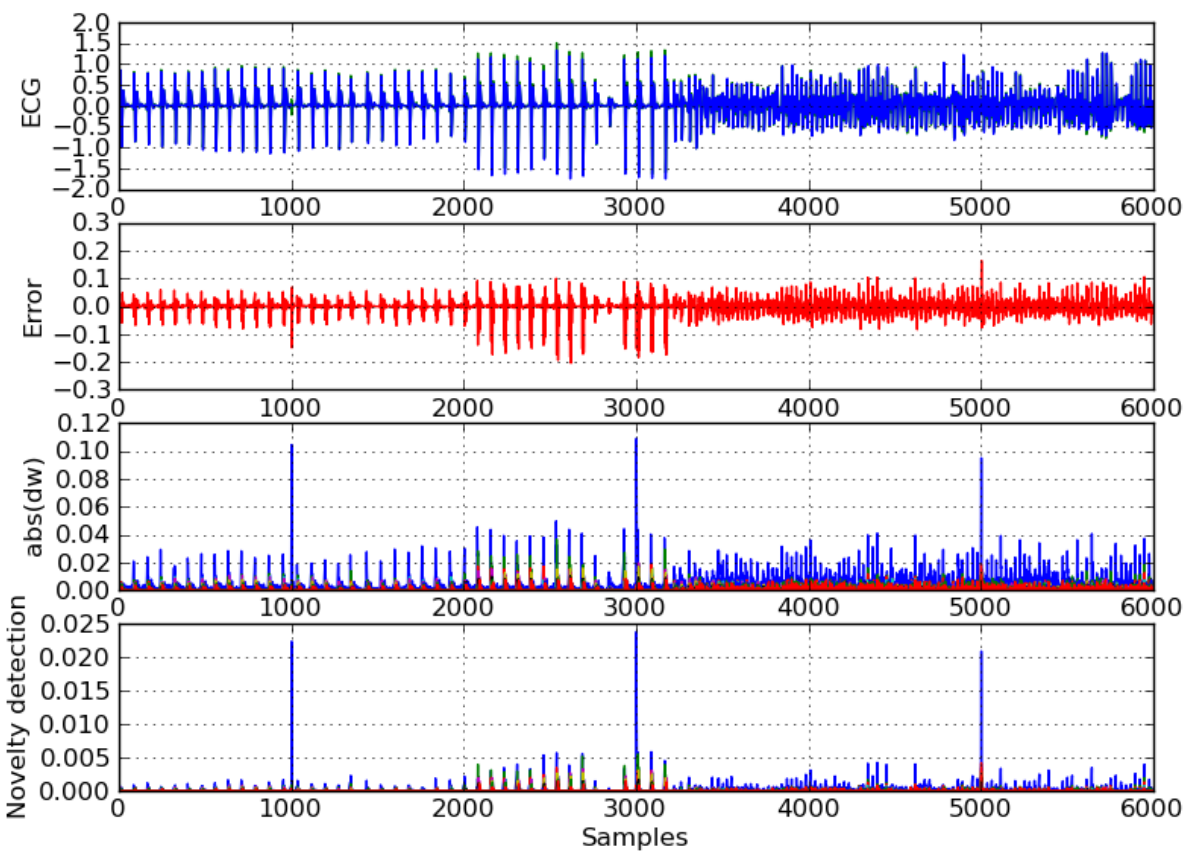

Figure 5. Novelty Detection used on real measured ECG signal
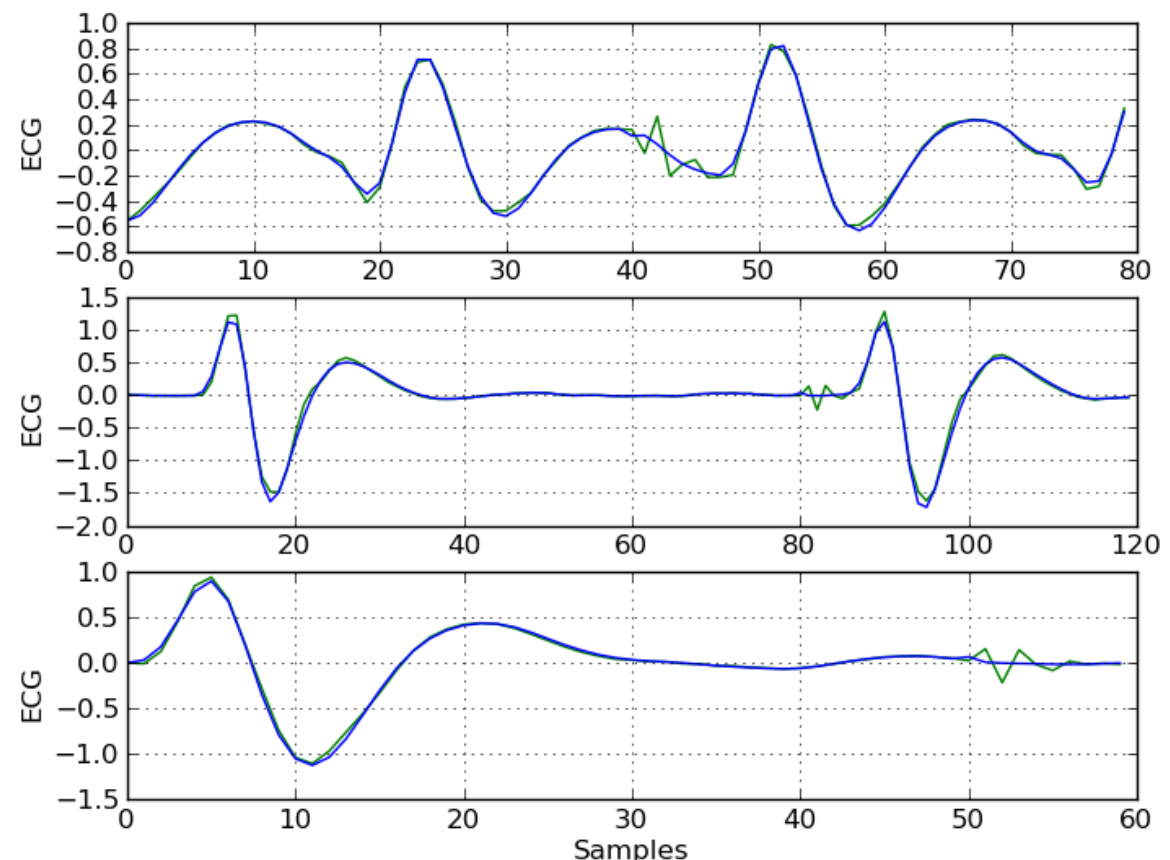

Figure 6. Details of prediction in areas of introduced perturbations in real measured ECG (blue - real signal, green - output of neural unit) 


\section{DISCUSSION}

As presented in previous chapters, the potentials of this method for novelty detection in biomedical signals, is quite promising. For demonstration, we chose a predictive model, which was relatively simple by structure. Here, such model was not capable to properly predict the measured ECG, yet the introduced novelty detection was able to distinguish the difference between prediction error caused by the model insufficiency and prediction error caused by novelty in the samples. This method also seems to be highly useful on artificial signals containing significant noise, where the model is unable to learn such noise, but can distinguish the noise from new phenomena in the measured data. Also on artificial data without noise we can certainly note, that novelty detection distinguishes the prediction error from the novelty in data, also with even a significantly small, signal perturbation.
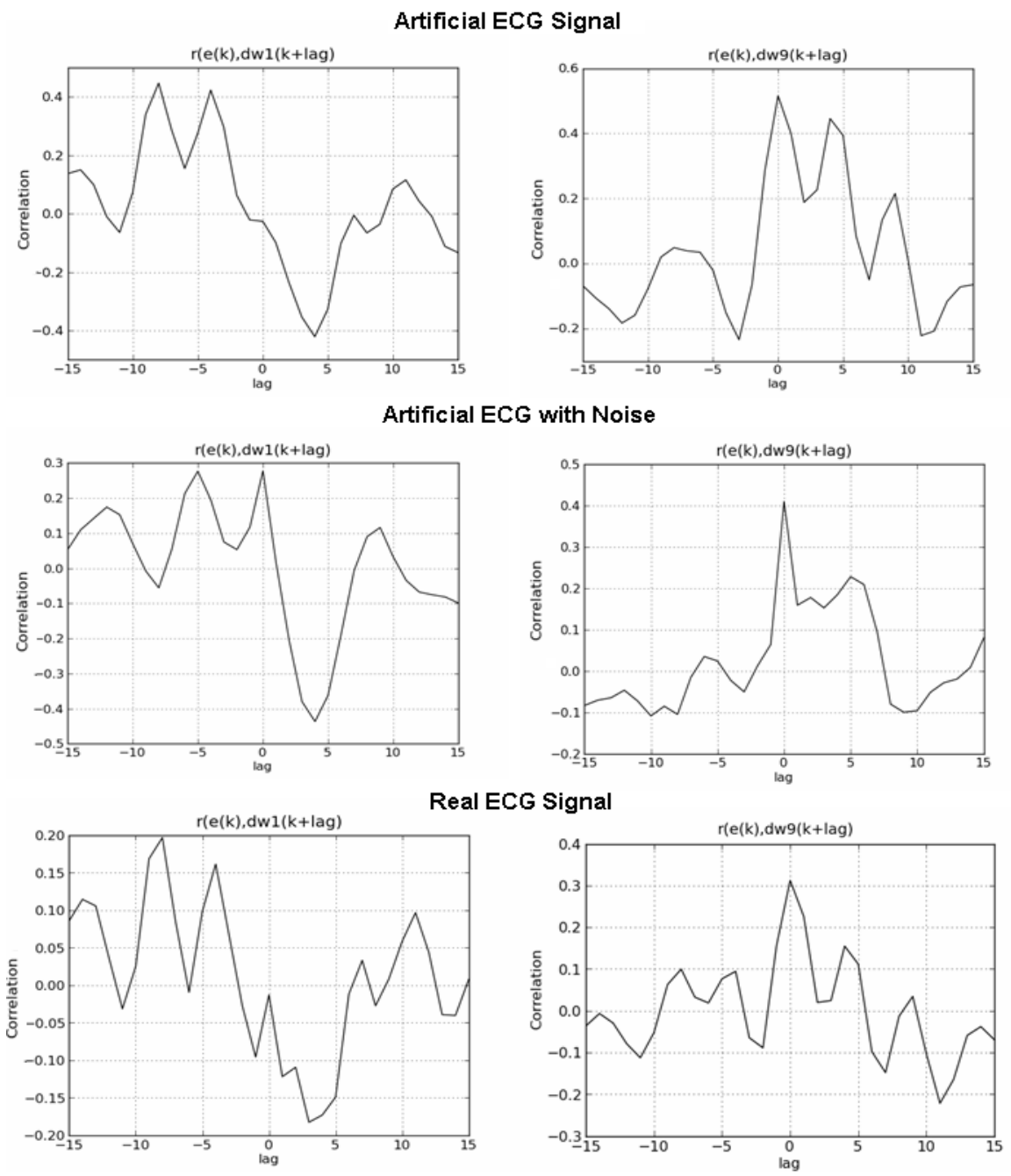

Figure 7. Correlation coefficients between adaptive weight increments and error, for $\Delta w_{1}$ and $\Delta w_{9}$ in the artificial ECG signal without noise (upper pair), artificial ECG signal with noise (middle pair) and real ECG signal (lower pair)

A further remark may be seen in Figure 7. Where, the correlation coefficients between the adaptive weight increments and error, over various samples of lag are analysed. For 
demonstrative purposes, Figure 7 only includes an analysis over the $\Delta w_{1}$ and $\Delta w_{9}$ adaptive weight increments with the error. Here, we may note that in all cases of the corresponding correlation coefficients, statistically insignificant correlation between the adaptive weight increments and error is seen and the same was observed for all other weight increments. This is justified by the notion that the prediction error of the model carries the information about the size of inaccuracy, while the adaptive weight increments carry information about how much the model tried to adapt on new data. Thus, even when the model error may be high, this may not necessarily be related with the adaptive weight increments (for more on this principle, please refer to the works of [20][22]). In case of a high error value, caused by common phenomena (what is out of the models capability to learn), novelty detection doesn't mark those particular samples, because the model has already recognized its inability to learn, which also further justifies the functionality of this method for novelty detection.

Another remark is in regards to the small time consumption in detection of this newly introduced method. This is due to the nature of the model being merely multiplication of values, which was calculated during the prediction of the data. Novelty detection doesn't slow down the process of prediction. This fact is a big advantage especially in processing the signal in real time, what could be a crucial feature in the case of ECG or other biomedical signals. Furthermore, as we can see on the results obtained by application of novelty detection on real ECG (Fig. 5), this technique of detection of unexpected data highlights the onset of arrhythmia from the other data. According to this method it is possible to see the onset of arrhythmia before it appears in the measured signal or prediction error. The first few samples show that there is inconsistency in the data, which appears approximately 1000 samples before the start of the arrhythmia signal. This model could be used for the detection of arrhythmia after proper optimizing and, it is an objective for further research.

\section{CONCLUSION}

Unusual behaviour of adaptable parameters and actual prediction error together, establishes novel learning-system-based measure of novelty that is introduced in this paper. The method presented in this paper is different from other methods and from the methods in [20] \& [21], because this method is using actual error of the predictive model, together with cognitive information in changes of adaptive weights of the model. This is an interesting approach, because the prediction accuracy and behaviour of a learning model are not necessarily correlated with each other [21]; however, both values together provide us with important information about consistency at every sample with temporary system dynamics. These facts are the reason why this and similar models of novelty detection are important for future research and development.

\section{ACKNOWLEDGEMENTS}

The authors would like to thank their colleagues from Tohoku University, namely from the Yoshizawa-Sugita Lab (formerly Yoshizawa-Homma Lab) and from the Department of Radiological Imaging and Informatics, for their vital, continuous cooperation and support. The authors would also like to acknowledge the SGS12/177/OHK2/3T/12 grant, for support of this work.

\section{REFERENCES}

[1] Markou, M.; Singh, S. Novelty detection: a review-part 1: statistical approaches, Signal Processing, Volume 83, Issue 12, 2003, pp. 2481-2497.

[2] Markou, M.; Singh, S. Novelty detection: a review-part 2: neural network based approaches, Signal Processing, Volume 83, Issue 12, 2003, pp. 2499-2521. 
[3] Pincus, S.M. Approximate Entropy as a Measure of System Complexity. Proc Natl Acad Sci, USA 88: 1991, pp. 2297-2301.

[4] Richman, J.S.; Moorman, J.R. Physiological time-series analysis using approximate entropy and sample entropy. Am. J. Physiol. Heart Circul. Physiol., 2000, 278, H2039-H2049.

[5] Costa, M.; Goldberger, A.L.; Peng, C.K. Multi-scale entropy analysis of complex physiologic time series. Phys. Rev. Lett. 2002, 89, 68102.

[6] Costa, M.; Goldberger, A.L.; Peng, C.K. Multi-scale entropy analysis of biological signals. Phys. Rev. E Stat. Nonlin Soft Matter Phys. 2005, 71, 021906.

[7] Yin, L.; Zhou, L. Function Based Fault Detection for Uncertain Multivariate Nonlinear Non-Gaussian Stochastic Systems Using Entropy Optimization Principle, Entropy 2013, 15, pp.32-52.

[8] Vorburger, P.; Bernstein, A.: Entropy-based Concept Shift Detection.,Proc. 6th Int. Conf. Data Min., 2006, pp.1113-1118.

[9] Willsky, A. A survey of design methods for failure detection in dynamic systems. Automatica, 1976, vol. 12, pp.601-611.

[10] Gertler, J. Survey of model-based failure detection and isolation in complex plants. IEEE Contr. Syst. Mag. 1988, vol. 8, pp.3-11.

[11] Isermann, R. Process fault detection based on modeling and estimation methods: A survey. Automatica, 1984, vol. 20, pp. 387-404.

[12] Frank, P.M. Fault diagnosis in dynamic systems using analytical and knowledge-based redundancyA survey and some new results. Automatica, 1990 vol. 26, pp.459-474.

[13] Widmer, G.: Kubat, M. Learning in the Presence of Concept Drift and Hidden Contexts, Machine Learning, April M. M. 1996, Volume 23, Issue 1, pp.69-101.

[14] Polycarpou, M.M.; Trunov, A.B. Learning Approach to Nonlinear Fault Diagnosis: Detectability Analysis. IEEE Transactions on Automatic Control, 2000, vol. 45, no. 4.

[15] Polycarpou, M.M.; Helmicki, A.J. Automated fault detection and accommodation: A learning systems approach. IEEE Trans. Syst., Man Cybern., 1995, vol. 25, pp.1447-1458.

[16] Demetriou, M.A: Polycarpou, M.M.: Incipient Fault Diagnosis of Dynamical Systems Using Online Approximators. IEEE Transactions on Automatic Control, 1998, Vol. 43, No. 11.

[17] Trunov, A.B.; Polycarpou, M.M. Automated fault diagnosis in nonlinear multivariable systems using a learning methodology. IEEE Transactions on Neural Networks. 2000, vol. 11, no. 1, pp.91-101.

[18] Alippi, C.; Roveri, M. Just-in-Time Adaptive Classifiers-Part I: Detecting Nonstationary Changes. IEEE Transactions on Neural Networks, 2008, Vol. 19, No. 7.

[19] Grossberg, S.; Adaptive Resonance Theory: How a brain learns to consciously attend, learn, and recognize a changing world. Neural Networks, 2013, 37, pp.1-47.

[20] Bukovsky, I.; Bila, J. Adaptive Evaluation of Complex Dynamic Systems using Low-Dimensional Neural Architectures. Advances in Cognitive Informatics and Cognitive Computing, Series: Studies in Computational Intelligence, Vol. 323/2010, eds. D. Zhang, Y. Wang, W. Kinsner, Springer-Verlag Berlin Heidelberg, 2010, pp.33-57.

[21] Bukovsky, I.; Kinsner, W.; Bila, J. Multi-scale Analysis Approach for Novelty Detection in Adaptation Plot. 3rd Sensor Signal Processing for Defence 2012 (SSPD 2012), Imperial College London, UK.

[22] Bukovsky, I.: "Learning Entropy: Multiscale Measure for Incremental Learning", manuscript ID entropy-39174, Entropy, special issue on Dynamical Systems, ISSN 1099-4300, (accepted paper 22/09/2013).

[23] B. Widrow and S. D. Stearns, Adaptive Signal Processing. Englewood Cliffs, NJ: Prentice-Hall, 1985.

[24] van Rossum, G., Drake, F. L. (eds): Python Reference Manual, PythonLabs, Virginia, USA, 2001. Available at http://www.python.org

[25] Ascher, D., Dubios, P., F., Hinsen, K., Hugunin, J., Oliphant, T.: Numerical Python, Lawrence Livermore National Laboratory, Livermore, California, USA, 2001.

[26] Yoshizawa-Sugita Lab and Homma Lab, Tohoku University, Japan. 


\section{Short Biography of Authors}

Matouš Cejnek received his Bachelor's Degree at Czech Technical University in Prague in 2012. Currently he is a Master's student, with expected $\mathrm{PhD}$ studies to follow in 2014 at CTU. His research focuses on non-conventional neural networks for adaptive systems and novelty detection in time series and biomedical applications. Matous's work has been awarded in local and international student competitions in 2013.

Peter Mark Beneš received his Bachelor's degree with honours at Czech Technical University in Prague (CTU) in 2012. Currently, he is a Master's student, with expected PhD studies to follow at CTU in 2014. His research focuses on non-conventional neural networks for adaptive identification and control of industrial systems including hoist mechanisms and skew control of rail-based mechanisms. Peter's work has been awarded in local and international student competitions also with an industrial BOSCH award in 2013.

Ivo Bukovsky graduated from Czech Technical University in Prague where he received his $\mathrm{Ph} . \mathrm{D}$. in the field of Control and System Engineering in 2007 and became associate professor since 2013. His research interests include higher-order neural networks, adaptive evaluation of time series and systems, multiscale-analysis approaches, control and biomedical applications. He was a visiting researcher at the University of Saskatchewan (2003), at the University of Manitoba in Canada (2010), and he was a visiting professor at Tohoku University in 2011. 


\title{
Resource Allocation USING METAHEURISTIC SEARCH
}

\author{
Dr Andy M. Connor ${ }^{1}$ and Amit Shah ${ }^{2}$ \\ ${ }^{1}$ CoLab, Auckland University of Technology, \\ Private Bag 92006, Wellesley Street, Auckland, NZ \\ andrew. connordaut. ac.nz \\ ${ }^{2}$ School of Computing \& Mathematical Sciences, Auckland University of \\ Technology,Private Bag 92006, Wellesley Street, Auckland, NZ
}

\begin{abstract}
This research is focused on solving problems in the area of software project management using metaheuristic search algorithmsand as such is research in the field of search based software engineering. The main aim of this research is to evaluate the performance of different metaheuristic search techniques in resource allocation and scheduling problemsthat would be typical of software development projects.This paper reports a set of experiments which evaluate the performance of three algorithms, namely simulated annealing, tabu search and genetic algorithms. The experimental results indicate thatall of themetaheuristics search techniques can be used to solve problems in resource allocation and scheduling within a software project. Finally, a comparative analysis suggests that overall the genetic algorithm had performed better than simulated annealing and tabu search.
\end{abstract}

\begin{abstract}
KEYWORDS
Evolutionary Computing, Genetic Algorithms, Simulated Annealing, Tabu Search, Resource Allocation, Scheduling, Project Management, Search Based Software Engineering
\end{abstract}

\section{INTRODUCTION}

In recent years, interest in the area of solving problems with optimisation techniques has increased considerably. Because of this it has led to the development of new algorithms, systems and methods. When the performances of these new developments are compared against the performance of traditional linear programming methods, it is clear that new optimisation techniques are often more robust and efficient. Harman \& Jones [1] observe that most of these techniques are currently implemented in disciplines like software \& mechanical engineering, biotic engineering, software testing, and many more. The importance of metaheuristics has been increasing over the years and to support that argument, many researchers have attempted to solve "real world" problems using a range of algorithms, including simulated annealing, tabu search and genetic algorithms. The application of search algorithms in the discipline of software engineering has resulted in the emergence of the term Search Based Software Engineering (SBSE) [1].

This paper investigates the performance of three metaheuristic algorithms on classes of problems that are drawn from the project management discipline and are representative of the types of

David C. Wyld et al. (Eds) : CCSIT, SIPP, AISC, PDCTA, NLP - 2014

pp. 353-364, 2014. (C) CS \& IT-CSCP 2014

DOI : $10.5121 /$ csit.2014.4230 
problems found in the management of software development projects. In particular there is a focus on resource and scheduling problems that have already been investigated in previously published work in order to allow a comparison of the results to be made.

\section{BACKGROUND \& RELATED WORK}

\subsection{Search Based Software Engineering}

The history of SBSE predates the term itself, with early research in representing software engineering challenges as a search problem dating back to 1976 [2]. Early approaches represented problems to be solved using classical techniques such as linear programming. However, Clark et al. [3] and Harman [4]suggest that linear programming models are not the best option for solving optimisation problems and this is because there are instances where the problem has certain objectives which cannot be represented with linear algorithms, furthermore, these problems also have multiple characteristics and fitness functions. Clarke et al. (2003) and Harman (2007) have identified three areas where problems could persist when implementing metaheuristics search techniques, but they have also provided potential solution to overcome the problems. One area in which there has been only limited interest is that of software project planning.

\subsubsection{Software Project Planning\& Resourcing}

The software engineering discipline has been in existence for a long time and since its introduction there have been substantial introduction of project management techniques to manage development projects. Over the years, there has been extensive publication in the area of project management and scheduling. Herroelen [5] has further suggested that there is an abundance of literature in this area, but for several reasons the theories have not been implemented into practice. Project management in the discipline of software engineering has always been problematic for many practitioners and there could be several reasons for it. Herroelen [5] argued that these problems are mainly caused because of the following reasons:

- Poor project management skills

- Poor leadership skills

- Size of the projects

- Lack of resources

- Inappropriate cost estimation and allocation methods

Furthermore, Herroelen[5] has also mentioned that these problems have been identified by literature in the past. To overcome the above mentioned problems, Herroelen has proposed a hierarchical project management model. In interest of solving the above mentioned problems, more effectively, it has been suggested to use heuristics approaches and there is a growing body of literature whereby researchers and practitioners have used algorithms to solve project management and scheduling problems.

Resource Constrained Project Scheduling Problems (RCPSP) is a subsection of the issue identified with in the software project planning and literature. This paper makes use of searchbased software engineering to resolve test examples that fall with in this class of problem. Kolisch \& Hartmann [6] have argued that the problem with software project planning is a high level problem and when the problems are analysed further, it turns out that in most cases the problems were caused because the resources were scarce. Furthermore, Pinto, Ainbinder \& Rabinowitz [7] have argued that there are three main resources which are usually scarce in a software project and they are as follows: 
- Lack of human resource

- Lack of funding

- Lack of available time

The above mentioned categories are similar to Herroelen [5] whereby he was trying to explain reasons for failure or escalation of a project, but having said irresolvable constraintscan also cause the project to fail or escalate. Kolisch and Hartmann [6] have suggested that literature in the past indicates that if a software project falls within the definition of RCPSP, then it is very likely that project will either fail or be escalated. This is the main justification stated by Kolisch and Hartmann [6] in support of their research to solve classes of RCPSP. Many researchers have argued that literature in the past suggest that researchers and practitioners have used several different methodologies to solve RCPSP, but unfortunately, none of the methodologies have been successful implemented in the "real-world".

Kolisch\& Hartmann [6] have clearly extended the thoughts of Clarke et al. [3] by conducting experiments to resolve this problem (i.e. implementing search techniques to solve RCPSP). Having said that, Kolisch\& Hartmann [6] have conducted experiments based on their assumptions and their own past research in 2001 which could make this research biased, but on the other hand Gueorguiev, Harman, \&Antoniol [8] have conducted experiments using data from the "realworld" and this could potentially return results which are not biased.

Kolisch\& Hartmann [6] and Gueorguiev, Harman, \&Antoniol[8] all have mainly focused on solving RCPSP using search-based software engineering approaches. The authors have clearly followed the guidelines provided by Harman and Jones [1] and Clarke et al. [3] whereby they reformulated the RCPSP as search problem. In the next stage authors have selected a representation of the problem and after that, authors have identified their fitness functions to evaluate candidate solutions. Having said that, each research had different criteria for fitness function and this mainly because the nature of the experiments was different.

\section{METHEURISTIC SEARCH ALgORITHMS}

Metaheuristic search algorithms have been an area of growing interest for several decades as the recent growth in computing power has resulted in the potential of these approaches being realised. A wide range of algorithms have been developed, each of which has its own merits. This research is not intended to be an exhaustive exploration of the performance of every algorithm and is restricted to three standard algorithms, namely Simulated Annealing, Tabu Search, and Genetic Algorithms.

\subsection{Simulated Annealing}

Simulated annealing is a metaheuristic search technique which can be used to solve optimisation problems. The technique has the ability to find solutions in large and small solution spaces. Unlike many other metaheuristic search techniques, this technique is a direct search method involving a single search trajectory [9]. The name and inspiration for this search technique was derived from the process of annealing metals. This annealing process involved heating and gradually cooling the solid material so that the defects are reduced. After the completion of this process, it can be concluded that the solid material has reached a global minimum state.

The simulated annealing algorithm is therefore very straight forward. When the algorithm is initiated, an initial solution to the problem is randomly generated. After initial value is selected, it is evaluated in accordance to the problem cost function and then changed slightly to generate a new candidate solution from the neighbourhood of the initial solution. After selecting a new 
candidate solution, the value of the cost function is obtained and if the value is better than previous candidate solutions then it is retained. However, if the value is worse than any other candidate solution, then there is small probability that the search will move to the next candidate solution and continue. The calculation of the probability is calculated using an analogy to the Maxwell-Boltzmann probability function.

When there is a change in the value of the cost function, it determines the change in energy. The units of temperature control parameters and cost function is the same. Additionally, the temperature control parameter also enables the probability of selection. During the initial stages of the execution process of the algorithm, the temperature is kept steady and this allows the system to gain momentum in searching. As the temperature drops, the probability of selecting a bad solution reduces. Hence towards the end, this algorithm tends to move towards an optimum solution. Previous work [10] has shown that Simulated Annealing and Tabu Search both have the capacity to solve complex problems, but with different solution trajectories.

\subsection{Tabu Search}

Tabu search has similar search method characteristics to simulated annealing and is generally implemented as a single search trajectory direct search method. The concept was originally coined by Glover $[11,12]$ and since then the application of this search technique has increased considerably. Tabu search has been successfully implemented to solve discrete combinatorial optimisation problems such as graph colouring and Travelling Salesman Problems, and has also been applied to a range of practical problems. In terms of operations, tabu search is initiated at a random starting point within a solution. After that, it identifies sequences of moves and whilst that process is executed, a tabu list is generated. Evaluation of cost function can determine whether the member belongs to the tabu list or not. Some members of the tabu list can belong to an aspiring set. The criteria for aspiring move are dependent on the size and the type of the problems; hence this could differ for each implementation. Additionally, tabu search also uses tabu restrictions and a number of flexible memories with different time cycles. The flexible memories allow search information to be exploited more thoroughly than rigid memory or memoryless systems, and can be used to either intensify or diversify the search to force the method to find optimum solutions. Previous work has shown that Tabu Search has the potential to find solutions to complex problems much more efficiently than Genetic Algorithms [13].

\subsection{Genetic Algorithms}

Unlike simulated annealing and tabu search, genetic algorithm is not a local search method. This search technique uses a population of solutions that are manipulated independently of the evaluation of the cost function. This algorithm was built on the principles of Darwinian Evolution [14]. Since its introduction, this search technique has been used in variety of disciplines and there is substantial research to identify its practical implementations.

Goldberg [14] further adds that genetic algorithmsare a non-derivative based optimisation technique and the outcome of this algorithm is based upon the principle of the survival of the fittest. When the algorithm is initiated, a candidate solution set is created on random and this is called a population. Using the existing population, new generation is created using genetic operators like crossover, mutation, and reproduction. Ideally as the algorithm progresses, the solutions are improved and optimum solutions can be achieved over time.

Genetic Algorithms are a broad and effective search method which has been applied to a wide range of practical problems. The term Genetic Algorithm is particularly broad and covers many 
variations in implementation ranging from the simple GA presented by Golberg[14] through to complex multi-objective algorithms such as NSGA-II [15].

\section{TEST PROBLEMS}

Each of the algorithms described in Section 3 have been implemented and tested on a number of different test problems. Prior to investigating resourcing and scheduling problems, the performance and scalability of the implementations were tested on numerical test functions and other discrete optimisation problems, such as the n-Queens problem. This is not reported in this paper but allowed for each algorithm to be suitably tuned to allow a fair comparison to be made.

\subsection{Resourcing Problem}

To schedule a project effectively, project planners must select appropriate costing and resourcing options. This selection will determine the duration of the project. In most cases, projects have multiple costing and resourcing options which lead to multiple due dates. The main objective in the evaluation is to schedule resource unconstrained and constrained project using metaheuristics search techniques.

Traditionally, project schedules can be generated using a critical path method and that project planners can also include resources and activities assigned to those resources. Unfortunately, such schedules have a down side whereby it is difficult for project planners to identify when the resources were freed from the previous activity. Hence this evaluation will overcome the limitation identified by using critical path method. Before the evaluation process starts, consider a small project presented in Table 1 by each activity with its early start, early finish, late start, late finish and total float.

Table 1.Project Scheduling Data [16]

\begin{tabular}{|c|c|c|c|c|c|c|c|c|}
\hline $\begin{array}{c}\text { Activity } \\
\text { no. }\end{array}$ & $\begin{array}{c}\text { Start } \\
\text { Node }\end{array}$ & \multicolumn{2}{c}{$\begin{array}{c}\text { End } \\
\text { Node }\end{array}$} & \multicolumn{2}{c}{ Successor } & \multicolumn{2}{c}{ Early Start } & \multicolumn{2}{c|}{$\begin{array}{c}\text { Early } \\
\text { Finish }\end{array}$} & $\begin{array}{c}\text { Late } \\
\text { Start }\end{array}$ & $\begin{array}{c}\text { Late } \\
\text { Finish }\end{array}$ & $\begin{array}{c}\text { Total } \\
\text { Float }\end{array}$ \\
\hline $\mathbf{l}$ & 0 & 2 & $7,8,9$ & 0 & 20 & 15 & 35 & 15 \\
\hline $\mathbf{2}$ & 0 & 5 & 7 & 0 & 33 & 45 & 78 & 45 \\
\hline $\mathbf{3}$ & 0 & 8 & 9 & 0 & 70 & 24 & 94 & 24 \\
\hline $\mathbf{4}$ & 1 & 3 & 8,9 & 0 & 40 & 0 & 40 & 0 \\
\hline $\mathbf{5}$ & 1 & 5 & 7 & 0 & 37 & 41 & 78 & 41 \\
\hline $\mathbf{6}$ & 1 & 6 & 9 & 0 & 56 & 41 & 97 & 41 \\
\hline $\mathbf{7}$ & 2 & 7 & 9 & 20 & 87 & 48 & 115 & 28 \\
\hline $\mathbf{8}$ & 2 & 8 & 9 & 20 & 79 & 35 & 94 & 15 \\
\hline $\mathbf{9}$ & 2 & 9 & - & 20 & 98 & 48 & 126 & 28 \\
\hline $\mathbf{1 0}$ & 3 & 8 & 9 & 40 & 94 & 40 & 94 & 0 \\
\hline $\mathbf{1 1}$ & 3 & 9 & - & 40 & 94 & 72 & 126 & 32 \\
\hline $\mathbf{1 2}$ & 4 & 5 & 7 & 0 & 29 & 49 & 78 & 49 \\
\hline $\mathbf{1 3}$ & 4 & 6 & 9 & 0 & 43 & 54 & 97 & 54 \\
\hline $\mathbf{1 4}$ & 5 & 7 & 9 & 37 & 74 & 78 & 115 & 41 \\
\hline $\mathbf{1 5}$ & 6 & 9 & - & 56 & 85 & 97 & 126 & 41 \\
\hline $\mathbf{1 6}$ & 7 & 9 & - & 87 & 98 & 115 & 126 & 28 \\
\hline $\mathbf{1 7}$ & 8 & 9 & - & 94 & 126 & 94 & 126 & 0 \\
\hline
\end{tabular}

This data was used by Christodoulou [16] to schedule the project using ant colony optimisation algorithm. The critical path calculations on the above mentioned case study topology and the resulting early start, early finish, late start, late finish and total float can be solved by applying traditional critical path planning methods. Based on the critical path method calculation and activities 4, 10 and 17 have been identified as critical and the total duration of the project is 126 time units. 
Christodoulou [16] has also solved the above mentioned case study using critical path method resource unconstrained and resource constrained environments. Results are presented in section 5 that can be compared to the work of Christodoulou [16].Figure 1 illustrates the critical path for this small project.

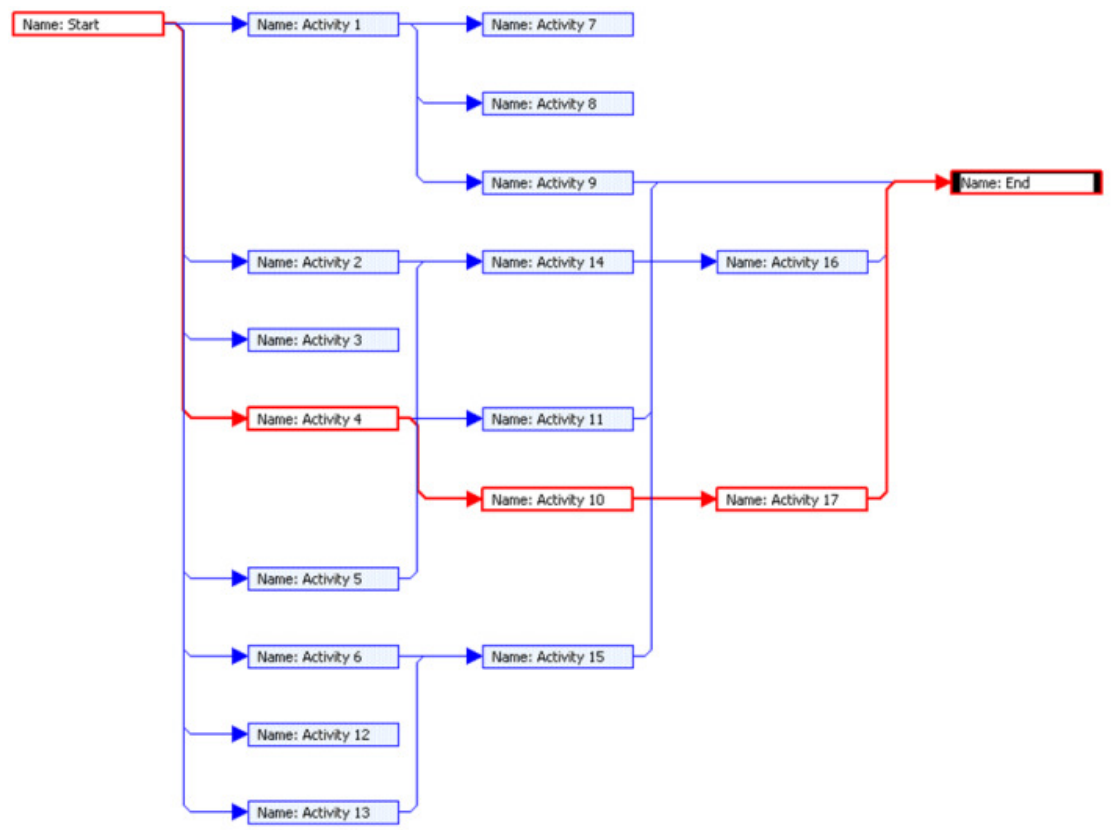

\subsection{Scheduling Problem}

Figure 1.Critical path

This paper also evaluates the performance of three meta-heuristic algorithms on a multi-objective time-cost trade-off project scheduling problem which is discrete in nature. The problem data is presented in Table 2. This data has also used by Elbeltagi, Hegazy\& Grierson [17] and Feng, Liu \& Burns [18] to solve discrete optimisation problem by implementing a number of different algorithms. 
Table 2.Project Scheduling Data [17]

\begin{tabular}{|c|c|c|c|c|c|c|c|c|c|c|c|}
\hline Activity & Depends & \multicolumn{2}{|c|}{ Option 1} & \multicolumn{2}{|c|}{ Option 2} & \multicolumn{2}{|c|}{ Option 3} & \multicolumn{2}{|c|}{ Option 4} & \multicolumn{2}{|c|}{ Option 5} \\
\hline 1 & & 14 & 2400 & 15 & 2150 & 16 & 1900 & 21 & 1500 & 24 & 1200 \\
\hline 2 & & 15 & 3000 & 18 & 2400 & 20 & 1800 & 23 & 1500 & 25 & 1000 \\
\hline 3 & & 15 & 4500 & 22 & 4000 & 33 & 3200 & 33 & 3200 & 33 & 3200 \\
\hline 4 & & 12 & 45000 & 16 & 35000 & 20 & 30000 & 20 & 30000 & 20 & 30000 \\
\hline 5 & 1 & 22 & 20000 & 24 & 17500 & 28 & 15000 & 30 & 10000 & 30 & 10000 \\
\hline 6 & 1 & 14 & 40000 & 18 & 32000 & 24 & 18000 & 24 & 18000 & 24 & 18000 \\
\hline 7 & 5 & 9 & 30000 & 15 & 24000 & 18 & 22000 & 18 & 22000 & 18 & 22000 \\
\hline 8 & 6 & 14 & 220 & 15 & 215 & 16 & 200 & 21 & 208 & 24 & 120 \\
\hline 9 & 6 & 15 & 300 & 18 & 240 & 20 & 180 & 23 & 150 & 25 & 100 \\
\hline 10 & 2,6 & 15 & 450 & 22 & 400 & 33 & 320 & 33 & 320 & 33 & 320 \\
\hline 11 & 7,8 & 12 & 450 & 16 & 350 & 20 & 300 & 20 & 300 & 20 & 300 \\
\hline 12 & $5,9,10$ & 22 & 2000 & 24 & 1750 & 28 & 1500 & 30 & 1000 & 30 & 1000 \\
\hline 13 & 3 & 14 & 4000 & 18 & 3200 & 24 & 1800 & 24 & 1800 & 24 & 1800 \\
\hline 14 & 4,10 & 9 & 3000 & 15 & 2400 & 18 & 2200 & 18 & 2200 & 18 & 2200 \\
\hline 15 & 12 & 12 & 4500 & 16 & 3500 & 16 & 3500 & 16 & 3500 & 16 & 3500 \\
\hline 16 & 13,14 & 20 & 3000 & 22 & 2000 & 24 & 1750 & 28 & 1500 & 30 & 1000 \\
\hline 17 & $11,14,15$ & 14 & 4000 & 18 & 3200 & 24 & 1800 & 24 & 1800 & 24 & 1800 \\
\hline 18 & 16,17 & 9 & 3000 & 15 & 2400 & 18 & 2200 & 18 & 2200 & 18 & 2200 \\
\hline Total & & 100 & 169820 & 131 & 136705 & 159 & 107650 & 166 & 101178 & 169 & 99740 \\
\hline
\end{tabular}

The data presented above relates to a project which constitutes 18 activities and has been presented with 5 options of different cost and duration. In each case, the first option is the most expensive option but it will take the least number of days to complete the project and the fifth option is the cheapest option and it will take the longest to complete. For each task the project managers would have to choose from five options and this could traditionally be done using heuristics approaches, but to get most optimised solution, one of the five options will be selected for each task using genetic algorithm, simulated annealing and tabu search. As mentioned earlier, this data is related to time-cost trade-off problem and as such there is likely to be a pareto-optimal set of solutions to the problem. Thepareto-optimal set of solutions is a unique line through the total set of solutions that represents what are considered to be non-dominated solutions. Each solution along the pareto-optimal front is equally valid in terms of how it trades off cost and time. Before the evaluations are carried out, a critical path must be established for the 18 tasks mentioned above and it illustrated in Figure 2.

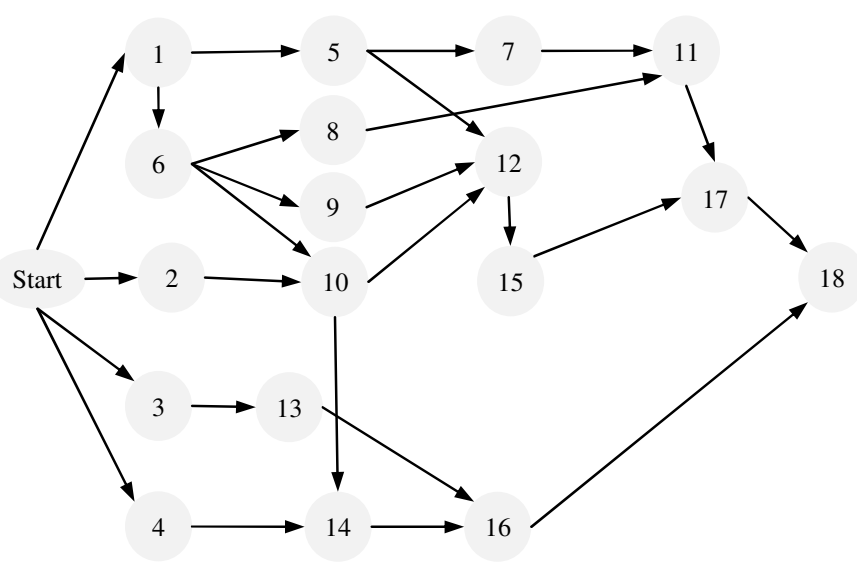

Figure 2.Task dependency network 
This task dependency network does not display a critical path as the selection of different options from Table 2 may result in different critical paths being generated. The objective of this problem is to minimise the total cost of the project and to do this a fitness function has been used against each algorithm. This fitness function is defined by Equation 1.

$$
f(x)=M I N\left((T \times I)+\sum_{i=1}^{n} C_{i j}\right)
$$

Equation 1: Cost Estimation Fitness Function

The variables mentioned in the above mentioned fitness function represents the following:

$\mathrm{n}=$ number of activities

$\mathrm{C}_{\mathrm{ij}}=$ direct cost of activity $\mathrm{i}$ using its method of construction $\mathrm{j}$

$\mathrm{T}=$ total project duration

$\mathrm{I}=$ daily indirect cost

The three different metaheuristic search algorithms are used to minimise the total cost of the project using the fitness function mentioned above. The underlying application and parameters for each algorithm is similar to the previous evaluations. The results generated from these evaluations will be compared against the results from evaluations carried out by Elbeltagi, Hegazy\& Grierson [17] and Feng, Liu \& Burns [18].

\section{EXPERIMENTAL RESULTS}

\subsection{Resourcing Problem}

Resource unconstrained scheduling is fairly straight forward and in most cases can be solved using critical path methods. For the purpose of this evaluation, the genetic algorithm, simulated annealing and tabu search algorithms will be used. Because the case study is relatively simple, all search techniques were able to find an optimum solution in a reasonable timescale. In this case the optimum solution is 126 time units for the project duration. When this solution is compared against the solution presented using critical path method it is the same.

Although in this case the solution is the same as critical path method, it may always not be the same. If the size of the project would be extensively large then finding an optimum project duration would take longer and may not be correct because of human intervention. In this case study, the critical path method calculation required ten conditional statements and 17 additions / subtractions for each forward or backward pass in the network. In contrast to that the metaheuristic search algorithms are more efficient in finding the optimum solution. The advantage of this might not be so obvious in this evaluation mainly because of the size of the data, but it is likely that for larger dataset these algorithms would generate results significantly faster and more efficiently.

Table 3. Unconstrained Resourcing Problem Results

Algorithm

Duration Critical Path Activities Iterations

\begin{tabular}{|l|r|r|r|}
\hline Ant Colony Optimisation [16] & 126 & $4,10,17$ & $<=50$ \\
\hline Genetic Algorithm & 126 & $4,10,17$ & $<=39$ \\
\hline Simulated Annealing & 126 & $4,10,17$ & $<=49$ \\
\hline Tabu Search & 126 & $4,10,17$ & $<=55$ \\
\hline
\end{tabular}


The results presented above for each algorithm are the same and that is mainly because there are no constraints on the project. However, some search techniques have found the optimum solution sooner than other search techniques. In this case, genetic algorithm was the quickest to find the best solution. In the evaluations carried out by Christodoulou [16], he has also achieved the same results as genetic algorithm, simulated annealing and tabu search. Although the size of the case is study is fairly small the overall process for calculating the total duration and identifying critical activities was very straight forward. The main idea behind this evaluation is to schedule the project as soon as possible, hence any constraints were not considered. However, if we were to assign resources constraint to each task and still wanted to same project due date, there would be some over allocated resources.

Scheduling a resource-unconstrained project is reasonably straightforward, but as soon as there is a constraint on resources for the project, the scheduling becomes very complicated and critical path method may not be sufficient to achieve an optimised project schedule. The lack of resources needed to start and complete an activity make certain critical paths unfeasible solutions.As a result, some of the activities in a project can be put on hold which in turn can impact the entire project schedule. In the standard critical path method the importance of activities are determined by its total float value. The importance of activity increases as the value of total float drops. Hence, when scheduling a project activities with fewer totals float value get preference in allocating resources.

In the unconstrained problem, it is assumed that each activity in the problem presented in Table 1 utilises one unit of resources for each day and based on that a resource histogram is can be generated. However for this evaluation it is assumed that the availability of resource is constrained to 7 units. As a result the need for resources has exceeded the available resource threshold. When the constraints are implemented the results shown in Table 4 are achieved.

Table 4.Constrained Resourcing Problem Results

\begin{tabular}{|l|r|r|r|}
\hline \multicolumn{1}{|c|}{ Algorithm } & Duration & Critical Path Activities & Iterations \\
\hline Ant Colony Optimisation [16] & 142 & $3,13,15$ & $<=50$ \\
\hline Genetic Algorithm & 139 & $4,7,17$ & $<=58$ \\
\hline Simulated Annealing & 147 & $5,9,17$ & $<=55$ \\
\hline Tabu Search & 143 & $2,9,17$ & $<=62$ \\
\hline
\end{tabular}

This table represents time taken in the duration column, and also highlights the critical activity. The first results are derived from the experiments of Christodoulou [16]. In his experiments, ant colony optimisation finds a solution that takes 142 time units to complete a project and in comparison that genetic algorithm implemented in this research will take 139 time units and the critical activities are 4, 7 and 17. Whilst the genetic algorithm has found the solution by projecting to complete the project in 139 time units, it took more iterations than ant colony optimisation and simulated annealing. Ant colony optimisation has outperformed simulated annealing and tabu search in terms of both duration of the outcome and the number of iterations required to find the solution.

\subsection{Scheduling Problem}

The summary of results generated from this evaluation is presented in Table 5. This table represents the minimum and average of project cost and duration over multiple runs of the algorithms. In addition to this, it also presents the percentage of success against the other algorithms. The percentage of success is calculated based on numbers of days and total cost of the project. Hence, the lower the total cost of project and duration, higher the success rate of the algorithm. 
Table 5.Scheduling Problem Results

\begin{tabular}{|l|c|c|c|c|c|c|c|}
\hline Algorithm & \multicolumn{3}{|c|}{ Minimum } & \multicolumn{3}{c|}{ Average } \\
\hline & Duration & Cost & Iterations & Duration & Cost & Iterations & \% Success \\
\hline Genetic Algorithm & 104 & 139,320 & 64 & 111 & 152,010 & 68 & 50 \\
\hline Simulated Annealing & 110 & 145,820 & 77 & 118 & 156,310 & 80 & 30 \\
\hline Tabu Search & 108 & 156,720 & 71 & 113 & 156,910 & 75 & 20 \\
\hline
\end{tabular}

The genetic algorithm was again the best performing algorithm by finding an option for project managers to complete the project in 104 days with total cost of $\$ 139,320$. The best combination found by simulated annealing was to complete the project in 110 days with total cost of $\$ 145,820$ and the best combination found by tabu search was to complete the project in 108 days with total cost of $\$ 156,720$. Although the combination found by tabu search enables the project to complete faster than simulated annealing, the cost of the proposed combination from tabu search is costlier than simulated annealing. Hence simulated annealing has a greater success rate than tabu search.

These results can be compared with those of Feng, Liu \& Burns [18] who utilised a Genetic Algorithm to solve this problem and they discovered two non-dominated solutions, 100 days $/ \$ 133,320$ and 101 days $/ \$ 129,320$. The best solution found in the current research is very close to the pareto-optimal front for this problem. The above solutions appear to be an improvement when compared with the results of Elbeltagi, Hegazy \& Grierson [17]. Their comparative study indicated that the Particle Swarm Optimisation algorithm was best at solving the problem; however the best solution it found was 110 days/ $\$ 161,270$. The overall results achieved for each algorithm is presented in a time-cost trade-off curve as illustrated in Figure 3.

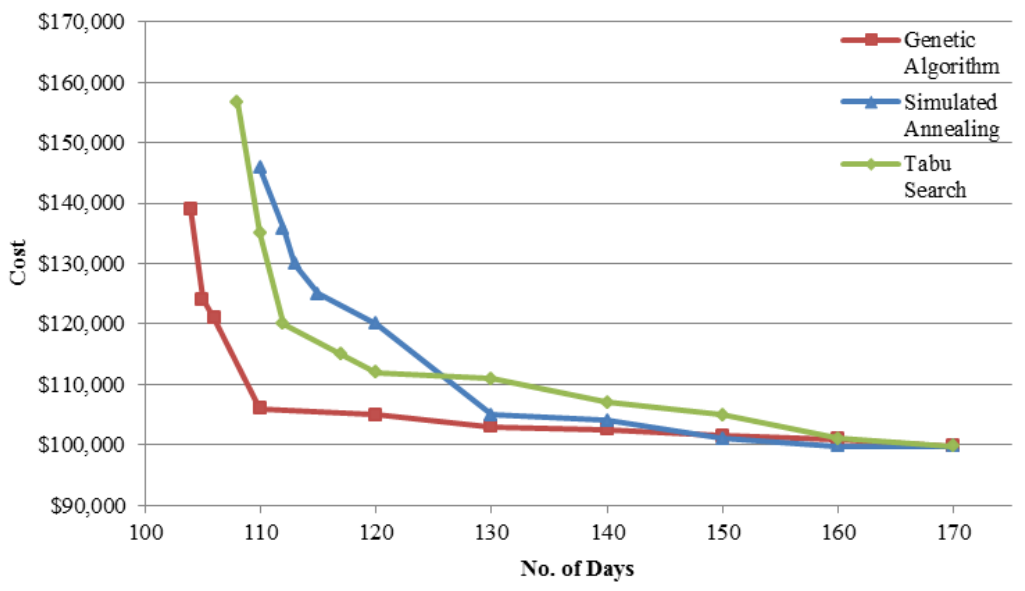

Figure 3.Pareto-optimal sets

Figure 3 illustrates the pareto-optimal fronts identified by the different algorithsm, where each point on the curve represents a unique time-cost trade-off that is non-dominated. During the initial stages of evaluation, trade-off curve are generated, but they are scattered all over the solution space and does not gather into one region, but as the evaluation progresses the trade-off curve takes shape. The Genetic Algorithm took 64 iterations to achieve final generation which produces the trade-off curve whereas simulated annealing took 77 iterations and tabu search took 71 iterations. An effective way to judge a performance of the algorithm is to ensure that the trade-off curve is closest to the axis. Hence looking at Figure 3, it is evident that trade-off curve for the genetic algorithm has performed better than that of simulated annealing and tabu search. 


\section{CONCLUSIONS}

This paper presents the results of applying three metaheuristic search algorithms to a number of problems that would be typical of those found in the management of software development projects. All three of the algorithms have the potential to solve scheduling and planning problems, though the genetic algorithm has performed consistently well when compared against the other algorithms. Simulated annealing was the second most favourable for this evaluation, and that it is evident that tabu search is the least favourable choice of algorithm to solve the problems presented in this paper. This isdifferent to conclusions of Elbeltagi, Hegazy\& Grierson [17] who mentioned that tabu search has been used widely by many researchers to solve not only time-cost trade-off problem, but many other NP-hard problems.

After finding the trade-off curve, project managers can determine the total cost of the project by summing up the estimated indirect cost and direct cost from trade-off curve. Using trade-off curve as the objective function allows for much more efficient evaluation of various other indirect costs.Future work will investigate the scalability of the approach to significantly larger problems.

\section{REFERENCES}

[1] Harman, M., \& Jones, B. F. (2001). Search-based software engineering. Information and Software Technology, 43(14), 833-839.

[2] Miller, W. \& Spooner, D.L. (1976). Automatic Generation of Floating-Point Test Data, IEEE Transactions on Software Engineering, Vol. 2, No. 3, pp. 223-226

[3] Clarke, J., Dolado, J. J., Harman, M., Hierons, R., Jones, B., Lumkin, M., ...Shepperd, M. (2003). Reformulating software engineering as a search problem. IEE Software, 150(3), 161-175.

[4] Harman, M. (2007). The Current State and Future of Search Based Software Engineering. presented at the meeting of the 2007 Future of Software Engineering.

[5] Herroelen, W. (2005). Project Scheduling-Theory and Practice. Production and Operations Management, 14(4), 413-432.

[6] Kolisch, R., \& Hartmann, S. (2006). Experimental investigation of heuristics for resource-constrained project scheduling: An update. European Journal of Operational Research, 174(1), 23-37.

[7] Pinto, G., Ainbinder, I., \&Rabinowitz, G. (2009). A genetic algorithm-based approach for solving the resource-sharing and scheduling problem. Computers \& Industrial Engineering, 57(3), 1131-1143.

[8] Gueorguiev, S., Harman, M., \&Antoniol, G. (2009). Software project planning for robustness and completion time in the presence of uncertainty using multi objective search based software engineering. presented at the meeting of the Proceedings of the 11th Annual conference on Genetic and evolutionary computation, Montreal, Quebec, Canada.

[9] Kirkpatrick, S., Gelatt, C. D., \&Vecchi, M. P. (1983). Optimization by Simulated Annealing. Science, 220(4598), 671-680.

[10] Connor, A.M. \& Shea, K. (2000). A comparison of semi-deterministic and stochastic search techniques.Evolutionary Design and Manufacture, Selected Papers from ACDM '00, 287-298.

[11] Glover, F. (1989). Tabu Search (Part I). ORSA Journal on Computing, 1(3), 190-206.

[12] Glover, F. (1990). Tabu Search (Part II). ORSA Journal on Computing, 2(1), 4-32.

[13] Connor, A.M. \& Tilley, D.G. (1999). A tabu search method for the optimisation of fluid power circuits.IMechE Journal of Systems and Control, 212(5), 373-381.

[14] Goldberg, D. E. (1989). Genetic Algorithms in Search, Optimization and Machine Learning: Kluwer Academic Publishers, Boston, MA.

[15] Deb, K., Agrawal, S., Pratap, A., \&Meyarivan, T. (2000). A Fast Elitist Non-dominated Sorting Genetic Algorithm for Multi-objective Optimization: NSGA-II Parallel Problem Solving from Nature PPSN VI. In M. Schoenauer, K. Deb, G. Rudolph, X. Yao, E. Lutton, J. Merelo, \& H.-P. Schwefel (Eds.), (Vol. 1917, pp. 849-858): Springer Berlin / Heidelberg.

[16] Christodoulou, S. (2010). Scheduling Resource-Constrained Projects with Ant Colony Optimization Artificial Agents. Journal of Computing in Civil Engineering, 24(1), 45-55

[17] Elbeltagi, E., Hegazy, T., \& Grierson, D. (2005). Comparison among five evolutionary-based optimization algorithms. Advanced engineering informatics, 19(1), 43-53. 
[18] Feng, C.-W., Liu, L., \& Burns, S. A. (1997). Using Genetic Algorithms to Solve Construction TimeCost Trade-Off Problems.Journal of Computing in Civil Engineering, 11(3), 184-189.

\section{AUTHORS}

Andy Connor is a Senior Lecturer in CoLab and has previously worked in the School of Computing \& Mathematical Sciences at AUT. Prior to this he worked as a Senior Consultant for the INBIS Group on a wide range of systems engineering projects. He has also worked as a software development engineer and held postdoctoral research positions at Engineering Design Centres at the University of Cambridge and the University of Bath.

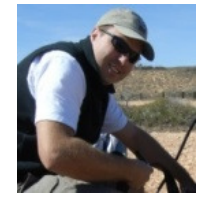

Amit Shah completed hisMasters degree in Computer \& Information Science at Auckland University of Technology, investigating the use of metaheuristic search algorithms applied in the management of software development projects. 


\title{
Are EVOlutionary AlgorithmS REQUiRED TO SOLVE SudoKu PROBlems?
}

\author{
Sean McGerty and Frank Moisiadis \\ University of Notre Dame Australia \\ sean.mcgerty@gmail.com \\ frank.moisiadis@nd.edu.au
}

\begin{abstract}
Sudoku puzzles are an excellent testbed for evolutionary algorithms. The puzzles are accessible enough to be enjoyed by people. However the more complex puzzles require thousands of iterations before a solution is found by an evolutionary algorithm. If we were attempting to compare evolutionary algorithms we could count their iterations to solution as a indicator of relative efficiency. However all evolutionary algorithms include a process of random mutation for solution candidates. I will show that by improving the random mutation behaviours I was able to solve problems with minimal evolutionary optimisation. Experiments demonstrated the random mutation was at times more effective at solving the harder problems than the evolutionary algorithms. This implies that the quality of random mutation may have a significant impact on the performance of evolutionary algorithms with sudoku puzzles. Additionally this random mutation may hold promise for reuse in hybrid evolutionary algorithm behaviours.
\end{abstract}

\section{KEYWORDS}

attention, adaption, artificial intelligence, evolution, exploitation, exploration, satisficing, sudoku, particle swarm, genetic algorithm, simulated annealing, mutation.

\section{INTRODUCTION}

Evolutionary algorithms attempt to iteratively improve a population of candidate solutions. Each solution is randomly mutated. Random mutations are applied to each solution, and a fitness function is used to assess if an improvement has occurred. Evolutionary out rhythms may then attempt to replicate attributes of the more successful candidates to the others. In this way we can solutions become more like the better solutions and the cycle continue. This behaviour can be seen in both particle swarm optimisation and genetic algorithm heuristics [1] [2].

The optimisation in this approach can be seen as an accumulating behaviour for solution candidates around optimal points in the namespace. The forces of random mutation and fitness function assessment bring more candidates around the best solution found so far. Diversification within the candidate population is being transferred into specificity. This accumulation of candidates can be seen as an exploitation strategy, which needs balancing against exploration [3][4]. We can describe non-optimal behaviours in evolutionary algorithms in these terms [5]. At higher levels of namespace complexity is inefficient to scan every possible candidate to know for sure which is the best. Doing so would be the ultimate in exploration, and in relational terms very little optimisation exploitation would be occurring. The strength in a heuristic is in the

David C. Wyld et al. (Eds) : CCSIT, SIPP, AISC, PDCTA, NLP - 2014

pp. 365-377, 2014. (C) CS \& IT-CSCP 2014

DOI : $10.5121 /$ csit.2014.4231 
expectation of being ample to find a good solution and potentially the best solution without checking all possible solutions.

Strengths in exploitation may lead to weaknesses in exploration. By replicating attributes among the solution candidates it is entirely possible that they may accumulate around a local maxima. In this case the desire to exploit has overpowered the entropy of the randomisation function, which now lacks the ability to break from the local maxima.

At this point the algorithm may relatively prioritise a repulsion factor between candidate neighbours [6]. The algorithm may de-prioritise the optimisation component allowing more random mutation. In either case the algorithm requires awareness that relative improvement is no longer occurring. There is also the question of how to parameterise these exploration modes, preferably in a non-namespace specific way.

This hybrid behaviour between exploration and exploitation is also seen when different evolutionary algorithms are combined [5]. If we compare particle swarm optimisation and simulated annealing we might consider particle swarm optimisation to be a relatively strong exploiter [7]. In the same terms simulated annealing may rely more on random mutation and therefore be a relatively strong explorer. If our implementation allowed each algorithm to share the same solution candidate population, then we would be able to swap between the two algorithms as needed. We would then be able to rebalance between exploitation and exploration at will.

\section{EXPLORATION VS. EXPLOITATION}

Evolutionary algorithms face the same problems that people often do. Should I continue to try and solve a problem where I am at the moment? Or should I diversify in case I am not making enough progress in the hope that there are better opportunities elsewhere?

The reality with most evolutionary algorithms is that they will only support one of these modes. For the most part evolutionary algorithms have their random mutation options for exploration inline with the rest of the optimisation. This means that the optimisation has to be held in balance with optimization. The act of sharing successful attributes makes the candidates look more similar, while the actions of random mutation pushes them further apart. If we take the assumption that we are working towards an achievable solution in a logical way then the exploitative action of optimisation will have to overpower the explorative desire of randomisation to move apart.

This balance may work well in most cases. However where there is little change occurring because we have reached a local maximum we have a problem. Those forces making the candidates exploit the incorrect but best solution so far hamper the ability of the randomisation to escape and find other better solutions.

At this point the algorithm may relatively prioritise a repulsion factor between candidate neighbours [6]. The algorithm may de-prioritise the optimisation component allowing more random mutation. In either case the algorithm requires awareness that relative improvement is no longer occurring. There is also the question of how to parameterise these exploration modes, preferably in a non-namespace specific way.

This hybrid behaviour between exploration and exploitation is also seen when different evolutionary algorithms are combined [5]. If we compare particle swarm optimisation and simulated annealing we might consider particle swarm optimisation to be a relatively strong 
exploiter [7]. In the same terms simulated annealing may rely more on random mutation and therefore be a relatively strong explorer.

The ideal partner for a normal evolutionary algorithm is therefore a randomisation algorithm that I optimised in such a way to preferentially find better solution candidates without traditional optimization.

\section{BROAD BASED ATTENTION}

I draw many similarities between human vision and evolutionary algorithms. Much of the processing power at the back of your eye is devoted to peripheral vision. The right hemisphere of your brain is most likely dedicated to qualitative processing and broad based attention.

In these modes coverage and efficiency of operation appear to be primary concerns. Your peripheral vision is optimised to detect unexpected motion and changes in light intensity. This allows the majority of your left hemisphere and the central aspects of your vision to focus on specific tasks while not losing the bigger picture. If nothing else, consider it a survival mechanism where autonomic processing can save you while you think about something else.

We can achieve many of the same goals in a heuristic if we first notice that simulated annealing's optimisation modes are a bit different than the others. Rather than replicate attributes from solution candidates with better fitness function scores to weaker ones, simulated annealing has a random mutation step that it discards if the result is a net loss. This is an example of being able to direct changes in a beneficial way. You can also see that not needing to select or correlate solution candidates might have efficiencies over normal processing modes. Efficiency is the main consideration for an exploration mode broad-based attention agent. The most comprehensive mechanism of this type would be scanning every possibility in the namespace, but as we mentioned earlier this rapidly becomes unworkable for large name spaces.

A broad based attention algorithm expects that we can disperse candidates through a data namespace and in so doing gain a better view. Each solution candidate is a mapping between causal input variables and a resulting fitness scalar. By varying these inputs as much as possible we gain a broader view of the distribution of this fitness curve.

Note too that the simplex algorithm is a use case for a select type of problem that evolutionary algorithms would be able to solve. The simplex algorithm understands that the best values will occur at the boundary values of one or more variables. This then leads to checks where correlated variables are set to boundary values and transitions between these combinations will maximise the fitness function. In a topographical sense we navigate the boundary of an $\mathrm{n}$ dimensional object for a corner value we prefer.

In the same way we could, for example, recognise that we could add more values than we remove during random mutation. This is similar to saying that we expect a solution to be more likely with cells added than removed, and as we are solving Sudoku puzzles this is the case.

Using these ideas we will create a population of randomly mutating solution candidates that will move about sampling the namespace in a directed way. With optimising mechanisms these candidates will disperse giving an aggregated view of a subsection of the problem. Note that we should be able to direct this mutator towards more interesting parts of the namespace without using evolutionary algorithm style optimisations. Visualise this as being more interested in the surface of a bubble than the air inside. We are beginning to make a case that there is benefit to thinking of random mutation as having a lifecycle. 
We can also optimise the fitness function to our needs. We will always need to check that any solution is valid and consistent. Also if we accept that we may find an endpoint solution through random change then we want to know if we have reached the endpoint solution. We do not need the fitness scalar for comparing the solution candidates within this population, as we do not optimise by exchanging attributes. We are interested in the scalar sometimes, but only when we are interested to know if we have found a better solution via random change if we are running a separate random mutation solution candidate population. For the most part though, our processing for broad attention modes is simplified with respect to optimisation.

Take for example Sudoku puzzles, which are completed when all cells have been filled [8]. We are restricted to adding digits such that each digit can only occur once with in a row or column or a region. We can separate these considerations: we are attempting to add digits, and solutions cannot be invalid.

The check for validity of the solution is a subset of the fitness function. Rather than returning a fitness score we can simply return true or false. We can combine our simplified fitness function with a random mutation agent with a bias for addition. I call this mechanism the greedy random. Solution candidates are spread through the namespace by the greedy random. This behaviour attempts to fill as many cells as possible. If we correlate to human vision, the greedy random moves around the boundaries of what a human can see flagging changes. Note as well that the greedy random uses less resources as a subset of an evolutionary algorithm, so we can run more of them with less effort.

\section{ATTENTION ADAPTION}

Darwin was asked for the most important attribute for continued success in his model of evolution. He avoided factors like strength, or speed, and instead suggested it was far more important to be able to adapt [9]. When an evolutionary algorithm collects around a local maximum we could see this specificity as a failure to adapt. Any candidate undergoing random mutation does not have the entropy to produce a candidate better than the current population. In these cases these insufficiently adapted mutations are removed or assimilated. I suggest that we need a mechanism for being aware of candidates sufficiently outside the local maxima to be able to escape.

Think of this as an attention mechanism, which allows the adaption away from local maximums to occur. By implementing this ability we gain understanding of a mechanism that has been known to plant sciences for most of a century. It is entirely possible to be able to separate changes into those based on internal factors from those that occur in response to their environment.

By being able to notice beneficial change in candidates undergoing random mutation we can adopt that change, even when it is outside the realm of experience for the evolutionary algorithm.

\section{FITNESS FUNCTION}

Evolutionary algorithms work by attempting to maximise a scalar fitness function by changing values within constraints [10]. For example we may attempt to maximise the sum of two numbers greater than zero but less than five. The constraints place acceptable input values from one to four. We may start the process with values of one, and a fitness of two. Over time we randomly change values and remember those pairs, which lead to improved fitness function scalars. Eventually as a result of random changes and using the best candidates so far as a reference, our 
paired values improve. Eventually we reach a stable solution at four and four of eight, and we no longer see improvement with any random change.

A random mutation component capable of integrating with evolutionary heuristics will need to interoperate with this evolutionary algorithm life cycle. As previously mentioned we do not include optimisation, but the question remains how much of the fitness remains relevant to random change function

At its simplest the fitness function returns a scalar value, which increases as our solution candidate improves if we are attempting maximisation. However there is also an expectation that candidate solutions, which violate the problem constraints, are invalid. In this case the fitness function may return a value equal to or less than zero to mark that this candidate is less valuable than any other current candidate. If we were to realise that we had produced an invalid candidate we could then choose to discard it, or to revert to the most recent valid version.

If we are not optimising, then we are not necessarily comparing candidates by fitness. It remains a serious issue however candidate should receive random mutations that render it invalid. Therefore we still have interest in a subset of the fitness function outcomes. The assumption is that in most cases it should require less processing to validate a candidate than produce the complete fitness scalar.

\section{RANDOM MUTATION}

We have used the phrase random mutation, however not all random changes have equal effect [11]. Consider our example from earlier though we had two numbers between zero and five. Random change can either the increase or decrease of value. In the case of a crossword puzzle this could be the addition or erasure of the character. In general terms if we are attempting to fill positions in a solution should attempt to add values in preference to removing them.

This leads to an idea that we call the greedy random. The greedy random understands in general terms that either setting values or removing values is preferential to the fitness function. For example we may set a probability to add as 0.8 , and a probability to remove at 0.2 . In this case before performing an operation we first choose whether we are in addition or removal mode. The net effect of this bias is to produce candidate solutions, which have added as many sales as possible before rendering the candidate invalid. We call this process the greedy random because of this perspective of attempting to fill as many cells as possible.

In this case the relative fitness function assessment of any candidate is less important than knowing if the candidate remains valid. So we can perform these greedy operations more efficiently as a result. In testing this represented as an opportunity for more random mutation cycles to each evolutionary algorithm cycle.

The risk of course is that a candidate solution may rapidly fill and lose degrees of freedom. This problem replicates the issue experienced by evolutionary algorithms around local maxima.

This was an important design consideration during testing. The solution to this problem became apparent during attempts to integrate with the evolutionary algorithm lifecycle. During each iteration the candidates in the population is assessed by the fitness function. In the case of attempting to maximise the process of filling a board such as Sudoku puzzle, performance was greatly improved by ensuring that the last change before fitness function assessment was a removal. 


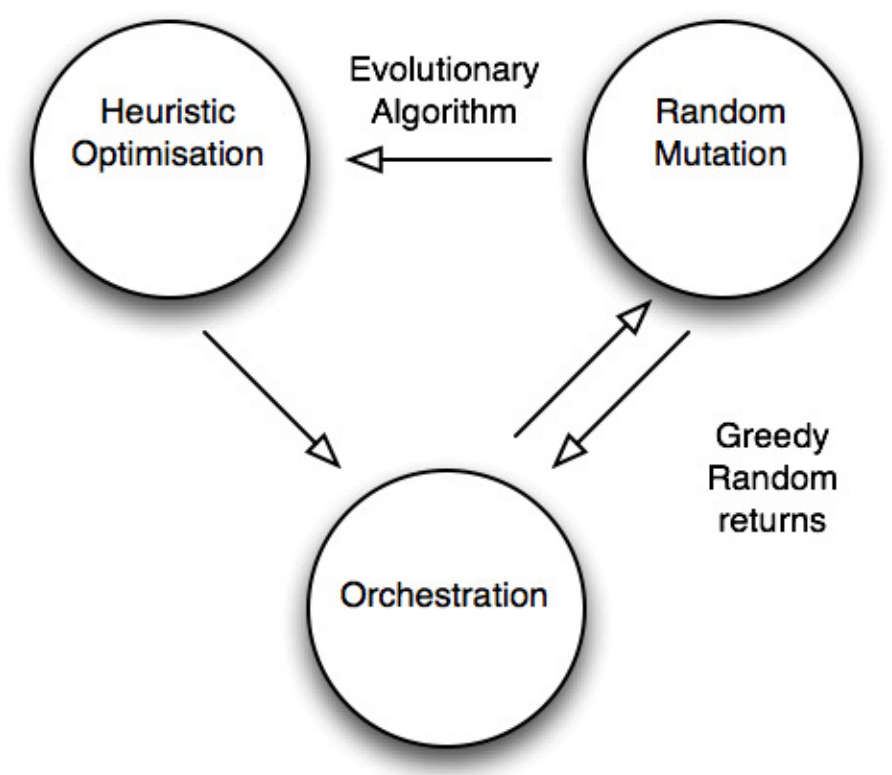

Figure 1 - This diagram shows the modified heuristic iteration lifecycle

I realised a further implication of the fitness function lifecycle. In one respect the fitness function tells us when we have reached a global maximum. In the case of a Sudoku puzzle we may have a valid candidate with all the cells occupied by digits. If not there is a subtle difference between asking which candidate is the best, and which candidate has the best chance of improving with more random mutations. It seems that a strong candidate with additional degrees of freedom can be as valuable as a stronger candidate with more cells filled.

It is important to check fitness with as many cells as possible filled in order to find completed solutions. However if we are attempting to measure potential for improvement in a process where we are filling as many cells is possible, testing showed it was more meaningful to rank the candidates after a single removal. Doing so concentrates the random mutation entropy around the boundary conditions of the solution. Once again we have a collection pressure, but while evolutionary heuristics concentrate around local maxima the greedy random collects the candidates around input boundary values.

We can also better conform to the problem namespace by prioritising changes with less degrees of freedom. In this way additions are validated against the cells that are already filled in this solution candidate. If we were to choose a more empty part of the name space we can choose from more values for a cell, however we may be introducing a combinatorial issue with later additions.

Therefore we reduce rework by using a fitness function that can be thought of as the count of neighbours that each filled cell has. For Sudoku we are checking each row, cell and region, so we are looking for $8 \times 8 \times 3 \times 9=2781$ as the score for a solved board and 0 for an empty one.

\section{COMPARING DIFFERENT EVOLUTIONARY ALgORITHMS}

Consider the situation where we are attempting to evaluate suitability of different evolutionary algorithms on the same problem. If we were simply reusing algorithms then we would find a way of encoding the problem in a format that each algorithm could recognise. This would give us a time to completion for each of the algorithms, but we would not be able to differentiate the 
performance factors within each algorithmic life cycle. This would leave us less capable of classifying performance by problem type, and less capable of predicting performance with other problems in the future. Finally we are also at the mercy of the quality of the implementation of each component. What might be a specialised optimisation forgiven example of a problem may be suboptimal in the more general case. I argue that when comparing the performance of evolutionary algorithms we need to separate those components, which are less related to optimisation and more likely to be shared between implementations.

I would make an argument that the fitness function that is better suited to the namespace topology has better alignment to the data than the optimisation. In this case it would make sense to rework the fitness function to each problem data type than to attempt to re-use the fitness function between different problem types. If we separate these shared functions from the optimisation then we can better evaluate the efficiency of the optimisations in isolation. All we need to do is create an encapsulating life cycle, which accepts differentiated optimisation components in the data management, and fitness functions should be reusable.

In the same way as we have managed to isolate the fitness function from the optimisations we can also isolate random mutation. As mentioned the reasons for differentiating random mutation are less obvious. When we look at randomisation it soon becomes apparent that not all random variations are the same. We have stated that in the middle time period of solving the problem it may make sense to add as many cells as we remove during random mutation. However as we fill more of the board we encounter reduced degrees of freedom and so more cell additions will fail consistency checks. In effect will be more successful removing cells than adding cells and random mutation may be detrimental to optimisation in that case. To remediate we may decide to bias in favour of cell additions than cell deletions, or we may retry additions until successful. If we follow this path we introduce another anti-pattern in that we may leave the solution candidates with no degrees of freedom entering the optimisation component processing. During experimentation we had greater success when we left deletion steps at the end of the process. We expect there are two factors for the observed behaviours. Firstly it may be preferential to leave a degree of combinatorial flexibility for the process of attribute replication during optimisation to occur. Secondly the question arises of the optimal time to evaluate fitness in the optimisation life cycle.

If we accept that producing a scalar for comparison and evaluating the possibility of an end point solution are different questions then we open the possibility that it may make sense to check for these conditions at different times in the life-cycle. Consider waves at a beach. Our endpoint condition may be a wave reaching a distant point up the shore. However the fitness of any wave might be better measured by the height of the swell before the wave approaches the beach as an indicator of future success. In these terms are fitness is the height of the swell and the endpoint condition is distance up the beach as a boolean consistent with a causal relationship. Increase the swell, the waves drive further up the beach. So if we are attempting to solve a Sudoku problem then it may be more valuable to rank candidate solutions with better fitness and degrees of freedom than fitness alone.

In any case if we are complicating the random mutation, particularly if we are doing so to suit conditions in the data namespace, then it also makes sense to separate the random mutation from the evolutionary algorithm component. We can see by a process of optimisation we have extracted reusable components and encapsulated complexity to the point where optimisation components have become more specialised. The fitness function and random mutations have become more specialised to the namespace topologies of the data. These components are orchestrated by an extensible component life cycle. We can now test different evolutionary algorithms by implementing their optimisations within the shared component framework. 
At this point we have evolved a component framework that allows us to differentially optimise and orchestrate discrete components:

- I have identified a common lifecycle among evolutionary algorithms

- I argue that the fitness function can be better suited to the data namespace than the optimisation. The fitness function is modal as the checks for consistency, endpoint solution and fitness scalar have different processing and usage modes.

- I argue that random mutation can be a collection of different random action types. I argue that differentiating these modes leads to performance optimisations and further that these can be orchestrated in their own lifecycle to optimise degrees of freedom.

- If we follow this path we come to the conclusion that optimization may be best implemented as a component within a framework that uses a fitness function and a random mutation that are detailed to each data namespace.

- A framework of this type allows us to compare and contrast the suitability and performance of different evolutionary algorithms.

- If we accept that random mutation can reach solutions then a subset of fitness function modes will allow bypassing of optimisation if an endpoint solution has been reached.

Most interestingly we have also gained the ability to test the efficiency of the random mutation in isolation by deactivating the optimisation components entirely. We have already made an argument that the random mutation can be better suited to the data than the optimisation, and so it makes sense that the random mutation be optimised independently for each dataset before integration with evolutionary optimisations. It was during this optimisation that I realised random mutation is capable of solving Sudoku puzzles without an optimisation component.

This question is an intriguing one. Sudoku has regularly been used to demonstrate the ability of evolutionary algorithms. On various occasions particle swarm, genetic algorithms and hybrid meta heuristics have been shown to be capable of solving Sudoku problems. Using the component framework above I managed to confirm that indeed, particle swarm, genetic algorithms and simulated annealing could solve these problems.

However as noted each of these heuristics has a random mutation component which is separately optimise able. My aim therefore was to improve this function in isolation, which would then improve the baseline performance of each of the evolutionary algorithms. Doing so involves operating life cycle with out the optimisation components. At this point it became apparent that the random mutation is capable of solving the problems by itself.

- This of course implies that all of the optimisations within this life cycle may be able to solve the same problems as the random mutation, as long as they do not sufficiently overpower the random mutation function.

- This also implies that an inability to solve Sudoku problems may be implementation specific.

Results indicated a benefit in using evolutionary algorithms for most problems, for which evolutionary algorithms received lower main iteration counts to endpoint solution. However, in the same way that some problems are more difficult for humans, the evolutionary algorithms also seem to struggle by concentrating around local maxima. In these cases the optimised random mutation lacked concentrating behaviour and achieved faster solution times in these cases. This result seemed counterintuitive. You would hope that by applying focused attention should be more capable of solving problems in all conditions. Yet we appear to see evidence that broader solution mode can lead to answers for more difficult problems in a shorter time frame. 
Consider too that the value of each solution candidate is a sample of the fitness of that point in the namespace. Aggregating the population of candidate solutions might be considered as analogous to an awareness of the solution candidates so far.

This brings an intriguing correlation to the anatomy of human vision, and the implications of having too narrow a focus. Split brain theory shows the left hemisphere has a predilection for focused mono-procedural tools based processing, much like the way optimisation acts as a concentrating force for solution candidates around the best solution found so far. The longer an evolutionary algorithm spends around a maxima the more attributes are copied from the better solutions to the weaker, the more similar the population becomes. We can see this as a concentration of attention focus around the best solution so far.

The human brain however uses both strategies at the same time. The left hemisphere has a preference for focused attention, much in the way evolutionary algorithms concentrate solution candidates around local maxima. The right hemisphere prefers a broader attention mode. Human vision in particular has peripheral perception modes, which have strengths in motion detection and changes in light intensity. These modes benefit from the widest possible distribution of attention, which correlates to the idea of reduced optimisation for solution candidates, and more of a bias towards random mutation. In the same way optimisation concentrates candidates, random mutation distributes them through the namespace topology. This matches how we look for something we've lost. The attempt to remember where we have left an object as though it were a problem to solve, and we are also broadly paying attention, as we look in case we have forgotten something or someone else may have moved the object in question.

\section{IMPLEMENTATION}

We will demonstrate these ideas with a python-based component framework implementation of heuristics for solving Sudoku problems. Sudoku problems are defined on a 9 x 9 grid where the digits from 1 to 9 are arranged such that no digit is repeated on any column, row or 3 by 3 cell grid of which there are 9. Sudoku puzzles are simple enough to be enjoyed as a diversion, and yet the more complex can occupy heuristics for thousands of iterations [12][13].

I have collected a sample of 60 or so Sudoku puzzles which were all solved by the evolutionary algorithms and greedy random. Most significantly I tested against 4 Sudoku puzzles, which have been known as some of the most difficult created: "The Easter Monster", the "Golden Nugget", "tarek071223170000-052" and "col-02-08-071".

My implementation shows the greedy random acting as the usual random mutation agent for each of the evolutionary algorithms. During testing each of the evolutionary algorithms can be selected or deselected individually via a command line option.

During initial testing the algorithm was run separately with each evolutionary algorithm selected and I verified that all the sample Sudoku puzzles could be solves with each.

It then became apparent that it would be a useful comparison to produce a baseline where no optimisation was selected. This would help identify the net benefit of the optimisation action above random mutation. 


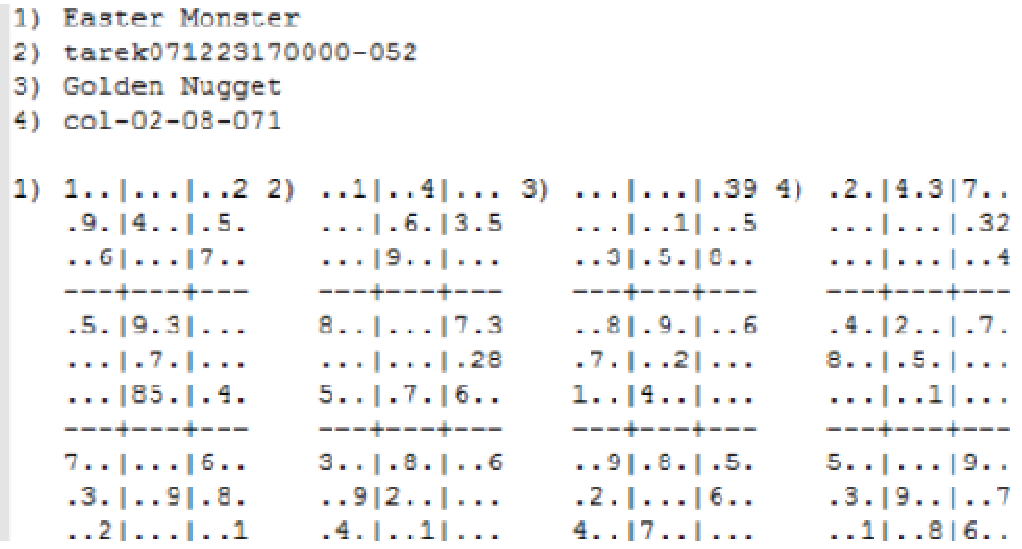

Figure 2 - The four most difficult sudoku puzzles tested.

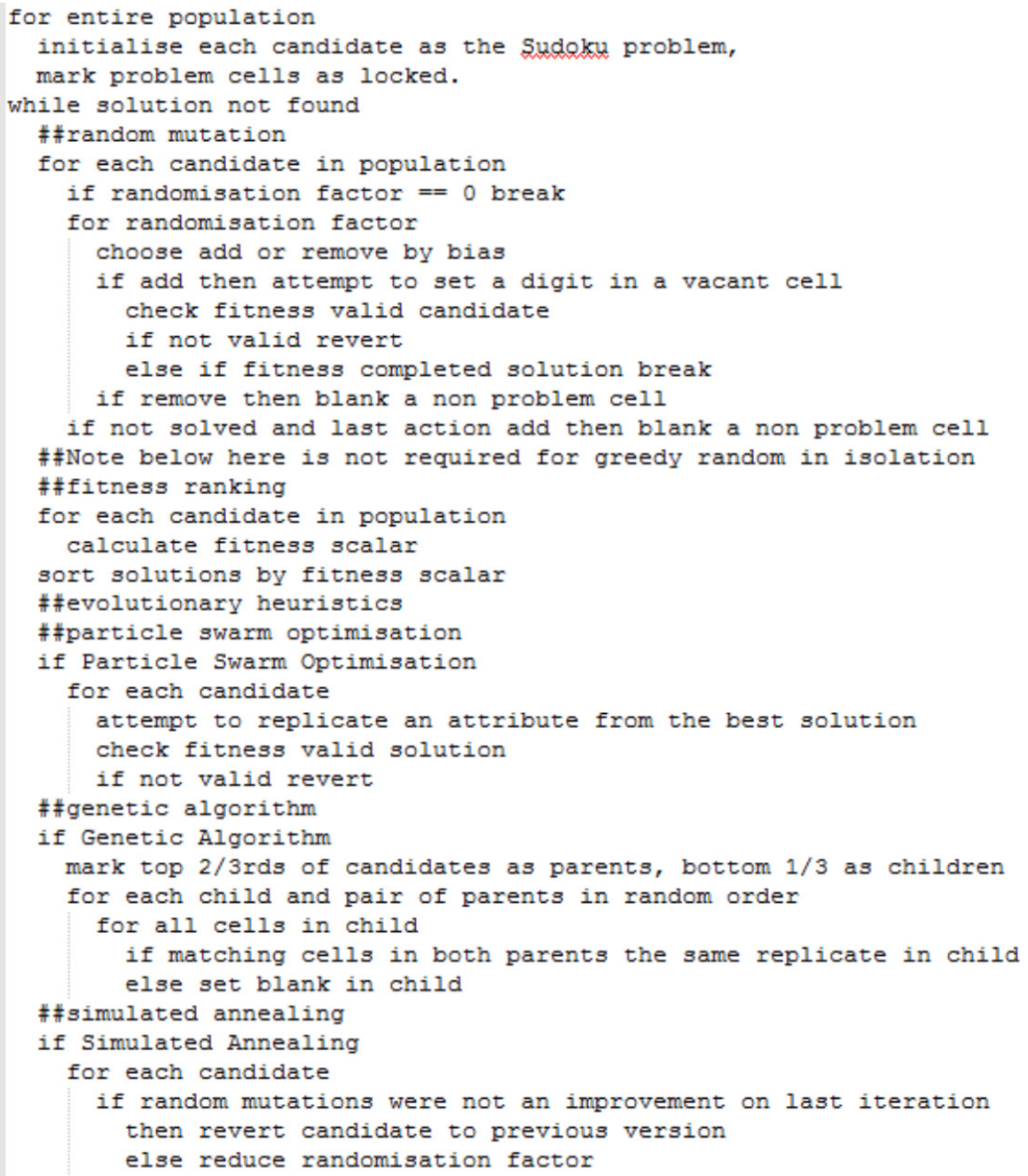

Figure 3 - The component hierarchy as pseudo code. 


\section{TESTING OUTCOMES}

\begin{tabular}{|c|c|c|c|c|c|c|c|c|c|}
\hline \multicolumn{3}{|c|}{ U01 "Easter Monster" - Iterations } & \multicolumn{2}{|c|}{ Populations } & \multicolumn{2}{|c|}{ U04 "col-02-08-071" - Iterations } & \multicolumn{3}{|c|}{ Populations } \\
\hline Heuristic & 8000 & 4000 & 2000 & 1000 & Heuristic & 8000 & 4000 & 2000 & 1000 \\
\hline Genetic Algorythm & 640 & 3030 & 2020 & 1680 & Genetic Algorythm & 3250 & 1290 & 1550 & 2230 \\
\hline Particle Swarm & 1080 & 1000 & 770 & 1460 & Particle Swarm & 780 & 580 & 1300 & 1550 \\
\hline Pure Random & 1140 & 1050 & 450 & 2290 & \begin{tabular}{|l|} 
Pure Random \\
\end{tabular} & 1440 & 780 & 8260 & 9530 \\
\hline Simulated Annealing & 250 & 1640 & 350 & 5520 & Simulated Annealing & 1620 & 1430 & 3000 & 4160 \\
\hline \multicolumn{3}{|c|}{ U02 "tarek071223170000-052" - Iterations } & \multicolumn{2}{|c|}{ Populations } & \multicolumn{2}{|c|}{ Average iterations for $\mathrm{U}^{*}$} & & \multicolumn{2}{|c|}{ Populations } \\
\hline Heuristic & 8000 & 4000 & 2000 & 1000 & Heuristic & 8000 & 4000 & 2000 & 1000 \\
\hline Genetic Algorythm & 3670 & 5920 & 1480 & 3690 & Genetic Algorythm & 2160.0 & 3025.0 & 1825.0 & 2655.0 \\
\hline Particle Swarm & 800 & 1260 & 1300 & 1050 & Particle Swarm & 862.5 & 810.0 & 1185.0 & 1465.0 \\
\hline Pure Random & 330 & 850 & 3070 & 380 & Pure Random & 910.0 & 980.0 & 3040.0 & 3182.5 \\
\hline Simulated Annealing & 440 & 1100 & 920 & 770 & Simulated Annealing & 765.0 & 1300.0 & 1172.5 & 2920.0 \\
\hline \multicolumn{3}{|c|}{ U03 "Golden Nugget" - Iterations } & \multicolumn{2}{|c|}{ Populations } & \multicolumn{3}{|c|}{ Thousand boards for solution of $U^{*}$} & \multicolumn{2}{|c|}{ Populations } \\
\hline Heuristic & 8000 & 4000 & 2000 & 1000 & Heuristic & 8000 & 4000 & 2000 & 1000 \\
\hline Genetic Algorythm & 1080 & 1860 & 2250 & 3020 & \begin{tabular}{|l|} 
Genetic Algorythm \\
\end{tabular} & 17280 & 12100 & 3650 & 2655 \\
\hline Particle Swarm & 790 & 400 & 1370 & 1800 & Particle Swarm & 6900 & 3240 & 2370 & 1465 \\
\hline Pure Random & 730 & 1240 & 380 & 530 & \begin{tabular}{|l|} 
Pure Random \\
\end{tabular} & 7280 & 3920 & 6080 & 3183 \\
\hline Simulated Annealing & 750 & 1030 & 420 & 1230 & Simulated Annealing & 6120 & 5200 & 2345 & 2920 \\
\hline
\end{tabular}

Figure 4 - Results

The pure random mutation (with no optimisation) and all three evolutionary algorithms were shown to be able to solve all 60 Sudoku puzzles. For results for the 4 hardest puzzles:

1) The effect of being caught in local maxima had a significant effect on average times. If the algorithm catches a local maxima on harder problems in $20 \%$ of runs average iteration counts can double or triple. The algorithms recover and complete, but at large time scales.

2) The genetic algorithm had median performance. This is thought to be of a consequence of a relatively higher complexity in the optimiser combined with a slower propagation rate for good attributes. This idea is correlated in the genetic algorithm showing less benefit from larger population sizes (17280 to 12100 to 3650 to 2655) thousand boards.

3) Where optimisation out performed random mutation on the harder problems it was usually particle swarm optimisation. If we multiple the size of the population by the number of iterations as a number of boards then particle swarm achieves end point solution in less than half the number of (1465 to 3138) thousand boards for populations of 1000 .

4) Simulated annealing held the closest correlation to pure random (2920 to 3183) thousand boards for populations of 1000. This is to be expected, as there is no real propagation of attributes in this optimization. Rather there is an additional random mutation, which is only significant on improvement.

\section{DISCUSSION}

We showed that an optimised random mutation is capable of solving Sudoku puzzles on its own. We also found that evolutionary algorithms, which used this random mutation, were also capable of solving the puzzles as well. 
The major danger to completion would therefore appear to be in the balance between the random mutation and the optimisation. If the action of copying attributes from the strongest candidates is capable of offsetting randomisation then any attempt to break away from a local maximum would be lost.

An amenable solution to this problem would appear to be the addition of a separate random mutation population to the action of the evolutionary algorithm. In this way one population would always be capable of random mutation. Whenever the random mutation population finds a better solution than the evolutionary algorithm then this can be replicated across. Optimisation will then replicate these new preferable attributes among the evolutionary algorithm population.

\section{FUTURE WORK}

This implementation was created to test optimisation of the evolutionary algorithms. In this case the random mutations are inline with the rest of the evolutionary algorithm, and the candidate population has one heuristic mode. During the discussion on satisficing behaviours we noted possibilities for additional modes.

We have seen that the greedy random has promise with problems that challenge evolutionary algorithms. Creating a hybrid with a population for each will allow us to displace the evolutionary algorithm from local maxima by replicating better candidates from the mutation population.

We would also be able to create a secondary population of mutation that was seeded from the evolutionary algorithm. This population:

1) Improves randomisation around the best exploitation targets.

2) Can operate as a satisficing cache [13][14][15] between different algorithms where the best candidates can be shared between populations.

Since this work began new evaluations of Sudoku puzzles have emerged and I would enjoy retesting against some of the newer higher ranked puzzles.

\section{ACKNOWLEDGMENTS}

I thank Mehmet Orgun of Macquarie University.

\section{REFERENCES}

[1] Carlos M Fonseca and Peter J Fleming. Genetic algorithms for multiobjective optimization: Formulation, discussion and generalization. 423:416-423, 1993.

[2] J Kennedy and R Eberhart. Particle swarm optimization. . . 1995 Proceedings, 1995.

[3] Sylvain Gelly and Yizao Wang. Exploration exploitation in go: UCT for Monte- Carlo go. 2006.

[4] ]Enrique Alba and Bernab'e Dorronsoro. The exploration/exploitation tradeoff in dynamic cellular genetic algorithms. Evolutionary Computation, IEEE Transactions on, 9(2):126-142, 2005.

[5] Urszula Boryczka and Przemyslaw Juszczuk. Solving the sudoku with the differential evolution. Zeszyty Naukowe Politechniki Bialostockiej. Informatyka, pages 5-16, 2012.

[6] Kyun Ho Lee, Seung Wook Baek, and Ki Wan Kim. Inverse radiation analysis using repulsive particle swarm optimization algorithm. International Journal of Heat and Mass Transfer, 51(11):2772-2783, 2008.

[7] Scott Kirkpatrick, D Gelatt Jr, and Mario P Vecchi. Optimization by simulated annealing. science, 220(4598):671-680, 1983.

[8] Rhyd Lewis. Metaheuristics can solve sudoku puzzles. Journal of Heuristics, 13(4):387-401, 2007.

[9] Charles Darwin. On the origin of the species by natural selection. 1859. 
[10] Dirk Buche, Nicol N Schraudolph, and Petros Koumoutsakos. Accelerating evolutionary algorithms with gaussian process fitness function models. Systems, Man, and Cybernetics, Part C: Applications and Reviews, IEEE Transactions on, 35(2):183-194, 2005.

[11] Ajith Abraham, Rajkumar Buyya, and Baikunth Nath. Nature's heuristics for scheduling jobs on computational grids. In The 8th IEEE international conference on advanced computing and communications (ADCOM 2000), pages 45-52, 2000.

[12] Sean McGerty. Solving Sudoku Puzzles with Particle Swarm Optimisation. Final Report, Macquarie University, 2009.

[13] Sean McGerty, Frank Moisiadis. Managing Namespace Topology as a Factor in Evolutionary Algorithms. Artificial Intelligence in Computer Science and ICT 2013.

[14] Herbert A Simon. Theories of bounded rationality. Decision and organization, 1:161-176, 1972.

[15] Herbert A Simon. Rationality as Process and as Product of Thought. The American Economic Review, 68(2):1-16, 1978.

[16] Francesco Grimaccia, Marco Mussetta, and Riccardo E Zich. Genetical swarm optimization: Selfadaptive hybrid evolutionary algorithm for electromagnetics. Antennas and Propagation, IEEE Transactions on, 55(3):781-785, 2007.

\section{AUTHORS}

Sean McGerty - A Research PHD Student at the University of Notre Dame Australia Sydney Campus

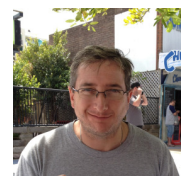


Computer Science \& Information Technology (CS \& IT)

INTENTIONAL BLANK 


\title{
HYBRID ANT COLONY OPTIMIZATION FOR REAL-WORLD DELIVERY PROBLEMS BASED ON REAL TIME AND PREDICTED TRAFFIC IN WIDE AREA ROAD NETWORK
}

\author{
Junichi Ochiai $^{1}$ and Hitoshi Kanoh ${ }^{2}$ \\ ${ }^{1}$ Department of Computer Science, Graduate School of Systems and \\ Information Engineering, University of Tsukuba, Tsukuba, Ibaraki, Japan \\ ochiai@kslab.cs.tsukuba.ac.jp \\ ${ }^{2}$ Division of Information Engineering, Faculty of Engineering, Information and \\ Systems, University of Tsukuba, Tsukuba, Ibaraki, Japan \\ kanoh@cs.tsukuba.ac.jp
}

\begin{abstract}
This paper presents a solution to real-world delivery problems for home delivery services where a large number of roads exist in cities and the traffic on the roads rapidly changes with time. The methodology for finding the shortest-travel-time tour includes a hybrid meta-heuristic that combines ant colony optimization with Dijkstra's algorithm, a search technique that uses both real-time traffic and predicted traffic, and a way to use a real-world road map and measured traffic in Japan. Experimental results using a map of central Tokyo and historical traffic data indicate that the proposed method can find a better solution than conventional methods.
\end{abstract}

\section{KEYWORDS}

Ant Colony Optimization, Dijkstra's Algorithm, Delivery Problem, Traffic, Real-World

\section{INTRODUCTION}

Ant colony optimization (ACO) is a stochastic search algorithm for problem solving that takes inspiration from the foraging behaviors of ants. The main idea of ACO rests on the indirect communication among individuals in an ant colony based on the pheromone trails that real ants use for communication. ACO has been formalized into a meta-heuristic for combinatorial optimization problems by Dorigo et al., and many applications are now available [1]-[3]. In particular, many studies on ACO have been performed using the traveling salesman problem (TSP [4]), and it has been shown that ACO is superior to other meta-heuristics [5] [6] for this type of problem.

In this paper, we deal with real-world delivery problems (RWDPs) for home delivery services as an extension of the TSP, where a large number of roads exist in cities and the traffic on the roads rapidly changes with time. This scenario reflects the typical traffic congestion in a wide area urban road network. 
The problems that deal with finding optimal tours with time-dependent travel time have been studied as TSPs, delivery problems [7], and vehicle routing problems [8]. Conventional problemsolving methods using ACO repeat a search when the traffic flow changes during movement [9][11]. However, finding the global optimal solution by this method is difficult because when the traffic changes rapidly, the information obtained from an old search may not be helpful. Furthermore, research based on real road maps and traffic information services in the real world is seldom found.

In this paper, we propose a new method to solve RWDPs using ACO and Dijkstra's algorithm. (DA). Search techniques based on only the predicted traffic have previously been presented for real-world time-dependent vehicle routing problems using ACO [12], an evolution strategy [13], and a genetic algorithm [14]. When these methods make mistakes with the prediction values, solution accuracy may deteriorate. The proposed method aims at improving the accuracy by combining real-time data with predicted traffic data that can actually be obtained.

In the following section, we start by describing the problem. Then, we detail the algorithm of the proposed method. Finally, we present the results of experiments using a map of central Tokyo and real traffic data.

\section{PROBLEM DESCRIPTION}

In this paper, we regard the RWDP as an extension of the TSP: a vehicle starts from a depot, visits all customers without any time constraint, and finally returns to the depot. This type of problem is also called a one-to-many-to-one delivery problem [7]. Here, we first describe the time-dependent TSP (TDTSP) and the calculation of the tour travel time and then explain the RWDP and a traffic information service in the real world. Finally, a brief account of ACO will be given.

\subsection{Time-Dependent Traveling Salesman Problem}

The TSP [4] can be represented by a complete graph $G=(N, A)$, where $N$ is a set of nodes, i.e. cities, $n=|N|$ is the number of nodes, and $A$ is the set of arcs fully connecting the nodes. Each arc $(i, j) \in A$ is assigned a value $d_{i, j}\left(=d_{j, i}\right)$, which represents the distance between nodes $i$ and $j$. The TSP then is the problem of finding the shortest closed tour that visits each of the nodes of $G$ exactly once. The TSP instances used in this paper are taken from the TSPLIB benchmark library [15].

The TDTSP extends the original TSP so that traffic congestion can be included. Let $T_{i, j}(t)$ be the travel time between nodes $i$ and $j$ at time $t ; T_{i, j}(0)$ means the original travel time of a given TSP, i.e. $T_{i, j}(0)=d_{i, j}$. Traffic congestion can be represented by a change in the travel time. Here, this change is defined by the following formula, where $\Delta t$ is an updated interval of travel time, $R_{\text {jam }}$ and $B_{\text {upper }}$ are constants showing the rate and the upper bound of traffic congestion, respectively, and rand $\in[-1,1]$ is a uniform random number. The time when a salesman leaves city 1 is set to $t$ $=0$, and he always starts from and returns to city 1 .

$$
\begin{aligned}
& T_{i, j}(t+\Delta t)=\left[T_{i, j}(t) \times\left(1+R_{\text {jam }} \times \text { rand }\right)\right]_{T_{\min }}^{T_{\max }} \\
& {[x]_{b}^{a}= \begin{cases}a & \text { if } x>a \\
b & \text { if } x<b \\
x & \text { otherwise }\end{cases} }
\end{aligned}
$$




$$
\begin{aligned}
& T_{\max }=d_{i, j} \times B_{\text {upper }} \\
& T_{\text {min }}=d_{i, j}
\end{aligned}
$$

In the following, the time required to travel around a tour is called the tour travel time. The tour travel time for solution $S$ can be calculated by the following formulas, where $t_{i}$ is the time when a salesman reaches node $i$ :

$$
\begin{aligned}
& T(S)=\sum_{i=1}^{n} T_{i, i+1}\left(t_{i}\right) \\
& t_{i}= \begin{cases}t_{1} & \text { if } i=1 \\
t_{i-1}+T_{i-1, i}\left(t_{i-1}\right) & \text { otherwise }\end{cases}
\end{aligned}
$$

\subsection{Real-World Delivery Problem}

The RWDP can be represented by a quadruple $G^{R W}=\left(N^{R W}, A^{R W}, M^{R W}, T^{R W}\right)$, where $N^{R W}=\left\{C_{i} \mid i=\right.$ $1, \ldots, n\}, C_{1}$ is a depot and $\left\{C_{2}, \ldots, C_{n}\right\}$ is a set of customers, $A^{R W}=\left\{R_{i, j}\left(t_{i}\right) \mid i, j=1, \ldots, n(i \neq j)\right\}$ is the set of optimal routes from $C_{i}$ to $C_{j}$ at time $t_{i}$ when a vehicle reaches $C_{i}$, and $M^{R W}$ and $T^{R W}$ are a road map and a set of time-series traffic data in the real world, respectively. Each $R_{i, j}\left(t_{i}\right)$, generally $\neq R_{j, i}\left(t_{i}\right)$, can be calculated using $T^{R W}$ on $M^{R W}$. It is necessary to calculate the optimal route between customers in RWDPs, while the distance between cities is given in TSPs. The RWDP then is the problem of finding a shortest-travel-time tour when a vehicle starts from $C_{1}$ and returns to $C_{1}$ visiting each of the customers $\left\{C_{2}, \ldots, C_{n}\right\}$ exactly once.

The road map $M^{R W}$ used in this paper is the standard map database that is used in actual car navigation systems. This map includes all drivable roads in Japan and its format was developed and established by the Navigation System Researchers' Association.

Historical time-series traffic data $T^{R W}$ are also used to calculate the travel time of a vehicle. In Japan, traffic meters are installed at more than 20,000 locations along principal roads throughout the country. These meters measure the average travel time of cars passing through specific road links at 5-minute intervals. The data so obtained is collected at a traffic information center and provided to subscribers in real time. Figure 1 shows an example of time-series traffic data. The vertical axis represents the average speed of cars on a link calculated directly from the traffic data. In this figure, data in the range of 0:00 to 5:00, 5:00 to 8:00, 8:00 to 18:00, and 18:00 to 20:00 correspond to no congestion, outbreak of congestion, heavy congestion, and dissolution of congestion, respectively. Thus, time-series traffic data is highly nonlinear, which makes it difficult to perform accurate predictions. Application to real-world scenarios must take the prediction error rate into consideration.

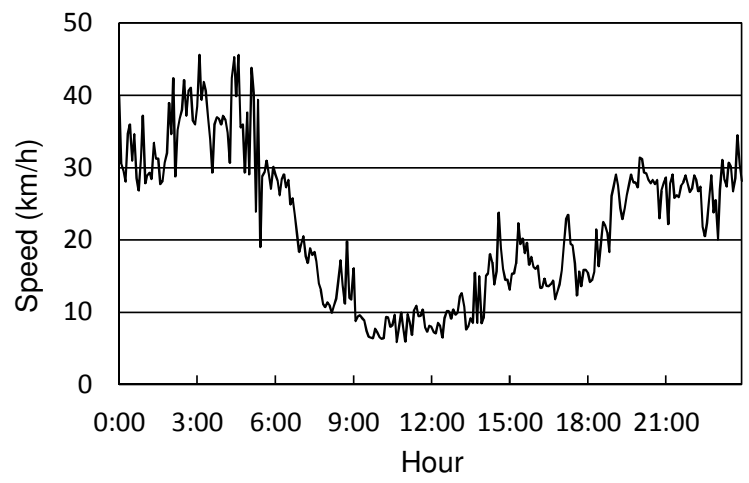

Figure 1. Example of time-series traffic data. 


\subsection{Ant Colony Optimization}

The generic ACO meta-heuristic [1] is shown below. After initialization, the meta-heuristic iterates over two phases. First, a number of solutions are constructed by the ants, and second, the pheromone trails are updated.

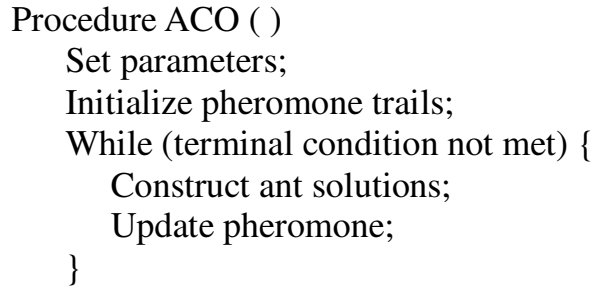

There have been many attempts to improve the performance of ACO. Max-Min Ant System (MMAS) has demonstrated an especially impressive performance [1], so we used the MMAS, except for constructing the ant solution described in section 3.3, as the ACO in the proposed method.

\section{PROPOSED METHOD}

\subsection{Configuration of System and Data Flow}

Figure 2 shows the configuration and the data flow of the proposed system. A target road map including the depot and all customers is prepared along with the historical traffic data for principal roads on the road map. The latter are required for the prediction. The real-time traffic data for the principal roads are input at $\Delta t$ intervals, so updating the travel time and recalculating the predicted traffic are performed at this interval. The prediction system, as we have already reported [17] [18], has an interpolation function as well as a prediction function. This system can estimate traffic on roads not installed with detectors from the traffic on roads installed with detectors. The other systems and data in Fig. 2 are described in the following sections.

\subsection{General Procedure}

The general procedure of the proposed method is shown in Fig. 3. The index $i$ in the outermost loop corresponds to the turn at which a vehicle visits customers. While the vehicle is moving, the search is repeated. When the vehicle reaches the next customer, if new traffic data is available, a new tour is constructed, where $k$ is an ant number, $\mathrm{C}_{l(i)}$ is the $i$-th customer to which the ant moves, $S^{i b}$ is the best solution in the current iteration, and $S^{g b}$ is the best solution found since the first iteration. Constructing a tour by hybrid ACO is described in the next section.

\subsection{Constructing a Tour by Hybrid ACO}

In the proposed method, the routes between customers are planned by Dijkstra's algorithm (DA) and the turn of visiting customers is constructed by ACO. The DA is widely used as a route planning method, and ACO is superior to other meta-heuristics including genetic algorithms [5] and simulated annealing [6] in terms of constructing a tour.

When the ant $k$ is at the customer $C_{i}$ in constructing a tour, the customer to which the ant $k$ moves next can be selected as follows. 
[Step 1] The DA calculates the shortest-travel-time routes using predicted traffic for all roads at time $t_{i}:\left\{R_{i, j}\left(t_{i}\right) \mid C_{j} \in N^{k}\right\}$, where $N^{k}$ is the set of customers not yet visited by the ant $k$ and $t_{i}$ is the time when a vehicle reaches $C_{i}$.

[Step 2] Selection probabilities for all $C_{j} \in N^{k}$ are calculated by the following formulas, where $\tau_{i, j}$ is a pheromone on $R_{i, j}\left(t_{i}\right), \eta_{i, j}\left(t_{i}\right)$ is a heuristic value, $T_{i, j}\left(t_{i}\right)$ is a travel time between $C_{i}$ and $C_{j}$, and $\alpha$ and $\beta$ are constants.

$$
\begin{aligned}
& p_{i j}^{k}\left(t_{i}\right)=\frac{\left[\tau_{i, j}\right]^{\alpha} \cdot\left[\eta_{i, j}\left(t_{i}\right)\right]^{\beta}}{\sum_{l \in N^{k}}\left[\tau_{i, l}\right]^{\alpha} \cdot\left[\eta_{i, l}\left(t_{i}\right)\right]^{\beta}} \\
& \eta_{i, j}\left(t_{i}\right)=\frac{1}{T_{i, j}\left(t_{i}\right)}
\end{aligned}
$$

[Step 3] Select a customer according to the probabilities above.

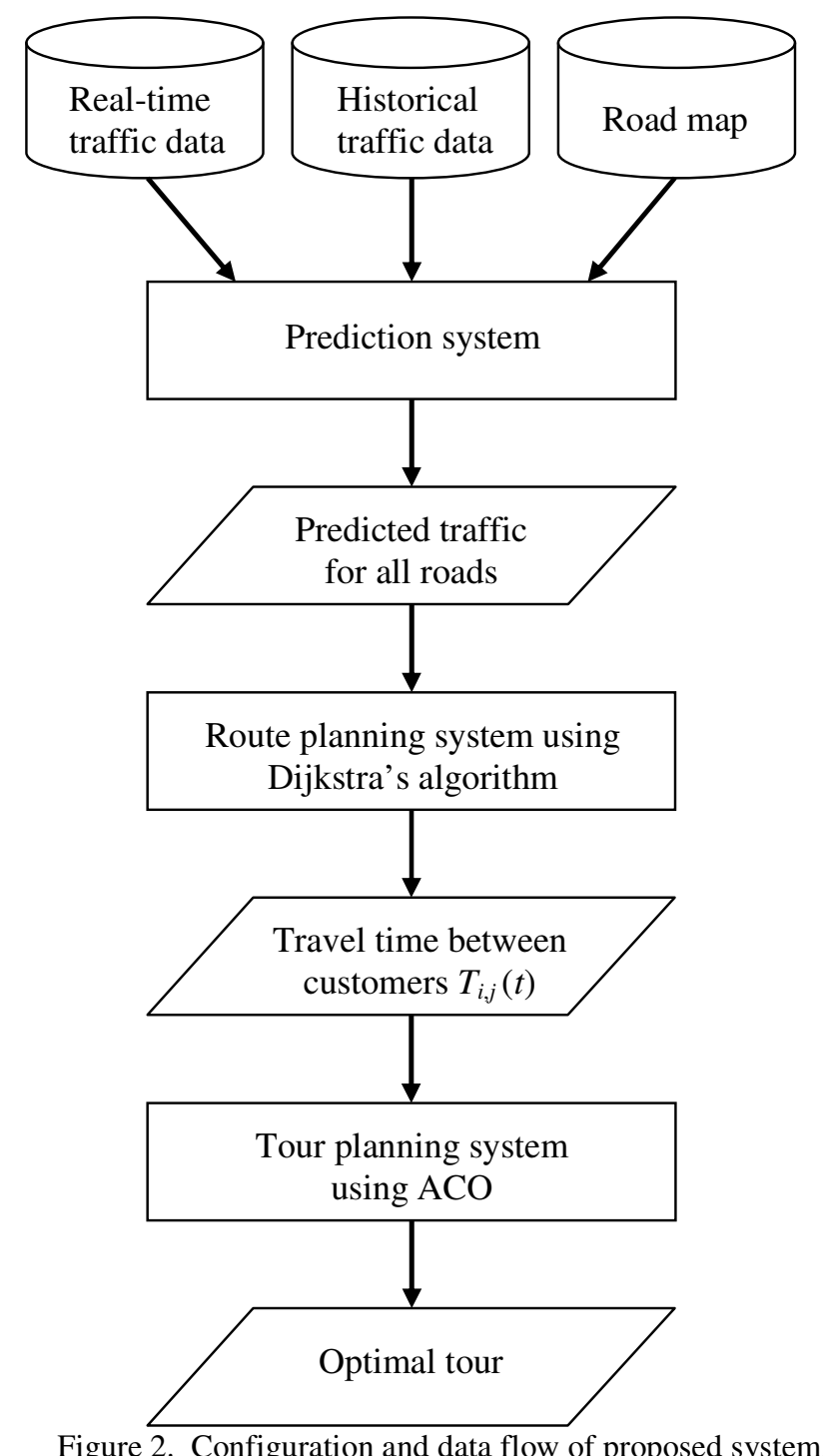




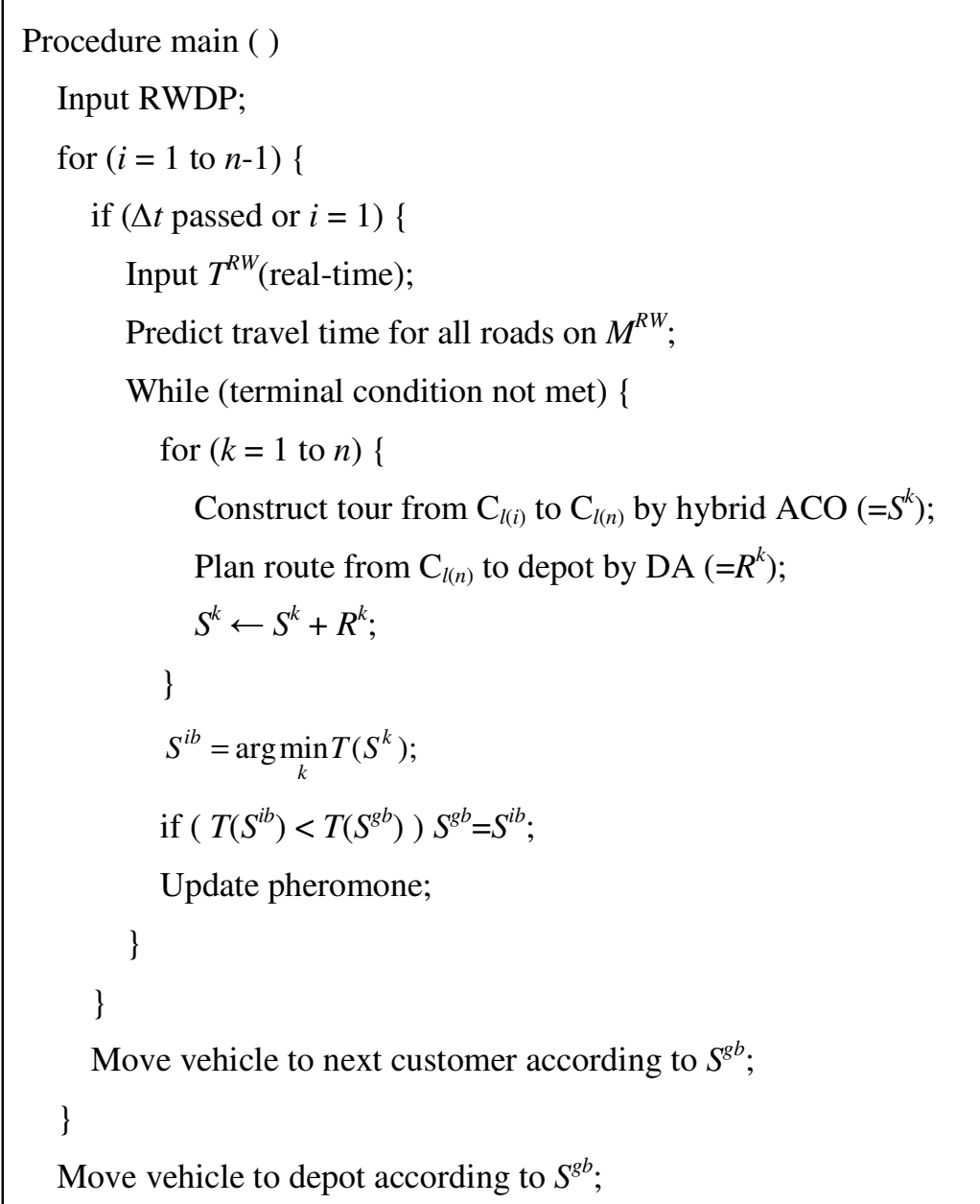

Figure 3. General procedure of proposed method.

\section{EXPERIMENTS}

\subsection{Experiments with TSP Instances (TDTSP)}

\subsubsection{Experimental Methods}

To evaluate how well the proposed method performs, we first conducted experiments using the TSP instances eil51, eil76, kroA100, u159, and d198 from the TSPLIB [15]. The number of cities seems small in TSPLIB. However, considering correspondence with real-world problems, since the number of cities (or customers) that a salesman (or a vehicle) can visit in one day is at most 200 , this number should be suitable as a scale for benchmark problems.

TDTSPs were generated by the method described in Section 2.1. Constants $R_{\text {jam }}$ and $B_{\text {upper }}$ were respectively set to 0.5 and 5 by reference to real-world traffic. Table 1 shows the number of cities, the optimal solution known in a static environment, the update interval of travel time in a dynamic environment, and the number of updates for each instance. Minutes and seconds are assumed as units of travel time for instances \{eil51, eil76\} and \{kroA100, u159, d198\}, 
respectively. The number of updates for each optimal solution was as shown in Table 1. The number of updates ranged from 53 to 140 -in other words, the traffic flow was changed very frequently.

We compared the minimal tour travel time obtained by the proposed method with those by the conventional methods below.

- Plain method: Search is conducted once before a vehicle starts using static traffic $T_{i, j}(0)$.

- Repeat method [9]-[11]: Search is repeated while a vehicle is moving using real-time traffic $T_{i, j}(t)$.

- Prediction method [12] [19]: Search is conducted once before a vehicle starts using predicted $\operatorname{traffic} T_{i, j}\left(t_{i}\right)$.

- Prediction and repeat method: The proposed method.

We assumed two kinds of prediction errors, i.e., $2 \%$ and $5 \%$ in Fig. 4. The predicted traffic with error rate can be calculated as follows, where $\operatorname{error}(t)$ is given by Fig. $4, t$ is the current time, and rand $\in[-1,1]$ is a uniform random number:

$$
\begin{aligned}
& T_{i, j}^{\prime}\left(t_{i}\right)=T_{i, j}\left(t_{i}\right) \times\left(1+\operatorname{error}\left(t_{i}\right) \times \text { rand }\right) \text { for prediction method } \\
& T_{i, j}^{\prime}\left(t, t_{i}\right)=T_{i, j}\left(t_{i}\right) \times\left(1+\operatorname{error}\left(t-t_{i}\right) \times \text { rand }\right) \text { for proposed method }
\end{aligned}
$$

This is based on experience in which short-term prediction has a smaller error rate than long-term prediction. For each solution, i.e. tour, obtained in this way, the tour travel time was calculated using predicted traffic without error. In addition, the values of parameters of the MMAS were those generally used [1].

Table 1. TSP instances used in experiments and updated travel time.

\begin{tabular}{|l|l|l|l|l|}
\hline TSP & $\begin{array}{l}\text { Number of } \\
\text { cities }\end{array}$ & $\begin{array}{l}\text { Optimal } \\
\text { solution }\end{array}$ & $\begin{array}{l}\text { Update } \\
\text { interval }\end{array}$ & $\begin{array}{l}\text { Number of } \\
\text { updates }\end{array}$ \\
\hline ei151 & 51 & 426 & 5 & 86 \\
\hline eil76 & 76 & 538 & 5 & 108 \\
\hline kroA100 & 100 & 21282 & 300 & 71 \\
\hline u159 & 159 & 42080 & 300 & 140 \\
\hline d198 & 198 & 15780 & 300 & 53 \\
\hline
\end{tabular}

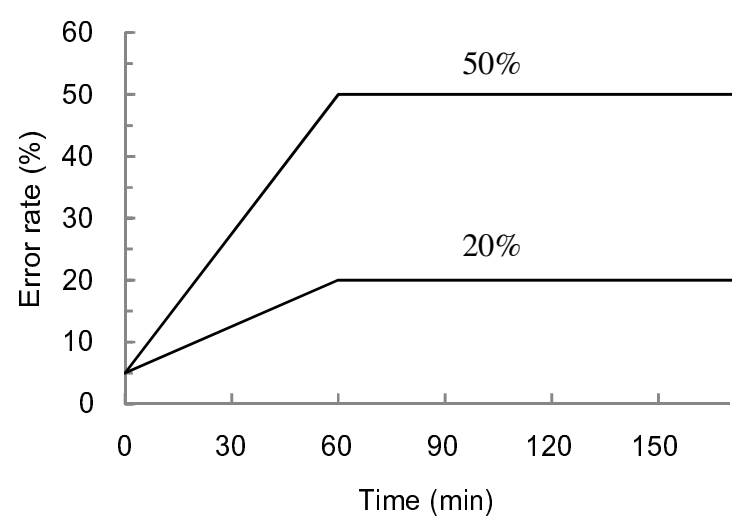

Figure 4. Relationship between prediction error and time. 


\subsubsection{Experimental Results}

Table 2 lists the ratio of the tour travel time of each method to the optimal tour travel time. Since the optimal solutions of TDTSPs are not known, we assumed that the solution obtained by the prediction method without error, which knows the exact travel time on all the links at all times, is the optimal solution. Each value in Table 2 is the average of 30 trials using different random number sequences; the standard deviation was about 5 to $10 \%$. Table 2 reveals three main findings.

- Compared with the plain method, the performances of the other methods are improved.

- When the prediction error rate becomes large $(50 \%)$, the prediction method is inferior to the repeat method.

- The proposed method is superior to the other methods, even when the error rate is large $(50 \%)$.

Table 2. Experimental results for TDTSP. Each value indicates the ratio of the tour travel time of each method to the optimal tour travel time.

\begin{tabular}{|l|l|l|l|l|l|l|}
\hline \multirow{2}{*}{ TSP } & \multirow{2}{*}{ Plain } & \multirow{2}{*}{ Repeat } & \multicolumn{2}{|l|}{ Prediction } & \multicolumn{2}{l|}{ Prediction + Repeat } \\
\cline { 4 - 7 } & & & $\mathbf{2 0 \%}$ & $\mathbf{5 0 \%}$ & $\mathbf{2 0 \%}$ & $\mathbf{5 0 \%}$ \\
\hline eil51 & 1.50 & 1.31 & 1.05 & 1.41 & 1.02 & 1.12 \\
\hline ei175 & 1.39 & 1.36 & 1.29 & 1.44 & 1.06 & 1.10 \\
\hline kroA100 & 1.27 & 1.18 & 1.16 & 1.17 & 1.03 & 1.05 \\
\hline u159 & 1.26 & 1.27 & 1.29 & 1.39 & 1.03 & 1.08 \\
\hline d198 & 1.55 & 1.24 & 1.06 & 1.26 & 1.03 & 1.04 \\
\hline (Mean) & 1.39 & 1.27 & 1.17 & 1.33 & 1.03 & 1.08 \\
\hline
\end{tabular}

\subsection{Experiments with Real-World Problems (RWDP)}

\subsubsection{Experimental Methods}

Next, to evaluate the proposed method in a real-world environment, we applied it to the RWDP described in section 2.2. Figure 5 shows a map of central Tokyo, which was the target area of this experiment. This map includes 58,222 links and 19,963 nodes and represents the most congested area in Japan. The positions of 100 customers are randomly selected from the nodes. The travel time of a vehicle was calculated from historical traffic data on June 17, 2003. An example of the data is shown in Fig. 1. We also assumed the prediction error rate in Fig. 4. We performed three experiments with the starting time of a vehicle at 6:00 (morning), 12:00 (afternoon), and 18:00 (night).

\subsubsection{Experimental Results}

Figure 6 shows examples of the tours obtained by the proposed method. The red and black circles indicate a depot and customers, respectively. As shown, the tour depends on the time period. Crosses and returns appear in some parts of the tour in 2D, but in the 3D real-world, the tour is a complete circuit. Table 3 lists the tour travel time (minutes) of each method. Each value in Table 3 is the average of 30 trials using different random number sequences, and the standard deviation was about 2 to $5 \%$. Table 3 reveals two main findings.

- The same results as the experiments for TDTSPs can be obtained in a real-world environment.

- The performance of the proposed method does not deteriorate when the prediction error rate is large $(50 \%)$, while that of the prediction method deteriorates. 


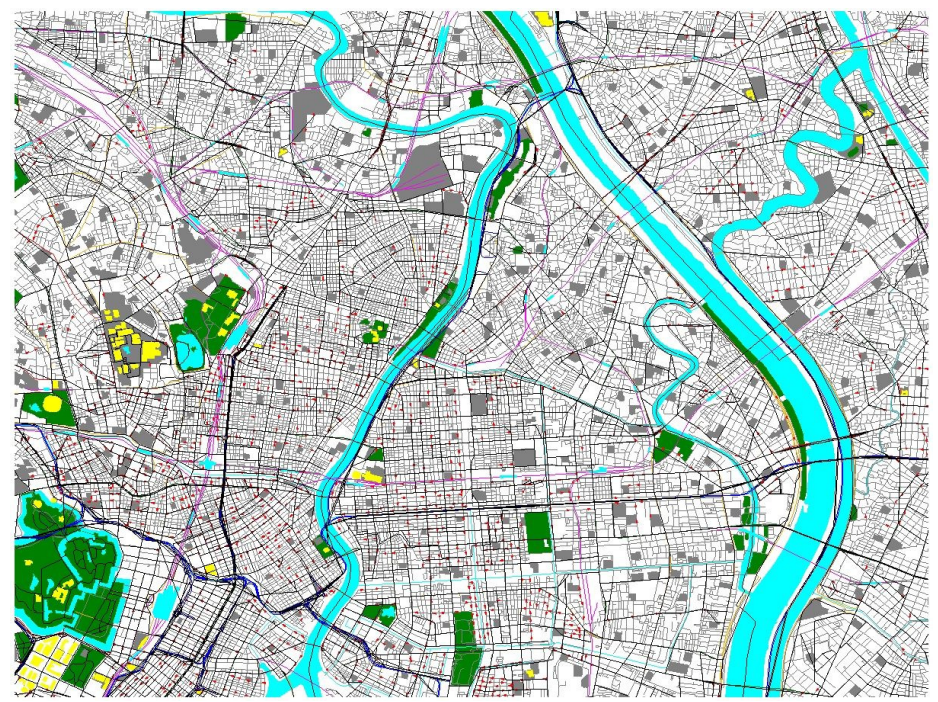

Figure 5. Map of central Tokyo. There are 58,222 links and 19,963 nodes.

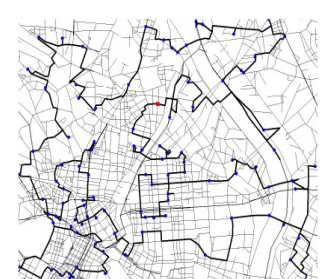

(a) Morning

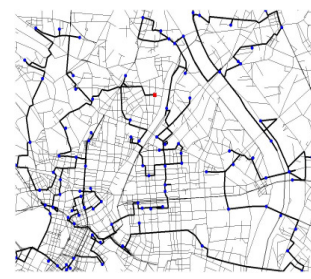

(b) Afternoon

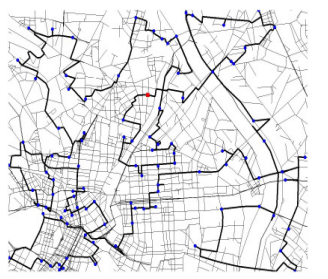

(c) Evening

Figure 6. Examples of tours obtained by proposed method.

Table 3. Experimental results for RWDP. Each value is shown in minute.

\begin{tabular}{|l|l|l|l|l|l|l|}
\hline \multirow{2}{*}{ Time } & \multirow{2}{*}{ Plain } & \multirow{2}{*}{ Repeat } & \multicolumn{2}{|l|}{ Prediction } & \multicolumn{2}{l|}{ Prediction + Repeat } \\
\cline { 4 - 7 } & & & $\mathbf{2 0 \%}$ & $\mathbf{5 0 \%}$ & $\mathbf{2 0 \%}$ & $\mathbf{5 0 \%}$ \\
\hline Morning & 371 & 336 & 308 & 323 & 294 & 294 \\
\hline Afternoon & 349 & 341 & 327 & 336 & 316 & 320 \\
\hline Night & 281 & 266 & 266 & 280 & 265 & 269 \\
\hline
\end{tabular}

\section{CONCLUSIONS}

In this paper, we presented three techniques: a hybrid meta-heuristic that combines ACO with Dijkstra's algorithm, a search method that combines repeat and prediction, and a way to use a real-world road map and measured traffic data in Japan. The experimental results suggest that the proposed method is effective in a wide area road network. The results presented in this paper are based on five benchmark problems and a real-world problem. Further investigation using other maps and traffic from additional days is necessary. Although the proposed method is for delivery problems, the basic idea can be used for other combinatorial optimization problems in networks. In future work, we will improve the ACO model and shorten the computational time. 


\section{ACKNOWLEDGEMENTS}

The authors wish to thank Mr. Yosuke Kameda for his considerable advice in carrying out this research. This research was partly supported by a Grant-in Aid for Scientific Research (C) of the Japan Society for the Promotion of Science (23500169).

\section{REFERENCES}

[1] Dorigo, M., Stutzle, T. , (2004) Ant colony optimization, The MIT press.

[2] Dorigo, M., Birattari, M., Stutzle, T., (2006) "Ant colony optimization - Artificial ants as a computational intelligence technique”, IEEE Computational Intelligence Magazine. Vol. 1, No. 4, pp 28-39.

[3] Dorigo, M., Stutzle, T., (2010) "Handbook of Metaheuristics", International Series in Operations Research \& Management Science 146, pp 227-263.

[4] Reinelt, G., (1994), "The Traveling Salesman: Computational Solution for TSP Applications", Lecture Notes in Computer Science 840.

[5] Albayrak, M., Allahverdi, N., (2011) "Development a new mutation operator to solve the Traveling Salesman Problem by aid of Genetic Algorithms", Expert Systems with Applications 38, pp 13131320.

[6] Genga, X., Chen, Z., Yang, W., Shi, D., Zhao, K., (2011) "Solving the traveling salesman problem based on an adaptive simulated annealing algorithm with greedy search", Applied Soft Computing 11, pp 3680-3689.

[7] Berbeglia, G., Cordeau, J., Laporte, G., (2010) "Dynamic pickup and delivery problems", European Journal of Operational Research 202, pp 8-15.

[8] Pillac, V., Gendreau, M., Gueret, C., Medaglia, A. L., (2013) "A review of dynamic vehicle routing problems", European Journal of Operational Research 225, pp 1-11.

[9] Mavrovouniotis, M., Yang, S., (2010) "Ant Colony Optimization with Immigrants Schemes in Dynamic Environments", Lecture Notes in Computer Science, vol. 6239, pp 371-380.

[10] Guntsch, M., Middendorf, M., (2001) "Pheromone modification strategies for ant algorithms applied to dynamic TSP”, Lecture Notes in Computer Science 2037, pp 213-222.

[11] Eyckelhof, C.J., Snoek, M., (2002) "Ant systems for a dynamic TSP: ants caught in a traffic jam", Lecture Notes in Computer Science 2463, pp 88-99.

[12] Donati, V.A., Montemanni, R., Casagrande, N., Rizzoli, E.A. and Gambardella, M. L., (2008) "Time dependent vehicle routing problem with a multi ant colony system”, European Journal of Operational Research, Vol. 185, No. 3, pp 1174-1191.

[13] Kanoh, H., Tsukahara, S., (2010) "Solving Real-World Vehicle Routing Problems with Time Windows using Virus Evolution Strategy", International Journal of Knowledge-Based and Intelligent Engineering Systems, Vol. 14, No. 3, pp 115-126.

[14] Haghani, A. and Jung, S., (2005) "A dynamic vehicle routing problem with time-dependent travel times”, Computers \& Operations Research, Vol. 32, No. 11, pp 2959-2986.

[15] Traveling Salesman Problem http://www.iwr.uniheidelberg.de/groups/comopt/software/TSPLIB95/

[16] Stutzle, T., Hoos, H. H., (2000) "MAN-MIN ant system”, Future Generation Computer System, Vol. 16, No. 8, pp 889-914.

[17] Kanoh, H, Furukawa, T., Tsukahara, S., Hara, K., Nishi, H., Kurokawa, H., (2005) "Short-Term Traffic Prediction Using Fuzzy C-Means and Cellular Automata in a Wide-Area Road Network", IEEE International Conference on Intelligent Transportation Systems (ITSC 2005), pp984-988.

[18] Ichiba, D., Hara, K., Kanoh, H., (2006) "Spatial Interpolation of Traffic Data by Genetic Fuzzy System”, IEEE 2nd International Symposium on Evolving Fuzzy Systems (EFS 2006), pp 280-285.

[19] Kanoh, H, Ochiai, J., (2012) "Solving Time-Dependent Traveling Salesman Problems using Ant Colony Optimization Based on Predicted Traffic", International Symposium on Distributed Computing and Artificial Intelligence (DCAI 2012), Advances in Intelligent and Soft Computing 151, pp 25-32. 


\section{AUTHORS}

Junich Ochiai received M.E. degree in computer science from University of Tsukuba, Japan, in 2011. He is with the Department of Computer Science, Graduate School of Systems and Information Engineering, University of Tsukuba. His research interests include ant colony optimization and evolutionary computation.

Hitoshi Kanoh received M.S. degree in physics and Ph.D. degree in computer science from University of Tsukuba, Japan, in 1980 and 1992, respectively. In 1980, he joined Hitachi Cable, Ltd. where he has been engaged in Research of expert systems, fuzzy systems and neural networks as a section chief. In 1993 he moved to the University of Tsukuba. He currently is a professor with the Division of Information Engineering, Faculty of Engineering, Information and Systems, University of Tsukuba. His main research interests include evolutionary computation, swarm intelligence, and soft computing with special application to intelligent transportation systems. 
Computer Science \& Information Technology (CS \& IT)

INTENTIONAL BLANK 


\title{
THE RESEARCH OF INDUCED CURRENT IN COILS WHEN THE PROCESS OF MOTION OF MAGNETICALLY LEVITATED PLANAR ACTUATORS
}

\author{
Rougang Zhou ${ }^{1, \mathrm{a}}$, Yunfei Zhou ${ }^{1, \mathrm{~b}}$, Guangdou Liu ${ }^{1, \mathrm{c}}, \mathrm{Xiao} \mathrm{Tu}^{1, \mathrm{~d}}$ \\ ${ }^{1}$ State Key Lab of Digital Manufacturing Equipment \& Technology, Huazhong \\ University of Science \& Technology, Wuhan, P.R.,430074,China ; \\ aemail:rougangzhou@hust.edu.com, bemail:_yfzhou@mail.hust.edu.cn, \\ cemail: lgd-5401@163.com, demail:_1006336923@qq.com
}

\begin{abstract}
In the process of motion magnetically levitated planar actuators, the magnetic flux through the coils been changed as the position changed, It produced inductive electromotive force which associated with the location and speed of coils. Induction electromotive force to produce induced current in the coils, which influence the stability of the actuators. This paper presents a analytical model of induction electromotive force in the coils of magnetically levitated a planar actuators, According to the analytical model it could predict control the compensation of drive currents in order to offset the induced current which produce by electromotive force, the stability of Magnetically levitated planar actuators have been improved. Finite element simulation had been used in this paper to approve the correct of the analytical model.
\end{abstract}

\section{KEYWORDS}

Induced Current, Magnetically Levitated Planar Actuators, Halbach Permanent Magnet array

\section{INTRODUCTION}

Magnetically levitated planar actuators can realize high-accuracy and wear-free high-speed and friction-less motion[1-6]. So, they have become the focus of the research on the high-accuracy and high-speed long-stroke motion system. Because the motion part of magnetically levitated planar actuator is six-degree-of-freedom levitation motion, a minor disturbance could cause the instability and kinematic accuracy. The drive current accuracy of coils is the one of critical point of control precision and stability of magnetically levitated planar actuators. The papers [2] have indicated that the magnetic flux in the coils have been changed which according to the speed and position of the actuators, hence it produce induction electromotive force. Induced current have been produced by induction electromotive force which influence the stability of the drive current accuracy. In order to solve this question the predict control offset should be adopted. The recent researches have not performed the analytical model of induced current in magnetically levitated planar actuators. For this reason, this paper theoretically analyzes the induced current regularity in the motion of magnetically levitated planar actuators. Also, the paper proposes the analytical model for the induced current by the coils when the motion of the actuators. The comparison of the analytical model results with the finite element calculation shows that the analytical model in 
this paper yields correct results and can further provide a induced current compensation method for the precise magnetic levitation motion control.

\section{The Characteristic of Permanent Magnet Planar ARRay}

The topology structure of Magnetically levitated planar actuators with moving coils as shown in figure(1)[1-3], It is composed of Halbach permanent magnet array and coils which suspended on it, the coils are arranged in an angle of $45^{\circ}$ with the Halbach magnet array in order to product the force applied to coils in the $\mathrm{x}$ and $\mathrm{y}$ direction is physically decoupling. The global coordinate system is defined at the stationary part of the Halbach permanent magnet array [2,3]:

$$
\vec{x}=\left[\begin{array}{lll}
x & y & z
\end{array}\right]^{T}
$$

A local coordinate system is defined in the volume center point of the coil, it is denoted with the superscript ${ }^{c}$

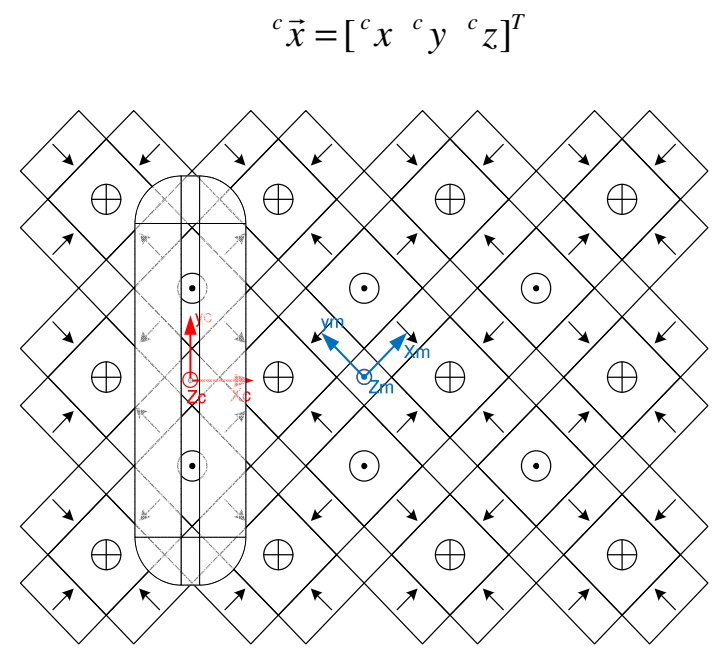

(a)

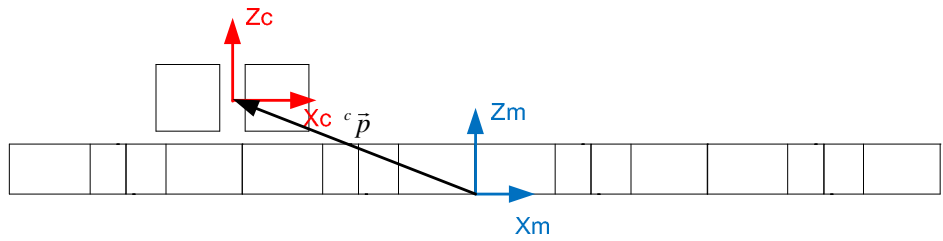

(b)

Fig. 2 Bottom and side view of the components of a magnetically levitated stage with moving coils a)cross section b) Bottom view.

The magnetic flux density expression in local coordinate system is simplified to[2]: 


$$
\overrightarrow{{ }^{c} B}=\left[\begin{array}{l}
-\frac{B_{x y}}{\sqrt{2}} e^{-\frac{\sqrt{2} \pi}{\tau} c^{c} z} \sin \left(\frac{{ }^{c} x \sqrt{2} \pi}{\tau}\right) \\
\frac{B_{x y}}{\sqrt{2}} e^{-\frac{\sqrt{2} \pi}{\tau}{ }^{c} z} \sin \left(\frac{{ }^{c} y \sqrt{2} \pi}{\tau}\right) \\
\frac{1}{2} B_{z} e^{-\frac{\sqrt{2} \pi}{\tau} c^{c}}\left(\cos \left(\frac{{ }^{c} x \sqrt{2} \pi}{\tau}\right)-\cos \left(\frac{{ }^{c} y \sqrt{2} \pi}{\tau}\right)\right)
\end{array}\right]
$$

The $B_{z}$ component of the magnetic flux density is the factor that produced induction electromotive force in the coils, as shown in Fig. (3).

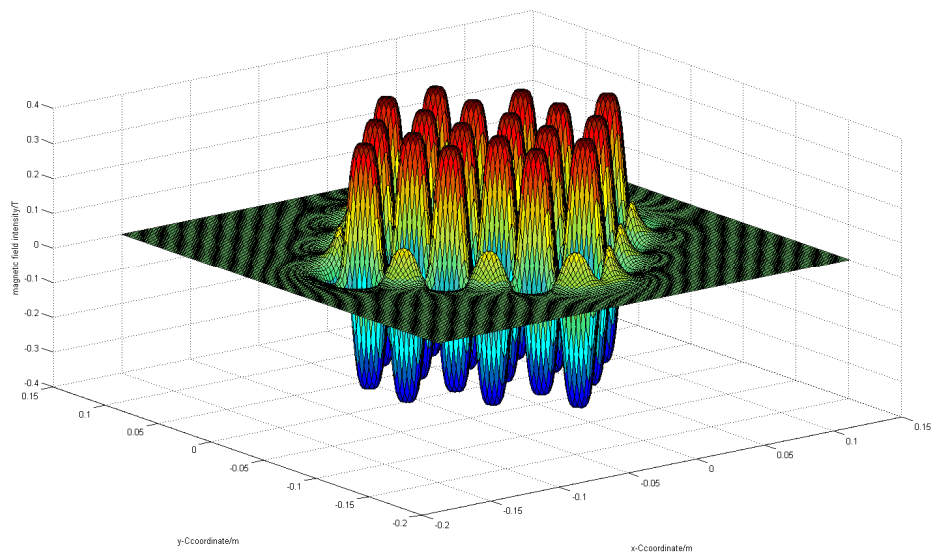

Figure 3: component in the $\mathrm{Z}$ direction of the magnetic field

\section{THE ANALYZE OF INDUCED CURRENT IN MOTION COILS}

According the paper[2,3], in the filaments model, current could been seen as through the center of the coils. And it could be adopted in the analyzed of induced current in motion coils. As show in figure(1), defining 4 lines in the center of the coils, and the $\mathrm{Z}$ coordinate of the lines is[23]:

$$
{ }^{c} r_{z}=c_{t}-{ }^{c} p_{z}-\frac{1}{\lambda}-\frac{\left(c_{b}-c_{t}\right) e^{\lambda c_{b}}}{e^{\lambda c_{b}}-e^{\lambda c t}}
$$

Because the motion range of coils in Z-direction is small (less than $1 \mathrm{~mm}$ ), it is not care the Induction electromotive force which has been produced by motion of coils in Z-direction, if the coils have horizontal motion. According the formula of inductive electromotive force:

$$
\xi_{a b}=\int_{a}^{b}(v \times B) \cdot d l
$$

The induction electromotive force of the coils when moving in $\mathrm{x}, \mathrm{y}$ direction could obtain. Combine the size and structure of the Halbach permanent magnet array it could deduce that the induction electromotive force be produced when the coils have y- velocity component, the zdirection of magnetic field produce induction electromotive force in 1,3 sides of the coils which can be simply added,2,4 sides don't produce induction electromotive force. When the coils have $\mathrm{y}$ - velocity component, 1,3 sides don't produce induction electromotive force and the z-direction of magnetic field produce induction electromotive force in 1,3 sides of the coils which is offset. So the induction electromotive force of the moving coils is: 


$$
\xi_{c o i l}=\int_{a 1}^{b 1}\left(v_{y} \times B_{z}\right) \cdot d l_{1}+\int_{a 2}^{b 2}\left(v_{y} \times B_{z}\right) \cdot d l_{2}
$$

And the induced current of the moving coils is:

$$
I=\frac{\boldsymbol{\xi}_{\text {coil }}}{R_{\text {coil }}}
$$

From Formula[BZ], it can be deduced that induced current is:

$$
I=v_{y} \frac{-2 \cos \left(\frac{{ }^{c} y \sqrt{2} \pi}{\tau}\right) \sqrt{2} \tau B_{z} e^{-\frac{\sqrt{2} \pi}{\tau} c z}}{R_{\text {coil }}}
$$

\section{VERTIFICATION RESULTS AND DISCUSSION}

The model structure is as shown in Fig. (1). For a coil and a Halbach magnet array with dimension as shown in Table (1),

Dimensions of coil and Halbach magnet array during calculation

\begin{tabular}{|c|c|c|}
\hline Parameter & Value & Unit \\
\hline Pole pitch magnet array, $\tau_{l}$ & 21 & $\mathrm{~mm}$ \\
\hline Pole pitch magnet array, $\tau_{n}$ & 10 & $\mathrm{~mm}$ \\
\hline Pole pitch of magnetic cycle, $\tau$ & $\tau=\tau_{l}+\tau_{n}$ & $\mathrm{~mm}$ \\
\hline Coil length,$l$ & 81.4 & $\mathrm{~mm}$ \\
\hline $\begin{array}{c}\text { Remanence of permanent } \\
\text { magnets }, B\end{array}$ & 1.24 & $T$ \\
\hline magnet height,$m h$ & 7.0 & $\mathrm{~mm}$ \\
\hline coil height, $h$ & 9.2 & $\mathrm{~mm}$ \\
\hline Coil width,$c w$ & 22.8 & $\mathrm{~mm}$ \\
\hline velocity y-direction,$x$ & 2 & $\mathrm{~m} / \mathrm{s}$ \\
\hline $\begin{array}{c}\text { Mechanical clearance }(z- \\
\text { direction), } m c\end{array}$ & 1 & $\mathrm{~mm}$ \\
\hline The thickness of the layers,$b$ & 7.23 & $\mathrm{~mm}$ \\
\hline
\end{tabular}

The induced current that the coil is subject to when it moves along the $\mathrm{x}$ axis at a speed of $2 \mathrm{~m} / \mathrm{s}$ is as shown in Fig.[], it can be seen that the results from the analytical model are consistent with the periodic rules of the simulation results and the amplitudes are identical. 


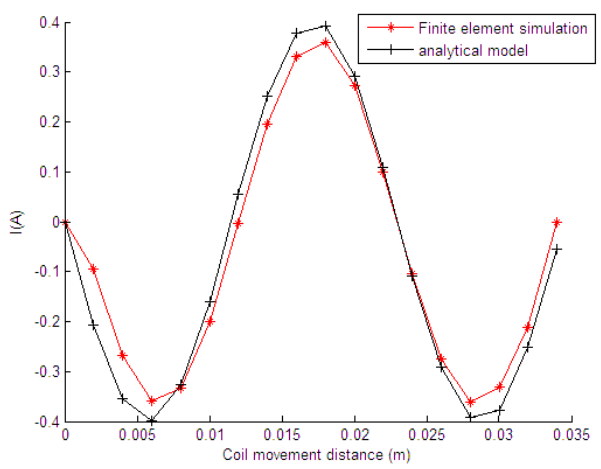

Fig. Comparison of induced current of the coil when the coil moves at $1 \mathrm{~m} / \mathrm{s}$ in $y$-direction.

\section{CONCLUSIONS}

In the magnetically levitated planar actuator, the induced current are generated in the moving foilwound coils and it can't be ignored which exert influence over the stability of the Magnetically levitated planar actuator. This paper analyzes and calculates the induced current generated in the coils when the coils move above the Halbach magnet array. The induced current analytical model has been given and finite element simulation deduced in this paper. According compare the results, it is demonstrates that the analytical model is correct. it is possible to conduct the compensation calculation in the precise motion control.

\section{REFERENCES}

[1] Jansen, J. W., Van Lierop, C. M. M., Lomonova, E. A., \& Vandenput, A. J. A. (2007). Modeling of magnetically levitated planar actuators with moving magnets. Magnetics, IEEE Transactions on, 43(1), 15-25.

[2] Jansen, J. W. (2007). Magnetically levitated planar actuator with moving magnets: Electromechanical analysis and design. Einhoven: Technische Universiteit Einhoven.

[3] Rovers, J. M. M., Jansen, J. W., Compter, J. C., \& Lomonova, E. A. (2012). Analysis method of the dynamic force and torque distribution in the magnet array of a commutated magnetically levitated planar actuator. Industrial Electronics, IEEE Transactions on, 59(5), 2157-2166.

[4] Rovers J M M, Stöck M, Jansen J W, et al. (2013)Real-time 3D thermal modeling of a magnetically levitated planar actuator. Mechatronics,240 - 246.

[5] Peng, Junrong, and Yunfei Zhou. (2013)"Modeling and Analysis of a New 2-D Halbach Array for Magnetically Levitated Planar Motor. IEEE Transactions on,: 1-1.

[6] Rovers J M M, Jansen J W, Compter J C, et al. (2012), Analysis method of the dynamic force and torque distribution in the magnet array of a commutated magnetically levitated planar actuator. Industrial Electronics, IEEE Transactions on, 59(5), 2157-2166.

\section{AUTHORS}

Rougang Zhou was born in Zhejing,China.. Now ,he is a PhD Candidate of State Key Lab of Digital Manufacturing Equipment \& Technology, Huazhong University of Science. 


\title{
BAYESIAN METHODS FOR ASSESSING WATER QUALITY
}

\author{
Khalil Shihab $^{1}$ and Nida Al-Chalabi ${ }^{2}$ \\ ${ }^{1}$ College of Engineering \& Science, Victoria University, Australia \\ Khalil.shihab@gmail.com \\ ${ }^{2}$ Department of Computer Science, SQU, Oman \\ nida@squ.edu.om
}

\begin{abstract}
This work presents the development of Bayesian techniques for the assessment of groundwater quality. Its primary aim is to develop a predictive model and a computer system to assess and predict the impact of pollutants on the water column. The process of the analysis begins by postulating a model in light of all available knowledge taken from relevant phenomenon. The previous knowledge as represented by the prior distribution of the model parameters is then combined with the new data through Bayes' theorem to yield the current knowledge represented by the posterior distribution of model parameters. This process of updating information about the unknown model parameters is then repeated in a sequential manner as more and more new information becomes available.
\end{abstract}

\section{KEYWORDS}

Bayesian Belief Networks, Water Quality Assessment, Data Mining

\section{INTRODUCTION}

Water is an essential requirement for irrigated agriculture, domestic uses, including drinking, cooking and sanitation. Declining surface and groundwater quality is regarded as the most serious and persistent issue and has become as a global issue effecting the people and the ecosystem. Anthropogenic sources of pollution such as agriculture, industry, and municipal waste, contribute to the degradation of groundwater quality, which may limit the use of these resources and lead to health-risk consequences. For these reasons, the need for intensive groundwater resources management has become more urgent.

In this work, we studied the Salalah area of Oman because the groundwater has been an important natural resource and the only available water source other than the seasonal rainfall.

Groundwater quality and pollution are determined and measured by comparing physical, chemical, biological, microbiological, and radiological quantities and parameters to a set of standards and criteria. A criterion is basically a scientific quantity upon which a judgment can be based [1]. In this work, however, we considered only the chemical parameters: total dissolved solids (TDS), electrical conductivity (EC) and water $\mathrm{pH}$. 


\section{UNCERTAINTY ANALYSIS}

The Ministry of Water Resources (MWR) maintains data on the concentration of the harmful substances in the groundwater at Taqah monitoring sites, which are located to the south of the Sultanate of Oman, in the Salalah plain [2,3]. We observed that good quality data were obtained from several monitoring wells in this region. Because of the lack of monitoring wells in certain areas in that region, we filled in the missing measurements with data obtained from Oman Mining Company (OMCO) and Ministry of Environmental and Regional Municipalities (MRME) [4].

Data for water quality assessment are normally collected from various monitoring wells and then analyzed in environmental laboratories in order to measure the concentration of a number of water quality constituents. We realized that the methods used by these laboratories do not emphasize accuracy. There is a lack of awareness among both laboratory and validation personnel regarding the possibility of false positives in environmental data. In order to overcome this problem and to have representative data, we, therefore, used the following modified Bayesian model to that developed by Banerjee, Planting and Ramirez [6], to preprocessing the datasets used for the development of the Bayesian Networks.

\subsection{Bayesian Models}

The formulation of the model is as follows:

Let $\mathbf{S}$ denote a particular hazardous constituent of interest. Since the concentration of the substance may vary from well to another, it is necessary to consider each well separately. Let $\mathrm{x}_{\mathrm{t}}=$ $\left(\mathrm{x}_{\mathrm{t} 1}, \mathrm{x}_{\mathrm{t} 2}, \mathrm{x}_{\mathrm{t} 3}, \mathrm{x}_{\mathrm{tm}}\right)$ be the vector of $\mathbf{m}$ measurements of the concentration of $\mathbf{S}$ in $\mathbf{m}$ distinct water samples from a given well at a given sampling occasion where $(\mathbf{m}>=1)$ and $(t=1,2, \ldots)$. Each measurement consists of the true concentration of $\mathbf{S}$ plus an error.

Let $X_{t}$ be the true concentration of $\mathbf{S}$ in the groundwater at sampling occasion $t$. If we assume that the true concentration $X_{t}$ is unknown and is a random variable, the model evaluates the posterior distribution of $X_{t}$ given the sample measurements $X_{t}$ at sampling occasion $t$.

Using the normality assumption and given $\mathrm{X}_{\mathrm{t}}=\mathrm{x}_{\mathrm{t}}$ and $\delta^{2}$, the concentration measurements in $\mathrm{x}_{\mathrm{t}}$ represent a random sample of size $m$ for random distribution with mean $x_{t}$ and variance $\delta^{2}$.

We assume that the parameters $x_{t}$ and $\delta^{2}$ of the normal distribution are random variables with certain prior probability distribution. Therefore, the model for prior distribution of $\mathrm{X}_{\mathrm{t}}$ and $\delta^{2}$ can be presented as follows:

For $\mathrm{t}=1,2 \ldots$ and given $\delta^{2}$ the conditional distribution of $\mathrm{X}_{\mathrm{t}}$ at sampling occasion $\mathrm{t}$ is a normal distribution with mean $\mu_{\mathrm{t}-1}$ and variance $\delta_{\mathrm{t}-1}^{2} \delta^{2}$. The marginal distribution of $\delta^{2}$ is an inverted gamma distribution with parameter $\beta_{\mathrm{t}-1}$ and $v_{\mathrm{t}-1}$.

This model uses the following prior distribution, which represents the concentration measurements before the first sampling.

The pdf of the prior distribution of $\mathrm{X}_{0}$ is: 


$$
f_{0}\left(x_{0}\right)=\left\{1+\frac{1}{2 v_{0}}\left[\frac{x_{0}-\mu_{0}}{\sigma_{0} \sqrt{\beta_{0} / v_{0}}}\right]\right\}^{2}
$$

which is the pdf of the student's t-distribution with $2 \mathrm{v}_{0}$ degrees of freedom, location parameters $\mu_{0}$ and variance $\delta_{0}^{2} \beta_{0} / v_{0}$.

Now suppose that the observations are available on the concentration of $\mathbf{S}$, given the sample $X_{t}$ the posterior marginal distribution of $X_{t}$ is a student's t-distribution with $2 v_{t}$ degree of freedom, location parameters $\mu_{\mathrm{t}}$ and variance $\delta^{\mathrm{t}} \beta_{\mathrm{t}} / v_{\mathrm{t}}$ where the pdf has the form:

$$
f_{t}\left(x_{t} / x\right)=\left\{1+\frac{1}{2 v_{t}}\left[\frac{x_{t}-\mu_{t}}{\sigma_{t} \sqrt{\beta_{t} / \nu_{t}}}\right]^{2}\right\}^{-\left(2 v_{t}+1\right) / 2}
$$

where:

$$
\begin{aligned}
& \qquad \beta_{t}=\beta_{t-1}+\sum_{j=1}^{m}\left(x_{t j}-\bar{x}\right) / 2+m\left(\mu_{t-1}-\bar{x}_{t}\right) /\left[2\left(1+m \sigma_{t-1}^{2}\right)\right] \\
& v_{t}=v_{t-1}+m / 2 \\
& \mu_{t}=\left(\mu_{t-1}+m \bar{x}_{t} \sigma_{t-1}^{2}\right) /\left(1+m \sigma_{t-1}^{2}\right) \\
& \sigma_{t}^{2}=\sigma_{t-1}^{2} /\left(1+m \sigma_{t-1}^{2}\right) \\
& \bar{x}_{t}=\sum_{j=1}^{m} x_{t j} / m
\end{aligned}
$$

It is obvious from the equation of $\mu_{\mathrm{t}}$ the sequential nature of this posterior distribution. Therefore, in order to present the true unknown concentration of the substance $\mathbf{S}$ in the well under consideration, it is frequently more convenient to put a range (or interval) which contains most of the posterior probability. Such intervals are called highest posterior density (HPD) intervals. Thus for a given probability content of $(1-\alpha), 0<\alpha<1$, a $100(1-\alpha)$ percent HPD interval for $X_{t}$, is given by:

$$
\mu_{t} \pm t_{2 v_{t}}(\alpha / 2) \sigma_{t} \sqrt{\beta_{t} / v_{t}}
$$

when $t_{2 v t}(\alpha / 2)$ is the $100(1-\alpha / 2)$ percentile of the student's t-distribution with $2 v_{t}$ degree of freedom.

\subsection{Bayesian Algorithm}

In brief, the monitoring algorithm, which is based on the Bayesian model, is as follows:

(1) Fix a value of $\alpha(0<\alpha<1)$ based on the desired confidence level. In this case, we chose $\alpha$ to be 0.01 .

(2) Since we do not have enough data to work with, we used the same parameters of the prior distribution used in the model of Banerjee, Plantinga and Ramirez. These parameters are :

$$
\beta_{0}=0.0073, v_{0}=2.336, \mu_{0}=9.53, \delta_{0}{ }^{2}=3056.34
$$


(3) At each sampling occasion $t,(t=1,2, \ldots)$, compute the parameters $\beta_{t}, v_{t}, \mu_{t}$ and $\delta_{t}$ of the posterior distribution $X_{t}$ given the set of observations in $\mathbf{x}_{\mathbf{t}}$ on the concentration of $S$ available from a given well in a given site using (2.3). Compute LHPD and UHPD using these parameter estimates and (2.4).

(4) Plot $\mu_{t}$, LHPD, and UHPD that are obtained in step 3 above against sampling occasion $t$.

(5) For the next sampling occasion, update the values of the parameters $\beta_{\mathrm{t}}, v_{\mathrm{t}}, \mu_{\mathrm{t}}$ and $\delta_{\mathrm{t}}$ using (2.3) and the datasets just obtained. Recomputed LHPD, and UHPD using the updated parameter values in (2.4) and repeat step 4 above.

Some of these datasets needed to be scaled down using the following normalization technique:

$$
x=\frac{\bar{x}-\mu}{\sigma}, \text { where } \bar{x}=\sum_{i}^{n} x_{i} / n \text {, and } \sigma=\sqrt{\frac{\sum_{i}^{n} x_{i}^{2}-n \bar{x}^{2}}{n-1}}
$$

\subsection{Implementation}

The pre-processing system is implemented on PC platform using Visual Basic programming language.

Table 1 presents the concentration data for TDS (Total Dissolved Solids) for Well 001/577 in the Taqah area. In particular, the table shows the true concentration data for TDS produced by our pre-processing system.

Table 1. Concentration Data of TDS for Well001/577 in the Salalah plain, where OC stands for Observed Concentration and ETC stands for Expected True Concentration.

\begin{tabular}{|c|c|c|c|c|}
\hline Te & OC & LHPD & ETC & UHPD \\
\hline 84 & 1.147 & 0.85 & 1.15 & 1.45 \\
\hline 85 & 1.106 & 1 & 1.13 & 1.26 \\
\hline 86 & 1.938 & 1.12 & 1.4 & 1.68 \\
\hline 87 & 2.237 & 1.33 & 1.61 & 1.88 \\
\hline 88 & 3.857 & 1.6 & 2.06 & 2.52 \\
\hline 89 & 3.834 & 1.91 & 2.35 & 2.79 \\
\hline 90 & 3.957 & 2.18 & 2.58 & 2.98 \\
\hline 91 & 3.761 & 2.38 & 2.73 & 3.08 \\
\hline 92 & 4.3 & 2.58 & 2.9 & 3.23 \\
\hline 93 & 3.958 & 2.72 & 3.01 & 3.3 \\
\hline 94 & 1 & 2.54 & 2.83 & 3.11 \\
\hline 95 & 3.714 & 2.64 & 2.9 & 3.16 \\
\hline 96 & 3.65 & 2.73 & 2.96 & 3.19 \\
\hline 97 & 3.381 & 2.78 & 2.99 & 3.2 \\
\hline 98 & 3.396 & 2.83 & 3.02 & 3.2 \\
\hline 99 & 3.477 & 2.87 & 3.04 & 3.22 \\
\hline 00 & 3.498 & 2.91 & 3.07 & 3.23 \\
\hline 01 & 3.23 & 2.93 & 3.08 & 3.23 \\
\hline 02 & 3.243 & 2.95 & 3.09 & 3.22 \\
\hline 03 & 3.267 & 2.97 & 3.1 & 3.22 \\
\hline 04 & 3.297 & 2.99 & 3.11 & 3.22 \\
\hline
\end{tabular}




\section{BAYESIAN NETWORKS}

After the pre-processing stage, we constructed a Bayesian Network $(\mathrm{BN})$ by using the Hugin system. We then used this BN as an initial building network for the construction of two Dynamic Bayesian Networks in order to predict the impact of pollution on groundwater quality.

\subsection{Dynamic Bayesian Networks (DBNs)}

DBNs extend Bayesian Networks from static domains to dynamic domains [7, 8]. This is achieved by introducing relevant temporal dependencies between the representations of the static network at different times.

The main characteristic of DBNs is as follows:

Let $\mathbf{X}_{\mathrm{t}}$ be the state of the system at time $\mathrm{t}$, and assume that

(1) The process is Markovian, i.e.,

$$
\mathrm{P}\left(\mathbf{X}_{\mathbf{t}} / \mathbf{X}_{\mathbf{0}}, \mathbf{X}_{1}, \ldots, \mathbf{X}_{\mathrm{t}-1}\right)=\mathrm{P}\left(\mathbf{X}_{\mathrm{t}} / \mathbf{X}_{\mathrm{t}-1}\right)
$$

(2) The process is stationary or time-invariant, i.e.,

$$
\mathrm{P}\left(\mathbf{X}_{\mathbf{t}} / \mathbf{X}_{\mathbf{t}-1}\right) \text { is the same for every } \mathrm{t} \text {. }
$$

Therefore, we just need $\mathrm{P}\left(\mathbf{X}_{\mathbf{0}}\right)$, which is a static Bayesian network $(\mathrm{BN})$, and $\mathrm{P}\left(\mathbf{X}_{\mathbf{t}} / \mathbf{X}_{\mathrm{t}-1}\right)$, which is a network fragment, where the variables in $\mathbf{X}_{\mathbf{t}-\mathbf{1}}$ have no parents, in order to have a Dynamic Bayesian Network (DBN).

\subsection{Bayesian Networks Development}

Among more than twenty wells in the Taqah area, we selected only four wells for this study. Those four wells have had, to the greatest extent, complete data measurements and provide sufficient information for the assessment of the groundwater quality for this area.

The electrical conductivity (EC) of the water has been used as a measure for the salinity hazard of the groundwater used for irrigation in the Salalah plain. The total dissolved solid (TDS) limit is $600 \mathrm{mg} / \mathrm{L}$, which is the objective of the current plan of the MWR. TDS contains several dissolved solids but $90 \%$ of its concentration is made up of six constituents. These are: sodium Na, magnesium $\mathrm{Mg}$, calcium $\mathrm{Ca}$, chloride $\mathrm{Cl}$, bicarbonate $\mathrm{HCO}_{3}$ and sulfate $\mathrm{SO}_{4}$. We, therefore, considered only these elements in the calculation of TDS.

We also used the following relationship between TDS and EC.

$$
\mathrm{TDS}=\mathrm{A} * \mathrm{EC} \text {; where } \mathrm{A} \text { is a constant with value between } 0.65 \text { and } 0.77 \text {. }
$$

Both TDS and EC can affect water acidity or water $\mathrm{pH}$. Solute chemical constituents are variable in high concentration at lower $\mathrm{pH}$ (higher acidity). On the other hand, acidity allows migration of hydrogen ions $(\mathrm{H}+)$, which is an indication of conductivity. Therefore, our work concentrated on the following relations.

$$
\mathrm{TDS} \rightarrow \mathrm{EC}, \mathrm{EC} \rightarrow \mathrm{pH}, \mathrm{TDS} \rightarrow \mathrm{pH}
$$

Reaching to these relations we used two learning approaches to construct and parameterize a simple static BN that have three nodes, each node represents a groundwater quality constituent 
(TDS, EC or $\mathrm{pH}$ ). Learning basically consists of two different components: 1) learning the network structure, 2) learning the conditional probability distributions.

For the first component, we used the Hugin system that supports structure and parameter learning in Bayesian networks. We also developed a program written in $\mathrm{C}++$ to generate the conditional probabilities for TDS, EC and $\mathrm{pH}$ using Table 2 as input.

Once the static BN model (static model) for each monitoring well was built, parameterized and tested, we used these models as initial building networks in the construction of OOBNs. Figure 1 models the time slices for each well characterizing the temporal nature of identical model structures, where the initial building network, see Figure 2, describes a generic time-sliced network.

Table 2. TDS, EC, and pH data for the well Well 001/577.

\begin{tabular}{|l|l|l|l|}
\hline Yr & $\begin{array}{l}\text { TDS } \\
\mathbf{m g} / \mathbf{L}\end{array}$ & $\begin{array}{l}\text { EC } \\
\boldsymbol{\mu S} / \mathbf{c m}\end{array}$ & $\mathbf{p H}$ \\
\hline 84 & 542.7 & 548 & 7.85 \\
\hline 85 & 525.5 & 548 & 7.8 \\
\hline 86 & 565.4 & 579 & 7.75 \\
\hline 87 & 604.2 & 588 & 7.57 \\
\hline 88 & 541.8 & 601 & 7.43 \\
\hline 89 & 565.9 & 625 & 7.34 \\
\hline 90 & 558.6 & 638 & 7.32 \\
\hline 91 & 640.4 & 798 & 7.27 \\
\hline 92 & 754.5 & 739 & 7.24 \\
\hline 93 & 798.7 & 758 & 7.28 \\
\hline 94 & 746.4 & 799 & 7.29 \\
\hline 95 & 615.8 & 514 & 7.3 \\
\hline 96 & 737.5 & 619 & 7.28 \\
\hline 97 & 753.6 & 869 & 7.19 \\
\hline 98 & 935.6 & 558 & 7.15 \\
\hline 99 & 1174 & 855 & 7.15 \\
\hline 0 & 1021 & 796 & 7.06 \\
\hline 1 & 1067 & 855 & 6.98 \\
\hline 2 & 1223 & 844 & 6.94 \\
\hline 3 & 1055 & 881 & 6.9 \\
\hline & & & \\
\hline
\end{tabular}




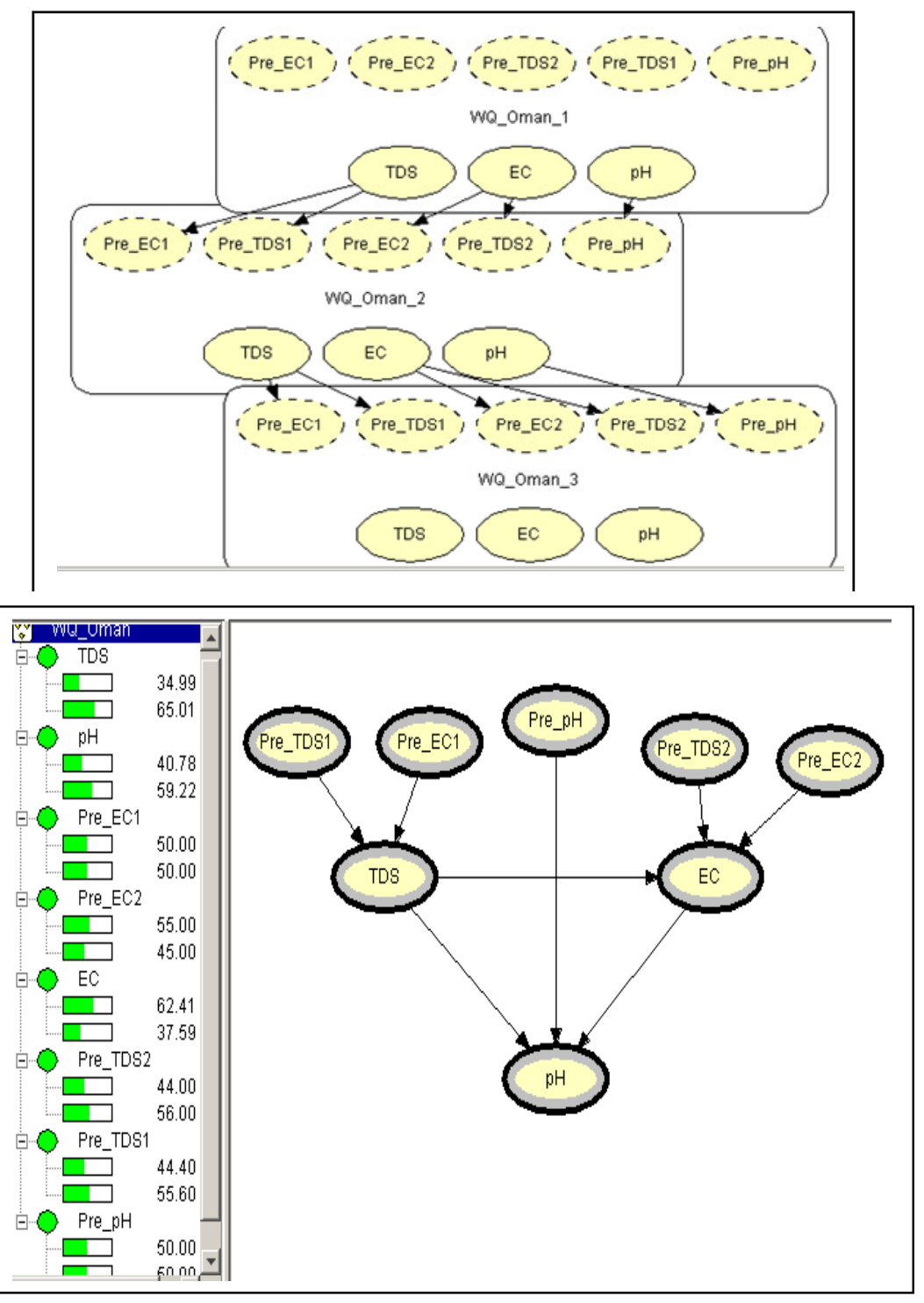

Figure 2. The initial building block representing one time-sliced network

\section{USING CLASSICAL TIME SERIES FOR THE ASSESSMENT OF GROUNDWATER QUALITY}

The purpose of this section is to apply the classical time series analysis to groundwater quality data and to compare the results with that obtained by the application of Dynamic Bayesian Networks (DBNs). The continuous and regular monitoring data of electrical conductivity (EC), total dissolved solid (TDS), $\mathrm{pH}$ measured by the Ministry of Water Resources (MWR) were also used here for the time series analysis.

Time series analyses of water supply wells with respect to the concentration of chemical constituents are presented in Figures 3-8. 
Total dissolved solids (TDS) are a measure of the dissolved minerals in water and also a measure of drinking water quality. There is a secondary drinking water standard of 500 milligrams per liter $(\mathrm{mg} / \mathrm{L})$ TDS; water exceeding this level tastes salty. Groundwater with TDS levels greater than $1500 \mathrm{mg} / \mathrm{L}$ is considered too saline to be a good source of drinking water. Figure 3 shows the concentration of TDS for the well Wel1001/577 for a period of twenty one years.

The fluctuation of the concentration of the chloride $(\mathrm{Cl})$, sodium $(\mathrm{Na})$, and calcium $(\mathrm{Ca})$ with respect to time is shown in Figure 5. The values were averaged during the initial analysis as there were no significant differences among the monthly data. Chloride values above $250 \mathrm{mg} / \mathrm{l}$ give a slight salty taste to water which is objectionable by many people.

Relationships between TDS, EC and $\mathrm{pH}$ are examined using multiple regression analysis, see Figure 5. Multiple regression analysis is used to explain as much variation observed in the response variable as possible, while minimizing unexplained variation from "noise". The results of this analysis are used to produce the moving average chart, Figure 7, and the linear regression chart, Figure 8. We used Excel Business Tools, Microsoft Excel, and Matlab for producing these and other charts.

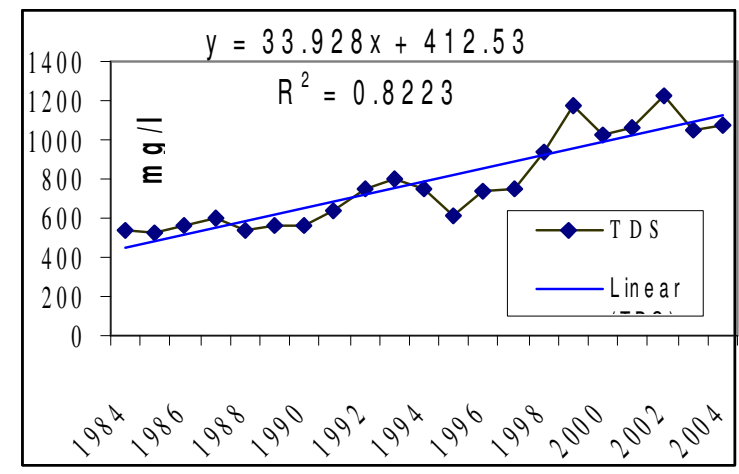

Figure 3. Fluctuation of TDS concentration for the well Well001/577

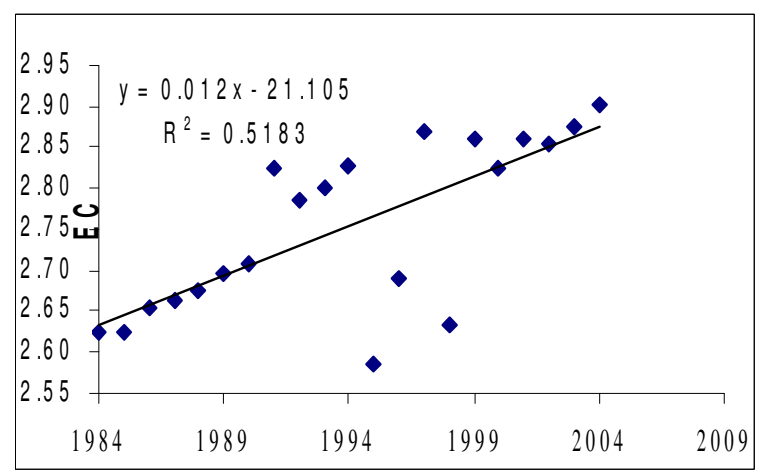

Figure 4. EC concentration is poorly represented for the well Well001/577 


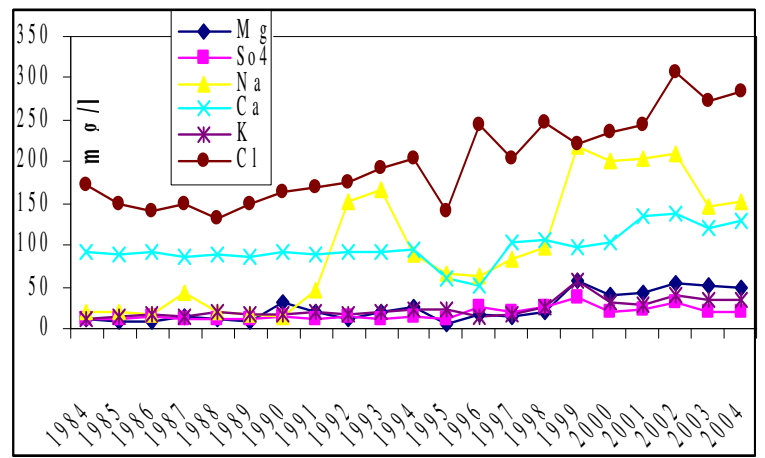

Figure 5. Fluctuation of the concentration of the major chemical constituents for Well001/577 for a period of 21 years

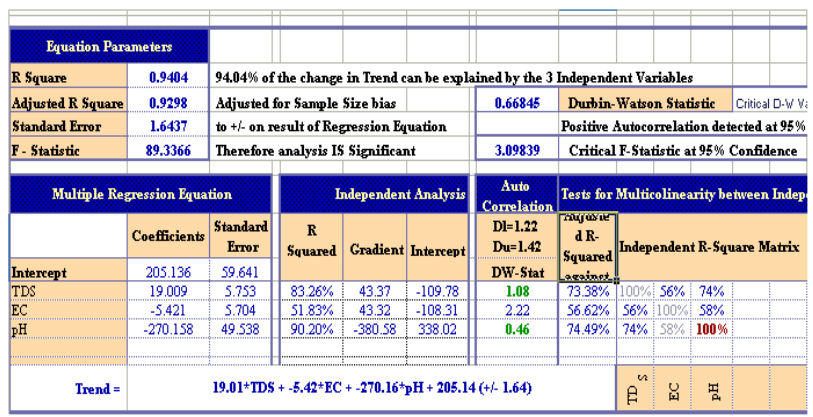

Figure 6. Excel templates for financial analysis and business productivity from Excel Business Tools

As is shown in Figure 5 that the trend is as follows:

TrendWQ=19.01*TDS $-5.42 * \mathrm{EC}-270.16 * \mathrm{pH}+205.14$

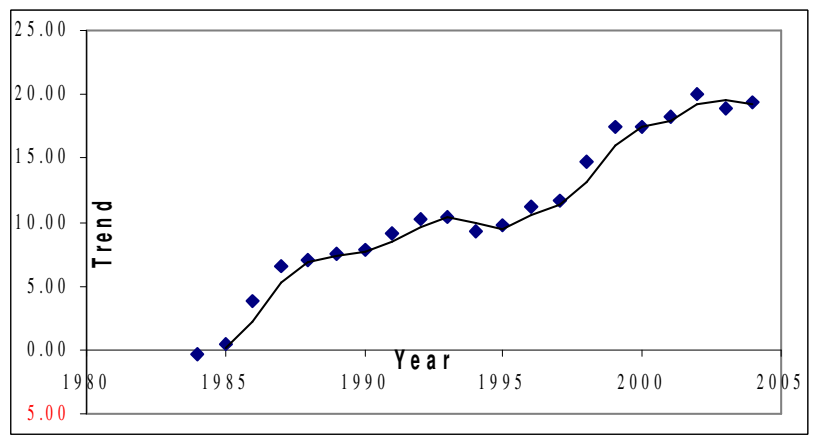

Figure 7. Moving average chart of 2-year period for groundwater quality trend 


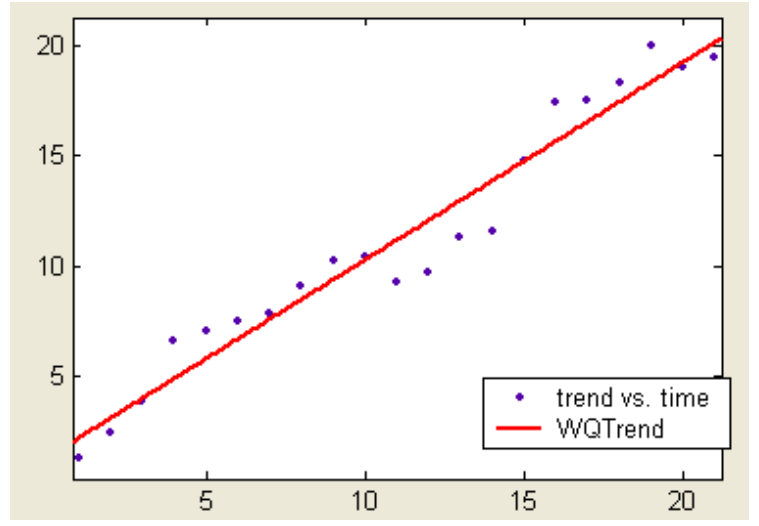

Figure 8. A curve fitting chart showing groundwater quality trend over time

Figure 7 shows the groundwater quality trend over time (linear regression). The trend has the following properties:

Linear model Poly1:

Coefficients (with $95 \%$ confidence bounds):

$$
f(x)=p 1 * x+p 2
$$

$$
\begin{aligned}
& \mathrm{p} 1=0.8954(0.7962,0.9947) \\
& \mathrm{p} 2=1.332(0.08589,2.579)
\end{aligned}
$$

Goodness of fit:

SSE: 32.91

R-square: 0.9494

Adjusted R-square: 0.9467

RMSE: 1.316

Although the classical time series models are used here to assess the presence and strength of temporal patterns of groundwater quality. These models are based on the assumption of stationary (i.e. time invariant). They have been widely used in many domains such as financial data and weather forecasting. Yet these models do not readily adapt to domains with dynamically changing model characteristics, as is the case with groundwater quality assessment. In addition to the above mentioned assumption, the classical models are restricted in their ability to represent the general probabilistic dependency among the domain variables and they fail to incorporate prior knowledge.

The observed groundwater quality data are irregularly spaced and not predetermined as in the case with ordinary time series. This may cause the traditional time series techniques to be ineffective (Prediction: what is the predicted value for one period a head). It is evident that the time series casts doubts on the positive or negative effects of any chemical constituent on the groundwater quality for the long run, and is thus not as clear and reliable as in the case of using Dynamic Bayesian Techniques. While some groundwater quality constituents, such as chloride and TDS, show an increasing trend, the other constituents, such as $\mathrm{pH}, \mathrm{Mg}$, and $\mathrm{SO} 4$ do not demonstrate obvious trends. Therefore, we can draw a reliable conclusion on the cause of the increasing trend of the groundwater quality and we cannot investigate the effect of the increasing or decreasing other constituents, such as $\mathrm{pH}$ and EC. In addition to this ignorance of the causeeffect relationships, classical time series models assume the linearity in the relationships among variables and normality of their probability distributions. 


\section{CONCLUSION AND FURTHER WORK}

This work presents the assessment of groundwater quality. Bayesian methods have been investigated and shown to offer considerable potential for use in groundwater quality prediction. These methods are based on reasoning under conditions of uncertainty. This work is the first step towards having a comprehensive network that contains the other variables that are considered by the researchers significant for the assessment of groundwater quality in the Salalah plain in particular.

Also we showed that the classical time series models do not readily adapt to domains with dynamically changing model characteristics, as is the case with groundwater quality assessment. This is mainly because these models are restricted in their ability to represent the general probabilistic dependency among the domain variables and they fail to incorporate prior knowledge.

\section{REFERENCES}

[1] Wu-Seng, L. 1993. Water Quality Modeling, CRC Press, Inc.

[2] Dames and Moore. 1992. Investigation of The Quality of Groundwater Abstracted from the Salalah Plain: Dhofar Municipality, Final Report.

[3] Ministry of Water Resources (MWR), Sultanate of Oman. 2004. Law on the Protection of Water Resources, promulgated by Decree of the Sultan No. 29 of 2004, and its implementing regulations (Regulations for the organization of wells and aflaj, and Regulations for the use of water desalination units on wells), (in Arabic).

[4] Shihab, K. and Al-Chalabi, N. 2004. Treatments of Water Quality Using Bayesian Reasoning, Lecture Notes in Computer Science, 3029, 728-738.

[5] Shihab, K and Nida Al-Chalabi, 2007. Dynamic Modeling of Groundwater Quality Using Bayesian Techniques, Journal of the American Water Resources Association (JAWRA), Blackwell Publishing (Online Blackwell Synergy), Vol. 43, No. 3, pp. 664-674.

[6] Banerjee A. K. et al. 1985. TR no. 773, Monitoring groundwater quality, Department of Statistics, University of Wisconsin.

[7] HUGIN Expert Brochure. 2005. HUGIN Expert A/S, P. O.Box 8201 DK-9220, Aalborg, Denmark, (http://www.hugin.com).

[8] Kjaerulff, U. 1995. dHugin: A computational system for dynamic time-sliced Bayesian Networks, International Journal of Forecasting, 11, 89-111.

[9] Shihab, K. 2008. Analysis of Water Chemical Contaminants: A Comparative Study, Applied Artificial Intelligence (AAI), Vol 22, No. 4, pp. 352-376. 


\title{
A RECOMMENDER SYSTEM SENSITIVE TO INTRANSITIVE CHOICE AND PREFERENCE REVERSALS
}

\author{
Amir Konigsberg and Ron Asherov \\ General Motors R\&D \\ amir.konigsberg@gm.com \\ rasherov@gmail.com
}

\begin{abstract}
One of the basic foundations for many recommender systems is the assumption of preference consistency and transitivity of choice. In this paper we challenge this assumption and argue that it should be revised. We also provide a method by which recommender systems can estimate preference reversals and choice intransitivity. Our general approach is to incorporate variants of choice-behavior such that recommender systems incorporate decision-sensitive factors within choice-sets that tend to influence decision making. After establishing why recommender systems ought to consider preference reversal phenomena, we provide a high-level outline of a mechanism for automatically discovering and predicting preference reversals. Thereafter we show how the discovered or predicted preference reversals can help in generating choice sets that 1) are better for the users all things considered (by being in line with their general preferences as exhibited over time) and 2) help users make better decisions.
\end{abstract}

\section{KEYWORDS}

Intransitive choice; preference reversals; recommender systems; rational choice

\section{INTRODUCTION}

The importance of understanding user preferences has been recognized by researchers and practitioners in many disciplines. The wide interest in this area in academia and in the industry over the past decade reflects a recognition of the abundance of practical applications that are possible with an increased understanding of user preferences (G. Adomavicius and Tuzhilin 2005). Moreover, from the practical standpoint understanding, what users prefer becomes ever more important as the amount of available information, services, and products continues to increase and personalizing services and filtering information becomes crucial for user experience and information management. Companies in a variety of fields have begun to recognize that they stand to benefit by matching relevant and timely information, products, or services to users based on an understanding of their preferences.

One of the disciplines in which an understanding of user preferences is most valuable is recommender systems. This paper deals with a specific yet important issue that we believe recommender systems ought to deal with - preference reversals. Our goal is to discuss the preference reversal problem in the context of intransitive choice behavior, and argue why it is important for recommender systems to consider. Our primary goal is to outline the phenomena, 
and to suggest a system by which preference reversals can be detected so as to generate more useful recommendations.

We also propose a way in which intransitive choice behavior can be detected and also a way of estimating the conditions under which user preferences will be reversed. We then make salient the central features of such a system. It is our belief that in dealing with this issue and making the necessary amendments, recommender systems can become more accurate in a wider range of cases. Perhaps more importantly, in incorporating the issues we draw attention to here, recommender systems can become sensitive to the context of choice.

While a substantial amount of research has already been done in the area of recommender systems (e.g., Celma and Herrera 2008; Herlocker et al. 2004; Shani and Gunawardana 2011; Cramer et al. 2008; Victor CODIN and Luigi CECCARON 2011; Ricci 2011; Gediminas Adomavicius and Tuzhilin 2011), underlying most of the existing approaches is the assumption that users are consistent in their choices in varying choice-environments (Brun et al. 2010, sec. 3.3; Brafman and Domshlak 2009, 2). It is assumed that if a recommender system, using content filtering strategies or collaborative filtering methods, or both ${ }^{1}$, were to recognize that in the past a particular user has always selected a hotel when offered a hotel and a B\&B in an accommodation selection website, then the assumption is that this user will also prefer a hotel to a $B \& B$ when short-term apartments are also on offer. That is, if $\mathrm{H}$ has always been the preferred choice from $\{\mathrm{H}, \mathrm{B}\}$, then $\mathrm{H}$ will be preferred to $\mathrm{B}$ in $\{\mathrm{H}, \mathrm{B}, A\},\{\mathrm{H}, \mathrm{B}, A, I\}$ and in any other choice set that includes $\mathrm{H}$ and $\mathrm{B}$, however many other items are in that set. And this assumption is in fact quite reasonable, since most of the time users behave consistently by upholding transitivity in the way described above. Hence assuming that users are consistent in their preferences is a useful methodology, most of the time. Nonetheless transitivity of choice can be influenced by affect dependence between items. These are cases when the presence of an item in a choice set will influence the utility of other items in that set whose utility would be different in the absence or substitution of that item.

We believe that attending to the influence that in a choice set can have on preference consistency is an important factor to consider in recommender systems, especially because in reality some user preferences are transient and vary with change of context and item availability (Gediminas Adomavicius and Tuzhilin 2011). It is our contention that recommender systems can be improved if they can predict under what conditions user preferences are likely to occur and incorporate these predictions in such a way that more useful choice sets can automatically be generated.

In what follows we demonstrate how dependency can arise between choice-options and how this dependency can induce changes in user preferences. We also suggest how the kind of choicedependency that we focus on can be handled by eliciting user preferences for items in all possible option-spaces. We believe that if it is reasonable to assume that the level of accuracy by which a recommender system can estimate user preferences depends on the degree to which it has incorporated variants of choice-behavior into its method, then the approach to handling the behavioral phenomena we focus on here will be a positive addition to current attempts to provide accurate recommendations to users based on their preferences.

\footnotetext{
${ }^{1}$ Existing approaches to recommender systems will typically rank items recommended to users based on one of two strategies (Koren, Bell, and Volinsky 2009); either based on the attributes of the available items (content filtering) or based on user-item/option associations inferred from similarities between different items and different users (collaborative filtering). In both cases attributing preferences to users is the means by which recommendations are made. Depending on the strategy, preferences can either be learned from past choice-behavior or explicitly stated, for instance by elicitation methodologies or questions (Brafman and Domshlak 2009).

* This research received no specific grant from any funding agency in the public, commercial, or not-forprofit sectors.
} 


\section{EXPECTING THE UNEXPECTED: THE DEPENDENCY OF INDEPENDENT ITEMS IN CHOICE SETS}

Recommender systems typically assume that a user's preferences are consistent. They assume that if so-and-so has repeatedly evaluated the set of options $\{x, y\}$ and has then repeatedly chosen $\mathrm{x}$, that he can then be registered as preferring $\mathrm{x}$ to $\mathrm{y}$. They also assume that he will continue to do so in all other instances where $\mathrm{x}$ and $\mathrm{y}$ are offered together. Additionally, most utility based systems assume that $\mathrm{x}$ has a greater utility than $\mathrm{y}$ and that this is why $\mathrm{x}$ is preferred to $\mathrm{y}$ whenever the two are offered together. But this is not to say that existing systems cannot also detect preference changes. They can. But this is only after they detect that the user has modified his choice a sufficient number of times to count as adequate for judging that his preference has changed. Yet existing systems don't identify the conditions under which an existing preference will change due to choice-context - i.e., due to the presence of a particular item (or items) with which they are offered - and remain the same in all other cases. Instead, the consistency assumption of current recommendation systems implies that if other items are added to a choice set - for instance in other contexts - then either 1) those other items will be chosen, or 2) the previous choice - i.e., $\mathrm{x}$ from $\{\mathrm{x}, \mathrm{y}\}-$ will remain as before.

Figure 1 (below) illustrates the consistency of preference assumption that we are referring to:

Figure 1

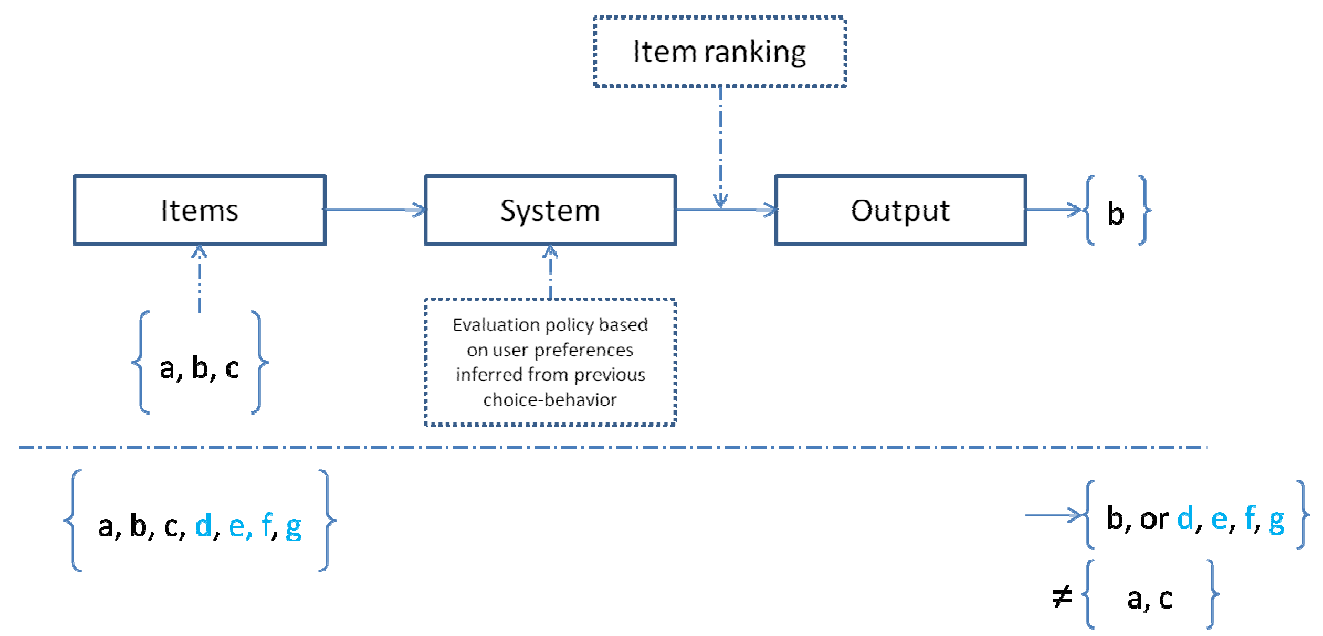

Below the dotted line in figure 1 is a set of items and the output that a recommender systems will typically give; either one of the new items that have been added to the choice set will be chosen, or the choice will remain as before (i.e., b). As can be seen, once choice $b$ has been made between $\{a, b, c\}$ the user will then be ascribed preferences that correspond to this choice and these preferences will be remembered by the system. Moreover, on a later occasion when a, b, c are given in conjunction with other items - d, e, f, g, in the same choice set, the system's output will either be b, consistent with the previous choice, or d, e, f, or g, if one of these happens to have a greater utility than $\mathrm{b}$. Most existing systems will not give either a or $\mathrm{c}$ as an output.

Yet users do sometimes reverse their preferences because additional items in the choice set, even if those additional items aren't themselves chosen.Because of such sensitivity to the context of choice, an item in a choice set can have a relative as well as an absolute value, and its relative 
value is often a function of how it relates to other items ${ }^{2}$. And it is natural to suppose that choices made between items under particular choice-conditions may change under other choiceconditions, even if those choice-conditions don't offer any items that offer tangible gain on a previously considered utility function or decision rule.

Because recommender systems that aim to anticipate user choice are modelled on the anticipated satisfaction of a user, it is natural to expect that the accuracy of a system's prediction will be reflective of the degree to which it has incorporated contextual variants such as the impact of items on other items in relation to choice behaviour and recommendation acceptance.

In what follows we propose a method for estimating preference reversals that result from inner set dependencies in between items. As noted above, we believe that a recommender system that uses this approach can deliver more accurate recommendations than existing systems.

\section{A MODEL FOR PREFERENCE REVERSAL BEHAVIOR AND ITEM DEPENDENCY}

In order to deal with the interacting options in an option space we define a matrix A, whose $i, j$ entry represents the additional utility, or gain, of the $I^{\text {th }}$ item, given the $j^{\text {th }}$ item. The entries on the main diagonal of the matrix represent the initial utility of the item, disregarding any other items with which it is presented. Otherwise put: the values in the diagonal represent the utility that item $\mathrm{x}$ has in and of itself, independent of the presence of any other item. Utility shall be measured in pure unit-free numbers. The scale is chosen such that the biggest number on the main diagonal is 10 , meaning that the largest value that can be attributed to an item is 10 . But this is only for demonstrative purposes. In essence, larger or smaller scales can be used.

Additionally, we assume that utilities are additive in this matrix, meaning that as a means of evaluating an item we add together the utilities it gets from every other available item. Hence, in order to find the final utility of an item (in some context or option space), we add up the entries of the item's row of values and the columns of the other available options. Then we compare these sums and determine which item has the highest utility, given the context of the specific option space (note: we are aware that assuming additivity is not trivial. We have chosen to presuppose it since we believe it serves our system best. Nonetheless, we discuss this assumption further below). We also assume that the user whose choices this matrix represents is rational, meaning that he maximizes a utility function by choosing the item, or row, with the highest measure of utility. Let us flesh out this model by way of an illustrative example.

You are on a business trip in an area that you are visiting for the first time, driving a car equipped with a point-of-interest (POI) recommendation system that offers you restaurants, museums, shops, and other services and points of interest upon request or proactively, based on your location and preferences. At some point on your journey the system offers you two options:

1) "Hank's" - a club with live music $(\mathrm{H})$

2) A local restaurant $(\mathrm{R})$.

You don't have too much information about the local music scene - e.g., what kind of music the local bars play, and you also feel quiet hungry, so you rate the options accordingly - you give Ra 10 , and $\mathrm{H}$ a 5, reflecting your order of preference, in favor of the restaurant. You now have the incomplete matrix:

\footnotetext{
${ }^{2}$ See Dan Ariely's now famous Economist subscription example in: (Ariely 2009, chap. 1)
} 


\begin{tabular}{|c|c|c|}
\hline & $\mathbf{H}$ & $\mathbf{R}$ \\
\hline $\mathbf{H}$ & 5 & \\
\hline $\mathbf{R}$ & & 10 \\
\hline
\end{tabular}

As can be seen, the utility scores in the matrix are distributed for each item $-\mathrm{H}$ and $\mathrm{R}$. Because the items that the system has offered are independent of one another and no information derived from one item can affect the other item, the remaining entries in the matrix are zero:

\begin{tabular}{|c|c|c|c|}
\hline & $\mathbf{H}$ & $\mathbf{R}$ & $\mathbf{\Sigma}$ \\
\hline $\mathbf{H}$ & 5 & 0 & 5 \\
\hline $\mathbf{R}$ & 0 & 10 & 10 \\
\hline
\end{tabular}

You calculate the sum of all entries in the same row, and, being rational (that is, a maximizer), you decide to go to the restaurant $(\mathrm{R})$, because this item has the greatest overall utility.

Now, before you manage to reveal your choice to the system, the system offers you a third item: a music festival (F). You evaluate this item at 7 , and then you also realize that now the item relating to the possibility of going to "Hank's" $(\mathrm{H})$ sounds much better, giving it an additional value of 15 , perhaps because the idea of going to a festival $(\mathrm{F})$ made listening to music in a bar $(\mathrm{H})$ more attractive. The important point here is that knowing about item $\mathrm{F}$ adds some utility to item $\mathrm{H}$.

You now have the following matrix:

\begin{tabular}{|c|c|c|c|c|}
\hline & $\mathbf{H}$ & $\mathbf{R}$ & $\mathbf{F}$ & $\boldsymbol{\Sigma}$ \\
\hline $\mathbf{H}$ & 5 & 0 & 15 & 20 \\
\hline $\mathbf{R}$ & 0 & 10 & 0 & 10 \\
\hline $\mathbf{F}$ & 0 & 0 & 7 & 7 \\
\hline
\end{tabular}

As can be seen, the offer of a third item F made you assign a higher utility to the first item $(\mathrm{H})$, and in practice it also changed the result of the inner comparison between the initial items $\mathrm{H}$ and $\mathrm{R}$ (we can also describe this change on a temporal dimension: whereas at time $t l$ you preferred item $\mathrm{R}$ to item $\mathrm{H}$, at time $t 2$, when $\mathrm{F}$ also became a possibility, you preferred $\mathrm{H}$. Your preference thus reversed between $t_{1}$ and $t_{2}$, as a result of the additional availability of $\mathrm{F}$ at $t 2$ ).

\section{GENERALIZING THE MODEL}

Let us generalize this phenomenon to a case in which one faces $n$ choices, denoted as $c_{1}, \ldots, c_{n}$. In such a case $A$ is an $n \times n$ matrix, whose elements are $a_{i, j}$. As explained above, the main diagonal of the matrix houses the basic independent utilities of the items:

\begin{tabular}{|c|c|c|c|c|}
\hline & $c_{1}$ & $c_{2}$ & $\cdots$ & $c_{n}$ \\
\hline$c_{1}$ & $U_{1}$ & & & \\
\hline$c_{2}$ & & $U_{2}$ & & \\
\hline$\vdots$ & & & $\ddots$ & \\
\hline$c_{n}$ & & & & $U_{n}$ \\
\hline
\end{tabular}


We proceed to fill in the rest of the matrix by examining the relations between the different items: $\mid a_{i, j}$ represents the additional utility of item $c_{i}$ when we know $c_{j}$ is present (i.e., when $c_{j}$ is also a viable possibility). Note that in most cases, the effect isn't symmetrical, so generally speaking $a_{i, j} \neq a_{j, i}$. We now get this full matrix:

1

$\mid$

\begin{tabular}{|c|c|c|c|c|}
\hline & $c_{1}$ & $c_{2}$ & $\cdots$ & $c_{n}$ \\
\hline$c_{1}$ & $U_{1}$ & $a_{1,2}$ & & $a_{1, n}$ \\
\hline$c_{2}$ & $a_{2,1}$ & $U_{2}$ & & \\
\hline$\vdots$ & & & $\ddots$ & $\vdots$ \\
\hline$c_{n}$ & $a_{n, 1}$ & & $\cdots$ & $U_{n}$ \\
\hline
\end{tabular}

What we want to suggest is that the total utility of an item in some option-space $\mathrm{C}$ is the sum of the relevant row and columns. And we believe that recommender systems can benefit by so calculating an item's utility:

$$
U\left(c_{k}\right)=\sum_{i \in C} a_{k, i}
$$

Where $\mathrm{C}$ is the relevant item space; $\mathrm{C}$ is a nonempty subset of the set of all possible items. As can be seen in the formula above, the utility of an item depends on its context, or on the options available besides that item. Understanding the effect of the context over the item's utility allows us to manipulate or predict user preference as a function of the item space C. In the last example, we saw that when $C=\{H, R\}$, item $R$ was preferred (i.e. $U(R)>U(H)$ ), but when we extended $C$ and made it $\{\mathrm{H}, \mathrm{R}, \mathrm{F}\}$, we got $\mathrm{U}(\mathrm{R})<\mathrm{U}(\mathrm{H})$. By adding more items $\mathrm{X}, \mathrm{Y}, \mathrm{Z}$, we can (perhaps) change the preference again.

The above equation can be rearranged to get the following result: the utility of an item in context is the sum of the basic independent utility of that item and the additional utility caused by the presence of all other items in the item space.

$$
U\left(c_{k}\right)=U_{k}+\sum_{\substack{i \in C \\ i \neq k}} a_{k, i}
$$

Henceforth our goal is to suggest a computational method that for every set of items $\left\{x_{1}, \ldots, x_{n}\right\}$, from which a particular choice $x_{1}$ is made, is able to determine the conditions under which that choice is reversed (to some different $x_{k}$ ) due to an expected reversal in the user's preferences.

To determine in what context (in what item space) the choice is reversed to $x_{k}$ - i.e. the conditions under which the total utility of $x_{k}$ is greater than the total utility of $x_{1}$ - we consider the difference $d=x_{k}-x_{1}$.

Because choice $x_{1}$ is the choice made at the outset, we assume that its utility is greater than $x_{k}$ 's and so $d<0$. The method we propose tries to reverse the choice by creating conditions within which $x_{k}$ is of greater value. Hence the method tries to maximize $d$, or at least make it positive.

The method enables establishing how any other $x_{m}$ affects both $x_{1}$ and $x_{k}$. As a rule, if $x_{m}$ affects $x_{k}$ more positively than it affects $x_{1}$, i.e. if $a_{k m}>a_{1 m}$ or if it has a positive d: $d_{m}=$ $a_{k m}-a_{1 m}>0$, then adding $x_{m}$ to our choice space will increase the total utility of $x_{k}$ relative to $x_{1}$. By gathering all such $x_{m}$ 's we reach the maximal difference between $x_{k}$ and $x_{1}$. 
However, this does not yet assure us that $x_{k}$ will be the selected item. Two additional cases need to be considered for the system to be able to predict the user's preference when other items are at hand:

1. Even with all such $x_{m}$ 's, the total utility of $x_{k}$ might still be lower than $x_{1}$ 's. Relating to the last example, if the music festival (F) is not my taste, it may add only 3 to C's utility, and then item $\mathrm{R}$ (at 10) is still preferred over item $\mathrm{H}$ (at 8).

\begin{tabular}{|c|c|c|c|c|}
\hline & $\mathbf{H}$ & $\mathbf{R}$ & $\mathbf{F}$ & $\boldsymbol{\Sigma}$ \\
\cline { 1 - 4 } $\mathbf{H}$ & 5 & 0 & 3 & 8 \\
\cline { 1 - 4 } $\mathbf{R}$ & 0 & 10 & 0 & 10 \\
\hline $\mathbf{F}$ & 0 & 0 & 7 & 7 \\
\hline
\end{tabular}

2. Every such $x_{m}$ affects not only $x_{k}$ and $x_{1}$, but other $x_{l}$ 's too. So it is possible that after taking all such $x_{m}$ 's, the total utility of $x_{k}$ will indeed surpass $x_{1}$ 's, but the total utility of some other item $x_{l}$ will be even higher. Following the last example, the idea of going to a music festival in a foreign land may sound so attractive to you, that you may prefer it over anything else.

\begin{tabular}{|c|c|c|c|c|}
\hline & $\mathbf{H}$ & $\mathbf{R}$ & $\mathbf{F}$ & $\boldsymbol{\Sigma}$ \\
\hline $\mathbf{H}$ & 5 & 0 & 15 & 20 \\
\hline $\mathbf{R}$ & 0 & 10 & 0 & 10 \\
\hline $\mathbf{F}$ & 0 & 0 & 30 & 30 \\
\hline
\end{tabular}

3. If we are looking at larger initial sets and we aim to reverse the choice to specific items in those sets then we may end up with a third item being chosen: for example, if the initial item space was $\{X, Y, Z\}$, out of which $X$ was chosen, and in order to change the preference to $\mathrm{Y}$ in that set we added $\mathrm{T}, \mathrm{U}, \mathrm{V}$, we might reach a situation in which the choice in $\{\mathrm{X}, \mathrm{Y}, \mathrm{Z}, \mathrm{T}, \mathrm{U}, \mathrm{V}\}$ is $\mathrm{Z}$.

Hence by adding or subtracting items in the item space a user may proceed to take one of a number of consequent actions:

a) Sticking with the previous choice;

b) Reversing to a previously available (but not chosen) option (the one we aimed to reverse the choice to);

c) Choosing one of the new options;

d) Reversing the preference such that the option that is chosen is another, different option.

We can now ask several questions about the collection of external items that satisfy the "positive $d$ ' condition (by "external items" we mean items that are part of the set of all possible items, but currently not in the presented item space). As a reminder, element $m$ is said to meet the "positive d' condition if $d_{m}=a_{k m}-a_{1 m}>0$, i.e. if it adds more value to the kth $x_{k}$ item than it adds to the first item $x_{1}$.

a. Which external items will give the greatest difference between the two items (the currently chosen option and the one we wish to reverse the option to)? i.e. what options will make me prefer the second item by the largest "gap"? The answer appears to be that all of the items 
that have a positive $d$-every one of them strengthens the utility of the kth option with respect to the first item, so adding them all up will give the greatest difference.

b. What is the set of basic sufficient combinations of choices (choice spaces) that span the remaining item spaces? Evidently if a certain set of items is sufficient to tip the scales, then any additional item with a positive $d$ will result in reversing the choice (making the difference even greater). Hence, for the collection of all item spaces that give us a total positive $d$, we may be interested in finding the minimal set of item spaces that spans all other combinations. This set shall be referred to as a "base" to the collection of all combinations that tip the scales. In other words, a set B will be a base if every item space in which the choice is reversed contains an element (i.e. a choice set) of B.

c. What external items will give the minimal yet positive difference? i.e. what items will be sufficient to change one's chosen item (to tip the scales). It should be made clear that the answer to this question is a member of a "base" of the collection of all preference-reversing combinations of items, as it is defined in answer to the previous question.

\section{A Note AbOUT AdDitivity}

In the system we proposed we assumed that utilities are additive. As a reminder, in order to get the total utility of an item in a choice space, our model sums the initial utility of the item and the additional utilities that it receives from the other items in the space. This method is distinct from other methods such as for instance combining utilities in non-additive ways.

As far as computation time and complexity are concerned, assuming that utility is additive has significant implications. Without this assumption, each and every set of items must be checked independently, since we cannot assume any connection between different item spaces; without assuming additivity, knowing the user chose $\mathrm{X}$ out of $\{\mathrm{X}, \mathrm{Y}, \mathrm{Z}\}$ tells us nothing about the choice set $\{\mathrm{X}, \mathrm{Y}, \mathrm{Z}, \mathrm{T}\}$. Under the linear assumption, it is sufficient to know the independent effect every item has on any other item, regardless of the current space; in this case it is sufficient to know the entries of the matrix. Hence in a given space, we sum these pairwise effects to get the total effect. Because we assume linearity, we can add up independent partial values to get the value of the whole. If we do not assume additivity, we cannot infer any information about the whole space, even when we have full information about its subsets.

What this means for the model is that with the additivity assumption, we can reduce the amount of item spaces (i.e. subsets of the collection of all items) to be checked from all possible subsets (the number of subsets of a set with n elements is $\left.2^{n}\right)^{3}$, to the number of elements in the matrix $\left(n^{2}\right)$. This reduction, from exponential runtime to polynomial, is extremely significant, since exponent grows much faster than any polynomial, and so the computation time of the nonadditive method will grow rapidly, and will be impractical for even relatively small values of $n$. Hence because we assume additivity we assume that the additional gain of a set equals the sum of the gain of the individual elements. We therefore ignore any internal relations within the external

group that may affect the total gain on a certain item. By considering every subset $\left(2^{n}\right)$ we take these internal relations within the external group into consideration. The effect could indeed be zero; in such a case the two methods will give similar results. But if the effect is nonzero, the "full method" (exponential runtime) is more accurate. For example, the utility of a hamburger with a good bun and quality beef exceeds the arithmetic sum of the utilities of a hamburger with a good bun and a hamburger with quality beef. That is, it could be that one quality $q_{1}$ adds some utility

\footnotetext{
${ }^{3}$ For example, if $n=3$ and the total option space is $\{X, Y, Z\}$, we have 8 subsets, or partial option spaces: \{\} , $\{\mathrm{X}\},\{\mathrm{Y}\},\{\mathrm{Z}\},\{\mathrm{X}, \mathrm{Y}\},\{\mathrm{Y}, \mathrm{Z}\},\{\mathrm{X}, \mathrm{Z}\},\{\mathrm{X}, \mathrm{Y}, \mathrm{Z}\}$.
} 
$u_{1}$ to an item $o$, and a different quality $q_{2}$ adds $u_{2}$ to $o$, yet there is something (an internal relation) in the combination of the two qualities $\left\{q_{1}, q_{2}\right\}$ that gives $o$ a utility greater than $q_{1}+q_{2}$. The "full method" will capture this; the method proposed here will not. Nonetheless, our system assumes additivity because of its relative simplicity and because the amount of data to be considered under the additivity assumption is significantly smaller, and hence the amount of data to be collected is also much smaller, which means much less for the system to learn.

\section{LEARNING CONDITIONAL UTILITIES}

The system learns the values of the conditional utilities of items (i.e. the entries of the matrix A) by monitoring users' selections in various item spaces. Every selection that a user makes of an item from an item space is translated to an inequality with A's entries, and using many such inequalities, a prediction for the exact values of the matrix may be made. Every selection made in an option space gives $\mathrm{N}$ inequalities ( $\mathrm{N}$ being the number of options in the option space). Large amounts of data give us large amounts of inequalities. Every such inequality poses a constraint; by considering all such constraints, the region (in the $\mathrm{n}^{\wedge} 2$ space) in which the actual Matrix's entries are to be found is narrowed down. With enough constraints it is therefore possible to give good estimates for the matrix entries.

For instance, if when being presented options 1 and 2 the user chooses 1 , then we may assume that $U\left(c_{1}\right)>U\left(c_{2}\right)$, i.e. $U_{1}+a_{1,2}>U_{2}+a_{2,1}$. However, if when option 3 is also present the user chooses 2 , that means that $U_{1}+a_{1,2}+a_{1,3}<U_{2}+a_{2,1}+a_{3,1}$. Every such inequality defines a region in an $n^{2}$-dimensional world (a dimension for every entry in the matrix). By considering many such regions, we can narrow down the options for every entry, and by that get a good estimation for their exact numerical values.

Several methods may be employed to improve this learning process:

- Since we do not consider the exact numerical utility, but just compare different utilities, we may normalize all values, such that, for example, $U_{1}=1$ (otherwise, we can multiply the whole matrix by a constant to get that, and the recommendation results will be identical. What we mean here is that it doesn't matter if $A=[12 ; 34]$ or $A=[24 ; 68]$; all that matters are the proportions. If with the first matrix we arrived at utility 8 , the second matrix will give utility 16 , and so on, i.e. it's all monotonous (if $\mathrm{X}>\mathrm{Y}$ with matrix $\mathrm{A}$, this inequality will remain true if we use $2 \mathrm{~A}$ or $6 \mathrm{~A}$ ). So we can divide all elements of the matrix by a constant and remain with the same properties, so we can assume $\mathrm{U} 1=\mathrm{a} 11=1$ (otherwise, if a1 $1=8$, we'll just divide it all by a factor of 8.)).

- If the system records many selections made in the same option space, the system may use that information in order to estimate the "gap" between options. For instance, if when given A and B the user chose A $97 \%$ of the time, then the gap may be big, but if the user chose A only $51 \%$ of the time, then the total utilities of A and B (in that context) are identical, or close to identical. This replaces the inequality with an equality, which is far better computationally, as it reduces the dimension of the problem by one.

- As explained before, the values on A's main diagonal $U_{k}=a_{k, k}$ represent the utility the option has in and of itself. Therefore, after a selection has been made, the system may ask to rate the selection in a context free environment, in order to get an estimated value of $U_{k}$, or at least an estimation for the effect cause by the context. For example, in book recommendations, out of three books $\mathrm{A}, \mathrm{B}$, and $\mathrm{C}$, book B seems very appealing, so you choose to read it and discover that it is in fact boring. You may feel cheated; in its 
context, the book seemed interesting, however when it was context-free, it was boring. That means that the presence of books $\mathrm{A}$ and $\mathrm{C}$ had a positive effect on the contextual (total) utility of book B.

\section{CONCLUSION}

We have proposed a recommender system that is sensitive to preference changes that result from dependencies between items or options in choice sets. The type of cases we have attended to are those in which choice transitivity is violated due to preference-sensitive information conferred by a new option (or options) in the option space. We have proposed a system that provides a framework that better reflects the way people do in fact behave - because seemingly independent items can impose meaning and can provide information that bears on other, seemingly independent and unrelated items. Consequently we believe that any system that possesses information relating to when and under what conditions preferences and choices will be reversed will be more intelligent and useful than a system that only operates according to consistency considerations by assuming transitivity of choice.

\section{REFERENCES}

[1] Adomavicius, G., and A. Tuzhilin. 2005. "Toward the Next Generation of Recommender Systems: a Survey of the State-of-the-art and Possible Extensions." IEEE Transactions on Knowledge and Data Engineering 17 (6): 734-749.

[2] Adomavicius, Gediminas, and Alexander Tuzhilin. 2011. "Context-Aware Recommender Systems." In Recommender Systems Handbook, edited by Francesco Ricci, Lior Rokach, Bracha Shapira, and Paul B. Kantor, 217-253. Boston, MA: Springer US.

[3] Ariely, Dan. 2009. Predictably Irrational, Revised and Expanded Edition: The Hidden Forces That Shape Our Decisions. Rev Exp. Harper.

[4] Brafman, Ronen, and Carmel Domshlak. 2009. "Preference Handling - An Introductory Tutorial." AI Magazine 30 (1) (January 18): 58.

[5] Brun, Armelle, Ahmad Hamad, Olivier Buffet, and Anne Boyer. 2010. "Towards Preference Relations in Recommender Systems." In In Proc. of the ECML/PKDD Workshop on Preference Learning.

[6] Celma, Òscar, and Perfecto Herrera. 2008. "A New Approach to Evaluating Novel Recommendations.” In , 179. ACM Press.

[7] Cramer, Henriette, Vanessa Evers, Satyan Ramlal, Maarten Someren, Lloyd Rutledge, Natalia Stash, Lora Aroyo, and Bob Wielinga. 2008. "The Effects of Transparency on Trust in and Acceptance of a Content-based Art Recommender.” User Modeling and User-Adapted Interaction 18 (5) (August 20): 455-496.

[8] Herlocker, Jonathan L., Joseph A. Konstan, Loren G. Terveen, and John T. Riedl. 2004. "Evaluating Collaborative Filtering Recommender Systems." ACM Transactions on Information Systems 22 (1) (January 1): 5-53.

[9] Hsee, Christopher K. 2012. "The Evaluability Hypothesis: An Explanation for Preference Reversals Between Joint and Separate Evaluations of Alternatives." SSRN eLibrary. Accessed April 3.

[10] Kahneman, Daniel, and Amos Tversky. 1979. "Prospect Theory: An Analysis of Decision Under Risk." Econometrica 47 (2) (March): 263.

[11] Kokinov, Boicho Nikolov. 1994. "The Context-Sensitive Cognitive Architecture DUAL." In Proceedings of the Sixteenth Annual Conference of the Cognitive Science Society.

[12] Kokinov, Boicho, Penka Petkova, and Georgi Petkov. 2004. "Does Irrelevant Information Play a Role in Judgment." In In: Proceedings of the 26th Annual Conference of the Cognitive Science Society, $720-725$.

[13] Koren, Y., R. Bell, and C. Volinsky. 2009. "Matrix Factorization Techniques for Recommender Systems." Computer 42 (8): 30-37.

[14] Kriesler, Kobi, and Shmuel Nitzan. 2007. "Is Context-Based Choice Due to Context-Dependent Preferences?" Theory and Decision 64 (1) (June 20): 65-80. 
[15] Payne, John W., James R. Bettman, and Eric J. Johnson. 1993. The Adaptive Decision Maker. Cambridge University Press.

[16] Ricci, Francesco. 2011. Recommender systems handbook. New York: Springer.

[17] Samuelson, P. A. 1938. "A Note on the Pure Theory of Consumer's Behaviour.” Economica 5 (17). New Series (February 1): 61-71.

[18] Sen, Amartya. 1993. "Internal Consistency of Choice." Econometrica 61 (3) (May 1): 495-521.

[19] Shani, Guy, and Asela Gunawardana. 2011. "Evaluating Recommendation Systems." In Recommender Systems Handbook, edited by Francesco Ricci, Lior Rokach, Bracha

[20] Shapira, and Paul B. Kantor, 257-297. Boston, MA: Springer US.

[21] Tversky, Amos, and Itamar Simonson. 1993. "Context-Dependent Preferences.” Management Science 39 (10) (October 1): 1179-1189.

[22] Victor Codin, A, and Luigi Ceccaron. 2011. "Extending Recommendation Systems with Semantics and Context-Awareness: Pre-Filtering Algorithms.” In. http://www.lsi.upc.edu/ vcodina/papers/ccia11.pdf.

\section{AUTHORS}

\section{Amir Konigsberg}

Amir a senior scientist at General Motors Research and Development Labs, where he works on advanced technologies in the field of artificial intelligence and human machine interaction. Amir gained his PhD from the Center for the Study of Rationality and Interactive Decision Theory at the Hebrew University in Jerusalem and the Psychology Department at Princeton University.

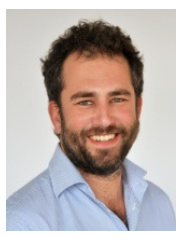

\section{Ron Asherov}

Ron is an intern researcher at the General Motors Advanced Technical Centre in Israel. Ron completed a degree in Mathematics and Computer Science at the Open University of Israel. 
Computer Science \& Information Technology (CS \& IT)

INTENTIONAL BLANK 


\title{
PARALLEL GUIDED LOCAL SEARCH AND SOME PRELIMINARY EXPERIMENTAL RESULTS FOR CONTINUOUS OPTIMIZATION
}

\author{
Nasser Tairan ${ }^{1}$, Muhammad Asif Jan $^{2}$ and Rashida Adeeb Khanum ${ }^{3}$ \\ ${ }^{1}$ College of Computer Science, King Khalid University, Abha, KSA \\ nmtairan@kku.edu.sa \\ ${ }^{2}$ Department of Mathematics, Kohat University of Science \& Technology, \\ Kohat 26000, KPK, Pakistan \\ majan@kust.edu.pk \\ ${ }^{3}$ Jinnah College for Women, University of Peshawar, Peshawar, KPK, Pakistan \\ adeeb_maths@yahoo.com
}

\begin{abstract}
This paper proposes a Parallel Guided Local Search (PGLS) framework for continuous optimization. In PGLS, several guided local search $(G L S)$ procedures (agents) are run for solving the optimization problem. The agents exchange information for speeding up the search. For example, the information exchanged could be knowledge about the landscape obtained by the agents. The proposed algorithm is applied to continuous optimization problems. The preliminary experimental results show that the algorithm is very promising.
\end{abstract}

\section{KEYWORDS}

Guided Local Search, Continuous Optimization Problems, Parallel Algorithms, Cooperative algorithms

\section{INTRODUCTION}

Many real-world applications can be modeled as optimization problems [1][2]. These problems may have many local optima and/or have no analytical form available. Traditional mathematical programming methods are not suitable for dealing with them. During the last two decades, many heuristic methods have been developed. Many of them such as simulated annealing [3], tabu search[4], and guided local search [5] are single point iteration based methods. The research work in the Evolutionary Computation and Particle Swarm Intelligence has demonstrated that a population-based search scheme often has some advantages over single-point based methods on efficiently utilizing computer memory and parallel computing process units. Therefore, a natural issue arises: how can a single point based heuristic be populationized? Mistunori and Ogura [6], E-Abd and Kamel[7] and Blum and Roli [8]have made some attempts on this issue.

GLS, proposed by Voudouris and Tsang in 1995 [5], is a single point based meta-heuristic approach. It escapes from local optima by penalizing some bad solution features. GLS has been applied successfully to a number of optimization problems [5]. This paper proposes a populationbased guided local search (PGLS). In PGLS, several agents run the GLS and exchange 
information, learned from the previous search about the problem, with each other periodically for speeding up the search. Some preliminary experiments have been carried out to study the effectiveness of the proposed PGLS up the search.

The rest of the paper is organized as follows: the proposed PGLS is described in Section 2. Sections 3 and 4 list the test instances and report experimental results. Section 5 concludes this paper.

\section{PARAllel Guided Local SEARCH}

\subsection{Guided Local Search}

Guided Local Search (GLS) is a very general strategy for guiding a local search algorithm to escape from local optima. In GLS, solution features are defined to distinguish between solutions with different characteristics, so that bad characteristics can be penalized and hopefully removed. The solution features depend on the problem and the local search. Features can be defined in a very natural way. For example, in the travelling salesman problem (TSP), a feature can be a link between two cities. When the search is trapped in a local optimum, GLS will use the following function as the objective function:

$$
\mathrm{h}(\mathrm{s})=\mathrm{g}(\mathrm{s})+\lambda \sum\left(\mathrm{p}_{\mathrm{i}} \times \mathrm{I}_{\mathrm{i}}(\mathrm{s})\right)
$$

where sis a candidate solution and $\mathrm{g}(\mathrm{s})$ is the original objective to minimize, $\lambda$ is a control parameter, I ranges over the features. $\mathrm{p}_{\mathrm{i}}$ is the penalty for feature i. $I_{i}(s)$ indicates whether or not solution s exhibits feature $\mathrm{i}$ :

$$
I_{i}(s)=\left\{\begin{array}{cc}
1 & \text { if } s \text { exhibits feature } i \\
0 & \text { otherwise }
\end{array}\right.
$$

All the $p_{i}$ are initialized to be 0 . When the local search traps at a local optimum $s *$, GLS computes the utility function of penalizing feature $\mathrm{i}$ :

$$
\operatorname{util}_{\mathrm{i}}\left(\mathrm{s}^{*}\right)=\mathrm{I}_{\mathrm{i}}\left(\mathrm{s}^{*}\right) \times \frac{\mathrm{c}_{\mathrm{i}}}{1+\mathrm{p}_{\mathrm{i}}}
$$

Where $c_{i}$ is the cost for the feature $\mathrm{i}$ (in TSP, the cost for a feature is the distance for that feature), $p_{i}$ is the current penalty value forfeature ${ }_{i}$. GLS penalizes the feature with the highest utility value, i.e., increase its $p_{i}$ value by 1 . The LS will then continue with the new augmented objective function.

\subsection{PGLS}

In PGLS, several agents run GLS in a parallel way and exchange information periodically for speeding up the search. Different agents start their searches from different points in the solution space. PGLS (manager) records the best solution $x^{*}$ found so far to the problem under consideration by these agents. After every $\mathrm{K}$ penalizations, each agent conducts a crossover operator on $x^{*}$ and its current solution to generate a new solution and then performs its search 
from this new solution. The $p_{i}$ values for each agent will be reset to zero. The algorithm with $n$ agents works as follows:

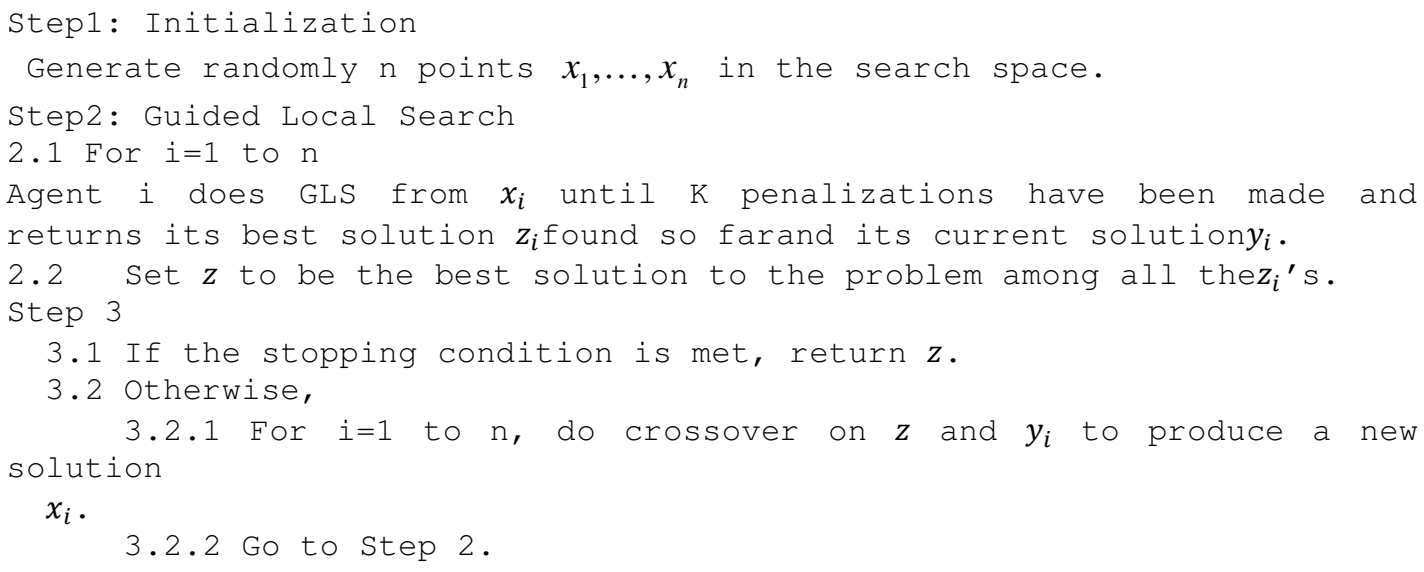

\subsubsection{Local Search}

Following Voudris [9], each real-valued variable in a continuous optimization problem is represented by a number of bits. Therefore, the flip bit mutation is used as a local search method. The local search moves from one solution to another by flipping the value of a bit in the solution. It starts from a random bit and then examines all the possible bits.

\subsubsection{Crossover}

In the crossover, $2 \mathrm{n}$ crossover sites are first randomly selected under the constraint that there are two crossover sites in each variable substring, and then 2 child solutions are generated by swapping along these sites. The child solution with lower original objective function value is returned as a new solution (Step 3.2.1).

\section{EXPERIMENTAL STUDIES}

We have tested the above PGLS algorithm to minimize Rastriging function, Rosenbrock function, and Schwefel function. These three functions are:

$$
\begin{gathered}
\mathrm{f}_{\text {Ra }}=10 \mathrm{n}+\sum_{\mathrm{j}=1}^{\mathrm{n}}\left(\mathrm{x}_{\mathrm{j}}^{2}-10 \cos \left(2 \pi \mathrm{x}_{\mathrm{j}}\right)\right) \\
\mathrm{f}_{\min }=0,\left[\mathrm{x}_{\mathrm{j}}=0\right], \quad-5.12 \leq \mathrm{x}_{\mathrm{j}} \leq 5.12 \\
\mathrm{f}_{\mathrm{Ro}}=\sum_{\mathrm{j}=1}^{\mathrm{n}-1}\left[100\left(\mathrm{x}_{\mathrm{j}+1}-\mathrm{x}_{\mathrm{j}}^{2}\right)^{2}+\left(\mathrm{x}_{\mathrm{j}}-1\right)^{2}\right] \\
\mathrm{f}_{\min }=0,\left[\mathrm{x}_{\mathrm{j}}=1\right],-2.048 \leq \mathrm{x}_{\mathrm{j}} \leq 2.048 \\
\mathrm{f}_{\mathrm{Sh}}=418.9829 \mathrm{n}-\sum_{\mathrm{j}=1}^{\mathrm{n}}\left(\mathrm{x}_{\mathrm{j}} \sin \sqrt{\left|\mathrm{x}_{\mathrm{j}}\right|}\right)
\end{gathered}
$$




$$
f_{\min }=0,\left[x_{j}=1\right],-500 \leq x_{j} \leq 500
$$

In our experiments, each real-valued variable is represented in 22 bits as in [9]. Therefore, each solution is represented in $22 \mathrm{n}$ bits when $\mathrm{n}$ is the number of variables.

The GLS used in our experiments is the same as in [9]. The number of agents=2. The value of $\mathrm{K}$ varies based on the problem as it is shown in Table 1. The algorithm stops after each agent has done $100 \times$ K penalizations.

\section{RESULTS}

The obtained results show that the proposed cooperative method, PGLS, was the most effective algorithm of the two algorithms. It showed a good performance and produced good results for the lunched functions in every run. Table 1 shows the experimental results for the run algorithms

Table 1. Comparison between the run algorithms

\begin{tabular}{|c|c|c|c|c|c|c|c|}
\hline \multirow[t]{2}{*}{$\begin{array}{c}\text { Test } \\
\text { Function }\end{array}$} & \multirow{2}{*}{$\begin{array}{c}\text { Number } \\
\text { of } \\
\text { Variable } \\
s \\
\end{array}$} & \multirow[t]{2}{*}{$\begin{array}{l}\text { Known } \\
\text { Optimum }\end{array}$} & \multicolumn{2}{|c|}{$\begin{array}{c}\text { Algorithm 1 } \\
\text { No cooperation }\end{array}$} & \multicolumn{2}{|c|}{$\begin{array}{c}\text { Algorithm } 2 \\
\text { (PGLS) }\end{array}$} & \multirow{2}{*}{$\begin{array}{c}\text { Number of } \\
\text { Penalization } \\
\text { per } \\
\text { generation }\end{array}$} \\
\hline & & & Average & STDEV & Average & STDEV & \\
\hline$f_{R a}$ & 2 & 0 & 3.24E-01 & 7.06E-01 & $5.91 \mathrm{E}-10$ & 0.0 & 300 \\
\hline$f_{R a}$ & 5 & 0 & 4.23E-01 & $5.50 \mathrm{E}-01$ & 1.47E-09 & 0.0 & 800 \\
\hline$f_{R a}$ & 10 & 0 & 7.93E-01 & $8.06 \mathrm{E}-01$ & $9.95 \mathrm{E}-02$ & 3.15E-01 & 1500 \\
\hline$f_{R o}$ & 2 & 0 & $1.34 \mathrm{E}-02$ & $3.94 \mathrm{E}-02$ & $1.34 \mathrm{E}-02$ & $3.94 \mathrm{E}-02$ & 500 \\
\hline$f_{R o}$ & 5 & 0 & 0.2634 & 0.4174 & 0.0002 & 0.0002 & 800 \\
\hline$f_{R o}$ & 10 & 0 & 0.6281 & 0.7416 & 0.0906 & 0.0498 & 1000 \\
\hline$f_{s h}$ & 2 & 0 & $6.31 \mathrm{E}-04$ & 4.04E-04 & 4.79E-04 & 2.67E-04 & 100 \\
\hline$f_{s h}$ & 5 & 0 & $1.00 \mathrm{E}-02$ & $3.88 \mathrm{E}-04$ & $6.60 \mathrm{E}-04$ & $5.32 \mathrm{E}-04$ & 500 \\
\hline$f_{s h}$ & 10 & 0 & $2.38 \mathrm{E}-02$ & $2.30 \mathrm{E}-02$ & $2.26 \mathrm{E}-02$ & 2.36E-02 & 1000 \\
\hline
\end{tabular}

\section{CONCLUSIONS}

A framework called PGLS for solving continuous optimization problems has been proposed. The idea is to build a cooperative learning environment through running a number of agents of GLS to efficiency explore the search space. After predetermined iterations the acquired information through the search is exchanged between the agents to speed up the search. The genetic crossover was exploited as an exchanging information operator between the agents. In order to allow different components (variables) from the continuous spaces to be selected we apply the crossover operation in each variable substring. Our experimental results on test continuous optimization problems show that our method is the most effective and performed better than the other mean of parallel -GLS: no coordination. Our future work is to expand the experiments with more number of agents and variables. Furthermore, theoretical analysis for the proposed algorithm should be conducted in order to study its convergence and computation time and thus to perform further improvement to the algorithm and subsequently make comparisons with others algorithms. 


\section{REFERENCES}

[1] R. Horst, N.V. Thoai and P.M. Pardalos, Introduction to Global Optimization, Second Edition, Kluwer Academic Publishers, Boston, MA, 2000.

[2] P.M. Pardalos and H. E. Romeijn (Eds.), Handbook of Global Optimization, Kluwer Academic Publishers, Boston, MA, 2002.

[3] Kirkpatrick, S., C. D. Gelatt Jr., M. P. Vecchi, "Optimization by Simulated Annealing",Science, 220, 4598, 671-680, 1983.

[4] Fred Glover, Fred Laguna, Tabu Search, Kluwer Academic Publishers, Norwell, MA, 1997.

[5] Voudouris, C, Guided local search for combinatorial optimization problems, PhD Thesis, Department of Computer Science, University of Essex, Colchester, UK, July, 1997.

[6] Hiroyasu, T., Miki, M. and Ogura M., "Parallel simulated annealing using genetic crossover", Proceedings of the IASTED International Conference on Parallel and Distributed Computing Systems, Las Vegas, pp. 145-150, 2000.

[7] El-Abd, M. and Kamel, M., "A Taxonomy of Cooperative Search Algorithms". Hybrid Metaheuristics (Springer Berlin - Lectures in Computer science), pp. 32-41, 2005.

[8] Blum, C. and Roli, A. "Metaheuristics in Combinatorial Optimization: Overview and Conceptual Comparison”. ACM Computing Surveys, vol. 35, no. 3, pp. 268- 308, 2003.

[9] Voudouris, C., Guided Local Search -- An illustrative example in function optimisation, BT Technology Journal, Vol.16, No.3, July 1998, 46-50.

\section{AUTHORS}

Nasser Tairan received B.Sc. degree in computer science from King Abdulaziz University, KSA in 1999, M.Sc. degree in Software Engineering from University of Bradford, UK in 2005, M.Sc. degree in Computer Science (AI) from University of Essex, UK in 2007 and P.hD. degree from University of Essex 2012. He is currently Assistance Professor in King Khalid University, College of Computer Science, KSA. His main research areas are evolutionary computation, single and multiobjective optimization and metaheuristics,

Muhammad Asif Jan received the M.Sc. degree in mathematics from University of Pehawar, Khyber Paktunkhwa, Pakistan in 1997 and the Ph.D. in mathematics from University of Essex, UK, in 2011. He is currently the Chairperson of the Department of Mathematics, Kohat Uiversity of Science \& Technology (KUST), Khyber Paktunkhwa, Pakistan. His main research areas are evolutionary computation, unconstrained/constrained single/multi- objective optimization by using evolutionary algorithms, decomposition based evolutionary methods for constrained multiobjective optimization, mathematical programming, and numerical analysis.

Rashida Adeeb Khanum received the M.Sc. degree from University of Peshawar, Pakistan in 1997and the Ph.D. from University of Essex, Colchester, United Kingdom in 2012.
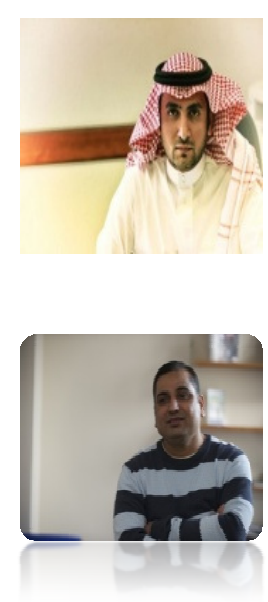


\title{
DESIGN AND IMPLEMENTATION OF A CACHE HIERARCHY-AWARE TASK SCHEDULING FOR PARALLEL LOOPS ON MULTICORE ARCHITECTURES
}

\author{
Nader Khammassi ${ }^{1}$ and Jean-Christophe Le Lann ${ }^{2}$ \\ ${ }^{1,2}$ Lab-STICC UMR CNRS 6285, ENSTA Bretagne, Brest, France \\ 1 nader.khammassidensta-bretagne.fr \\ 2 jean-christophe.le_lanndensta-bretagne.fr
}

\begin{abstract}
Effective cache utilization is critical to performance in chip-multiprocessor systems (CMP). Modern CMP architectures are based on hierarchical cache topology with varying private and shared caches configurations at different levels. Cache-aware scheduling has become a great design challenge. Many scheduling strategies have been designed to target specific cache configuration. In this paper we introduce a cache hierarchy-aware task scheduling (CHATS) algorithm which adapt to the underlying architecture and its cache topology. The proposed scheduling policy aims to improve cache performance by optimizing spatial and temporal data locality and reducing communication overhead without neglecting load balancing. CHATS has been implemented in the parallel loop construct of XPU framework introduced in previous works [1,7]. We compared CHATS to several popular scheduling policies including dynamic and static scheduling and task-stealing. Experimental results on synthetic and real workloads shows that our scheduling policy achieves up to $25 \%$ execution speed up compared to OpenMP, $T B B$ and Cilk++ parallel loop implementations. We use our parallel loop implementation in two popular applications from the PARSEC benchmark suite and we compare it to the provided OpenMP, TBB and PThreads version on different architectures.
\end{abstract}

\section{KEYWORDS}

Cache-aware Scheduling, Cache Locality, Parallel Loops, Multicore, Hierarchical Cache

\section{INTRODUCTION}

This Chip Multiprocessor (CMP) architectures are becoming widely available on many scales: from personal computers to embedded systems to high performance supercomputers...[17,18,19]. CMP cores count is growing continuously and their cache topologies are becoming increasingly hierarchical and deeper. Cache-aware scheduling has become a great design challenge in parallel programming for recent multicore architectures. Chip Multiprocessor (CMP) may exhibit different cache topologies with varying numbers of hierarchical shared and private caches at different levels. An effective task scheduling policy must take into account cache sharing not only at the SMT (Simultaneous Multi-Threading), CMP and SMP (Symetric Multi-Processor) levels but also at the different cache levels of a same chip. 
Task scheduling is critical for execution efficiency especially in the case of parallel loops which are often a great performance multiplier. An efficient cache-aware scheduling policy for recent CMP should take into consideration three major parameters: spatial and temporal data locality in caches, communication and load-balancing. Hierarchical cache topology determines communication latencies between cores at the different levels of cache and thus, has a direct impact or these three critical scheduling factors. In this paper, we present a cache hierarchy-aware task scheduling (CHATS) policy which target to provide efficient hierarchical cache utilization without neglecting load-balancing in parallel loop implementation. CHATS consider spatial and temporal data locality (data reuse and communication) and load-balancing as the most critical parameters for delivering high performance and execution efficiency. We implemented this scheduling algorithm in the parallel loop construct "parallel_for" of the XPU framework and we compared it to parallel programming frameworks using different scheduling techniques. We used both synthetic workloads and real application from the PARSEC and Intel RMS Benchmarks [16].

In the next section, we gives an overview of several prior works on cache-aware scheduling, section 3 give a brief overview of some cache hierarchies used by modern general-purpose CMP and how they can be unified by an abstract model. Section 4 presents the design and implementation our cache-aware scheduling strategy. This scheduling policy is evaluated and compared to several popular scheduling strategies through an experiment using synthetic workloads in section 5. Section 6 shows benchmark results on two real applications: "Blackscholes" and "Fluid Animate" from the PARSEC Benchmark in comparison to different parallel prorgamming models.

\section{RELATED WORKS}

Traditional scheduling techniques such as dynamic scheduling [8] or task-stealing $[9,10,11]$ make different trade-offs between data locality and load-balancing but does not take into consideration cache hierarchy and communication latencies. Some prior works $[20,15,5,14,13]$ target to design cache-aware scheduling policies which target to improve cache-utilization by focusing on one or more cache-related considerations. Processor-cache affinity scheduling [20] focused on temporal data locality and data reuse between threads. Thread clustering scheduler [15] detect sharing patterns between threads online using monitoring techniques and attempts to reduce cachecoherence overhead by clustering threads sharing same data onto close cores. CAB [5] aims to improve task stealing on hybrid SMP-CMP by reducing memory footprint and cache misses, It focus mainly on data sharing at the SMP level and try to reduce inter-socket communication. Constructive cache sharing [14] aims to reduce the memory footprint through exploiting potential overlap of shared data among co-scheduled threads. CATS [13] target to improve cache performance by considering data reuse, memory footprint and coherency misses. None of these prior works take into consideration the cache hierarchy of CMPs.

\section{OVERVIEW OF CMP ARCHITECTURES}

Multicore processor employs a cache structure to simulate a fast common memory. This cache structure may display different cache sharing degrees at different levels. It is mainly composed of hierarchical private and shared caches.

Figure 1 shows a set of CMP architectures from different vendors with varying cache configurations. For example, while the Intel Nehalem architecture associates a private L1 cache for each core, a private L2 cache and a shared L3 cache between all cores, Intel Dunnington architecture is uniformly hierarchical: a private $\mathrm{L} 1$ cache is associated to each core, an L2 cache 
is shared between each 2 cores and finally an common L3 cache is shared between all cores. The Sun UltraSPARC T2 Processor uses a private L1 cache for each core and a shared L2 caches between all cores.

Cache level count and cache sharing degrees at each level are key information for our scheduling policy. The variation of sharing degree at different levels force programmer to make explicit and architecture-specific program optimization in order to get efficient execution.

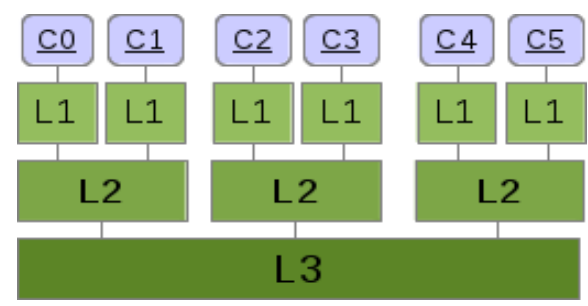

Figure 1. Intel Dunnington

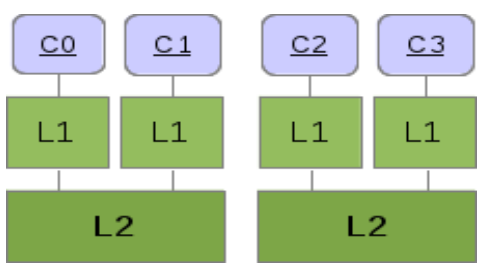

Figure 2. Intel "Hapertown"

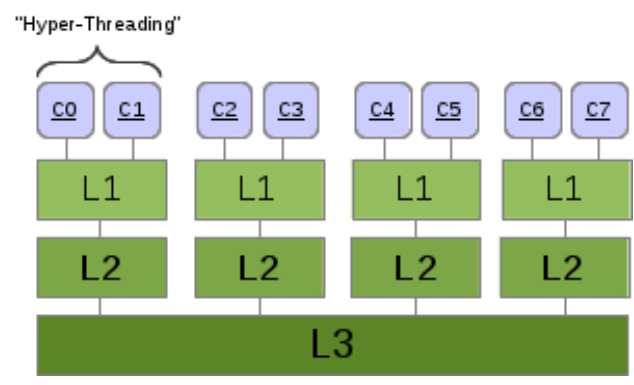

Figure 3. Intel "Nehalem"

In order to be provide an efficient execution on various possible underlying architectures with different cache topology, a cache-aware scheduling algorithm should be dynamically adaptable to the target architecture. Consequently, such scheduling algorithm should have a detailed description of the cache topology of the underlying CMP. This description can be established through dynamic exploration of the target platform at the initialization of the run-time system. Modern operating systems provide means to obtains cache hierarchy details at high level either through system files such as in the Linux OS or through native API such as Windows [21].

Variation of the cache level count and cache sharing degrees raise the need to unify them under a single abstract description. The Unified Multicore Architecture Model (UMAM) [22] that can be used to provide a unified description for different CMP architectures. 
Memory hierarchy including cache levels and main shared memory can be described using UMAM as shown in Tab 1 which gives an example of three different platforms. The two first columns gives the cache-levels and cores count, the next columns gives the count of cores sharing $\boldsymbol{L}_{\boldsymbol{i}}$ caches or $\boldsymbol{M}_{\boldsymbol{i}}$ memory $(\boldsymbol{i} \in \mathbf{1} . . n, \boldsymbol{n}$ : Memory hierarchy levels count).

Table 1. Description of Cache and Memory Hierarchy of some CMP Architectures in UMAM

\begin{tabular}{cc|c|c|c|c|c|}
\hline Architectures & \multicolumn{7}{c|}{ Parameters } \\
\hline $\begin{array}{c}\text { Nehalem } \\
\text { Core i7 Q920M Levels }\end{array}$ & Cores & $\mathbf{L}_{\mathbf{1}}$ & $\mathbf{L}_{\mathbf{2}}$ & $\mathbf{L}_{\mathbf{3}}$ & $\mathbf{M}_{\mathbf{4}}$ \\
\hline $\begin{array}{c}\text { Nehalem } \\
2 \mathrm{x} \text { Xeon E5620 }\end{array}$ & 4 & 8 & 2 & 2 & 8 & 8 \\
\hline $\begin{array}{c}\text { Dunnington } \\
\text { Xeon X7460 }\end{array}$ & 3 & 8 & 2 & 2 & 8 & 16 \\
\hline
\end{tabular}

\section{CACHE HIERARCHY-AWARE TASK SCHEDULING}

The design and implementation of CHATS rely a several basic building blocks which allows partitioning of the main work of a parallel loop into a set a little works which can be executed concurrently by several threads. We start by defining the components of our run-time system.

\subsection{Runtime System}

The run-time system is based on a worker (thread) pool able to execute tasks. Each worker have a FIFO (First-In-First-Out) work (task) queue. A scheduler can submit tasks to the workers through this work-queue. A worker remains idle until a task is pushed into its work queue so it wake up and execute its task. Submitting a task follows a one-to-one communication scheme between the main thread holding the scheduler and each worker to reduce communication overhead. Figure 4 gives and overview of the run-time system.

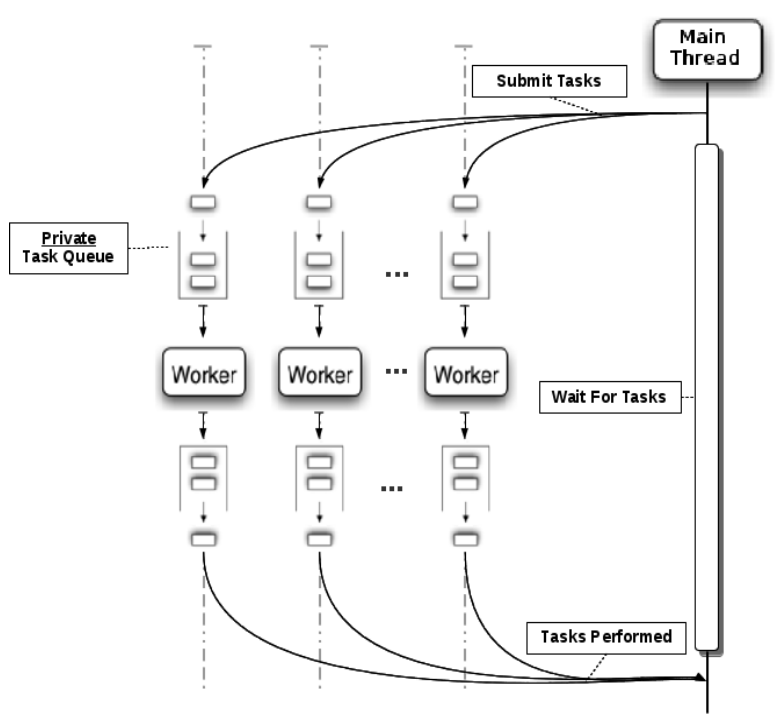

Figure 4. Worker Pool-Based Run-Time System With Private Work-Queue 


\subsection{Work Unit}

A work unit is a task which should be executed on a range of iteration then a range of shared iterations. In XPU "parallel_for" loop, a work unit is composed of:

A Range : Specifies a range of iterations to process (min, max, progression step)

A Shared Range : As "Range", it specify a range of iteration, however, it allows "stealing" of iterations by concurrent threads.

A Task : The code which will process each iteration of a given range and/or shared range(s).

\subsection{Data Partitioning}

Data partitioning is a primordial step in parallelization of a loop. In our case we use basic a quasifair partitioning algorithm which decompose a "Range" into $\mathbf{N}$ "Range" and $\mathbf{M}$ "Shared Range". The algorithm ensure that the generated partitions are quasi-equals.

\subsection{Parallel For Loop : Data Partitionning and Cache Topology}

Let's consider a "for loop" $\mathbf{F}$ defined by : $\mathbf{i}=\mathbf{0}$..n by $\mathbf{1}$, where $\mathbf{F}$ corresponds to a "Range" which can be partitioned into $\mathbf{N}$ "Range" and $\mathbf{M}$ "Shared Range". Determining $\mathbf{M}$ and $\mathbf{N}$ depends directly on the underlying architecture:

A $\mathbf{N}=$ Workers count $\sim$ Cores count

A $\mathbf{M}=$ Number of shared caches at all levels (consecutive cache levels shared by the same cores are considered as one)

A $\mathbf{P}=\mathbf{N}+\mathbf{M}$ : Total partitions count

Let's consider a Nehalem Intel Core i7 Q720 with 8 Hardware Threads and 4 Physical Cores (Fig.3). Data partitioning is described in Figure 5, so $\mathbf{P}$ is equal to $\mathbf{1 3}$ in this case. Green ranges are private "Range" so a worker doesn't share them with other workers. Orange box correspond to "Shared Ranges" which are shared among two co-scheduled SMT workers (threads) sharing L1/L2 caches. Finally, red box is a "Shared Range" from which all workers can steal iterations, it corresponds to the L3 cache.

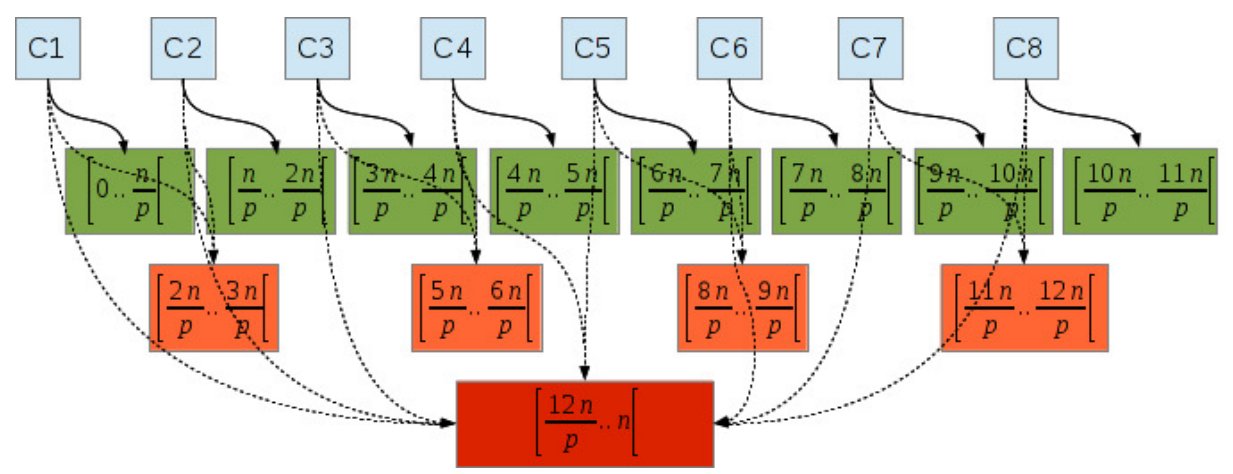

Figure 5. CHATS Data Partitioning on an 8 Hardware Threads Intel Nehalem Processor 


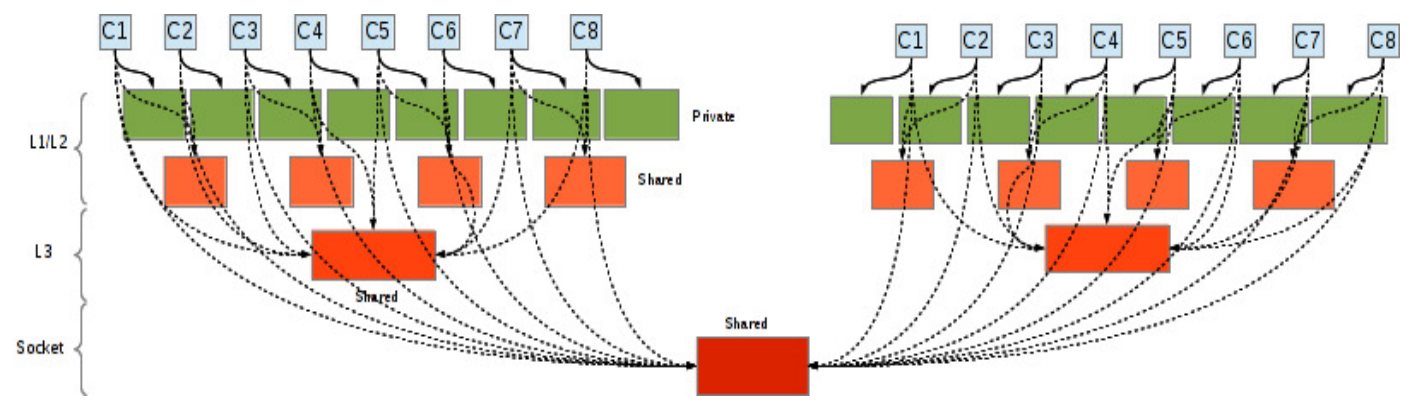

Figure 6. CHATS Data Partitioning on an on hybrid SMT-CMP-SMP platform with 16 Hardware threads (2 x Intel Xeon E5620 at $2.4 \mathrm{GHz})$

Figure 6 shows the data partitioning scheme for an SMP platform containing two Intel Nehalem Processors having eight hardware threads (4 Physical cores with Hyper-Threading enabled).

\subsection{Workload Scheduling}

Once data partitioned into "Ranges" and "Shared Ranges", we can submit works to our workers running on the different cores. Submitted work will specify a Task, a Range and one ore more Shared Ranges. If we take the partitioning pattern of the Figure 5. "Worker 0" running on "Core 0" will get a work containing:

A One "Range" : [0 .. n/p[

A Two "Shared Ranges" : [ 2n/p .. 3n/p[ and [ 12n/p .. n[

Analogously, the other workers will gets their three ranges of iterations.

\subsection{Execution Semantic}

"Worker 0" will execute the task code on each iteration of its private range without any communication with the other threads. Once finished, it will try to steal iterations from the shared ranges if available. Iteration stealing requires communication (locking) between threads working on the same shared range. This communication overhead is reduced by the fact that threads communicates through shared caches. So, the communication introduced buy concurrent accesses to "Shared Range" $[\mathbf{1 2 n} / \mathbf{p}$.. $\mathbf{n}[$ is more costly than the one introduced by concurrent accesses to [2n/p .. 3n/p[ . However, we note that low level caches-associated "Shared Range" are fewer than those associated to high level caches (1 associated to $\mathbf{L 3}$ and 4 associated to $\mathbf{L 1 / L 2}$ ).

We outline that "Shared Ranges" aims to provide good load-balancing at the lower possible cost in term of communication overhead : when worker finish their work on private works, they does not remain idle, instead, they steal works from shared ranges or more precisely the "closed" shared range to their high level caches.

\subsection{XPU: Implementation and programming Interface}

The modified the default scheduler of XPU [7] to implement CHATS. Parallel for loop can be easily implemented using XPU. Figure 7 shows how to express data parallelism through a parallel for loop. 


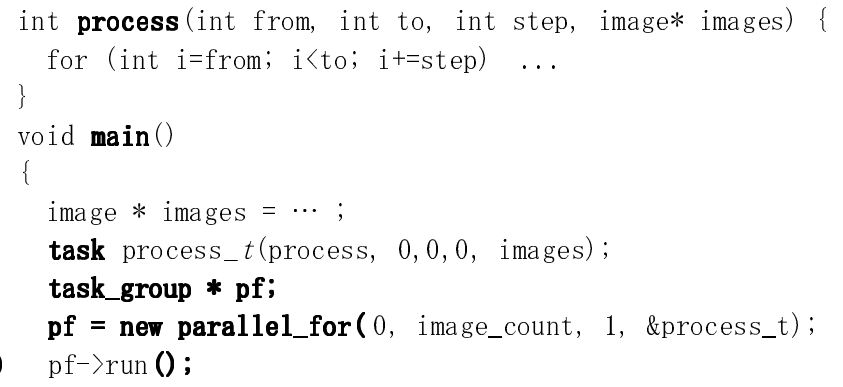

Figure 7. An example of parallel for loop implementation using XPU

\section{Performance Evaluation}

We compare our CHATS implementation to several popular programming models implementing static scheduling, dynamic scheduling and task stealing which we present briefly.

\subsection{Common Scheduling Policies}

\subsubsection{Static scheduling}

Static scheduling is the most straightforward scheduling technique: data is statically partitioned into $N$ equal or pseudo-equal chunks, these chunks are then processed respectively by $N$ parallel threads. This scheduling scheme avoid communication between threads, offer good data reuse when the parallel loop is executed several time. However, this method may result in load unbalancing, especially in the case of heavy workload, since faster threads remains idle, waiting for other threads until finishing their work.

\subsubsection{Dynamic scheduling}

Dynamic scheduling provide better load balancing since threads does not remains idle as long as chunks are available in the common work queue. Unfortunately, while improving workload distribution, this technique may introduces a costly communication between threads accessing concurrently to the common work queue (Many-To-Many Communication). This may results into ineffective uses of processor caches. Also, this technique provide poor data reuse since a same chunks may be processed by different threads on different cores when the parallel loop is executed multiple time. Bad data reuse may amplify consequently cache-miss rate.

\subsubsection{Task Stealing}

Task-stealing is a popular scheduling algorithm which is introduced in Cilk [10]. Task-stealing attempts to combines advantages of the two previous scheduling policies by making another trade-off between efficient cache utilization and load-balancing, In task stealing, each thread (worker) has a task pool in which its tasks are stored. Whenever a worker finishes its current task, the worker try to get another task from its task pool. If there's no more works to perform (its task pool is empty), the worker select randomly a "victim" worker and try to steal a work from its task pool. If succeeded, it execute the stolen task, otherwise, it try to steal a task from another randomly-chosen worker [5].

Task-stealing performs good load-balancing since no thread (worker) remains idle as long as there is available "works", i.e. ,available tasks in the task pools of workers. However, task- 
stealing may introduce significant communication overhead since "victim" threads are chosen randomly without considering cache-hierarchy or communication latencies. Deep cache hierarchies introduce non-uniform communications between cores, consequently, the choice of the "victim" thread becomes critical for performance: stealing a task from a "close" thread (sharing high-level cache with the stealer) is much cheaper than stealing a task from a "far" thread (running on a core which does not share any cache with the stealer).

\subsection{Experiment : Parallel For On Synthetic Workloads}

In order to evaluate the performances of our approach we designed an experiment which aims to evaluate cache utilization efficiency and global performance of a configurable target application. We generate a synthetic work load witch allow us to control unit workload and global workload and simulate data reuse. Thus, in order to achieve efficient execution, a scheduling strategy should provides good spatial and temporal data locality and an efficient load balancing.

The used unit workload is a sequential function performing a "quicksort" on a small vector. We control the unit workload by varying the size of this small vector. So, our input data is a set of small vector, our program perform a "quicksort" in each of these small vector. "quicksort" sort a vector performing multiple compare and swap so intensive intensive read/write accesses to data. This make it good candidate to evaluate efficient cache utilization.

In this experiment we try to evaluate the efficiency of our scheduling policy CHATS to: static scheduling, dynamic scheduling and task stealing. We run our synthetic workloads on an hybrid SMT-CMP-SMP platform with 16 Hardware threads ( 2 x Intel Xeon E5620 at $2.4 \mathrm{GHz}$ ) and we measure average execution time for different workload as well as cache-misses for each of the scheduling policies.

\subsection{Results}

As shown in Figure 8, results shows that XPU processes the heaviest workload about $20 \%$ faster than the second fastest candidate. We notes also that XPU become more efficient as the workload is bigger.

Figure 9 shows that XPU generate a low cache-miss rate in comparison to the other candidate. XPU cache-miss rate remains close to the static scheduling based candidate. Static scheduling is known to offer verv good data localitv and doesn't introduces communication overhead.

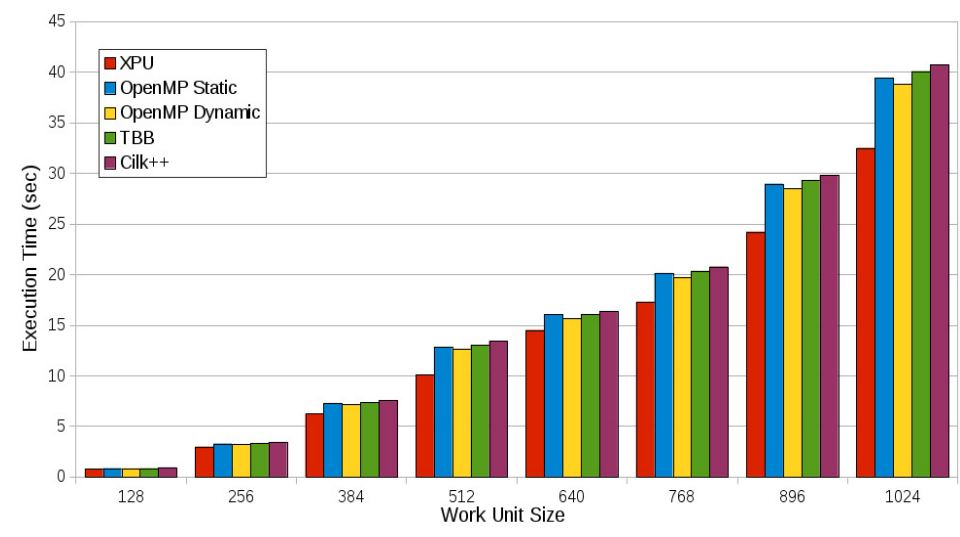

Figure 8. Average processing time of different workload sizes. 


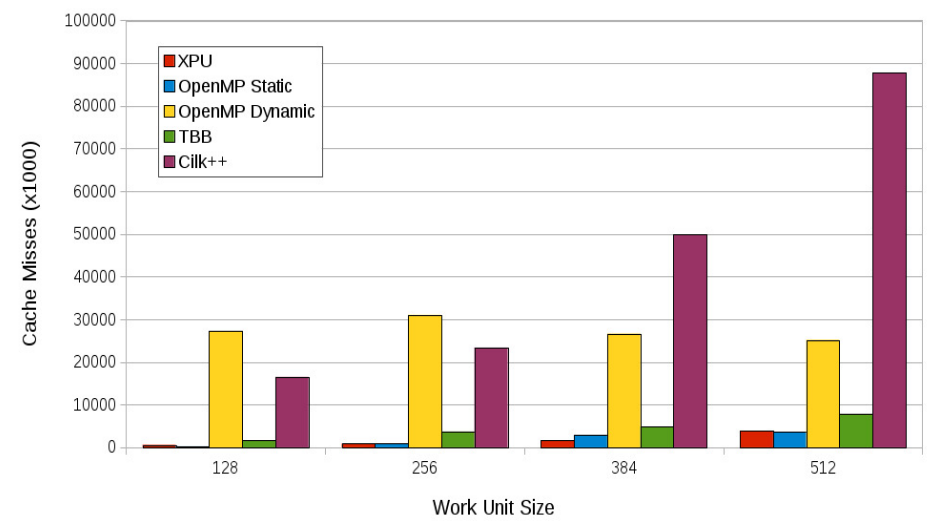

Figure 9. Cache-miss rate for different problem sizes.

\section{APPLiCATiOnS}

In order to evaluate performances of our scheduling policy, we use our parallel loop implementation to parallelize two popular applications from the PARSEC benchmark suite [16] which are also a part of the Intel RMS benchmark. The first application is the Blackscholes options pricing application and the second one is the fluid animation application.

For each application, the benchmark includes a serial version and several parallel versions using different parallel programming models including POSIX Threads [6], OpenMP [8] and Threading Building Blocks [12]. We use the serial version to build our parallel applications using the XPU framework [7]. More precisely, we use the "parallel_for" pattern to parallelize the main loop of both applications at thread level. We modified the default scheduler to implement our cacheaware scheduling algorithm.

The Blackscholes application exhibits massive parallelism thanks to its main parallel loop which process options with almost no communication between threads. The Fluid Animation workload processes large amount of particles through a parallel loop but suffer from significant inter-thread communication overhead.

\subsection{Blackscholes}

We parallelize the "Black-Scholes" workload at thread level using the XPU's "parallel_for" construct running on top of the cache hierarchy-aware scheduler. At the instruction level, we use the vectorization capability provided by XPU through a built-in vectorized type (vec4f) implemented in top of SSE to support SIMD. We used the sequential code of the "blackscholes" application as provided in PARSEC Benchmark Suite. The main processing loop is parallelized at the cost of 3 lines of extra-code. Vectorization is introduced simply by replacing regular float type by the XPU's "vec4f" vectorized type. We compare the performance achieved by our application to the five parallel versions provided in the same benchmark suite: OpenMP, TBB, Pthreads, OpenMP/SSE and POSIX Threads/SSE. We use the Intel C++ Compiler v12.0.5 and we executed our benchmark on different multicore platforms.

Fig. 10 and 11 shows the measured execution time for each parallel version. The XPU-based application provides higher performance than the other versions and execute up to $25 \%$ faster than POSIX Thread/SSE one. It takes advantage of the ability of the scheduler to provide both 
load-balancing, efficient cache utilization and low communication overhead to outperform the POSIX Thread version which use basic static scheduling achieving good cache utilization but poor load-balancing. The impact of this poor load-balancing issue becomes more visible as workload grows.

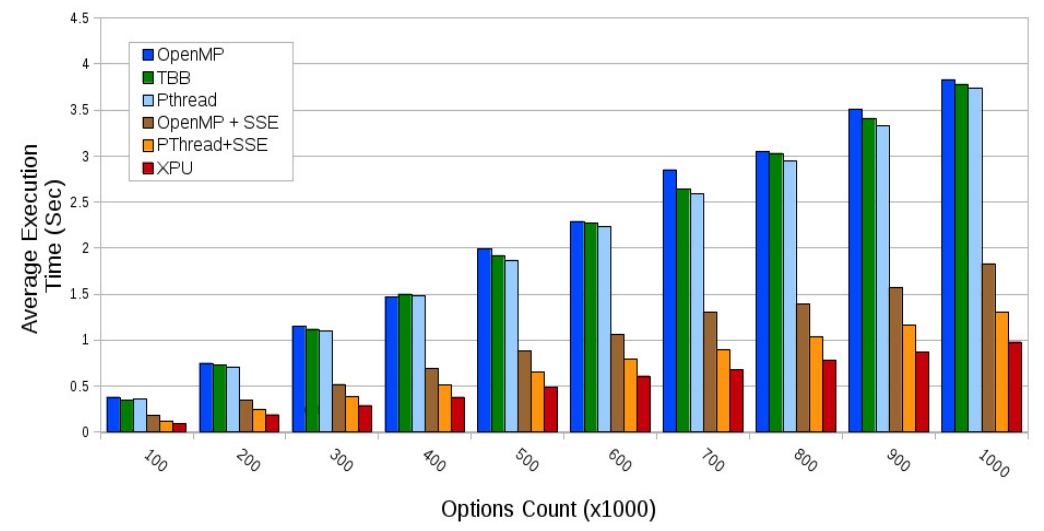

Figure 10. Execution time of the "Blackscholes" application for different problem sizes on hybrid SMTCMP Nehalem Processor with 8 Hardware threads (Intel Core i7 Q720)

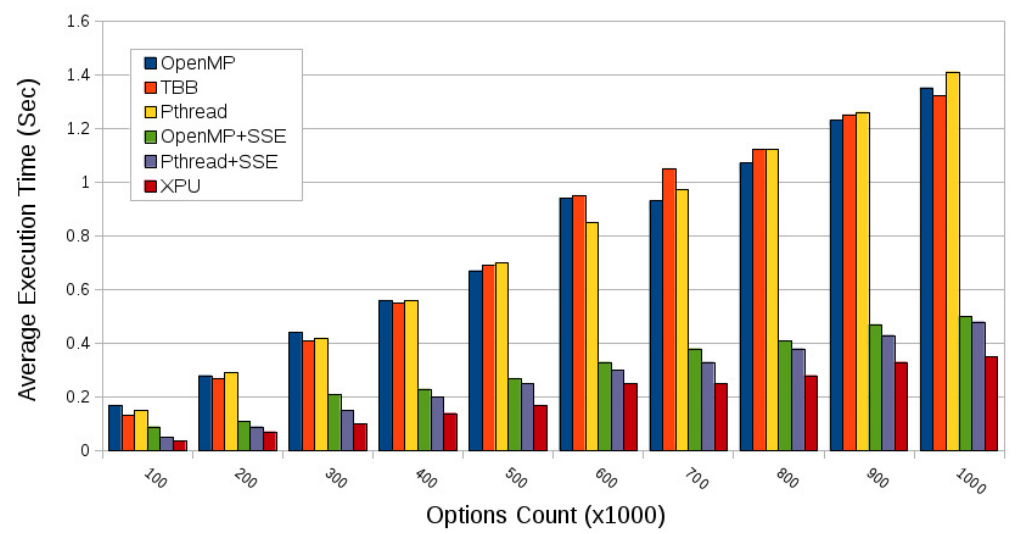

Figure 11. Execution time of the "Blackscholes" application for different problem size on hybrid SMTCMP-SMP platform with 16 Hardware threads (2 x Intel Xeon E5620 at $2.4 \mathrm{GHz}$ )

\subsection{Fluid Animation}

The fluid animation application is parallelized the same way as the Blackscholes one. Fig. 12 and 13 shows the measured execution time on two different platforms. The first one is an SMT-CMP processor which displays two cache level sharing. The second one is an hybrid SMT-CMP-SMP platform containing two Intel Nehalem processors and exhibiting three levels cache sharing.

The parallel "fluidanimate" program introduces significant communication between threads making spatial and temporal data locality in caches critical for achieving high performances. This gives an advantage to static scheduling techniques but doesn't reduces the impact of efficient load-balancing. 


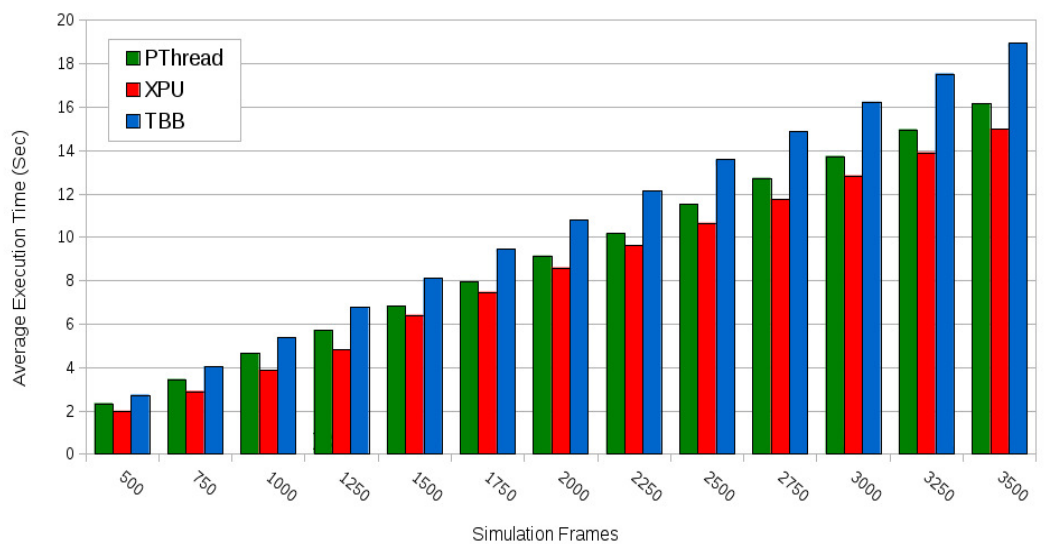

Figure 12. Execution time of the "Fluid Animation" application for different problem sizes on hybrid SMTCMP Nehalem Processor with 8 Hardware threads (Intel Core i7 Q720)

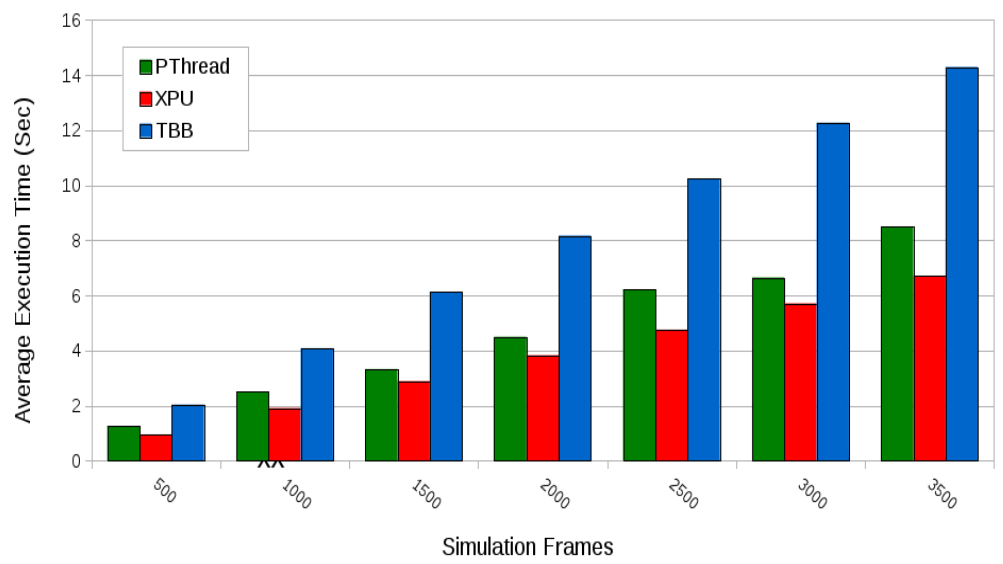

Figure 13. Execution time of the "Fluid Animation" application for different problem sizes on hybrid SMTCMP-SMP platform with 16 Hardware threads (2 x Intel Xeon E5620 at $2.4 \mathrm{GHz}$ )

\section{CONCLUSION AND FUTURE WORKS}

In this paper, we presented a cache-hierarchy aware scheduling which aims to provide efficient cache utilization without neglecting load-balancing. We described the CHATS scheduling policy and how it can improve spatial and temporal data locality in hierarchical caches. We shown how we can provide good load-balancing without generating significant communication overhead. Our experimental results on synthetic workloads outlined the high cache-misses rate introduced by some traditional scheduling policies implying arbitrary threads communications such as taskstealing or dynamic scheduling. These experiments demonstrated also that channelizing interthread communications through hierarchical sharing groups which communicates through shared caches reduces significantly this communication overhead and generates much lower cache-miss rate. 
Our implementation of CHATS algorithm in the XPU scheduler and its use on popular real applications from the PARSEC benchmark confirms our experimental results on synthetic workload and shows high performances in comparison to many others popular parallel programming models implementing different scheduling policies such OpenMP, POSIX Threads or Threading Building Blocks.

CHATS has been designed to adapt dynamically to the underlying CMP architecture by exploring its cache-topology at run-time. At the moment of writing this paper, dynamic cache topology exploration and CHATS scheduler have been developed separately. These two pieces will be put together to make CHATS adapt dynamically to various multicore architectures.

We can conclude that CHATS scheduling strategy may be good alternative to traditional scheduling techniques especially in the cases where spatial and temporal data locality in processor caches are critical for execution efficiency.

\section{REFERENCES}

[1] N. Khammassi, J.C. Le Lann, J.P. Diguet and A. Skrzyniarz, "MHPM: Multi-Scale Hybrid Programming Model: A Flexible Parallelization Methodology", HPCC '12 Proceedings of the 2012 IEEE 14th International Conference on High Performance Computing and Communication, 71-80, Liverpool UK, June 2012

[2] G. Blake, R. G. Dreslinski and T. Mudge, "A Survey of Multicore Processors", IEEE Signal Processing, vol. 26, n. 6, pp. 26-37 November 2009

[3] L. J. Karam, I. Alkamal, Alan Gatherer, G. A. Frantz, D. V. Anderson and B. L. Evans, "Trends in Multicore DSP platforms", IEEE Signal Processing, vol. 26, n. 6, pp 38-49, November 2009

[4 W. Wolf, "Multiprocessor System-on-Chip Technology", IEEE Signal Processing vol. 26, n. 6, November 2009

[5] Q. Chen, Z. Huang and M. Guo, "CAB: Cache Aware Bi-tier Task Stealing in Multi-socket Multicore Architecture", International Conference on Parallel Processing (ICPP), 2011

[6] D. Butenhof, Programming with POSIX Threads. Addison-Wesley Longman Publishing Co., Inc. Boston, MA, USA, 1997.

[7] XPU Framework, "http://www.xpu-project.net/"

[8] E. Ayguadé, N. Copty, A. Duran, J. Hoeflinger, Y. Lin, F. Massaioli, X. Teruel, P. Unnikrishnan, and G. Zhang, "The design of openmp tasks," IEEE Transactions on Parallel and Distributed Systems, vol. 20, no. 3, pp. 404-418, 2009.

[9] G. Cong, S. Kodali, S. Krishnamoorthy, D. Lea, V. Saraswat, and T. Wen, "Solving large, irregular graph problems using adaptive work-stealing," in 37th International Conference on Parallel Processing, pp. 536-545, IEEE, 2008.

[10] M. Frigo, C. E. Leiserson, and K. H. Randall, "The implementation of the Cilk-5 multithreaded language," in Proceedings of the ACM SIGPLAN '98 Conference on Programming Language Design and Implementation, (Montreal, Quebec, Canada), pp. 212-223, ACM, June 1998.

[11] C. Leiserson, "The Cilk++ concurrency platform," in Proceedings of the 46th Annual Design Automation Conference, pp. 522-527, ACM, 2009.

[12] J. Reinders, Intel threading building blocks. O'Reilly, 2007.

[13] T. F. Yang, C. H. Lin and C. L. Yang, "Cache-Aware Task Scheduling on Multi-Core Architecture", International Symposium on VLSI Design Automation and Test (VLSI-DAT), 2010

[14] S. Chen, P. B. Gibbons, M. Kozuch, V. Liaskovitis, A. Ailamaki, G. E. Blelloch, B. Falsas, L. Fix, N. Hardavellas, T. C. Mowry, and C. Wilkerson., "Scheduling threads for constructive cache sharing on CMPs", In Proceedings of the 19th Annual Symposium on Parallel Algorithms and Architectures, pages 105-115. ACM, 2007.

[15] D. Tam, R. Azimi, and M. Stumm, "Thread clustering: Sharing-aware scheduling on smp-cmp-smt multiprocessors", In Proceedings of the 2nd SIGOPS/EuroSys European Conference on Computer Systems, pages 47-58. ACM, 2007.

[16] C. Bienia, S. Kumar, J. P. Singh and K. Li, “The PARSEC Benchmark Suite: Characterization and Architectural Implications", Proceedings of the 17th International Conference on Parallel Architectures and Compilation Techniques, October 2008. 
[17] G. Blake, R. G. Dreslinski and T. Mudge, "A Survey of Multicore Processors", IEEE Signal Processing, vol. 26, n. 6, pp. 26-37 November 2009

[18] L. J. Karam, I. Alkamal, Alan Gatherer, G. A. Frantz, D. V. Anderson and B. L. Evans, "Trends in Multicore DSP platforms", IEEE Signal Processing, vol. 26, n. 6, pp 38-49, November 2009

[19] W. Wolf, "Multiprocessor System-on-Chip Technology", IEEE Signal Processing vol. 26, n. 6, November 2009

[20] M. S. Squillante and E. D. Lazowska, "Using processor-cache affinity information in shared-memory multiprocessor scheduling", IEEE Transactions on Parallel and Distributed Systems, Vol 4, pp.131143

[21] Processor information, "http://msdn.microsoft.com/en-us/library/windows/desktop/ms683194\%28v=vs.85\%29.aspx"

[22] J. E. Savage, M. Zubair, "A unified model for multicore architectures", IFMT '08 Proceedings of the 1st International Forum on Next-generation multicore/manycore Technologies

\section{AUTHORS}

Nader Khammassi received its engineering degree from the National Engineering School of Brittany in France. He is currently a research engineer at Thales Airborne Systems in the department of Radar and Electronic Warfare Systems and is pursuing its $\mathrm{PhD}$ degree in computer sciences at ENSTA Bretagne. His major research interests include developing high-performance parallel software technologies and applications for multicore and manycores architectures. His recent works include the design and development of the XPU parallel programming framework and the development of several high performance applications in the field of signal processing and numerical linear algebra.

Jean-Christophe Le Lann is researcher and lecturer at ENSTA Bretagne since 2008, in the area of parallel embedded systems, electronic-system level (ESL) and associated tooling (compiler, interpreters). Previously he was senior engineer with Thomson during 10 years, and was responsible for subsystems design in advanced system-on-chip (SoC) for real-time multi-standard video encoding and decoding $(\mathrm{H} .264, \mathrm{DV}, \ldots)$. He is also cofounder of a start-up Modaë Technologies, a french ESL-oriented company.
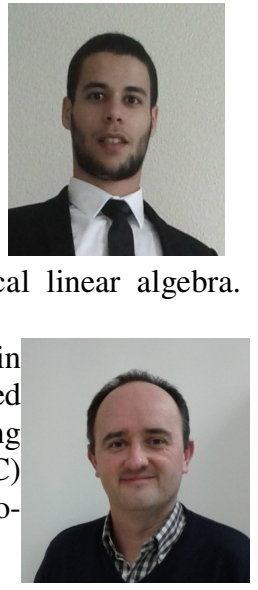
Computer Science \& Information Technology (CS \& IT)

INTENTIONAL BLANK 


\title{
REAL TIME FACE DETECTION ON GPU USING OPENCL
}

\author{
Narmada Naik ${ }^{1}$ and Dr Rathna.G.N ${ }^{2}$ \\ ${ }^{1,2}$ Department of Electrical Engineering \\ Indian Institute of science \\ Bangalore-560012 \\ India \\ ${ }^{1}$ nnreema22@gmail.com \\ ${ }^{2}$ rathnadee.iisc.ernet.in
}

\begin{abstract}
This paper presents a novel approach for real time face detection using heterogeneous computing. The algorithm uses local binary pattern $(L B P)$ as feature vector for face detection. OpenCL is used to accelerate the code using GPU[1]. Illuminance invariance is achieved using gamma correction and Difference of Gaussian(DOG) to make the algorithm robust against varying lighting conditions. This implementation is compared with previous parallel implementation and is found to perform faster.
\end{abstract}

\section{KEYWORDS}

Heterogeneous Computing, OpenCL, Image Processing, LBP, Histogram, Classifier.

\section{INTRODUCTION}

Face detection finds its application in various research discipline such as image processing, computer vision, pattern recognition. Face detection can be done using many algorithms such as Eigen faces, Fisher faces, Local Binary Pattern etc. Applications of human face detection algorithms, such as computer assisted surveillance, need to be in real time. These algorithms, being highly parallel, are more suited for platforms like GPU which have an inherent parallel execution architecture and higher computing capacity compared to CPU. In this paper we have implemented a parallelized variant of LBP on GPU using OpenCL, a heterogeneous computing platform.

In this paper Face detection is done based on efficient grey scale invariant texture Local Binary Pattern (LBP) Histogram, which is parallelized and processed by using Heterogeneous CPU-GPU based platform. The work is based on converting colored images(captured from camera) to grey scale, preprocessing the image and extraction of LBP feature and its histogram on GPU to reduce the computation time. LBP operator is a computationally simple and efficient texture analysis operator which labels the pixels of an image by thresholding the neighbourhood of each pixel and considers the result as a binary number. In real time application the success of LBP is due to its uncomplicated computation and robustness due to illumination variations [2].

Rest of the paper is organized into five more sections. Section 2 gives a brief overview about Heterogeneous computing and OpenCL. In Section 3, we have discussed the LBP algorithm.

David C. Wyld et al. (Eds) : CCSIT, SIPP, AISC, PDCTA, NLP - 2014

pp. 441-448, 2014. @ CS \& IT-CSCP 2014

DOI : $10.5121 /$ csit.2014.4238 
Section 4 discusses the implementation details on GPU. Experimental results are shown in section 5. In Section 6, we give a brief conclusion of the paper.

\section{HETEROGENEOUS COMPUTING WITH OPENCL}

OpenCL[3] is an industry standard writing parallel programs targeting heterogeneous platforms. In this section a brief overview of heterogeneous computing with OpenCL programming model is given. Programming model of OpenCL is classified into 4 models[4] Platform model, Execution model, Memory model, Programming model.

\section{1. platform model}

The OpenCL platform model defines a high-level representation of any heterogeneous platform used with OpenCL. This model is shown in the Fig 1. The host can be connected to one or more OpenCL devices(DSP, FPGA, GPU, CPU etc), the device is where the kernel execute. OpenCL devices are further divided into compute units which are further divided into processing elements(PEs), and computation occurs in this PEs. Each PEs is used to execute an SIMD.

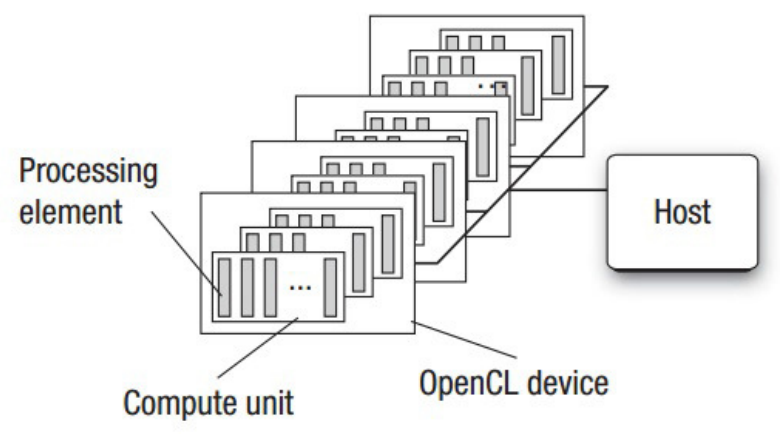

Fig. 1. OpenCL Platform Model .

\subsection{Execution model}

OpenCL execution consist of two parts - host program and collection of kernels. OpenCL abstracts away the exact steps for processing of kernel on various platforms like CPU, GPU etc. Kernels execute on OpenCL devices, they are simple functions that transforms input memory object into output memory objects. OpenCL defines two types of kernel, OpenCL kernels and Native kernels.

Execution of kernel on a OpenCL device:

1. Kernel is defined in the host,

2. Host issues a command for execution of kernel on OpenCL device,

3. As a result OpenCL runtime creates an index space.

4. An instance of the kernel is called work item, which is defined by the coordinates in the indexspace (NDRange) as shown in Fig 2. 


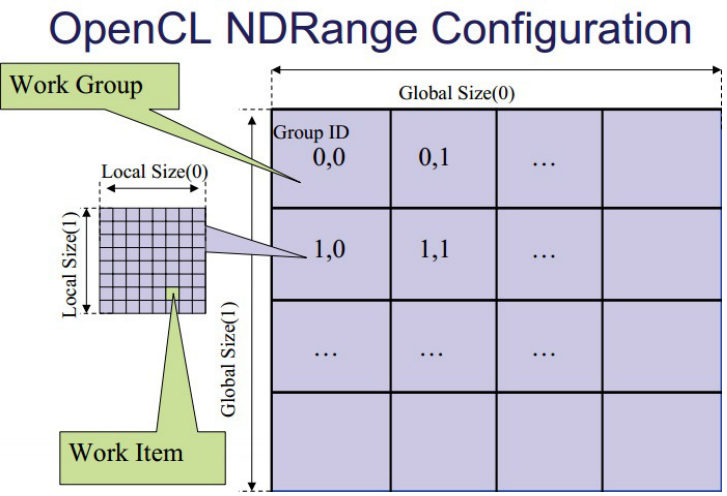

Fig. 2. Block diagram of NDRange.

\subsection{Memory model}

OpenCL defines two types of memory objects, buffer objects and image objects. Buffer object is a contiguous block of memory made available to the kernel, whereas image buffers are restricted to holding images. To use the image buffer format OpenCL device should support it. In this paper buffer object is used for face detection. OpenCL memory model defines five memory region:

- Host memory: This memory is visible to host only.

- Global memory: This memory region permits read/write to all work items in all the work groups.

- Local memory: This memory region, local to the work group, can be accessed by work items within the work group.

- Constant memory: This region of global memory remains constant during execution of the kernel. Workitems have read only access to these objects.

- Private memory: This memory region is private to a work item i.e variables defined private in one work item are not visible to the other work item. Block diagram of memory model is shown in Fig 3.

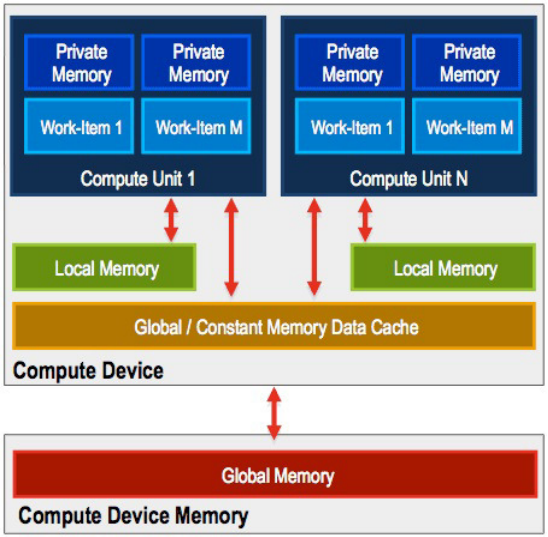

Fig. 3. Block diagram for memory model 


\subsection{Programming model}

Programming model is where the programmer will parallelize the algorithm. OpenCL is designed both for data and task parallelism. In this paper we have used data parallelism which will be discussed in section 4 .

Basic work flow of an application in OpenCL frame work is shown below in block diagram Fig.4

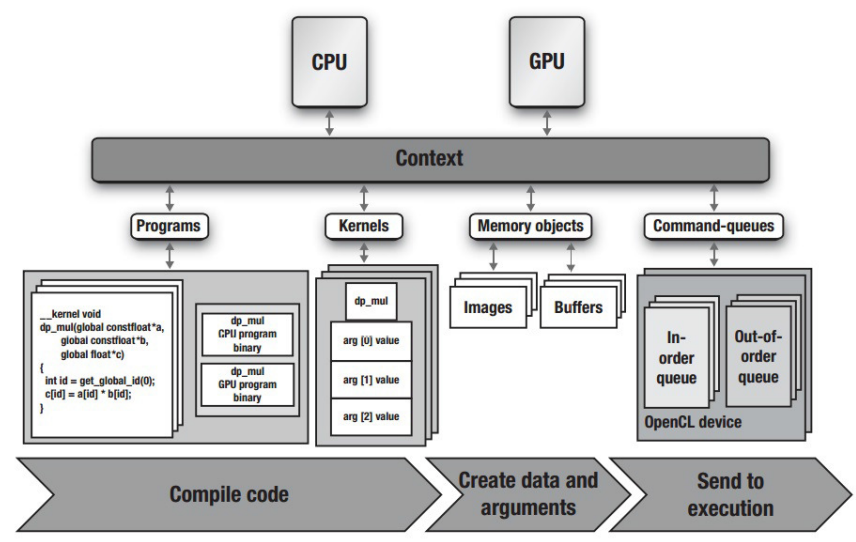

FIG. 4. WORK FlOW DiAgRAM OF OPENCL.

Here we start with the host program that defines the context. The context contains two OpenCL devices, a CPU and a GPU. Next the command queue is defined, one for GPU and the other for CPU. Host program then defines the program object to compile and generate the kernel object both for OpenCL devices. After that host program defines the memory object required by the program and maps them to the arguments of the kernel. Finally the host program enqueue the commands to the command queue to execute the kernels and then the results are read back.

\section{OVERVIEW OF THE ALGORITHM}

In this section it is discussed about how the image is captured from camera and converted to grey scale used gamma operation and DOG operation in preprocessing[1] and LBP feature extraction, Histogram and classifier.

\subsection{Face detection using LBP:}

LBP operator labels the pixel by thresholding the $3 \times 3$ neighbourhood of each pixel with the center value. This generic formulation of the operator puts no limitations to the size of the neighbourhood [5]. Here each pixel is compared to its 8 neighbours (on its left-top, left-middle, left bottom, right-top, right-middle, right-bottom) followed in clockwise direction. Wherever the center pixel value is greater than the neighbour write 1 to the corresponding neighbour pixel otherwise write 0 . This gives an 8-digit binary number as shown in Fig.5. This 8-digit binary number is then converted to a decimal. 


example
\begin{tabular}{|c|c|c|}
\hline 6 & 5 & 2 \\
\hline 7 & 6 & 1 \\
\hline 9 & 8 & 7 \\
\hline
\end{tabular}

Pattern $=11110001$

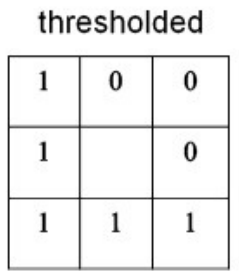

LBP $=1+16+32+64+128=241$

\section{weights}

\begin{tabular}{|c|c|c|}
\hline 1 & 2 & 4 \\
\hline 128 & & 8 \\
\hline 64 & 32 & 16 \\
\hline
\end{tabular}

Fig. 5. LPB Thresholding

After getting the LBP of the block, histogram of each block is calculated in parallel and is concatenated as shown in Fig.6. This gives the feature vector used for training the classifier. LBP operator[6] is defined as

$$
L B P_{P, R}\left(x_{c}, y_{c}\right)=\sum_{p=0}^{p-1} s\left(g_{p}-g_{c}\right) 2^{p}
$$

where $g_{p}$ is the intensity of the image at the $p_{t h}$ sample point where $p$ is the total number of the sample point at a radius of $\mathrm{R}$ denoted by $(\mathrm{P}, \mathrm{R})$. The $\mathrm{P}$ spaced sampling points of the window are used to calculate the difference between center $\mathrm{gc}$ and its surrounding pixel[5]. The feature vector of the image obtained after cascading the histogram is,

$$
H_{s}(p, k)=\sum_{i=1}^{I} \sum_{j=1}^{J} f\left(L B P_{P, R}, P\right)
$$

where $\mathrm{k}$ is an integer to represent the sub histogram that is obtained from each block $\mathrm{k}=1,2 \ldots \mathrm{K} . \mathrm{K}$ is the total no of histograms , and $\mathrm{f}(\mathrm{x}, \mathrm{y})=\left(\begin{array}{l}1, x=y \\ 0 \text { othorwisd }\end{array}\right)$ where $\mathrm{f}(\mathrm{x} ; \mathrm{y})$ is the LBP calculated value at pixel $(\mathrm{x}, \mathrm{y})$.

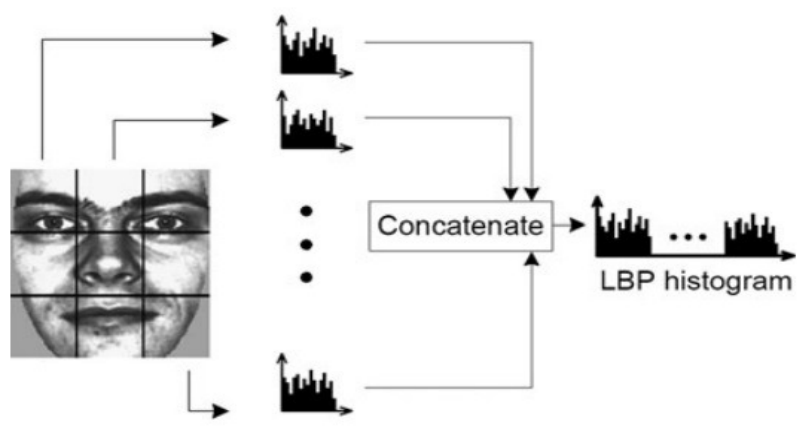

Fig. 6. LBP in Face detection 


\section{2. classifier}

There are many methods to determine the dissimilarity between the LBP pattern, here chi-square method is used and further work is going on with SVM for training and classifying the LBP feature. The chi-square distance used to measure the dissimilarity between two LBP images $\mathrm{S}$ and $\mathrm{M}$ is given by

$$
\mathrm{D}(\mathrm{S}, \mathrm{M})=\sum_{\mathrm{i}=1}^{\mathrm{L}}\left(S_{x}-M_{x}\right)^{2} /\left(S_{x}+M_{x}\right)
$$

where $L$ is the length of the feature vector of the image and $S_{x}$ and $M_{x}$ are respectively the sample and model image in the respective bin.

\section{IMPLEMENTATION}

The goal of this paper is to implement LBP algorithm on a cpu, gpu based heterogeneous platform using OpenCL, to reduce computation time. The process of LBP feature extraction and histogram calculation from the image is computationally expensive $\left(\mathrm{N}^{2} \mathrm{xW}^{2}\right.$, where $\mathrm{NxN}$ is size of image and $\mathrm{WxW}$ is size of LBP block) and it is easy to figure out that the extraction in different parts are independent as discussed in section 3 . Thus it can be efficiently parallelized [7].

For real time implementation, first the image is captured using OpenCV and is converted to a grey scale image. Then preprocessing of the image is done. To figure out different features in the image, various algorithms are implemented on the image. For parallel processing of the algorithm, the image is subdivided into smaller parts. In this case the image is divided into $16 \mathrm{x}$ 16 pixels blocks. The task of calculating LBP for each block is given to work items. So there are different work items processing different blocks in parallel. Each work item processes 256 pixels and different work items work in parallel, reducing the processing time up to a significant extent. So, to calculate the LBP of 16x16 pixels blocks the image is converted to an one dimensional array. A global reference of the image is used by each work item for creating one $18 \mathrm{x} 18$ two dimensional matrix to find out the LBP for each block. From the 18 x 18 matrix, LBP is calculated as discussed in section 3 for $16 \times 16$ pixels not considering the boundary pixel of $18 \mathrm{x}$ 18 matrix . Afterwards the calculated values for LBP are processed to form a histogram ranging from 0 to 255. Each work item formulates a histogram accordingly for one block and the different histograms are cascaded to form the actual histogram for the complete image in order to get the LBP feature vector as shown in Fig.7. This feature vector is then classified using nearest neighbourhood method.

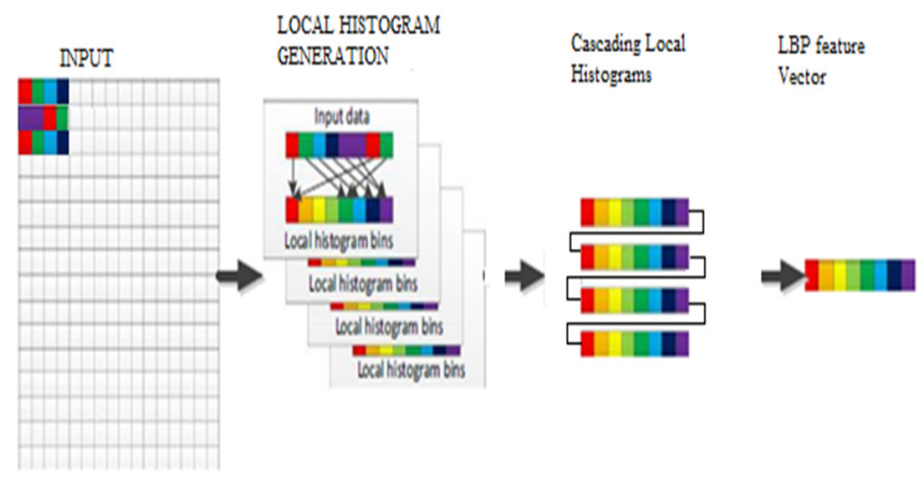

Fig. 7. Histogram Calculation on GPU 
The Block Diagram Of The Overall Method Is Shown In Fig.8

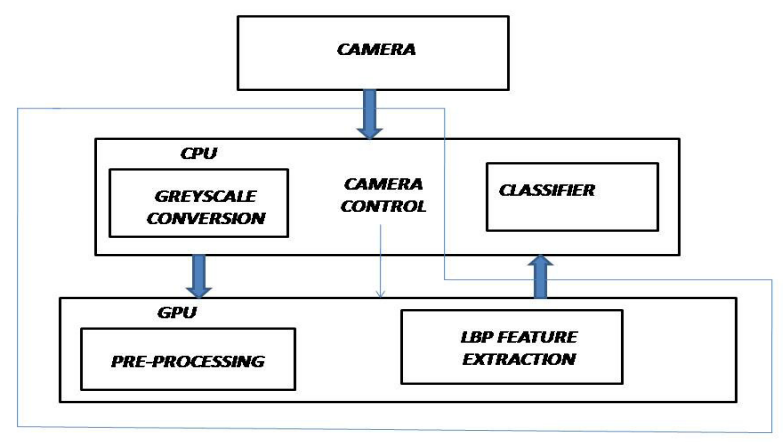

Fig. 8. Block diagram of the overall method

\section{RESULTS}

In the proposed paper we have calculated each block in different compute units. Since the calculation of histogram depends on all the pixels within a block thus it is better to do the whole calculation within one compute unit. Additionally, the amount of computation per compute unit shouldn't be too small otherwise the overhead associated with managing a compute unit will be more than the actual computation. Since the whole computation is done in the GPU and only the input image and the final histogram are transferred between the CPU and GPU thus overheads associated with data transfer are minimal. As a result the computational time is $20 \mathrm{~ms}$. The performance table of the implementation is shown in Table 1.

Table 1. Performance Table

\begin{tabular}{|l|l|}
\hline Input Resolution & $640 \times 480$ \\
\hline Sub Histograms & 256 \\
\hline CPU(i5 3rd generation) & $109 \mathrm{~ms}$ \\
\hline AMD(7670M) & $20 \mathrm{~ms}$ \\
\hline
\end{tabular}

Table 2. Comparison With Previous Work

\begin{tabular}{|l|l|l|}
\hline & PREVIOUS WORK[8] & OUR WORK \\
\hline Image size & $512 \times 512$ & $640 \times 480$ \\
\hline Sub Histograms & 256 & 256 \\
\hline Feature Extraction & $36.3 \mathrm{~ms}$ & $20 \mathrm{~ms}$ \\
\hline
\end{tabular}

Compared to previous work[8], the image is grabbed from camera and processed in real time. As can be seen from TABLE 2, computational time for our implementation is less. Performance of the proposed paper is tested on AMD 7670M GPU, i5 3rd generation CPU based system. Total time for feature extraction on CPU was $108 \mathrm{~ms}$ and on GPU was $20 \mathrm{~ms}$ for $640 \mathrm{X} 480$ resolution input image. Thus we get a $5 x$ improvement in speed using GPU implementation. 


\section{CONCLUSION}

In this paper real time face detection using LBP feature extraction is done and is classified using nearest neighbourhood method. We have parallelized the existing LBP algorithm to make it suitable for implementation on SIMD architecture such as GPGPU. Performance gain has been achieved over previous implementations.

\section{REFERENCES}

[1] Xiaoyang Tan; Triggs, B., "Enhanced Local Texture Feature Sets for Face Recognition Under Difficult Lighting Conditions," Image Processing, IEEE Transactions on , vol.19, no.6, pp.1635,1650, June 2010 doi: 10.1109/TIP.2010.2042645

[2] Computational Imaging and Vision, Vol. 40 Pietikinen, M., Hadid, A.,Zhao, G., Ahonen, T. 2011, XVI, 212 p.

[3] KHRONOS:OpenCLoverviewwebpage, http://www.khronos.org/opencl/,2009.

[4] Aaftab Munshi, Benedict Gaster, Timothy G. Mattson, James Fung, Dan Ginsburg ISBN: 978-0-3217 4964-2

[5] TY - JOUR T1 - Facial expression recognition based on Local Binary Patterns: A comprehensive study JO - Image and Vision Computing VL - 27 IS - 6 SP - 803 EP - 816 PY - 2009/5/4/ T2 - AU Shan, Caifeng AU - Gong, Shaogang AU - McOwan, Peter W. SN - 0262-8856 DO http://dx.doi.org/10.1016/j.imavis.2008.08.005

UR-http://www.sciencedirect.com/science/article/pii/S0262885608001844 KW - Facial expression recognition KW - Local Binary Patterns KW - Support vector machine KW - Adaboost KW - Linear discriminant analysis KW - Linear programming ER

[6] Caifeng Shan; Shaogang Gong; McOwan, Peter W., "Robust facial expression recognition using local binary patterns," Image Processing, 2005. ICIP 2005. IEEE International Conference on , vol.2, no., pp.II,370-3, 11-14 Sept. 2005 doi: 10.1109/ICIP.2005.1530069

[7] Miguel Bordallo Lpez ; Henri Nyknen ; Jari Hannuksela ; Olli Silvn and Markku Vehvilinen "Accelerating image recognition on mobile devices using GPGPU", Proc. SPIE 7872, Parallel Processing for Imaging Applications, 78720R (January 25, 2011); doi:10.1117/12.872860; http://dx.doi.org/10.1117/12.872860

[8] Parallel Implementation of LBP Based Face recognition on GPU Using OpenCL Dwith, C.Y.N. ; Rathna, G.N. Parallel and Distributed Computing, Applications and Technologies (PDCAT), 2012 13th International Conference on Digital Object Identifier: 10.1109/PDCAT.2012.107 Publication Year: 2012 , Page(s): 755 - 760 


\title{
POSITIVE IMPRESSION OF LOW-RANKING MICRORN AS IN HUMAN CANCER CLASSIFICATION
}

\author{
Feifei Li, Yongjun Piao, Meijing Li, Minghao Piao, Keun Ho Ryu \\ DataBase\&Bioinformatics Laboratory, Dept. of Computer Science, \\ Chungbuk National University, Cheongju, South Korea \\ \{feifeili, pyz, mjlee, bluemhp, khryu\}@dblab.chungbuk.ac.kr
}

\begin{abstract}
Recently, many studies based on microRNAs (miRNAs) showed a new aspect of cancer classification, and feature selection methods are used to reduce the high dimensionality of miRNA expression data. These methods just consider the problem of where feature to class is 1:1 or n:1. But one miRNA may have influence to more than one type of cancers. However, these miRNAs are considered to be low ranked in traditional feature selection methods and they are removed at most of time. Therefore, it is necessary to consider the problem of 1:n or m:n during feature selection. In our wok, we considered both high and low-ranking features to cover all problems $(1: 1, n: 1,1: n, m: n)$ in cancer classification. After numerous tests, information gain and chi-squared feature selection methods were chosen to select the high and low-ranking features to form the m-to-n feature subset, and LibSVM classifier was used to do the multi-class classification. Our results demonstrate that the m-to-n features make a positive impression of low-ranking microRNAs in cancer classification since they lead to achieve higher classification accuracy compared with the traditional feature selection methods.
\end{abstract}

\section{KEYWORDS}

low-ranking features, feature selection, cancer classification, microRNA

\section{INTRODUCTION}

Feature selection, as we know, is aimed to remove the redundant and irrelevant features to find a subset of features. Feature selection method involves two aspects: evaluation of a candidate feature subset using some evaluation criterion, and searching through the feature space to choose a minimum subset of features. Usually, the categories of feature selection algorithms can be identified based on their theoretical basis: correlation, distance, information, dependence, consistency and classifier error rate measures.

The correlation-based feature selection method uses some measures like information gain [1], gain ratio, or linear correlation coefficient [2] to find the good features that highly correlated with the class but not highly correlated with other features. Then these features will be relevant to the class concept but not redundant to any of the other relevant features. And the correlation-based feature selection method has been widely used for many kinds of classification analysis. For the

David C. Wyld et al. (Eds) : CCSIT, SIPP, AISC, PDCTA, NLP - 2014

pp. 449-461, 2014. (C) CS \& IT-CSCP 2014

DOI : $10.5121 /$ csit.2014.4239 
mutual-information-based feature selection, the largest mutual information reflects the largest dependency in the target class, so the top features are often selected. In research of [3], they proposed a minimal-redundancy-maximal-relevance (mRMR) method, and that can use either mutual information, correlation, distance scores to select features, then tested it with different classifiers, i.e., naive Bayes (NB), support vector machine (SVM), and linear discriminant analysis (LDA). Their results showed that the mRMR can improve the classification accuracy for both discrete and continuous data sets and multiple types of classifiers. Consistency-based search approach [4] uses the inconsistency rate to solve the problem that two instances have the same feature values but have different class labels. This measure is aimed to search in the set of features and find a minimal set of features which are consistent. Feature ranking method sorts features based on the criterion measure, and the criterion measure can be the information, the relevance, or the relation of the features.

Recently, these feature selection methods have been used for cancer classification. With the discovery of microRNAs (miRNAs), a class of small non-coding RNAs, which have been proved that the abnormal expression data can indicate human cancer $[5,6]$, many feature selection and classification methods have been used to do the miRNA expression data analysis for cancer classification. However, from the year 1993 when the first identified miRNA [7] has been discovered until now, only more than one thousand miRNAs have been discovered. One special characteristic of miRNA expression data is the high dimensionality. The high dimensionality may cause a series of problems for cancer classification, such as add noise, reduce the accuracy rate, and increase the complexity. Although we can use both feature selection and feature extraction to reduce the dimensionalities, feature selection is a better choice than feature extraction for miRNA expression data analysis: feature selection is used in the area where there are a large number of features compared with the small number of samples which is just the characteristic of miRNA expression data; the feature extraction is aimed to create new features using some transform functions of the original features, but these new features maybe cannot be explained in the physical aspect.

However, these methods just consider the condition that the relationship between feature and class is $1: 1$ or $\mathrm{n}: 1$, but not consider the condition that the relationship between feature and class is 1:n or m:n. But since the miRNA expression data is a special kind of data, one miRNA may has influence to more than one type of cancers [8]. If using the traditional feature selection algorithms, these miRNAs may be deleted, since they will be considered as the low-ranking features. But this kind of miRNAs are also very important, removing them may lead to the loss of important information. In Lu et al.'s work [9], they used bead-based flow cytometric miRNA expression profiling method to analyze the 217 mammalian miRNAs from 334 samples including human cancers. And the result showed the potential of miRNA profiling in cancer diagnosis. Based on this data resource, many works using different feature selection methods and classification methods have done to do the cancer classification [10-12]. Most of them based on the binary-class classification, and they showed very high accuracy results. However, these work just considered the high-ranking microRNAs. Therefore, in our study, we made a new hypothesis that consider both of the high and low-ranking features to cover all the cases (1:1, n:1, 1:n, m:n) can get better accuracy in the cancer classification. We used the data resource from Lu et al.'s work, also used different kinds of feature ranking methods with different classifiers to do the analysis. Finally, the results proved that the m-to-n features can get higher classification accuracy compared with the traditional feature selection methods, and it is reasonable to take the lowranking features into consideration for cancer classification.

The remainder paper is organized as follows. The methods used in the work are discussed in Section 2. Section 3 is about the data set and performance evaluation. The conclusion of our work is presented in Section 4. 


\section{FeATURE SELECTION AND ClASSIFICATION METHODS}

Since there is no evidence to show which kind of feature selection and classification method would fit for miRNA expression data, we chose many different kinds of methods to do the analysis and compared their results.

For feature selection, we used the correlation-based feature selector (CFS) with different search algorithms: re-ranking search algorithm [13], best first search algorithm, particle swarm optimization (PSO) search algorithm [14, 15], and tabu search algorithm [16, 17]. We also used the ranker search method with different attribute evaluators: Pearson's correlation, chi-squared distribution, information gain, and gain ratio.

Re-ranking search algorithm first uses a filter measure to rank all the attributes in decreasing order, the ranking is split in many blocks, and then runs a filter-wrapper algorithm over the first block to select some attributes. Then the remaining attributes are re-ranked again. And the filterwrapper algorithm is run again on the first current block, and so on. The process is iterated until no attribute is selected in current block. The re-ranking search algorithm can reduce the CPU time and wrapper evaluations compared with the incremental feature subset selection algorithms.

Best first search algorithm first evaluates all the features regard them as a separate subset. And the feature subset which has the highest object function is selected. Then the algorithm updates the subset by adding all the possible combinations of new single features. After that the algorithm evaluates the new subset, if the result is improved then the features are retained otherwise the process searches other features to expend the subset. The process will stop when there is no improvement by adding new features.

Particle swarm optimization (PSO) is usually applied to continuous search spaces. It is a population-based heuristic global optimization algorithm for feature selection. In the PSO algorithm, particle swarm involves n particles, each particle has a randomized velocity based an objective function. And the particle iteratively changes its position to find most optimist position of particle itself and swarm. The algorithm stops until a termination criterion is met.

Tabu search is a metaheuristic search method used for solving mathematical optimization problems. It is a form of local neighborhood search. The tabu search algorithm starts with a current solution, and evaluates the criterion function for that solution. Then the algorithm stores the neighboring solutions in the candidate list. And then it finds the best candidate from the candidate list, if the candidate has a higher criterion function value than the current best, its features are add to the tabu list and it is viewed as the new best. The process is looped until the stop criterion is met. At last the best solution obtained so far is the solution of the tabu search.

The Pearson's correlation coefficient is used to compute the correlation between the feature and the class variable. It is defined by the following equation (1):

$$
\operatorname{PCC}=\frac{\sum\left(\mathrm{x}_{\mathrm{i}}-\overline{\mathrm{x}}\right)\left(\mathrm{c}_{\mathrm{i}}-\overline{\mathrm{c}}\right)}{\sqrt{\sum\left(\mathrm{x}_{\mathrm{i}}-\overline{\mathrm{x}}\right)^{2} \sum\left(\mathrm{c}_{\mathrm{i}}-\overline{\mathrm{c}}\right)^{2}}}
$$

where the index ci is the class label. The Pearson's correlation coefficient ranges from -1 and 1. If the value is 1 (or -1) that means the feature and class label have a perfect positive (or negative) 
linear relationship. However, if the value is 0 , then there is no linear relationship between the feature and class label.

Chi-squared attribute evaluation evaluates a feature by computing the chi-squared statistic of the feature with respect to the class label. First the hypothesis $\mathrm{H} 0$ is assumed as the two features are unrelated. Then it is tested using the following equation (2):

$$
\chi^{2}=\sum_{i=1}^{r} \sum_{j=1}^{c} \frac{\left(O_{i j}-E_{i j}\right)^{2}}{E_{i j}}
$$

where Oij is the observed frequency and Eij is the expected frequency, the larger the value of $\chi^{2}$ is, the more evidence to show that the hypothesis $\mathrm{H} 0$ is true.

Information gain measures the expected reduction in entropy. The entropy is a measure of the uncertainty in a random variable. Based on it, the information gain of a feature is defined as the following equation (3):

$$
\Delta_{\text {info }}=\operatorname{Entropy}(C)-\sum_{j=1}^{k} \frac{\left|C_{\mathrm{v}}\right|}{|C|} \operatorname{Entropy}\left(C_{v}\right)
$$

where $\mathrm{k}$ is the number of attribute values, $\mathrm{C}$ is a collection of samples and $\mathrm{Cv}$ is the subset of collection $\mathrm{C}$ for attribute which has the value of $\mathrm{v}$. The higher value indicates the higher purity of class.

Gain ratio is aim to maximize the information gain of feature and minimize the number of its value. Gain ratio is the ratio between the information gain and intrinsic value defined as the following equation (4):

$$
\text { Gain Ratio }=\frac{\Delta_{\text {info }}}{-\sum_{\mathrm{j}=1}^{\mathrm{k}} \frac{\left|\mathrm{C}_{\mathrm{V}}\right|}{|\mathrm{C}|} \log _{2} \frac{\left|\mathrm{C}_{\mathrm{V}}\right|}{|\mathrm{C}|}}
$$

For classification methods, we chose four different kinds of classifiers: support vector machine $(\mathrm{SVM})$, naive Bayes, k-nearest neighbors $(\mathrm{KNN})$, and decision tree. The SVM constructs a hyperplane or sets of hyperplanes in a high dimensional space, and aims to find the largest margin to separate the objects of different classes. To build the SVM classifier, we used the LibSVM package [18] since it can support both 2-class and multi-class classification. A naive Bayes classifier assumes that each feature is independent to others. And a naive classifier is based on the Bayes' theorem and is very efficiently in supervised learning. For the naive Bayes classifier, we adopted the Aggregating One-Dependence Estimators (A1DE) algorithm [19]. This algorithm can solve the attribute-independence problem of the traditional naive Bayed classifier. The k-nearest neighbors classifier can predict the class label of the object based on the $\mathrm{k}$ closest objects in the feature space. The KNN algorithm is almost the simplest of all machine learning algorithms. Decision tree classifier is a form of a tree structure. In the tree, each internal node represents a test on an attribute, each branch represents the outcome of the test, and each leaf node represents a class label. The path from the root to the leaf represents classification rules. And we chose the 
C4.5 algorithm to build the decision tree classifier. This algorithm uses information gain as the splitting criterion.

\section{EXPERIMENTAL RESULTS}

\subsection{Data Set}

The miRNA expression data used in this paper is from Lu et al.'s work. It is used to build a multiclass classifier, it consists of five kinds of tumor samples from colon, uterus, pancreas, T-cell ALL, and B-cell ALL which totally includes 73 samples with the expression value of 217 miRNAs for multiple cancer types. The detail of the cancer types shows in Table 1.

Table 1 . The number of the samples for each cancer type

\begin{tabular}{lc}
\hline Cancer Name & Number of Tumor Samples \\
\hline Colon & 10 \\
Pancreas & 9 \\
Uterus & 10 \\
B Cell ALL & 26 \\
T Cell ALL & 18 \\
\hline SUM & 73 \\
\hline
\end{tabular}

\subsection{Performance Evaluation}

To get a reliable result, 10 -fold cross validation is performed on the whole data set. And the data set is randomly divided into 10 parts, nine of them are used as training set, the rest part is used as test set.

In our study, we first used the correlation-based feature subset selection methods with four different search methods: re-ranking search, best first search, tabu search and PSO search method. Using these search methods can automatically select the features with the exactly number. For comparison, we tested these features on four classifiers including LibSVM algorithm of SVM classifier, A1DE algorithm of naive Bayes classifier, J48 algorithm of decision tree classifier and IBK algorithm of k-nearest neighbor classifier. Table 2 shows the final results, after feature selection, the re-ranking search method resulted in 15 top-ranking features, the best search method resulted in 16 top-ranking features, the tabu search method resulted in 17 top-ranking methods, and the PSO search method resulted in 50 top-ranking features. The LibSVM algorithm shows the better results with the accuracy of $91.78 \%$ without feature selection. However, after we reduced the dimensionality of miRNA expression data, the result became quite different. With the feature selection, most of the accuracies of the classification methods have been increased except LibSVM classifier. The A1DE classifier got better result when using the re-ranking, best first, and tabu search method. And when using the PSO search method, the results of J48 and IBK classifiers have been increased. The result indicated that feature selection is very necessary for cancer classification. However, these methods just selected the fit number of features, since the number is very small, we cannot find how the feature number influences the classification accuracy. Therefore, we need to use other methods to find the relationship between feature number and classification accuracy. 
Table 2. Classification accuracy (\%) of four classification algorithms (FS: feature selection).

\begin{tabular}{lllll}
\hline Method & LibSVM & A1DE & J48 & IBK \\
\hline Without FS & 91.78 & 86.30 & 86.30 & 83.56 \\
Re-ranking & 90.41 & 89.04 & 84.93 & 83.56 \\
Best First & 90.41 & 89.04 & 84.93 & 83.56 \\
Tabu & 90.41 & 90.41 & 84.93 & 82.91 \\
PSO & 89.04 & 84.93 & 87.67 & 86.30 \\
\hline
\end{tabular}

Classification algorithms: LibSVM, A1DE, J48 and IBK. Feature selection method: correlationbased subset selection algorithm with different search methods including re-ranking search, best first search, tabu search and PSO search method. The selected high-ranking feature number with these four methods is : $15,16,17$ and 50 .

In order to find the relationship, we did another experiment used the Pearson's correlation, chisquared distribution, information gain, and gain ratio as the attribute evaluators to do the feature selection. The LibSVM package solved the quadratic problems and used shrinking and caching methods to reduce the size of working problem, also in the last experimental results the LibSVM got better accuracy compared with other classification methods. Therefore, the LibSVM package was chosen as the classifier. Figure 1, 2, 3, 4 shows the classification accuracy for the four kinds of feature selection methods (i.e. Pearson's correlation, chi-squared, gain ratio, information gain) with LibSVM classifier. The top-ranking feature number that we chose for test is from 10 to 210. Compared both the results of the four feature selection methods, the Pearson' correlation method and gain ratio method show the similar results, and the chi-squared method and information gain method show the similar results. For Pearson's correlation method and gain ratio method, when the feature number is very small, the accuracy is very low, but the accuracy of chi-squared method and information gain method is high. For both of these feature selection methods, there is a same trend that with the increase of the feature numbers the accuracy is also been improved. Also figure 5 is the histogram of these four feature selection methods. When the feature number is smaller than 20, the chi-squared and information gain methods show higher accuracy comparing with the Pearson's correlation and gain ratio methods. When the feature number is between 50 and 140, the Pearson's correlation and gain ratio methods show the better result. When the feature number is larger than 170, these methods show the similar high accuracy.

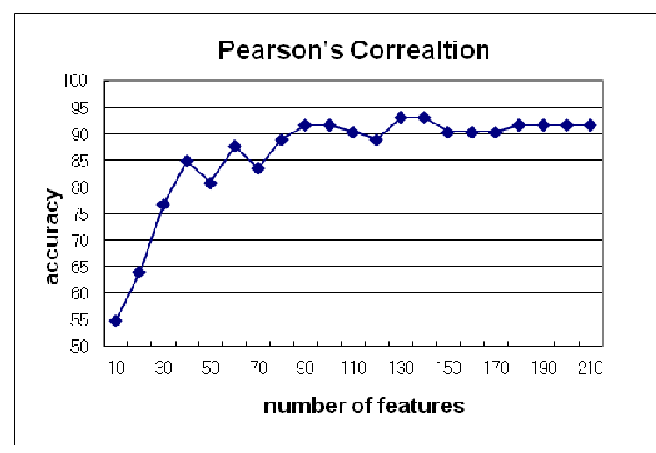

Figure 1. Classification accuracy (\%) for the Pearson's correlation feature selection method with LibSVM classifier. The number of the selected high-ranking features is from 10 to 210 . 


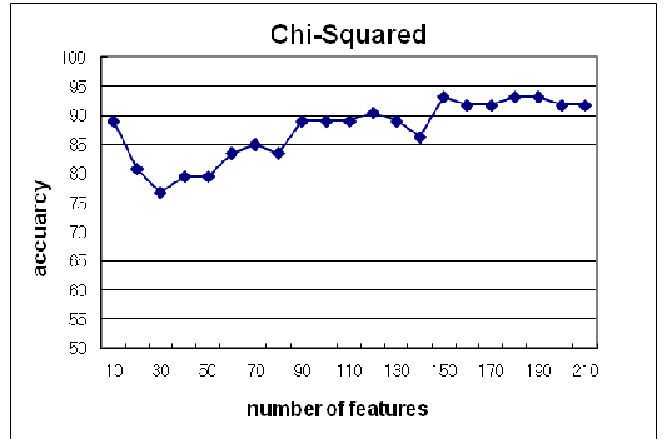

Figure 2. Classification accuracy (\%) for the chi-squared feature selection method with LibSVM classifier. The number of the selected high-ranking features is from 10 to 210 .

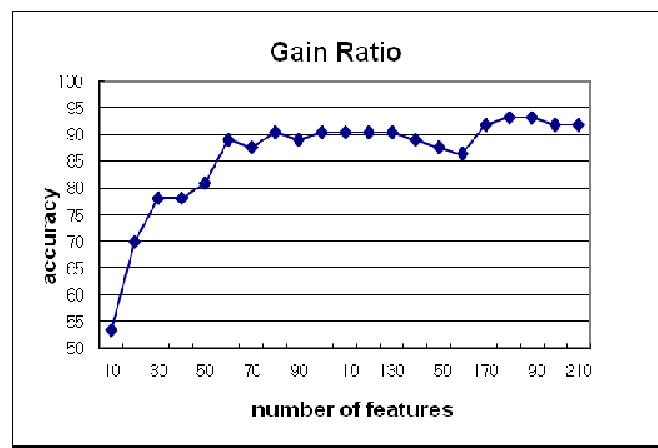

Figure 3. Classification accuracy for gain ratio feature selection method with LibSVM classifier. The number of the selected high-ranking features is from 10 to 210.

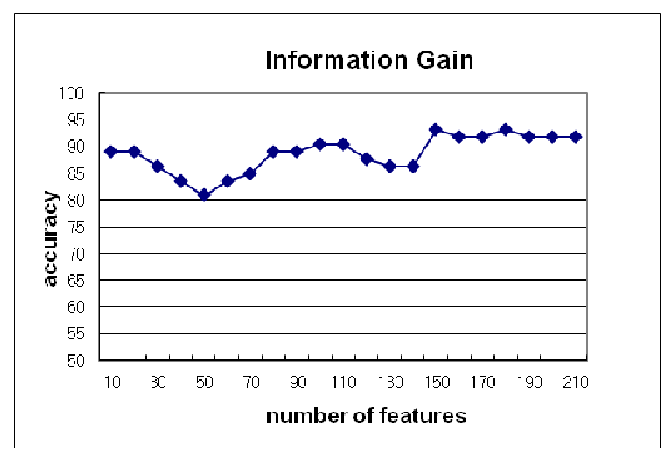

Figure 4. Classification accuracy for information gain feature selection method with LibSVM classifier.

The number of the selected high-ranking features is from 10 to 210 . 


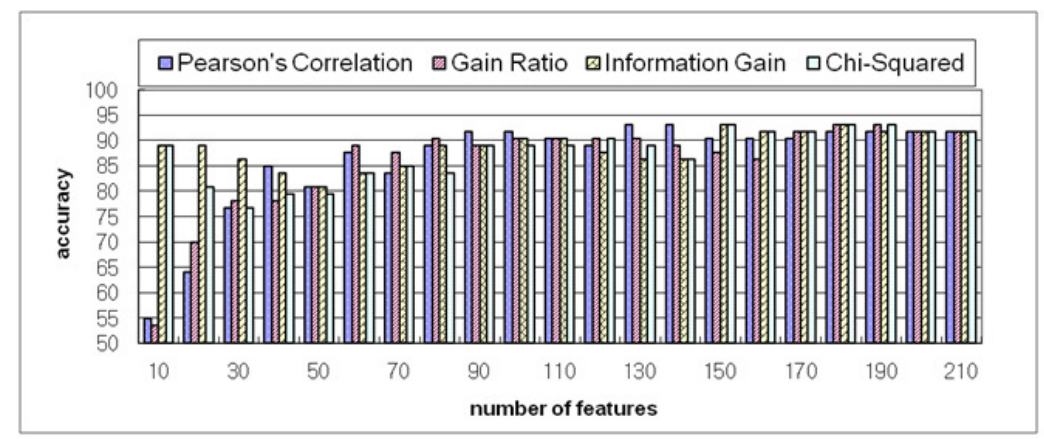

Figure 5. The histogram of the four feature selection methods (i.e. Pearson's correlation, gain ratio, information gain, chi-squared) with LibSVM classifier. The number of the selected high-ranking features is from 10 to 210 .

Both of these feature selection methods select the high-ranking features, if we want to get the higher accuracy, the feature number should be large enough, but the large number is meaningless. Therefore, we considered both the high and low-ranking features to form the m-to-n feature subset. The previous experiment shows that the information gain and chi-squared feature selection methods are totally better compared with the other two methods. Because when the feature number is small, the Pearson's correlation and gain ratio feature selection methods show very low classification accuracy which means these selected top-ranking features cannot excellent classify the miRNA data. Considering this reason, the information gain and chi-squared feature selection methods were used to form the feature subsets with both the high and low-ranking features, and as well the LibSVM package of SVM classifier was chosen for the multiple classification problems.

The result is showed in Table 3. First we chose 10 high-ranking features, and that means the relationship between feature and class is 1 to 1 or $n$ to 1 . The information of selected high-ranking microRNA is showed in table Table 4 and Table 5 . The classification accuracy is $89.04 \%$ for both of the two feature selection methods. Then we considered the case of the feature to class is $1: \mathrm{n}$, in this case we selected 17 low-ranking features. The information of selected low-ranking microRNA is showed in Table 6 and Table 7. The classification accuracy of information gain method is $52.05 \%$ while the classification accuracy of chi-squared method is $50.68 \%$. Obviously the accuracy is very low since the low-ranking features would lead to the impurity of the class. At last, we considered the m-to-n features with both the high-ranking and low-ranking features, and in this condition feature to class is m:n. We combined both the 10 high-ranking features and 17 low-ranking features together, totally 27 features, and used them to do the classification, surprisingly, we got a very good result, with the classification accuracy of information gain method is $94.52 \%$ and the classification accuracy of chi-squared method is $93.14 \%$. In the work of [11], they used the Default ARTMAP as the classifier to do the multi-class cancer classification with the same data set as in our work. But the best result only has the accuracy of $88.89 \%$. Compared with this work and our first experiment in Table 2, feature selection with the m-to-n features got the highest classification accuracy. The result also proved that it is reasonable to take the low-ranking features into consideration when doing cancer classification. 
Table 3. Classification accuracy for LibSVM classifier considering the high-ranking and low-ranking features. 1:1, n:1, 1:n and m:n indicate the relationship between feature and class.

\begin{tabular}{lll}
\hline Relationship & Information Gain & Chi-Squared \\
\hline $1: 1$ or $\mathrm{n}: 1$ & 89.04 & 89.04 \\
$1: \mathrm{n}$ & 52.05 & 50.68 \\
m:n & 94.52 & 93.14 \\
\hline
\end{tabular}

Table 4. The information of 10 high-ranking microRNA selected by Information Gain method.

\begin{tabular}{lll}
\hline Probe ID & Target Sequence & MicroRNA Name \\
\hline EAM250 & AUGACCUAUGAAUUGACAGAC & hsa-miR-215 \\
EAM330 & UGUAAACAUCCUCGACUGGAAGC & hsa-miR-30a-5p \\
EAM105 & UCCCUGAGACCCUAACUUGUGA & hsa-miR-125b \\
EAM348 & CAUCAAAGUGGAGGCCCUCUCU & mmu-miR-291-5p \\
EAM190 & UACCCUGUAGAACCGAAUUUGU & hsa-miR-10b \\
EAM288 & CCCUGUAGAACCGAAUUUGUGU & mmu-miR-10b \\
EAM366 & UUCAGCUCCUAUAUGAUGCCUUU & mmu-miR-337 \\
EAM261 & AUCACAUUGCCAGGGAUUACCAC & hsa-miR-23b \\
EAM260 & AUCACAUUGCCAGGGAUUUCC & hsa-miR-23a \\
EAM381 & UCGAGGAGCUCACAGUCUAGUA & rno-miR-151* \\
\hline
\end{tabular}

Table 5. The information of 10 high-ranking microRNA selected by Chi-Squared method.

\begin{tabular}{lll}
\hline Probe ID & Target Sequence & MicroRNA Name \\
\hline EAM250 & AUGACCUAUGAAUUGACAGAC & hsa-miR-215 \\
EAM190 & UACCCUGUAGAACCGAAUUUGU & hsa-miR-10b \\
EAM288 & CCCUGUAGAACCGAAUUUGUGU & mmu-miR-10b \\
EAM105 & UCCCUGAGACCCUAACUUGUGA & hsa-miR-125b \\
EAM366 & UUCAGCUCCUAUAUGAUGCCUUU & mmu-miR-337 \\
EAM381 & UCGAGGAGCUCACAGUCUAGUA & rno-miR-151* \\
EAM303 & UACAGUAGUCUGCACAUUGGUU & hsa-miR-199a* \\
EAM336 & AGGCAGUGUAGUUAGCUGAUUGC & hsa-miR-34c \\
EAM339 & CACCCGUAGAACCGACCUUGCG & hsa-miR-99b \\
EAM260 & AUCACAUUGCCAGGGAUUUCC & hsa-miR-23a \\
\hline
\end{tabular}


Table 6. The information of 17 low-ranking microRNA selected by Information Gain method.

\begin{tabular}{lll}
\hline Probe ID & Target Sequence & MicroRNA Name \\
\hline EAM247 & UAACAGUCUCCAGUCACGGCC & hsa-miR-212 \\
EAM252 & UACUGCAUCAGGAACUGAUUGGAU & hsa-miR-217 \\
EAM254 & UGAUUGUCCAAACGCAAUUCU & hsa-miR-219 \\
EAM259 & UGUCAGUUUGUCAAAUACCCC & hsa-miR-223 \\
EAM283 & UUCCCUUUGUCAUCCUUUGCCU & mmu-miR-211 \\
EAM293 & CAUCCCUUGCAUGGUGGAGGGU & hsa-miR-188 \\
EAM306 & UACUCAGUAAGGCAUUGUUCU & mmu-miR-201 \\
EAM308 & UGGAAUGUAAGGAAGUGUGUGG & hsa-miR-206 \\
EAM309 & GCUUCUCCUGGCUCUCCUCCCUC & mmu-miR-207 \\
EAM328 & CAGUGCAAUAGUAUUGUCAAAGC & hsa-miR-301 \\
EAM331 & UGUAAACAUCCUUGACUGGA & hsa-miR-30e \\
EAM337 & CAAAGUGCUGUUCGUGCAGGUAG & hsa-miR-93 \\
EAM340 & CUAUACGACCUGCUGCCUUUCU & mmu-let-7d* \\
EAM341 & CAAAGUGCUAACAGUGCAGGUA & mmu-miR-106a \\
EAM346 & CUCAAACUAUGGGGGCACUUUUU & mmu-miR-290 \\
EAM352 & AAAGUGCUUCCCUUUUGUGUGU & mmu-miR-294 \\
EAM361 & CCUCUGGGCCCUUCCUCCAGU & hsa-miR-326 \\
\hline
\end{tabular}

Table 7. The information of 17 low-ranking microRNA selected by Chi-Squared method.

\begin{tabular}{lll}
\hline Probe ID & Target Sequence & MicroRNA Name \\
\hline EAM247 & UAACAGUCUCCAGUCACGGCC & hsa-miR-212 \\
EAM252 & UACUGCAUCAGGAACUGAUUGGAU & hsa-miR-217 \\
EAM254 & UGAUUGUCCAAACGCAAUUCU & hsa-miR-219 \\
EAM259 & UGUCAGUUUGUCAAAUACCCC & hsa-miR-223 \\
EAM283 & UUCCCUUUGUCAUCCUUUGCCU & mmu-miR-211 \\
EAM290 & UGGACGGAGAACUGAUAAGGGU & hsa-miR-184 \\
EAM293 & CAUCCCUUGCAUGGUGGAGGGU & hsa-miR-188 \\
EAM308 & UGGAAUGUAAGGAAGUGUGUGG & hsa-miR-206 \\
EAM309 & GCUUCUCCUGGCUCUCCUCCCUC & mmu-miR-207 \\
EAM324 & CAUUGCACUUGUCUCGGUCUGA & hsa-miR-25 \\
EAM328 & CAGUGCAAUAGUAUUGUCAAAGC & hsa-miR-301 \\
EAM331 & UGUAAACAUCCUUGACUGGA & hsa-miR-30e \\
EAM337 & CAAAGUGCUGUUCGUGCAGGUAG & hsa-miR-93 \\
EAM340 & CUAUACGACCUGCUGCCUUUCU & mmu-let-7d* \\
EAM341 & CAAAGUGCUAACAGUGCAGGUA & mmu-miR-106a \\
EAM346 & CUCAAACUAUGGGGGCACUUUUU & mmu-miR-290 \\
EAM352 & AAAGUGCUUCCCUUUUGUGUGU & mmu-miR-294 \\
\hline & & \\
\hline
\end{tabular}




\section{CONCLUSIONS}

The right choice of feature selection and classification method is very important to cancer classification since the special characteristic of miRNA expression data. After numerous tests, the information gain and chi-squared feature selection methods were chosen to do the dimensionality reduction. Different with the traditional feature selection, we considered all cases (1:1, n:1, 1:n, $\mathrm{m}: \mathrm{n})$ in cancer classification. Our work has proved the usefulness of the m-to-n features in cancer classification, since the results showed that considering both the high-ranking and low-ranking features can get higher classification accuracy than just considering the high-ranking features. And the selected low-ranking miRNAs in Table 6 and Table 7 provide cancer researchers some very useful information for further research analysis of their function in human cancer. However, there have some shortcomings: we have tested for many times to find a relatively good number of the m-to-n features to do the analysis, but in fact it is very difficult to determine the best number of the selected features.

In the future work, we will do our best to discover some feature selection algorithms which can choose the appropriate m-to-n feature number automatically. Also we will try to use this idea to test for other kinds of data not only the miRNA expression data.

\section{ACKNOWLEDGEMENTS}

This research was supported by Basic Science Research Program through the National Research Foundation of Korea (NRF) funded by the Ministry of Science, ICT \& Future Planning (No.2013R1A2A2A01068923) and the National Research Foundation of Korea (NRF) grant funded by the Korea government (MEST) (No. 2008-0062611).

\section{REFERENCES}

[1] Lei Yu, Huan Liu: Feature selection for high-dimensional data: a fast correlation-based filter solution. AAAI. 856-863 (2003).

[2] Rajdev Tiwari, Manu Pratap Singh: correlation-based attribute selection using genetic algorithm. IJCA. 4, 28-34 (2010).

[3] Hanchuan Peng, Fuhui Long, Chris Ding: Feature selection based on mutual information: criteria of max-dependency, max-relevance, and min-redundancy. IEEE. 27, 1226-1238 (2005).

[4] Manoranjan Dash, Huan Liu: Consistency-based search in feature selection. Artificial Intelligence. 151, 155-176 (2003).

[5] He L, Thomson JM, Hemann MT, Hernando-Monge E, Mu D, Goodson S, Powers S, Cordon-Cardo C, Lowe SW, Hannon GJ, Hammond SM: A microRNA polycistron as a potential human oncogene. Nature. 435, 828-833 (2005).

[6] Mraz M, Pospisilova S, Malinova K, Slapak I, Mayer J: MicroRNAs in chronic lymphocytic leukemia pathogenesis and disease subtypes. Leuk Lymphoma. 50, 506-509 (2009).

[7] Lee RC, Feinbaum RL, Ambros V: The C. elegans heterochronic gene lin-4 encodes small RNAs with antisense complementarity to lin-14. Cell. 75, 843-854 (1993).

[8] Moller HG, Rasmussen AP, Andersen HH, Johnsen KB, Henriksen M, Duroux M: A systematic review of microRNA in glioblastoma multiforme: micro-modulators in the mesenchymal mode of migration and invasion. Mol Neurobiol. 47, 131-144 (2013).

[9] Lu J, Getz G, Miska EA, Alvarez-Saavedra E, Lamb J, Peck D, Sweet-Cordero A, Ebert BL, Mak RH, Ferrando AA, Downing JR, Jacks T, Horvitz HR, Golub TR: MicroRNA expression profiles classify human cancers. Nature. 435, 834-838 (2005). 
[10] Rosenfeld N, Aharonov R, Meiri E, Rosenwald S, Spector Y, Zepeniuk M, Benjamin H, Shabes N, Tabak S, Levy A, Lebanony D, Goren Y, Silberschein E, Targan N, Ben-Ari A, Gilad S, Sion-Vardy N, Tobar A, Feinmesser M, Kharenko O, Nativ O, Nass D, Perelman M, Yosepovich A, Shalmon B, Polak-Charcon S, Fridman E, Avniel A, Bentwich I, Bentwich Z, Cohen D, Chajut A, Barshack I: MicroRNAs accurately identify cancer tissue origin. Nat Biotechnol. 26, 462-469 (2008).

[11] Xu R, Xu J, Wunsch DC 2nd: MicroRNA expression profile based cancer classification using Default ARTMAP. Neural Networks. 22, 774-780 (2009).

[12] Kyung-Joong Kim, Sung-Bae Cho: Exploring features and classifiers to classify microRNA expression profiles of human cancer. Neural Information Processing. 6444, 234-241 (2010).

[13] Pablo Bermejo, Luis de la Ossa, Jose A. Gamez, Jose M. Puerta: Fast wrapper feature subset selection in high-dimensional datasets by means of filter re-ranking. Knowledge-Based Systems. 25, 35-44 (2012).

[14] Alberto Moraglio, Cecilia Di Chio, Julian Togelius, Riccardo Poli: Geometric particle swarm optimization. Journal of Artificial Evolution and Application. 2008, 14 pages (2008).

[15] J.Garcia-Nieto, E.Alba, L.Jourdan, E.Talbi: Sensitivity and specificity based multiobjective approach for feature selection: Application to cancer diagnosis. Information Processing Letters. 109, 887-896 (2009).

[16] Abdel-Rahman Hedar, Jue Wang, Masao Fukushima: Tabu search for attribute reduction in rough set theory. Soft Computing. 12, 909-918 (2008).

[17] Hongbin Zhang, Guangyu Sun: Feature selection using tabu search method. Pattern Recognition. 35, 701-711 (2002).

[18] Chih-Chung Chang, Chih-Jen Lin: LIBSVM: A library for support vector machines. ACM Transactions on Intelligent System and Technology. 2 (2011).

[19] Geoffrey I.Webb, Janice R.Boughton, Zhihai Wang: Not So Naive Bayes: Aggregating OneDependence Estimators. Machine Learning. 58, 5-24 (2005).

\section{AUTHORS}

Feifei Li was born in Shandong Province, China, in 1989. She received an Undergraduate degree in 2012 from Harbin Institute of Technology, China. Now she is pursuing the Master degree at Electrical and Computer Engineering, Chungbuk National University, South Korea. Her research fields include data mining application to human cancer, classification analysis of high-dimensional data, and feature reduction for microRNA expression data.

Yongjun Piao was born in Jilin Province, China, in 1987. He received a Master degree in Computer Science in 2013 from Chungbuk National University, South Korea. Currently, he is pursuing the Ph.D. degree at the Computer Science, Chungbuk National University, South Korea. His research activities include bioinformatics, computational biology, data mining.

Meijing Li received a MS degree at Database and Bioinformatics Laboratory, Chungbuk National University, Cheongju, South Korea in 2010. She received BS degree in the School of Information and Computing Science from Dalian University, China, in 2007. Currently, she is a Ph.D. candidate at the same laboratory of the Department of Computer Science, Chungbuk National Univ., Rep. of Korea since 2010. Her major research interests include database, bioinformatics and data mining.

Minghao Piao was born in Jilin Province, China, in 1983. He received a Master degree in Bioinformatics in 2009 from Chungbuk National University, South Korea. Currently, he is pursuing the Ph.D. degree at the Computer Science, Chungbuk National University, South Korea. He became a member of IEEE in April, 2012.His research activities include electrical customer classificati on, data mining application to energy data, discovery of emerging patterns in clustering analysis, and reduction of information redundancy for building classifiers.
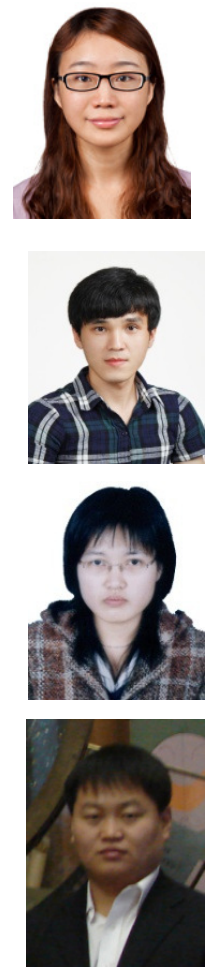
Keun Ho Ryu received the Ph.D. degree from Yonsei University, Seoul, Korea, in 1988. $\mathrm{He}$ is a Professor in the School of Electrical and Computer Engineering, the Chungbuk National University, Cheongju, Korea. He was a Postdoctoral Researcher at the University of Arizona and also a Research Scientist at Electronics and Telecommunications Research Institute, Daejeon,Korea. His research interests include temporal databases, spatiotemporal databases, temporal GIS, ubiquitous computing and stream data processing, knowledgebase information retrieval, database security, data mining, and bioinformatics. Dr. Ryu is a

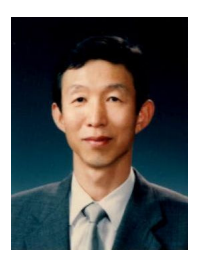
member of the IEEE and ACM since 1983. 
Computer Science \& Information Technology (CS \& IT)

INTENTIONAL BLANK 


\title{
LATENT SEMANTIC WORD SENSE DISAMBIGUATION USING GLOBAL CO-OCCURRENCE INFORMATION
}

\author{
Minoru Sasaki \\ Department of Computer and Information Sciences, Faculty of Engineering, \\ Ibaraki University, 4-12-1, Nakanarusawa, Hitachi, Ibaraki, Japan \\ msasaki@mx.ibaraki.ac.jp
}

\begin{abstract}
In this paper, I propose a novel word sense disambiguation method based on the global cooccurrence information using NMF. When I calculate the dependency relation matrix, the existing method tends to produce very sparse co-occurrence matrix from a small training set. Therefore, the NMF algorithm sometimes does not converge to desired solutions. To obtain a large number of co-occurrence relations, I propose to use co-occurrence frequencies of dependency relations between word features in the whole training set. This enables us to solve data sparseness problem and induce more effective latent features. To evaluate the efficiency of the method of word sense disambiguation, I make some experiments to compare with the result of the two baseline methods. The results of the experiments show this method is effective for word sense disambiguation in comparison with the all baseline methods. Moreover, the proposed method is effective for obtaining a stable effect by analyzing the global co-occurrence information.
\end{abstract}

\section{KEYWORDS}

Word Sense Disambiguation, Global Co-occurrence information, Dependency Relations, NonNegative Matrix Factorization

\section{INTRODUCTION}

In natural language processing, acquisition of sense examples from an example set that contain a given target word enables to construct an extensive data set of tagged examples to demonstrate a wide range of semantic analysis. For example, using the obtained data set, we can construct a classifier that identifies its word sense by analyzing co-occurrence statistics of a target word. Also, we can make a wide-coverage case frame dictionary automatically and construct thesaurus for each meaning of a polysemous word. To construct large-sized training data, language dictionary and thesaurus, it is increasingly important to further improve to select the most appropriate meaning of the ambiguous word.

If we have training data, word sense disambiguation (WSD) task reduces to a classification problem based on supervised learning. This approach is generally applicable to construct a classifier from a set of manually sense-tagged training data. Then, this classifier is used to identify the appropriate sense for new examples. A typical method for this approach is the

David C. Wyld et al. (Eds) : CCSIT, SIPP, AISC, PDCTA, NLP - 2014

pp. 463-468, 2014. (C) CS \& IT-CSCP 2014

DOI : $10.5121 /$ csit.2014.4240 
classical bag-of-words (BOW) approach [5], where each document is represented as a feature vector counting the number of occurrences of different words as features. By using such features, it becomes easy to adapt many existing supervised learning methods such as Support Vector Machine (SVM) [1] for the WSD task. However, when the general vector space model, in which a document is represented as a vector using term frequency based weighting methods, is applied to the WSD, the local context words are typically used as features and the global context information without dictionary information is not employed in the previous research.

In this paper, I propose a novel word sense disambiguation method based on the global cooccurrence information using NMF. In previous research, [2] proposes a novel WSD method of particular word instances using the automatically extracted sense information. When we calculate the dependency relation matrix, the existing method tends to produce very sparse co-occurrence matrix from a small training set. Therefore, the NMF algorithm sometimes does not converge to desired solutions. To obtain a large number of co-occurrence relations, I propose to use cooccurrence frequencies of dependency relations between word features in the whole training set. This enables us to solve data sparseness problem and induce more effective latent features.

\section{NON-NEGATIVE MATRIX FACTORIZATION}

Non-Negative Matrix Factorization (NMF) is a popular decomposition method for multivariate data [4]. NMF decomposes the $m \times n$ non-negative matrix $X$ to the $m \times k$ matrix $W$ and the $k \times n$ matrix $H$, while these matrixes have no negative elements. Usually, $k$ is chosen to be smaller value than $n$ and $m$.

$$
X \approx W H
$$

Using the NMF for a term-document matrix $X$, the matrix $H$ represents the clustering result with $k$ topics.

For quantifying the quality of this approximation, cost functions based on Kullback-Leibler divergence is used and minimized using iterative update rules as follows:

$$
\begin{gathered}
W_{i j} \leftarrow W_{i j} \frac{(X H)_{i j}}{\left(W H H^{T}\right)_{i j}} \\
H_{i j} \leftarrow H_{i j} \frac{\left(X^{T} H\right)_{i j}}{\left(H W^{T} W\right)_{i j}},
\end{gathered}
$$

where $W_{i j}$ and $H_{i j}$ are the $i$-th row and the $j$-th column element respectively. These matrices $W$ and $H$ are initialized randomly with non-negative data and these above update rules are iteratively applied until the max iteration number (or convergence) is reached.

\section{WSD USING GLOBAL CO-OCCURRENCE INFORMATION}

\subsection{Latent Semantic WSD Using Local Co-occurrence Information}

In previous research, [3] proposes a novel WSD method of particular word instances using the automatically extracted sense information. This method induces latent features for three matrices. The first matrix $A$ contains co-occurrence frequencies of words that the target word co-occurs 
with. The second matrix $B$ contains term frequencies of words that appear in the context window. The third matrix $C$ contains co-occurrence frequencies of words that the co-occurring context words of the target word co-occur with. Then, NMF is applied to the three matrices to factorize each matrix into two non-negative matrices, while the former results are used to initialize the next factorization, as shown in Figure 1.

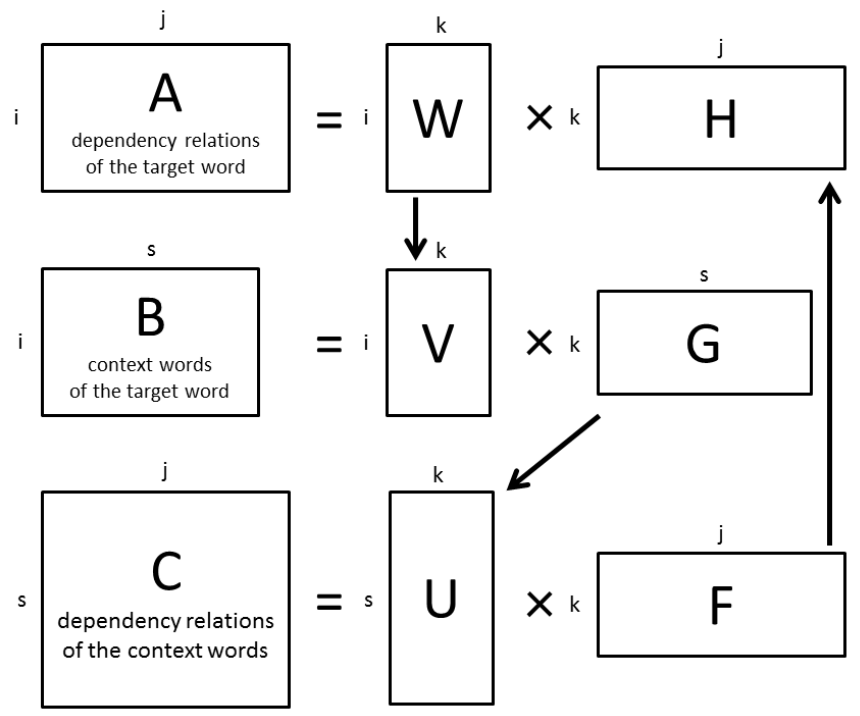

Figure 1. Interleaved NMF algorithm for Latent Semantic WSD

Given a non-negative matrices $A, B$ and $C$ in the beginning of this method, matrices $W, H, G$ and $F$ are initialized randomly with non-negative values. Then, it decomposes the matrix $A$ into the matrices $W$ and $H$ using NMF. In the decomposition of the matrix $B$, the updated matrix $W$ is copied to matrix $V$ and the updated matrices $V$ and $G$ is computed using NMF. In the decomposition of the matrix $C$, the transpose of the updated matrix $G$ is copied to matrix $U$ and the updated matrices $U$ and $F$ are obtained using NMF. At the last step of the iteration, the matrix $F$ is copied to matrix $H$. This iteration is repeated until the maximum number of iterations is reached or the objective function of all NMF decomposition no longer improves.

In order to perform this method for WSD, it needs to fold each sense of the target word into semantic space using the matrix $H$. For each sense in training data, centroid vector $c$ is calculated and this centroid is mapped into the semantic space using the matrix $H$ as follows:

$$
b=c H^{T}
$$

For input example of the target word, its context words are extracted to construct a vector $f$ and the vector $f$ is also mapped into the same semantic space using the matrix $G$ as follows:

$$
d=f G^{T}
$$

Then, cosine similarity between the vector $d$ and each of the sense vectors $b$ are calculated and the sense that is the largest cosine similarity is selected. 


\subsection{Latent Semantic WSD Using Global Co-occurrence Information}

This Latent Semantic WSD method is efficient for finding a reduced semantic space. However, problem arises when we apply this method. When we calculate the third matrix $C$, this method tends to produce very sparse co-occurrence matrix from a small training set. To obtain a large number of co-occurrence relations, I propose to use co-occurrence frequencies of dependency relations between word features in the whole training set. This enables us to solve data sparseness problem and induce more effective latent features. Like the above method, the proposed method needs three matrices, but the third matrix is different from the previous method. The third matrix $D$ contains co-occurrence frequency of context words that co-occur in dependency relations to context words in a large document set. The proposed method induces latent features for these three matrices $A, B$ and $D$.

\section{EXPERIMENT}

To evaluate the efficiency of the proposed WSD method using the global Co-occurrence information, I conduct some experiments to compare with the result of the existing methods. In this section, I describe an outline of the experiments.

\subsection{Data}

I used the Semeval-2010 Japanese WSD task data set, which includes 50 target words comprising 22 nouns, 23 verbs, and 5 adjectives [2]. In this data set, there are 50 training and 50 test instances for each target word. Moreover, to obtain a large number of co-occurrence relations, I use 22,832 documents chosen from the Japanese BCCWJ corpus ${ }^{1}$.

\subsection{Evaluation Method}

\subsubsection{Baseline System 1}

As the first baseline method, I only use the first matrix $A$ described in section 3.1. To construct the matrix $A$, I represent each sentence with the target word in the training set as a highdimensional vector where each component represents the co-occurrence frequency of the target word in the sentence. Then, NMF is applied to the matrix $A$ to factorize each matrix into two nonnegative matrices $W$ and $H$. Each vector is tagged with the sense of the target word in that sentence. So centroid $c_{i}$ of the co-occurrence vectors that are assigned the same sense $i$ is calculated and each centroid $c_{i}$ is mapped into the semantic space using the matrix $H$ as follows:

$$
b_{i}=c_{i} H^{T}
$$

For input example of the target word, its context words are extracted to construct a vector $f$ and the vector $f$ is also mapped into the same semantic space using the matrix $H$ as follows:

$$
d=f H^{T}
$$

Then, cosine similarity between the vector $d$ and each of the sense vectors $b$ are calculated and the sense that is the largest cosine similarity is selected.

\footnotetext{
${ }^{1}$ http://www.ninjal.ac.jp/english/products/bccwj/
} 


\subsubsection{Baseline System 2}

In the second baseline system, I use the latent semantic WSD using local co-occurrence information described in Section 3.1. I construct the three matrices $A, B$ and $C$ to induce latent semantic dimensions using NMF.

Table 1. Experimental Results of Each Execution

(highest precision rates among all the run are written in bold font)

\begin{tabular}{|c|l|l|l|}
\hline System & Run 1 & Run 2 & Run 3 \\
\hline Baseline System 1 & $53.28 \%$ & $\mathbf{5 3 . 8 8 \%}$ & $51.80 \%$ \\
\hline Baseline System 2 & $59.68 \%$ & $\mathbf{6 1 . 0 8 \%}$ & $\mathbf{6 1 . 0 8 \%}$ \\
\hline Proposed Method & $60.44 \%$ & $\mathbf{6 1 . 4 8 \%}$ & $61.04 \%$ \\
\hline
\end{tabular}

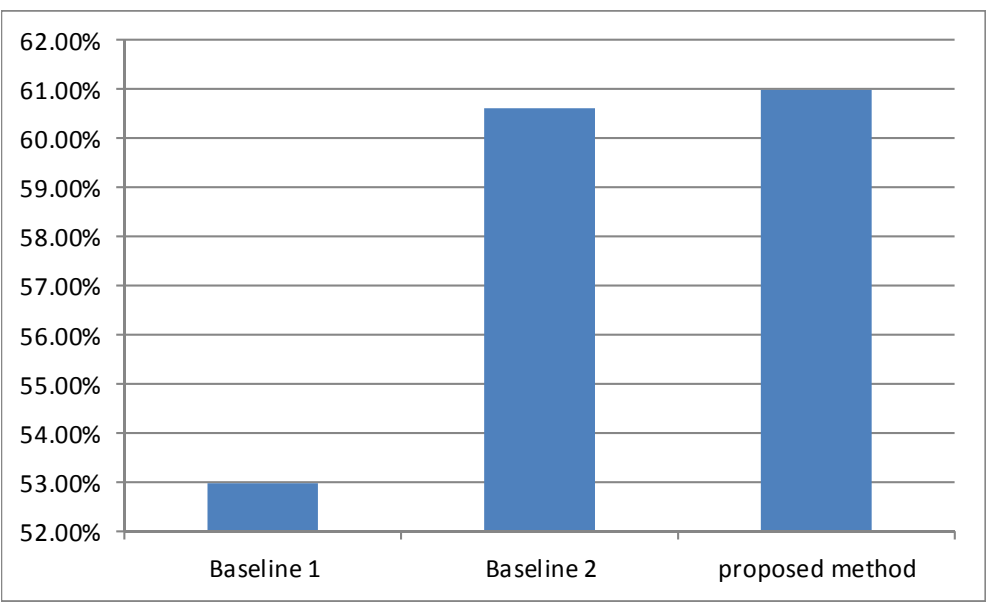

Figure 2. Highest Precision of Each system

\section{EXPERIMENTAL RESULTS}

Figure 2 shows the experimental results of the baseline methods and the proposed method. Computational experience reported shows that the choice of initial point is quite important to the NMF's goal. In practice, the algorithms are run several times with different initial points and the NMF is chosen as the feasible solution. In our experiments, each method is executed three times and average precision of all execution is calculated.

In this Figure 2, the proposed method shows higher precision than the other baseline methods so that this approach is effective for word sense disambiguation. In comparison with the baseline system 1, the proposed method can obtain better precision so that it is effective for WSD to use context information and co-occurrence information. In comparison with the baseline system 2 , the proposed method provides slightly better precision than the baseline system 2 . As shown in Table 1 , the proposed method can obtain the highest precision and can be stable at high precision value. However, the baseline system 2 cannot achieve stable precision because of the lack of the number of co-occurrence information. Therefore, the proposed method is effective for obtaining a stable effect by analyzing the global co-occurrence information. 


\section{ACKNOWLEDGEMENT}

In this paper, I propose a novel word sense disambiguation method based on the global cooccurrence information using NMF. To evaluate the efficiency of the method of word sense disambiguation, I conduct some experiments to compare with the result of the two baseline methods. The results of the experiments show this method is effective for word sense disambiguation in comparison with the all baseline methods. Moreover, the proposed method is effective for obtaining a stable effect by analyzing the global co-occurrence information.

Further work would be required to consider a larger sized training data to obtain a large amount of co-occurrence information.

\section{REFERENCES}

[1] Corinna Cortes \& Vladimir Vapnik, (1995) "Support-Vector Networks", Machine Learning, Vol. 20, No. 3, pp273-297.

[2] Okumura, Manabu \& Shirai, Kiyoaki \& Komiya, Kanako \& Yokono, Hikaru, (2010) "SemEval-2010 task: Japanese WSD", Proceedings of the 5th International Workshop on Semantic Evaluation, pp6974.

[3] Van de Cruys, Tim \& Apidianaki, Marianna, (2011) "Latent Semantic Word Sense Induction and Disambiguation", Proceedings of the 49th Annual Meeting of the Association for Computational Linguistics: Human Language Technologies-Volume 1, pp1476-1485.

[4] Lee, Daniel D. \& Seung, Sebastian, (2001) "Algorithms for Non-negative Matrix Factorization", Advances in Neural Information Processing Systems 13: Proceedings of the 2000 Conference, MIT Press, pp556-562.

[5] Witten, Ian H. \& Moffat, Alistair \& Bell, Timothy C., (1999) "Managing Gigabytes (second edition): Compressing and Indexing Documents and Images", Morgan Kaufmann Publishers Inc.

\section{AUTHORS}

Minoru Sasaki: received his B.E., M.E. and D.Eng. degrees in information science and intelligent systems from the University of Tokushima in 1996, 1998 and 2000. He is now a lecturer in the department of computer and information sciences at Ibaraki University. His research interests include natural language processing and information, information retrieval and text min ing.

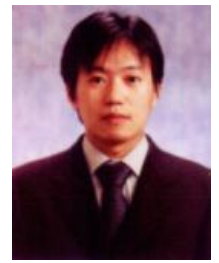




\title{
MODELING OF SPEECH SYNTHESIS OF STANDARD ARABIC USING AN EXPERT SYSTEM
}

\author{
Tebbi Hanane ${ }^{1}$ and Azzoune Hamid ${ }^{2}$ \\ ${ }^{1,2}$ LRIA, Option: Representation of Knowledge and systems of Inference, \\ USTHB, Algiers \\ ${ }^{1}$ tebbi_hanane@yahoo.fr, ${ }^{2}$ azzoune@yahoo.fr
}

\begin{abstract}
In this work we present our expert system of speech synthesis based on a text written in Standard Arabic, our work is carried out in two great stages: the creation of the sound data base, and the transformation of the written text into speech (Text To Speech TTS). This transformation is done firstly by a Phonetic Orthographical Transcription (POT) of any written Standard Arabic text that to transform it into his corresponding phonetics sequence, and secondly by the generation of the voice signal which corresponds to the chain transcribed. We spread out the different steps of conception of the system, as well as the results obtained compared to others manners of works studied to realize TTS based on Standard Arabic.
\end{abstract}

\section{KEYWORDS}

Engineering knowledge, modelling and representation of vocal knowledge, expert system, (TTS Text To Speech), Standard Arab, PRAAT.

\section{INTRODUCTION}

Generate the voice is a complex work because of the variability intra and interlocutor of the voice signal. In computer science the difficulty of modeling the speech signal is for the reason that we don't know yet how to model very well the enormous mass of knowledge and information useful to the signal synthesis. Thus we have made a choice to use an expert system to modeling that knowledge to build a robust system which can really read a text written in a language chosen especially in Standard Arabic. To obtain a better organization of our work, we defined our direct aims. We divided our modeling into three essential stages; the signal analysis, the phonetic orthographical transcription (POT) and finally the synthesis of the textual representation written in Standard Arabic. So the finality considered here is that the user can understand the different phrases transcribed and synthesized which will be pronounced with a clear and high quality manner.

\section{PREVIOUS WORKS}

At the current time, we can judge that works emanated in the same context of ours are still not really colossal, and this is because of the complexity of the language itself. And if this some works exists, they are based on the same principle as transcribers of the other languages (French,

David C. Wyld et al. (Eds) : CCSIT, SIPP, AISC, PDCTA, NLP - 2014 
English, etc.) [1], nevertheless, the efforts undertaken are encouraging and open a large window to follow research task in this field.

- TTS system MBROLA [2] which use the code SAMPA during the stage of transcription, in this case the user must respect the form of the SAMPA code which is not a universal code;

- Work of S. BALOUL [3] who represents a concrete example of transcription of words; based on morphological analysis, and on the studies of pauses to generate the pronunciation of the texts.

- SYAMSA (SYstème d'Analyse Morphosyntaxique de l'Arabe), realized by SAROH [4]. According to him, "the phonetisation of the Arabic language is based in particularly on the use of lexicons and on a morphological analyzer for the generation of the different forms of a word. In addition, they are the phenomena of interaction among the words (connection, elision, etc.) and the phenomena of assimilation which suggest the uses of phonological rules "[5]. This tool ensures for each word in entry, the root which correspond to it as well as the morphological and phonetic representations of the root.

- The GHAZALI [6] project which was carried out within the IRSIT (Institut Régional des Sciences Informatiques et des Télécommunications) of Tunisia, it is based on a transcription work which fits inside the framework of the realization of a TTS system. The characteristic of this system is shown in the use of a set of rules in the emphasis propagation.

- SYNTHAR+ [7] which is a tool studied by Z. ZEMIRLI within the NII (the National Institute of Informatics of Algiers), it ensures the transcription step for a TTS system so that it transmits the phonetic representation to the MULTIVOX synthesizer. It should be known that SYNTHAR+ is based on a morphological analysis before realizing the transcription.

\section{STRUCTURE OF THE SYSTEM}

The uses of the concept of code and the introduction of high levels in analysis (morphological, syntactic, and pragmatic.) makes the transcription task so difficult and requires deepened studies of the language itself. The difference in our work compared to all that exists is in the transcription using graphemes, i.e. the uses of the Arabic characters as basic units to transcribe directly the text, indeed modeled by an expert system.

\section{Definition of an expert system:}

An expert system is an informatics tool of AI (Artificial Intelligence), conceived to simulate the knowhow of an human expert, in a precise and delimited field, this thanks to the good exploitation of a set of knowledge provided explicitly by expert of the field [5].

The E.S is a system where the data (the knowledge database) is quite separated from program which handles it (the inference engine). In our case, we built our expert system basing on the two following components:

A knowledge database: It contains a database of facts and a database of rules, represents the knowledge (we speaks here about the sound database) and knowledge - to make (a set of 
rewriting rules). The fact database integrate two types of facts: permanent facts of the field and deduced facts by the inference engine which are specific to the field considered (voice synthesis).

The inference engine: It is capable to reason starting from the information contained in the knowledge database and to make deductions. The inference engine uses the facts and rules to produce new facts.

Thus the role of an expert system is then to infer generally rules like if/then. To build this system our work targets the following steps:

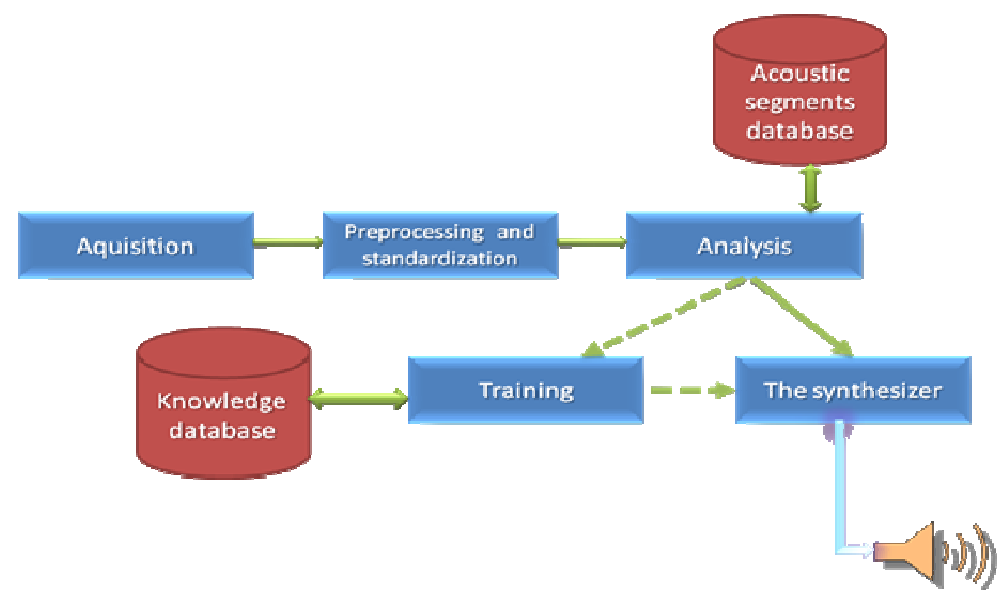

Figure 1. General schema of our system of speech synthesis

\subsection{Acquisition}

It is the first step of our process of voice synthesis; it plays the role of the interface intermediate between the user and the system. During this time the user have to enter his set of phrases to be pronounced by the system. After that the automatic process can begin.

\subsection{Preprocessing and standardization of the text}

In this phase any form of text is transformed to it literal, perfectly disambiguated form [1]. In particular, this module draft problems of formats, dates and hours, abbreviations, numbers, currencies, addresses, emails...etc. It was shown that majority errors of conversion grapheme/phoneme, for the best operational systems came from the proper names and the exceptions that they pose [8]. Here are some examples of the processing carried out in our case:

- The replacement of each composed character by its equivalents Example: $\forall \longrightarrow 1 \mathrm{~J}$

- Consultation of the exceptions lexicon to eliminate the special words.

- The application of the transcription rules established for the language. This module must be able to process grammatical complexes tasks to identify the rules of transcription which will be used in a considered context. 


\subsection{Analysis (the extraction of the characteristics)}

The aim of the analysis of the voice signal is to extracts the acoustic vectors which will be used in the stage of synthesis follows. In this step the voice signal is transformed into a sequence of acoustic vectors on the way to decrease redundancy and the amount of data to be processed. And then a spectral analysis by the discrete Fourier transform is performed on a signal frame (typically of size 20 or $30 \mathrm{~ms}$ ) [9]. In this frame, the voice signal is considered to be sufficiently stable and we extract a vector of parameters considered to be enough for the good operation of the voice signal. In the speech synthesis, the characteristics extraction step, commonly known as the step of analysis, can be achieved in several ways. Indeed, the acoustic vectors are usually extracted using methods such as temporal encoding predictive linear (Linear Predictive Coding LPC) or Cepstrales methods as the MFCC encoding (Mel Frequency Cepstral Coding), as well as the process of segmentation, etc. This process delimits on the acoustic signal a set of segments characterized by labels belonging to the phonetics alphabet of the language under consideration.

At present, the segmentation completely automatic of a voice signal remains a fastidious task. Indeed, looking at the complexity acoustico-phonetics phenomena being studied, this activity requires often a manual intervention. Generally, the methods that perform the segmentation of the acoustic wave are divided into two great classes:

- The first include all the methods which allow segmenting a voice signal without a priori knowledge of the linguistics content of this signal. These methods split the voice signal into a set of zones homogeneous and stable;

- The second class includes all the methods which allow segmenting the voice signal basing on a priori linguistic description (typically on phonemes) of this signal. These methods of segmentation are revealed like methods with linguistically constraints. In our state, we have opted for the second technique using a tool of voice signal analysis which is PRAAT [10] so to slice manually the speech signal in a succession of segments, each one associated with an element acoustic unit (phoneme or diaphone).

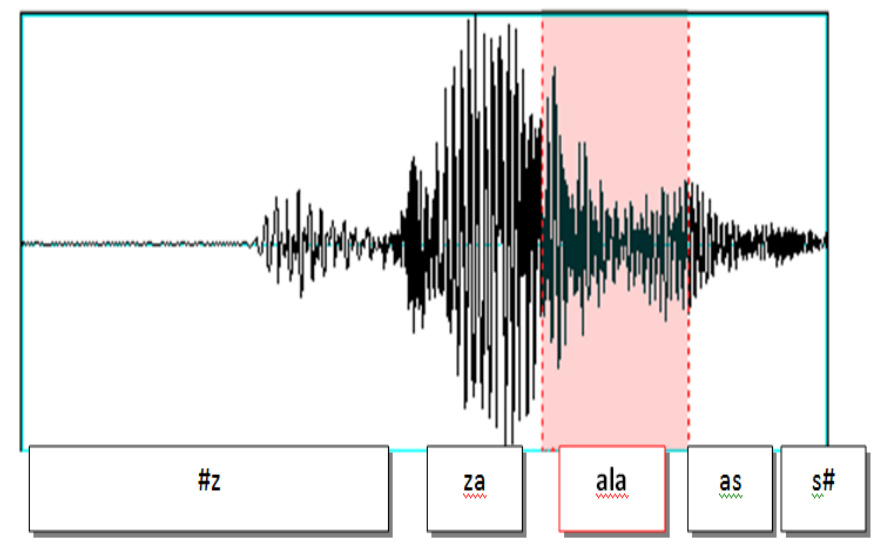

Figure 2. Decomposition in polysound of the word [ جلس] 


\subsection{Modeling of the sound database}

Majority of work curried out in the field of the spoken communication required often the recording, and the handling of corpuses of continuous speech, and that to curry out studies on the contextual effects, one the phonetic indices, and variability intra and inter-speaker. Our corpus is modeled by the diagram of class 1 as follow:

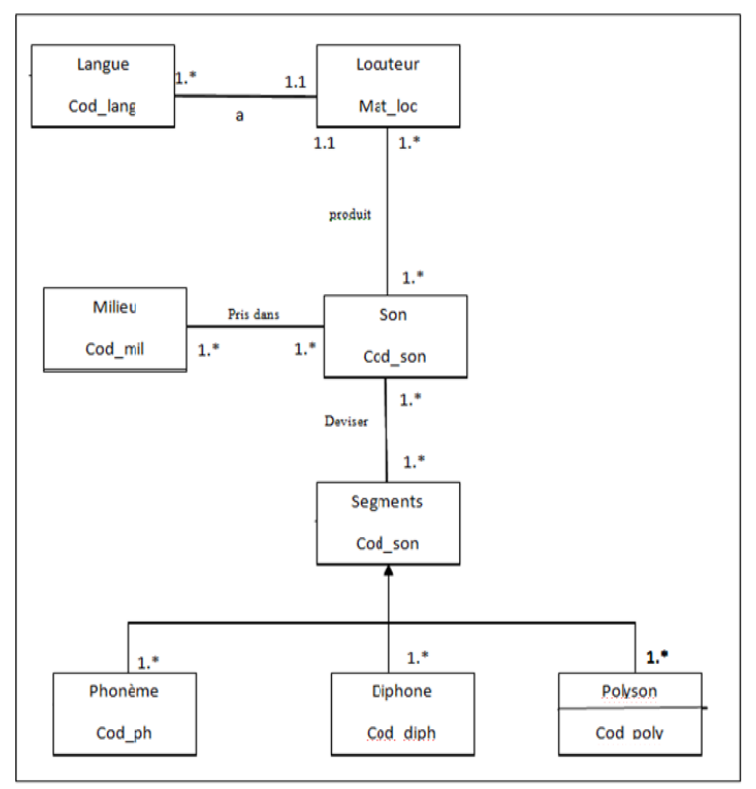

\subsection{The training}

Figure 3. The diagram of class of our sound database

Here we referred to a POT module to convert each grapheme in phoneme according to the context and that is possible using a set of rewriting rules. The principal advantage of this approach is the possibility to modeling the human linguistic knowledge by a set of rules which can be integrated in expert systems. Each one of these rules has the following form:

$[$ Phoneme $]=\{$ LC $($ Left Context $)\}+\{\mathrm{C}($ Character $)\}+\{$ RC (Right Context $)\}$

Here is concrete example of transcription rules [11]:

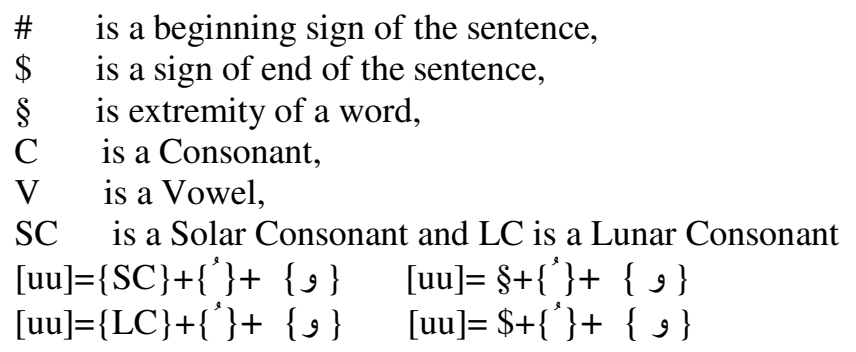

When the " "و is preceded by the vowel /" / and followed by a consonant, we obtain the long vowel [ uu ].

1: It is a diagram used in UML(Unified Modeling Language) which is used in object oriented modeling 
When the " is preceded by the vowel /" / in final position, we obtain the long vowel [ uu ].

\subsection{The synthesizer (the voice generation)}

The generation of the voice signal is the real synthesis of speech. This operation consist of a transform of the phonetic sequence (which represents the pronunciation of the written text) resulting from the transcription step to its substance, i.e. to it acoustics realization. During this step we choose from our sound database the units (phonemes, diphones) most suitable to build the sentence to generate, this means that we will create a automatic function of reading, so that in the end, the user has only to listen to the synthetic sentences. To improve the quality of the generated synthesis, we increase each time the sound unit only. The general silhouette of our expert system can be as follow:

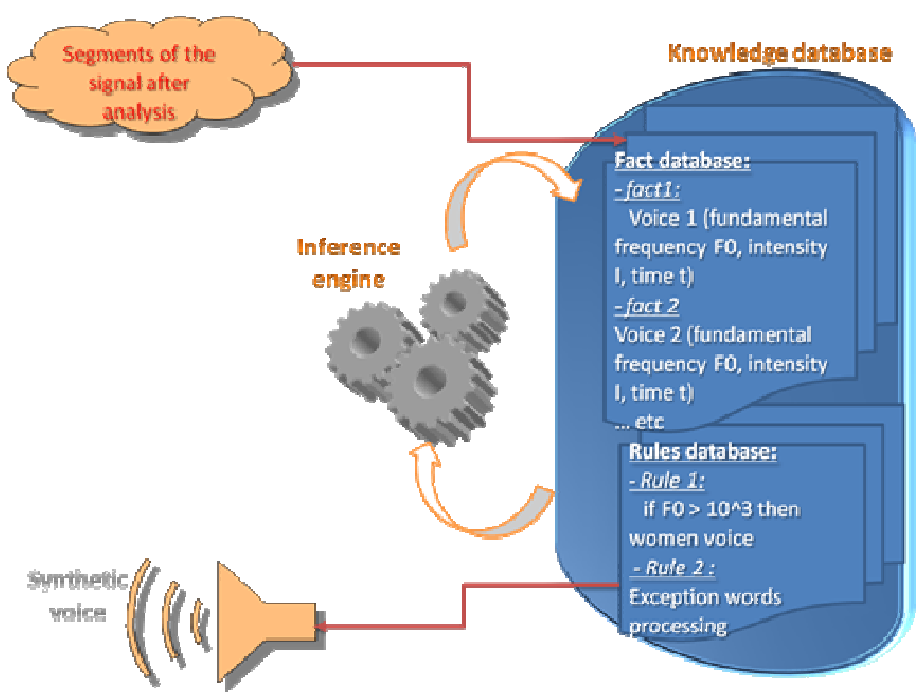

Figure 4. General diagram of our expert system

\section{TESTS AND RESULTS}

To test the performances of our TTS system based on Standard Arabic language, we have chosen a set of sentences which we judged like reference since they contain almost the different possible combinations specific to the language itself. To calculate the success rate (SR) associated with each sentence tested; we got the following formula:

$$
S R=\frac{\text { Numberofphrasewellprononced }}{\text { Numberofphrasestested }} * 100 \%
$$

The system present in general a SR of $96 \%$ for the set of the sentences tested. Results obtained are summarized by the following table: 
Table 1. Rate of success for a sample of selected sentences

\begin{tabular}{|l|l|l|l|}
\hline Majority content & \multicolumn{1}{|c|}{ POT } & Synthesis by Phonemes & Synthesis by Diphones \\
\hline Short vowels & $100 \%$ & $95 \%$ & $/$ \\
\hline Long vowels & $100 \%$ & $95 \%$ & $/$ \\
\hline Solar consonants & $100 \%$ & $97 \%$ & $/$ \\
\hline Lunar consonants & $100 \%$ & $95 \%$ & $/$ \\
\hline Isolated words & $100 \%$ & $80 \%$ & $90 \%$ \\
\hline Sentences & $100 \%$ & $75 \%$ & $85 \%$ \\
\hline Numbers & $90 \%$ & $95 \%$ & $100 \%$ \\
\hline Exception Words & $100 \%$ & $/$ & $/$ \\
\hline
\end{tabular}

\section{CONCLUSION AND PROSPECTIVE}

A system for synthesizing Arabic speech has been developed based on an expert system which uses a set of subphonetic elements as the synthesis units to allow synthesis of limited-vocabulary speech of good quality. The synthesis units have been defined after a careful study of the phonetic properties of modern Standard Arabic, and they consist of central steady-state portions of vowels, central steady-state portions of consonants, vowel-consonant and consonant-vowel transitions, and some allophones. A text-to-speech system which uses this method has also been explored. The input of the system is usual Arabic spelling with diacritics and/or simple numeric expressions. Synthesis is controlled by several text-to-speech rules within the rule database of the expert system, which are formulated and developed as algorithms more suited for computer handling of the synthesis process. The rules are required for converting the input text into phonemes, converting the phonemes into phonetic units, generating the synthesis units from the phonetic units, and concatenating the synthesis units to form spoken messages. The suitability of the method for synthesizing Arabic has been shown by realizing all its functions on a personal computer and by conducting understandability test on synthesized speech. So, we have detailed the different components which represents the basic blocks of our TTS system based on a written text in Standard Arabic and our modeling of it with the use of an expert system, this tool which is a fruits of the artificial intelligence remain less used in the field of automatic speech processing. This fact encouraged us to explore this world trying to give a little push to the research done in this multidisciplinary field. Like prospective to our work we show the following points:

- Improvement of the quality of voice generated by using methods of modification of the prosody;

- The use of others techniques of synthesis such as the Synthesis using the unit's selection;

- Make, if possible, the signal segmentation totally automatic.

\section{REFERENCES}

[1] Tebbi Hanane, « Transcription orthographique phonétique en vue de la synthèse de la parole a partir du texte de l'Arabe », Mémoire de magister en Informatique, Blida, Algérie, 2007

[2] http//:tcts.fpms.ac.be/ synthesis/ Mbrola.html.

[3] Baloul, « Développement d'un système automatique de synthèse de la parole à partir du texte arabe standard voyellé ». Thèse de doctorat d'université, Le Mans, France, 27 Mai 2003.

[4] SAROH Abdelghani, Base de données lexicales dans un système d'analyse morpho-syntaxique de l'arabe : SYAMSA, Toulouse 3, 1989.

[5] Guerti Mhania, Tebbi Hanane, La Conversion Graphèmes Phonèmes En Vue D'une Lecture Automatique de Textes en Arabe Standard, LANIA, Chlef, Algérie, 2007 S.

[6] Dichy J. \& Hassoun M.O. (1998), Some aspects of the DIINAR-MBC research programme, in Ubaydly A., ed., 1998: 2.8.1-5. 
[7] SYNTHAR+: Arabic speech synthesis under multivox. (SYNTHAR+: Synthèse vocale arabe sous Multivox.) (English) RAIRO, Tech. Sci. Inf. 17, No. 6, 741-761 (1998).

[8] Mehdi Braham, Oumaya Abbes, Maroua Trabelsi, Mehdi Dahmen, Rania Nouaari, « Intelligence artificielle : Diagnostic par système expert, Modèle d'analyse d'acteurs : MACTOR »

[9] P. Boula de Mareüil, «Synthèse de la parole à partir de courriers et évaluation de la conversion graphème-phonème ». LIMSI-CNRS http://www.limsi.fr/ Individu/ mareuil/

[10] Paul Boersma and David Weenink, "Praat: doing phonetics by computer" Phonetic Sciences, University of Amsterdam Spuistraat 210, 1012VT Amsterdam The Netherlands.

[11] T. Saidane, M. Zrigui, \& M. Ben Ahmed, « La transcription orthographique phonétique de la langue Arabe ». RECITAL 2004, Fès, 19-22 Avril 2004. 


\title{
A MODEL OF CORRELATED AGEING PATTERN FOR AGE RANKING
}

\author{
Onifade O.F.W. ${ }^{1}$ and Akinyemi J.D. ${ }^{2}$ \\ ${ }^{1}$ Department of Computer Science University of Ibadan \\ olufadeo@gmail.com \\ ${ }^{2}$ Department of Computer Science University of Ibadan \\ akinyemijd@gmail. com
}

\begin{abstract}
In this paper, we propose a framework for Age Estimation which uses a correlated ageing pattern to rank images and makes necessary inferences from the image ranks to estimate the exact age of images. We use AAM and LBP as complementary feature extraction techniques for extracting facial features in low dimensionality. Our correlated ageing pattern model learns the ageing patterns of different individuals across ages and uses these to determine an agerank for each image. Subsequently, the learned age rank of a reference image set is used to determine the ranks of test images in order to deduce relevant inferences for age estimation. Our approach is significantly different from the previous ranking approaches in that it determines age ranks that do not only represent the correlation of ages of different individuals but also the correlation of ageing patterns of different individuals. Our initial findings look promising with the intuitive manner with which we employ correlated ageing patterns.
\end{abstract}

\section{KEYWORDS}

Age Estimation, Ageing Pattern, Age Rank, Ranking

\section{INTRODUCTION}

Human age estimation is a challenging task for humans as well as for machines. Although, humans possess the ability early in life to estimate the age of a person from his/her appearance [1], [2], the task is a subjective one which is largely based on the previous experience of the estimator. On the part of the estimated face image, several factors - external (eating habits, drugs, sickness, injuries, weather etc.) as well as internal (genetic or hereditary factors, ethnicity, gender) - could greatly cause variations in the pattern of aging of different individuals, thus making it more challenging to find a unique solution to the Age estimation problem. Therefore, whatever solution is to be proffered to the Age Estimation problem must be an adaptive one.

Human Age Estimation has recently received attention in the research community and as such, several approaches and insights have been developed over the years to combat the problem. It continues to gain research interest especially due to its wide application in Adaptive Computing Methodologies such as Age-Specific Human Computer Interaction (ASHCI) [3], [4], [5]. A major motivation for this research from our own point of view is the fact that certain professions (Sports, Military etc.) require the knowledge of the actual age of individuals/professionals, hence, a medium of verifying the ages presented in such professions will be invaluable as it could be able to reduce the compromise in the ages supplied by these professionals.

David C. Wyld et al. (Eds) : CCSIT, SIPP, AISC, PDCTA, NLP - 2014

pp. 477-485, 2014. (C) CS \& IT-CSCP 2014

DOI : $10.5121 /$ csit.2014.4242 
Some areas where this research could be applicable include the use of Automatic Vending Machines which could restrict a customer's purchase of alcoholic products based on the estimated age of the customer, National Youth Service Corps (NYSC) Scheme in which Nigerian Youth (below the age of 30 years) are sent to various states of the country to serve their Fatherland in their respective disciplines. The NYSC is also one of the primary motivations for this work as it will be very resourceful in reducing age falsification for participation in the scheme.

As much as this research area is gaining a wider span of interest and applicability, it is still a challenging research area that has left research gaps, particularly in terms of its accuracy. The accuracy of any prediction or estimation algorithm/system largely determines the extent to which it will be widely adopted in real-world applications. Hence, this work proposes a model for ranking images based on the correlation between the ages and the ageing pattern of individuals. Subsequently, the ranks of images can be determined and used to make relevant inferences for age estimation. Our significant contribution with this model is the correlation between the ageing pattern of different individuals and their ages which is used to determine the age rank of images. Possible applications of this model in real world domains include age estimation and age learning from facial image.

\section{RELATED WORKS}

From our analysis of previous works on age estimation, we deduced that they can be classified into five major categories based on the approach employed in the research. We have the Anthropometric Models [6], [7], [8] which adopt knowledge from Facial Wrinkle Analysis and Craniofacial Research for modeling the growth (change in shape) of the face. This approach is mostly suitable for young faces and often requires high resolution images with minimal head pose. Some other research approaches use the aging pattern of faces [9], [10], [11] by learning the aging pattern of individuals and trying to synthesize a facial image for this individual at some other ages not present in the training sample. This approach performed greatly especially due to the fact that aging factors could be personalized. However, its flaws are quickly exposed when it is applied to images not closely represented (in terms of age, gender or ethnicity) in the training set, thus it required a very large training set with a well-spread age distribution to perform well. The third category is of those which treat the Age Estimation problem as a classification problem. This research approach assumes the age labels to be independent classes into which face images can be classified thus resulting in a multi-class classification problem [12], [13], [14]. This approach also met with great success, especially due to the use of the Support Vector Machine; an excellent Machine learning algorithm for classification. Unfortunately, the assumption that ages are independent is, however, not too realistic. A person may have similar looks across different age classes and two different people might have, to some extent, similar facial features at the same age thus flawing the classification approach. Some other research approaches have handled age estimation as a regression problem in which the age labels are learned by a function which fits the face images to their corresponding estimated ages [15], [16], [17]. This is intuitive as the age labels are integers and their relationship with the ageing features, expressed as real numbers can be learn, but this is after some rigorous training. Support Vector Regression (SVR) has been very successful in this approach, thus researchers have applied several modifications of it to improve the model fitting function. The last category of research approaches in age estimation are those which treat the age labels as ordinal pairs and therefore calculate a rank for each face image which is compared against the set of already ranked images [18], [19], [20]. In this work, we employ this approach to age estimation but with an improvement over the existing rank-based frameworks.

In [18], Yang et al. used Harr-like features to represent the face and then used a combination of a ranking model and personal aging pattern to reduce the dimensionality of the feature set obtained. 
Pair-wise samples were built for the ranking model by organizing the age sequence according to individual ageing patterns within each subject and RankBoost [21], which employs a ranking model with boosting learning, was used to select relevant features, thereafter, they used SVR with the Radial Basis Function kernel to estimate the age of a facial image. Chang et al. [19] also applied a rank-based framework to age estimation with an intuition that it is easier to estimate the age of a subject by comparing his face with the faces of other people whose ages are known. Their work used the relative order of age labels to build a rank model. To avoid exhaustive comparison with all face images in the database, they only used a subset of the database ( 80 images) to build the rank model which was used as a reference for comparison with test images. For each image compared against the set of ranked images, the age estimation problem is eventually reduced to a binary classification problem and a combination of binary decisions is used to make inferences which guide the age prediction. Cao et al. in [20], proposed a Ranking SVM for human age estimation by building a set of images used as a reference set to which images are compared before they are then classified into their corresponding age labels. They improved upon the ranking model of Yang et al. by including what they called 'consistent pairs' (images of the same age) in their reference set. Also, based on the intuition that Humans age differently, they ranked images of the same age such that they reflect their slight differences as well as their common trend, but these differences do not reflect the true variation in the ageing pattern of these individuals because it is not derived based on a trend of ageing patterns along ages.

\section{PROPOSED METHODOLOGY}

\subsection{Age Estimation Framework}

Our proposed ranking approach makes intuitive improvements on the existing ones by employing the correlation between individual ageing patterns for determining age ranks. The generic processes involved in our proposed model are the major processes involved in face processing but with different techniques.

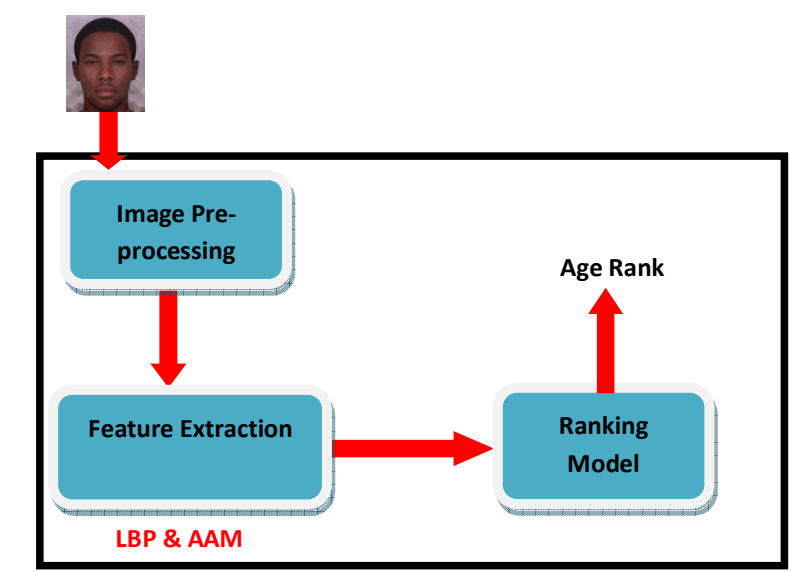

Figure 1: A General Overview of the Age Ranking Framework

The processes shown in figure 1 are involved in both the training and testing phases. As in most face processing systems, in order to improve performance, there will be need to pre-process our input images by first converting them to grayscale, detecting the facial part of the images and then cropping this facial part. Thus after pre-processing, the image is expected to contain just the face which is sufficient to provide the necessary facial features required for face representation. Feature extraction is to be carried out by Local Binary Pattern (LBP) and Active Appearance 
Model (AAM). LBP [22] is a powerful texture operator which is robust to illumination and grayscale changes, but more importantly, it extracts image features with reduced dimensionality and low computational time. For each pixel in an image, LBP extracts pixel information from by computing a binary code from its neighbouring pixels. It has found success in many face processing tasks including facial age estimation [23], [24]. AAM [25], known for appearance modeling, is an extension of the Active Shape Model (ASM) [26] which represents shape and contour features in a single appearance model. AAM uses annotated facial landmarks to model the contours/shape of the face and constructs a shape free patch of the face which it later uses to create a texture model. By learning the correlation between these two face models; AAM is able to create a single representation of the face which represents both its shape and contour.

Our choice of AAM and LBP is based on the fact that they have achieved significant success in previous face processing tasks including facial age estimation. More importantly, we chose to use the two techniques as complementary techniques so that the results obtained from both techniques can be compared to arrive at more accurate age estimates. Due to the relatively low dimension of features extracted by the two techniques mentioned above (when compared to other techniques such as Gabor and Haar features), using them concurrently is not as expensive as one would expect. LBP features can be collected into histograms of length 256 or less and AAM features could extract 68 landmark features. Taking advantage of multi-core processors, we consider that the concurrent use of these techniques is worth the expected age estimation results. Our proposed Feature Extraction technique is explained in details in figure 2.

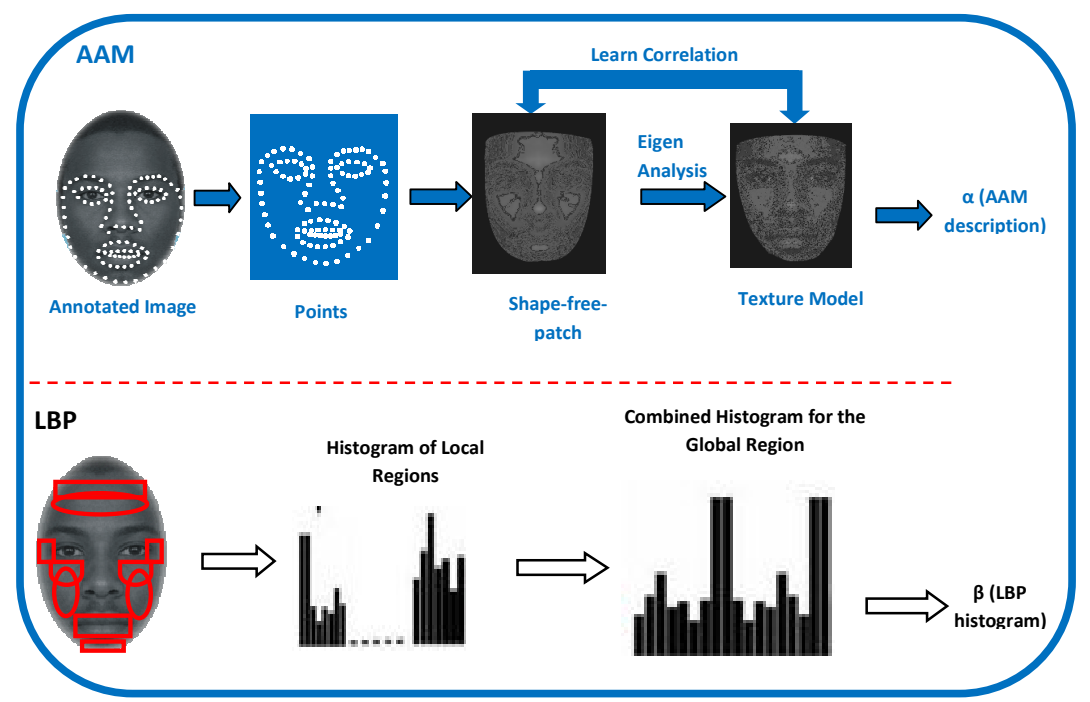

Figure 2: Feature Extraction and Selection using AAM, LBP and LARS

\subsection{Proposed Ranking Model}

Our usage of the ranking approach is based on its intuitive nature in helping to learn the correlation between the ages of images. The faces of two different people at the same age may not look completely similar, however, they possess similar characteristics common to their age; at the same time, the pattern of ageing of different individuals is personalized and thus different across different individuals. For instance three people of age 17 each; are of the same age but might reflect different ageing patterns. Although, they are different individuals, we expect that their different ageing patterns should also reflect certain traits common to their age (e.g. initial appearance of facial hair). Thus, inferences can be made from the correlation between individual ageing patterns along each age for determining the ranks of individuals and these inferences can be used to enhance age estimation. 
We present mathematical formulations for our proposed model below

Definition 1: Given a set of image, $X$ and an outcome space, $Q$ (in this case, age labels) and a set of ranks $\mathrm{R}$, we have the following definitions:

$$
\begin{aligned}
& X=\left(x_{1 j}, x_{2 j}, \ldots, x_{n m} \mid \Lambda j=1,2, \ldots, m\right) \\
& Q=\left\{q_{1 j}, q_{2 j}, \ldots, q_{n m} \mid \Lambda q_{i}>q_{i-1}, j=1,2, \ldots, m\right\} \\
& R=\left\{r_{1 j}, r_{2 j}, \ldots, r_{n m} \mid \Lambda r_{i j}>r_{i-1 j}, j=1,2, \ldots, m ; r \in \mathbb{R}\right\}
\end{aligned}
$$

Definition 2: For the given definition above, we wish to find a space of ranking functions, $H$, a mapping of images to ranks, such that each $h($.) $\epsilon H$ is a function which appropriately ranks $X$.

$H=\{h()$.

$h()=.X \rightarrow R$

We expect $h($.) to construct $R$ such that there is a one-to-one mapping between $Q$ and $R$ and that each rank in $R$ appropriately represents its corresponding age in $Q$. However, the age labels in $Q$ are integers while the ranks are real values in order to capture the variations in ageing patterns along ages (this is further illustrated in the following definitions and equations).

Yang et al. [18], Chang et al. [19] and Cao et al. [20] all used pair-wise ranks of images for comparison with test images. This reduces the substantiality of information available for making inferences about the age to be estimated. Cao et al [20] ranked images using a reference set containing ordinal and consistent pairs (images of different individuals of the same age), however, they abandoned the ageing pattern of individuals, an important factor which facilitated the success of many age estimation algorithms [9],[10],[11],[1]. Although, they provided a variation between the ranks of images of different individuals for each age, the use of images of the same individual in our model allows us to properly represent this variation as a true difference between the ages of individuals.

For our proposed model, we use a subset of the image database as a reference set (with known ranks) and organize it such that individual ageing patterns can be learned along different ages. In organizing our reference set, we maintain sets of images of the same individuals at different ages for learning the ageing pattern of each individual and arrange these sets in a dimension that groups together, images of different individuals at the same ages. Thus we have a matrix of images with the rows corresponding to images of different individuals at the same ages and the columns corresponding to the images of the same individuals at different ages, the images in our reference set are labeled with ground-truth ages so that their age ranks are pre-determined relative to their true ages. Mathematically,

Definition 3: suppose we have a particular individual $x$, with $n$ different images and an arbitrary individual $w$; then for $j$ such individuals and a function age(.) which returns the age of an individual, we define the set $\chi$ as follows;

$\chi=\left\{x_{i, j} \mid \operatorname{age}\left(x_{i, j}\right)>\operatorname{age}\left(x_{i-1, j}\right)\right.$ and $\operatorname{age}\left(x_{i, j}\right)=$

$\left.\operatorname{age}\left(w_{i, j-1}\right), \Lambda \quad i=1,2, . ., n, j=1,2, \ldots, m ; x \neq w\right\}$

For individuals with missing images at certain ages, in order to complement the row of same ages, we fix in images of other individuals belonging to the same age and gender. 
Definition 3: Thus, for a test image $x_{i, j}$ such that age $\left(x_{i, j}\right)$ is greater than an age $k$ (chosen arbitrarily) in $Q, R$ is divided into two subsets,

$R_{l}=\left\{r_{i, j}, q_{i+1, j}, \ldots, q_{k m}\right\}$

$R_{2}=\left\{q_{k+1, j}, q_{k+2, j}, \ldots, q_{n m}\right\}$

Where $R_{l}$ is the set of ageranks less than or equal to the rank of the test image $x_{i, j}$ and $R_{2}$ is the set of ageranks greater than the rank of the test image.

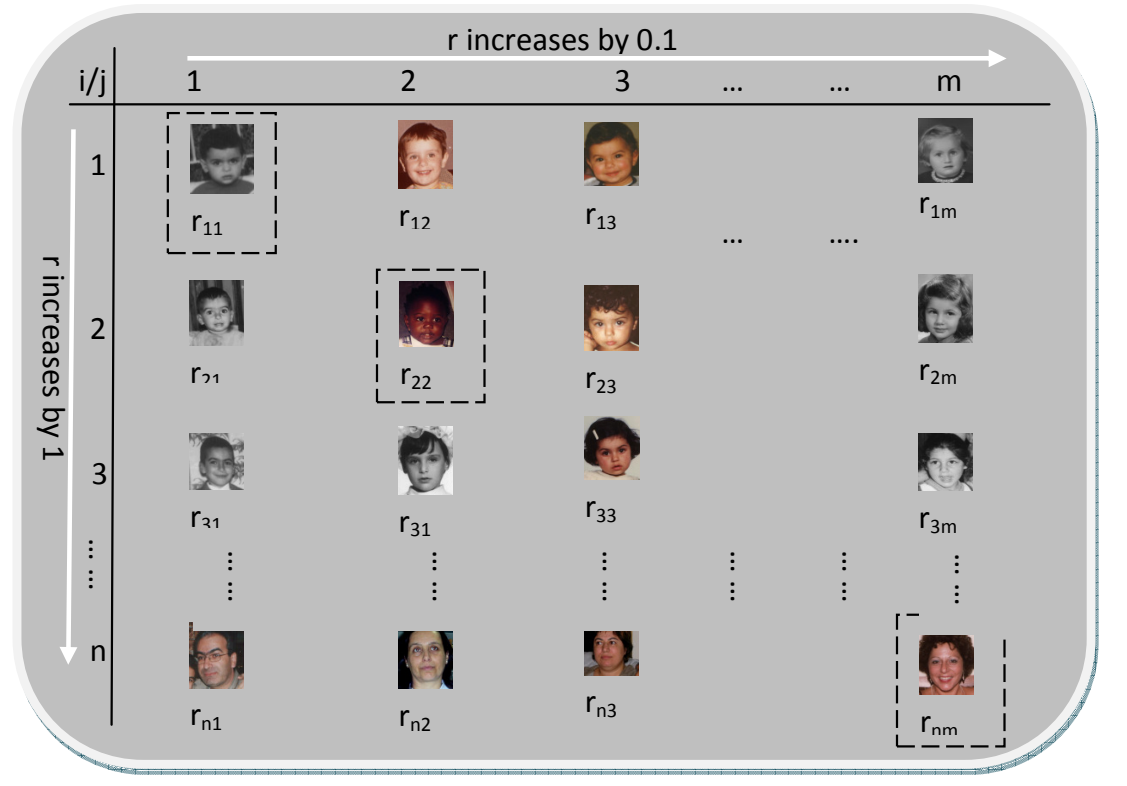

Figure 3: Proposed Correlated Ageing Pattern Ranking Model

Subsequently, the problem is reduced to a binary classification where we only need to compare the rank of $x_{i, j}$ with the ranks of images in either $R_{1}$ or $R_{2}$ and by an iteration of such binary classifications, we are able to further reduce each sub-problem to a smaller one until the appropriate rank of $x_{i, j}$ is found.

Inspired by the work of Li and Lin [27] in stating an equation for calculating the rank of a data set in an ordinal regression problem for the purpose of binary classification, we define our ranking equations. For each test image in $X$, we compare the rank of the image against the ranks in $R$ (comparing against $d$ different individuals in each age, and $d$ is less than the number of images in an age) until the test image is found to be less than or equal to the range of ranks of a particular row (of ages) $i$; we then compare along rows $i-1, i$ and $i+1$ until the rank of the test image satisfactorily falls within a range of ranks in one of these ages. The essence of this kind of comparison is to cater for the relative correlation in between neighbouring ages and to ascertain that an image truly belongs to the age to which it is ranked.

Definition 4: For each image $x_{i j}$, and an arbitrarily chosen $k$, we assume a rank comparison function $f\left(x_{i j}, k\right)$, a threshold $d$ and then calculate its rank as follows:

$$
r_{i j}=1+\sum_{k=1}^{k-1} g\left(\sum_{j=1}^{d} f\left(x_{i j}, k\right)\right)
$$


Where

$f\left(x_{i j}, k\right)=\Phi$ if the rank of $x_{i j}$ is greater than $k$ and 0 otherwise $(\Phi=1 / m)$

$g()=$.1 if the inner summation is equal to $(d \times \Phi) \pm \phi$ otherwise it is equal to 0 . (In our model, we chose $\Phi=0.1$ and $\phi=0.05$ with an assumption that there are 10 images for each age).

Thus, the age ranks of images for each age differs from those of images in neighboring ages by an approximate value of 1 while images within the same age differ in rank by 0.1 . Thus individual ageing patterns are reflected within each age but the ranks of images in each age are still kept within the same range.

As shown in our model in figure 3, images surrounded with dashed lines are substituted for those individuals whose images for that particular age are not present in the dataset used. As mentioned earlier, we used images of the same gender. Each $r_{i j}$ denotes the rank of the labeled image. This approach is superior to the ranking models discussed earlier in that it utilizes the correlation between aging patterns across different individuals alongside the discriminative features within the same age while maintaining relative similarities within the ages. For the purpose of illustration, we have used images from the popular FG-NET [28] database and our locally collected FAGE database in constructing the model shown in figure 3. FG-NET is a database of 1002 images of 82 different individuals with ages ranging from 0-69 years. Due to the wide range of age separated images for each individual in this database, we considered it suitable for use with our proposed model.

\section{CONCLUSION}

The intuitive approach used to construct our ranking model in this paper is based on the fact that, if the rank of facial images can be determined relative to the ages to which they belong, we can provide inferences for estimating exact ages more accurately. Thus, we have embedded in our model, a correlation between individual ageing patterns as well as a relative discrimination between ages. With the use of AAM and LBP for feature extraction, age estimation algorithms will find our proposed model applicable for estimating ages more accurately by using the age estimates of features extracted using both techniques to determine a more exact age estimate for a given image. With the intuition employed in our proposed model, it will be possible to reduce the Mean Absolute Error (MAE) and increase the Cumulative Score (CS) (the two mostly used benchmarks in age estimation) of most age estimation algorithms thus producing age estimation algorithms that compare favourably with (or even performs better than) human prediction and the state-of-the-art algorithms in age estimation.

\section{REFERENCES}

[1] G. Guo and T. S. Huang, "Human Age Estimation Using Bio-inspired Features," in IEEE Conference on Computer Vision and Pattern Recognition, 2009, pp. 112-119.

[2] G.J. Hole and P.A. George, "Factors influencing the accuracy of age estimates of unfamiliar faces". Perception, vol. 24, pp. 1059-1073, 1995.

[3] G. Guo and G. Mu, "Simultaneous dimensionality reduction and human age estimation via kernel partial least squares regression," in IEEE Computer Vision and Pattern Recognition (CVPR), 2011 ..., 2011, pp. 657-664.

[4] G. Guo, Y. Fu, C. R. Dyer and T. S. Huang, "Image-Based Human Age Estimation by Manifold Learning and Locally Adjusted Robust Regression,” vol. 17, no. 7, pp. 1178-1188, 2008.

[5] A. Lanitis, C. Draganova, and C. Christodoulou, "Comparing Different Classifiers for Automatic Age Estimation,” IEEE Trans. Syst. Man, Cybern. Part B Cybern., vol. 34, no. 1, pp. 621-628, 2004. 
[6] Y. H. Kwon and V. Lobo, "Age Classification from Facial Images," Comput. Vis. Image Underst., vol. 74, no. 1, pp. 1-21, 1999.

[7] W. Horng, C. Lee, and C. Chen, "Classification of Age Groups Based on Facial Features," Tamkang J. Sci. Eng., vol. 4, no. 3, pp. 183-192, 2001.

[8] N. Ramanathan and R. Chellappa, "Face Verification across Age Progression Age Progression in Human faces," IEEE Trans. Image Process., vol. 15, no. 11, pp. 3349 - 3361, 2006.

[9] M. R. Gandhi, "A Method for Automatic Synthesis of Aged Human Facial Images," 2004.

[10] X. Geng, Z. Zhou, and K. Smith-miles, "Automatic Age Estimation Based on Facial Aging Patterns," IEEE Trans. Image Process., vol. 29, no. 12, pp. 2234-2240, 2007.

[11] M. Tsai, Y. Liao, and I. Lin, "Human face aging with guided prediction and detail synthesis," Multimed. Tools Appl., 2013.

[12] Y. Tang and B. Lu, "Age Classification Combining Contour and Texture Feature," 2010.

[13] S. Agrawal, R. Raja, and S. Agrawal, "Support Vector Machine for age classification," Int. J. Emerg. Technol. Adv. Eng., vol. 2, no. 5, pp. 128-131, 2012.

[14] A. Lanitis, C. Draganova, and C. Christodoulou, "Comparing Different Classifiers for Automatic Age Estimation,” IEEE Trans. Syst. Man, Cybern. Part B Cybern., vol. 34, no. 1, pp. 621-628, 2004.

[15] K. Ricanek, Y. Wang, C. Chen, and S. J. Simmons, "Generalized Multi-Ethnic Face Age-Estimation," in IEEE BTAS, 2009.

[16] Y. Fu and T. S. Huang, "Human Age Estimation With Regression on Discriminative Aging Manifold," IEEE Trans. Multimed., vol. 10, no. 4, pp. 578-584, 2008.

[17] G. Guo, Y. Fu, C. R. Dyer, and T. S. Huang, "Image-Based Human Age Estimation by Manifold Learning and Locally Adjusted Robust Regression,” IEEE Trans. Image Process., vol. 17, no. 7, pp. $1178-1188,2008$.

[18] P. Yang, L. Zhong, and D. Metaxas, "Ranking Model for Facial Age Estimation," in International Conference on Pattern Recognition, 2010, pp. 3408-3411.

[19] K. Chang, C. Chen, and Y. Hung, "A RANKING APPROACH FOR HUMAN AGE ESTIMATION BASED ON FACE," in International Conference on Pattern Recognition, 2010.

[20] D. Cao, Z. Lei, Z. Zhang, J. Feng, and S. Z. Li, "Human Age Estimation Using Ranking SVM,” Lect. Notes Comput. Sci., pp. 324-331, 2012.

[21] Y. Freund, R. Iyer, R. E. Schapire, and Y. Singer, "An efficient boosting algorithm for combining preferences," J. Mach. Learn. Res., vol.4, pp. 933-969, 2003.

[22] Matti Pietikäinen. (2010). "Local Binary Patterns". Machine Vision Group, Department of Electrical and Information Engineering, University of Oulu, Finland. [Online] Available: http://www.scholarpedia.org/articles/Local_Binary_Patterns. Accessed February, 2013.

[23] Z. Yang and H. Ai, "Demographic Classification with Local Binary Patterns," in International Conference on Biometrics, 2007, pp. 464-473.

[24] C. Shan, S. Gong, and P. W. Mcowan, "Facial expression recognition based on Local Binary Patterns : A comprehensive study,” Image Vis. Comput., vol. 27, no. 6, pp. 803-816, 2009.

[25] T. F. Cootes, G. J. Edwards, and C. J. Taylor, "Active Appearance Models," IEEE Trans. Pattern Anal. Mach. Intell., vol. 23, no. 6, pp. 681-685, 2001.

[26] T.F. Cootes, C.J. Taylor, D. Cooper, and J. Graham, "Active Shape Models - their training and application," Computer Vision and Image Understanding, vol. 61, no. 1, pp. 38-59, 1995.

[27] L. Li and H. Lin, "Ordinal Regression by Extended Binary Classification," Advaces Neural Inf. Process. Syst., vol. 19, pp. 865-872, 2007.

[28] FG-NET Ageing Database. (2013). [Online] Available: http://sting.cycollege.ac.cy/ alanitis/ fgnetaging/index.htm. Accessed June, 2013 


\section{Authors}

Onifade, Olufade Falade Williams holds a Ph.D. in Computer Science from Nancy 2 University, France. He is a seasoned academic and researcher per excellence who currently lectures at the University of Ibadan, Nigeria. His research interests include Information Retrieval, Fuzzy Learning, Biometrics, Image Processing and Computer Vision.

Akinyemi, Joseph Damilola holds a B.Sc. in Computer Science from University of Ilorin and is currently a Second Year M.Sc. student at the department of Computer Science, University of Ibadan. His research interests include Computer Vision, Image Processing and Pattern Recognition.
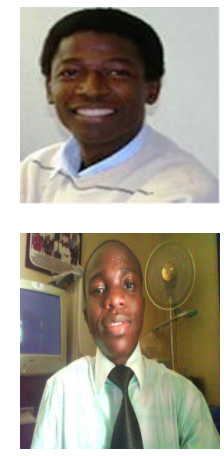


\section{AUTHOR INDEX}

Abdul Azim Abd. Ghani 115

Ahmed Sahloul 227

Akinyemi J.D 477

Alghanmi Ali Omar 153

Ali Mehdi 317

Amir Konigsberg 409

Amit Shah 353

Andrew Stranieri 297

Andy M. Connor 13, 353

Ashraf A. Shahin 53

Aymen Achouri 123

Ayumi Kawai 169

Azzoune Hamid 469

Bala Srinivasan 139

Brian Regan 245

ChangmingSun 245

Cheng Suen 227

ChongGun Kim 153

Craig M. Gelowitz 27

Eric Hitimana 255

Feifei Li 449

Frank Moisiadis 365

Guangdou Liu 391

Hamdi Yalin Yaliç 275

Harsh Jain 307

Hazlina Haron 115

Heba Kurdi 187

Hiba Shahid 203

Hitoshi Kanoh 379

Ittipong Khemapech 99

Ivo Bukovsky 327, 341

Jacqui Finlay 13

Jan Kalivoda 327

Jean-Christophe Le Lann 427

Jefferson Tan 139

Jiunn-Tsair Fang 215

Julien Ugon 297

Junichi Ochiai 379

Keshav Mathur 307

Keun Ho Ryu 449

Khalil Shihab 397

Kwan Hee Han 175

Luigi Benedicenti 27

Manish Mittal 297

Matouš Cejnek 327, 341

Meijing Li 449

Melih Kirlidog 65

Minghao Piao 449

Minoru Sasaki 463

Mohammad A. U. Khan 203
Mohammed Golam Kaosar 77

Mohammed Saeed Al-kahtani 01

Muhammad Asif Jan 421

Nada Alfaifi 187

Nader Khammassi 427

Narmada Naik 441

Nasser Tairan 421

Nida Al-Chalabi 397

Noureddine Bouhmala 37

Nuseiba M. Altarawneh 245

Onifade O.F.W 477

Orhio Mark Creado 139

Oubong Gwun 255

Pao-Chi Chang 215

Peter Mark Beneš 327, 341

Phu Dung Le 139

Pradnya Kulkarni 297

Raman Paranjape 27

Rashida Adeeb Khanum 421

Rathna.G.N 441

Ron Asherov 409

Rougang Zhou 391

Russel Pears 13

Sara Alkhaider 187

Sean McGerty 365

Shankarayya G. Kambalimath 267

Sharol Sibongile Mkhomazi 85

Shinichi Goto 283

Siddhivinayak Kulkarni 297

Sokyna Al-Qatawneh 317

SuhuaiLuo 245

Tad Gonsalves 169

Tariq M. Khan 203

Tebbi Hanane 469

Terumasa Aoki 283

Thamer Al Rawashdeh 317

Tiko Iyamu 85

Tsai-Ling Liao 215

Wadee Al-Halabi 203

Xiao Tu 391

Yinan Kong 203

Yongjun Piao 449

Yongsun Choi 175

Yunfei Zhou 391

Zong-Yi Chen 215 